\title{
Experimental Investigation of Catalytic Soot Oxidation and Pressure Drop Characteristics in Wall-Flow Diesel Particulate Filters
}

\author{
THESIS \\ submitted in partial fulfillment of the requirements \\ for the degree of Doctor of Philosophy in Mechanical Engineering \\ of the Department of Mechanical and Industrial Engineering \\ University of Thessaly
}

\author{
By \\ GEORGIOS A. STRATAKIS \\ Dipl. Mechanical Engineer, \\ University of Thessaly \\ Born on $25^{\text {th }}$ Feb 1974 \\ in Chania, Crete
}

Advisory Committee:

Assoc. Prof. A. M. Stamatelos, supervisor

Prof. V. Bontozoglou

Prof. N. Vlachos

Volos, January 2004 


\section{Contents}

$1 \quad$ INTRODUCTION 15

$\begin{array}{lll}1.1 & \text { General } & 15\end{array}$

$\begin{array}{lll}1.2 & \text { Diesel Engine Emissions } & 15\end{array}$

$\begin{array}{lll}1.2 .1 & \text { Diesel particulate matter } & 16\end{array}$

$\begin{array}{lll}1.3 & \text { Diesel Emissions Reduction Legislation } & 19\end{array}$

$1.4 \quad$ Diesel Exhaust Gas Treatment Technologies $\quad 21$

$\begin{array}{lll}1.4 .1 & \text { Diesel catalysts } & 21\end{array}$

1.4.2 The wall-flow particulate filter 24

1.4.3 DPF regeneration systems based on catalysts 26

1.5 Scope and Objectives of this Thesis 33

$2 \quad$ PHYSICAL \& CHEMICAL PROCESSES INSIDE THE FILTER 36

$2.1 \quad$ Loading 36

$\begin{array}{lll}2.2 & \text { Regeneration } & 37\end{array}$

2.2.1 Thermal regeneration 37

2.2.2 Fuel doped catalytic regeneration 38

2.2.3 Modeling catalytic regeneration 39

2.3 Exhaust Gas Flow Inside Filter Channel 46

2.3.1 General 46

2.3.2 The problem of soot maldistribution in the determination of pressure drop 47

2.4 The Role of VOF $\quad 50$

2.4.1 Adsorption-desorption phenomena 50

$\begin{array}{lll}2.4 .2 & \text { Erratic regeneration behaviour } & 51\end{array}$

3 EXPERIMENTAL PART I: CATALYTIC REGENERATION KINETICS 53

3.1 Thermogravimetric Analysis of Soot - VOF Oxidation 53

3.1.1 Experimental 54

3.1.2 Results and discussion 57

$\begin{array}{lll}\text { 3.1.3 Concluding remarks } & 65\end{array}$

3.2 Experimental Investigation of the Role of Soot VOF 66

3.2.1 Experimental 66

3.2.2 Results and discussion 69

$\begin{array}{ll}\text { 3.2.3 Concluding remarks } & 76\end{array}$

$\begin{array}{lll}\text { 3.3 Modes of Catalytic Regeneration in Diesel Filters } & 77\end{array}$

$\begin{array}{lll}\text { 3.3.1 Experimental } & 77\end{array}$

$\begin{array}{lll}\text { 3.3.2 Experimental results and discussion } & 79\end{array}$ 
3.4 Experimental Validation of the 1D Model $\quad 83$

3.4.1 Design of the validation experiments 83

3.4.2 1D model validation results $\quad 85$

3.4.3 Discussion 90

$\begin{array}{lll}\text { 3.4.4 Concluding remarks } & 97\end{array}$

$3.5 \quad 3 D$ ANSYS - CATWALL Model Validation $\quad 98$

3.5.1 3D model validation results and discussion 99

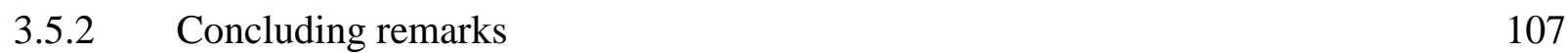

\section{EXPERIMENTAL PART II: PRESSURE DROP, FLOW AND SOOT} DISTRIBUTION

4.1 Experimental Investigation of the Pressure Drop Behavior of Porous Ceramic Filters.

4.1.1 Experimental 109

4.1.2 Computational assistance of experiments 115

4.1.3 Computer calculation procedure 116

4.1.4 Results and discussion $\quad 118$

4.1.5 Concluding remarks 125

4.2 Flow Distribution Effects During Loading and Catalytic Regeneration (Pitot Tubes Experiments) $\quad 126$

4.2.1 Experimental 126

4.2.2 Results and discussion 129

4.2.3 Concluding remarks 136

4.3 Cold Flow Distribution Experiments in a Wall Flow Diesel Particulate Filter 137

$\begin{array}{lll}\text { 4.3.1 Experimental } & 137\end{array}$

4.3.2 Results and discussion 140

4.3.3 Concluding remarks 151

4.4 Soot Deposition Characteristics as Function of Engine Operation Point and Filter Loaded History.

4.4.1 Experimental 152

4.4.2 Results and discussion 153

4.4.3 Concluding remarks 159

5 COMPARATIVE DISCUSSION 160

5.1 Reaction Scheme and Kinetics of the Catalytic Regeneration Process 161

5.2 The Role of the Volatile Organic Fraction of the Particulate. 162

5.3 3D Effects During Filter Loading 162

5.4 3D Effects During Catalytic Regeneration 162

5.5 Correlation Between Pressure Drop and Accumulated Soot Mass. 162 
6 CONCLUSIONS AND FUTURE OUTLOOK

$7 \quad$ APPENDIX: EXPERIMENTAL 165

$\begin{array}{lll}7.1 & \text { Equipment } & 165\end{array}$

$\begin{array}{lll}7.2 & \text { Materials } & 175\end{array}$

$\begin{array}{lll}7.3 & \text { Experimental Devices } & 176\end{array}$

\section{REFERENCES}

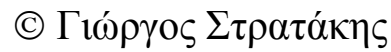

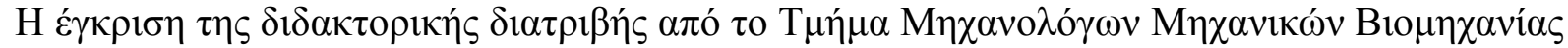

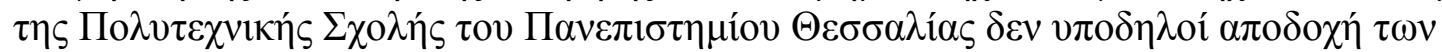

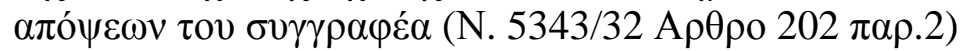




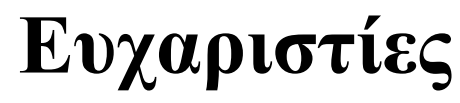

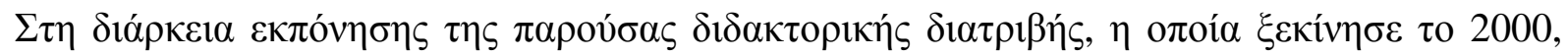

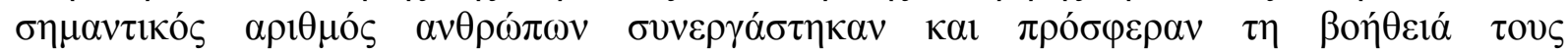

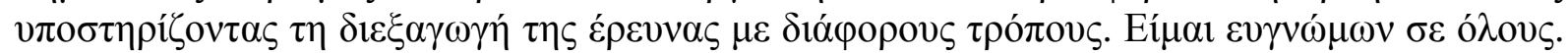

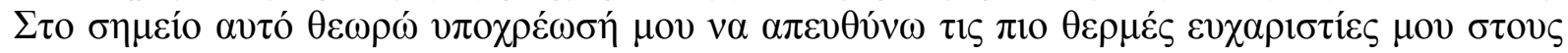
$\pi \alpha \rho \alpha \kappa \alpha ́ \tau \omega:$

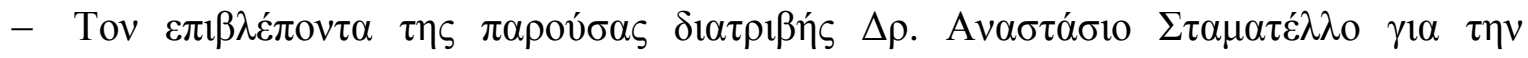

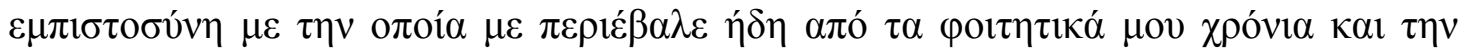

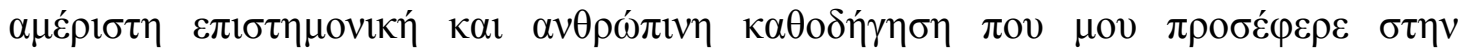

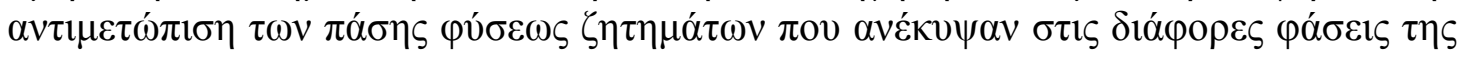

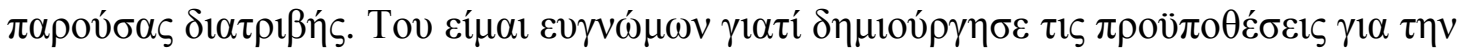

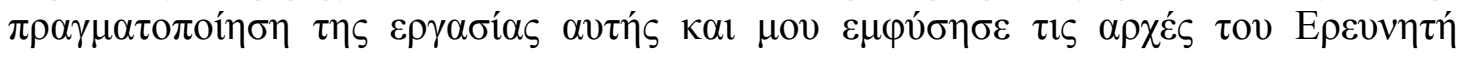

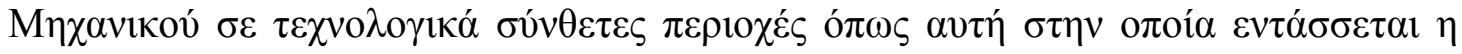

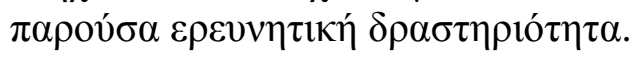

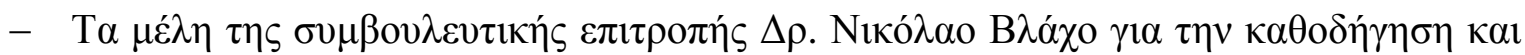

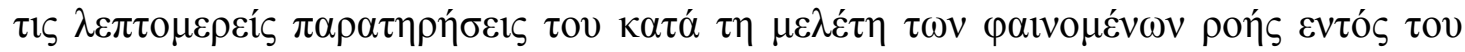

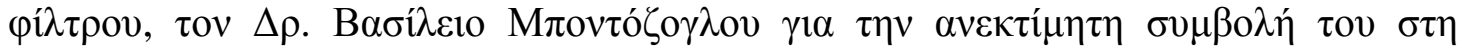

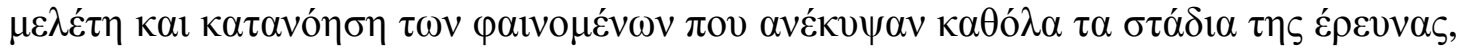

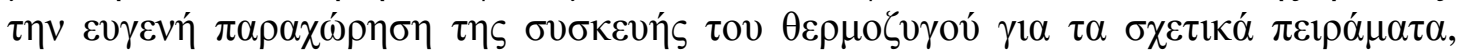

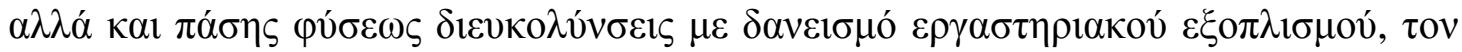

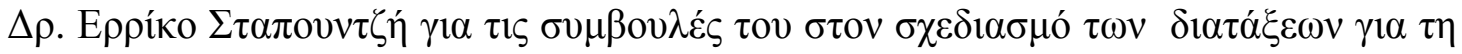

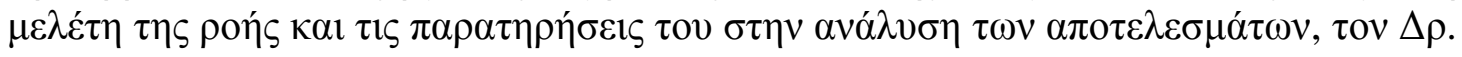

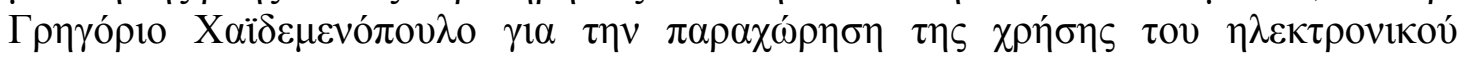

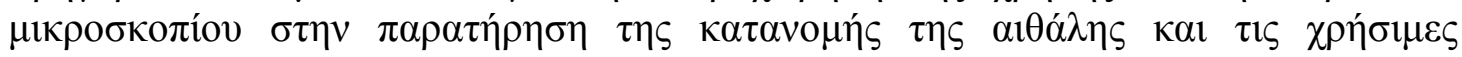

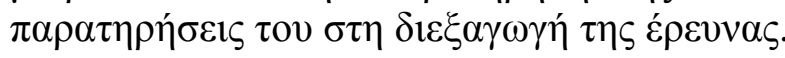

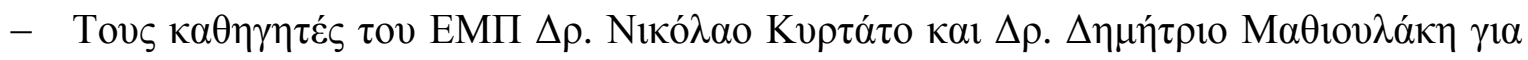

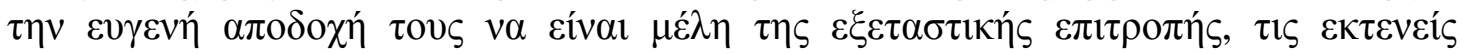

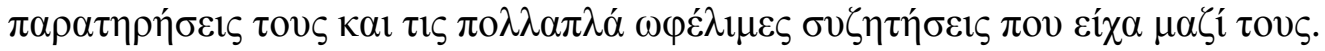

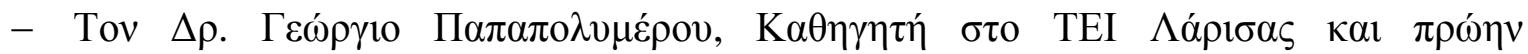

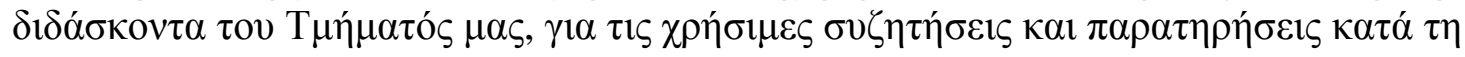

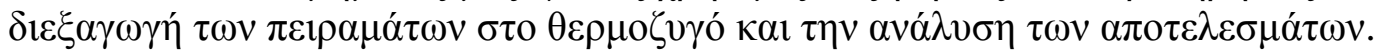

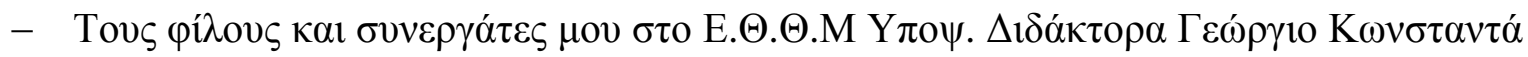

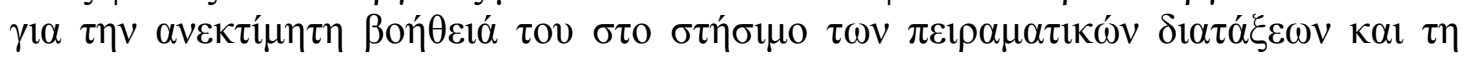

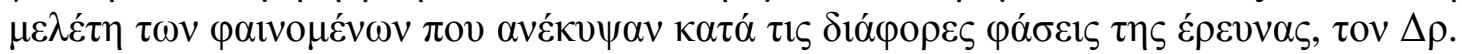

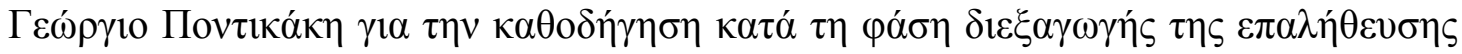

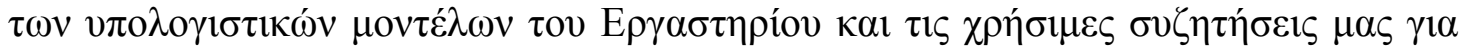

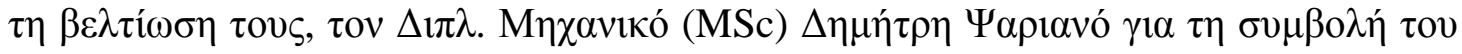

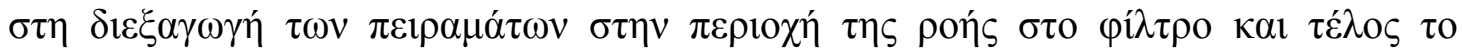

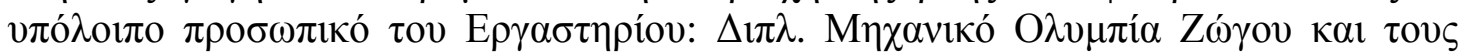

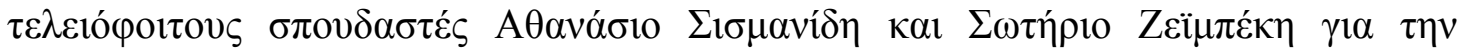

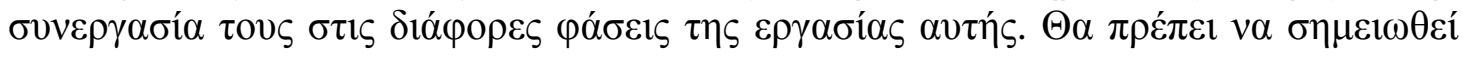

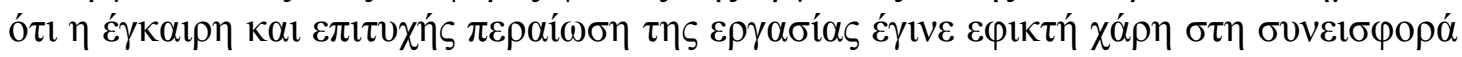

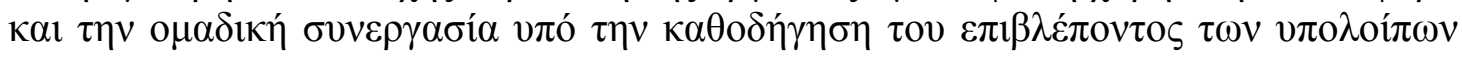

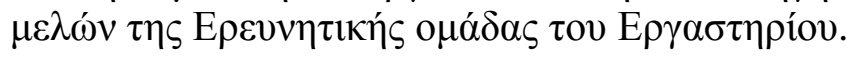




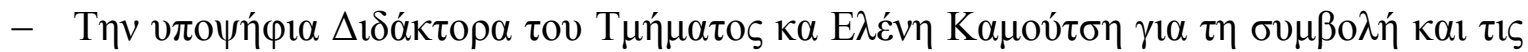

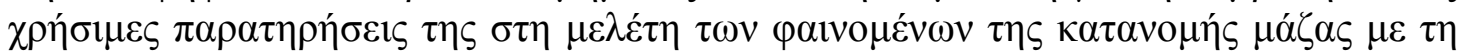

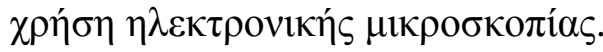

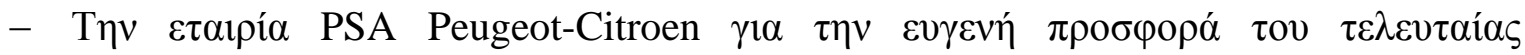

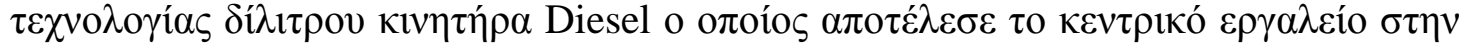

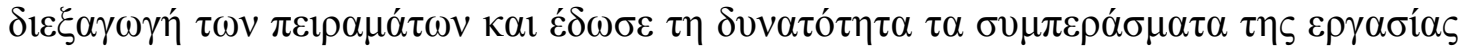

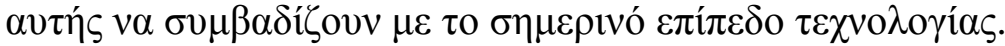

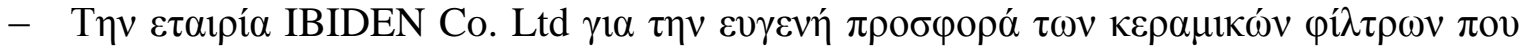

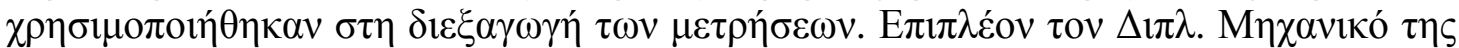

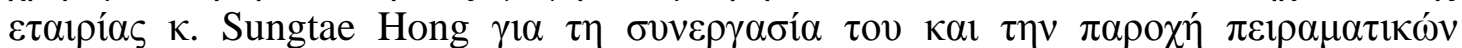

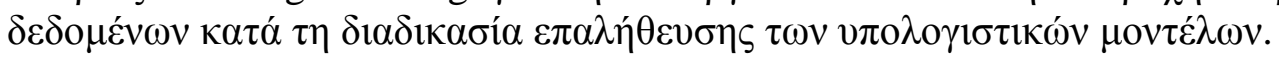

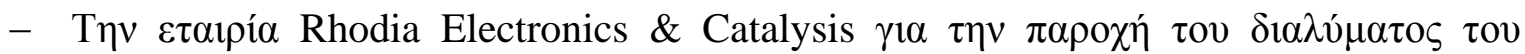

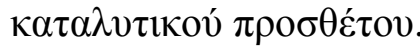

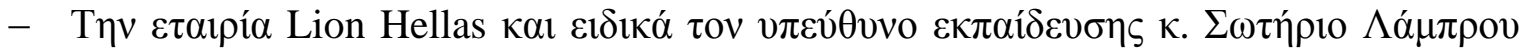

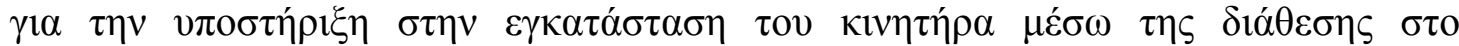

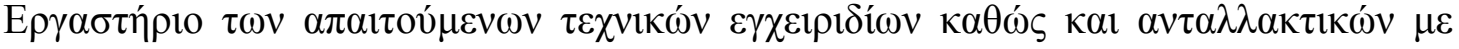

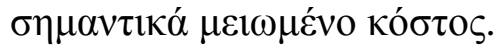

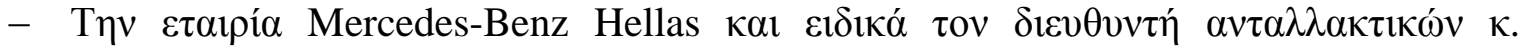

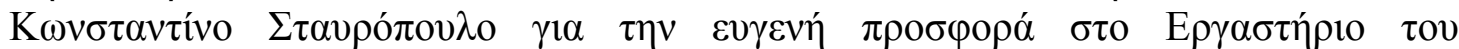

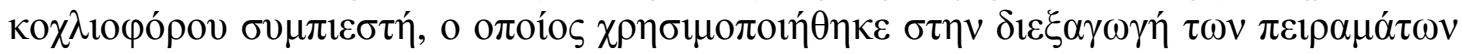

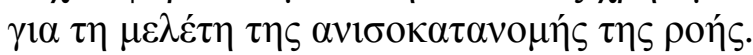

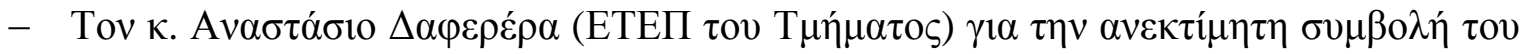

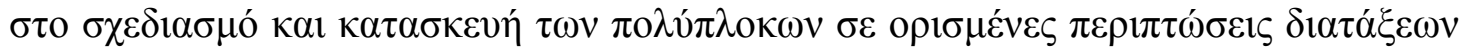

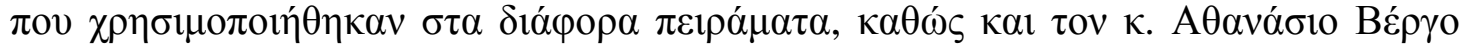

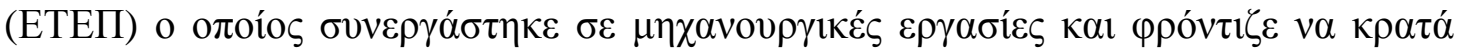

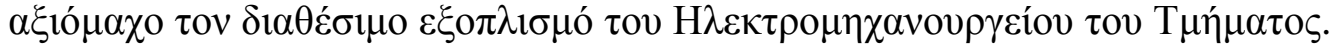




\section{Acknowledgements}

Whilst undertaking my $\mathrm{PhD}$, a variety of people helped and supported me in various ways. In this section, I would like to acknowledge those mentioned below and express my gratitude to them.

My supervisor, Dr. Anastassios Stamatelos, for his confidence from the first years of my undergraduate studies and his invaluable support in all phases of this work. Through these years, he guided me, contributed in every bit of this work and offered his thoughtful advice and knowledge in the field of Mechanical Engineering. I am grateful to him because he generated the conditions for the completion of this work and inspired me the values of the Research Engineer in this complex area of Engineering.

The members of the supervising committee Dr. Nikolaos Vlachos for his guidance during the study of the flow phenomena inside the filter, Dr. Vasilios Bontozoglou for his contribution to the study and understanding of various fundamental issues that came up during the various phases of this work and the kind supply of the thermobalance equipment for the TGA tests and other laboratory equipment, Dr. Errikos Stapountzis for his advice to the design of the experimental devices for the study of the flow phenomena and the profitable discussions during the analysis of the results, Dr. Gregory Haidemenopoulos for the kind supply of the SEM for the study of the soot distribution inside the channel and his useful advice during the research.

Dr. Nikolaos Kyrtatos of the Naval Architecture and Marine Engineering Faculty, NTUA, and Dr. Dimitrios Mathioulakis, of the Mechanical Engineering Faculty, NTUA, for their valuable advice and remarks.

Dr. Georgios Papapolymerou, Technological Education Institute of Larissa, for his valuable advice and rewarding discussions during the experimental work performed on the thermobalance equipment and the analysis of the results.

My colleagues in the Lab, Dipl.-Ing. George Konstantas for his invaluable help to the preparation of the experimental devices and the useful discussions during the study of the phenomena. Dr. Georgios Pontikakis for his important feedback in the validation of the CATWALL DPF code and the dynamic discussions for the capitalization of the experimental results in the improved models, the Dipl-Ing. Dimitri Psarianos, MSc, for his contribution to the experimental study of pressure drop and soot mass distribution inside the filter, and finally the other members of the Laboratory, Dipl-Ing. Olympia Zogou, Athanasios Sismanidis and Sotirios Zeimbekis, for the smooth collaboration during the various phases of this work. The $\mathrm{PhD}$ candidate of the Mechanical Engineering Department Ms Eleni Kamoutsi for her contribution and the productive cooperation during the study of the soot distribution phenomena inside the filter channels with the use of electronic microscopy. Ing. Anastassios Dafereras, support staff of the Department, for his invaluable contribution during the design and construction of the electronic parts of experimental devices employed in this work and Mr. Athanassios Vergos for the smooth cooperation and support during the various technical works and manufacturing operations performed in the Machine Shop of the Dept.

The Companies PSA Peugeot-Citroen, for the supply to the Lab of a modern HDI Diesel engine, Ibiden Co Ltd for the supply of Diesel filters and Rhodia Electronics \& Catalysis for the supply of fuel additives employed in the loading and regeneration experiments. 


\section{Abstract}

In Europe and other regions where Diesel vehicles are common, the development of clean diesel systems that reduce $\mathrm{CO}_{2}$ levels in the atmosphere is attracting considerable attention. However, the diesel exhaust aftertreatment presents significant challenges to the automotive manufacturers, due to the difficulty to oxidize diesel particulate and reduce NOx. The diesel filter is a workable device that can be employed to reduce particulate emissions about one order of magnitude. However, although its first, unsuccessful, pilot series application by a big European manufacturer in California dates back to 1985, it was necessary to continue development for about 20 years until a workable model equipped with a diesel filter system was series produced by another European manufacturer starting 2001. This system exploited the additional potential of the modern diesel powertrain, with a common rail, high-pressure injection system, to attain the required exhaust temperature levels for filter regeneration. The assistance of the process by a catalyst seems to be unavoidable, and this first system employed the catalyst as a fuel additive. Based on the extensive vehicular experience with this pioneering system (more than 300,000 cars produced), which is now being followed by other diesel vehicle manufacturers, it came out that our understanding of the process is not to the level required for the design of reliable and durable systems. This $\mathrm{PhD}$ thesis examines certain aspects of diesel filter regeneration by use of fuel additives that proved to need further investigation.

These include the reaction scheme and kinetics parameters of the catalytic regeneration, the role of the Volatile Organic Fraction (VOF) of the particulate, the study of soot and exhaust flow maldistribution in the filter and the study of pressure drop parameters as the permeability of the ceramic wall and the permeability - density of the particulate layer. All these studies were based on specially designed experiments, on the engine bench and on purpose designed test rigs.

The experimental results of this study confirmed the complexity of the process. As regards the reaction scheme, the range of catalytic activity of Ceria with regard to the soot and VOF oxidation, as well as the Ceria re-oxidation by exhaust gas oxygen was determined, along with the respective apparent kinetics parameters (activation energy values). As regards the VOF, the VOF content of soot produced by the specific engine was mapped in the operation range of the engine. Also, the role of VOF in the low-temperature regeneration process was demonstrated and explained. Early in this study, it was found that the exhaust gas flow distribution between the filter channels was interacting with the soot loading distribution and VOF adsorption - desorption. For this reason, specific series of experiments were designed and conducted. The results demonstrated an unstable behaviour of soot maldistribution during loading and regeneration. This behaviour was found responsible for a major part of the significant discrepancies observed between 1D and 2D (axisymmetric) models and experimental findings by numerous researchers. Moreover, the above-mentioned maldistribution behaviour prohibits any valid correlation between pressure drop across the filter and soot loading mass. This is a problem for the control system of the engine exhaust treatment, because the filter pressure drop signal was traditionally an essential sensor input signal for the engine management. In addition to the soot maldistribution between center and periphery of the filter, it was found that the soot layer thickness in a single channel could also present a certain degree of inhomogeneity. Another major objective of this study was to support improved modeling of the process. This was partly succeeded as one may deduce from the extensive validation results of $1 \mathrm{D}$ and 3D codes, which are now routinely being applied as engineering design tools. 


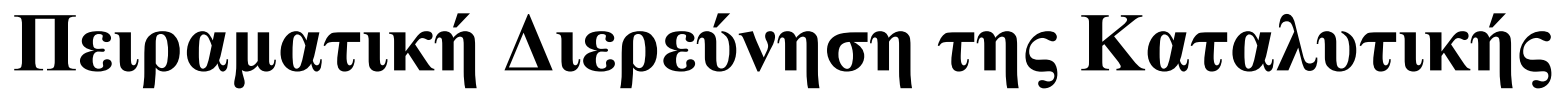 O $\xi \varepsilon i ́ \delta \omega \sigma \eta \varsigma \Sigma \omega \mu \alpha \tau \iota \delta i ́ \omega v$ Kal $\tau \omega v$

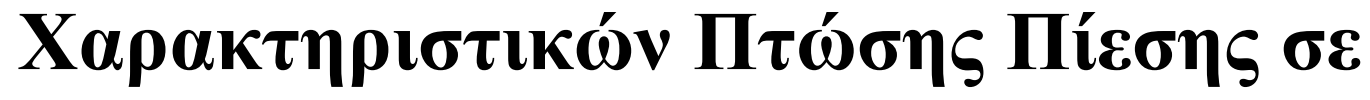

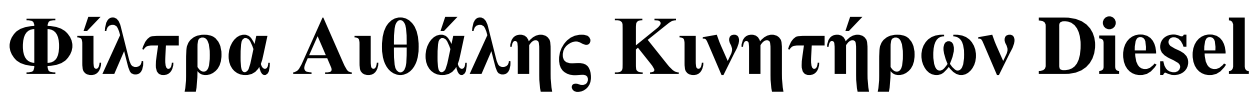

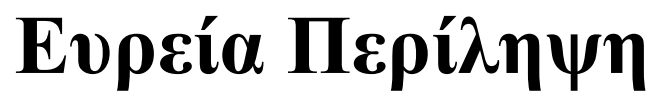

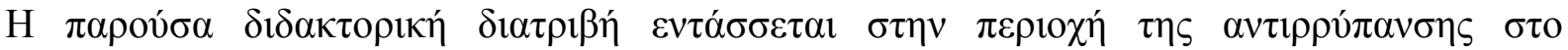

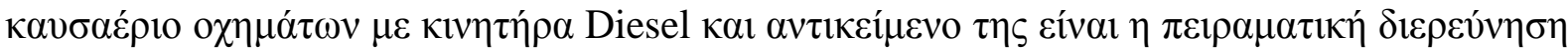

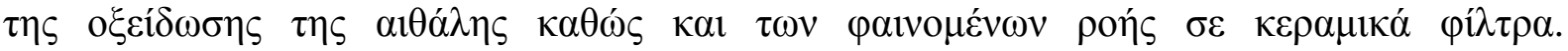

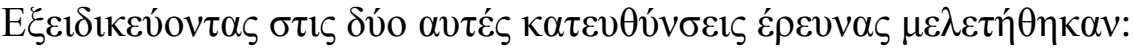

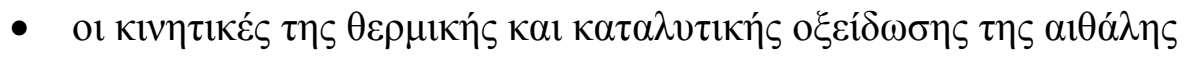

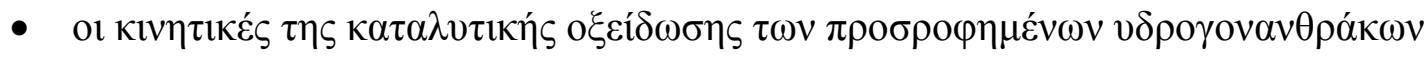

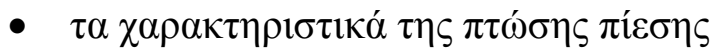

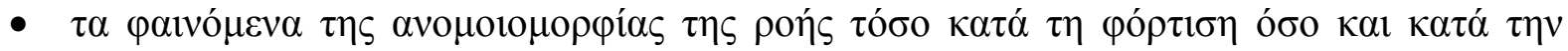
$\alpha \nu \alpha \gamma \varepsilon \dot{\varepsilon} v \eta \eta \sigma \eta$

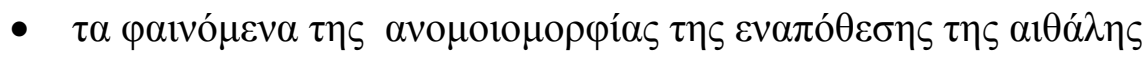

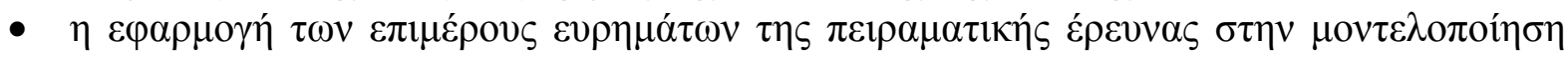

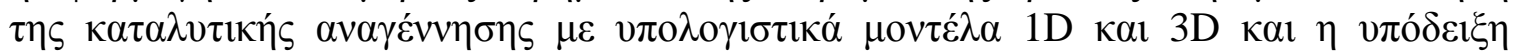

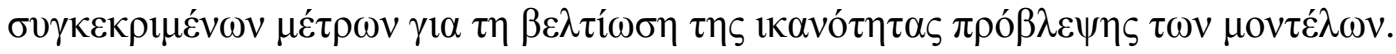

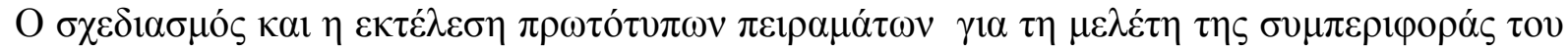

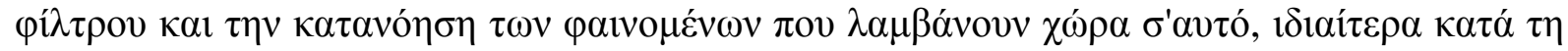

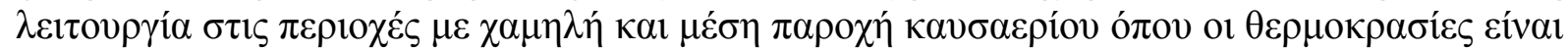

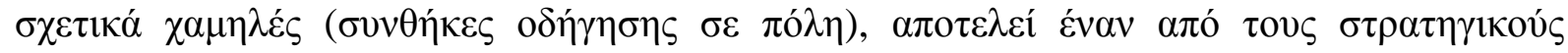

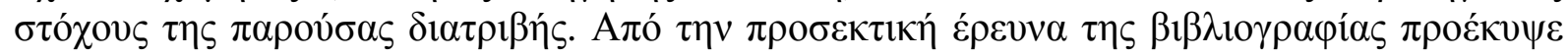

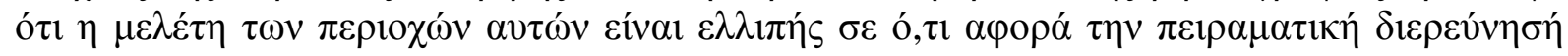

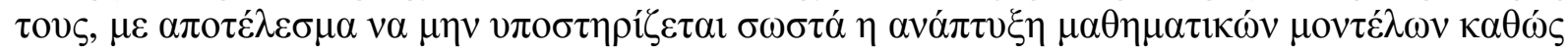

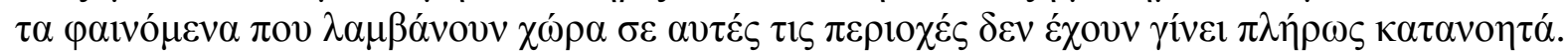

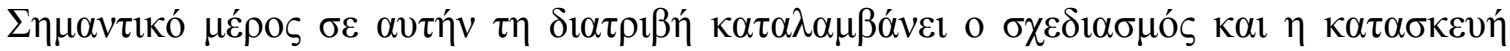

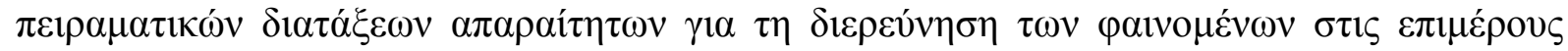

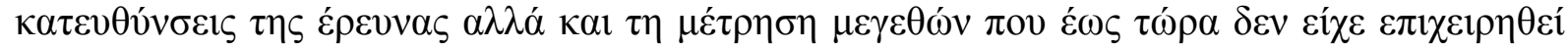

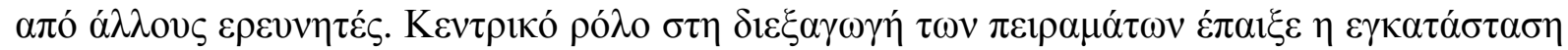

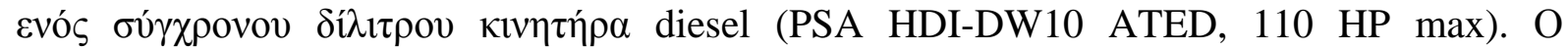

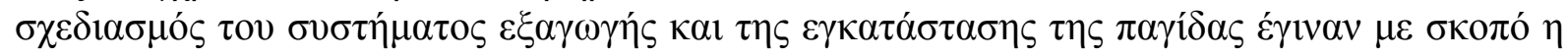

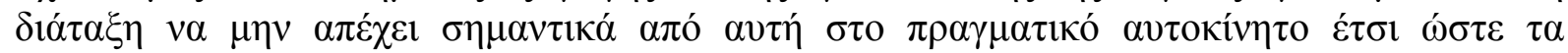

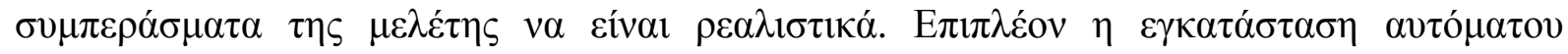

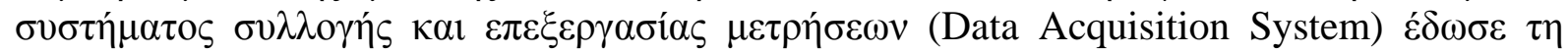

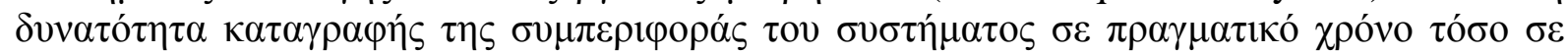

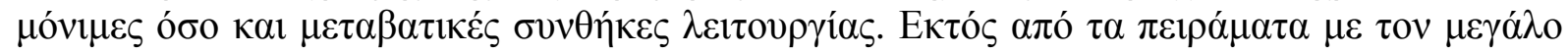




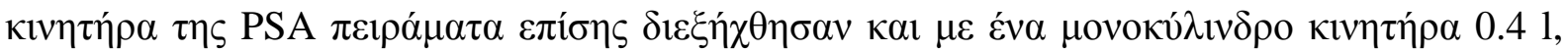

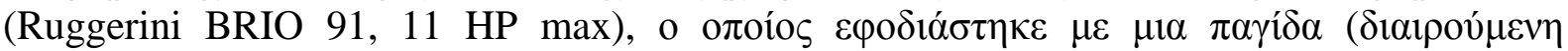

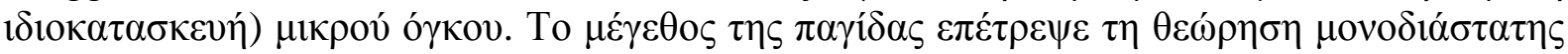

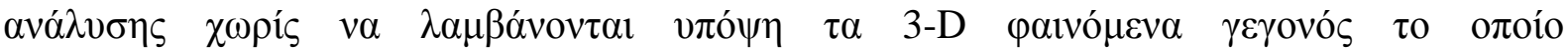

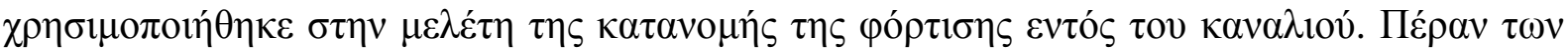

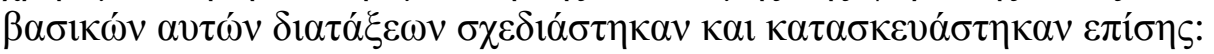

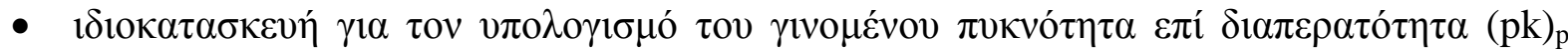

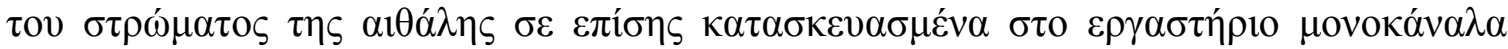

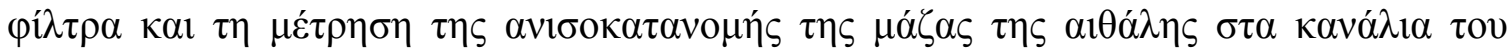
$\pi \lambda \eta \dot{\rho o v \varsigma} \varphi \hat{i} \lambda \tau \rho o v$.

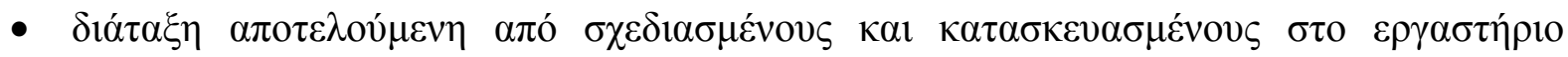

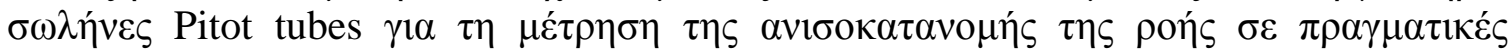

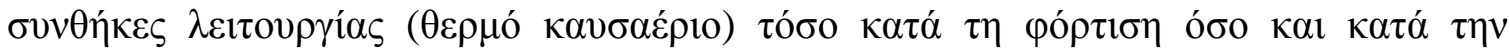
$\alpha \vee \alpha \gamma \varepsilon ́ v v \eta \sigma \eta$.

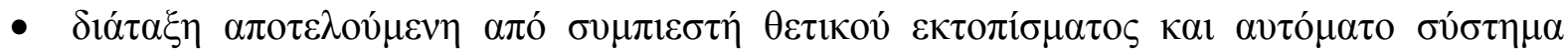

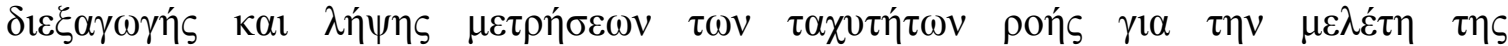

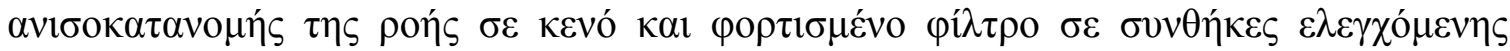
$\pi \alpha \rho \circ \chi \eta ́ s$.

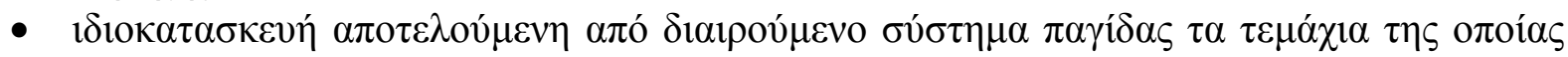

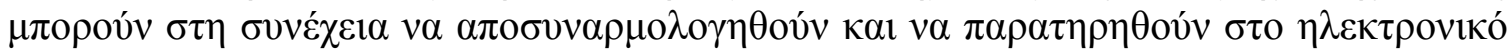

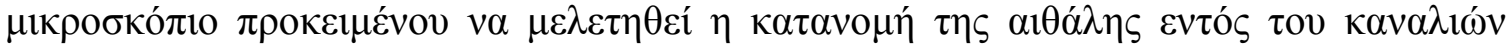

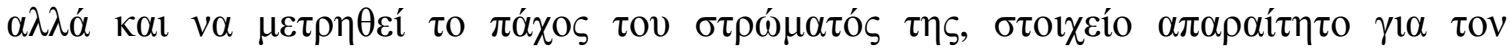

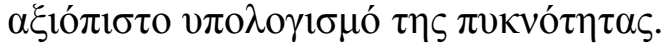

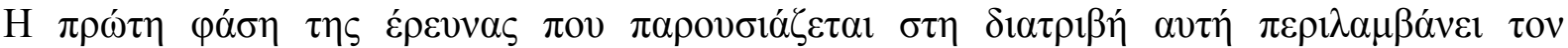

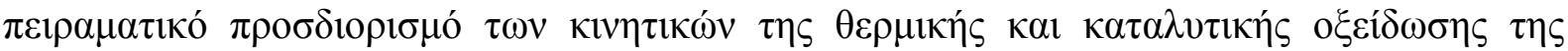

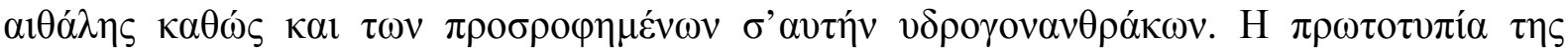

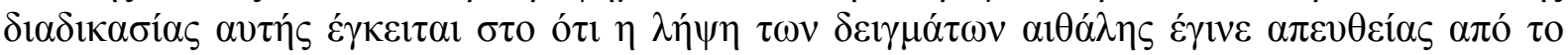

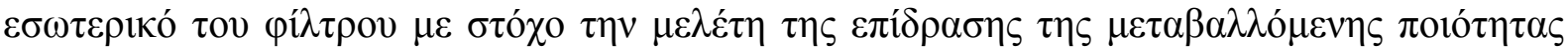

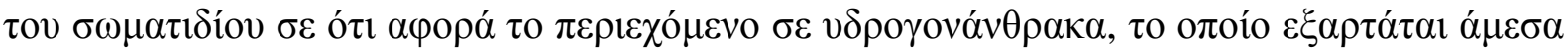

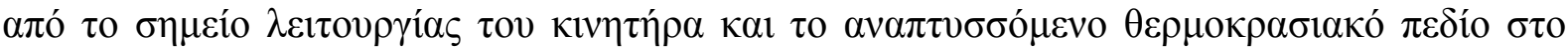

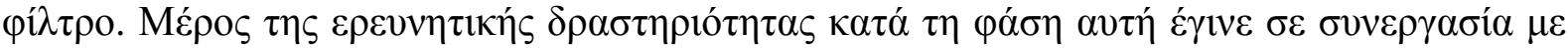

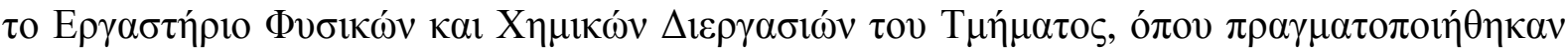

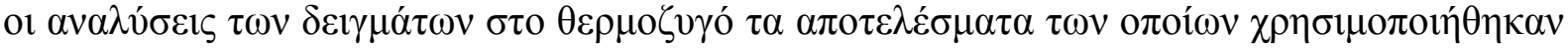

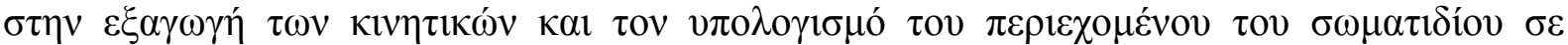

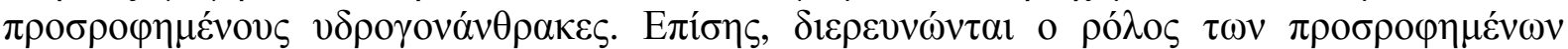

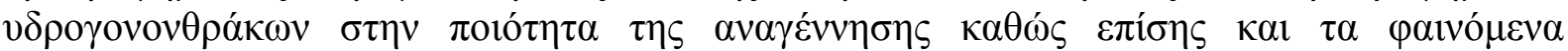

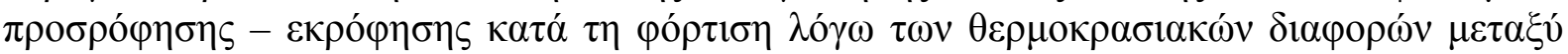

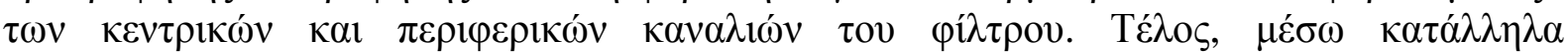

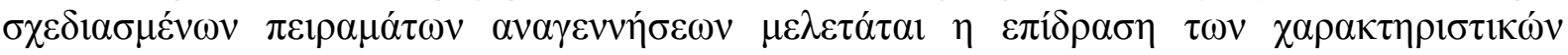

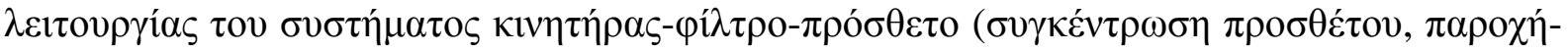

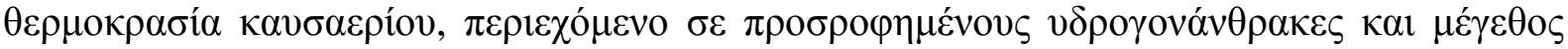

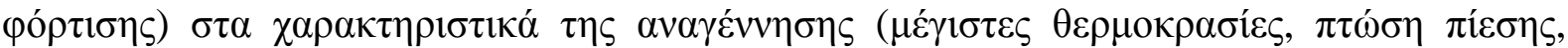

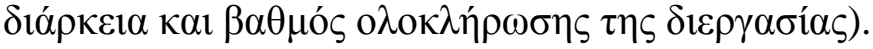

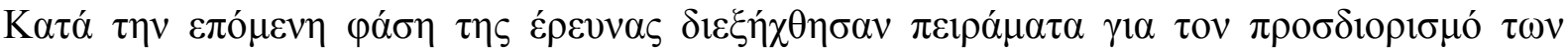

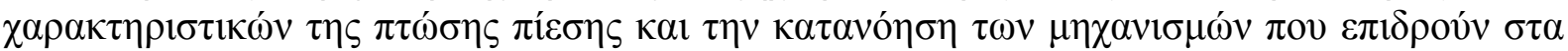

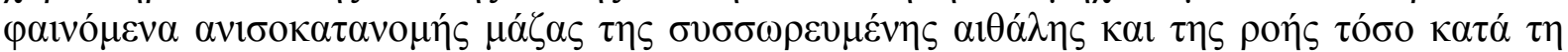

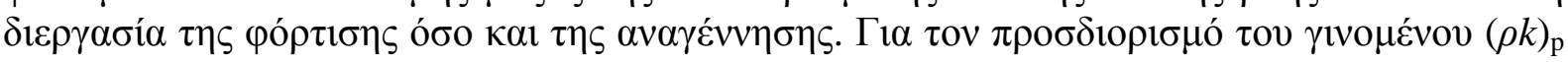




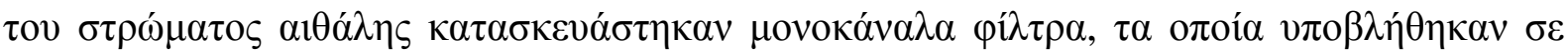

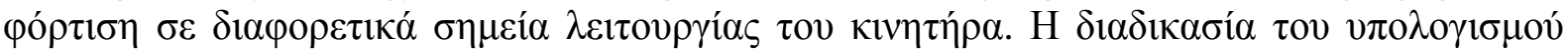

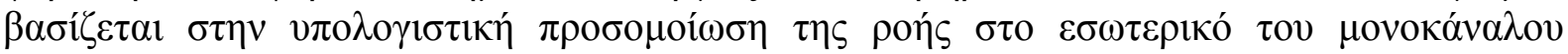

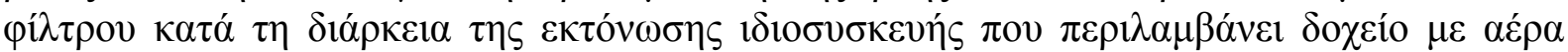

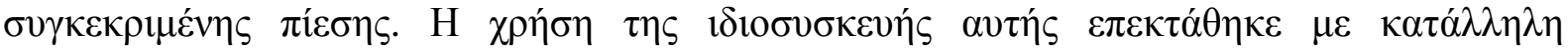

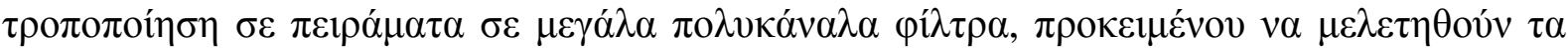

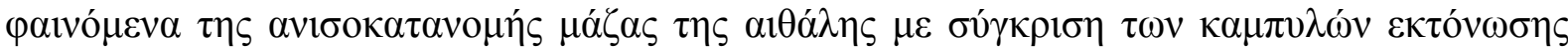

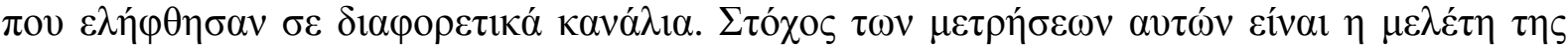

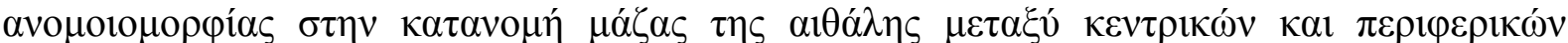

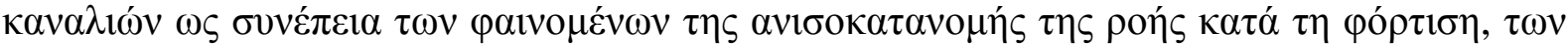

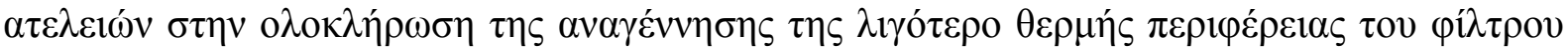

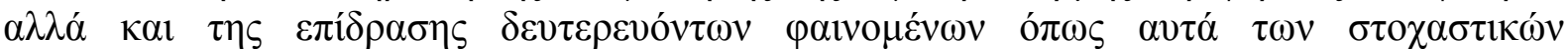

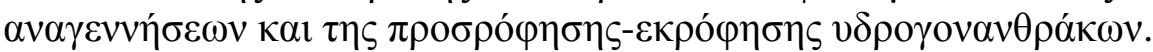

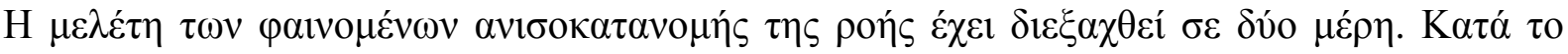

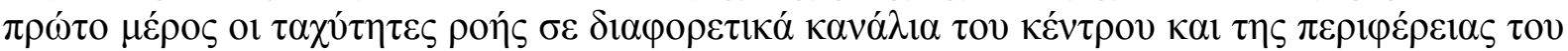

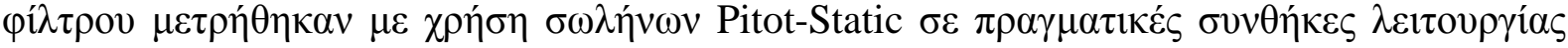

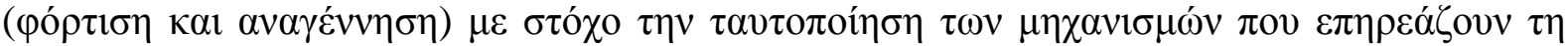

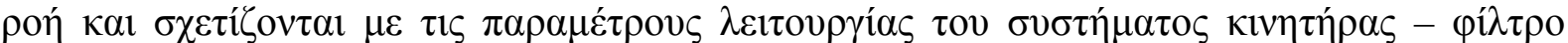

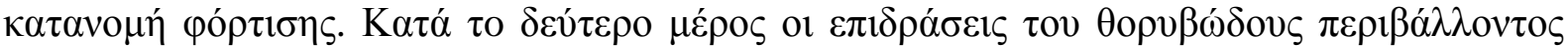

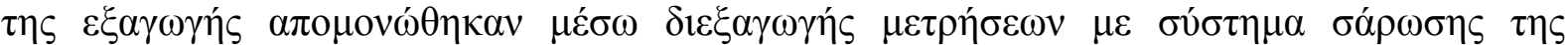

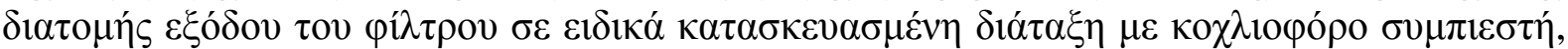

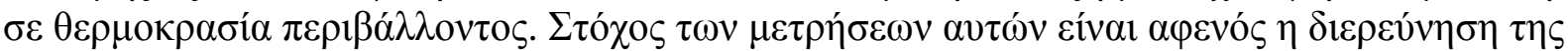

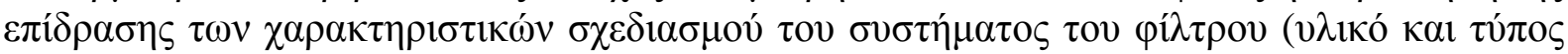

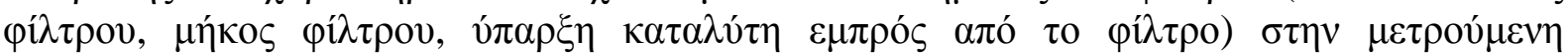

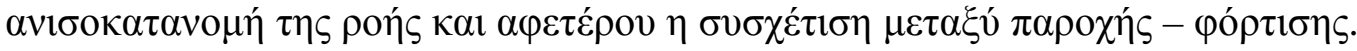

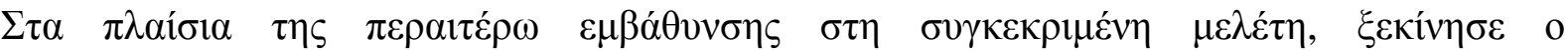

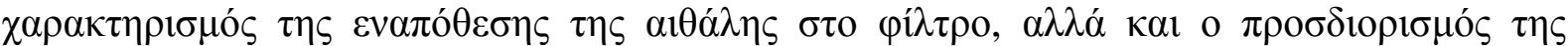

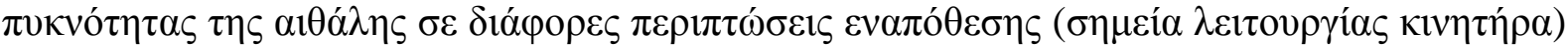

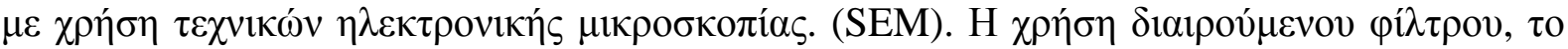

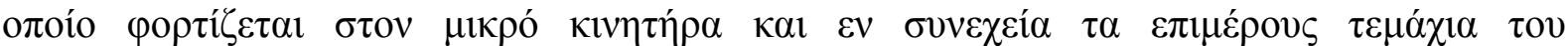

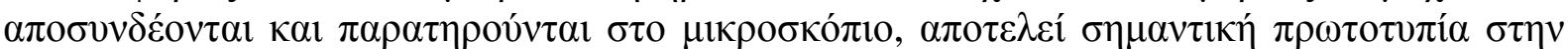

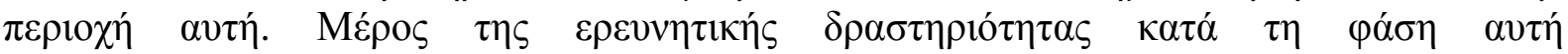

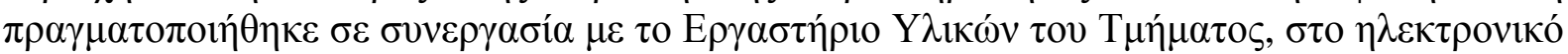

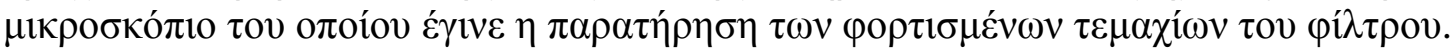

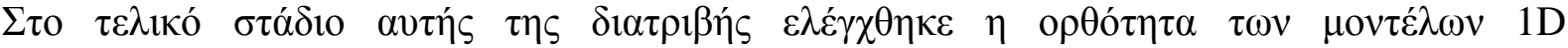

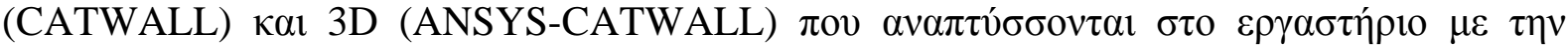

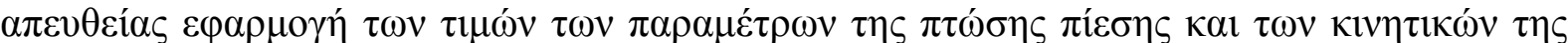

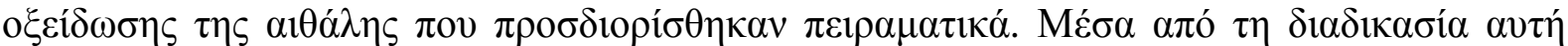

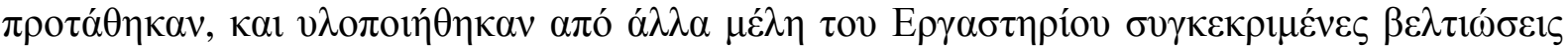

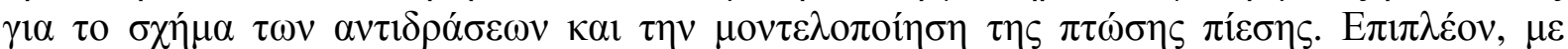

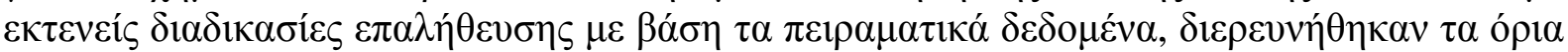

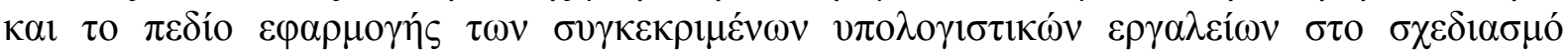

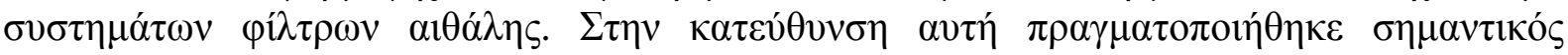

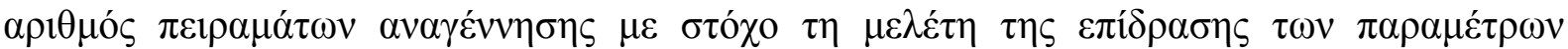

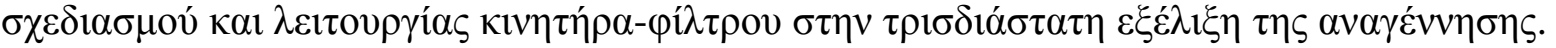




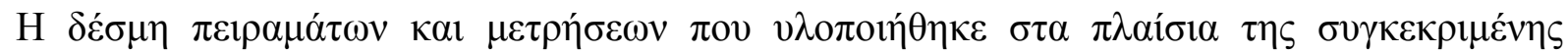

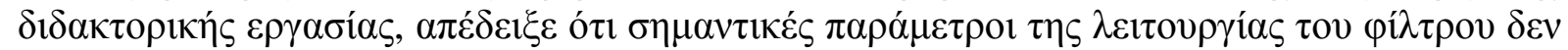

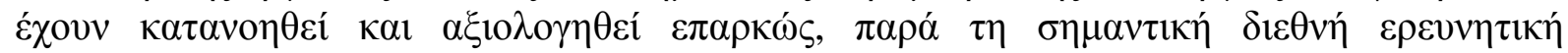

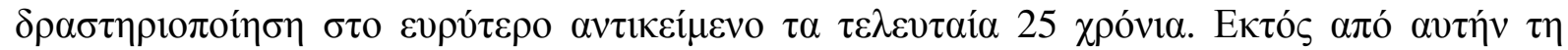

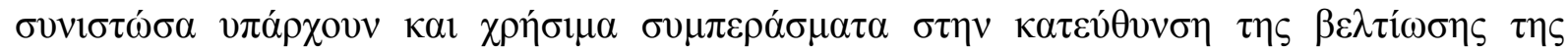

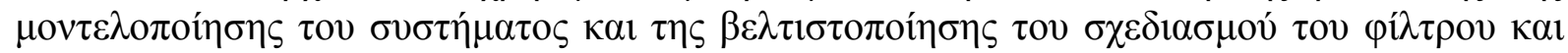

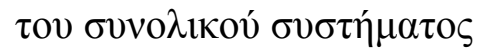

$\Sigma \tau \alpha$ кúpı

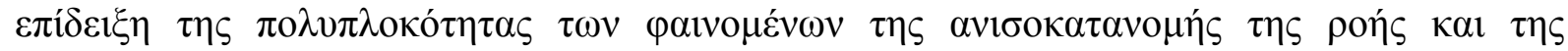

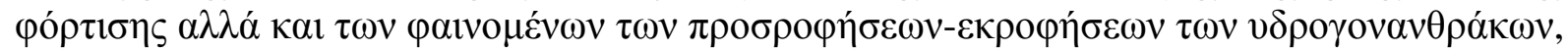

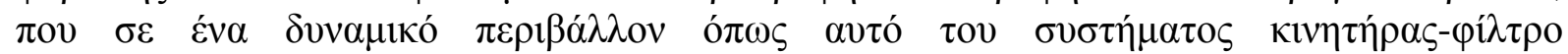

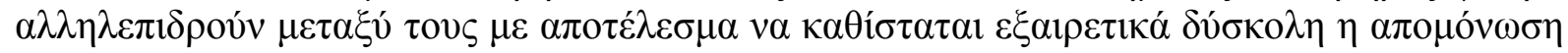

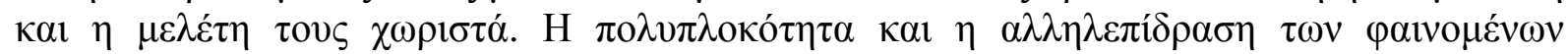

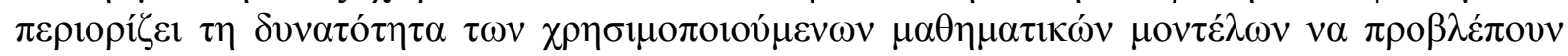

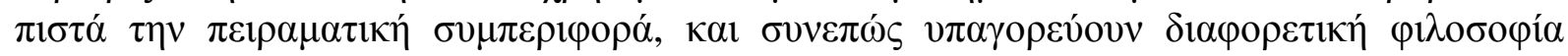

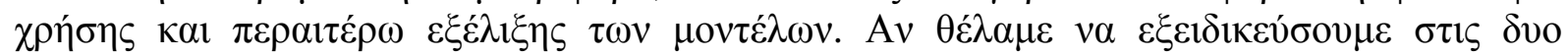

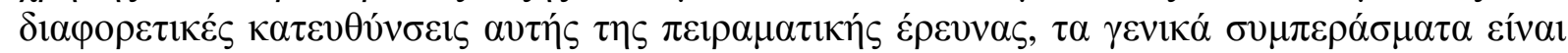
$\tau \alpha \varepsilon \xi \eta ́ n:$

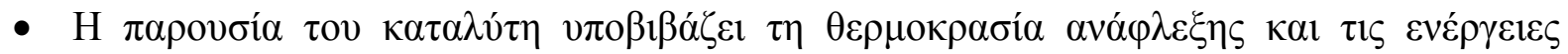

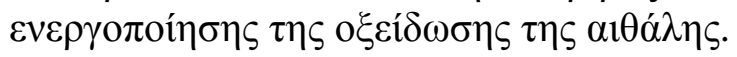

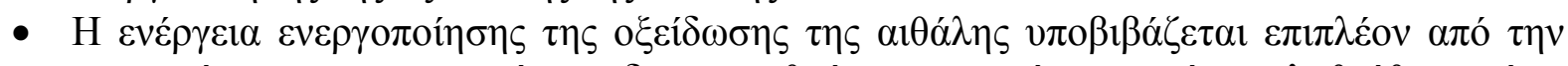

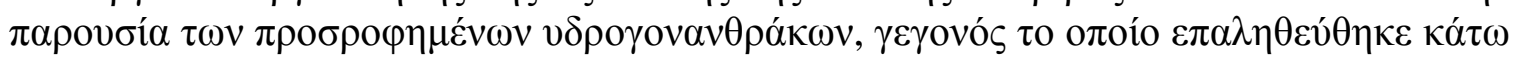

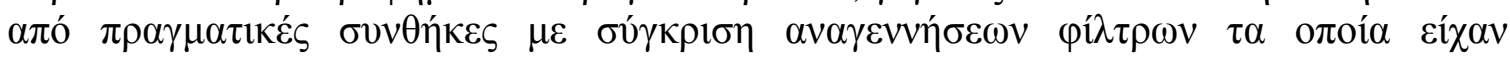

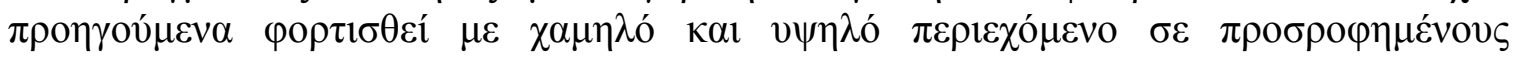

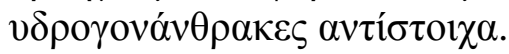

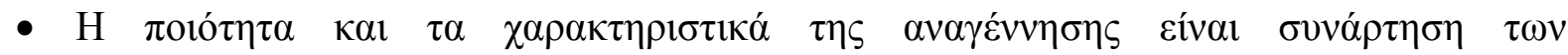

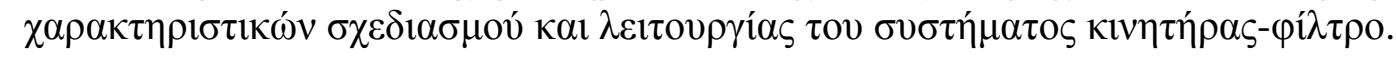

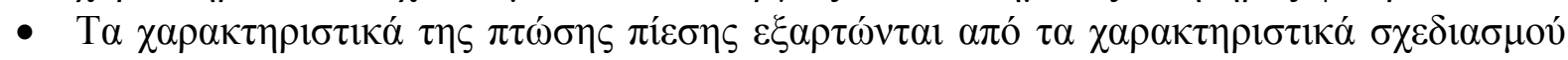

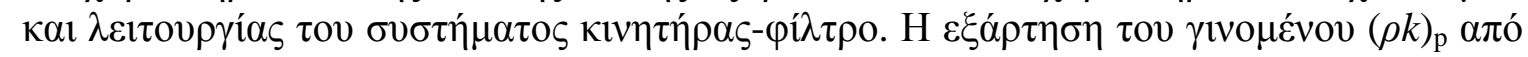

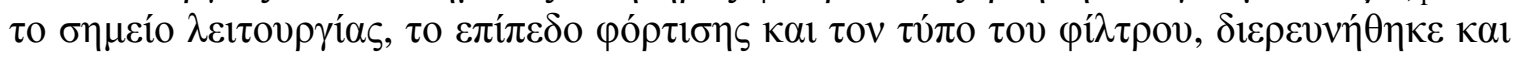

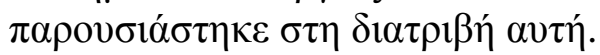

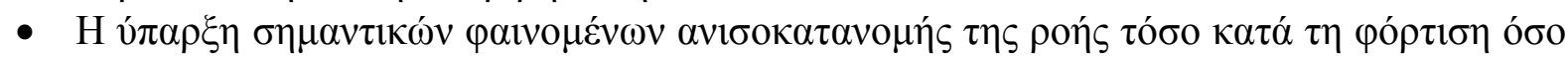

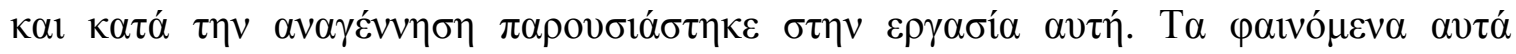

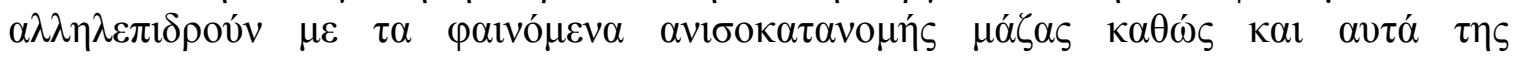

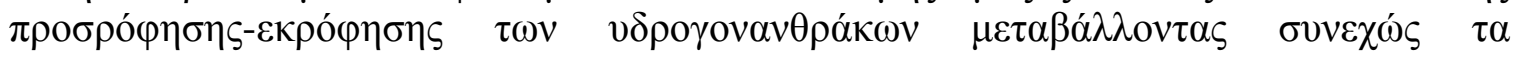

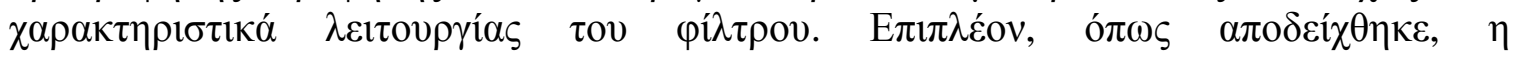

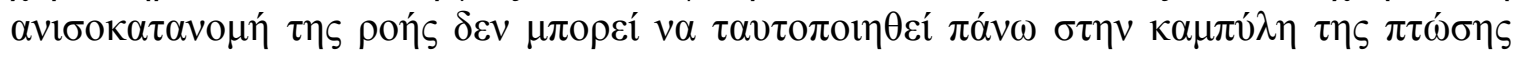

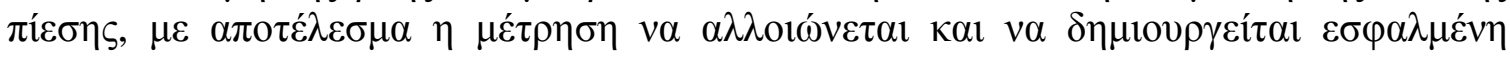

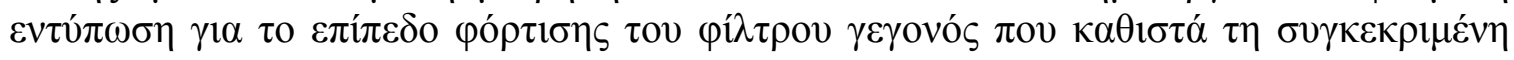

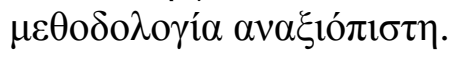




\section{List of Symbols}

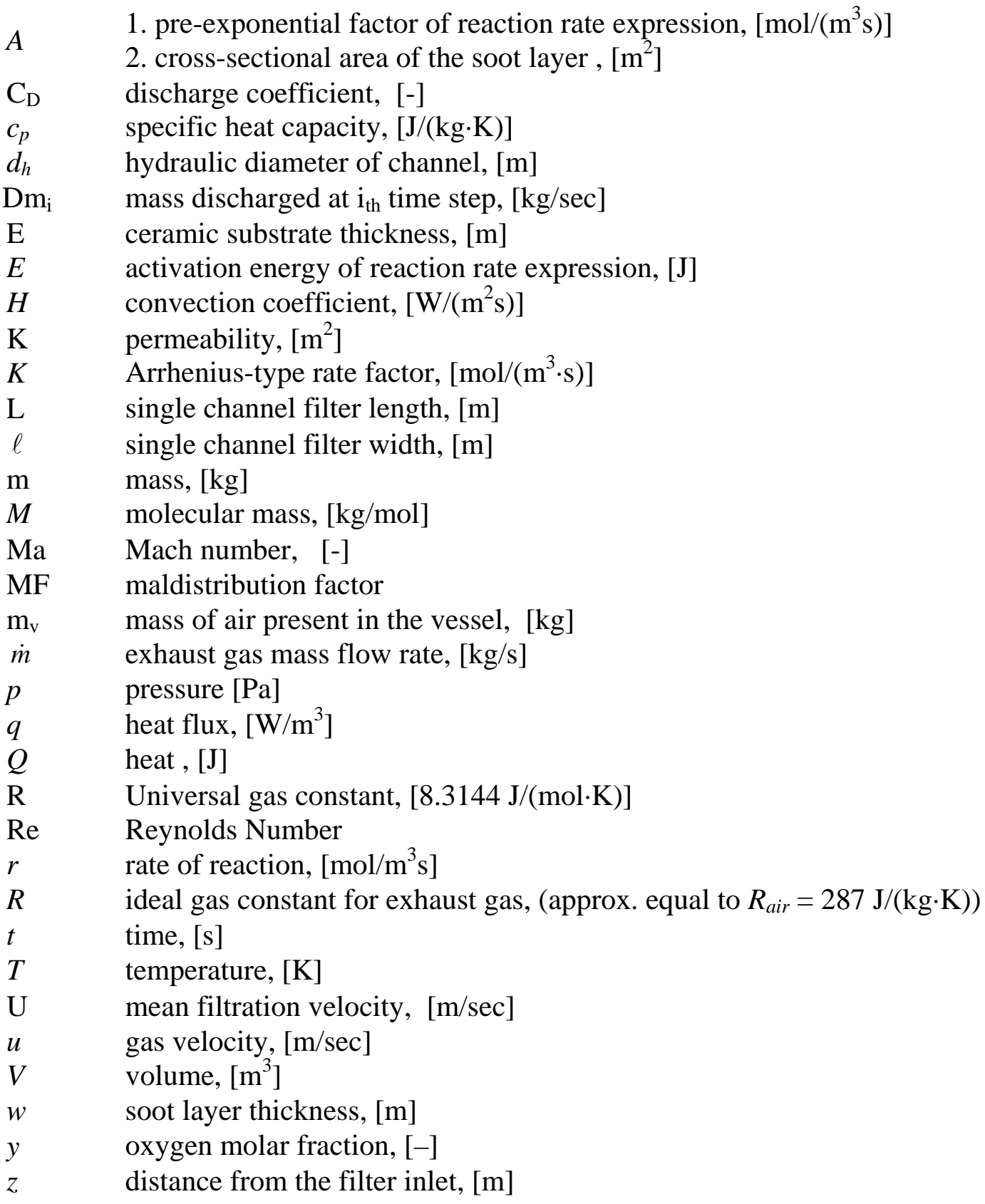

\section{Greek Letters}

$\gamma \quad$ specific heat ratio $\mathrm{c}_{\mathrm{p}} / \mathrm{C}_{\mathrm{v}},[-]$

$\Delta H \quad$ molar heat of reaction, $[\mathrm{J} / \mathrm{mol}]$

$\Delta p \quad$ pressure drop across filter, $[\mathrm{Pa}]$

$\lambda \quad$ thermal conductivity, $[\mathrm{W} /(\mathrm{m} \cdot \mathrm{K})]$

$\lambda$ equivalence ratio of combustion, $\lambda=(\mathrm{A} / \mathrm{F}) /(\mathrm{A} / \mathrm{F})_{\mathrm{st}}$

$\mu \quad$ dynamic viscosity, [Pa·s]

$\rho \quad$ density, $\left[\mathrm{kg} / \mathrm{m}^{3}\right]$

$(\rho \mathrm{k})_{\mathrm{p}} \quad$ soot layer density times permeability product, $[\mathrm{kg} / \mathrm{m}]$

$\psi \quad$ fractional extent of the catalytic additive oxidation, [-] 
$\xi \quad$ total concentration of catalytic additive in the soot layer, $\left[\mathrm{mol} / \mathrm{m}^{3}\right]$

\section{Subscripts}

$\begin{array}{ll}\text { atm } & \text { Atmospheric } \\ \text { c } & \text { Calculated } \\ \text { ch } & \text { Channel } \\ \mathrm{f} & \text { Filter } \\ \mathrm{G} & \text { Guessed } \\ g & \text { Gas } \\ \mathrm{i} & \text { time step } \\ \text { in } & \text { gas flow through the inlet channel } \\ \text { out } & \text { gas flow through the outlet channel } \\ \mathrm{p} & \text { particulate } \\ \text { real } & \text { not ideal } \\ \mathrm{s} & \text { Ceramic } \\ \mathrm{T} & \text { Throat } \\ \mathrm{V} & \text { Vessel } \\ W & \text { gas flow through the wall } \\ 0 & \text { Stagnation }\end{array}$

\section{Abbreviations}

bmep Break mean effective pressure

DI Direct Injection

DPF Diesel Particulate Filter

DPX9 Commercial name of Cerium fuel additive

EGR Exhaust Gas Recirculation

EN590 European Standard for diesel fuel

HC Hydrocarbons

HDI High pressure Direct Injection

LTTE Laboratory of Thermodynamics and Thermal Engines

Mfr Exhaust gas Mass flow rate

PAH Polycyclic Aromatic Hydrocarbons

TF Temperature of filter

T/C Thermocouple fitted on experimental layout

TGA Thermo-Gravimetric Analysis

VOF Volatile Organic Fraction 


\section{Introduction}

\subsection{General}

Since the invention of Diesel engine in 1893 its application has become very widespread over the world. The main reasons for its popularity are the following:

- the combustion efficiency loss is reduced (higher fuel economy)

- the amount of expansion stroke extracted is greater, due to the high compression ratio and the operation with lean fuel-air mixtures.

- the pumping work at partial load is essentially eliminated

- low maintenance requirements

- high durability

Although diesel engines do exhibit clear efficiency advantages, on a worldwide basis, diesel vehicle emission standards in US and Europe are becoming increasingly stringent [1]. The first location of diesel filters on car was made in California by Mercedes-Benz in 1985 [2]. At that time, the diesel engine technology could not profit from the potential of computerized engine management systems and advanced common rail injection systems. However, the possibility of retrofitting diesel filters to existing diesel vehicles, especially heavy-duty trucks and buses was promising and led to a significant number of pilot applications [3]. For this reason, manually activated systems able to raise exhaust gas temperature on-demand [4] were applied in combination with catalytic aids. During the nineties, the significant advances in diesel engine technology, in combination with diesel catalysts, were fully exploited in the direction of minimizing particulate emissions without the use of diesel filters (due to the significant difficulties faced with diesel filter applications).

Starting in 2000, the interest in diesel filter systems by automotive manufacturers is reestablished and ever growing in the frame of the currently proposed EURO IV and Tier 2 (USA) emission standards that are expected to be effective from 2005. As regards particulate emissions, the wall-flow diesel particulate filter (DPF) is today the most efficient aftertreatment device [5], attaining filtration efficiencies over 90\% (for dry particulate) under normal operating conditions. Since 2001, PSA Peugeot-Citroen produces in large numbers, passenger cars with a particulate filter fitted as standard equipment. Recently, other car producers start to introduce diesel filters in certain models. This leads to further enhancement in the research in this field.

An overview of the operating conditions, requirements and limitations of the exhaust treatment devices for automotive diesel engines is given in this chapter. Technologies in series production or at an advanced pre-production stage will be briefly discussed.

\subsection{Diesel Engine Emissions}

Diesel engine emissions are highly complex mixtures. The composition of diesel exhaust varies considerably depending on engine type and operating conditions, fuel, lubricating oil, and whether an emissions control system is present. Various researchers (see for example [6,7]), have studied the effects of fuel composition and properties on particle formation.

Diesel emissions consist of a wide range of organic and inorganic compounds distributed among the gaseous and particulate phases: $\mathrm{CO}, \mathrm{CO}_{2}, \mathrm{NO}, \mathrm{NO}_{2}, \mathrm{~N}_{2} \mathrm{O}, \mathrm{NH}_{3}$, Volatile organic compounds and water vapor, Hydrocarbons (HC), Polynuclear aromatic hydrocarbons, Carboxyl compounds, Organic acids, Halogenated organic compounds, Sulfur dioxide, and 
Dioxins. The majority of these pollutants are toxic at high concentrations and are suspected for a series of adverse effects on human life, on the physiology of plants and the integrity of building construction materials. Table 1.1 presents typical concentrations of the exhaust gas components.

Nowadays the focus for the reduction of harmful diesel emissions is mainly on particulate matter (PM) and NOx. Hydrocarbons and carbon monoxide can be removed by use of wall flow oxidation catalysts. Furthermore the SOx concentration of the exhaust gas is directly proportional to the sulfur content of the fuel and thus it could be removed with measures during the distillation fuel process.

\begin{tabular}{||c|c|c|c||}
\hline \hline Component & Concentration & Component & Concentration \\
\hline $\mathrm{CO}$ & $100-10000 \mathrm{ppm}$ & Aldehydes & $0 \mathrm{mg} / \mathrm{mile}$ \\
\hline $\mathrm{HC}$ & $50-500 \mathrm{ppm} \mathrm{C}_{1}$ & Ammonia & $2 \mathrm{mg} / \mathrm{mile}$ \\
\hline $\mathrm{NO}_{\mathrm{x}}$ & $30-1000 \mathrm{ppm}$ & Cyanide & $1 \mathrm{mg} / \mathrm{mile}$ \\
\hline $\mathrm{SO}_{\mathrm{x}}$ & Proportional to fuel S content & Benzene & $6 \mathrm{mg} / \mathrm{mile}$ \\
\hline $\mathrm{PM}$ & $20-200 \mathrm{mg} / \mathrm{m}^{3}$ & Toluene & $2 \mathrm{mg} / \mathrm{mile}$ \\
\hline $\mathrm{CO}_{2}$ & $2-12 \mathrm{vol} \%$ & $\mathrm{PAH}$ & $0.3 \mathrm{mg} / \mathrm{mile}$ \\
\hline
\end{tabular}

Table 1.1 Typical exhaust gas components [8]

\subsubsection{Diesel particulate matter}

The term 'particle' generally applies to aerosols created by the dispersion in air of atomised solids and liquids, powders or droplets, and hence includes the terms "fine dust, soot, mist, fog and smog”. Their diameter is smaller than $10 \mu \mathrm{m}$.

Inside an engine, the complete combustion of motor fuels composed exclusively of carbon and hydrogen, would only generate $\mathrm{CO}_{2}$ and $\mathrm{H}_{2} \mathrm{O}$, to the exclusion of any other harmful product. However, the very short time allowed for chemical oxidation processes integration in combustion chambers, the lack of homogeneity in the carburetted mixtures, and the heterogeneity and rapid variations in temperature do not allow for the state of ideal thermodynamic equilibrium to be reached.

Diesel exhaust particles consist mainly of highly agglomerated solid carbonaceous material and ash, and volatile organic and sulfur compounds. Solid carbon is formed during combustion in locally rich regions. Much of it is subsequently oxidized. The result is exhausted in the form of solid agglomerates. A tiny fraction of the fuel as well as atomized and evaporated lubrication oil escape oxidation and appearing as volatile or soluble organic compounds (Soluble Organic Fraction, SOF) in the exhaust. Metal compounds in the fuel and lubrication oil lead to a small amount of inorganic ash [9]. The US EPA defines diesel particulate matter as the mass collected on a fiber filter from exhaust that has been diluted and cooled to $52{ }^{\circ} \mathrm{C}$ or below [10]. The composition of exhaust particulates depends on where and how they are collected. As the exhaust gas is diluted and cooled, nucleation, condensation and adsorption transform volatile materials to solid and liquid particulate matter. In the tailpipe, where temperatures are high, most of the volatile materials are of gaseous phase. The details of the dilution and cooling processes determine the elative amounts of material that adsorb or condense onto existing particles and nucleate to form new particles $[11,12]$. The Soluble Organic Fraction of the particulate is determined by chemical extraction (Soxlet method), and 
it is usually employed in the determination of adsorbed hydrocarbon speciation. If one is just interested in the percentage of volatile compounds, it is preferable to determine, instead, the Volatile Organic Fraction (VOF), by means of vacuum evaporation. The composition of PM varies greatly depending on the engine technology, test conditions and, in the case of sulfate particulates, the sulfur content in the fuel. An example of DPM composition from a post-1994 US heavy-duty engine is illustrated in Figure 1.1.

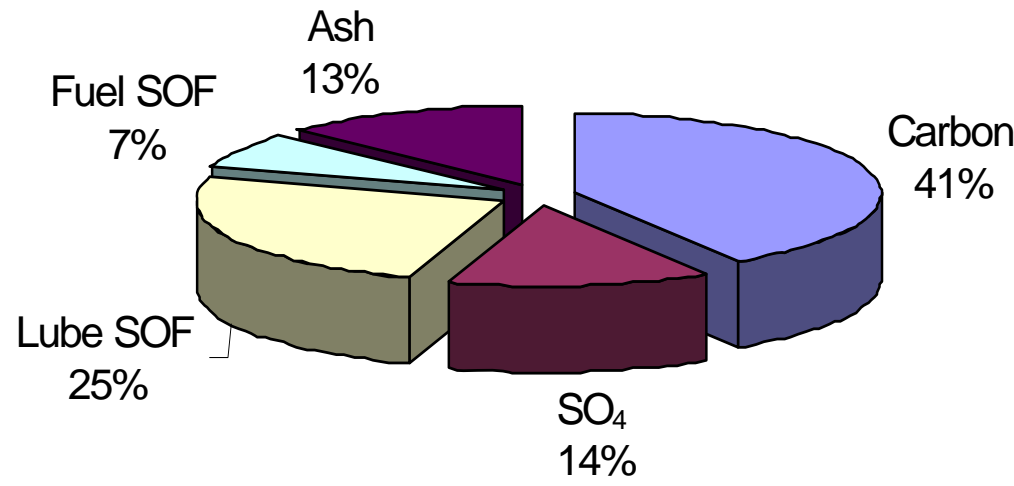

Figure 1.1 Diesel particulate matter composition

Diesel particulates have a bimodal size distribution $[9,13]$. They are a mixture of nuclei mode and accumulation mode particles (Figure 1.2). The nuclei mode particles are very small - their diameters are usually between 0.007 and $0.04 \mu \mathrm{m}$ (micron). These particles originate from the primary carbon spherules, which are generated in the combustion chamber. As the exhaust gases cool down after leaving the engine cylinder, nucleation of hydrated sulfuric acid also produces nuclei mode particles, which are known as sulfate particulates. Under certain conditions, nucleation of hydrocarbons may also occur. The nuclei mode typically contains $1-20 \%$ of the particle mass and more than $90 \%$ of the particle number. The accumulation mode particles are formed by agglomeration of carbonaceous, nuclei mode particles. It's about typical long chain or cluster of carbon particles that contain adsorbed hydrocarbons.

In the burning of liquid fuels of the diesel type, the size of the droplets and hence the quality of spraying are extremely important because the formation of soot increases with droplet size [6, 14]. Of course, the non-homogenous nature of the mixture and the duration of the injection and its overlap with combustion are parameters that influence the process of soot formation. Table 1.1 summarizes the parameters that favour diesel particulate emissions.
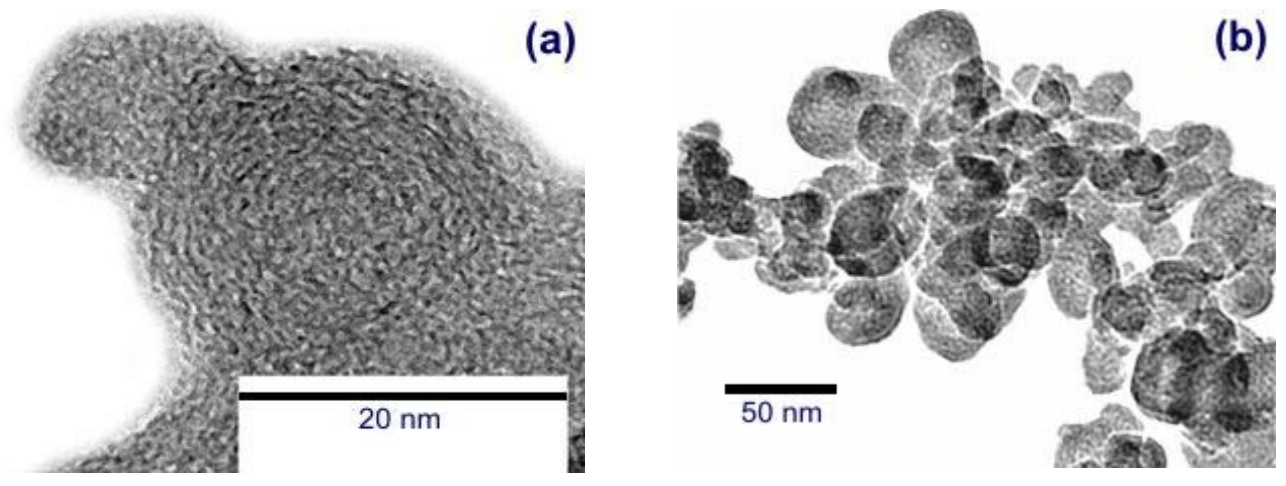
Figure 1.2 TEM pictures of nuclei mode (a) and agglomerated particles (b)

Table 1.2 Main parameters influencing particulate formation [15]

\begin{tabular}{|c|c|}
\hline $\begin{array}{l}\text { Formation of insoluble fraction } \\
\text { favored by: }\end{array}$ & $\begin{array}{ll}\text { - } & \text { Elevated temperature } \\
\text { - } & \text { High pressure } \\
\text { - } & \text { Absence of oxygen }\end{array}$ \\
\hline $\begin{array}{l}\text { Oxidation of insoluble fraction } \\
\text { favored by: }\end{array}$ & $\begin{array}{ll}\text { - } & \text { Elevated temperature } \\
\text { - } & \text { High pressure } \\
\text { - } & \text { Presence of Oxygen }\end{array}$ \\
\hline $\begin{array}{l}\text { Formation of HC particles } \\
\text { favored by: }\end{array}$ & $\begin{array}{ll}\text { - } & \text { Lean mixture zones } \\
\text { - } & \text { Temperatures below the flammability limit } \\
\text { - } & \text { Hydrocarbons layers on the walls of the bowl } \\
\text { - } & \text { Fuel droplets seeping at the nozzle tip }\end{array}$ \\
\hline $\begin{array}{l}\text { Sources of particles from } \\
\text { lubricant: }\end{array}$ & $\begin{array}{ll}\text { - } & \text { Surface of cylinder liners } \\
\text { - } & \text { Valve stem gaskets } \\
\text { - } & \text { Turbo-compressor gaskets } \\
\text { - } & \text { Recycling of crankcase ventilation gases to inlet }\end{array}$ \\
\hline
\end{tabular}

\section{Soluble Organic Fraction Components}

The SOF is typically composed of lube oil derived hydrocarbons, with a small contribution from the higher boiling end diesel fuel hydrocarbons. Diesel fuel is composed of hydrocarbons having boiling range equal to that of aliphatic hydrocarbons with 12 to 20 atoms of carbon in their molecules. Diesel lubrication oil consists of many compounds with boiling points comparable to normal aliphatic hydrocarbons in the range between C18 and C36. The SOF fraction contains most of the polynuclear (or polycyclic) aromatic hydrocarbons (PAH) emitted with diesel exhaust gases. PAHs are aromatic hydrocarbons with two or more (up to five or six) benzene rings joined in various, more or less clustered forms generated by pyrosynthesis in the engine cylinder, or transformed by fuel that appears to be the dominant source of PAH to the lubrication oil during engine operation [16]. They attracted special attention because many of PAH compounds have been shown to have carcinogenic effects. Figure 1.3 shows an example chromatogram of diesel particulate SOF compared with chromatograms of diesel lube oil and diesel fuel. 


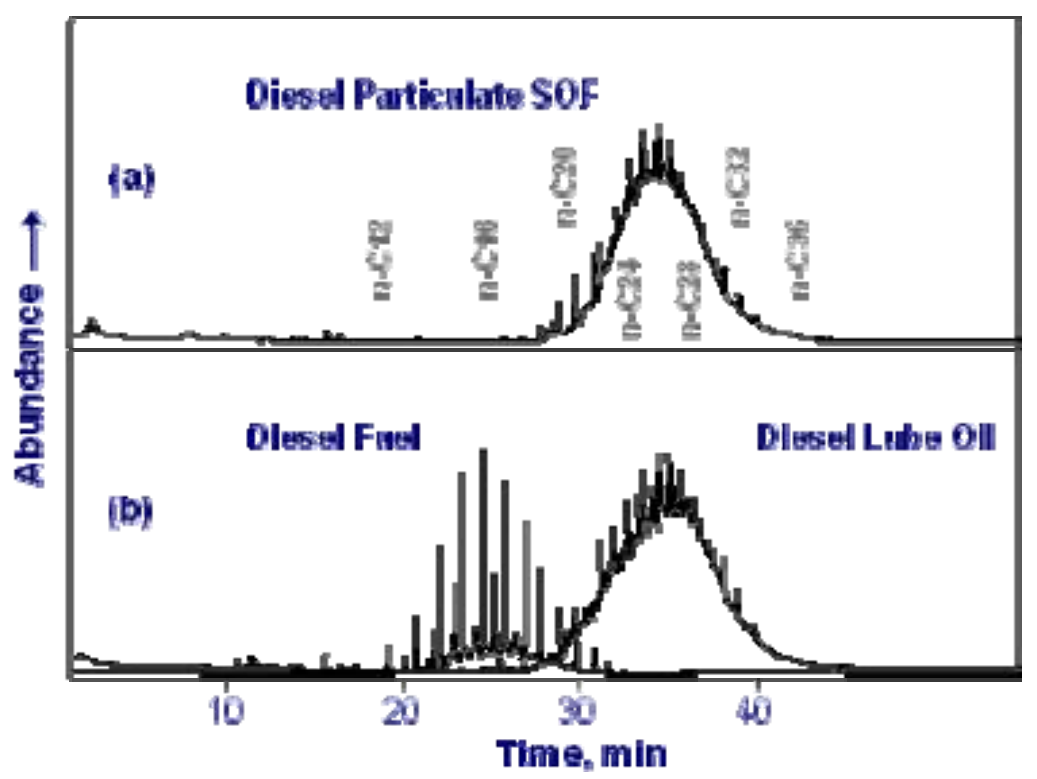

Figure 1.3 SOF composition analysis performed on the chromatograph [25].

\subsection{Diesel Emissions Reduction Legislation}

It has been reported [17] that Diesel exhaust emissions of $\mathrm{PM}, \mathrm{NO}_{\mathrm{x}}, \mathrm{HC}$ and $\mathrm{CO}$ are responsible for health and environmental hazards. Thus the international legislation from the early 80's has acknowledged the problem and posed limits on these emissions. The emission standards have become more stringent in time.

In Europe since 1992 a series of five steps referred as Euro Standards (Euro I to Euro V) was adopted for the reduction of the particulate matter and the other harmful gas components in the exhaust of the passenger cars and light and heavy-duty trucks. These standards are given in Table 1.3. As it can be seen from 2005 only light duty passenger cars with PM emissions under $0.025 \mathrm{~g} / \mathrm{km}$ and heavy duty trucks with PM emissions under $0.02 \mathrm{~g} / \mathrm{kWh}$ will be certified for driving in the EU. These values indicate a lowering to the PM emissions standard of the order of $80 \%$ than these of Euro I. In the case of passenger and light-duty trucks the standards are referred to emission measurements performed during the ECE + EUDC test cycle. This is a combination of four ECE 15 also known as UDC cycles (Urban Driving Cycle) that simulate city driving conditions and one EUDC cycle (Extra Urban Driving Cycle) that simulates highway driving conditions. In the case of heavy-duty trucks, according to the current EURO III and the next EURO IV and EURO V standards engine emissions are measured during either the European Stationary Cycle (ESC) or the European Transient Cycle (ETC) that replaces the EURO I ECE R-49 cycle (mixture of 13 steady-state engine operation points). In other European countries as Germany and Sweden, additional requirements are instituted for vehicles in specific areas such as city centres and mines. In Germany, engines used in mines are required to be equipped with particulate filters. The same applies for engines of a certain age to enter in the centres of the big cities of Sweden. 
Table 1.3 European emission standards for diesel passenger cars and light-duty and heavy-duty trucks.

\begin{tabular}{|c|c|c|c|c|c|}
\hline \multicolumn{6}{|l|}{ Diesel cars/ [g/km] } \\
\hline Standard & Year & $\mathrm{HC}+\mathrm{NO}_{\mathrm{x}}$ & $\mathrm{NO}_{\mathrm{x}}$ & CO & PM \\
\hline Euro I & 1992 & 0.97 & - & 2.72 & 0.14 \\
\hline Euro I & 1996 & 0.7 & - & 1.0 & 0.08 \\
\hline Euro II-DI & 1999 & 0.9 & - & 1.0 & 0.10 \\
\hline Euro III & 2000 & 0.56 & 0.50 & 0.64 & 0.05 \\
\hline Euro IV & 2005 & 0.3 & 0.25 & 0.50 & 0.025 \\
\hline \multicolumn{6}{|c|}{ Light duty trucks [g/km] } \\
\hline Class & Year & $\mathrm{HC}+\mathrm{NO}_{\mathrm{x}}$ & $\mathbf{N O}_{\mathrm{x}}$ & CO & PM \\
\hline \multirow{3}{*}{ I $(<1305 k g)$} & 1994 & 0.97 & - & 2.72 & 0.14 \\
\hline & 2000 & 0.56 & 0.50 & 0.64 & 0.05 \\
\hline & 2005 & 0.30 & 0.25 & 0.50 & 0.025 \\
\hline \multirow{3}{*}{ II (1305-1760kg) } & 1994 & 1.40 & - & 5.17 & 0.19 \\
\hline & 2001 & 0.72 & 0.65 & 0.80 & 0.07 \\
\hline & 2006 & 0.39 & 0.33 & 0.63 & 0.04 \\
\hline \multirow{3}{*}{ III (>1760kg) } & 1994 & 1.70 & - & 6.90 & 0.25 \\
\hline & 2001 & 0.86 & 0.78 & 0.95 & 0.10 \\
\hline & 2006 & 0.46 & 0.39 & 0.74 & 0.06 \\
\hline \multicolumn{6}{|c|}{ Heavy duty diesel trucks / [g/kWh] } \\
\hline Standard & Year\&category & $\mathrm{HC}+\mathrm{NO}_{\mathrm{x}}$ & $\mathrm{NO}_{\mathrm{x}}$ & $\mathrm{CO}$ & $\mathbf{P M}$ \\
\hline \multirow{2}{*}{ Euro I } & $1992<85 \mathrm{~kW}$ & 1.1 & 8.0 & 4.5 & 0.612 \\
\hline & $1992>85 \mathrm{~kW}$ & 1.1 & 8.0 & 4.5 & 0.36 \\
\hline \multirow{2}{*}{ Euro II } & 1996.10 & 1.1 & 7.0 & 4.0 & 0.25 \\
\hline & 1998.10 & 1.1 & 7.0 & 4.0 & 0.15 \\
\hline Euro III & 2000.10 & 0.66 & 5.0 & 2.1 & 0.10 \\
\hline Euro IV & 2005.10 & 0.46 & 3.5 & 1.5 & 0.02 \\
\hline Euro V & 2008.10 & 0.46 & 2.0 & 1.5 & 0.02 \\
\hline
\end{tabular}

In USA two sets of standards, Tier1 and Tier2, have been defined for passenger and lightduty vehicles. The Tier1 regulations were published as a final rule on 1991 and fully implemented in 1997. The Tier 2 standards which are based in large part on California's LEV II program were issued on December 1999 to be phased in beginning in 2004 and fully phased in by 2007 with the requirement all passenger cars and light trucks to eventually meet the same stringent standards by 2009. Table 1.4 shows the Tier 2 standards for passenger and light duty vehicles. As it can be seen the structure of Tier 2 standard incorporates eight permanent standards bins and two temporary that are available only during the interim period and will be deleted until the final phase in of the program. An eleventh bin is available only for MDPV (Medium Passenger Vehicles) and expires in 2008. Tier 2 standards are applicable in FTP 75 driving cycle and emissions are measured in $\mathrm{g} / \mathrm{mile}$. Manufacturers may certify to one of the available bins in order to meet the required corporate average 120000 miles $\mathrm{NO}_{\mathrm{x}}$ standards of $0.07 \mathrm{~g} / \mathrm{m}$ for passenger and light, light-duty vehicles and $0.2 \mathrm{~g} / \mathrm{m}$ for heavy, lightduty vehicles that are phased-in on the following schedule: $2004-25 \%$, $2005-50 \%$, $2006-$ $75 \%$ and $2007-100 \%$. In $2008,50 \%$ of the heavy, light-duty trucks would be required to meet the corporate average 120000 mile 0.07 gpm NOx standard and in 2009 100\% would be required to meet the corporate average $0.07 \mathrm{NO} x$ standard.

Table 1.4 US Tier 2 standards for passenger and light duty vehicles. 


\begin{tabular}{|c|c|c|c|c|c|c|c|c|c|c|}
\hline \multirow{2}{*}{ Bin\# } & \multicolumn{5}{|c|}{50,000 miles } & \multicolumn{5}{|c|}{120,000 miles } \\
\hline & NMOG & CO & NOx & PM & НСНО & NMOG & CO & NOx* & PM & НСНО \\
\hline \multicolumn{11}{|c|}{ Temporary Bins (expire in 2006) } \\
\hline $\mathrm{MDPV}^{\mathrm{C}}$ & & & & & & 0.280 & 7.3 & 0.9 & 0.12 & 0.032 \\
\hline $10^{\mathrm{a}, \mathrm{b}, \mathrm{d}, \mathrm{f}}$ & $0.125(0.160)$ & $\begin{array}{l}3.4 \\
1\end{array}$ & 0.4 & - & $\begin{array}{l}0.015 \\
(n) 101\end{array}$ & 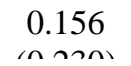 & $\begin{array}{l}4.2 \\
\text { ( } 11\end{array}$ & 0.6 & 0.08 & $0.018(0.027)$ \\
\hline $9^{\mathrm{a}, \mathrm{b}, \mathrm{e}}$ & $0.075(0.140)$ & 3.4 & 0.2 & - & 0.015 & $\begin{array}{c}0.090 \\
\text { In } 1 \text { וnn }\end{array}$ & 4.2 & 0.3 & 0.06 & 0.018 \\
\hline \multicolumn{11}{|c|}{ Permanent Bins } \\
\hline $8^{\mathrm{b}}$ & $0.100(0.125)$ & 3.4 & 0.14 & - & 0.015 & $\begin{array}{l}0.125 \\
1 \sim 1 \Gamma r\end{array}$ & 4.2 & 0.20 & 0.02 & 0.018 \\
\hline 7 & 0.075 & 3.4 & 0.11 & - & 0.015 & 0.090 & 4.2 & 0.15 & 0.02 & 0.018 \\
\hline 6 & 0.075 & 3.4 & 0.08 & - & 0.015 & 0.090 & 4.2 & 0.10 & 0.01 & 0.018 \\
\hline 5 & 0.075 & 3.4 & 0.05 & - & 0.015 & 0.090 & 4.2 & 0.07 & 0.01 & 0.018 \\
\hline 4 & - & - & - & - & - & 0.070 & 2.1 & 0.04 & 0.01 & 0.011 \\
\hline 3 & - & - & - & - & - & 0.055 & 2.1 & 0.03 & 0.01 & 0.011 \\
\hline 2 & - & - & - & - & - & 0.010 & 2.1 & 0.02 & 0.01 & 0.004 \\
\hline 1 & - & - & - & - & - & 0.000 & 0.0 & 0.00 & 0.00 & 0.000 \\
\hline \multicolumn{11}{|c|}{ *- average manufacturer fleet NOx standard is $0.07 \mathrm{~g} / \mathrm{mi}$} \\
\hline \multicolumn{11}{|c|}{ a - Bin deleted at end of 2006 model year (2008 for HLDTs) } \\
\hline \multicolumn{11}{|c|}{ b - The higher temporary NMOG, CO and HCHO values apply only to HLDTs and expire after 2008} \\
\hline \multicolumn{11}{|c|}{ c - An additional temporary bin restricted to MDPVs, expires after model year 2008} \\
\hline \multirow{2}{*}{\multicolumn{11}{|c|}{$\begin{array}{l}\text { d - Optional temporary NMOG standard of } 0.195 \mathrm{~g} / \mathrm{mi}(50,000) \text { and } 0.280 \mathrm{~g} / \mathrm{mi}(120,000) \text { applies for } \\
\text { qualifying LDT4s and MDPVs only }\end{array}$}} \\
\hline & & & & & & & & & & \\
\hline \multirow{2}{*}{\multicolumn{11}{|c|}{$\begin{array}{l}\text { e - Optional temporary NMOG standard of } 0.100 \mathrm{~g} / \mathrm{mi}(50,000) \text { and } 0.130 \mathrm{~g} / \mathrm{mi}(120,000) \text { applies for } \\
\text { qualifying LDT2s only }\end{array}$}} \\
\hline & & & & & & & & & & \\
\hline \multicolumn{11}{|c|}{ f - 50,000 mile standard optional for diesels certified to bin 10} \\
\hline \multicolumn{11}{|c|}{ MDPV: Medium Passenger Vehicles, LLDT: Light, Light-Duty trucks (LDT1 \& LDT2), HLDT: } \\
\hline Heav & y, Light-Duty T & ucks & DT3 & 及 LI & & & & & & \\
\hline
\end{tabular}

Additional regulations concerning the sulfur level in fuel were applied by both European and USA legislations. Lowering the fuel sulfur level will be beneficially for the particulate emissions and for the implementation of certain aftertreatment technologies, since sulfur may inhibit catalysts activity (see next section). The currently permitted fuel sulfur level is $350 \mathrm{ppm}$ but it will be lowered in Europe to 50ppm by 2005 and in USA to 15 to 30ppm by the mid of 2006.

\subsection{Diesel Exhaust Gas Treatment Technologies}

\subsubsection{Diesel catalysts}

\section{Oxidation Catalyst}

Oxidation catalysts are the presently preferred emission control system for passenger and light duty vehicles in Europe as well as heavy-duty diesel engines in USA. The diesel 
oxidation catalyst is an effective device to control carbon monoxide and hydrocarbon emissions from diesel engines, including the $\mathrm{PAH}$ and hydrocarbon derivatives such as aldehydes. Furthermore it converts a large part of the constituents of the SOF of diesel particulates. Although the particulate conversion efficiency is much less (does not exceed the 25-35\%) than the filtration efficiency of a wall-flow filter, it is sometimes enough for many current developed engines to pass the existing emission standards. The relatively low particulate conversion efficiency is attributed to the limited operating conditions, which are met during engine operation at medium loads. At low loads the exhaust gas temperature is not sufficient to activate the catalyst, whereas at high loads, the SOF part of the particulate, which may be oxidized in the converter, is relatively low.

The most widespread type of oxidation catalyst employs a ceramic honeycomb structure or monolith held in a metal can in the exhaust stream. The noble metals are impregnated into a highly porous alumina washcoat about $20-40 \mu \mathrm{m}$ thick that is applied to the passageway walls (Figure 1.4). Most of the oxidation catalysts used in the international market contain Pt and $\mathrm{Pd}$ in a ratio of about $5 / 2$ at a typical loading of $50-70 \mathrm{~g} / \mathrm{ft}^{3}$. A drawback of the diesel oxidation catalyst is the formation of sulfate emissions. The precious metal catalyze the reaction of $\mathrm{SO}_{2}$ to $\mathrm{SO}_{3}$ which can further react with water to form sulfates and sulfuric acid. In order to minimize sulfate production a silica washcoat is prefered over alumina and Pd is prefered over Pt as the noble metal. On the other hand research on zeolite based diesel catalysts (catalysts for selective $\mathrm{NO}_{\mathrm{x}}$ reduction) shows that a decrease to the amount of particulate matter is achieved by reducing the SOF, and exhibit no significant $\mathrm{SO}_{2}$ to $\mathrm{SO}_{3}$ at elevated catalyst temperatures [18].

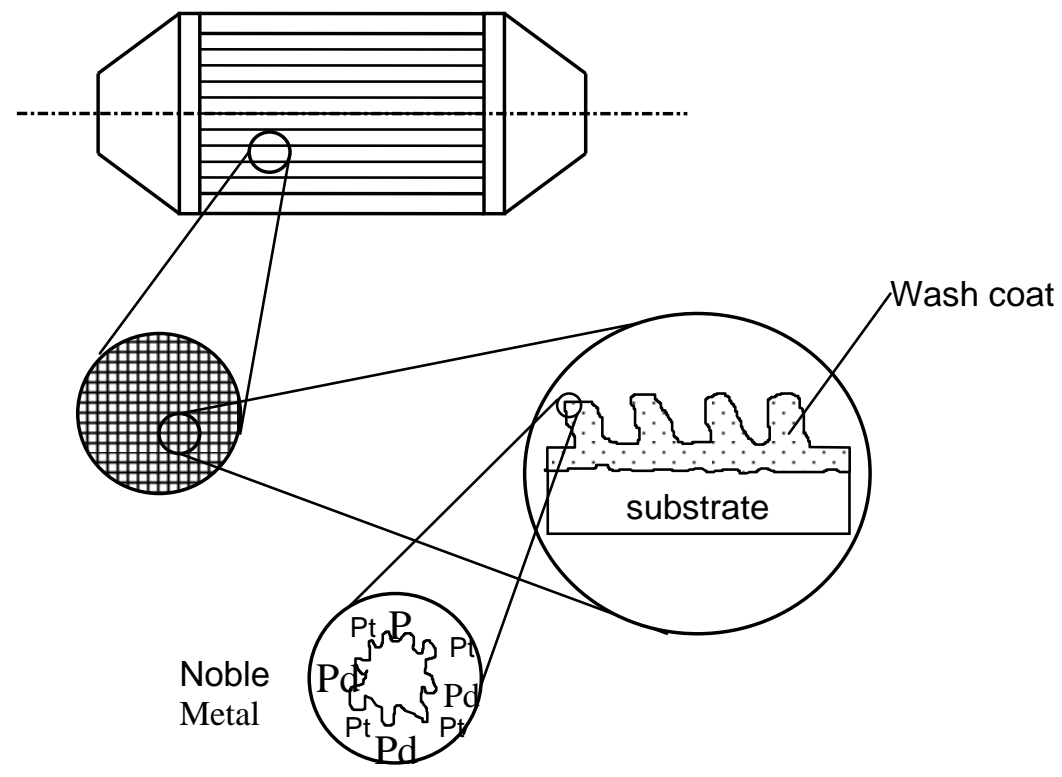

Figure 1.4 Structure of the monolithic catalytic converter

The tendency of the precious metal catalyst to convert $\mathrm{SO}_{2}$ to particulate sulfates requires the use of low sulfur fuel in order to avoid deactivation of the catalyst by poisoning from sulfur oxides. Europe, US and Japan have already decided to reduce the sulfur content of diesel fuel.

\section{NOx adsorber catalyst}

The catalytic reduction of $\mathrm{NO}_{\mathrm{x}}$ in diesel engines presents considerable difficulties due to the lean exhaust environment. The reducing CO presents in low concentrations while NO should further compete with oxygen to react with hydrocarbons. Numerous catalysts have 
been studied over the last years capable of reducing $\mathrm{NO}_{\mathrm{x}}$ in diesel exhaust $[19,20]$. This is achieved by selective reduction of $\mathrm{NO}_{\mathrm{x}}$ by $\mathrm{HC}$ existing in exhaust gases using families of catalysts including zeolite, alumina and complex oxides. However these catalysts faced a lot of problems such as low conversion efficiency, narrow temperature window, and low heat resistance. In their present status, these types of catalysts are difficult to commercialise and require further development.

A promising technique for the catalytic reduction of $\mathrm{NO}_{\mathrm{x}}$ is based on the principle of storing $\mathrm{NO}$ in the catalyst during engine operation and converting it during short duration, carefully controlled, rich excursions. This technique was presented and realized by Myoshi et al [21]. In a Pt-Ba- $\mathrm{Al}_{2} \mathrm{O}_{3}$ catalyst, $\mathrm{NO}$ was found to be oxidized with oxygen and then react with $\mathrm{Ba}$ (storage component) to form a nitrate. The amount of NO storage capacity in such catalysts is strongly affected by the basicity results in more stable nitrates. When the engine runs under excessive fuel conditions or at elevated temperatures the nitrate species become thermodynamically unstable and decompose, producing $\mathrm{NO}$ or $\mathrm{NO}_{2}$. Under rich conditions, these nitrogen oxides are subsequently reduced by $\mathrm{CO}, \mathrm{H}_{2}$, and $\mathrm{HC}$ to $\mathrm{N}_{2}$ over the reduction catalyst, in a conventional three-way catalyst process. The NO storage-reduction mechanism is presented in

Figure 1.5.
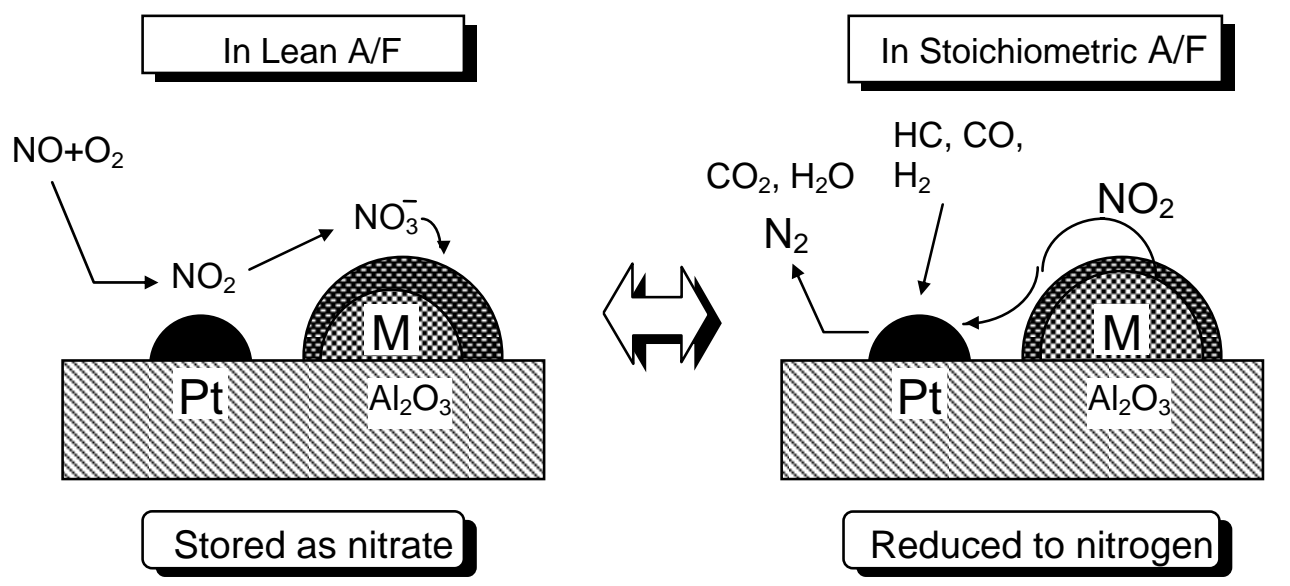

Figure 1.5 NOx storage-reduction mechanism in $\mathrm{NO}_{\mathrm{x}}$ adsorber catalysts.

The generation of rich air-to-fuel mixtures in the diesel engine, which normally operates at equivalence ratio $\lambda>1.3$ and leaner, is a complex task since there is the danger of appearance of torque fluctuations and high soot emissions. Thus a specially designed fuel control system that periodically causes short enrichments is necessary. In the modern diesel engines this is done with the aid of common rail system, which is able to electronically control the fuel quantity and timing in combination with EGR and turbocharger adjustment to meet the above conditions. The operation strategy of such a system is presented in Figure 1.6.

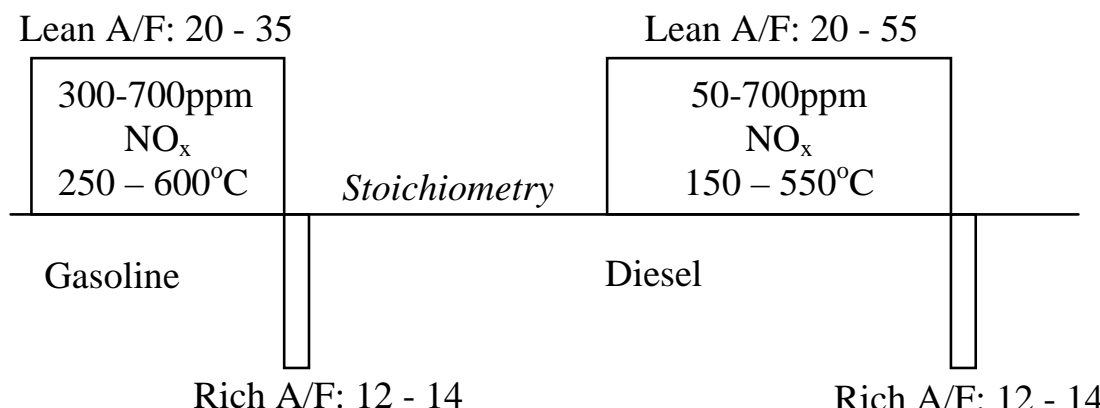


Figure 1.6 Operation strategies in gasoline and diesel engines for the regeneration of the $\mathrm{NO}_{\mathrm{x}}$ adsorber catalyst.

\subsubsection{The wall-flow particulate filter}

Wall flow ceramic monoliths, which are derived from the flow-through cellular supports used for catalytic converters, became the most common type of diesel filter substrate (originally reported by Howitt and Montierth [22]). It is a honeycomb structure made from porous ceramic material. The adjacent channels of the honeycomb are alternatively plugged at each end in order to force the diesel aerosol through the porous substrate walls, which act as a mechanical filter. The particulates cannot flow through the wall and are deposited in the channel walls. To reflect this flow pattern, (Figure 1.7) the substrates are referred to as the wall-flow monoliths. The main advantage of the wall flow particulate filter is the high surface area per unit volume, which combined with the high collection efficiencies (over 95\%) makes this technique very attractive. The filter walls have a porous structure that is carefully controlled during the manufacture process. Typical values of material porosity are between 45 and 50\% while the medium pore size varies in the range between 10 and $20 \mu \mathrm{m}$.

Two types of filter materials are commonly established: cordierite and silicon carbide. Cordierite is a synthetic ceramic developed for flow-through catalyst substrates and subsequently adapted for the filter application. Silicon carbide has been used for a long time in a number of industries for such applications as semiconductors, abrasives, or high temperature/molten metal contact materials. More recently, it was successfully introduced as a diesel filter material. Cordierite substrates perform satisfactory in most heavy-duty applications with high exhaust temperature. However, in low-temperature operation which may induce "uncontrolled regenerations", cordierite is more susceptible for thermal stress related damage. Silicon carbide has higher maximum operating temperature limits and better durability in high thermal stress applications. Disadvantages of SiC include higher weight and higher cost.

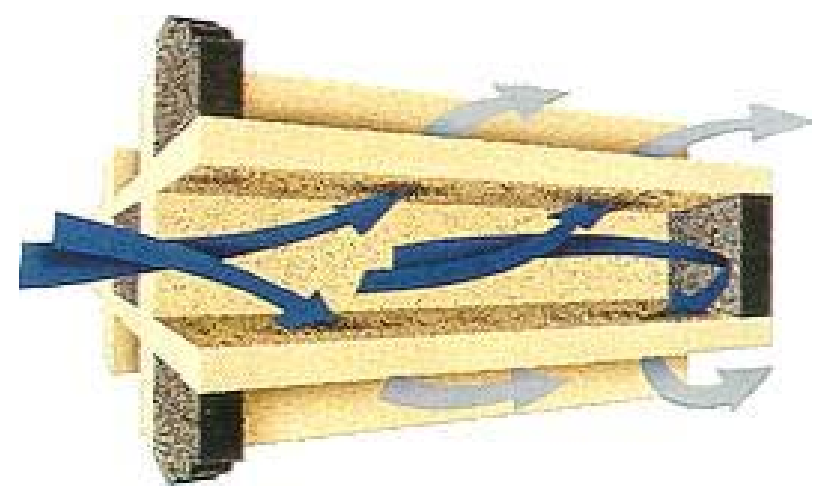

Figure 1.7 Schematic representation of flow pattern in a wall flow monolith (source: Corning).

The particulate collection mechanisms may categorized as follows:

- depth filtration where the particulates with mean diameter size lower than the filter porosity are deposited inside the porous material with a combination of mechanisms that are driven by various force fields and

- cake filtration where the particulates are deposited on the channel wall through sieving. 
The above two mechanisms may be discerned in the SEM photo of Figure 1.8 where the cross section of a loaded filter is presented.

The depth filtration relies on three mechanisms of aerosol deposition [23]:

- Diffusional deposition,

- Inertial deposition and

- Flow streamline interception.

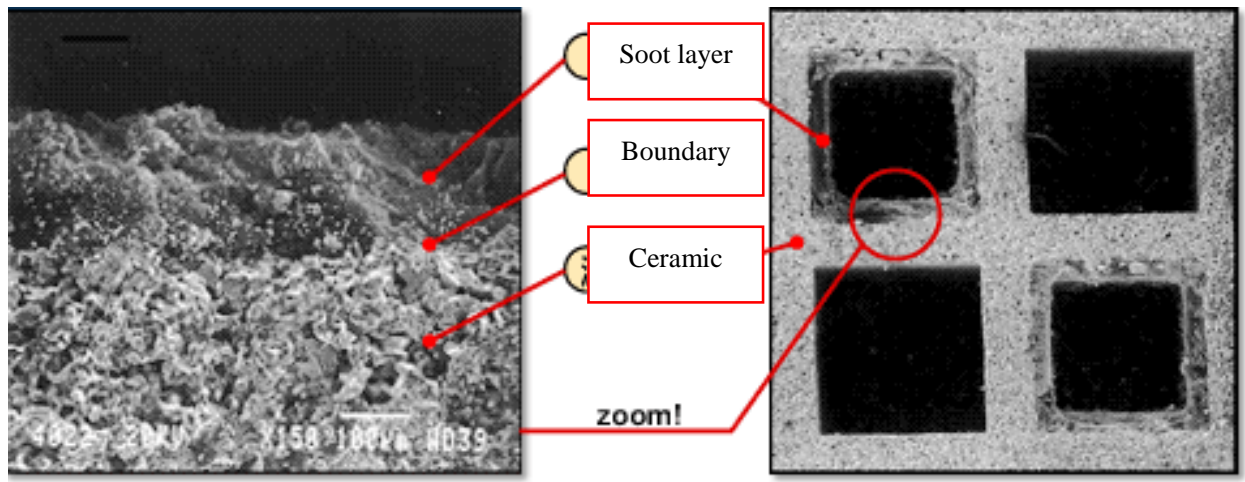

Figure 1.8 SEM photo of the cross section of a loaded DPF (source: Ibiden).

These mechanisms are illustrated in Figure 1.9, Figure 1.10 and Figure 1.11. The large circle in the middle represents a collecting body in the filter media, say a fiber in a ceramic fiber filter. Diesel exhaust gas flows around the filter media, as indicated by the streamlines. The small black-red spheres represent diesel exhaust particles traveling with the gas stream.

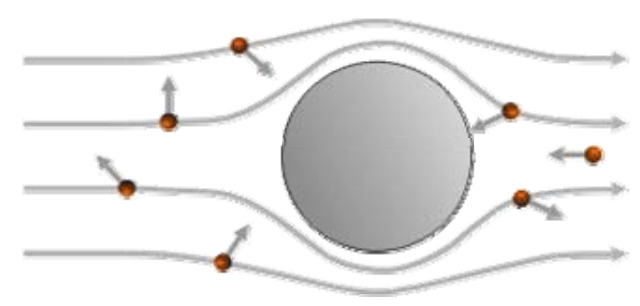

Figure 1.9 Diffusional deposition

Diffusional deposition (Figure 1.9) depends on the Brownian movement exhibited by smaller particulates, particularly those below $0.3 \mu \mathrm{m}$ in diameter. Those particulates do not move uniformly along the gas streamlines. Rather, they diffuse from the gas to the surface of the collecting body and are collected.

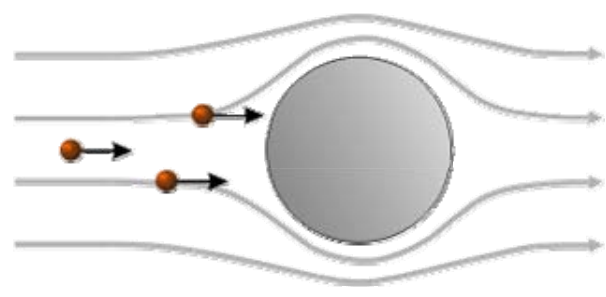


Figure 1.10 Inertial deposition

Inertial deposition (Figure 1.10), also called inertial interception, becomes more important with increasing particle size (mass). On approaching the collecting body, particles carried along by the gas stream tend to follow the stream but may strike the obstruction because of their inertia.

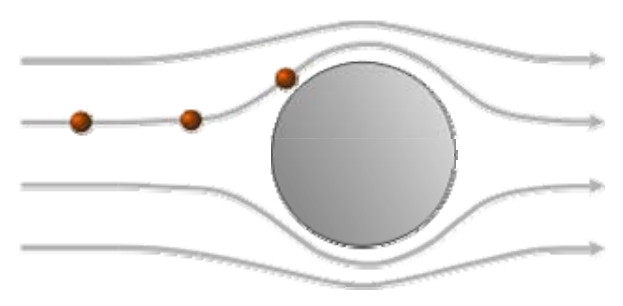

Figure 1.11 Streamline interception

Flow-streamline interception (Figure 1.11) may occur when a fluid streamline passes within one particle radius of the collecting body. Then, a particle traveling along the streamline will touch the body and may be collected without the influence of Brownian diffusion or inertia.

\subsubsection{DPF regeneration systems based on catalysts}

\section{Active regeneration systems}

A trap oxidizer system mechanically filters and collects the particulate matter from the exhaust gas, thus increasing filter backpressure. Backpressure increase is undesirable since it increases fuel consumption and reduces available torque [24]. Thus, it is necessary to clean the trap periodically by burning off (oxidizing) the collected particulate (filter regeneration). Under the conditions met in diesel exhaust systems regarding exhaust flow and oxygen concentration, the required reaction rates for complete regeneration are attained at temperatures above $550^{\circ} \mathrm{C}$. These conditions are scarcely attained under normal urban driving conditions. Numerous regeneration techniques have been suggested over the last 20 years, the most simple and effective being based on catalysts (catalytically coated filter $[25,26,27,28]$ or fuel doping [29, 30]). The significant activity in the development of advanced regeneration systems is also depicted in the numerous patent references (see for example [31, 32, 33]).

Lowering of soot combustion temperature by doping the fuel with catalytic additives (usually in the form of organometallic compounds) is a workable catalytic regeneration technique. The organic part of the solution is oxidized in the engine combustion chamber and the metallic part is incorporated in the soot in the form of fine distributed metal compounds that are in close contact with soot. Many types of metals have been suggested as fuel additives. Most popular are cerium [34, 35, 36] and iron [37, 38] due to their low cost and low toxicity. Although other metals like copper [39, 40] and molybdenum [41] are characterized by higher activity than cerium and iron, their possible adverse effects on health and environment (formation of dioxins) make them not favoured. Although the oxidation rate of soot is significantly increased, additional heating of the exhaust gas is necessary due to the low exhaust gas temperatures met in the modern diesel engines under all operation conditions. Engine controlled heating is the most practical way of temporarily raising exhaust temperatures. The preferred method is the fuel injection and timing control with the appropriate tuning of turbocharger to ensure constant bmep and to render the regeneration process unnoticeable to the driver. PSA Peugeot-Citroen was the first car manufacturer that 
commercialised such a system for serial production [42]. Figure 1.12 presents this system. It is composed of:

- a wall flow silicon carbide filter

- an oxidation catalyst in front of the filter

- a dosing system for the fuel additive (the additive is stored on the vehicle and automatically mixed to the fuel)

- a number of sensors associated with a specific engine ECU software to activate through the engine management system the regeneration process and insure the system diagnosis.

The regeneration of the filter, which occurs every $400-500 \mathrm{~km}$, is initiated by a special mode of operation of the common rail injection system. During the regeneration mode three injections are performed:

- a pilot fuel injection to control the initiation of the heat release and therefore the combustion noise

- a second injection that is the nominal injection to participate in bmep formation and to insure stable and late combustion

- a third injection at the top end of the cylinder's expansion stroke (post injection) to create the necessary exhaust gas temperature and complete bmep.

Further increase of the exhaust gas temperature is produced by the oxidation of the unburned hydrocarbons of the third injection by the oxidation catalyst placed in front of filter. The role of the oxidation catalyst is more significant at low engine loads and speeds where the increase of exhaust gas temperature up to $450^{\circ} \mathrm{C}$ is difficult without the increase of BMEP. In order to keep the engine hydrocarbons emissions at low level the efficiency of the catalyst is evaluated by the engine ECU by monitoring the catalyst inlet and exit temperatures to generate the appropriate strategy of operation of Common Rail system which controls the composition of the exhaust gas.

The functional and technical characteristics of the system have been developed and adapted for optimal vehicle integration and compatibility with the other engine components as well as for meeting requirements for health and environmental friendliness that are significant for the final customer acceptance. 


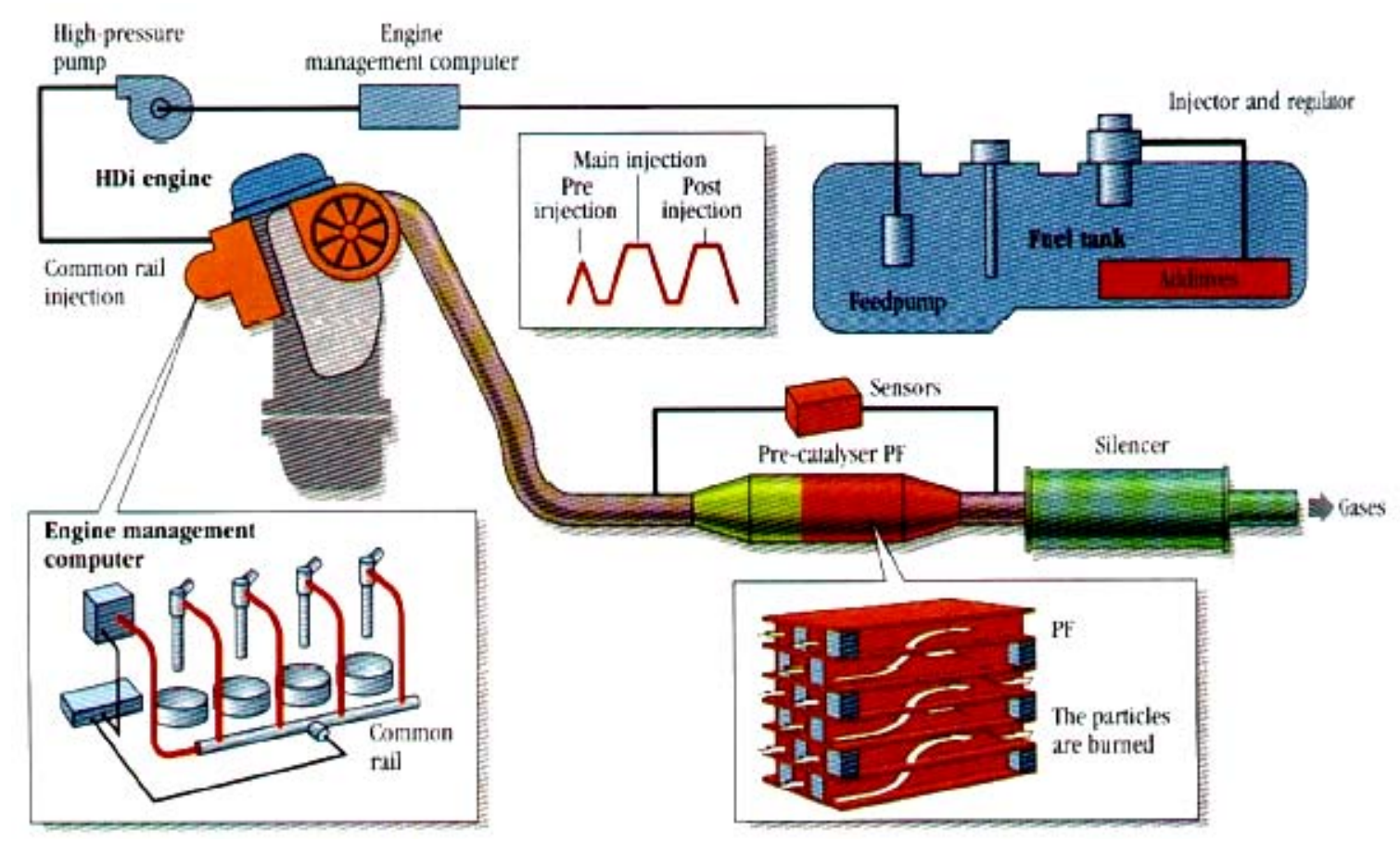

Figure 1.12 Schematic representation of an active regeneration system introduced from PSA in passenger cars in 2000 .

The design of successful catalytic fuel additive assisted trap systems depends on the solution of problems related to filter durability and additive ash accumulation. The appearance of high thermal stresses resulting from temperature gradients during low- space- velocity regeneration may cause microcracks, which reduce filter durability [43]. A common type of destructive regeneration happens upon vehicle deceleration from a high engine load to idle with a heavily loaded filter. The presence of significant quantities of adsorbed hydrocarbons is thought to have a role in this process. The disadvantage of the additive currently being used is that it causes extra ash build up in the diesel particulate filter and therefore, with increasing mileage accumulation, makes it necessary to clean the filter or even to replace it. Furthermore an extra maintenance effort is necessary to periodically replenish the on-vehicle additive tank. After every $80,000 \mathrm{~km}$ the filter must be cleaned from the additive ashes by flushing with water, and the additive replenished. However, in contrast with the precious metal catalytic filter systems, this system allows the use of fuels with sulfur levels currently legislated in the EU (350 ppm). Recently, another car manufacturer with a long tradition of diesel filter pilot applications, VW, have announced the introduction of fuel additive DPF system with Iron and Ceria based fuel additives as an option in medium to large class of diesel passenger cars [44].

\section{The Washcoated filter}

The Wash-Coated filters (Catalyzed ceramic traps) were developed in the early 80's. Their first applications included diesel powered cars and, later, underground mining machinery. Catalyzed filters were commercially introduced for Mercedes cars sold in California in 1985. The main component of the filter is the ceramic (cordierite, SiC) wall-flow monolith. The porous walls of the monolith are coated with an active catalyst. Many materials active in soot oxidation catalysts have been studied over the last 20 years as coating of a particulate filter. Mainly oxides of base metals ( $\mathrm{Ba}, \mathrm{Ca}, \mathrm{Ce}, \mathrm{Co}, \mathrm{Cr}, \mathrm{Cu}, \mathrm{Fe}, \mathrm{La}, \mathrm{Mn}, \mathrm{V}$ ) and noble metals (Pd, 
Pt) as well as mixtures of base and noble metals. A schematic of a catalyzed ceramic diesel filter is shown in Figure 1.13.

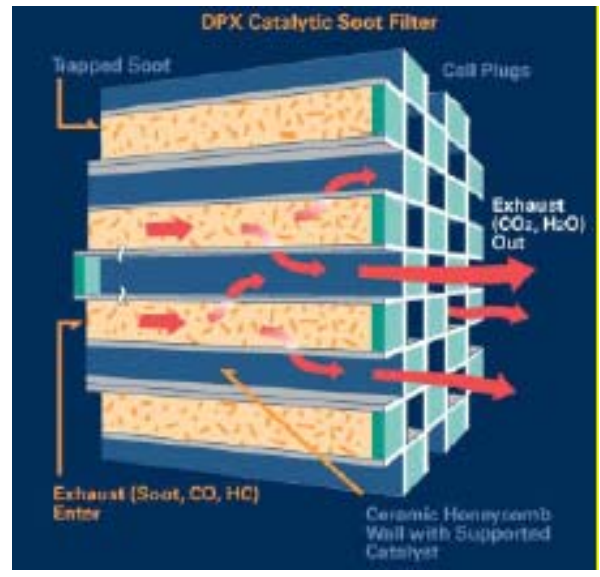

Figure 1.13 Washcoated filter (source: Engelhard Corporation)

The main drawback of these systems is the pure contact between the catalytic coating and the soot particles. The distance is too high to enforce catalytic action. Only the particulates that are in direct contact with the catalytic coating are able to oxidize. Another problem of catalyzed filters is the generation of sulfates by catalytic oxidation of the exhaust SO2 to SO3, at high exhaust temperatures. The gaseous SO3 can penetrate the porous walls and freely leave the filter. Combining with water it forms sulfate particulates that increase the total particulate matter output especially in case of fuels with high sulfur content.

Some catalytic coatings may overcome the above limitations. The liquid phase catalysts like molten salts belong to this category. These catalysts are based on eutectic mixtures of metal oxides that are in the liquid phase at relative low temperatures. Due to the liquid state the soot particles may be wetted by the catalyst improving the contact between soot - catalyst $[45,46,47]$. This type of catalysts are more suitable for foam filters [48]

Recent developments, already present in the patent literature, make the washcoated filter, combined with an advanced engine control system, a viable alternative to the fuel additive assisted systems $[49,50]$. Toyota is the first to introduce a washcoated filter system in series production (redesigned Avensis, beginning of 2004 in the United Kingdom and Germany), with the Diesel Particulate NOx reduction (DPNR) NOx catalyst used in Toyota's lean-burn petrol engine [51]. It is a combination of a diesel particulate filter with a NOx adsorbercatalyst system. The NOx adsorber-catalyst is coated onto the walls of a wall-flow, monolithic particulate filter substrate. Combination of these two components in the DPNR results in a compact size of the system. As presented in Figure 1.14 the DPNR unit is installed in the close-coupled position on the engine. Its effective operation relies on the engine management system being able to vary the fuel-air ratio in the exhaust gases. This has been made possible with the addition of a fifth injection nozzle, located in the exhaust port. At a critical moment, fuel is injected downstream of the exhaust manifold to create the right operating conditions in the DPNR catalyst. This 'rich' spike allows the DPNR catalyst to release and reduce the NOx it has stored. The system requires low-sulphur diesel, fuel that is now available in the UK, Germany and other members of the European Union.

The D-CAT system makes its debut with the 2.0-litre D-4D engine, which uses Toyota's second-generation high-pressure common rail direct injection system. This is capable of developing injection pressure of $180 \mathrm{MPa}$, regardless of engine speed, and achieves an interval between the end of pre-injection and the start of the main injection of 0.4 
milliseconds - the shortest of any engine currently available. D-CAT takes its NOx and PM emissions to extremely low levels - 50 and 90 per cent respectively below Euro IV levels.

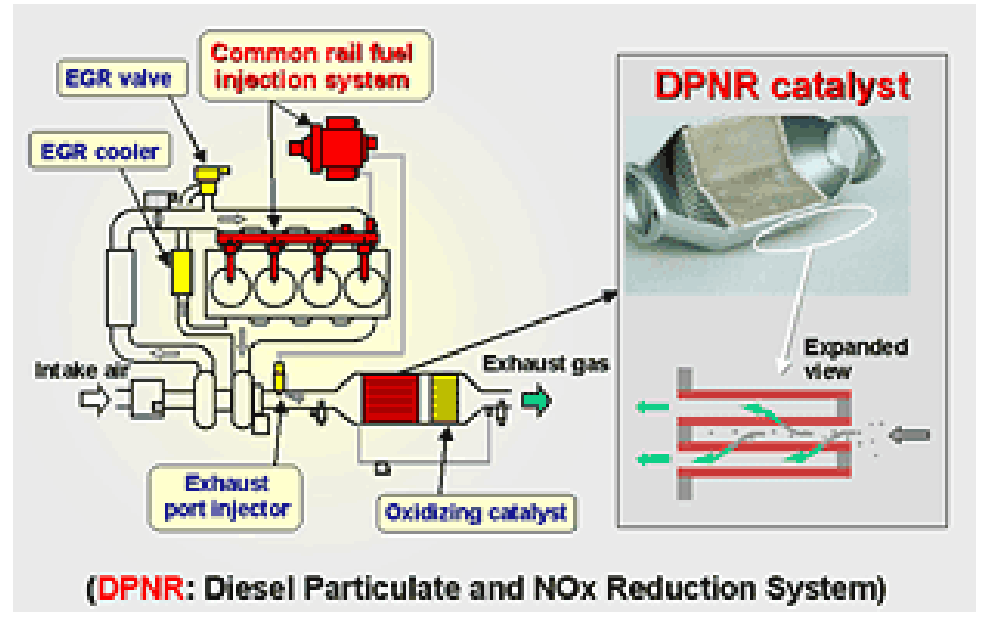

Figure 1.14 Illustration of the DPNR system developed by Toyota

The Automotive Catalyst Division of Umicore (formerly Degussa/dmc²/OMG), has also developed a new catalyst technology for washcoated diesel particulate filters [52]. These systems will initially be used in DaimlerChrysler's C-Class and E-class models with 4cylinder diesel engines, to comply with the 2005 EU IV legislation. The catalytically activated diesel particulate filters reduce harmful emissions contained in exhaust fumes from diesel engines, i.e. carbon monoxide (CO), hydrocarbons (HC) and diesel particulates, in particular those harmful emissions generated during the regeneration of diesel particulate filters due to soot burn up. The active regeneration strategy for catalytically activated diesel particulate filters, developed jointly with DaimlerChrysler, makes it possible to guarantee filter regeneration without extra additives. The opportunity to be able to adapt the catalytically activated filter to various vehicle and engine characteristics, offers further potential. Recently, VW/Audi has also announced the introduction of catalyzed DPF systems in large class vehicle models [44].

\section{Passive Regeneration systems: The CRT Filter}

The CRT (Continuously Regenerating Trap) particulate filter is a patented (initial patent by Johnson Matthey [53]) emission control technology that is based on the presence of Nitrogen oxides in the exhaust gases. As presented in Figure 1.15, the main parts of the CRT filter are a Platinum (Pt) catalyst and a particulate filter. It is modularly engineered as a totally passive emission control system, which does not require the use of supplemental heat. The CRT particulate filter requires ultra low sulfur fuel $(15 \mathrm{ppm})$ for maximum PM reduction. For reliable regeneration at lower operating temperatures, $50 \mathrm{ppm} \max , 30$ average is recommended. The CRT filter can operate at sulfur levels up to $500 \mathrm{ppm}$ but such operation requires significantly higher exhaust temperatures. 


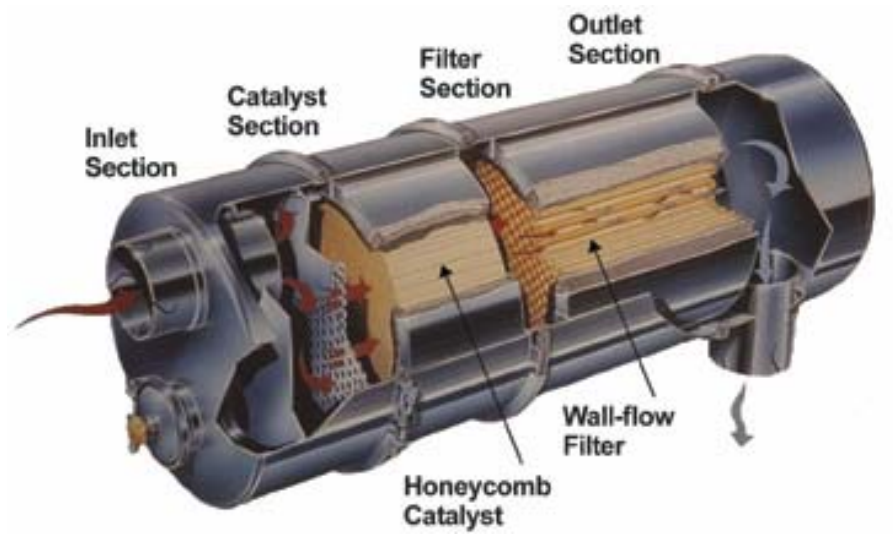

Figure 1.15 CRT filter system [53].

The CRT particulate filter uses a patented process that functions on the basis that soot will oxidize in the presence of $\mathrm{NO}_{2}$ at a lower temperature than with oxygen. In fact, this lower temperature is compatible with the typical exhaust temperature from diesel engines.

The device is made up of two chambers where the oxidation step is separate from the soot collection/combustion process. The first chamber contains a substrate coated with a proprietary, highly active Pt oxidation catalyst which is designed to oxidize a portion of the $\mathrm{NO}$ in the exhaust to $\mathrm{NO}_{2}$, which is the key to the oxidation of soot collected by the CRT filter. The catalyst also converts $\mathrm{CO}$ and $\mathrm{HC}$ into $\mathrm{CO}_{2}$ and $\mathrm{H}_{2} \mathrm{O}$. In the second chamber, the exhaust flows through a particulate filter, where gaseous components pass through but soot is trapped on the walls of the filter. The trapped soot then is destroyed by the $\mathrm{NO}_{2}$ produced by the catalyst in the first chamber. This particulate filter may be uncoated or it may be coated with Pt to further enhance the reaction of soot with $\mathrm{NO}_{2}$. The basic requirements for maximum PM reduction with the CRT particulate filter are ultra low sulfur fuel, an average exhaust temperature of $260^{\circ} \mathrm{C}$ and a NOx/PM ratio of at least 25 . For use on higher sulfur fuels the basic requirements are exhaust temperatures in excess of $350 \mathrm{C}$ with $>25 \mathrm{NOx} / \mathrm{PM}$ ratio, however, total PM reduction is reduced due to the sulfates produced.

\section{Plasma Regeneration systems}

This is a more advanced regeneration system capable to reduce diesel particulate matter attaining low oxidation temperatures. The oxidation behaviour of soot in air that have been ionised by an electric arc (thermal plasma) at temperatures in the range from 200 to $450^{\circ} \mathrm{C}$ was investigated by Levendis et al [54]. It was found that the oxidation rate might increase more than $100 \%$. This is attributed to some reactive species generated in plasma, such as $\mathrm{O}$ and $\mathrm{OH}$ radicals or $\mathrm{NO}_{2}$ that facilitate the oxidation of soot particles at low temperatures.

Two reactor configurations have been proposed to achieve this objective in a plasma device:

- Two-stage reactor, where a "classic" diesel particulate filter (DPF) is positioned downstream of the plasma generator. Such a configuration is described in a patent by Johnson Mattey [55]. The role of plasma in the two-stage configuration is to generate $\mathrm{NO}_{2}$ and, possibly, ozone, which can oxidize particulates in the downstream filter. However the applicability of the system by itself is questionable, due to the production of $\mathrm{NO}_{\mathrm{x}}$. In order to attain simultaneous reduction of particulates and $\mathrm{NO}_{\mathrm{x}}, \mathrm{a} \mathrm{NO}_{\mathrm{x}}$ reduction catalyst downstream of the filter is needed.

- Single-stage reactor, where a packed bed plasma reactor acts as the PM trapping device. A full-scale prototype of a single-stage plasma reactor for a diesel car was developed by AEA Technology. The system, shown in Figure 1.16, utilizes a bed of ceramic pellets 
placed between two cylindrical electrodes. Perforation and a system of channels in the inner electrode direct the exhaust gas flow through the pellet bed [56]. It was found that the chemistry in the case of the single-stage plasma PM-reducing device appears to be quite different. The presence of carbonaceous particulates in the reactor influences plasma reactions. When particulates are present, less hydrocarbon oxidation is observed and less $\mathrm{NO}_{2}$ is generated. Furthermore, PM reductions can be achieved at $\mathrm{HC}_{1}: \mathrm{NO}_{\mathrm{x}}<1$, a mode where $\mathrm{NO}_{2}$ production is not very efficient. Although the reaction mechanisms are not understood, it is speculated that particulates can be oxidized by different plasma species than $\mathrm{NO}_{2}$, presumably by $\mathrm{O}$ and $\mathrm{OH}$ radicals.

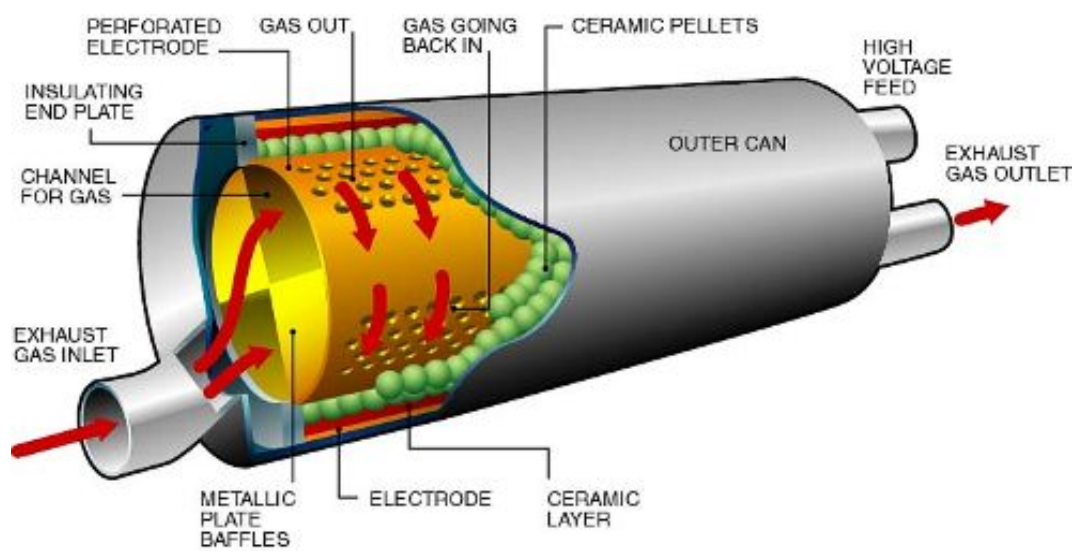

Figure 1.16 Illustration of a single-stage reactor Plasma regeneration system. Courtesy of AEA Technology.

In both configurations the plasma generator may be controlled and actuated by the engine management system, to operate intermittently according to certain engine operation conditions, which are characterized by different soot emissions.

\section{Recent trends in Diesel Emissions Control}

As we approach the year 2004-2005, (EURO 4 and corresponding US legislation significant reduction in diesel particulate emissions levels) the significant advances made in new components that are integrated into automotive powertrain systems are accelerating. These include, for the case of the diesel engine, diesel filters and diesel NOx traps, electronic throttles, and advanced common rail injection systems. In addition, the on board computers embodied in the Engine Control Units (ECU) are becoming faster and better equipped with memory and networking capabilities. Thus, more detailed physical models of complex systems, like the diesel filter, are now demonstrating real time capability, and control system tools and theories are becoming more applicable to these complex systems.

Furthermore, optimisation of the specific components is also an issue. This comprises the ceramic filter itself, the fuel additive dosing device and the post-injection system [57]. The overall design objectives for the filter as a soot reactor are the following:

- Low pressure drop,

- High filtration efficiency, for all particles independent of size, and

- Low additional system cost without the use of excessive maintenance. The cost of the system must be kept at low levels comparing with the engine total cost.

As presented above, catalytic aids are important to the success of the above objectives, and the following alternatives exist:

- catalytic activation of the soot itself by the use of fuel additives (fuel borne catalysts)

- filter coating with catalytic washcoat, and

- gas phase catalytically activated (CRT system) 
In the case of fuel additive activation, which is studied in this thesis, the dosing of the metalbased catalyst is critical for the system durability since the ash produced from the soot oxidation is also trapped in the filter differentiating its filtration characteristics.

The system integration is very important in all the above systems. This comprises the sizing of the filter, placement of filter in the exhaust, use of converter upstream, use of post injection, effect of turbocharger characteristics and position, EGR, system monitoring and control.

Detailed physical models of the diesel filter operation, especially the regeneration process, are absolutely necessary as analysis and design tools in this process. For this reason, the main findings of the experimental work carried out in the frame of this thesis, are always cross checked with the Catwall [68] diesel filter modeling software, in order to improve understanding of the processes involved and also improve the capacity of our computational software.

One main goal of this chapter is to demonstrate the synergy between the various experimental investigations carried out in this work in order to develop improved engineering models that enhance our understanding of the diesel filter operation and can be used as component, system and control design aids.

The main points where the regeneration process lacks a good understanding and modeling capability can be categorized as follows:

- Reaction schemes and kinetics of the catalytic regeneration process

- The role of the volatile organic fraction of particulates (adsorption, desorption, stochastic regeneration)

- 3D effects during filter loading (flow maldistribution, instabilities during soot loading, soot maldistribution)

- 3D effects during catalytic regeneration (flow maldistribution at filter inlet, soot maldistribution, effect of filter material and design), and

- Correlation between pressure drop and accumulated soot mass.

\subsection{Scope and Objectives of this Thesis}

- Although diesel filter applications date back to 1980's, the diesel engine was subjected to revolutionary design advances during the 1990's (HDI, common rail etc). This changed the characteristics of diesel particulate emissions. Especially the performance with fuel additives was severely affected (VOF content, etc.) An extensive search in the specialized publications and patent literature shows a lack in reliable estimation of specific particulate properties (morphology, density, permeability etc). The effects of engine type and operation point are important and not yet well investigated and understood.

- Traditionally, soot oxidation kinetics was determined with the use of Thermogravimetric Analysis (TGA) either with carbon black or with soot collected on Pallflex filters according to the particulate emissions measurement legislation (diluted exhaust, low temperatures). Such kinetics are not representative of the behavior of soot deposited on the filter wall at high temperatures, which is loaded with varying percentages of adsorbed VOF. It became clear that a method to sample directly from the filter was necessary to develop and apply.

- The effect of VOF on the catalytic soot oxidation kinetics was considered important based on experience, but was never attempted to understand and model. Recent 
developments on modeling of this effect reported in Pontikakis Thesis [70], allowed the pilot application of a scheme of oxidation reactions for unburned hydrocarbons. This was only initiated in the frame of this work, because of problems with tuning and validating such a complex model. Also the effect of the upstream diesel oxidation catalyst on the VOF content needs to be addressed.

- Although significant work has been done internationally in filter pressure drop characteristics (due to the importance in the vehicle application) the results are not satisfactory, mainly due to unrealistic assumptions regarding the homogeneity of soot and flow distribution across the different channels. The real problem is extremely complex and its better understanding would require the application of CFD in 3D for the filter, comprising at least the inlet diffuser. In this work a starting point is set up by the study of soot and flow maldistribution characteristics in typical channels.

- An important overall objective in terms of systems applicability is to correlate the collected soot mass to the pressure drop and other variables (exhaust temperature, flow distribution etc). This is important for the improvement of the design of the system control and its adaptation to the engine management. Some progress in this direction was done in the frame of this work. The results are not so promising, because of the complex effects of flow and soot maldistribution that have been demonstrated in the experiments.

- Although certain aspects of the regeneration process are already well understood and modelled, there remain important aspects that are not yet well understood. At the same time these aspects are most critical for the reliability of the system. Filter failure is happening at low flowrates, usually associated with stochastic regeneration events that are triggered by the catalytic fuel additive. These critical conditions of filter operation are studied in this work by means of carefully designed experiments.

- A final objective of this thesis is to support improved modelling of the regeneration process. A systematic attempt was made to capitalize the findings of the experimental research in the Catwall $1 \mathrm{D}$ and 3D regeneration models. The models were subjected to a validation procedure, against full-scale tests of the regeneration behaviour of a diesel filter fitted to a modern diesel engine run on catalyst-doped fuel under carefully designed and controlled operating conditions. The extensive validation process over a wide range of filter design and operation parameters resulted in significant improvements of the models.

The interrelation of the above-mentioned battery of experimental subtasks of this work with the main fields of research in Diesel particulate filters is schematically presented below: 
Fields of Research in Diesel Filters

Experimental Subtasks

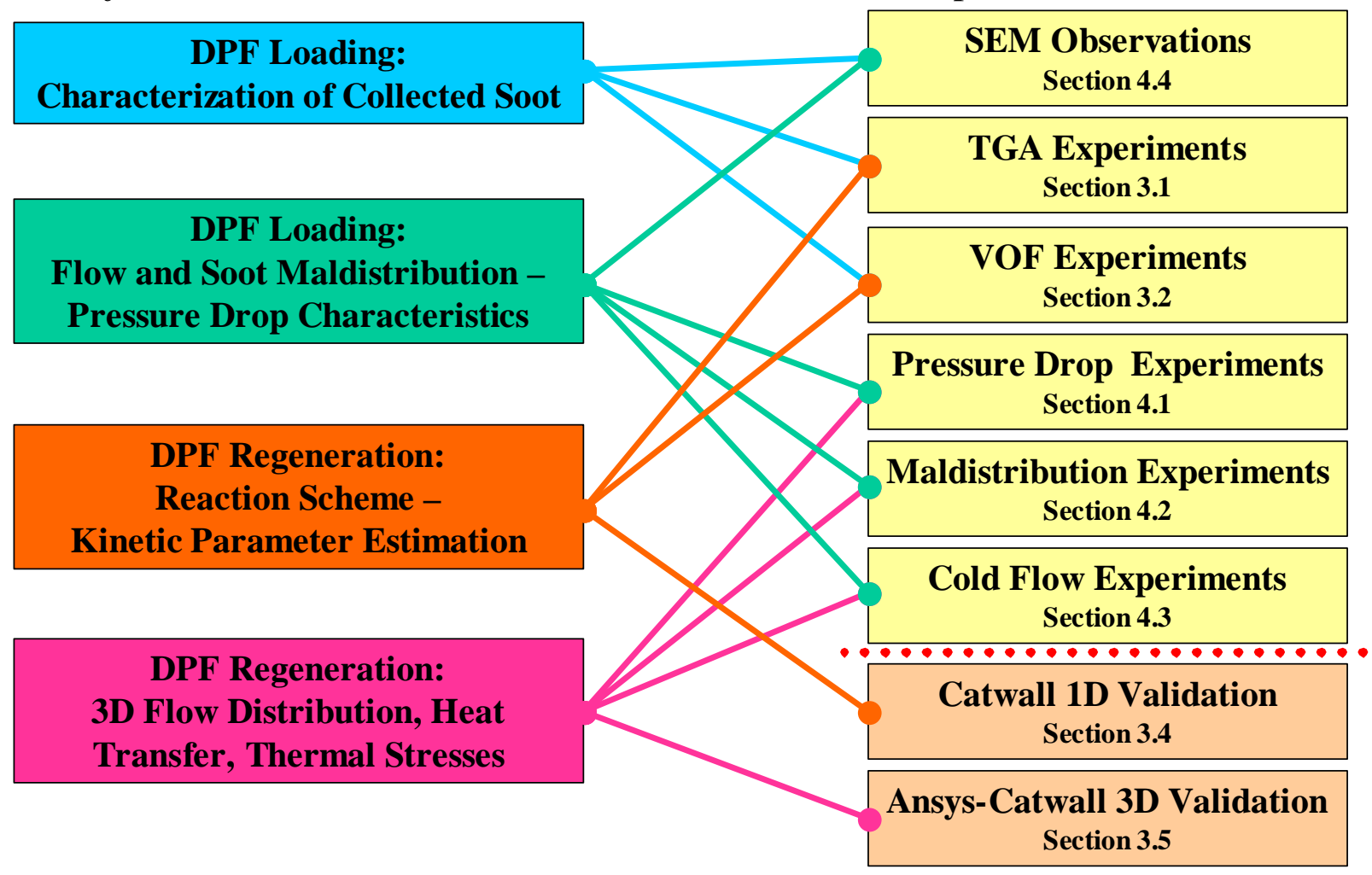




\section{Physical \& Chemical Processes Inside the Filter}

Diesel Particulate Filters are porous media. The processes such as the combustion of soot during regeneration and, more generally, the transport phenomena that occur in inert catalytic and combustible porous media are non-equilibrium processes [58]. The phenomena related with porous media occur under the influence of a large range of geometric length scales, thermophysical and thermochemical properties, and flow, heat and mass transfer conditions as a result a large range of phenomenological length and time scales control the extent of departure from local thermal and chemical process. The analysis is based on the continuum viewpoint [59], since the Knudsen number, $\mathrm{Kn}$, is small compared to unity (the mean free path of the exhaust molecules is about $0.3 \mu \mathrm{m} \alpha \tau 1000 \mathrm{~K}$, whereas the mean pore size is $10-20$ $\mu \mathrm{m}$ for the ceramic and a few microns for the soot).

\subsection{Loading}

As mentioned in previously section the wall flow particulate filter is today the most efficient device for reducing diesel soot emissions, attaining filtration efficiencies of the order of $95 \%$ at nominal operation conditions. The particulate filter concept is based on a durable temperature resistant filter, which mechanically removes particulate matter from the exhaust before it is emitted to the atmosphere. The accumulated particles raise the filter backpressure (the pressure drop across the filter), where the exhaust through it. The typical backpressure levels depend on the filter type [60,61] and increases as the collected soot mass also increases. High backpressure is undesirable since it increases fuel consumption and reduces the available power. A typical filter loading curve is presented in Figure 2.1.

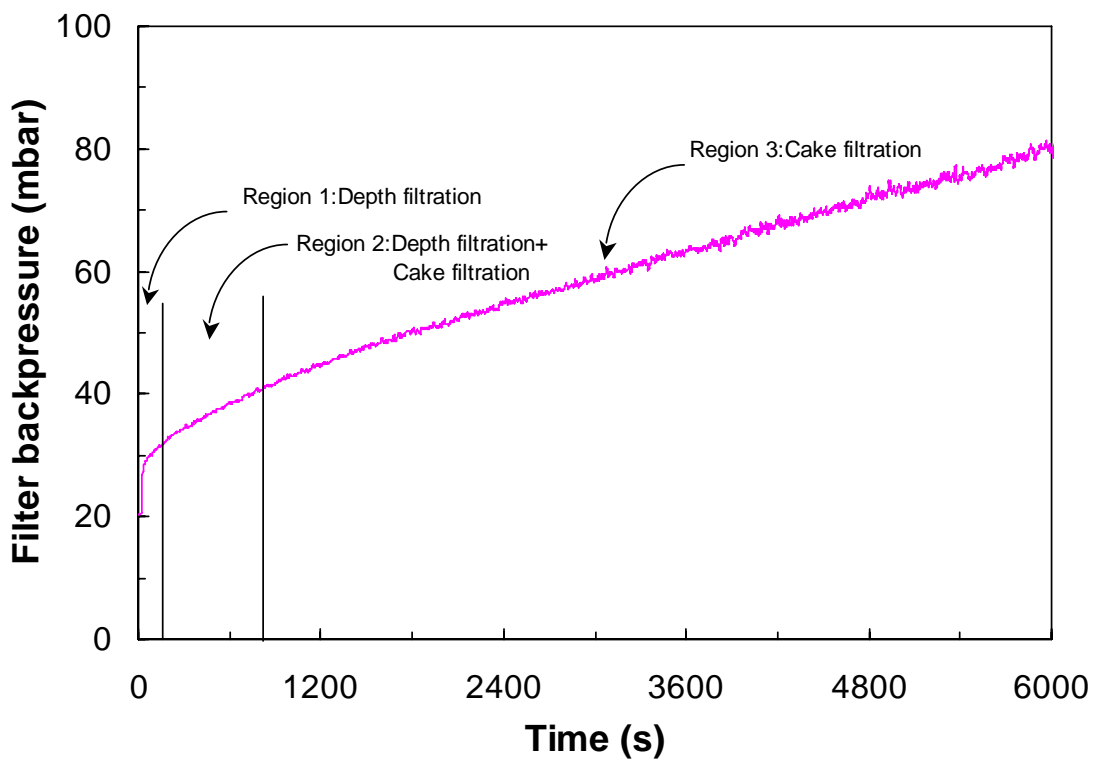

Figure 2.1 Recording of a filter loading process of a SiC 14/200 filter with the engine operating at a medium speed and load operation point.

We could observe three characteristic parts in the recording of the loading process: 
- The first incorporates the phase of the beginning of loading where the particulates with size lower than the material pores accumulated inside the porous wall. As described in the previous section the mechanisms of depth filtration governs the soot accumulation during this phase. It can be observed that the pressure drop increases very rapidly due to the reduction of the wall porosity.

- In the second part the wall porosity decreases enough and the particles with size higher than this of pores begin to deposit also on the wall. This is a rather short transition phase where the mechanisms of depth and cake filtration are active. As soon as a finite particulate layer is formed, the porous wall begins to act as a filter.

- In the third part the porous wall reaches a 'steady state' such that the porosity of the trap remains unchanged. Following this condition, particles continue to accumulate more and more on the surface of the wall, rather within the wall. The cake filtration mechanism governs this phase. Within this region the pressure drop becomes a linear function of the soot mass trapped in the filter. Consequently soot layer thickness changes in a quasiisotropic mode.

However variations on soot loading or quality among different channels exist, during the loading phase as result of flow maldistribution phenomena and the effects of

- volatile organic fraction adsorption-desorption phenomena and

- stochastic regeneration phenomena that are triggered by the temperature differences between the central and peripheric channels of the filter.

These phenomena are discussed in detail in the next sections of this thesis.

\subsection{Regeneration}

Diesel particulate filters must be periodically cleaned, that is, the particulate matter that is accumulated in the filter must be removed, otherwise the filter becomes clogged and its operation is inhibited. The filter regeneration process depends on the particular application, especially on the filter type and the collected matter. The most straightforward method of diesel particulate removal is combustion. The special regeneration techniques that employed fall into the following categories:

- Thermal regeneration by use of engine measures or by the supply of external energy

- Catalytic regeneration (catalytically coated filter or fuel doping)

\subsubsection{Thermal regeneration}

Thermal regeneration of diesel particulate filters involves the oxidation of regular solid particulates to gaseous products. The rate of thermal regeneration is limited by the kinetics of soot oxidation. The oxidation of soot, as any other chemical process, has a reaction rate, which depends on the temperature, and concentration of reactants, and can be accelerated by the presence of catalysts. The rate of soot oxidation is significantly lower than that of the oxidation of gaseous exhaust pollutants, such as carbon monoxide. This is one of the reasons why carbonaceous particulates are not oxidized in flow-through catalysts, which provide relatively short particle residence times. In the case of diesel filters the physical process of capturing the particulates extends the residence time and the oxidation is easier. Two requirements must be satisfied in order to begin a thermal regeneration :

- $\quad$ the temperature of the exhaust gas is high enough (over $550^{\circ} \mathrm{C}$ ) 
- and the oxygen concentration in the exhaust flow is enough to oxidize the accumulated soot

Due to the non-uniform distribution of temperature and flow the regeneration starts at certain points where the conditions are favourable. The propagation of the regeneration process depends on the exhaust gas flow, the distribution of the temperatures and the amount of the accumulated soot mass. If the collected amount of soot in the filter is too high, regeneration of the filter may result in a thermal runaway. During such a runaway, temperatures higher than $1350 \mathrm{~K}$ may occur, not only depending on the regeneration conditions but also on the thermal conductivity of the filter. At the high temperatures that can occur during a regeneration under high soot mass loading and low exhaust flowrate conditions, cracks may occur in the filter channels. Consequently, too high temperatures during regeneration should be prevented by keeping low the total amount of the collected particulates. In this direction, catalysts can be applied to increase the oxidation rate of soot at low temperatures.

\subsubsection{Fuel doped catalytic regeneration}

The most effective application of catalysts in diesel particulate filter systems is based on the mixing of organometallic compounds of various metals in the fuel in very small quantities. The additive is oxidized in the combustion chamber and its oxides form the kernels of particulates. The soot particles bonded with the metal oxides are deposited on the filter wall and oxidized at lower temperatures than those with oxygen. These temperatures in the case of transition metals such as $\mathrm{Cu}, \mathrm{Fe}, \mathrm{Ce}$ and $\mathrm{Pb}$ that finally dispersed during the fuel combustion process throughout the soot particles, may be as low as $350^{\circ} \mathrm{C}$ [62]. A simplified consideration of the mechanism of fuel additive action during regeneration is presented in Figure 2.2. The active oxygen atoms of the additive oxides may react with the carbon at exhaust temperatures of the order of $350^{\circ} \mathrm{C}$ and initiate the oxidation process. After this initial phase of ignition the oxidation process is expanded and the additive oxide particles begin to precipitate as the carbon mass is decreased. The resulting additional exothermic reactions may increase local the temperature to above $550^{\circ} \mathrm{C}$, thus allowing fast oxidation of carbon by exhaust gas oxygen. Finally all the carbon is oxidized and the precipitated additive is accumulated on the channel wall forming a layer of ash.

Catalytic regeneration by use of fuel additives is a quite complex process, which is not yet well understood and modelled. The instability to the appearance and evolution of regeneration at temperatures down to $300^{\circ} \mathrm{C}$ under favourable engine and filter operating conditions (city part load driving conditions), is one of the most significant problems since it is related to filter durability as mentioned below. The role of particulate volatile organic fraction that tends to oxidize in the presence of catalyst at low temperatures (lower than $300^{\circ}$ ) and its relation to the flow and mass maldistribution phenomena is proved to be significant for the appearance of such instabilities. This behaviour known as erratic or stochastic regeneration behaviour is discussed in the next sections.
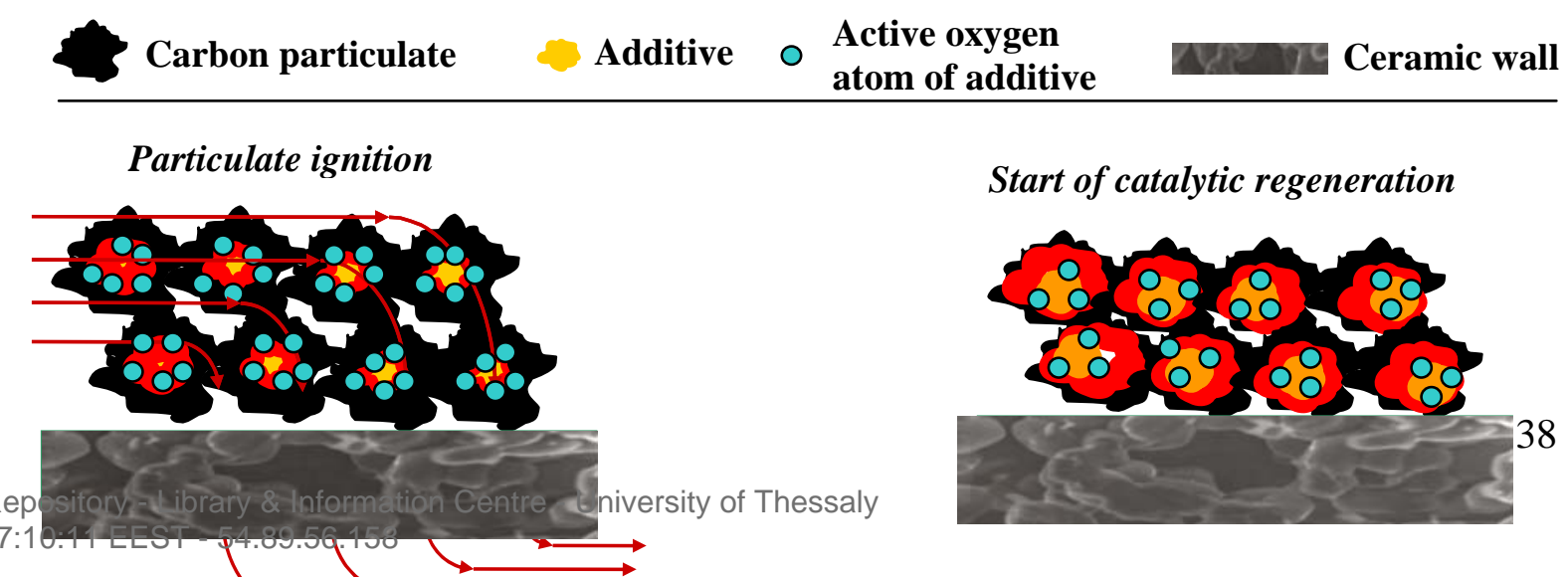
Figure 2.2 Schematic representation of a simplified consideration of the mechanism of catalytic regeneration in wall flow ceramic filters.

The design of successful catalytic fuel additive assisted trap systems depends on the solution of problems related to filter durability and additive ash accumulation. The appearance of both high temperature and high thermal stresses resulting from temperature gradients during a low flow rate regeneration of a usually non uniform loaded filter may cause local melting or cracking of the filter thus reducing its durability [43]. A common type of uncontrolled regeneration happens upon vehicle deceleration from a high engine load to idle with a heavily loaded filter. The presence of significant quantities of adsorbed hydrocarbons is thought to have a role in this process.

\subsubsection{Modeling catalytic regeneration}

\section{General}

Design optimization of diesel particulate filter systems is increasingly dependent on efficient modeling of the filter regeneration process [63]. The basic tools in this process are 1$\mathrm{D}$ models, because of their speed and simplicity, while multi-dimensional models are employed during the final stages of the design process for the detailed component design. In principle, a 1-D model should incorporate the effects of the following parameters:

- Filter material and filtration characteristics (material density, mean pore size, porosity, permeability)

- Filter thermophysical properties (specific heat capacity, thermal conductivity etc)

- Filter geometry (cell density, wall thickness, plug length, filter size)

- Soot deposit filtration characteristics (soot density, permeability)

- Soot thermophysical properties (specific heat and thermal conductivity)

- Soot composition (VOF content)

- Hydrocarbon adsorption/ desorption - combustion characteristics

- Fuel additive type and concentration in fuel and soot

- Engine operation point (exhaust gas mass flow rate, soot emissions, fuel consumption) 
Although a fairly large variety of models are emerging during the last years in the literature, the pioneering work of Bisset and Shadmann [64] remains a valid, mathematically proper and well-documented approach for thermal regeneration modeling that deserves careful study by any newcomer in the field. This work has been adopted and extended in [65], allowing the reliable study and experimental validation of high space velocity thermal regeneration events, with a systematic methodology of filter loading assessment by energy balances. It was further extended to cover catalytic regeneration [66], and its 1-D version [67], formed the basis of commercial software [68], currently employed in diesel exhaust systems design by several automotive and filter manufacturers.

There are two aspects in diesel filter modeling that challenge very much the accuracy and predictive ability of models:

- The first is the modeling of filter backpressure that is important for correct assessment of soot loading [69].

- The second one is the study of kinetic scheme and parameters of catalytic soot oxidation, also comprising adsorbed hydrocarbon oxidation.

A main difficulty encountered in the validation of diesel filter regeneration models, especially as regards the filter backpressure prediction, lies in the extraction of realistic particulate properties by the experiments. Tests with full-scale filters, loaded and regenerated under real-world operating conditions are more realistic than those with mini scale filters and synthetic soot accumulation, although the operating conditions (particulate loading, ash accumulation, exhaust mass flow rate, exhaust gas properties) are more difficult to control. For this reason, model validation in this thesis is based on the simulation of standardized, fullscale filter regeneration experiments under various scenarios with varying soot loading, VOF content and fuel additive concentration in fuel.

Together with the pressure drop calculation, reaction kinetic scheme and kinetic parameters form the core of every diesel filter regeneration model. Nevertheless, out of the numerous works cited in the literature on diesel filter modeling, only a few employ an inclusive reaction scheme that introduces all necessary degrees of freedom in order to model real-world filter operation. This is a severe drawback, especially when one deals with the complex problem of fuel additive assisted regeneration at low temperatures. Therefore, the model presented in [70] includes a reaction scheme that accounts for catalytic chemical reactions, and its validation process focuses especially on the reaction kinetics.

As already mentioned, the improved 1-D model update developed in [70] is considered as a basic design tool that is employed in the initial design stage of the DPF, to carry out kinetic and pressure drop parameter estimation, and to perform parametric optimization studies. When one moves to the detailed design of components, then other tools as 3-D stress modeling and CFD modeling come to the foreground.

\section{Geometry of the channels and the soot layer}

The DPF consists of a ceramic grid of parallel channels. Adjacent channels are alternatively plugged at each end in order to force the diesel aerosol through the porous substrate walls which act as a mechanical filter. Thus, diesel particulate matter is deposited on the four sides of the inlet channels. Because of the repeating geometry of the DPF, the mathematical treatment may be restricted to a fundamental channel volume of the trap as defined in Figure 2.3. That is, this fundamental volume consists of one inlet channel and four quarters of the four adjacent outlet channel. The boundaries of the volume are assumed 
adiabatic and no flow occurs through them to the rest of the outlet channel. From the standpoint of modeling, the whole trap is constructed by repeating this fundamental volume.

To formulate the balance equations pertinent for regeneration modeling, we consider a small part of this fundamental volume, of length $\Delta z$ (Fig, 2.4). At this part of the channel, a layer of soot of mass $m$ and thickness $w$ has already accumulated. The wall's thickness is $w_{s}$ and the length of the channel edge is $\ell$. Exhaust gas flow is perpendicular to the soot layer and wall. Exhaust gas species are consumed or produced as gas flows through the soot layer. In this work, we shall use the subscript $p$ to denote the physical properties of the soot layer and $s$ to denote the corresponding properties of the wall.

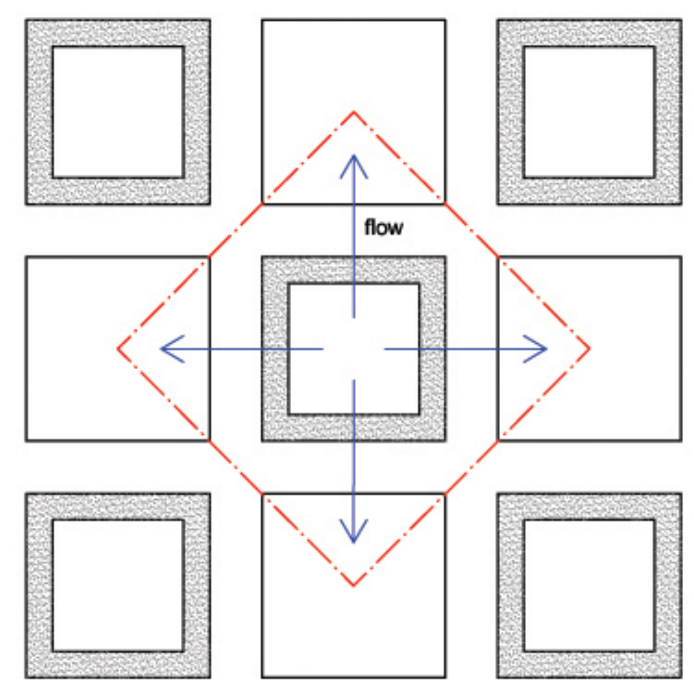

Figure 2.3 Fundamental volume of a wall flow diesel particulate filter consisting of one inlet channel and four adjacent outlet channels.

It is evident from Figure 2.4 that the soot layer consists of four trapezoids. On earlier works [67], the trapezoids were approximated as rectangles but the approximation yields considerable error regarding the soot layer thickness, especially for highly loaded filters.

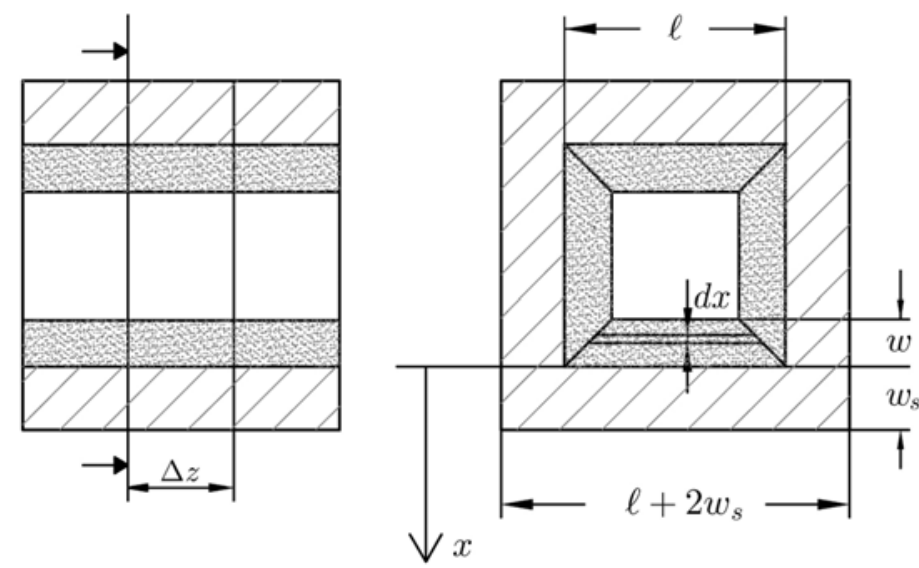

Figure 2.4 Schematic representation of the cross section of a loaded wall flow channel assuming that the soot layer consists of four trapezoids.

The layer thickness calculation is important especially in the calculation of the model's backpressure. Therefore, in this work, the exact description of the soot layer geometry is 
introduced. Assuming that some soot mass $m$ of density $\rho_{p}$ is deposited on a filter channel with inner side length $\ell$, the deposit thickness can be calculated as:

$$
w=\frac{1}{2}\left(\ell-\sqrt{\ell^{2}-\frac{m}{\rho_{p} \Delta z}}\right)
$$

This relation is used by the model to compute the thickness of the deposit, which, in its turn, is needed for the calculation of the filter pressure drop and flow field distribution. As a consequence of the trapezoid distribution of the soot layer, the area perpendicular to the flow $A(x)$ (i.e. the area that the flow 'faces' at each $x$ ) is also changing along $x$ :

$$
A(x)=\left\{\begin{array}{cc}
4(2 x+\ell) \Delta z, & -w \leq x \leq 0 \\
4 \ell \Delta z, & 0 \leq x \leq w_{s}
\end{array}\right.
$$

The volume of the soot layer may be readily calculated from the above relationship.

$$
V_{p}=4 \Delta z(l-w) w
$$

\section{Reaction kinetics}

As previously mentioned, when a fuel additive is used to assist regeneration, additive is present in the formation process of the particles in the engine combustion chamber. Therefore meatal bonded soot particles are produced that are deposited in the trap. When the conditions of temperature are favourable the additive oxides may react with the carbon and VOF contained in soot setting up the oxidation process.

The kinetic model that will be described in this section accounts for catalytic combustion of soot owing to a Ce-based fuel additive, but does not include the effect for the VOF. The model for catalytic oxidation of carbon was first formulated by Koltsakis and Stamatelos [65]. The implemented mechanism is only valid when the catalytic oxide has two oxidation states, which is true for Ceria. When Ceria is in a low oxidation state, it can form a 3-valent Ce oxide $\left(\mathrm{Ce}_{2} \mathrm{O}_{3}\right)$; when it is in a high oxidation state, it can form a 4-valent Ce oxide $\left(\mathrm{CeO}_{2}\right)$. The first step of the mechanism is oxidation of $\mathrm{Ce}_{2} \mathrm{O}_{3}$ by $\mathrm{O}_{2}$ to produce $\mathrm{CeO}_{2}$. The second step involves reaction of $\mathrm{CeO}_{2}$ with soot carbon. Ceria returns to its 3-valent state, while carbon monoxide is produced. The presence of additive does not prevent direct oxidation of carbon by Oxygen, if the temperature is sufficient for this reaction. The reactions are given along with their reaction rates in Table 2.1.

The rates for all reactions are assumed to follow first order Arrhenius-type expressions. For the thermal carbon oxidation and the $\mathrm{Ce}_{2} \mathrm{O}_{3}$ oxidation the rate is proportional to the oxygen concentration in the exhaust gas. In the two reactions of catalyst assisted regeneration the quantity $\psi$ is employed, representing the percentage of mol Ce at its higher oxidation state $\mathrm{CeO}_{2}$ :

$$
\psi=\frac{\mathrm{mol} \mathrm{CeO}_{2}}{\mathrm{~mol} \mathrm{Ce}}=\frac{\mathrm{mol} \mathrm{CeO}_{2}}{2 \times \mathrm{mol} \mathrm{CeO}_{2}+\mathrm{mol} \mathrm{CeO}_{2}}
$$

The term $\xi$ will also be needed in this study. It is defined as the concentration of catalyst in the soot layer and is a function of metal additive concentration in the fuel. It may be expressed as:

$$
\xi=\frac{\mathrm{mol} \mathrm{Ce}}{\mathrm{mol} \mathrm{soot}}=\frac{2 \times \mathrm{mol} \mathrm{Ce}_{2} \mathrm{O}_{3}+\mathrm{mol} \mathrm{CeO}_{2}}{\mathrm{~mol} \mathrm{C}}
$$


It should be noted that both $\psi$ and $\xi$ are viewed as averaged quantities along the soot layer thickness.

Table 2.1 Reaction and rate expressions of the regeneration model

\begin{tabular}{lll}
\hline & Reaction & Rate expression \\
\hline 1 & $\mathrm{C}+\mathrm{O}_{2} \longrightarrow \mathrm{CO}_{2}$ & $r_{1}=k_{1} y$ \\
2 & $\mathrm{C}+0.5 \mathrm{O}_{2} \longrightarrow \mathrm{CO}$ & $r_{2}=k_{2} y$ \\
3 & $\mathrm{C}+4 \mathrm{CeO}_{2} \longrightarrow 2 \mathrm{Ce}_{2} \mathrm{O}_{3}+\mathrm{CO}_{2}$ & $r_{3}=k_{3} \psi$ \\
4 & $\mathrm{C}+2 \mathrm{CeO}_{2} \longrightarrow \mathrm{Ce}_{2} \mathrm{O}_{3}+\mathrm{CO}$ & $r_{4}=k_{4} \psi$ \\
5 & $\mathrm{Ce}_{2} \mathrm{O}_{3}+0.5 \mathrm{O}_{2} \longrightarrow 2 \mathrm{CeO}_{2}$ & $r_{5}=k_{5}(1-\psi)$ \\
& Where: $k_{i}=A_{i} e^{-E / R_{g} T} i=1 \ldots 5$ & \\
\hline
\end{tabular}

Mass and heat balances in the washcoat (regeneration submodel)

As gas flows through the soot layer, the oxygen is gradually depleted because of reaction with carbon and Ceria. An oxygen concentration profile is thus established through the soot layer, which is expressed by an oxygen mass balance:

$$
\frac{\dot{m}_{g}}{A(x)} \frac{d y}{d x}=-M_{g}\left(k_{1}+0.5 k_{2}+0.5 k_{5}(1-\psi)\right) y
$$

This mass balance equation may be solved to get the oxygen concentration profile $y(x)$, for the initial condition $y(x=-w)=y_{\text {in }}$, provided that the temperature of the washcoat is considered uniform along the soot layer. This is a fundamental assumption that is supported by the original work of Bissett and Shadmann [64] (see also the boundary conditions below). The solution yields the total consumption of oxygen $\Delta y=y(x=-w)-y(x=0)$ through the soot layer:

$$
\Delta y=y_{i n}\left[1-\exp \left(-\frac{M_{g}\left(k_{1}+0.5 k_{2}+0.5 k_{5}(1-\psi)\right)}{\dot{m}_{g}} V_{1}\right)\right]
$$

The total consumption of oxygen may be used in order to calculate the rate of soot consumption because of both thermal oxidation with oxygen and catalytic oxidation with Ceria. Specifically, the mass balance for the soot mass consumption is:

$$
\frac{\rho_{p}}{M_{C}} \frac{d V_{p}}{d t}=-\int_{-w_{p}}^{0}\left[\left(k_{1}+0.5 k_{2}\right) y+\left(k_{3}+k_{4}\right) \psi\right] A(x) d x
$$

or, splitting the integral:

$$
\frac{\rho_{p}}{M_{C}} \frac{d V_{p}}{d t}=-\left(k_{1}+0.5 k_{2}\right) \int_{-w_{p}}^{0} y A(x) d x-\left(k_{3}+k_{4}\right) \psi \int_{-w}^{0} A(x) d x
$$


The first integral contained in the right hand side of the above equation may be calculated from the oxygen mass balance (2.2.6):

$$
\int_{-w_{p}}^{0} y A(x) d x=\frac{\dot{m}_{g}}{M_{g}\left(k_{1}+0.5 k_{2}+0.5 k_{5}(1-\psi)\right)} \Delta y
$$

The second integral of the soot mass balance is the volume of the soot layer $V_{p}$. Substituting the above, we get the following rate of soot mass consumption:

$$
\rho_{p} \frac{d V}{d t}=\frac{M_{C}}{M_{g}} \frac{k_{1}+0.5 k_{2}}{k_{1}+0.5 k_{2}+0.5 k_{5}(1-\psi)} \dot{m}_{g} \Delta y-M_{C} k_{5} \psi V_{p}
$$

A mass balance must also be formulated for $\psi$, which expresses the continuous transition of the catalytic additive between $\mathrm{CeO}_{2}$ and $\mathrm{Ce}_{2} \mathrm{O}_{3}$ states. According to the $\mathrm{CeO}_{2}$ mass balance, the rate of change depends on the additive oxidation and reduction reactions, i.e.:

$$
\left(\int_{-w_{p}}^{0} \frac{\rho_{p} A(x) d x}{M_{C}}\right) \xi \frac{d \psi}{d t}=\int_{-w_{p}}^{0}\left[-\left(4 k_{3}+2 k_{4}\right) \psi+k_{5} y(1-\psi)\right] A(x) d x
$$

The above equation may be simplified and, with the substitution of (2.2.10), yields the total rate of change for $\psi$ :

$$
\xi \frac{d \psi}{d t}=-\frac{M_{C}}{\rho_{p}}\left(4 k_{3}+2 k_{4}\right) \psi+\frac{k_{5}(1-\psi)}{k_{1}+0.5 k_{2}+k_{5}(1-\psi)} \frac{\dot{m}_{g}}{\rho_{p} V_{p}} \frac{M_{C}}{M_{g}} \Delta y
$$

The two mass balance equations for soot and Ceria should be solved for the following initial conditions: For $t=t_{0}$ we have $V_{1}=V_{1,0}$ and $\psi=\psi_{0}$.

To complete the model, a heat balance equation should be formulated. This expresses the accumulation of heat in the washcoat layer and the ceramic wall because of (a) heat generation owing to the chemical reactions, (b) heat conduction perpendicular to the soot layer and wall and (c) heat exchange between the gaseous and the solid phase.

$$
\rho_{j} c_{p, j} A(x) \frac{\partial T}{\partial t}=-\dot{q}(x) A(x)+\frac{\partial}{\partial x}\left(A(x) \lambda_{j} \frac{\partial T}{\partial x}\right)-\dot{m}_{g} c_{p, g} \frac{\partial T_{g}}{\partial x}
$$

where

$$
\dot{q}(x)=\left\{\begin{array}{cc}
\sum_{n=1}^{9} r_{n} \Delta H_{n} & \text { if }-w_{p} \leq x \leq 0 \\
0 & \text { if } \quad 0 \leq x \leq w_{s}
\end{array}\right.
$$

Obviously, no reactions occur as the gas flows through the ceramic wall, and thus $\dot{q}$ is zero in this region.

The boundary conditions for the heat balance equation are very important in the total mass balance formulation:

$$
\begin{aligned}
\text { At } x=-w_{p}: & A(x=-w) \lambda_{p} \frac{\partial T}{\partial x} & =m_{g} c_{p, g}\left(T-T_{g, \text { in }}\right) \\
\text { At } x=+w_{s}: & \frac{\partial T}{\partial x} & =0
\end{aligned}
$$


The boundary condition at $x=-w$ deserves some comments. It implies that the gas and the solid temperatures are equal everywhere except at the inlet face of the deposit layer and, thus, heat conduction in the direction perpendicular to the soot layer and wall is zero. This boundary condition was introduced by Bissett and Shadmann [64].

Under these boundary conditions, the heat balance (2.2.14) is integrated to yield the rate $d Q / d t$ of heat released (a) by chemical reactions and (b) by convection because of flow normal to the soot layer and wall.

$$
\begin{aligned}
& \frac{d Q}{d t}=\left(\rho_{p} c_{p, p} V_{p}+\rho_{s} c_{p, s} V_{s}\right) \frac{\partial T}{\partial t}= \\
& =-\frac{\left(\Delta H_{1} k_{1}+\Delta H_{2} k_{2}\right)}{k_{1}+0.5 k_{2}+0.5 k_{5}(1-\psi)} \frac{\dot{m}_{g}}{M_{g}} \Delta y-\left(\Delta H_{3} k_{3}+\Delta H_{4} k_{4}\right) \psi V_{p}+\dot{m}_{g} c_{p, g}\left(T-T_{g}^{i n}\right)
\end{aligned}
$$




\subsection{Exhaust Gas Flow Inside Filter Channel}

\subsubsection{General}

Any active regeneration system [71] requires some kind of monitoring of accumulated soot mass on the filter. An intensively sought objective in this context is to derive a valid correlation of soot mass, which cannot be directly measured, with pressure drop and other directly measurable variables (exhaust temperature, pressure etc). This brings into the foreground the need to define the parameters affecting pressure drop and the respective expressions allowing their calculation or estimation. As shown in Figure 2.5, the determination of pressure drop involves a complex interaction between design and operating parameters of engine and filter. The pressure drop signal is affected by numerous parameters and carries a lot of information that is difficult to be deciphered to extract the necessary information with respect to the soot mass loading level.

In chapter 4 and [69], measurements of pressure drop resistance of individual channels of a filter were carried out. The results indicated that there exist significant variations in soot loading or quality [72] among different channels (centre versus periphery of the filter), during the loading phase. This fact hints to the existence of flow maldistribution at filter inlet during the loading phase (centre versus periphery).

Similar flow maldistribution in catalytic converters for gasoline cars have been investigated by numerous researchers [73, 74, 75]. Flow maldistribution in catalytic converters are shown to be affected by the geometry of the inlet devices (piping and diffuser) that creates an initial non-uniform distribution of the gas flow before its entrance to the converter. Although this irregularity is reduced along the cell of the catalyst due to the pressure drop, a certain degree of non-uniformity is always transmitted downstream. Some researchers [76] report that cell geometry produces by itself some internal maldistribution, even in case of initial uniform distribution of the gas flow. Despite the significant literature studies for catalytic converters, no studies are known to date regarding flow maldistribution in diesel filters.

The physical mechanism affecting flow distribution is different from that of catalytic converter. In the case of diesel particulate filters, the soot deposition modes that result from the two-phase flow produce variations in the soot loading profile along the channel.

Apart from the factors that affect the soot packing on the channel wall, a series of secondary factors such as

- $\quad$ volatile organic fraction adsorption-desorption phenomena and

- $\quad$ stochastic regeneration phenomena,

triggered by temperature differences between the central and peripheric channels of the filter, should also be taken into account. These phenomena lead to a continuous redistribution of the properties of the accumulated soot layer that affect the flow distribution across the filter channels.

\section{FILTER OPERATION}

PARAMETERS

- wall temperature

Universagt doading grofile 
Figure 2.5 Principle of interaction among design and operation parameters related to the loading and regeneration of diesel filters.

\subsubsection{The problem of soot maldistribution in the determination of pressure drop}

A starting point for the calculation of the pressure drop across the particulate filter would be the generally accepted correlation of Darcy law [77], that takes into account flow resistance of the filter wall plus accumulated soot layer. According to this law, the pressure drop due to flow through the ceramic wall and the soot layer can be approximated by the following simplified relation:

$$
\Delta p=\frac{\mu U E_{s}}{k_{s}}+\frac{\mu U E_{p}}{k_{p}}
$$

By using the concept of effective particulate layer thickness, the latter formula can be rewritten as follows [78]:

$$
\Delta p=\frac{\mu U E_{s}}{k_{s}}+\frac{\mu U m_{p}}{A_{f}(\rho k)_{p}}
$$

Where $A_{f}$ denotes the total filtration area of the channel that could be approximated by the sum of the inlet channel areas, after subtraction of the area blocked by the filter plugs.

Both factors of the above product are widely variable, thus, the product of $(\rho \mathrm{k})_{\mathrm{p}}$ varies depending on engine type, injection pressure, filter type, operation point, possible use of fuel additives etc. In the frame of the current experimental thesis, we attempted to narrow the range of variation of this parameter by a specific experimental test rig (see chapter 4 and [69]). The results of this study provide some hints on how the product $(\rho \mathrm{k})_{\mathrm{p}}$ depends on the 
- $\quad$ Prevailing engine operation point during loading (VOF content - exhaust temperature) and

- $\quad$ Loading history of the filter - filter channel (soot mass etc).

The equation 18 allows, in principle, the backward approximate calculation of collected soot mass as function of measured filter backpressure at a certain engine and filter loading operation point, once an approximate value for the product $(\rho \mathrm{k})_{\mathrm{p}}$, that is, (soot layer density) $\mathrm{x}$ (soot layer permeability) was known for the specific combination of engine, filter and operation point. However, this formula is zero-dimensional, considering the total filtration surface of the filter as a homogeneous membrane. Use of the above equation would only be justified if the superficial velocity be assumed absolutely uniform. This assumption could only rarely represent the real situation. Soot maldistribution phenomena caused by inlet geometry and soot loading history (residual soot mass from previous incomplete regeneration), severely affect the pressure drop signal. Such a situation is presented in Figure 2.6 where the periphery of a $\mathrm{SiC}$ filter is incompletely regenerated (presumably due to lower temperatures).

As explained above, in reality, the situation is 3-dimensional and the usefulness of the above, simplified relations is very small. Now, if a more accurate calculation of the pressure drop of a full-sized filter is attempted, the added complexity depends on how many dimensions are inserted in the calculation. As a preliminary step, one could attempt to add one more dimension to the calculation. Based on experimental evidence, the radial dimension (differences between filter center and periphery) would be the most significant.

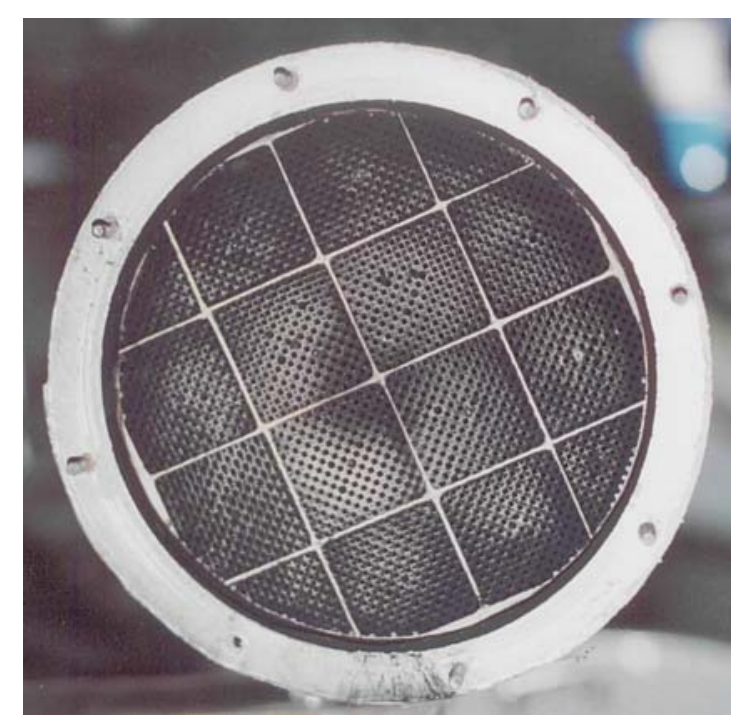

Figure 2.6 Front view of a partially regenerated filter. The appearance of a zone that becomes gradually darker as we move from the central to the outer cells of the filter indicates that the filter is incompletely regenerated at the periphery. This type of behaviour could be the cause of exhaust flow maldistribution between center and periphery of the filter.

Let us assume a simplified, radial stepwise variation of soot layer thickness (soot density permeability assumed uniform throughout), which distinguishes between two zones in the filter: A central zone (core) and a peripheric zone (outer), which are characterized by two distinct soot layer thickness values. The purpose of this simplified calculation would be to visualize the effect of stepwise radial maldistribution on the pressure drop (attempting to study situations resembling that of Figure 2.6) under typical flow rates in a typical full-sized filter.

Starting by the expression for equal pressure difference through the two zones of the filter,

$$
\Delta p_{\text {core }}=\Delta p_{\text {outer }}
$$


we may employ the above zero-dimensional correlations for each of the two distinct zones:

$$
\frac{\mu U_{\text {core }} E_{s}}{k_{s}}+\frac{\mu U_{\text {core }} E_{p, \text { core }}}{k_{p}}=\frac{\mu U_{\text {outer }} E_{s}}{k_{s}}+\frac{\mu U_{\text {outer }} E_{p, \text { outer }}}{k_{p}}
$$

This results to a correlation between the superficial velocities of the two zones.

$$
U_{\text {outer }}=\frac{a+b E_{p, \text { core }}}{a+b E_{p, \text { outer }}} U_{\text {core }}
$$

where $a=\frac{\mu E_{s}}{k_{s}}$ and $b=\frac{\mu}{k_{p}}$

On the other hand, the continuity equation can be profitably employed, if one makes the realistic assumption that the exhaust gas mass flowrate is more or less dictated by the engine characteristics at the specific operation point, that are only slightly affected by backpressure at low levels:

$$
m f r=\rho_{\text {gas }}\left(A_{\text {core }} U_{\text {core }}+A_{\text {outer }} U_{\text {outer }}\right)
$$

Using equation 22, one may deduce a correlation between the superficial velocities of the two zones, provided that the exhaust mass flowrate is known and kept constant:

$$
U_{\text {core }}=\frac{m f r}{A_{\text {core }} \rho_{\text {gas }}}-\frac{A_{\text {outer }}}{A_{\text {core }}} U_{\text {outer }}
$$

The system of algebraic equations (2.21) and (2.23) maybe employed, for example, to calculate the evolution of pressure drop if one keeps a constant, low soot layer thickness in the core zone of the filter, and assumes a variation of soot layer thickness in the periphery, starting from low and reaching extremely high values.

The results of the respective calculations are presented in Table 2.2 and the characteristic evolution of filter pressure drop as function of total soot loading is presented in Figure 2.7.

This figure explains why a filter that is heavily loaded in the periphery, continues to demonstrate reasonable backpressure values, if its core zone were kept relatively clean by frequent partial regenerations.

Obviously, the rate of filter pressure drop increase as a function of the total soot loading in the specific, simplified loading scenario, is directly affected by the relative dimensions of the two filter zones, which can be expressed by the ratio between the core and the total filtration area. In the example calculation of Figure 2.7, this ratio is assumed to be of the order of 0.6 (assuming a situation similar to that of Figure 2.6 where the central part up to the marked circle remains lightly loaded and the rest of the filter is gradually loaded up to high values).

Table 2.2 Summarized results of calculated filter backpressure and wall flow velocity at the central and peripheric channels of a non uniform loaded filter assuming constant exhaust gas mass flow rate and variable soot layer thickness only for the cells of the outer area. The ratio of the central to outer area is fitted to the value of 1.5 according to the situation described in Fig. 2.6 for the case of a filter loaded with an exhaust gas mass flow rate of the order of $70 \mathrm{~g} / \mathrm{s}$.

\begin{tabular}{|c|c|c|c|c|c|c|}
\hline $\begin{array}{c}\text { Soot layer } \\
\text { thickness at } \\
\text { core (m) }\end{array}$ & $\begin{array}{c}\text { Soot layer } \\
\text { thickness at } \\
\text { periphery (m) }\end{array}$ & $\begin{array}{c}\text { Core } \\
\text { velocity } \\
\mathbf{( m / s )}\end{array}$ & $\begin{array}{c}\text { Outer } \\
\text { velocity } \\
\mathbf{( m / s )}\end{array}$ & $\begin{array}{c}\text { Total soot } \\
\text { mass loading } \\
\mathbf{( k g )}\end{array}$ & $\begin{array}{c}\Delta \mathbf{P} \text { for } \\
\text { maldistributed } \\
\text { loading (kPa) }\end{array}$ & $\begin{array}{c}\Delta \mathbf{P} \text { for } \\
\text { homogenous } \\
\text { loading (kPa) }\end{array}$ \\
\hline $5.0 \mathrm{E}-05$ & $5.0 \mathrm{E}-05$ & 0.0542 & 0.0542 & 0.0073 & 25.22 & 25.22 \\
\hline $5.0 \mathrm{E}-05$ & $1.0 \mathrm{E}-04$ & 0.0662 & 0.0361 & 0.0102 & 30.77 & 33.55 \\
\hline
\end{tabular}




\begin{tabular}{|c|c|c|c|c|c|c|}
\hline $5.0 \mathrm{E}-05$ & $1.5 \mathrm{E}-04$ & 0.0721 & 0.0271 & 0.0131 & 33.55 & 41.88 \\
\hline $5.0 \mathrm{E}-05$ & $2.0 \mathrm{E}-04$ & 0.0757 & 0.0217 & 0.0160 & 35.21 & 50.21 \\
\hline $5.0 \mathrm{E}-05$ & $2.5 \mathrm{E}-04$ & 0.0781 & 0.0181 & 0.0189 & 36.32 & 58.54 \\
\hline $5.0 \mathrm{E}-05$ & $3.0 \mathrm{E}-04$ & 0.0798 & 0.0155 & 0.0219 & 37.11 & 66.86 \\
\hline $5.0 \mathrm{E}-05$ & $3.5 \mathrm{E}-04$ & 0.0811 & 0.0135 & 0.0248 & 37.71 & 75.19 \\
\hline $5.0 \mathrm{E}-05$ & $4.0 \mathrm{E}-04$ & 0.0821 & 0.0120 & 0.0277 & 38.17 & 83.52 \\
\hline $5.0 \mathrm{E}-05$ & $4.5 \mathrm{E}-04$ & 0.0829 & 0.0108 & 0.0306 & 38.54 & 91.85 \\
\hline $5.0 \mathrm{E}-05$ & $5.0 \mathrm{E}-04$ & 0.0835 & 0.0099 & 0.0335 & 38.84 & 100.18 \\
\hline
\end{tabular}

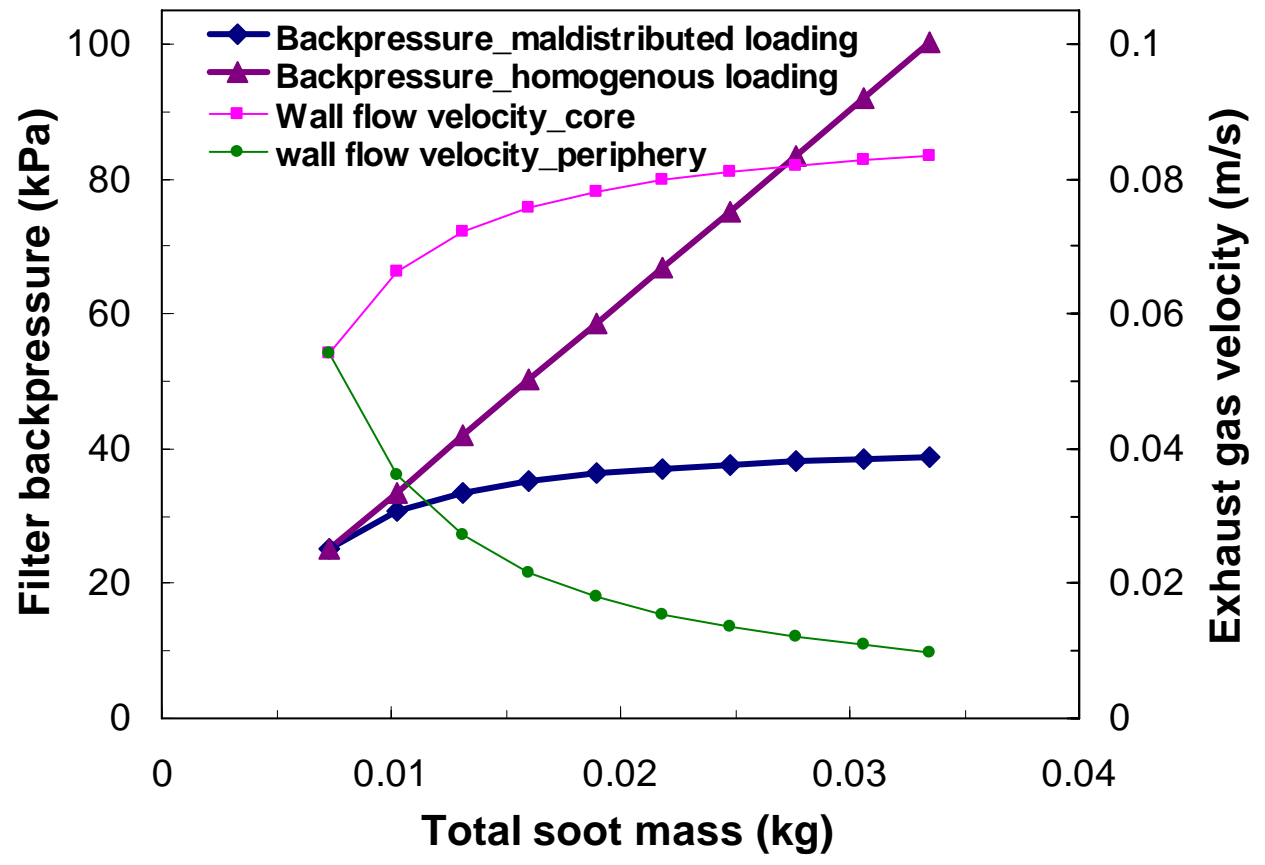

Figure 2.7 Calculated effect of soot maldistribution on filter backpressure and wall flow velocities at the central core and the outer area of the filter assuming constant exhaust gas mass flow rate and constant soot layer thickness at the core. The soot layer thickness at the outer cells is varied from 0.05 to $0.5 \mathrm{~mm}$.

\subsection{The Role of VOF}

\subsubsection{Adsorption-desorption phenomena}

The complex processes of VOF adsorption-desorption and their role to the regeneration behaviour are not yet well understood. It is well known that the percentage of VOF in the particulate emitted by a diesel engine significantly depends on the following engine design factors:

- combustion chamber design (DI, IDI, turbocharged),

- fuel injection system characteristics (injection pressure and timing, nozzles characteristics),

- the presence of exhaust gas recirculation (EGR) rate, and

- engine operation point $[79,86]$. 
Another important factor is diesel fuel composition. High boiling point components contained in fuel such as higher boiling point paraffines or aromatics adsorbed from particulates emitted by the engine, tend to increase the VOF content [80, 81]. Figure 2.8 compares distillation curves for typical diesel fuels and distillates, with different compositions and constituents' boiling points. Most diesel fuel components evaporate in the range between 200 and $380^{\circ} \mathrm{C}$ (when vapour is in its own presence).

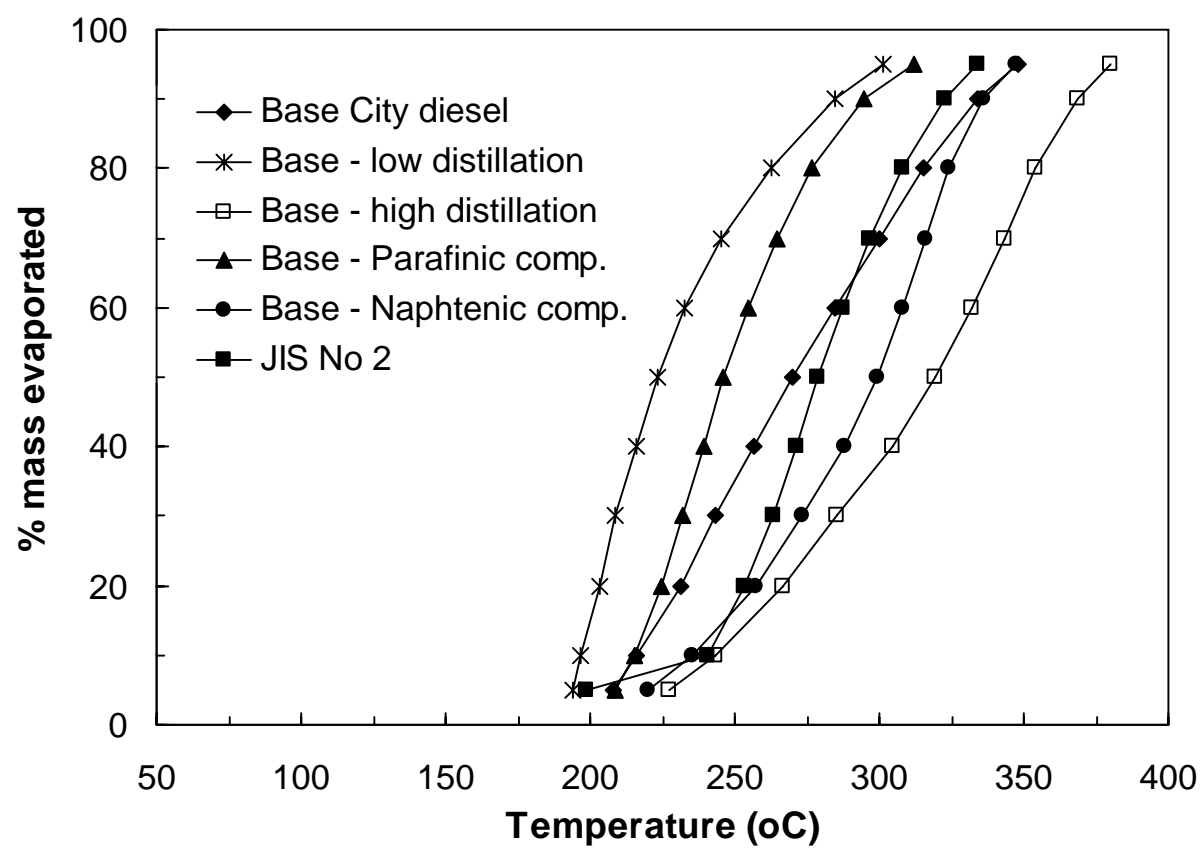

Figure 2.8 Distillation curves of various commercially available diesel fuels (JIS No 2: Japanese standard for automotive diesel fuel).

As a first approximation, one could assume that the particulate VOF evaporation and adsorption equilibrium, follows the distillation curve of the diesel fuel from which it originates. Now, VOF adsorption and desorption is a dynamic process continuously happening during the filter operation. The percentage of VOF that will be adsorbed on the soot layer at any specific time, depends at least on:

- the filter temperature and

- the filter operation history between complete regenerations.

Whenever filter temperature exceeds $380-420^{\circ} \mathrm{C}$, most VOF is expected to oxidize or vaporize, thus leaving mainly dry soot accumulated on the filter wall above $420^{\circ} \mathrm{C}$.

On the other hand, one could expect that prolonged operation of a highly loaded filter in low load and low speed (that is, high VOF content) conditions, would lead to the adsorption of heavy hydrocarbons from the exhaust gas to the particulate layer, thus increasing its VOF content. Although the adsorbate consists of multi-component hydrocarbons [79, 82], one could assume that the sorption behaviour of diesel exhaust hydrocarbons could be approximately represented by that of a single, typical hydrocarbon component.

\subsubsection{Erratic regeneration behaviour}

As previous mentioned the instability to the appearance and evolution of regeneration at temperatures down to $300^{\circ} \mathrm{C}$ under favourable engine and filter operating conditions, is one of the most significant problems since it leads to the incomplete regeneration of the filter and the 
non uniform distribution of the loaded soot mass. This behaviour is known as erratic regeneration behaviour and observed at low temperatures [83] and depends on the presence of volatile hydrocarbons. The volatile fraction originates from unburned fuel and lubricating fuel and is found adsorbed /condensed on the soot particles at temperatures below $190^{\circ} \mathrm{C}$. Due to the very close contact with the catalyst metal oxides these hydrocarbons may be oxidized at temperatures below $250^{\circ} \mathrm{C}$. Thus, a slow regeneration procedure may start in specific points where the local soot loading, composition, and temperatures are favourable. Under certain circumstances the heat release from the oxidized VOF leads to further carbon oxidation by the additive oxides at temperatures higher than $350^{\circ} \mathrm{C}$, which may lead to a more or less complete regeneration of the filter. Such behaviour is mapped in Figure 2.9 as observed by Lepperhoff et al. [84] on a particulate trap installed on a 1.9 litre IDI diesel engine by use of cerium fuel additive. Furthermore, an experimental work [85] on two passenger cars and a light truck clearly shows the erratic behaviour to the initiation temperature of regeneration that is probably due to VOF content.

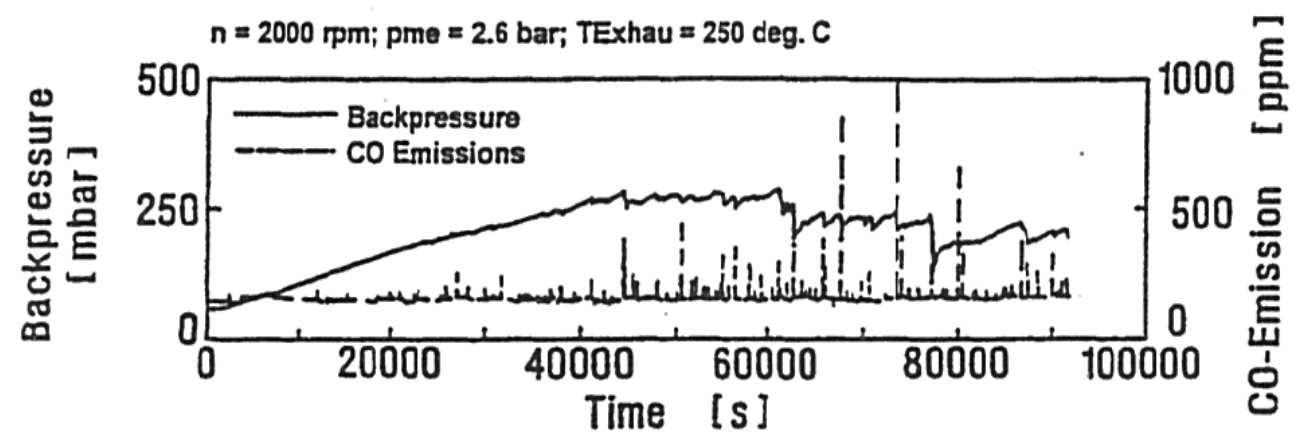

Figure 2.9 Stochastic Regeneration behaviour at a diesel filter fitted on a 1.9 litre engine as measured by Lepperhoff et al [84] in steady exhaust temperature conditions. Stochastic regenerations are also marked by the CO peaks.

In the literature, experimental studies of hydrocarbon adsorption are limited to experiments with diluted exhaust gas filtration on Teflon-coated glass fibre filters (according to the legislated diesel particulate mass measurement procedure). Catalytic oxidation of adsorbed hydrocarbons and the apparent kinetics determined from such experiments with filter soot samples are studied in chapter 3 and [86]. The TGA analysis performed in that work indicates that VOF is oxidized by Ceria in the temperature range of 300 to $420^{\circ} \mathrm{C}$.

Modelling of VOF oxidation and sorption behaviour requires the determination of adsorption - desorption kinetics. As a first step in this process, one could start from Arrhenius type expressions. This is supported by the fact that desorption of adsorbed liquids in microporous solids is an activated process [87]. The shape of the distillation curves of several diesel fuels that are given in Figure 2.8 which indicate an exponential dependence on temperature, also hints to such type of expressions. 


\section{Experimental Part I: Catalytic Regeneration Kinetics}

Understanding of the phenomena involved in catalytic regeneration, as they are observed in a wide range of engine and reactor operation points, is essential to the successful prediction of the onset and the evolution of catalytic regeneration. Moreover, the study of the particulate Volatile Organic Fraction adsorption - desorption - oxidation behavior is of major importance, due to the role of the VOF in soot maldistribution phenomena, 3D evolution of the regeneration and stochastic regeneration leading occasionally to filter failure. The initial part of this work focused on the experimental investigation of kinetics parameters for the thermal and catalytic soot oxidation, as well as for the VOF desorption - oxidation by Ceria by means of thermogravimetric and differential scanning calorimetry analysis (TGA-DSC). A novelty introduced in this work involved the sampling of soot directly from the filter channels, to study the effect of engine operation point during loading and the position of channel across the filter face on the VOF content and soot and VOF oxidation kinetics. The TGA analysis of the soot samples improved our understanding of the kinetic behavior and parameters of real soot, also as a function of the fuel additive dosimetry. A subsequent part of this work, investigated the role of VOF in the regeneration of the filter, as well as adsorption - desorption phenomena during filter loading. The effect of temperature differences between filter center and periphery on the VOF content of soot was studied. The onset of stochastic regeneration events during loading in low exhaust temperatures engine operation points with high VOF content was demonstrated. In the third part of this chapter, a systematic attempt is made to capitalize the previous findings in the LTTE in-house $1 \mathrm{D}$ and 3D regeneration models. The extensive validation process suggested significant model improvements. Moreover, the experimentally determined values of ceramic and soot permeability parameters and soot oxidation kinetics parameters are directly applicable to the models. During the validation process, an inclusive reaction scheme was developed, in cooperation with other group members. A more compact, reduced scheme that proved more workable with the specific models and parameter estimation procedures is applied in the simulations carried out in this work. The reduced scheme does not directly take into account the VOF oxidation from Ceria. VOF oxidation is lumped into the catalytic soot oxidation kinetics. The scope and extents of applicability of the 1D and 3D models in diesel filters and filter systems design is updated based on the experimental results of this work, which included a series of regeneration experiments under carefully designed and controlled conditions with the digitally controlled engine dyno and electronic throttle.

\subsection{Thermogravimetric Analysis of Soot - VOF Oxidation}

Thermal analysis methods (TGA-DSC) are commonly used in the investigation of diesel soot and VOF oxidation behavior and the determination of kinetic parameters. Although these methods are simple to use the results must be carefully analyzed because of the influence of parameters such as instrumental factors and sample characteristics. These parameters are summarized below, because they must be taken into account in interpreting the results of our investigations in order to be applicable in the further development of our computational tools [88]: 
- Instrumental (thermobalance) factors (furnace heating rate, furnace atmosphere, geometry of sample holder and furnace, sample holder material, thermocouple location)

- Sample characteristics (amount of sample, particle size, sample packing, nature of sample, thermal conductivity, heat capacity of the sample material)

A considerable amount of research has been carried out in recent years to investigate the influence of the above parameters [89, 90]. Another significant factor that must be considered to the estimation of kinetic parameters is the oxygen transport mechanism inside the bed. In the case of thermobalance the oxygen is supplied to the bed by diffusion only. So the conditions on the thermobalance do not represent the real conditions during filter regeneration where the oxygen is supplied mainly by forced convection. Further attention is necessary to the determination of the kinetic parameters since a TGA curve may consist of more than one usually overlapping processes.

\subsubsection{Experimental}

The collection of particulate samples was performed directly from the filter without dilution of the exhaust gas flow. The filter fitted to the exhaust system of a 2.0 liter displacement HDI turbocharged passenger car engine, running on the LTTE engine test bench on carefully selected steady state operation points. The engine and diesel filter specifications are presented in Table 3.1. Figure 3.1 presents the experimental layout of the filter loading experiments.

Table 3.1 Engine technical data and diesel filter specifications

\begin{tabular}{|l|l|}
\hline Engine manufacturer & PSA \\
\hline Engine type & HDI turbocharged engine (DW 10 ATED) \\
\hline Cylinders & 4, in-line \\
\hline Displacement & $1997 \mathrm{~cm}^{3}$ \\
\hline Rated power /rpm & $80 \mathrm{~kW} / 4000 \mathrm{rpm}$ \\
\hline Rated torque/rpm & $250 \mathrm{Nm} / 2000 \mathrm{rpm}$ \\
\hline Diesel Filter Manufacturer & Ibiden \\
\hline Filter Type & SiC $14 / 200 \mathrm{cpsi}$ (cells per square inch) \\
\hline Filter Diameter x length & $143.8 \mathrm{~mm}$ x $150 \mathrm{~mm}$ \\
\hline Cell pitch & $1.89 \mathrm{~mm}$ \\
\hline Filter Wall thickness & $0.4 \mathrm{~mm}$ \\
\hline
\end{tabular}

First, a mapping process is performed to assess the engine exhaust temperature under the low and medium load operating range. Temperatures were measured simultaneously at the exhaust pipe $50 \mathrm{~mm}$ after turbine exit, at the inlet of the filter, and inside the filter, along a filter diameter $1.5 \mathrm{~cm}$ deep from the end (Figure 3.1). Figure 3.2 shows a map of filter wall temperatures near filter exit, at center and periphery. Comparison of wall temperatures at center and periphery shows that periphery is systematically colder than the center by from about $25{ }^{\circ} \mathrm{C}$ (low exhaust gas flowrate-low engine rpm) to about $10{ }^{\circ} \mathrm{C}$ (high exhaust gas flowrate-high engine rpm). Of course, the observed temperature differences may be suppressed by an improved insulation of the filter shell. 


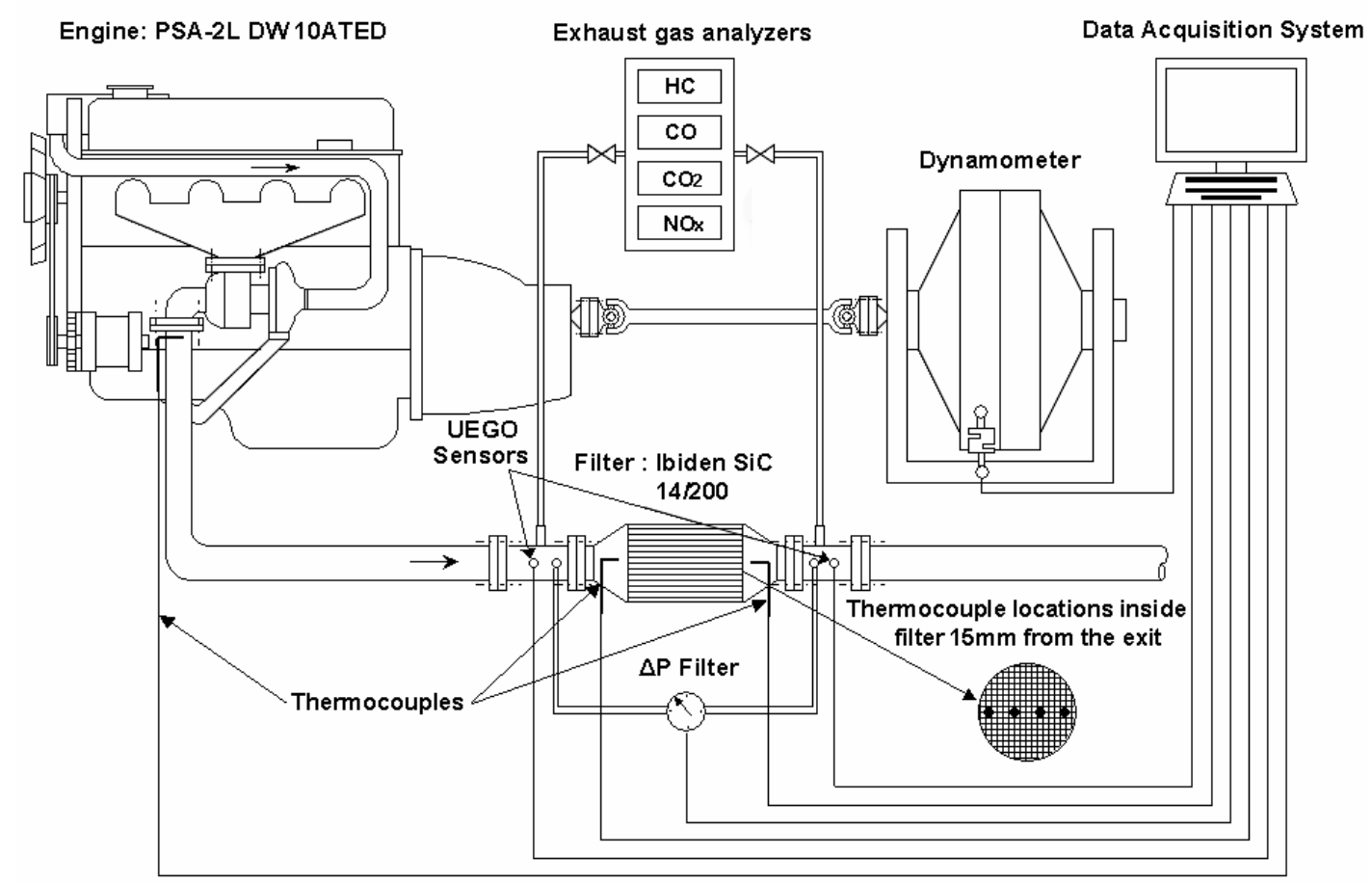

Figure 3.1 Experimental layout. Engine and digitally controlled dynamometer installation is shown along with exhaust gas analysers, main diesel filter measurement lines and data acquisition system.

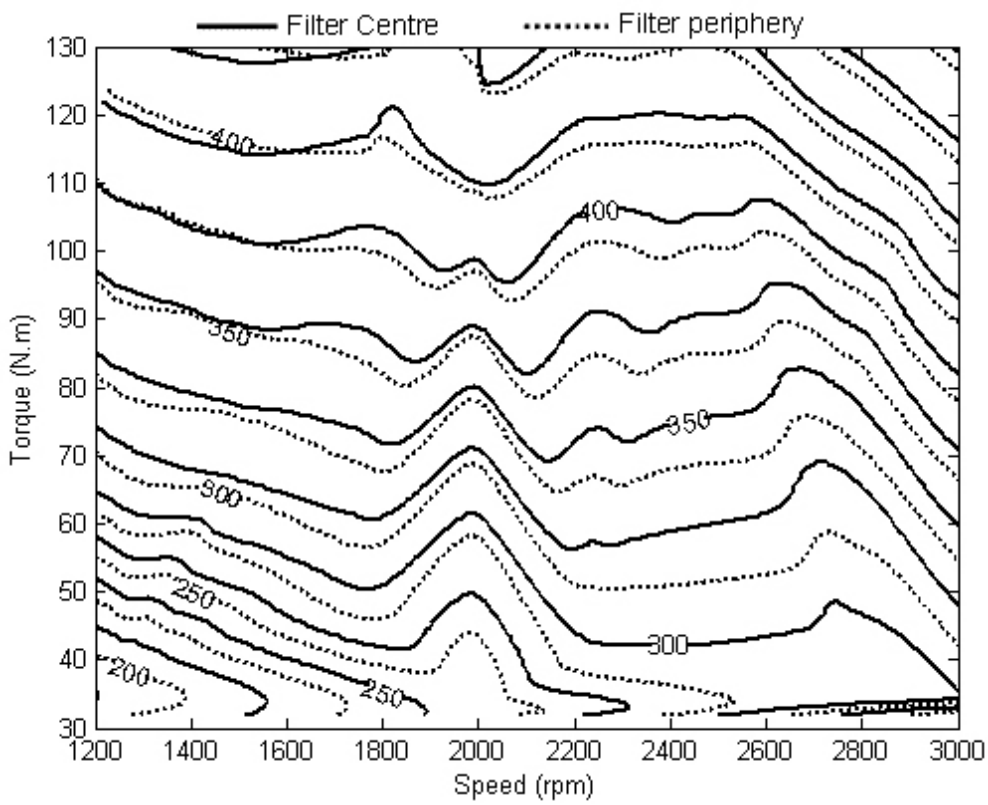

Figure 3.2 Map of channel wall temperatures $\left({ }^{\circ} \mathrm{C}\right)$ measured at the centre and periphery of the filter, at a distance of $15 \mathrm{~mm}$ from the end of the respective channels.

The following strategy is applied in the determination of the exact protocol for filter loading tests:

Low engine speed points are selected as more representative of city driving, and engine load is varied to result in a range of filter wall temperatures from $200^{\circ} \mathrm{C}$ to $420^{\circ} \mathrm{C}$. This range of filter wall temperatures results in varying VOF content of collected particulate. The test protocol, as it designed based on engine mapping, is presented in Table 3.2. Loading experiments with various fuel additive concentration levels in fuel, were performed in order to 
investigate the influence of additive concentration on soot oxidation behavior. The shift to a set of experiments with different fuel additive concentration was performed after filter cleaning and continuous running of engine for 6 hours to prevent possible memory effects to the soot composition. After completion of the particulate collection procedure at each operation point, the filter was regenerated.

Table 3.2 Experimental protocol

\begin{tabular}{|c|c|c|c|c|c|c|c|}
\hline $\begin{array}{l}\text { Speed } \\
\text { (rpm) }\end{array}$ & $\begin{array}{c}\text { Torque } \\
\text { (Nm) }\end{array}$ & $\begin{array}{c}\text { Exhaust gas } \\
\text { mass flowrate } \\
\text { (g/sec) }\end{array}$ & $\begin{array}{c}\text { T exhaust } \\
\left({ }^{\circ} \mathrm{C}\right)\end{array}$ & $\begin{array}{c}\text { TF inlet } \\
\left({ }^{\circ} \mathrm{C}\right)\end{array}$ & $\begin{array}{c}\text { TF wall } \\
\text { center } \\
\left({ }^{\circ} \mathrm{C}\right)\end{array}$ & $\begin{array}{c}\text { TF wall } \\
\text { periphery } \\
\left({ }^{0} \mathrm{C}\right)\end{array}$ & ppm Ce \\
\hline \multirow{2}{*}{1800} & 30 & 23 & 275 & 254 & 250 & 235 & \multirow{2}{*}{0} \\
\hline & 75 & 34 & 375 & 355 & 350 & 328 & \\
\hline 1500 & 30 & 23 & 240 & 226 & 220 & 206 & \multirow{6}{*}{25} \\
\hline \multirow{5}{*}{1800} & 30 & 28 & 274 & 256 & 250 & 237 & \\
\hline & 50 & 31 & 317 & 302 & 300 & 280 & \\
\hline & 75 & 34 & 371 & 353 & 350 & 327 & \\
\hline & 110 & 37 & 429 & 401 & 400 & 373 & \\
\hline & 125 & 41 & 440 & 415 & 415 & 388 & \\
\hline 1500 & 30 & 21 & 238 & 223 & 218 & 200 & \multirow{5}{*}{50} \\
\hline \multirow{4}{*}{1800} & 30 & 28 & 277 & 257 & 250 & 235 & \\
\hline & 50 & 31 & 323 & 304 & 300 & 283 & \\
\hline & 75 & 34 & 375 & 356 & 350 & 329 & \\
\hline & 110 & 36 & 430 & 400 & 400 & 375 & \\
\hline
\end{tabular}

The TGA-DSC tests performed with a Rheometric-Scientific STA $1500 \mathrm{H}$ model thermogravimetric analyzer (Temperature range Ambient $-1500{ }^{\circ} \mathrm{C}$, Temperature ramp rates $0.2-60{ }^{\circ} \mathrm{C} / \mathrm{min}$, Crucible size $50-100 \mu \mathrm{l}$ ). Samples ranging from 3.5 to $5.5 \mathrm{mg}$ were collected each time from the central and peripheral channels of the filter through a special designed blade used for the detachment of the soot layer pieces from the channel wall. In order to give an idea of the temperatures prevailing inside the filter during loading, Figure 3.3 presents the results of a filter loading experiment comprising an initial filter preheating - loading at 2000 rpm - no load conditions (500 seconds duration), followed by a step load change $60 \mathrm{Nm}$, with the engine speed remaining at $2000 \mathrm{rpm}$. Backpressure is observed to increase from 40 to 120 mbar in about 4500 seconds during the loading at $60 \mathrm{Nm}$ engine load. Exhaust gas temperature at filter inlet, (turbine exit), and at two points at filter exit channels (center and periphery) is measured. The clearly observable exhaust gas and filter temperature increase trend is due to the effect of backpressure increase on the engine cycle (reduction of break mean effective pressure due to the increase in pumping losses). According to this figure, temperature difference between center and periphery during loading is of the order of $25^{\circ} \mathrm{C}$ at the specific operation point. This temperature difference decreases at higher flowrates as explained above. Generally, due to this difference, the VOF content is expected to be higher at the periphery. 


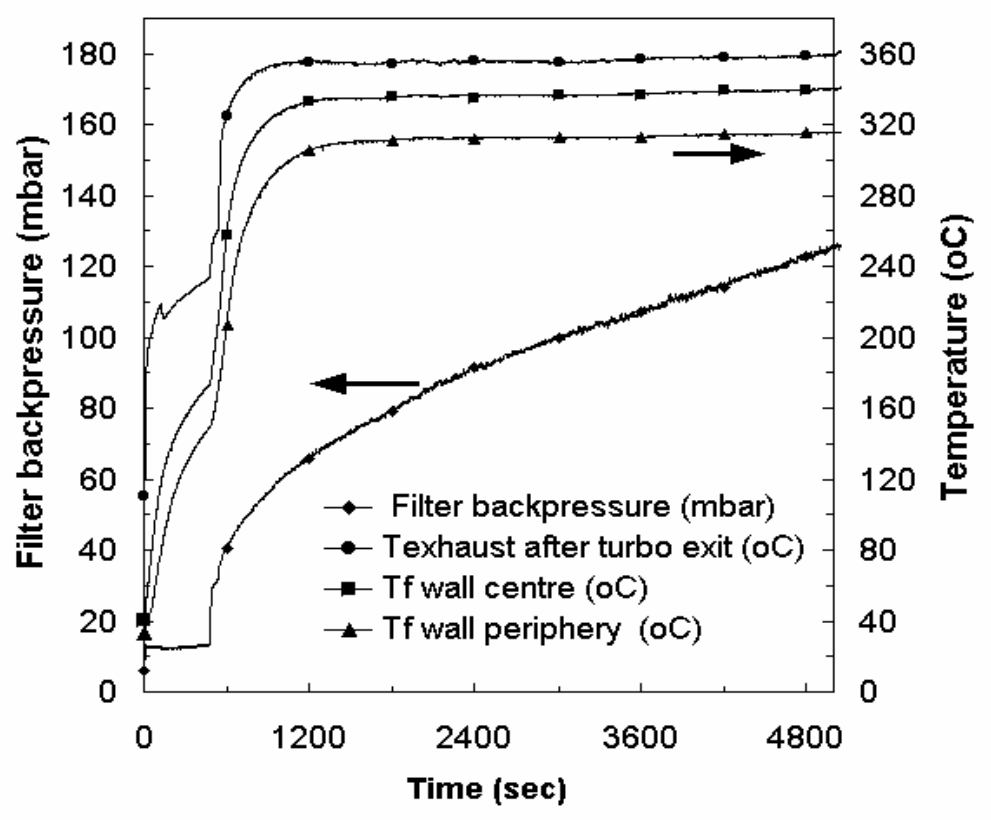

Figure 3.3 Evolution of exhaust gas and filter exit channels (centre and periphery) temperatures during a typical loading procedure, which comprises two steps: first, $2000 \mathrm{rpm}$ - no load for about 500 seconds. Then, step change to $2000 \mathrm{rpm},-60 \mathrm{Nm}$, and loading for about 4500 seconds. Evolution of filter backpressure is also recorded.

Each sample was packed in a platinum receptacle, immediately after the end of the filter loading at the respective operation point. The packing procedure was performed very carefully to achieve uniform distribution of sample mass inside the receptacle. Nonisothermal tests were performed with each sample, with a heating rate of $10^{\circ} \mathrm{C}$ per minute. The experiments were carried out in air and also in nitrogen environment (for the determination of VOF content). A gas flow of $10 \mathrm{ml} / \mathrm{min}$ in the case of synthetic air experiments and of $35 \mathrm{ml} / \mathrm{min}$ in the case of nitrogen was maintained over the receptacle chamber. The scan rate was adjusted to $1 \mathrm{sec}$.

\subsubsection{Results and discussion}

First, TGA/DSC analysis was performed on diesel soot samples collected with the engine operating on regular (not fuel additive - doped) fuel at the following two characteristic points:

- $\quad$ Engine speed :1800rpm, Engine Load: $30 \mathrm{Nm}$ resulting filter wall temperature ca. $250^{\circ} \mathrm{C}$

- Engine speed :1800rpm, Engine Load: $70 \mathrm{Nm}$ resulting filter wall temperature ca. $350^{\circ} \mathrm{C}$

The mass reduction-heat release graphs are comparatively presented in Figure 3.4. Starting from the soot sample collected at $250{ }^{\circ} \mathrm{C}$, we observe, in the mass reduction curve, a first decrease up to about $150{ }^{\circ} \mathrm{C}$, which we assume to be mainly due to the water evaporation desorption. A second mass decrease is observed between 200 and $420{ }^{\circ} \mathrm{C}$. This is assumed to be due to hydrocarbon desorption. From the associated heat release curve, we could possibly suspect a slight hydrocarbon oxidation to start above $300{ }^{\circ} \mathrm{C}$. Continuing with the soot sample collected at $350{ }^{\circ} \mathrm{C}$, we do not see, in the mass reduction curve, an observable mass decrease in the temperature range between 200 and $350{ }^{\circ} \mathrm{C}$. This could be expected, since the particulate collection was at $350{ }^{\circ} \mathrm{C}$. We observe a small decrease in the temperature range from 350 to $420{ }^{\circ} \mathrm{C}$, from which we can deduce a significantly lower VOF content for this second soot sample. Proceeding to higher temperatures, we could observe that the TGA/DSC 
behaviour of the two samples converges very much above $450{ }^{\circ} \mathrm{C}$. Both DSC curves indicate an ignition temperature (temperature at which the soot oxidation rate is maximized) of the order of $630^{\circ} \mathrm{C}$. Arrhenius plots were calculated based on the recorded mass reduction graphs in the temperature range between $550-630{ }^{\circ} \mathrm{C}$.

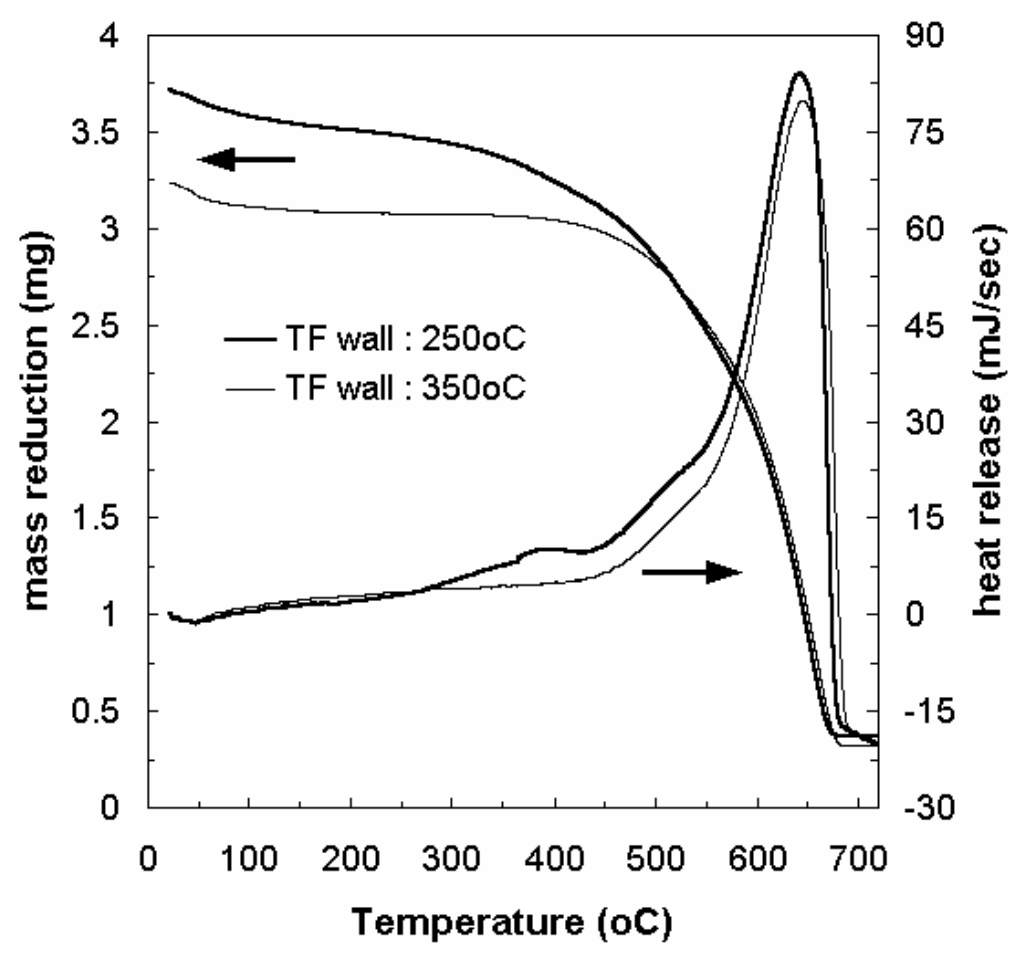

Figure 3.4 TGA (mass reduction) and DSC (heat release) curves for regular (no additive) soot samples taken directly from the filter channel, after moderate particulate loading at 250 and $350^{\circ} \mathrm{C}$ filter channel wall temperatures.

The results of Table 3.4 indicate activation energy levels of the order of $190 \mathrm{~kJ} / \mathrm{mole}$ for both cases. The different VOF content of the two samples does not seem to affect the kinetics of soot oxidation. This could be expected because of the separate temperature ranges of the two processes (HC desorption - soot oxidation by exhaust gas oxygen). In the literature the published values of activation energy of thermal soot oxidation is found to cover a range between 140 and $210 \mathrm{~kJ} / \mathrm{mole}$. Low values of the order of $140-150 \mathrm{~kJ} / \mathrm{mole}$ reported by Ciambelli et al [91] and Otto et al [92]. Neeft et al [93], report a somewhat lower value of $170 \mathrm{~kJ} / \mathrm{mol}$, for the oxidation of flame soot in a flow reactor with a different atmosphere $(10 \%$ $\mathrm{O} 2$ in $\mathrm{Ar}$ ). Stanmore et al [94] report higher values of $210 \mathrm{~kJ} / \mathrm{mole}$ obtained under isothermal tests of soot samples collected from a filter fitted to the exhaust of a naturally-aspirated, indirect injection 1.9litre diesel engine.

As a next step, TGA/DSC experiments with soot emitted by the engine operating with 25ppm Cerium doped fuel (DPX9 commercial fuel additive, containing an organometallic Ceria compound) were performed. Figure 3.5 presents a comparison of the results (mass reduction and heat release) of TGAIDSC analysis, with soot collected at the following six engine operating points:

- $\quad$ Speed: $1500 \mathrm{rpm}$, Load: $30 \mathrm{Nm}$, resulting filter wall temperature ca. $220^{\circ} \mathrm{C}$

- Speed: $1800 \mathrm{rpm}$, Load: $30 \mathrm{Nm}$, resulting filter wall temperature ca. $250^{\circ} \mathrm{C}$

- Speed: $1800 \mathrm{rpm}$, Load: $50 \mathrm{Nm}$, resulting filter wall temperature ca. $300^{\circ} \mathrm{C}$

- $\quad$ Speed: $1800 \mathrm{rpm}$, Load: $70 \mathrm{Nm}$, resulting filter wall temperature ca. $350^{\circ} \mathrm{C}$

- Speed: $1800 \mathrm{rpm}$, Load: $100 \mathrm{Nm}$, resulting filter wall temperature ca. $400^{\circ} \mathrm{C}$ 
- Speed: $1800 \mathrm{rpm}$, Load: $120 \mathrm{Nm}$, resulting filter wall temperature ca. $420^{\circ} \mathrm{C}$

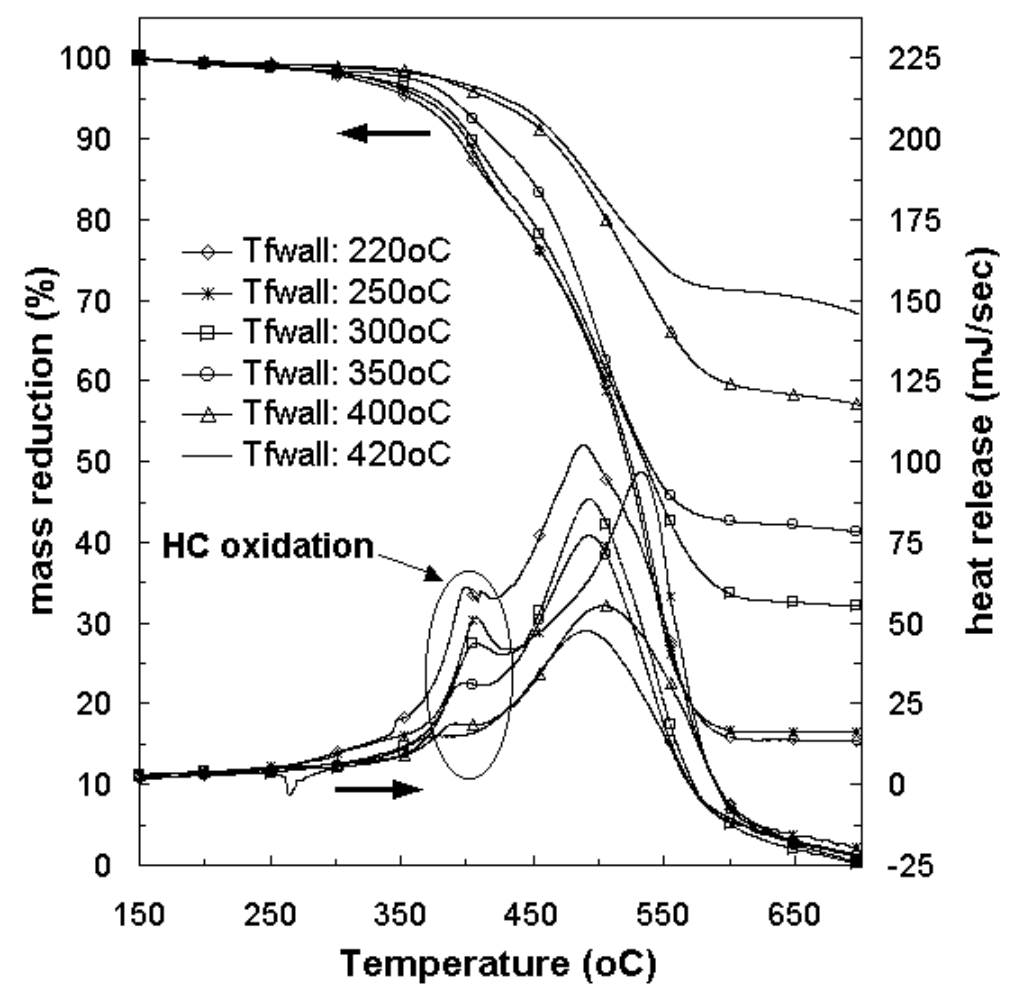

Figure 3.5 TGA (mass reduction) and DSC (heat release) curves for soot samples taken directly from the filter channel, after moderate particulate loading at various filter channel wall temperatures. All soot samples are taken from a filter loaded with the engine running with 25ppm Ce- doped diesel fuel.

We could observe two characteristic regions of catalytic soot oxidation in this figure:

- the first in the range of 300 to $420^{\circ} \mathrm{C}$, in which a new peak on the DSC curve appears. This peak precedes the regular peak of soot oxidation by oxygen, and is observed to gradually diminish as we shift to soot samples collected with increasing filter wall temperature. This region should be related to the catalytic oxidation of hydrocarbons adsorbed on the particulate (VOF). The new peak is clearly observable in the DSC curves of all six samples, which indicates that the presence of the catalyst in soot significantly promotes adsorbed hydrocarbon oxidation. An analogous behaviour has been reported in [95] with other types of catalysts mixed with plain soot in a U-type quartz glass microreactor.

- the second in the range of 420 to $490^{\circ} \mathrm{C}$, in which the dry soot oxidation takes place with an ignition temperature of the order of $490^{\circ} \mathrm{C}$ irrespectively of VOF content. Thus, the presence of the catalyst in soot is now associated with a shift of the regular soot oxidation peak towards lower temperatures $\left(490\right.$ vs $630^{\circ} \mathrm{C}$ ).

An exception can be observed in the temperature location of this second peak, for the sample from soot collected at $250^{\circ} \mathrm{C}$. The DSC curves from five samples taken this loading point, systematically demonstrate the same behaviour, with the peak shifted to $520^{\circ} \mathrm{C}$. This could be attributed to the fact that engine emissions at the specific loading operation point were increased compared to the standard emissions levels (see Table 3.3). Thus, ceria concentration in the particulate was reduced (assumed constant fuel consumption). 
Another observation is related to the percentage residual that is left at high temperatures that can be deduced from the right side of Figure 3.5. The respective calculations are summarized in Table 3.3. The composition of this is considered to be mainly fuel additive ash, but also heavy metallic compounds originating from lube oil [3, 9]. Now, if we compare the last two columns of this Table, we clearly observe that the percentage of residual to the overall soot mass, is at least an order of magnitude higher than an indicative percentage that would be expected based on the extremely simplifying assumptions that

- the ash is $100 \%$ Ceria

- $100 \%$ of the particulate emitted by the engine during the loading duration remained collected in the filter at the time we took the sample, and

- no ceria remained in the filter from previously regenerated soot due to incomplete cleaning of the filter.

Table 3.3 Fuel additive ash computations

\begin{tabular}{|c|c|c|c|c|c|c|c|}
\hline Speed (rpm) & Torque (Nm) & Ce (ppm) & $\begin{array}{c}\text { Fuel } \\
\text { Consumption } \\
(\mathbf{g} / \mathbf{h})\end{array}$ & $\begin{array}{c}\text { Soot } \\
\text { Emissions } \\
(\mathrm{g} / \mathrm{h})\end{array}$ & $\begin{array}{c}\text { Calculated Ce } \\
\text { Accumulation } \\
(\mathrm{mg} / \mathrm{h})\end{array}$ & $\begin{array}{c}\text { Calculated } \\
\text { Ce in soot } \\
(\%)\end{array}$ & $\begin{array}{c}\text { TGA Ash } \\
\text { residual } \\
(\%)\end{array}$ \\
\hline 1500 & 30 & \multirow{6}{*}{25} & 1840 & 5 & 46.00 & 0.92 & 16.04 \\
\hline \multirow{5}{*}{1800} & 30 & & 2440 & 5.6 & 61.00 & 1.09 & 17.22 \\
\hline & 50 & & 3120 & 5 & 78.00 & 1.56 & 32.48 \\
\hline & 75 & & 3980 & 4.9 & 99.50 & 2.03 & 40.03 \\
\hline & 110 & & 5010 & 4.84 & 125.25 & 2.59 & 55.56 \\
\hline & 125 & & 5860 & 4.11 & 146.50 & 3.56 & 65.6 \\
\hline \multirow[t]{4}{*}{1500} & 30 & \multirow{6}{*}{50} & 1780 & 5.9 & 89.00 & 1.78 & 35.62 \\
\hline & 30 & & 2440 & 5.6 & 122.00 & 2.18 & 33.84 \\
\hline & 50 & & 3120 & 5 & 156.00 & 3.12 & 36.75 \\
\hline & 75 & & 3980 & 4.9 & 199.00 & 4.06 & 57.84 \\
\hline \multirow{2}{*}{1800} & 110 & & 4990 & 4.84 & 249.50 & 5.15 & 62.67 \\
\hline & 125 & & - & - & - & - & - \\
\hline
\end{tabular}

The significant discrepancy between the two columns denies the validity of at least one of these assumptions. It is also observed that the percentage of the residual increases with exhaust temperature levels prevailing during filter loading (that is, increases with engine load during filter loading). This observation could be at least partially justified by the fact that this engine is characterized by decreasing particulate emissions per kg fuel consumed, in the range of part engine load that we are studying.

The role of Ceria in the VOF oxidation in the range of $300-420^{\circ} \mathrm{C}$ is apparent in all these results. As a next step, we focus on the effect of VOF content on the kinetics of catalytic soot oxidation by Ceria. This effect should be assessed by a comparison of the Arrhenius plots performed on TGA analysis results from Figure 3.5 for the soot samples collected at 250, 300, 350, $400{ }^{\circ} \mathrm{C}$ filter wall temperatures. The Arrhenius plots are presented in Figure 3.6. These plots have been calculated with reaction order assumed equal to 1 also for the catalytic oxidation [96]. Each plot comprises two distinct temperature ranges, in which the linear regression was of an acceptable quality ( $\mathrm{R}$ squared value higher than 0.98 ). We prefer not to perform the regression in the range between $410-440 \mathrm{C}$, because in this range the regression is not good. Obviously, the group of Arrhenius plots at the left side of the figure corresponds to the dry soot oxidation by Ceria, whereas the group at the right side corresponds to the VOF oxidation by Ceria. A certain degree of overlap between these two processes, with a gradually 
diminishing VOF role due to desorption - oxidation, could be happening between the two temperature ranges.

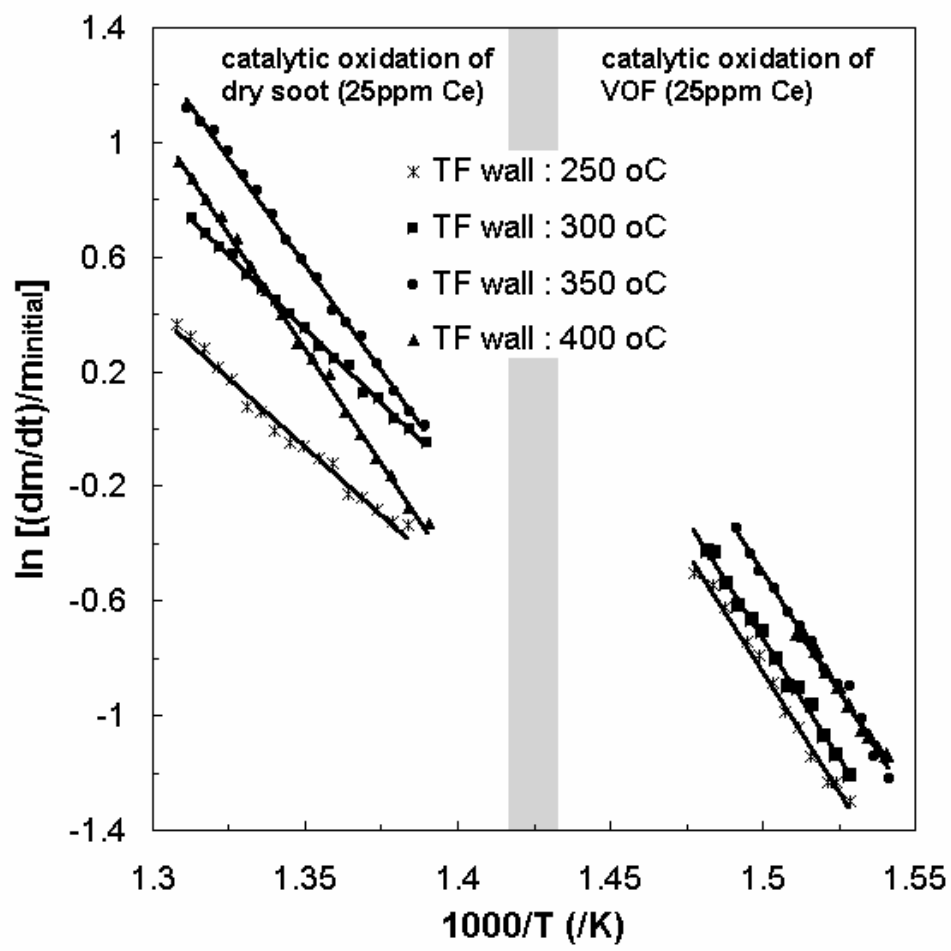

Figure 3.6 Non-isothermal kinetic analysis of the TGA curves of Figure 5. All soot samples taken from filter loaded by engine running on $25 \mathrm{ppm} \mathrm{Ce}$ - doped fuel. Arrhenius plots are performed in the two temperature ranges where high quality linear regression is possible. These two ranges correspond to dry soot (left group) and VOF (right group) oxidation. Each group corresponds to four different filter wall temperature levels during loading ( $250,300,350,400{ }^{\circ} \mathrm{C}$ ).

The overall results indicate that the presence of the catalyst in soot significantly lowers the activation energy of dry soot oxidation (Arrhenius plots at left side of the figure). This observation agrees with the results reported by Summers et al [97] and Lahaye et al [98] who found that the addition of Ce in the fuel is lowering the activation energy of soot oxidation from 170 to $120 \mathrm{~kJ} / \mathrm{mole}$. Ciambelli et al [99] also reported lower values of activation energy for soot oxidation after its impregnation with $\mathrm{Cu} / \mathrm{V} / \mathrm{K}$ catalyst. In contrast with the above observation Stanmore et al [94] reported unchanged activation energy of $210 \mathrm{~kJ} / \mathrm{mole}$ (obtained for the uncatalyzed oxidation) for the soot oxidation with cerium present. In the same direction Jelles et al. reported unchanged values of activation energy for the oxidation of cerium activated soot which, however, have been taken with a different type of reactor and a flow containing 10vol\% O2 in Argon [100]. According to our results, the presence of VOF reduces activation energy for soot oxidation by Ceria in the range of 420 to $490^{\circ} \mathrm{C}$. Thus, soot collected at 250 and $300^{\circ} \mathrm{C}$ filter wall temperature (rich in VOF) is oxidized with $\mathrm{E}$ of order of $80-85 \mathrm{~kJ} / \mathrm{mole}$, whereas soot collected at 350 and $400^{\circ} \mathrm{C}$ filter wall temperature (dry-soot) is oxidized with E of order of 120-130 kJ/mole. The effect of VOF to the apparent kinetics of diesel soot oxidation is related with the modifications which take place to the particulate structure during the oxidation of VOF. According to Alhstrom and Odenbrand [101] an increase of the surface area of the soot after VOF oxidation is observed and leads to a more porous structure which allows the easier access of oxygen through the pores. The VOF content on these points was calculated from the TGA curves obtained in Nitrogen environment as the percentage of mass reduction in the range of $150^{\circ} \mathrm{C}$ (water free) to $380^{\circ} \mathrm{C}$ 
according to the process presented in the next section. The results indicate a variation of the VOF content in the range from 2.5 to $8 \%$ for samples collected from filter center and 3 to 9.5\% for samples collected from filter periphery (see Figure 3.14) with the minimum values to be observed at high load engine operation, presumably due to the higher exhaust gas flowrates caused by the activation of the turbocharger.

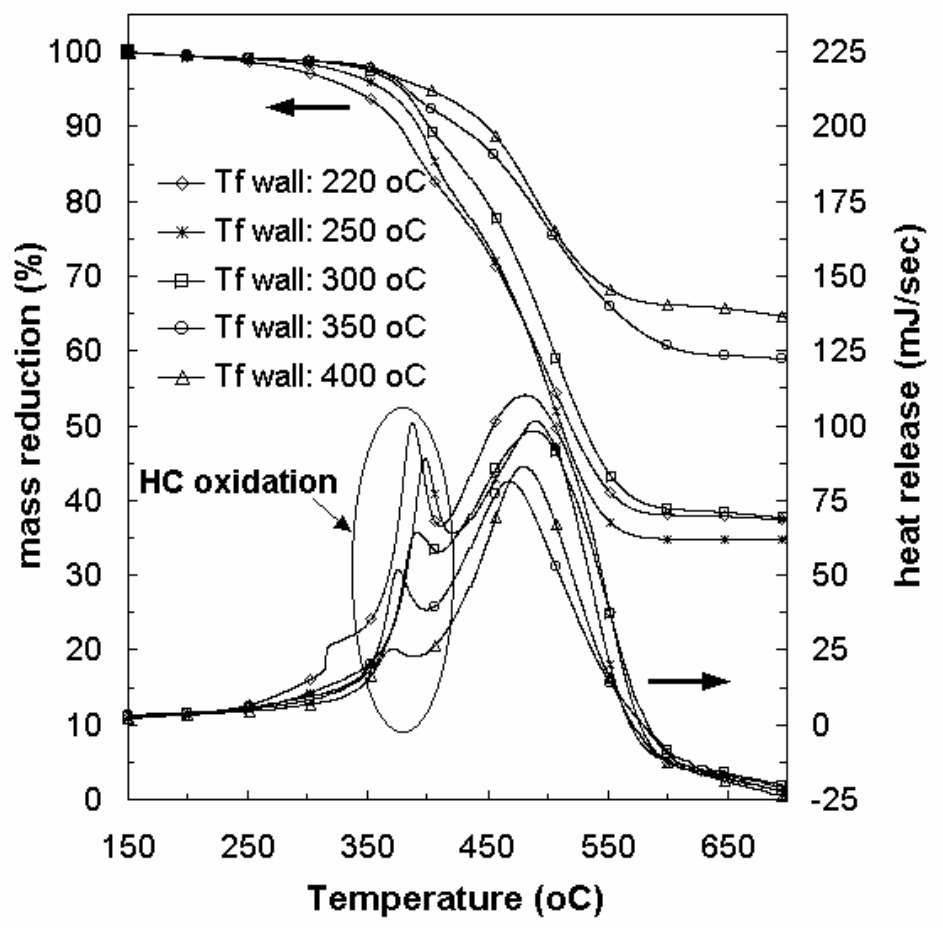

Figure 3.7 TGA (mass reduction) and DSC (heat release) curves for soot samples taken directly from the filter channel, after moderate particulate loading at various filter channel wall temperatures. All soot samples are taken from a filter loaded with the engine running with 50ppm Ce- doped diesel fuel.

As a next step, a more detailed analysis of soot oxidation in the temperature range between $350-420^{\circ} \mathrm{C}$ (adsorbed hydrocarbons oxidation) is performed. The group of Arrhenius plots presented at the right side of Figure 3.6. In this temperature range indicates activation energy for the catalytic VOF oxidation of the order of 130-140 kJ/mole irrespective of soot VOFcontent 
Table 3.4). We are not aware of analogous published results to compare with.

In order to confirm and further extend the above-mentioned observations, a new set of measurements was performed with soot collected on the engine operating with 50ppm Cerium doped fuel. We executed the same series of filter loading experiments except the last (1800 rpm, $120 \mathrm{Nm}$ ) where the regeneration process was started earlier because of the increase to the Cerium concentration in fuel. The results are presented in Figure 3.7, which is analogous to Figure 3.5 with 50ppm instead of 25ppm Cerium concentration in fuel. From a comparison between the two Figures, it is obvious that VOF oxidation activity at low temperatures is intensified with the higher Ce concentration.

For a quantitative assessment of the effect of increased Ce concentration on the kinetics of the reactions of interest, Arrhenius plots are performed for the new sets of TGA analysis data. The results presented in Figure 3.8. By a comparison of these results with those of Figure 3.6, it should become apparent that the calculated activation energies of catalytic oxidation of dry soot are not affected by Cerium concentration in the range of 420 to $490{ }^{\circ} \mathrm{C}$ for soot collected at 220,250 and $300^{\circ} \mathrm{C}$ filter wall temperature. On the other hand, a reduction of the order of 10 and $20 \mathrm{~kJ} / \mathrm{mole}$ was observed for soot collected at 350 and $400{ }^{\circ} \mathrm{C}$ respectively.

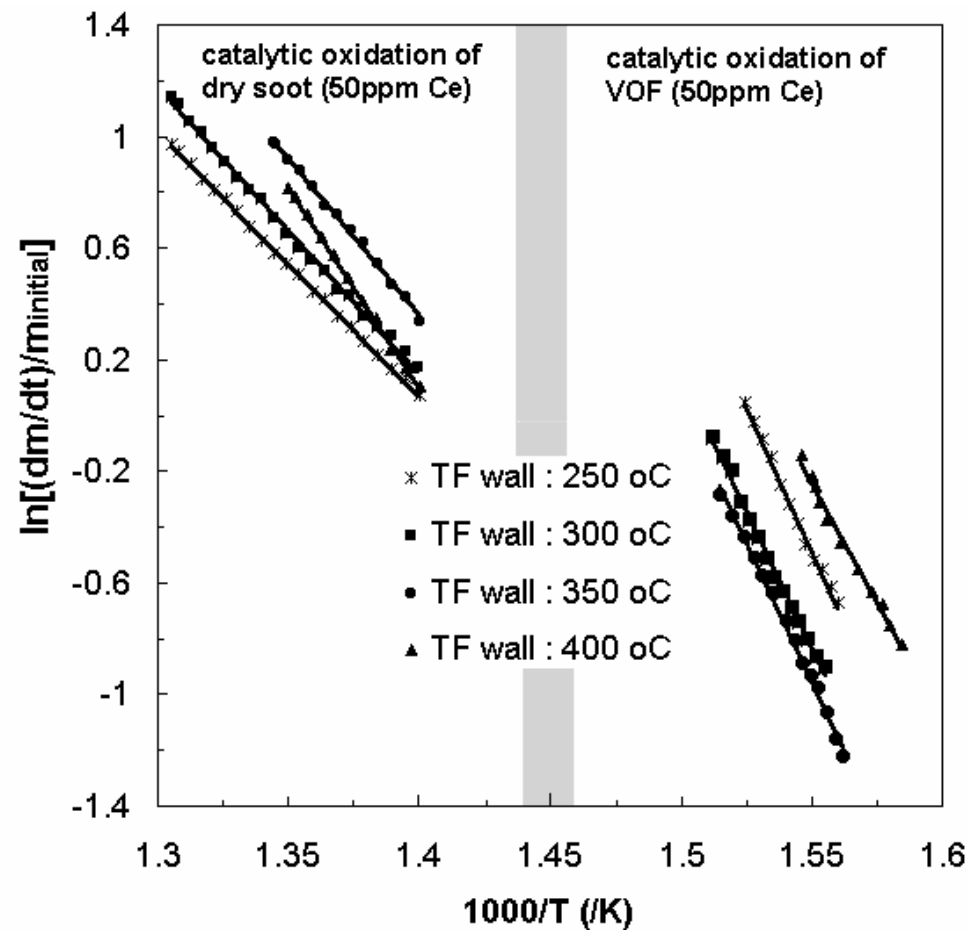

Figure 3.8 Non-isothermal kinetic analysis of the TGA curves of Figure 3.7. All soot samples taken from filter loaded by engine running on 50ppm Ce- doped fuel. Arrhenius plots are performed in the two temperature ranges where high quality linear regression is possible. These two ranges correspond to dry soot (left group) and VOF (right group) oxidation. Each group corresponds to four different filter wall temperature levels during loading $\left(250,300,350,400{ }^{\circ} \mathrm{C}\right)$.

The situation is different with the region between 350 to $420^{\circ} \mathrm{C}$, where the catalytic VOF oxidation is assumed to happen. The resultant activation energies from the Arrhenius plots in the range of 350 to $420^{\circ} \mathrm{C}$, are of the order of 160 to $180 \mathrm{~kJ} / \mathrm{mole}$ (Table 3.4). Again, we are not aware of analogous published results to compare with. 
Table 3.4 Ignition temperature and kinetic parameters of regular and catalysed soot oxidation for samples collected at various filter loading temperatures.

\begin{tabular}{|c|c|c|c|c|c|c|c|c|c|c|c|c|c|}
\hline \multirow{3}{*}{$\begin{array}{c}\text { TF Wall } \\
\text { at centre } \\
\left({ }^{\circ} \mathrm{C}\right)\end{array}$} & \multicolumn{3}{|c|}{$\begin{array}{l}\text { Ignition temperature } \\
\qquad\left({ }^{\circ} \mathrm{C}\right)\end{array}$} & \multicolumn{4}{|c|}{$\begin{array}{l}\text { Kinetic parameters of catalysed } \mathrm{HC} \\
\text { oxidation in the range of } 350-420^{\circ} \mathrm{C}\end{array}$} & \multicolumn{6}{|c|}{ Kinetic parameters of dry soot oxidation } \\
\hline & \multirow{2}{*}{0 ppm } & \multirow[b]{2}{*}{25 ppm } & \multirow[b]{2}{*}{$50 \mathrm{ppm}$} & \multicolumn{2}{|c|}{25 ppm } & \multicolumn{2}{|c|}{50 ppm } & \multicolumn{2}{|c|}{$0 \mathrm{ppm}^{1}$} & \multicolumn{2}{|c|}{$25 \mathrm{ppm}^{2}$} & \multicolumn{2}{|c|}{$50 \mathrm{ppm}^{2}$} \\
\hline & & & & $\begin{array}{c}\mathrm{E} \\
(\mathrm{kJ} / \mathrm{mole})\end{array}$ & $\underset{\left(\sec ^{-1}\right)}{\mathrm{A}}$ & \begin{tabular}{|c|}
$\mathbf{E}$ \\
$(\mathbf{k J} / \mathbf{m o l e})$
\end{tabular} & $\begin{array}{c}\mathrm{A} \\
\left(\sec ^{-1}\right)\end{array}$ & \begin{tabular}{|c|}
$\mathbf{E}$ \\
$(\mathbf{k J} / \mathbf{m o l e})$
\end{tabular} & $\begin{array}{c}\mathrm{A} \\
\left(\sec ^{-1}\right)\end{array}$ & $\begin{array}{c}\mathrm{E} \\
(\mathrm{kJ} / \mathrm{mole})\end{array}$ & $\begin{array}{c}\mathrm{A} \\
\left(\mathrm{sec}^{-1}\right)\end{array}$ & $\begin{array}{c}\mathrm{E} \\
(\mathrm{kJ} / \mathrm{mole})\end{array}$ & $\begin{array}{c}\mathrm{A} \\
\left(\sec ^{-1}\right)\end{array}$ \\
\hline 220 & - & 490 & 480 & $140 \pm 5$ & $0.83 \mathrm{E}+8$ & $160 \pm 5$ & $1.3 \mathrm{E}+10$ & - & - & $80 \pm 5$ & $0.08 \mathrm{E}+4$ & $80 \pm 5$ & $0.07 \mathrm{E}+4$ \\
\hline 250 & 630 & 520 & 490 & $140 \pm 5$ & $0.78 \mathrm{E}+8$ & $160 \pm 5$ & $1.02 \mathrm{E}+10$ & $190 \pm 5$ & $2.25 \mathrm{E}+8$ & $80 \pm 5$ & $0.08 \mathrm{E}+4$ & $80 \pm 5$ & $0.08 \mathrm{E}+4$ \\
\hline 300 & - & 490 & 480 & $140 \pm 5$ & $0.57 \mathrm{E}+8$ & $165 \pm 5$ & $1.44 \mathrm{E}+10$ & - & - & $85 \pm 5$ & $0.17 \mathrm{E}+4$ & $85 \pm 5$ & $0.21 \mathrm{E}+4$ \\
\hline 350 & 630 & 490 & 480 & $135 \pm 5$ & $0.22 E+8$ & $165 \pm 5$ & $0.81 \mathrm{E}+10$ & $190 \pm 5$ & $2.25 E+8$ & $120 \pm 5$ & $0.53 E+6$ & $100 \pm 5$ & $0.25 E+5$ \\
\hline 400 & - & 490 & 480 & $135 \pm 5$ & $0.17 \mathrm{E}+8$ & $140 \pm 5$ & $0.63 E+8$ & - & - & $130 \pm 5$ & $1.66 \mathrm{E}+6$ & $120 \pm 5$ & $0.77 \mathrm{E}+6$ \\
\hline 420 & - & 490 & 480 & - & - & - & - & - & - & $130 \pm 5$ & $1.92 \mathrm{E}+6$ & - & - \\
\hline
\end{tabular}

The role of Ce concentration to the initiation of catalytic soot oxidation was investigated too. The comparison of TGA results between the two different Cerium concentrations in fuel (25 \& 50ppm), shows a slight shift of the initiation of catalytic soot oxidation in the case of 50ppm to lower temperatures, presumably due to the earlier oxidation of VOF. This can be deduced from Figure 3.9 in which the oxidation rates for the 25ppm and 50ppm additive concentration at 220 and $400^{\circ} \mathrm{C}$ filter wall temperatures are presented.

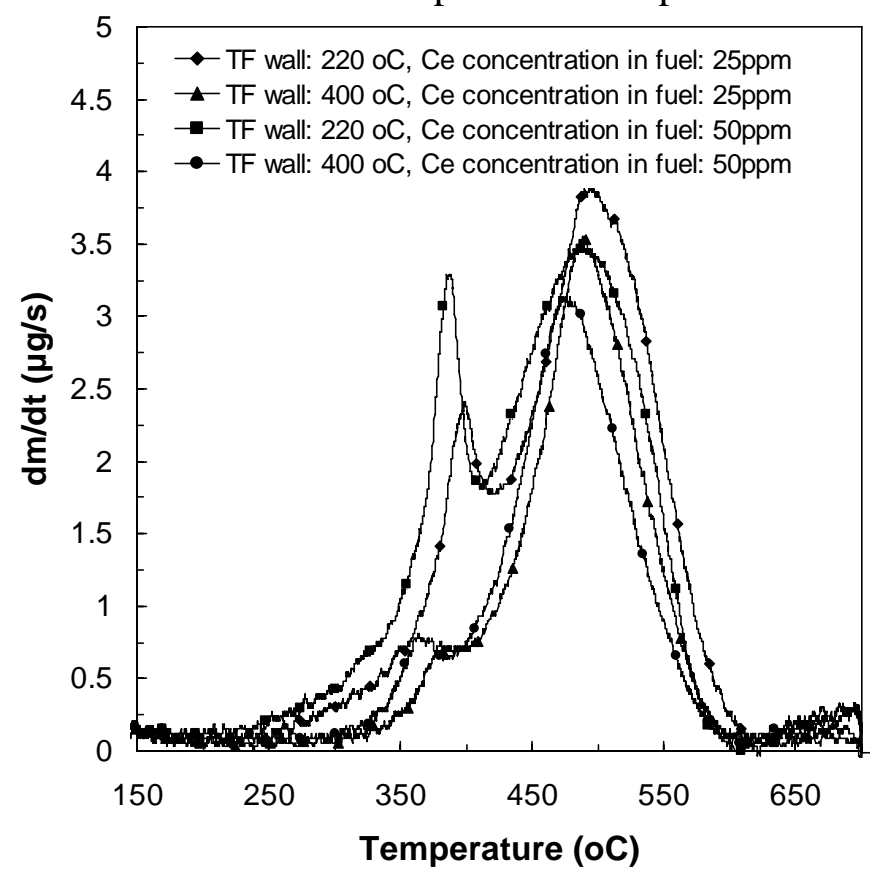

Figure 3.9 Oxidation rates produced by processing of TGA (mass reduction) curves, for a matrix of soot samples corresponding to two levels of Ce concentration in fuel (25 and $50 \mathrm{ppm})$ and two different filter wall temperatures during loading (lowest and highest temperatures with respect to VOF content in soot). A marked increase in VOF oxidation rates for the soot produced by combustion of the $50 \mathrm{ppm}$ Cerium-doped fuel is observed. Also, an observable shift to lower ignition temperatures, both for VOF and dry soot, maybe seen with the $50 \mathrm{ppm}$ soot samples.

In the same figure a significant increase to the reaction rate of catalytic VOF oxidation for the soot produced by combustion of the $50 \mathrm{ppm}$ Cerium-doped fuel is observed. Any attempt to explain these complex effects on the kinetics of soot oxidation by Ceria, should take first 
into account in-cylinder particulate formation by injection and combustion of additive-doped fuel at different fuel injection rates and profiles that correspond to the different levels of engine load. This type of investigations would be necessary especially for engines equipped with modern common-rail injection systems, as is our case. Further interactions by the fuel additive effect on the combustion of the particulate are suspected. According to [98], increasing Cerium concentration in fuel diminishes the mean particle size of the soot during formation and lowers the ignition temperature under oxidation.

The variation of particulate VOF content at the filter periphery due to the lower wall temperatures seems to have an observable effect on the calculated activation energies. Calculated activation energies for catalytic soot oxidation in the temperature range 420$490^{\circ} \mathrm{C}$, of soot samples collected from the filter periphery (higher VOF content) were lower than those of soot samples collected from filter center. This observation needs to be further investigated by additional experimental work, before an attempt is made to interpret such effects.

\subsubsection{Concluding remarks}

- A systematic effort was made to study the sorption and oxidation behaviour of soot emitted by a modern diesel engine run on catalyst-doped fuel, collected on a diesel particulate filter, in a number of steady state operation points. For this purpose, samples have been taken directly from the filter surface.

- The presence of Cerium in diesel fuel was observed to lower the TGA ignition temperature of soot emitted by a modern passenger car diesel engine from 630 to $490^{\circ} \mathrm{C}$. The observed catalytic ignition temperature was unchanged with 25 and 50ppm Ceriumdoped fuel.

- The initiation of soot oxidation occurs at lower temperatures as the Cerium concentration in the fuel increases. This may be attributed to the faster catalytic oxidation of adsorbed hydrocarbons.

- The VOF content does not appear to affect the oxidation behaviour of soot emitted by the engine run without fuel additive. The kinetic parameters are unchanged (E: $190 \mathrm{~kJ} / \mathrm{mole}$ )

- The presence of adsorbed hydrocarbons seems to result in a variation of activation energy $(80-130 \mathrm{~kJ} / \mathrm{mole})$ for soot oxidation by Ceria in the range of 420 to $490^{\circ} \mathrm{C}$. The activation energy in this range decreases as VOF increases for each Cerium concentration.

- The variation of Cerium concentration from 25 to 50ppm was not observed to have any effect on the catalytic soot oxidation activation energy in the range of 420 to $490^{\circ} \mathrm{C}$ (dry soot oxidation) for soot samples collected at $220-300^{\circ} \mathrm{C}$ filter wall temperatures. However a decrease of the activation energy was observed in the cases of samples collected at 350 and $400^{\circ} \mathrm{C}$. Furthermore, an increase of the activation energy was observed in the range of $300-420^{\circ} \mathrm{C}$ (range where the adsorbed hydrocarbons oxidation is assumed to take place).

- The wall temperature difference between center and periphery of the filter results in a variation of the VOF content, probably due to the condensation of the high boiling components to the soot particulate surface. 
- A continuation of this work with further investigation of the mechanisms of VOF - soot interaction on a diesel filter during loading and regeneration is necessary to enhance our understanding and improve our modeling of diesel particulate filter operation.

\subsection{Experimental Investigation of the Role of Soot VOF}

In this section an experimental study of the VOF adsorption-desorption phenomena and their effects on the regeneration behavior is presented. TGA analysis is used for the calculation of particulate VOF content at various engine operation points and the investigation of VOF adsorption-desorption phenomena during real filter loading experiments. The effect of VOF to the regeneration behavior is assessed by comparing regenerations of filter loaded at points with different VOF content and by reproducing the conditions for the occurrence of stochastic regenerations. This work aims at better understanding of sorption and oxidation behavior of soot produced by modern high-pressure direct injection engines when run on catalyst doped fuel for the support of improved modeling of fuel-additive assisted regeneration at low temperatures.

\subsubsection{Experimental}

Steady - State loading experiments

First objective was to map VOF content as function of engine operation point. Steady state loading experiments were performed on the LTTE (Laboratory of Thermodynamics and Thermal Engines) diesel engine test bench for this purpose. A SiC filter was fitted on the 2.0 litre displacement HDI turbocharged engine running with 25ppm Ce DPX9- doped fuel (engine specifications in Table 3.1). The collection of the particulate samples was performed directly from the filter channels' inlet. Figure 3.1 presents the experimental layout of the filter loading experiments. The characteristics of the SiC filter are shown also in Table 3.1.

\section{Filter loading test protocol}

The following strategy is applied in the determination of the exact protocol for filter loading tests: A mapping process is performed to assess the engine exhaust mass flow rate, the exhaust temperatures and HC emissions characteristics in the low- and medium- load operating range, as more representative of city driving conditions. In this work, temperatures were measured simultaneously at the exhaust pipe $50 \mathrm{~mm}$ after turbine exit, at the inlet of the filter, and inside the filter, at 4 points along a filter diameter, $15 \mathrm{~mm}$ deep from filter exit (Figure 3.1 ). The results are presented in Figure 3.10. Comparison of filter wall temperatures closer to center and periphery shows that periphery is systematically colder than the center by $25^{\circ} \mathrm{C}$ (low exhaust gas flowrate-low engine rpm) to about $10^{\circ} \mathrm{C}$ (high exhaust gas flowratehigh engine rpm). 


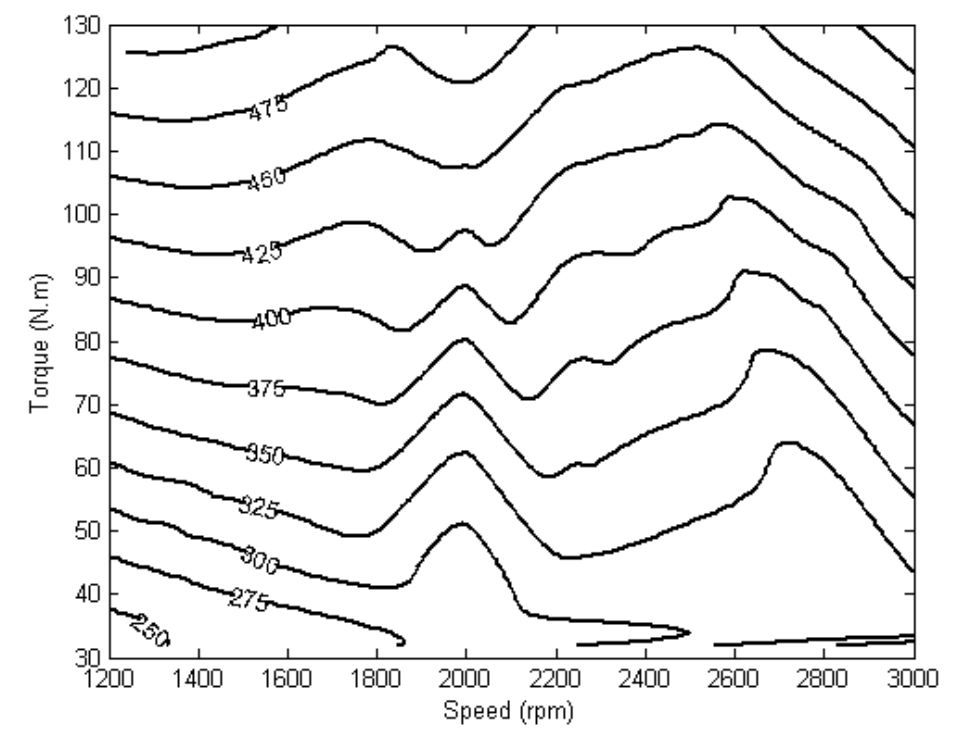

Figure 3.10 Map of engine exhaust gas temperature $\left({ }^{\circ} \mathrm{C}\right)$ measured in the exhaust pipe $50 \mathrm{~mm}$ after turbine exit (medium-to-low speed and load part of engine operation map).

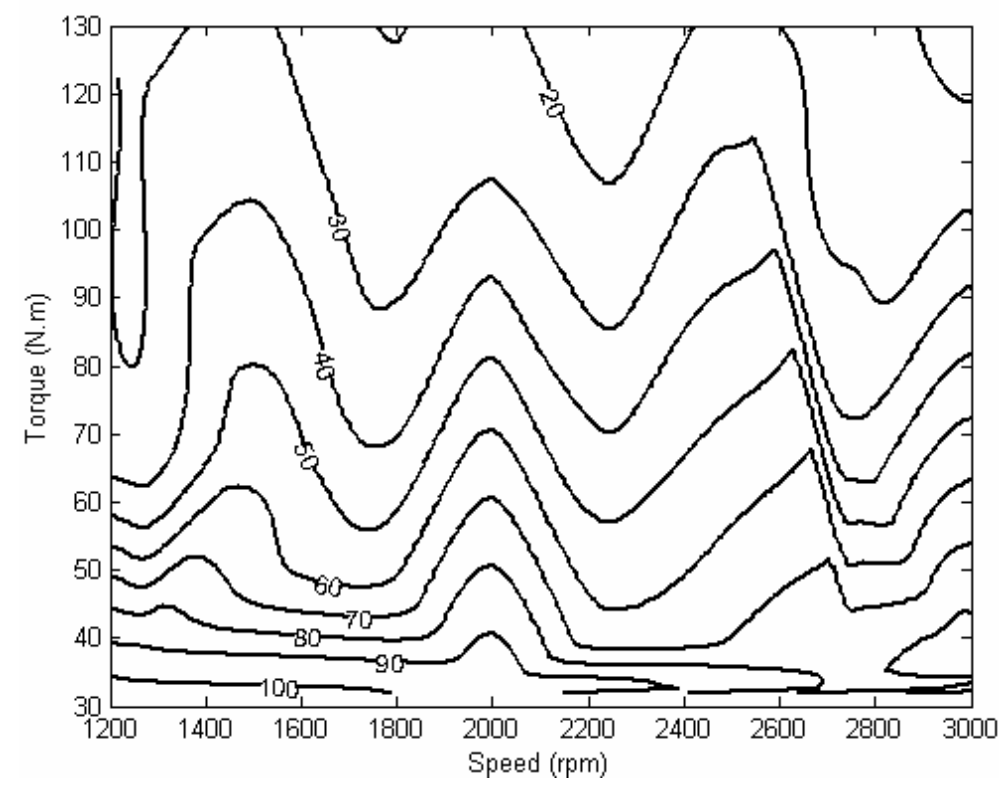

Figure 3.11 Medium-to-low speed and load part of engine map: Hydrocarbon emissions measured upstream filter as $\mathrm{CH}_{4}(\mathrm{ppm})$.

HC emissions before the filter were also measured, as additional evidence to the study of VOF sorption behaviour. Figure 3.11 shows a map of HC emissions near filter inlet. A lowering of $\mathrm{HC}$ emissions as the engine load increases is observed. Due to this behaviour, VOF adsorption is expected to be less significant at higher engine loads. The final design of the test protocol, based on the results of the engine mapping is presented in Table 3.5. It may be observed that the selected engine speed and engine load operation points cover a range of filter wall temperatures from 200 to $415^{\circ} \mathrm{C}$. This range was expected to result in a wide range of variation of VOF content.

Table 3.5 Experimental protocol 


\begin{tabular}{|c|c|c|c|c|c|c|}
\hline $\begin{array}{l}\text { Speed } \\
(\mathrm{rpm})\end{array}$ & $\begin{array}{l}\text { Torque } \\
\text { (Nm) }\end{array}$ & $\begin{array}{c}\text { Mass flowrate } \\
\text { (g/s) }\end{array}$ & $\begin{array}{c}\text { Exhaust gas } \\
\text { temperature }\left({ }^{\circ} \mathrm{C}\right)\end{array}$ & $\begin{array}{l}\text { Temperature, } \\
\text { Filter inlet }\left({ }^{\circ} \mathrm{C}\right)\end{array}$ & $\begin{array}{c}\text { Filter } \\
\text { temperature, } \\
\text { T/C9 }\left({ }^{\circ} \mathrm{C}\right) \\
\end{array}$ & $\begin{array}{c}\text { Filter } \\
\text { temperature, } \\
\text { T/C8 }\left({ }^{\circ} \mathrm{C}\right) \\
\end{array}$ \\
\hline \multirow{4}{*}{1250} & 30 & 18 & 225 & 198 & 193 & 178 \\
\hline & 60 & 21 & 300 & 270 & 261 & 240 \\
\hline & 80 & 25 & 355 & 317 & 310 & 285 \\
\hline & 100 & 28 & 419 & 385 & 380 & 364 \\
\hline \multirow{4}{*}{1800} & 30 & 30 & 254 & 236 & 234 & 216 \\
\hline & 60 & 35 & 340 & 317 & 314 & 291 \\
\hline & 80 & 38 & 400 & 370 & 370 & 344 \\
\hline & 100 & 42 & 433 & 400 & 400 & 372 \\
\hline \multirow{4}{*}{2200} & 30 & 34 & 276 & 257 & 255 & 238 \\
\hline & 60 & 38 & 360 & 340 & 340 & 315 \\
\hline & 80 & 43 & 415 & 388 & 388 & 365 \\
\hline & 100 & 50 & 423 & 392 & 392 & 371 \\
\hline \multirow{4}{*}{3000} & 30 & 69 & 305 & 300 & 297 & 287 \\
\hline & 60 & 74 & 373 & 364 & 364 & 342 \\
\hline & 80 & 79 & 395 & 384 & 384 & 377 \\
\hline & 100 & 83 & 427 & 415 & 415 & 407 \\
\hline
\end{tabular}

TGA tests on soot samples taken directly from the central and peripheral filter channels

TGA tests were performed on the TGA, to calculate the soot VOF content after each loading experiment. The methodology followed to the collection and packing of the samples was described in the previous section. Non-isothermal oxidation mode with a heating rate of $10^{\circ} \mathrm{C}$ per minute was selected in order to predict the acceleration temperature of soot oxidation [98] and to fully oxidize the soot VOF according to the experimental prediction of temperature ranges for catalytic hydrocarbon oxidation. It was assumed that VOF is oxidized in the temperature range $150^{\circ} \mathrm{C}-380^{\circ} \mathrm{C}$ and the mass reduction observed on the TGA curve in this range is considered as the VOF content of the soot collected. According to Zinbo et al [102] in this temperature range the TGA method yields a comparable measure of volatile components with those obtained by solvent extraction. This is supported by the previous TGA experiments presented in section 3.1 and [86] where a first peak value of oxidation rate was shown in this temperature range. Those experiments were carried out in synthetic air environment with a gas flow of the order of $10 \mathrm{ml} / \mathrm{min}$.

\section{Adsorption-desorption experiments}

In order to investigate the VOF adsorption-desorption behaviour, a new set of loading experiments was performed. In this series of experiments the filter was loaded at a steady state operation point with known VOF content until the filter backpressure reached a specific value. Then the operation point was shifted to effect an exhaust temperature step of the order of $100^{\circ} \mathrm{C}$ (increase in the case of desorption - decrease in the case of adsorption). In order to exclude any secondary effects from engine operation point variation, the exhaust mass flow rate was kept broadly unchanged. The engine was held at this operation point until steady state conditions were reached. Due to the variation in exhaust gas and filter wall temperatures the soot VOF content was expected to be higher or lower depending on the new operation point. After the end of each experiment, samples were collected from the central channels of the filter and analyzed on the TGA. By comparing the soot VOF content before and after the change of engine operation point, the VOF adsorption - desorption behaviour could be assessed. 


\section{Regenerations with stepwise load increase}

In another series of experiments, the filter was loaded at operation points with varying levels of VOF content. A regeneration process was initiated after the loading at each respective operation point, by a step increase in engine load. On specific temperature levels, soot oxidation was observed to start, presumably on the VOF content of the collected soot. By comparing the resultant curves of pressure drop and filter wall temperature, one could indirectly assess filter regeneration quality.

\section{Stochastic regenerations}

Loading experiments at low exhaust temperatures (lower than $300^{\circ} \mathrm{C}$ ) with relatively high VOF content were performed in order to investigate the onset of stochastic regeneration. A number of stochastic regeneration events were recorded. The conditions in which they appeared could be related to real city driving conditions [85]. A correlation of these events with VOF content of accumulated soot was attempted.

\subsubsection{Results and discussion}

First, a set of indicative TGA tests was performed to assess the fuel and engine lubrication oil behavior in the TGA to be employed as a reference point for the particulate behavior. The results are presented in Figure 3.12. EN-590 (European Norm) diesel fuel was found to 50\% evaporate at $240 \mathrm{C}$, and $95 \%$ evaporate at $350^{\circ} \mathrm{C}$. SAE-30 SG lubricant was found to $50 \%$ evaporates at $370{ }^{\circ} \mathrm{C}$ and $95 \%$ evaporate at $430^{\circ} \mathrm{C}$.

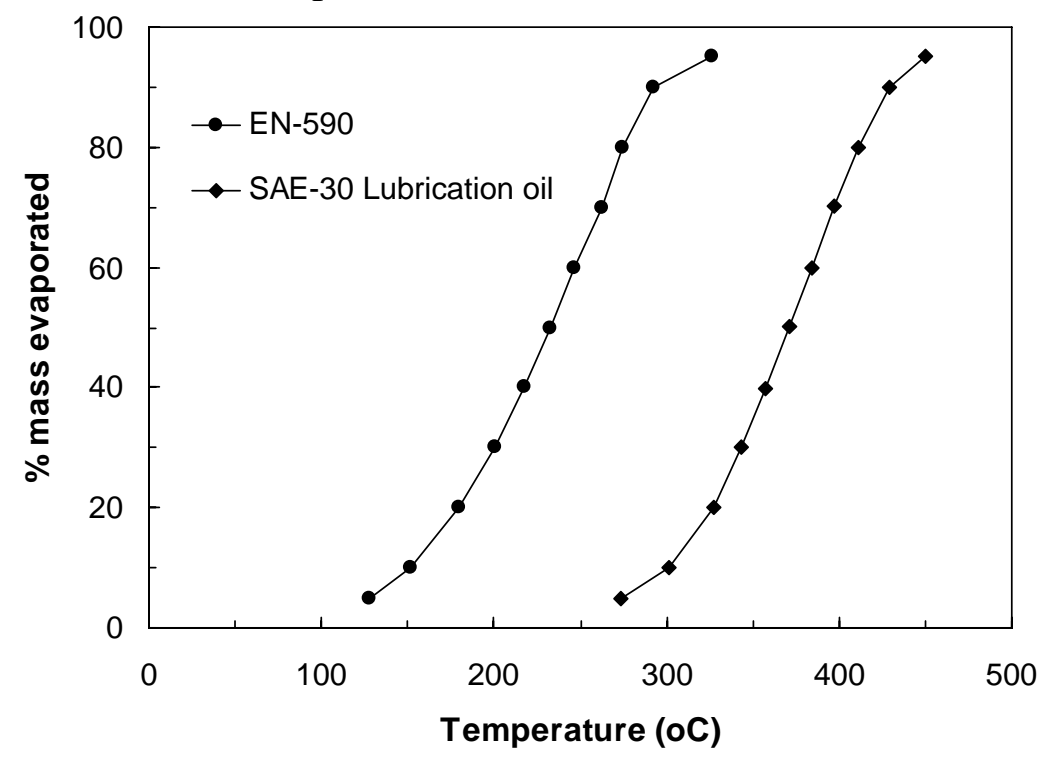

Figure 3.12 Diesel fuel EN-590 (European standard for automotive diesel fuel) and SAE_30 lubrication oil mass reduction curves as measured on the TGA.

Steady - State loading experiments - TGA tests results

The steady state filter soot loading experiments were performed with the engine operating on 25ppm DPX9-doped fuel at the previously described operation points (see Table 3). After the loading procedure, particulate samples were collected from a central and a peripheric channel of the filter, and analyzed on the TGA. The VOF content on these operation points was calculated from the TGA curves as the percentage of mass reduction in the range of $150^{\circ} \mathrm{C}$ (water free) to $380^{\circ} \mathrm{C}$. Figure 3.13 presents a comparison of the TGA curves obtained 
for samples collected from the central channels of the filter for various filter wall temperatures at $2000 \mathrm{rpm}$.

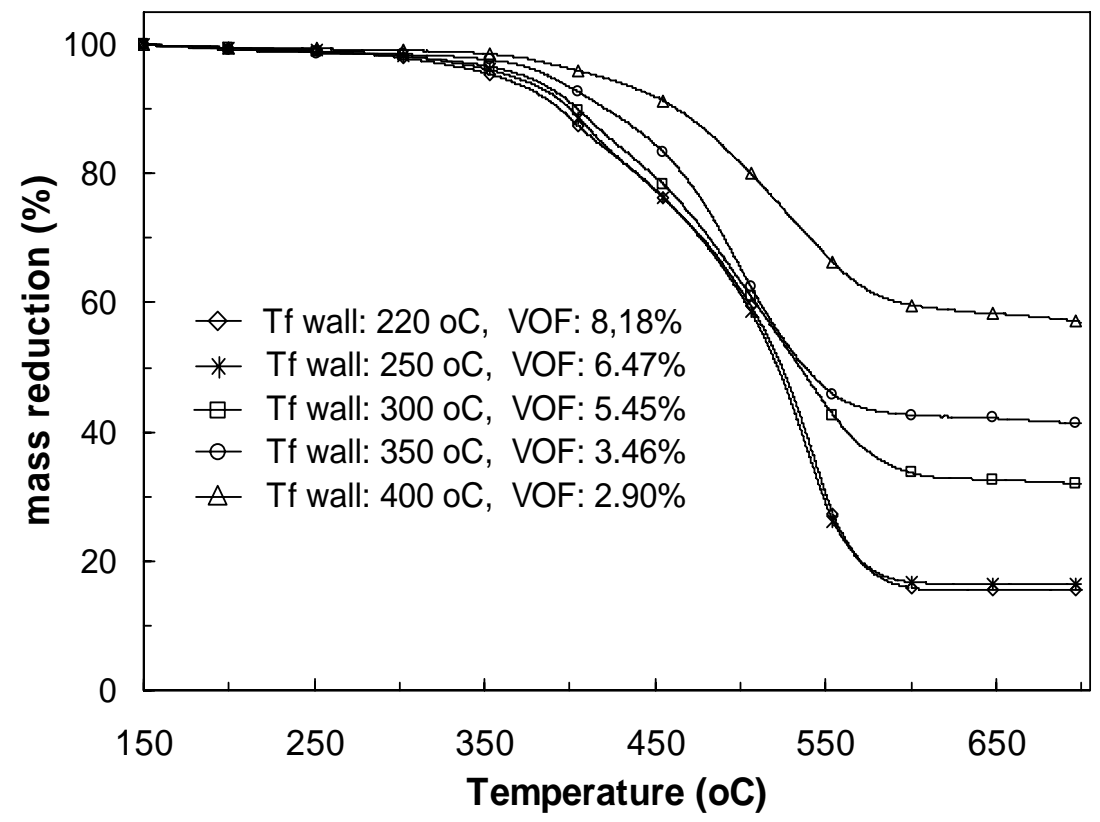

Figure 3.13 TGA (mass reduction) curves for soot collected from the central channels of the filter after moderate particulate loading at various filter channel wall temperatures. All soot samples are taken from a filter loaded with the engine running at 2000 rpm with 25ppm Ce-doped diesel fuel.

The lowering of VOF content as filter wall temperature increases is apparent. A more detailed study of the TGA curves shows that VOF evaporates and catalytically oxidized in the range of 300 to $420^{\circ} \mathrm{C}$. After this range and until about $500^{\circ} \mathrm{C}$ the catalytic oxidation of the dry soot takes place as presented in the previous section.

The results for the calculated VOF content values are summarized in Table 3.6 and are presented in the form of a map as function of engine operation point for the central and side channels of the filter in Figure 3.14. The results indicate a variation of the VOF content between 2 and $11 \%$. The role of the wall temperature difference during loading in the distribution of VOF along the filter diameter is apparent in all these results. The lowering of the filter wall temperature as one moves from filter center to periphery seems to lead to the readsorption of heavy hydrocarbons emitted in the particulate layer, thus increasing its VOF content. 
Table 3.6 VOF content of soot collected from central and peripheric filter channels (various operation points).

\begin{tabular}{|c|c|c|c|}
\hline \multicolumn{2}{|c|}{ Operation point } & \multicolumn{2}{c|}{ VOF Content (\%) } \\
\hline \multirow{3}{*}{ Speed (rpm) } & Torque (Nm) & Center & Side \\
\hline \multirow{4}{*}{1250} & 30 & 11.10 & 11.69 \\
\cline { 2 - 4 } & 60 & 6.32 & 7.43 \\
\cline { 2 - 4 } & 80 & 4.27 & 4.71 \\
\hline \multirow{4}{*}{1800} & 100 & 2.78 & 3.43 \\
\cline { 2 - 4 } & 30 & 8.18 & 9.37 \\
\cline { 2 - 4 } & 60 & 4.88 & 5.31 \\
\hline \multirow{4}{*}{2200} & 80 & 3.98 & 4.49 \\
\cline { 2 - 4 } & 100 & 2.67 & 3.23 \\
\cline { 2 - 4 } & 30 & 6.36 & 7.11 \\
\cline { 2 - 4 } & 60 & 4.51 & 4.86 \\
\hline \multirow{3}{*}{3000} & 100 & 3.76 & 4.50 \\
\hline & 30 & 3.18 & 3.30 \\
\cline { 2 - 4 } & 60 & 9.03 & 9.86 \\
\cline { 2 - 4 } & 80 & 7.74 & 8.28 \\
\hline & 100 & 2.43 & 6.71 \\
\hline
\end{tabular}

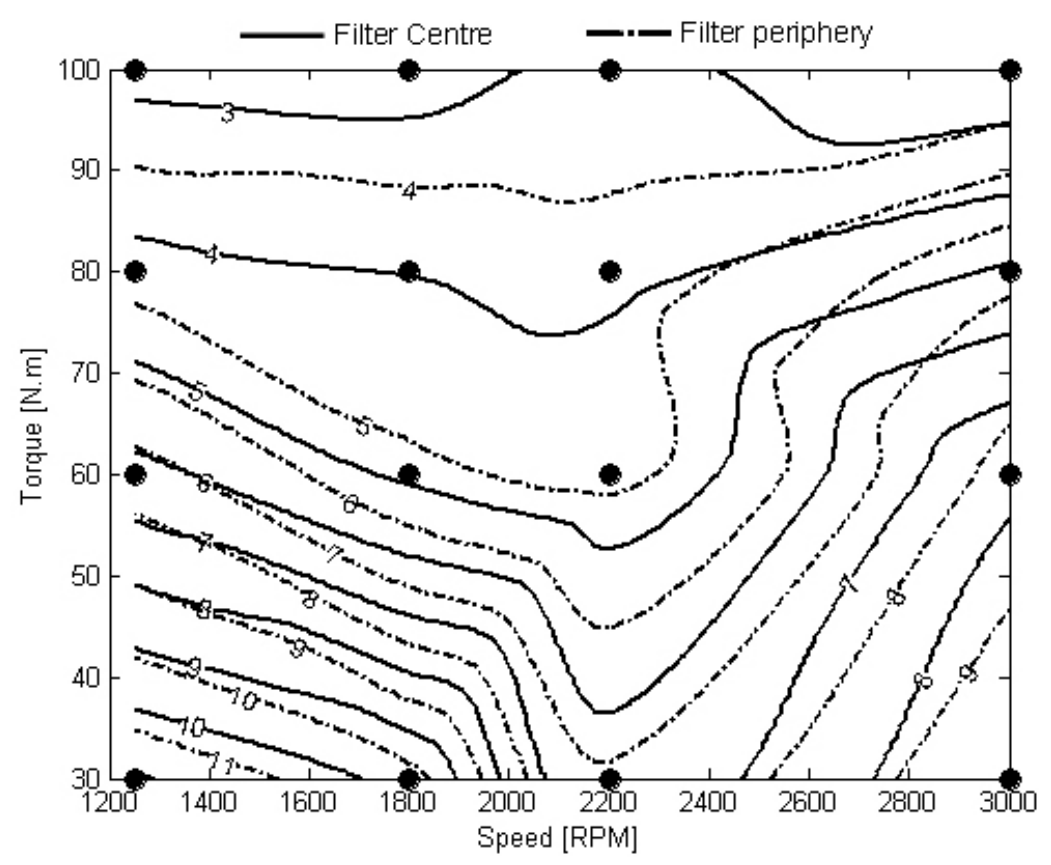

Figure 3.14 VOF map produced by processing of TGA (mass reduction curves) for soot samples collected from the central and side channels of the filter, for a matrix of engine operation points corresponding to different levels of filter wall temperatures during loading. All soot samples taken from filter loaded by engine running on 25 ppm Ce. (medium-to-low speed and load part of engine operation map).

\section{Desorption experiments}

To get a first impression of desorption events inside the filter, a series of loading experiments with step increase of filter wall temperature of the order of $100^{\circ} \mathrm{C}$ were 
performed. The scenario is presented in Figure 3.15 (step variation of engine operation point from $(3000 \mathrm{rpm}, 40 \mathrm{Nm})$ to $(2450 \mathrm{rpm}, 100 \mathrm{Nm})$, which results in near-centre filter wall temperature increase from $290^{\circ} \mathrm{C}$ to $390^{\circ} \mathrm{C}$ ). It must be mentioned that the new operation point is characterized by reduced particulate emissions that result in reduced soot accumulation rate inside the filter. Furthermore, it can be assumed that the effect of soot collected after the varying the engine operation point is negligible.

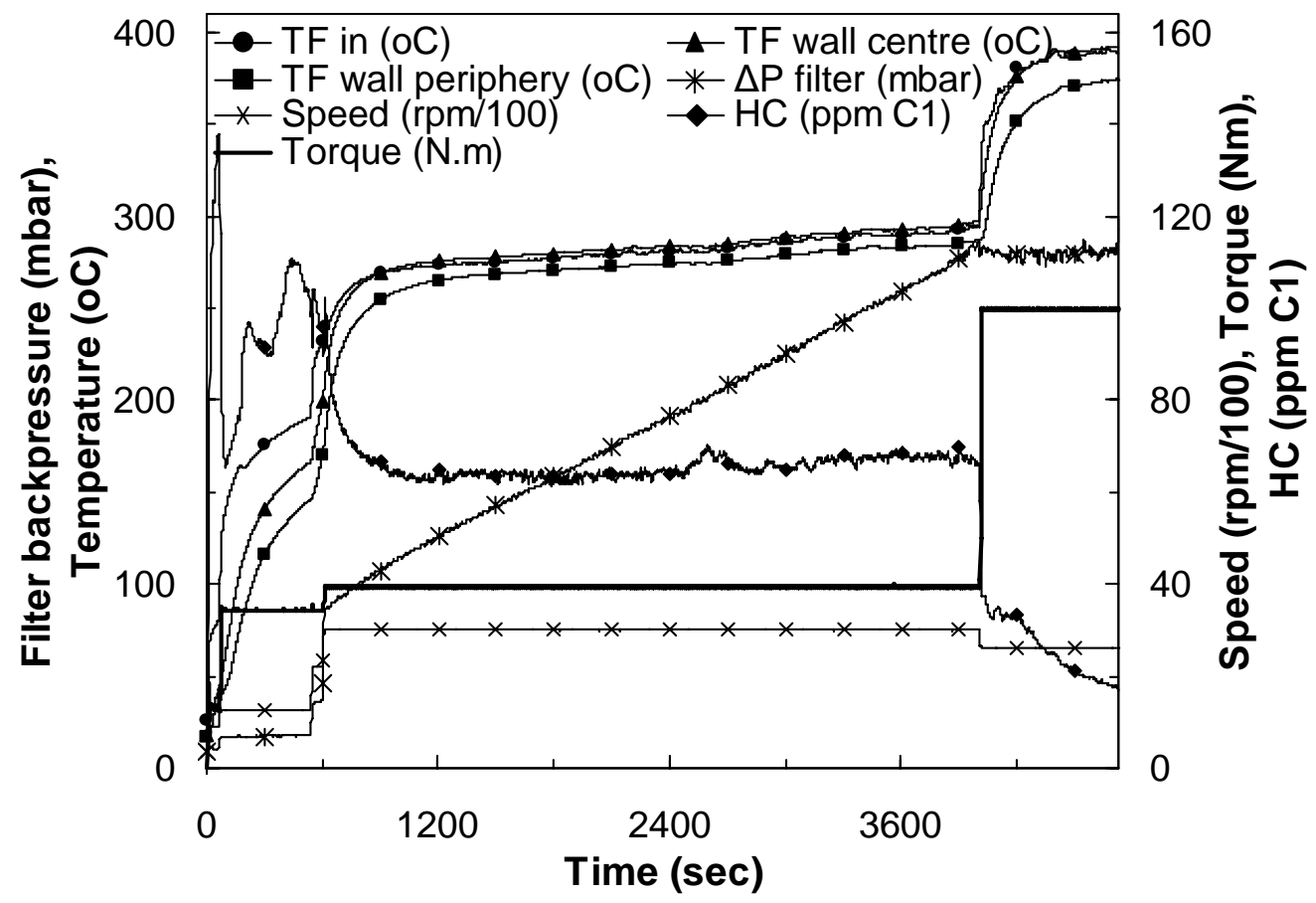

Figure 3.15 VOF desorption scenario. Result of a step increase in inlet temperature, produced by changing the engine operation point from speed: $3000 \mathrm{rpm}$, load: $40 \mathrm{Nm}$ to speed: $2450 \mathrm{rpm}$, load: $100 \mathrm{Nm}$. The effects on the filter wall temperatures are measured by thermocouples T/C9, which is closer to center and T/C8 (periphery).

A further observation is related to the hydrocarbon emissions that are monitored after the filter. Although the new operation point is characterized by lower HC emissions, there is an observable, minor delay to the initiation of decrease. This could be attributed to the evaporation and desorption of VOF accumulated in the dry soot and the porous ceramic filter due to the higher wall temperatures. In order to confirm this observation, samples were collected from the central channels of the filter and analyzed on the TGA. The TGA curves of Figure 3.16 indicate a decrease in soot VOF content from $7.7 \%$ (soot collected from filter loaded at speed: $3000 \mathrm{rpm}$, load: $40 \mathrm{Nm}$ ) to $4.4 \%$ (soot collected after desorption). 


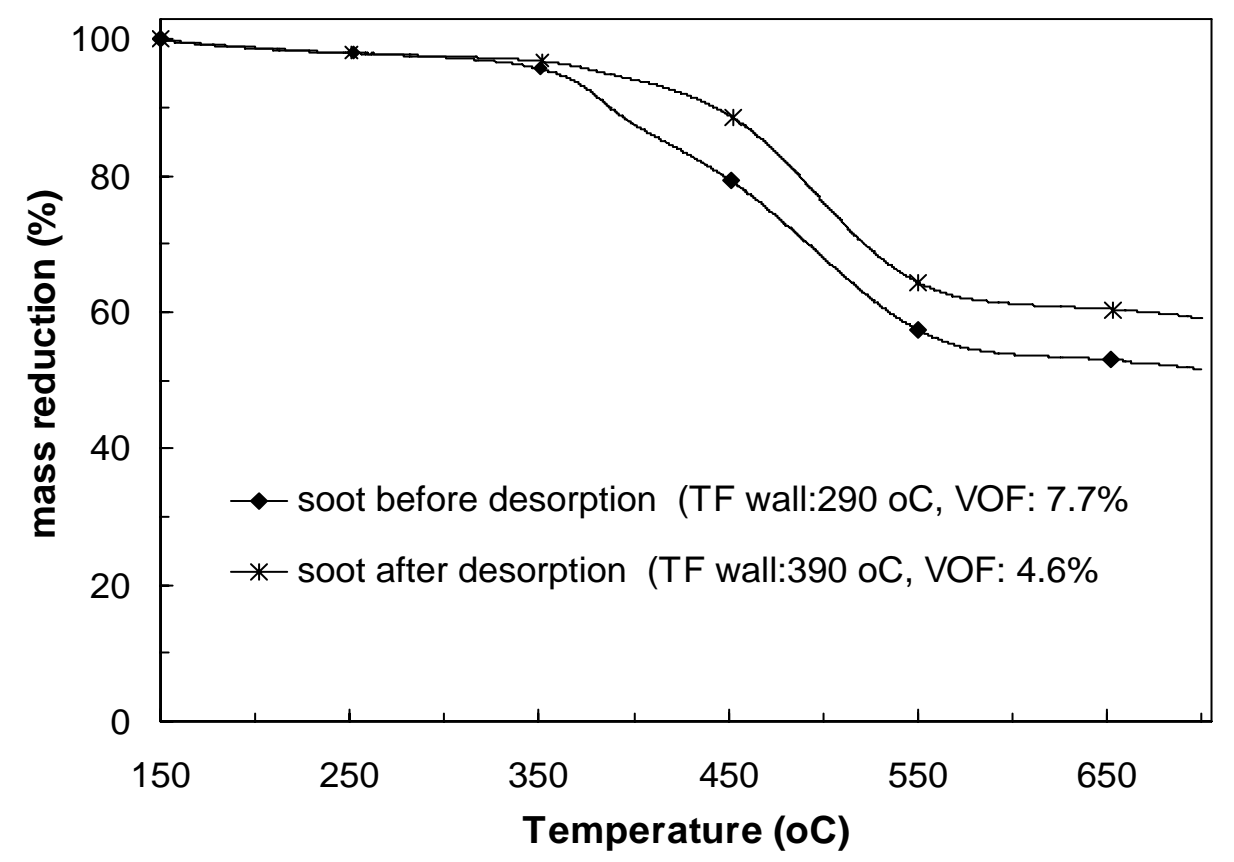

Figure 3.16 TGA curves for soot collected from the central channels of the filter before and after desorption.

\section{Adsorption experiments}

In this series of experiments, after prolonged filter loading at high temperatures and low engine $\mathrm{HC}$ emissions, the engine operation point was suddenly varied resulting in a step decrease in filter inlet temperature, resulting in a final decrease of filter wall temperature of the order of $100^{\circ} \mathrm{C}$ and a significant increase in engine out HC emissions. As presented in Figure 3.17, the engine operation point was varied from 2000rpm- $90 \mathrm{Nm}$ to $2500 \mathrm{rpm}-40 \mathrm{Nm}$, resulting in a decrease of the central filter wall temperature from $385^{\circ} \mathrm{C}$ to $290^{\circ} \mathrm{C}$. In this case, the shifted engine operation point is characterized by increased particulate emissions, which result in an increase in filter loading rate. Based on diminishing of the slope of the pressure drop increase curve after the engine operation point shift $(t=4000 \mathrm{~s})$, one could deduce that the collected mass of soot with the engine running on the new operation point is negligible compared to the soot mass collected before the change of operation point. Furthermore the faster increase to the filter backpressure could be attributed to the effect of VOF present in the thick particulate layer, which condenses and readsorbs due to the decrease of filter wall temperature, thus differentiating soot permeability characteristics. In order to confirm the readsorption of hydrocarbons on the soot, particulate samples collected from the central channels of the filter and analyzed in the TGA. The TGA curves of Figure 3.18 indicate an increase to the VOF content from $2.5 \%$ (soot collected from filter loaded at speed: 1800rpm load: $90 \mathrm{Nm}$ ) to $4.1 \%$ (soot collected after adsorption). 


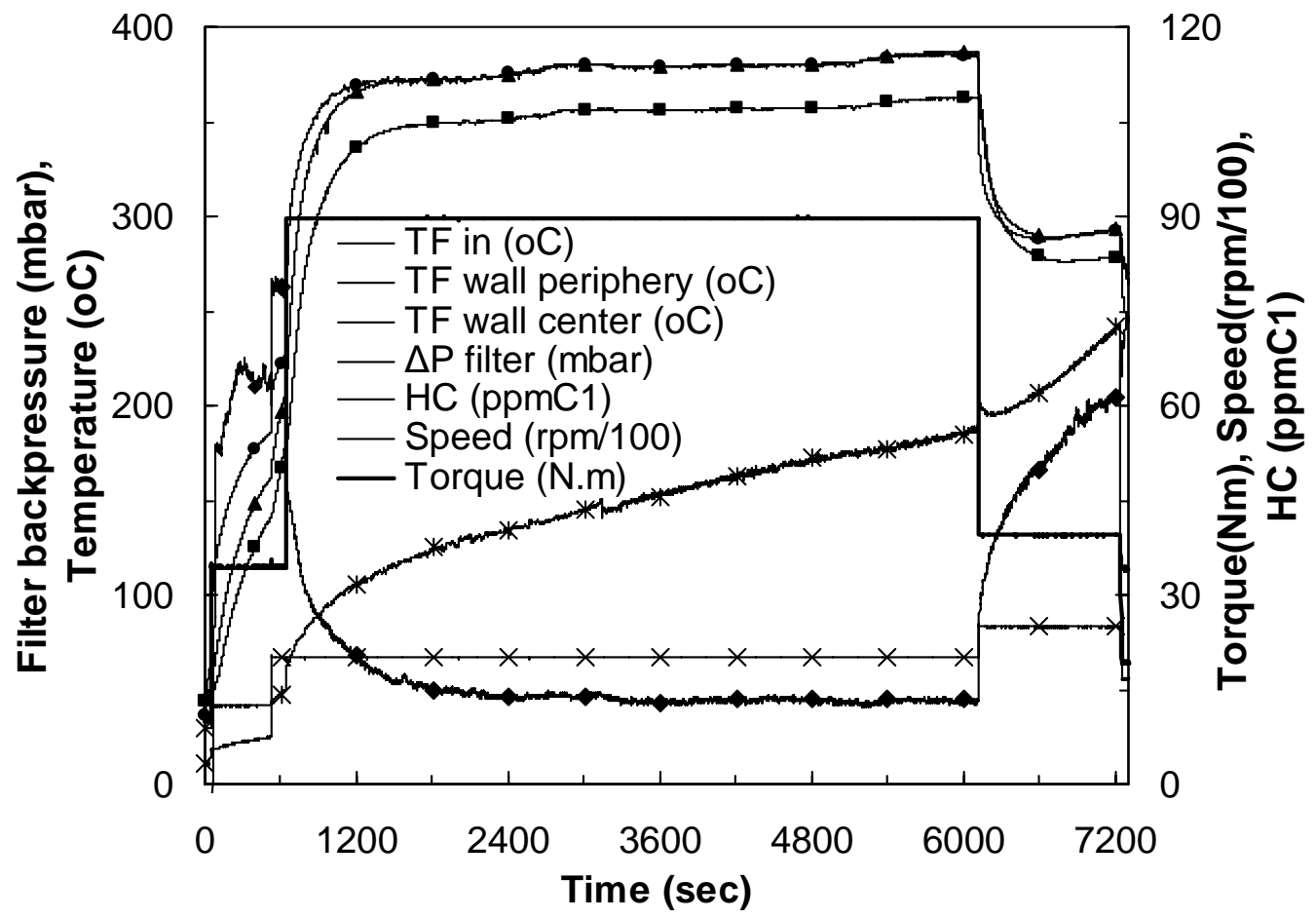

Figure 3.17 VOF adsorpion scenario. Result of a step increase in inlet temperature, produced by changing the engine operation point from speed: $2000 \mathrm{rpm}$, load: $90 \mathrm{Nm}$ to speed: $2500 \mathrm{rpm}$, load: $40 \mathrm{Nm}$. The effects on the filter wall temperatures are measured by thermocouples T/C9, which is closer to center and T/C8 (periphery).

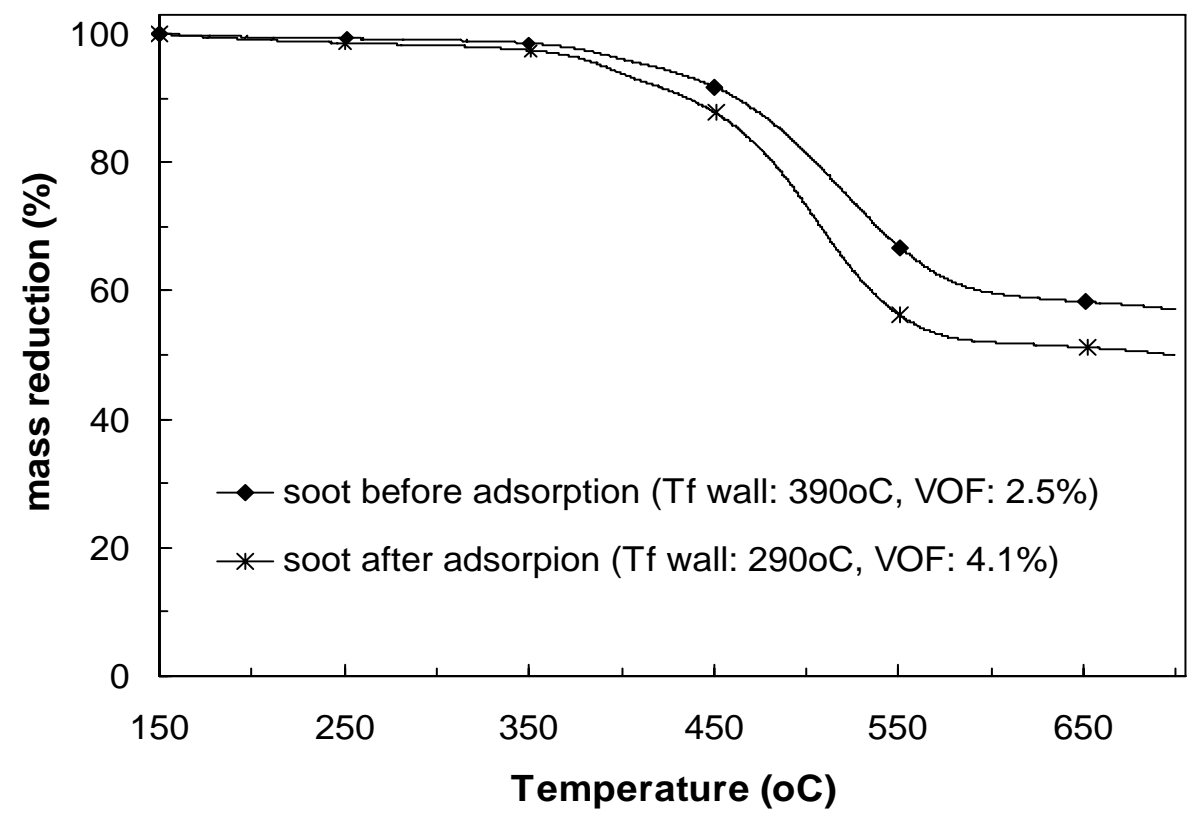

Figure 3.18 TGA curves for soot collected from the central channels of the filter before and after adsorption. 


\section{Regenerations with stepwise load increase}

In order to investigate the effect of the VOF content in the quality of regeneration, another set of experiments was performed with the filter loaded at two operation points: 1250rpm 30Nm (VOF: 10\%), and 1250rpm - 60Nm (VOF: 6\%) respectively. The filter was subsequently regenerated at 2000rpm with stepwise load increase from 20 to $190 \mathrm{Nm}$, resulting in a temperature increase of the order of $300^{\circ} \mathrm{C}$. A comparison of two typical recordings is shown in Figure 3.19.

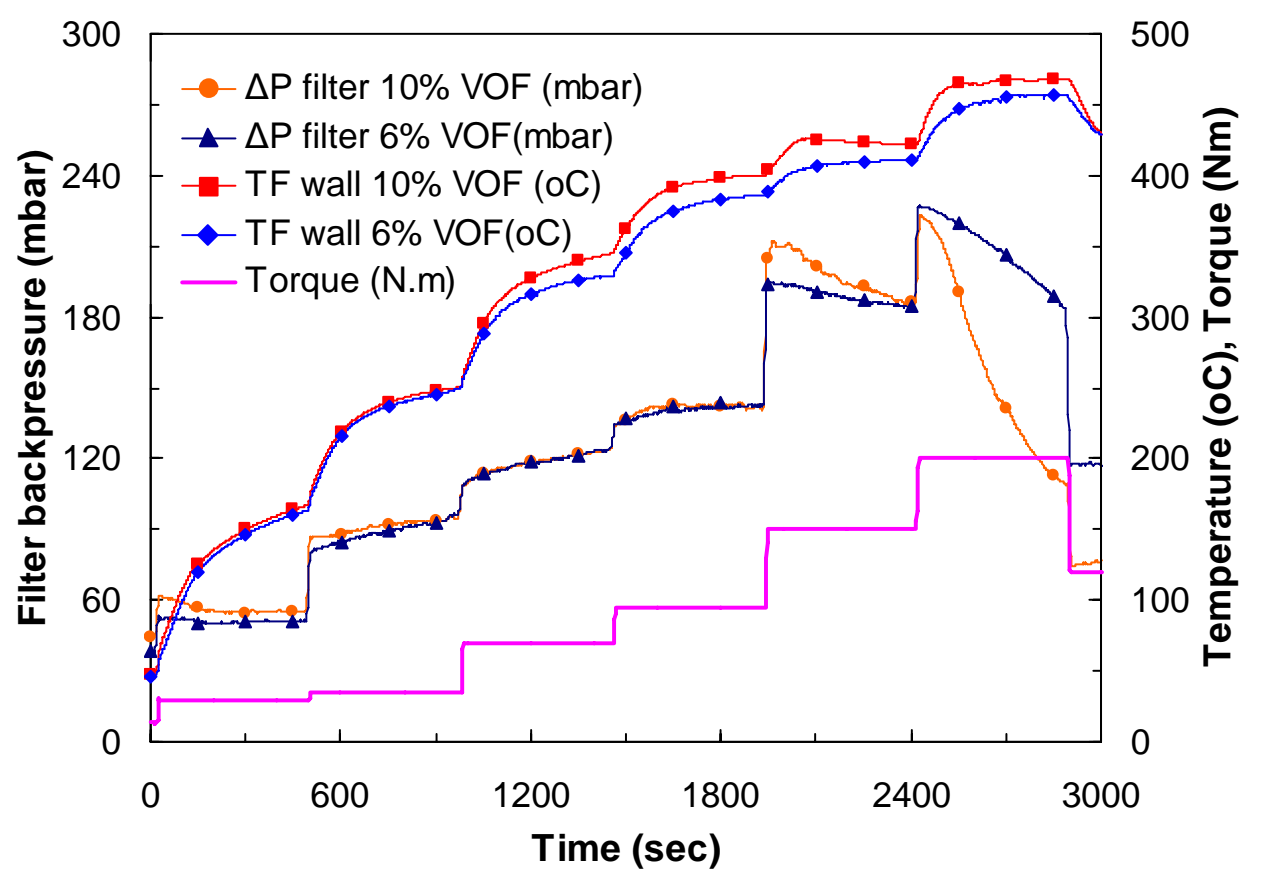

Figure 3.19 Comparison of two regenerations for a SiC 14/200 filter. In the first, the filter was loaded at a point with high VOF content (1250rpm -30Nm-VOF:10\%). In the second, filter loaded at low VOF operation point (1250rpm-60Nm-VOF:6\%). Temperature inside filter measured by thermocouple T/C9 (closer to filter centre).

It can been seen that for specific levels of filter backpressure and temperature, regeneration initiates in both cases and the shape of filter pressure drop directly correlates with the VOF content of each case. The filter loaded with higher VOF content seems to have a more rapid pressure drop decrease during regeneration, in comparison with the lower- VOF- contentloaded filter. In addition, a higher heat release rate was recorded in the first case, apparently due to the higher exothermy of hydrocarbon oxidation which resulted by the higher quantity of hydrocarbons adsorbed on the filter's soot. This results in higher wall temperature and enhances regeneration rates. This effect is pronounced at the end of this procedure, when a decrease in load is applied in order to stop the regeneration. At this moment, filter backpressure drops and it is clear that the filter loaded in higher VOF content was more efficiently regenerated.

Pressure drop recordings of this type could, in principle, be employed in extracting apparent kinetics parameters for modelling. This would require better knowledge of pressure drop as function of soot permeability and collected soot mass in the filter, but also of VOF content [80]. Assuming a good knowledge of the above dependencies [69], one could correlate oxidation rates of VOF content (that is represented qualitatively by the pressure drop), with the results of simplified Arrhenius-type curves, and thus determine apparent activation energies and frequency factors for VOF oxidation [86]

Stochastic regenerations 
Stochastic regenerations are frequently observed in city driving conditions. In the above series of engine bench experiments, during a filter loading at $3000 \mathrm{rpm}, 40 \mathrm{Nm}$, VOF: $6.8 \%$ a rapid pressure drop was observed, with the appearance of a stochastic regeneration.

As can been seen in Figure 3.20, an abrupt decrease in backpressure occurs, accompanied by a peak in the wall temperature of a peripheric group of filter channels. This fact hints the initiation of regeneration process in periphery, in this case. Heat release seems to be high enough to propagate regeneration in a transverse direction, thus burning a part of the rest of the soot accumulated in the other groups of channels, with a characteristic phase shift in the events. This event could be attributed to a higher VOF content in the periphery than in the center of the filter. It is observed that the regeneration in the filter center is responsible for the rapid pressure drop. While oxidation seems to stop in the center, filter temperature is high enough to assist regeneration of soot accumulated in periphery that is shown by the high temperature peaks of thermocouples 8 and 11.

It should be noted that the filter wall temperature is the regulative parameter for the initiation of a stochastic regeneration since it related with the adsorption-desorption phenomena that determines the soot VOF content. If the filter temperature exceeds the 380$420^{\circ} \mathrm{C}$ most VOF is vaporized and only dry soot remains accumulated on the filter wall. On the other hand, prolonged operation of a heavily loaded filter with dry soot under low load and speed conditions can lead to the readsorption of heavy hydrocarbons emitted in the particulate layer, thus increasing its VOF content. This stochastic behaviour of regeneration is quite useful in city driving conditions where low exhaust temperatures prevail.

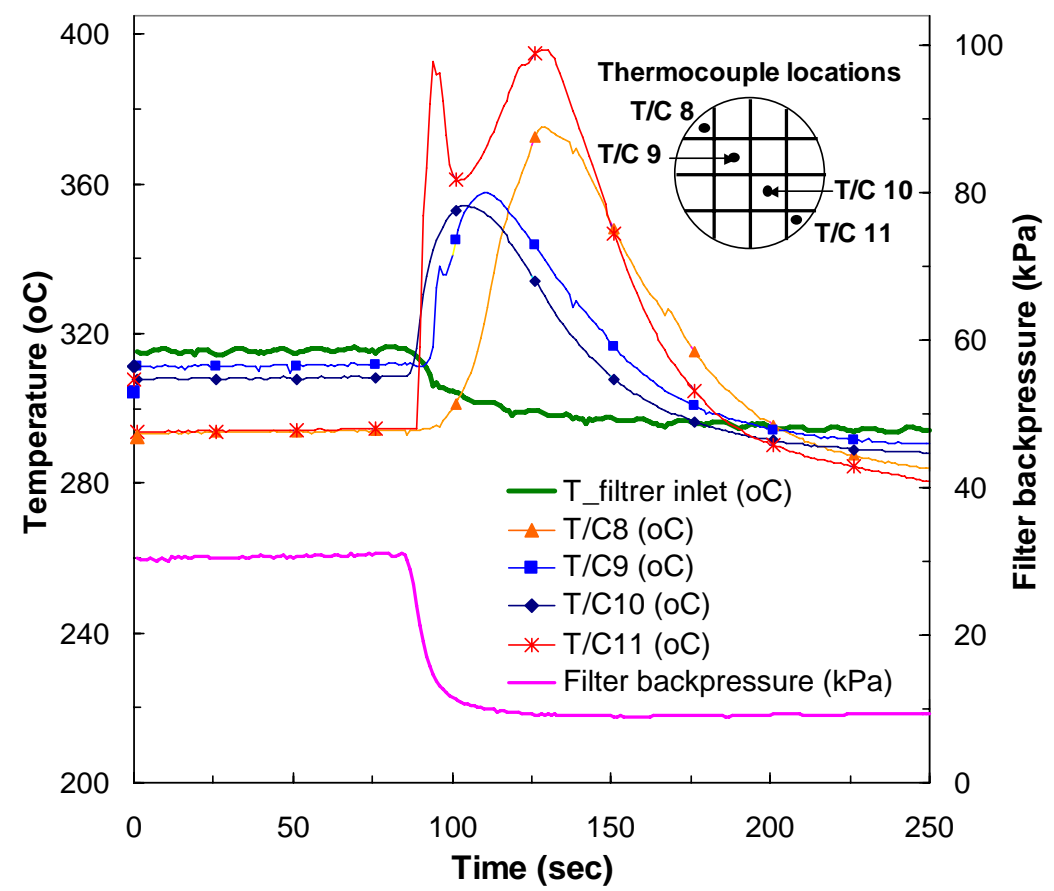

Figure 3.20 Stochastic regeneration scenario. Measured temperatures along a filter diameter near channels exit together with filter backpressure are presented during a stochastic regeneration initiated at 3000rpm, 40Nm after an approximate filter loading of $8 \mathrm{~g} / \mathrm{l}$.

\subsubsection{Concluding remarks}

- The role of volatile organic fraction of diesel particulate collected on a SiC filter installed on a modern DI diesel engine run on DPX9 doped fuel was investigated. 
- Starting from a mapping of exhaust gas and filter center and periphery temperatures as function of engine speed and load, a mapping of VOF content of particulate accumulated on the filter is derived.

- In addition to the steady state experiments that were necessary for the above mapping processes, a number of transient experiments were carried out to investigate the readsorption or desorption of VOF from the soot deposit.

- The results of above-mentioned steady state and transient tests, facilitate also the design and understanding of additional experiments with stochastic filter regeneration behaviour.

- In addition to improving understanding of diesel soot VOF adsorption - desorption behaviour, the experiments presented above set the scene for the determination of apparent kinetic parameters for hydrocarbon adsorption - desorption, and VOF and soot oxidation from Ceria. This is quite useful in the direction of further improving mathematical models of diesel particulate filter loading and regeneration.

\subsection{Modes of Catalytic Regeneration in Diesel Filters}

In this section, the results of characteristic full-scale regeneration tests that represent scenarios met under real driving conditions are presented. Two sets of regeneration experiments were performed. In the first set regeneration tests were performed at steady state engine operation points with markedly different levels of engine exhaust gas mass flowrate. In the second set the transient scenarios of uncontrolled and stochastic regeneration were reproduced on the engine bench. The main objective is to demonstrate the complexity of the phenomena involved to the process of catalytic regeneration (flow and soot maldistribution VOF) and their effects to the temperature distribution across the filter channels, and, consequently the evolution of the regeneration at the central and peripheric area of the filter.

\subsubsection{Experimental}

The regeneration tests were performed on a SiC 200cpsi filter (diameter x length: 5.66' 'x 6') fitted to the exhaust system of a 2.0 liter displacement, turbocharged passenger car engine about 600mm after turbo, running on one of the Laboratory of Thermodynamics and Thermal Engines (LTTE) engine test benches. The engine and diesel filter specifications are presented in Table 3.1. The temperatures were measured simultaneously, at the inlet and the exit of the filter, and inside the filter, along a filter diameter $15 \mathrm{~mm}$ deep from filter exit. The exhaust emissions of $\mathrm{CO}, \mathrm{CO}_{2}, \mathrm{NO}_{\mathrm{x}}$ and $\mathrm{HC}$ were measured simultaneously before and after the filter. The $\mathrm{O} 2$ concentration was calculated by the $\mathrm{A} / \mathrm{F}$ ratio obtained from the UEGO sensor installed before the filter inlet. Figure 3.1 presents the experimental layout of the filter loading and regeneration experiments.

\section{Steady state regeneration experiments}

The design of regeneration experiments takes into account the exhaust temperature and flowrate maps of the specific engine - filter combination presented in [86]. Three typical regeneration engine operation points that represent the real conditions met under City and Extra-Urban driving conditions were studied: 
- The first one involves a high speed of 4000rpm and a low load of 30Nm engine operation that result to a high mass flow rate of $92 \mathrm{~g} / \mathrm{s}$ and a high exhaust temperature of $620 \mathrm{oC}$.

- The second involves a medium speed of $2250 \mathrm{rpm}$ and a medium load of 60Nm engine operation that result to a medium flow rate of $58 \mathrm{~g} / \mathrm{s}$ and a medium exhaust temperature of $550^{\circ} \mathrm{C}$.

- The third involves a low speed of $1500 \mathrm{rpm}$ and a medium load of $80 \mathrm{Nm}$ engine operation that result to a low flow rate of $28 \mathrm{~g} / \mathrm{s}$ and a lower exhaust temperature of $500{ }^{\circ} \mathrm{C}$.

Each regeneration process was initiated by a combined step variation in engine load and engine intake airflow obtained with adjustment of turbocharger and EGR valves. The engine was left to run for 10 minutes on steady state conditions before the regeneration strategy is applied, duration considered long enough for thermal and chemical equilibrium to be reached. The filter loading was performed with the engine running on 3000rpm, 40Nm with 25ppm Cerium doped fuel. This point is characterized by low soot VOF content in order to exclude any secondary effects due to the dynamic phenomena of adsorption-desorption and stochastic regeneration.

\section{Transient regeneration experiments}

In this series of experiments the conditions resulting to the appearing of uncontrolled and stochastic regeneration were reproduced. Under real driving conditions an uncontrolled regeneration is possible to happen during a sudden deceleration of the vehicle from a high speed, high load engine operation point to idle. On the engine bench this scenario was reproduced with a step variation to the engine operation point from speed 2000rpm and load $180 \mathrm{Nm}$ that is a high flowrate $(80 \mathrm{~g} / \mathrm{s})$-high exhaust temperature $\left(550^{\circ} \mathrm{C}\right)$ point to speed 900rpm and no load that represents the idle conditions. The filter was previous heavily loaded $(18 \mathrm{~g} / \mathrm{l})$ at $1800 \mathrm{rpm}, 80 \mathrm{Nm}$ with 25ppm Cerium doped fuel.

Table 3.7 Experimental protocol of the catalytic regeneration experiments.

\begin{tabular}{|c|c|c|c|c|c|c|c|c|}
\hline \multicolumn{2}{|c|}{ Regeneration scenario } & $\begin{array}{c}\text { Speed } \\
(\mathbf{r p m})\end{array}$ & $\begin{array}{c}\text { Torque } \\
(\mathbf{N m})\end{array}$ & $\begin{array}{c}\mathbf{m f r} \\
(\mathbf{g} / \mathbf{s})\end{array}$ & $\begin{array}{c}\text { Soot } \\
\text { mass }(\mathbf{g})\end{array}$ & $\begin{array}{c}\text { TF inlet } \\
\left({ }^{\mathbf{0}} \mathbf{C}\right)\end{array}$ & $\begin{array}{c}\text { TF wall } \\
\mathbf{c e n t e r}\left(\mathbf{(}^{\mathbf{0}} \mathbf{C}\right)\end{array}$ & $\begin{array}{c}\text { TF wall } \\
\text { side }\left({ }^{\mathbf{0}} \mathbf{C}\right)\end{array}$ \\
\hline \multirow{3}{*}{$\begin{array}{c}\text { Steady } \\
\text { state }\end{array}$} & High flowrate & 4000 & 30 & 92 & 14 & 650 & 643 & 610 \\
\cline { 2 - 9 } & Medium flowrate & 2250 & 60 & 52 & 14 & 560 & 545 & 496 \\
\cline { 2 - 9 } & Low flowrate & 1500 & 80 & 28 & 14 & 535 & 517 & 486 \\
\hline \multirow{3}{*}{\begin{tabular}{c} 
Transient \\
\cline { 2 - 9 }
\end{tabular}} & Uncontrolled & $\begin{array}{c}2000 \\
\text { to 900 }\end{array}$ & $\begin{array}{c}180 \\
\text { to no load }\end{array}$ & 14 & 20 & 520 & 511 & 470 \\
\cline { 2 - 9 } & Stochastic & 3000 & 40 & 70 & 16 & 320 & 311 & 294 \\
\hline
\end{tabular}

In order to investigate the onset of stochastic regeneration loading experiments at low exhaust temperatures (lower than $350^{\circ} \mathrm{C}$ ) with a relatively high VOF content were performed. A number of stochastic regeneration events were recorded during loading at speed: 3000rpm, load: $40 \mathrm{Nm}$ that is an operation point associated with a high VOF content (6.8\%) after an approximate loading level of $6 \mathrm{~g} / \mathrm{l}$. The conditions in which the stochastic regenerations appeared could be related to real city driving conditions. The details of the regeneration experiments are presented in Table 3.7. 


\subsubsection{Experimental results and discussion}

\section{Steady state regeneration experiments}

\section{High flowrate regeneration}

This is a typical regeneration in real driving conditions occurring during engine operation at high speed - high load where the exhaust gas temperature is sufficiently high. A characteristic high- flowrate (92g/s) regeneration, is shown in Figure 3.21.

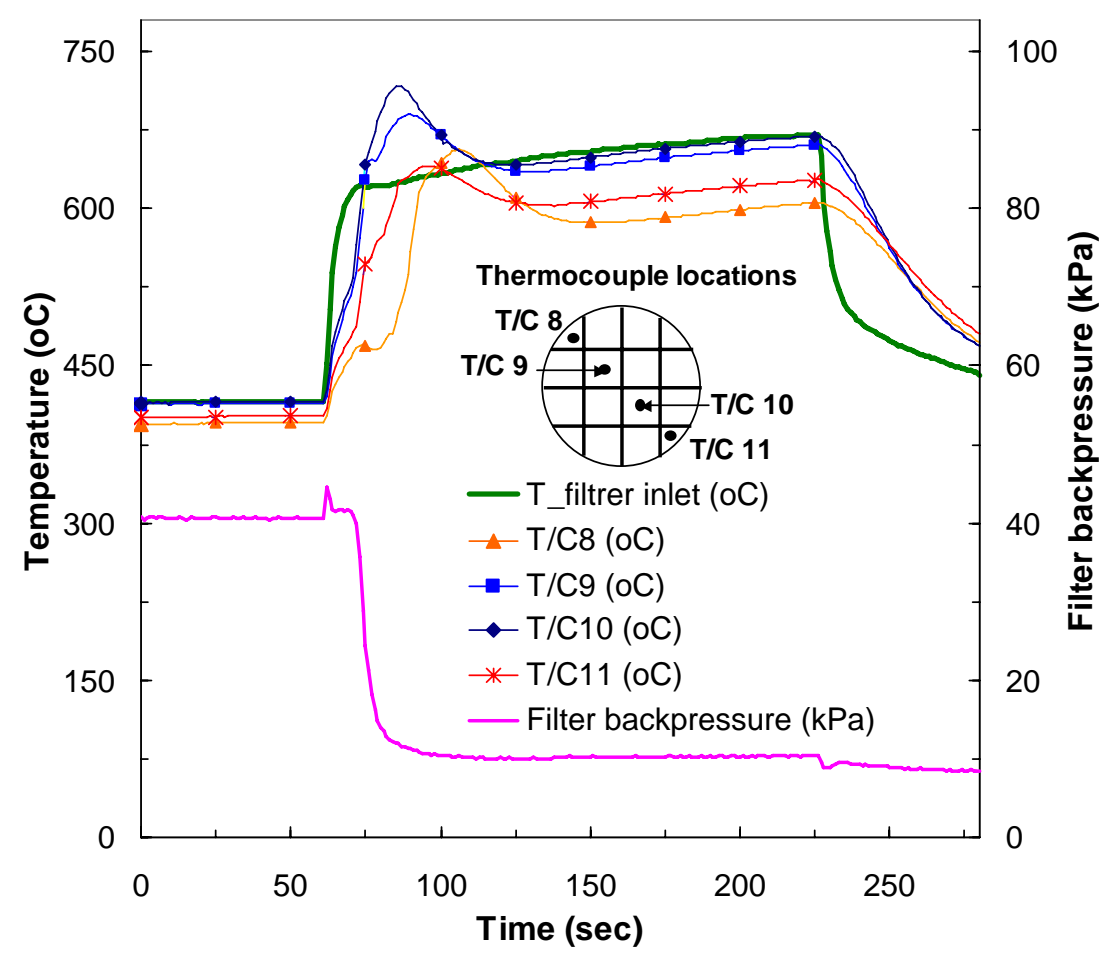

Figure 3.21 High flowrate regeneration scenario (92g/s). Measured temperatures along a filter diameter near channels exit together with filter backpressure are presented during a high mass flowrate regeneration performed at 4000rpm, 30Nm for a medium mass loaded filter (6g/l) at 3000rpm, 40Nm with 25ppm Ce-doped fuel.

As it can be seen a simultaneously elevation to the temperatures measured by the thermocouples located at the center and periphery near the filter exit is observed. This indicates a uniform evolution of regeneration across the different filter channels. The convective heating is the dominating phenomenon responsible for the initiation and the uniform propagation of the soot oxidation. Due to the high flowrate the filter is heated faster resulting to a short duration of the total regeneration. The soot starts to oxidize when a temperature level of $480^{\circ} \mathrm{C}$ is achieved. The temperatures in the periphery are lower, because of the heat losses through the periphery to the ambient air.

\section{Medium flowrate regeneration.}

A characteristic medium flowrate regeneration is shown in Figure 3.22. Initial soot mass is approximately the same. Exhaust gas oxygen content is also selected to be the same level, by careful selection of the operation point. As the space velocity is reduced, the onset of 3D behaviour is observed, and flow maldistribution phenomena prevail. In the specific example, we observe the onset of regeneration at the central channels at about $t=110$ seconds. The onset 
of regeneration at the peripheric channels is observed at about $\mathrm{t}=150-200 \mathrm{~s}$. This is a significant delay between the onset of regeneration of the central and peripheric filter blocks. A second remark refers to the maximum temperatures. Here, we observe that the increase of the maximum temperature levels over the inlet temperatures augments as flowrate decreases. This is in line with the energy balance equations [103].

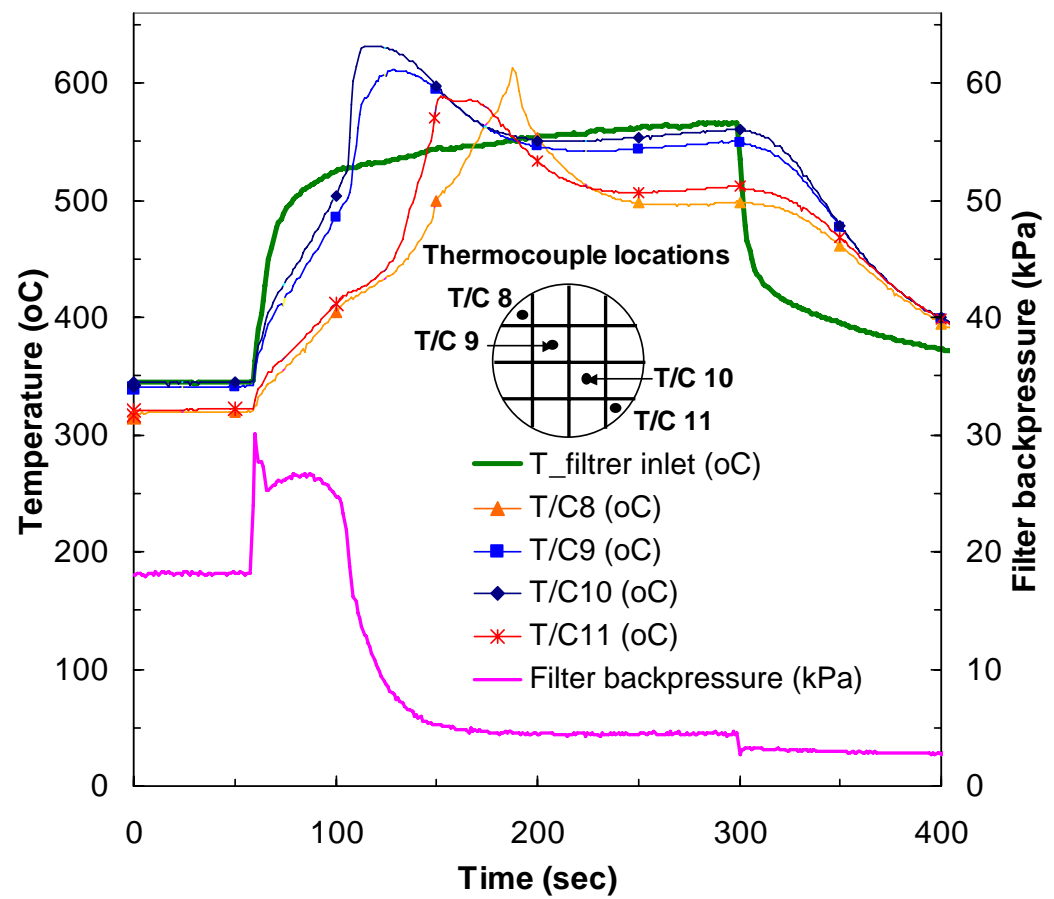

Figure 3.22 Medium flowrate regeneration scenario (52g/s). Measured temperatures along a filter diameter near channels exit together with filter backpressure are presented during a high mass flowrate regeneration performed at 4000rpm, 30Nm for a medium mass loaded filter (6g/l) at 3000rpm, 40Nm with 25ppm Ce-doped fuel.

\section{Low flowrate regeneration: 3-D effects}

This is a common mode of regeneration encountered in practice. It is frequently met in a filter system operating with additive-doped fuel, during city driving with frequent vehicle braking. A characteristic low-flowrate $(28 \mathrm{~g} / \mathrm{s})$ regeneration, is shown in Figure 3.23. The temperatures measured by the thermocouples located at the center and periphery near the filter exit, indicate that the regeneration initiate at the central channels of the filter where the specific levels of filter wall temperature achieved first. As in the case of high flow rate regeneration the soot layer begins to burn at $480^{\circ} \mathrm{C}$ indicating that the soot ignition temperature is not affected by the flow rate. However the lower oxygen content of this operation point ( $8 \%$ vs $12 \%$ of high and medium flowrate regeneration) results to lower peak temperatures.

Another observation is related with the very low oxidation rates observed in the periphery. This is due to the heat loses to the ambient that not allow the wall temperatures to reach the necessary level for regeneration. It is apparent that for the levels of inlet temperature and soot loading of the experiment the heat release from the regeneration of the central channels and the convective heating of the low flow rate exhaust gases are not able to compensate the losses to the ambient. This indicates that the filter periphery is incompletely regenerated making this type of regeneration responsible for the occurrence of partial regenerations. As came up from measurements of pressure drop resistance of individual channels of a previous incomplete regenerated filter there exist significant variations in soot loading or quality 
among different channels (center -vs- periphery of the filter) [69]. This fact hints to the existence of flow maldistribution at filter inlet during the loading phase (center -vsperiphery). The existence of flow maldistribution is investigated in [106] by means of flow velocity measurements at filter exit, during the loading phase and the regeneration phase.

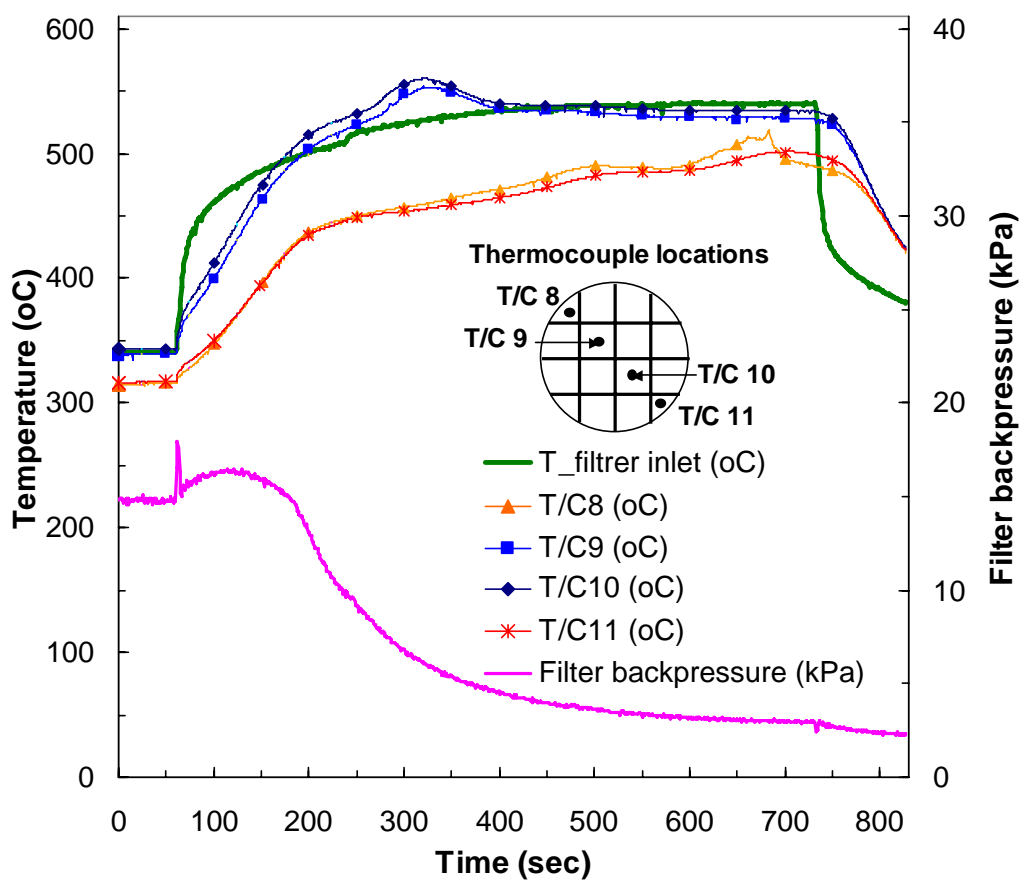

Figure 3.23 Low flowrate regeneration scenario (28g/s). Measured temperatures along a filter diameter near channels exit together with filter backpressure are presented during a high mass flowrate regeneration performed at $1500 \mathrm{rpm}, 80 \mathrm{Nm}$ for a medium mass loaded filter $(6 \mathrm{~g} / \mathrm{l})$ at $3000 \mathrm{rpm}, 40 \mathrm{Nm}$ with 25ppm Ce-doped fuel.

\section{$\underline{\text { Transient regeneration experiments }}$}

\section{Uncontrolled Regeneration}

A characteristic uncontrolled regeneration, that can result from a transient scenario is shown in Figure 3.24. This is a recording of the regeneration behavior of a SiC diesel filter during a sudden engine deceleration. It is generally considered that this kind of so-called 'filter failure scenarios' may gradually damage a filter. In the case of cordierite filters, such events usually damage the filter, due to the low thermal diffusivity of cordierite. A SiC filter generally demonstrates superior performance in such instances, due to the material's high thermal diffusivity and melting point. However, $\mathrm{SiC}$ has a higher thermal expansion coefficient, (4.3 x 10-6/K) and thus it could suffer from high thermal stresses. Such stresses may be enhanced by the modular structure of these filters that consist of a number of monolithic modules connected by special adhesive cement. Thus, in transient regeneration experiments where the operation point suddenly changes to very low flowrate, 3-D effects become prevalent, with a well-distinguished evolution of regeneration in different channels, as shown in [104].The onset of regeneration is again observed in the central channels. Although the observed delay in the onset of regeneration in the periphery is quite significant, (order of several minutes as shown in the Figure), the maximum temperatures observed in the periphery are equivalent, or even higher, than those observed at the central channels. 


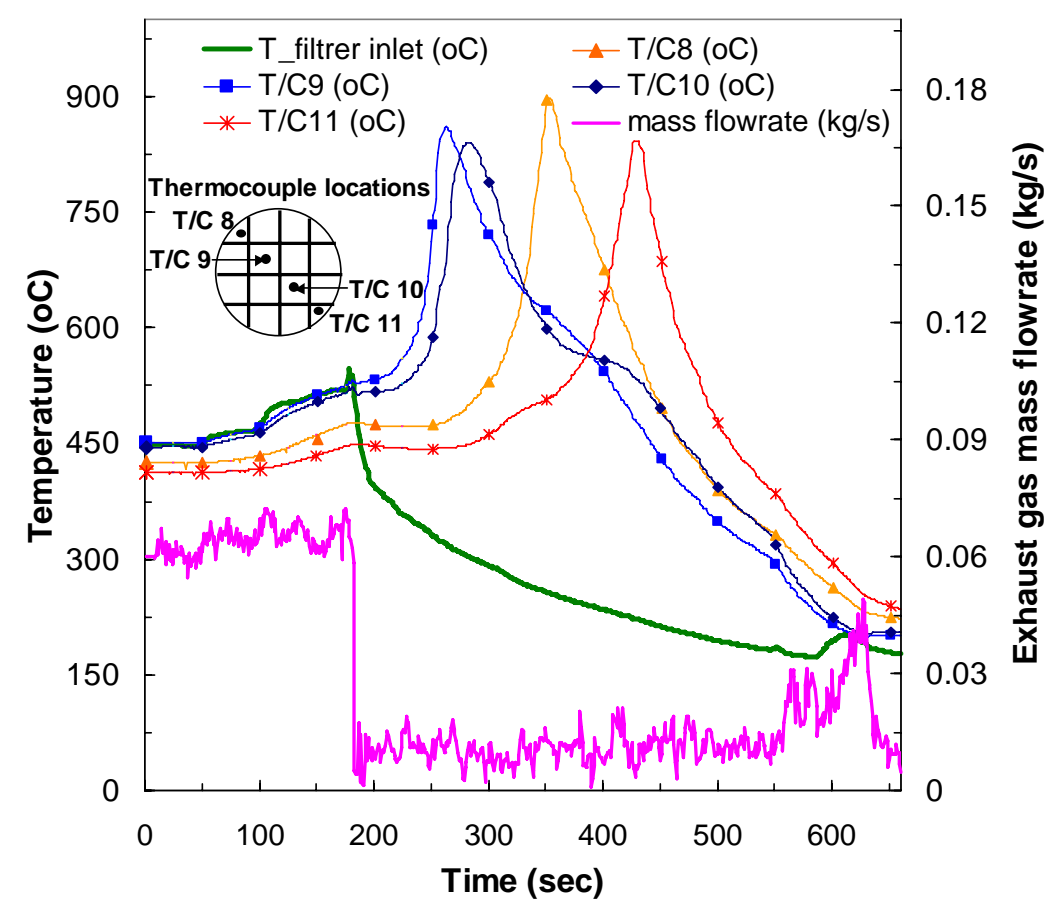

Figure 3.24 Uncontrolled regeneration scenario. Measured temperatures along a filter diameter near channels exit together with exhaust mass flow rate are presented during a deceleration scenario performed with a step variation to the engine operation point from a sped of $2000 \mathrm{rpm}$ and a torque of $180 \mathrm{Nm}$ to a speed of $900 \mathrm{rpm}$ and no load (idle conditions). The filter was previous highly loaded (12g/l) at 3000rpm, 40Nm with 25ppm Ce-doped fuel.

The 3-dimensional evolution of the regeneration is now obvious, and the previously observed grouping of central and periphery channels response is no longer valid. The role of the catalytic fuel additive in these phenomena is important and this was first reported by Wiedemann et al [105].

Stochastic regeneration: VOF and $3 D$ effects.

The appearance of stochastic regeneration phenomena during loading at engine points with relative high VOF content and low exhaust gas temperatures has already mentioned in section 3.2.2. The stochastic regeneration events recorded during filter loading at speed: 3000rpm and load: 40Nm (VOF: 6.8\%) are presented in Figure 3.20. As discussed these events are associated with an abrupt decrease to the filter backpressure, started from a peripheral group of filter channels (recording of thermocouple 11) due the higher VOF content of the colder outer channels which could be oxidized by the catalyst oxides when the temperatures and soot loading conditions become favorable.

In this type of regeneration there is a complex interaction between VOF and flow maldistribution affecting to the evolution of regeneration and the temperature distribution across the filter channels. As can been seen a difference of the order of $90^{\circ} \mathrm{C}$ is appeared between the temperatures measured at the periphery where the higher peak temperatures recorded and those measured at the center. This is attributed to the flow maldistribution phenomena that enforce the flow to pass mainly through the central channels of the filter [106]. The higher flowrate through the central part means higher convection that is responsible for the lower wall temperatures of this region.

Also it can be observed that the regeneration in the filter center is responsible for the rapid pressure drop. 


\subsubsection{Concluding remarks}

- An experimental study of the fuel additive assisted catalytic regeneration process with focus to the most frequently regeneration scenarios met under real driving conditions is presented in this work.

- Steady state regeneration tests were performed at 3 engine operation points with markedly different levels of engine exhaust gas mass flow rate. The evolution of regeneration was also studied in the transient cases of uncontrolled and stochastic regeneration.

- Especially in the low flowrate tests, it becomes apparent that there exist significant 3D effects that affect the propagation of the regeneration. Maldistribution of flow interacts with maldistribution of soot and VOF content, thus producing a complex overall behaviour.

- In all experiments, it was observed that the catalytic soot oxidation initiates at about $480 \mathrm{oC}$ irrespective of the exhaust gas conditions (25 ppm Ceria fuel additive was used in all loading and regeneration tests).

- The observed maximum peak temperatures become lower as the mass flow rate increases.

\subsection{Experimental Validation of the 1D Model}

In this section, we demonstrate how an improved 1D filter regeneration model (described in [70]), is validated against full-scale experimental data aiming at further model and code development. The software is subjected to a validation procedure, against full-scale tests of the regeneration behaviour of a diesel filter fitted to a modern diesel engine run on catalystdoped fuel. The main objectives of the validation procedure concern the assessment of the model's ability to predict the effects of additive concentration in fuel, initial soot loading mass, volatile organic fraction of the soot and exhaust gas mass flow rate.

\subsubsection{Design of the validation experiments}

The validation was based on the results of engine bench regeneration experiments performed on one of the LTTE engine benches. The filter employed in these experiments was a SiC 200 cpsi filter (diameter x length: 5.66”x 6”) fitted in the exhaust pipe of a 2-liter displacement HDI turbocharged passenger car engine about $600 \mathrm{~mm}$ after turbo. The engine and diesel filter specifications are presented in Table 3.1. The temperatures were measured simultaneously, at the inlet and the exit of the filter, and inside the filter, along a filter diameter $15 \mathrm{~mm}$ deep from filter exit. The exhaust emissions of $\mathrm{CO}, \mathrm{CO} 2, \mathrm{NO}_{\mathrm{x}}$ and $\mathrm{HC}$ were measured simultaneously before and after the filter. The $\mathrm{O} 2$ concentration was calculated by the $\mathrm{A} / \mathrm{F}$ ratio obtained from the UEGO sensor installed before the filter inlet. The experimental layout of the filter loading-regeneration experiments is presented in Figure 3.1. The model's capacity to address the effect of the following design and operation parameters was validated:

- Fuel additive concentration

- Initial filter soot loading 
- Collected soot characteristics (VOF content [72], etc) and, indirectly, also the effect of

- Exhaust gas mass flow rate

Fuel additive concentration. The effect of fuel additive concentration on the regeneration was checked at a characteristic medium operation point of $2250 \mathrm{rpm}-60 \mathrm{Nm}$ for the catalytic regeneration, employing as reference for the thermal regeneration the non-catalytic regeneration. The effects of 0,25 and 50ppm Cerium fuel additive concentration in fuel were studied. The non-catalytic regeneration was performed at the increased speed point of 3800 rpm-60 Nm in order to meet the high exhaust temperatures levels that are necessary for the initiation of regular soot oxidation. The filter was previous loaded at the medium point of $3000 \mathrm{rpm}-40 \mathrm{Nm}$ until a medium soot mass loading of the order of $6 \mathrm{~g} / \mathrm{l}$ filter is obtained. The shift to a set of experiments with different fuel additive concentration was performed after filter cleaning and engine running for about $6 \mathrm{~h}$ to prevent possible memory effects to the fuel and soot composition.

Steady state loading experiments at specific combinations of engine speed and load were performed in order to validate the model's ability to predict the effect of initial soot loading and VOF content.

Initial filter soot loading. Indirect assessment of the effect of the soot deposit filtration characteristics and soot oxidation kinetics was based on the variation of initial soot loading (3, 6 and $9 \mathrm{~g} / \mathrm{l}$ filter), obtained with adjustment of filter loading process duration assuming constant particulate emissions and no effects of the increased backpressure to the engine exhaust mass flow rate. The filter loading was performed with the engine running on 3000rpm, 40Nm with 25ppm Cerium doped fuel.

Soot accumulation conditions. Two characteristic engine operation points were selected for the assessment of differences in VOF content of soot: $3000 \mathrm{rpm}-40 \mathrm{Nm}$ (6.8\% VOF), versus $1800 \mathrm{rpm}-90 \mathrm{Nm}$ (2\% VOF). These points are characterized by equivalent total particulate matter emissions and cover a range of filter wall temperatures from $300^{\circ} \mathrm{C}$ to $400^{\circ} \mathrm{C}$ that is responsible for the VOF variation [72]. Secondary effects due to the dynamic phenomena of adsorption-desorption may also affect soot oxidation kinetics.

Exhaust gas mass flow rate. The engine operation points for the regeneration experiments were specially selected for the assessment of the effect of exhaust gas flow rate. The variation of exhaust gas flow rate was effected through the combination of engine speed of 1500, 2250, 3000 and $4000 \mathrm{rpm}$ with engine load of 80,60, 60 and $30 \mathrm{Nm}$ respectively, in order to obtain during the regeneration approximately equivalent filter inlet temperature levels in the range of 500 to $550^{\circ} \mathrm{C}$. These points result to a variation in exhaust gas mass flow rate in the range from 28 to $100 \mathrm{~g} / \mathrm{s}$ and represent the real conditions met under City and Extra-Urban driving conditions. The appearance of secondary effects related with the flow maldistribution phenomena affected by the flow rate during regeneration is expected to play significant role on the simulation results [106].

Table 3.8 Experimental protocol 


\begin{tabular}{|c|c|c|c|c|}
\hline & Uి & 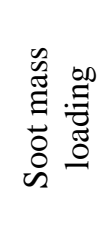 & 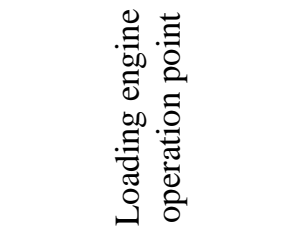 & 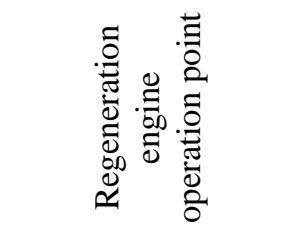 \\
\hline \multirow{3}{*}{ Catalyst concentration } & 0ppm & \multirow{3}{*}{ 6g/L } & \multirow{3}{*}{ 3000rpm x 40Nm } & 3800rpm x 60Nm \\
\hline & *25ppm & & & \multirow{2}{*}{ 2250rpm x 60Nm } \\
\hline & 50ppm & & & \\
\hline \multirow{3}{*}{ Soot mass loading } & \multirow{3}{*}{ 25ppm } & $3 g / L$ & \multirow{3}{*}{ 3000rpm x 40Nm } & \multirow{3}{*}{ 2250rpm x 60Nm } \\
\hline & & $* 6 \mathrm{~g} / \mathrm{L}$ & & \\
\hline & & $9 g / L$ & & \\
\hline \multirow{2}{*}{ Filter accumulation condition } & \multirow{2}{*}{ 25ppm } & \multirow{2}{*}{$6 g / L$} & $\begin{array}{c}\text { *3000rpm x 40Nm } \\
\text { (VOF:10\%) }\end{array}$ & \multirow{2}{*}{ 2250rpm x 60Nm } \\
\hline & & & $\begin{array}{l}\text { 1800rpm x 90Nm } \\
\text { (VOF:2\%) }\end{array}$ & \\
\hline \multirow{4}{*}{ Filter regeneration condition } & \multirow{4}{*}{ 25ppm } & \multirow{4}{*}{ 6g/L } & \multirow{4}{*}{$3000 \mathrm{rpm}$ x 40Nm } & $\begin{array}{c}\text { 1500rpm x 80Nm } \\
(28 \mathrm{~g} / \mathrm{s})\end{array}$ \\
\hline & & & & $\begin{array}{c}\text { *2250rpm x 60Nm } \\
(52 \mathrm{~g} / \mathrm{s})\end{array}$ \\
\hline & & & & $\begin{array}{c}3000 \mathrm{rpm} \times 60 \mathrm{Nm} \\
(70 \mathrm{~g} / \mathrm{s})\end{array}$ \\
\hline & & & & $\begin{array}{c}\text { 4000rpm x 30Nm } \\
(92 \mathrm{~g} / \mathrm{s})\end{array}$ \\
\hline
\end{tabular}

*Reference Experiment

Each regeneration process was initiated after loading at the respective engine operation point, until the same level of filter loading is achieved (6g/l filter) by a combined step variation in engine load and engine intake airflow obtained with adjustment of turbocharger and EGR valves. The engine was left to run for 10 minutes on steady state conditions before the regeneration strategy is applied, duration considered long enough for thermal and chemical equilibrium to be reached.

The experimental protocol is summarized in Table 3.8. The regeneration experiments were performed in combination with TGA experiments of soot samples taken directly from the filter loaded at the above operation points in order to improve our understanding of the kinetics of soot oxidation by Ceria and exhaust gas [86]. Previous experimental work in the direction of determination of pressure drop parameters as function of collected soot mass [69] was employed to support pressure drop parameter selection.

\subsubsection{D model validation results}

Before carrying out the main validation process, it is necessary to determine reference values for pressure drop and kinetic parameters. The source for this information was our previous experimental work in the subjects of pressure drop and soot oxidation kinetics, along with the published results of experimental work of other researchers. Starting from the pressure drop parameters, the determination of the product of soot permeability times soot density $(\rho k)_{\mathrm{p}}$ was based on experiments with single channel filters described in detail in [69]. A range between $3.5 \mathrm{E}-12$ and $1.15 \mathrm{E}-11 \mathrm{~kg} / \mathrm{m}$ is reported for the $(\rho \mathrm{k})_{\mathrm{p}}$ product over the 
medium range of engine speed and load. However these values are necessary to be reduced in order to match the pressure drop behaviour of the validation experiments. As is shown in Table 3.9 a constant, typical value of $(\rho k)_{\mathrm{p}}=2.25 \mathrm{E}-13$ was proven successful in most simulations. The discrepancy between the experimental and calculated values could be partly attributed to soot and flow maldistribution effects [106] that cannot be taken into account in the $1 \mathrm{D}$ model. Concerning the wall permeability, a constant value of the order of $2 \mathrm{E}-13$ was found capable to match the pressure drop curves of the whole range of validation experiments. This value is in accordance with these reported by other researchers [107]. On the other hand, the lack of measured data for collected soot density (values reported in the literature between 60 and $120 \mathrm{~kg} / \mathrm{m}^{3}$ ), made necessary the assumption of a typical value of $80 \mathrm{~kg} / \mathrm{m}^{3}$.

A summary of the validation runs and the respective kinetic parameter values inserted in the model is presented in Table 3.9.

Baseline for the determination of $\mathrm{E}$ for thermal and catalytic regeneration, have been the extensive TGA analysis experiments of soot samples taken directly from the filter [86]. The following values for activation energy were determined from the above-mentioned experimental work:

- $\mathrm{E}=190 \mathrm{~kJ} / \mathrm{mole}$ for the complete thermal soot oxidation to $\mathrm{CO} 2$ and $\mathrm{E}=150 \mathrm{~kJ} / \mathrm{mole}$ for the incomplete thermal soot oxidation to CO.

- $\mathrm{E}=120 \mathrm{~kJ} / \mathrm{mole}$ for the complete catalytic soot oxidation to $\mathrm{CO} 2$ and $\mathrm{E}=80 \mathrm{~kJ} / \mathrm{mole}$ for the incomplete catalytic soot oxidation to CO.

The values of activation energies for catalytic oxidation correspond to dry soot oxidation for samples where the VOF content varies in the range between 2.5 and $8 \%$.

Keeping constant the above values of activation energies, a certain amount of tuning of the frequency factors (A's) was allowed. First, the thermal Carbon oxidation frequency factors were tuned to represent the experimentally determined thermal regeneration behaviour of the filter.

The tuning of the frequency factor values assumes that the catalytic regeneration prevails in the temperature range from $400^{\circ} \mathrm{C}$ to $550^{\circ} \mathrm{C}$. Thermal regeneration is more active at higher temperatures. This situation is schematically presented in Figure 3.26 by means of comparison between the calculated thermal and catalytic reaction rates as function of temperature. As it can be seen, for the case of catalytic regeneration the reaction of soot oxidation by $\mathrm{CeO}_{2}$ to $\mathrm{CO} 2$ is assumed to be more active after the temperature of $500^{\circ} \mathrm{C}$. This assumption is in accordance with our previous experimental work that indicates an ignition temperature for the catalytic soot oxidation of the order of $500^{\circ} \mathrm{C}$. Following the same reasoning in the case of thermal regeneration we may assume that the reaction of soot oxidation by the exhaust gas oxygen to $\mathrm{CO} 2$ is more active above $630^{\circ} \mathrm{C}$ where the ignition temperature for thermal oxidation is observed. 


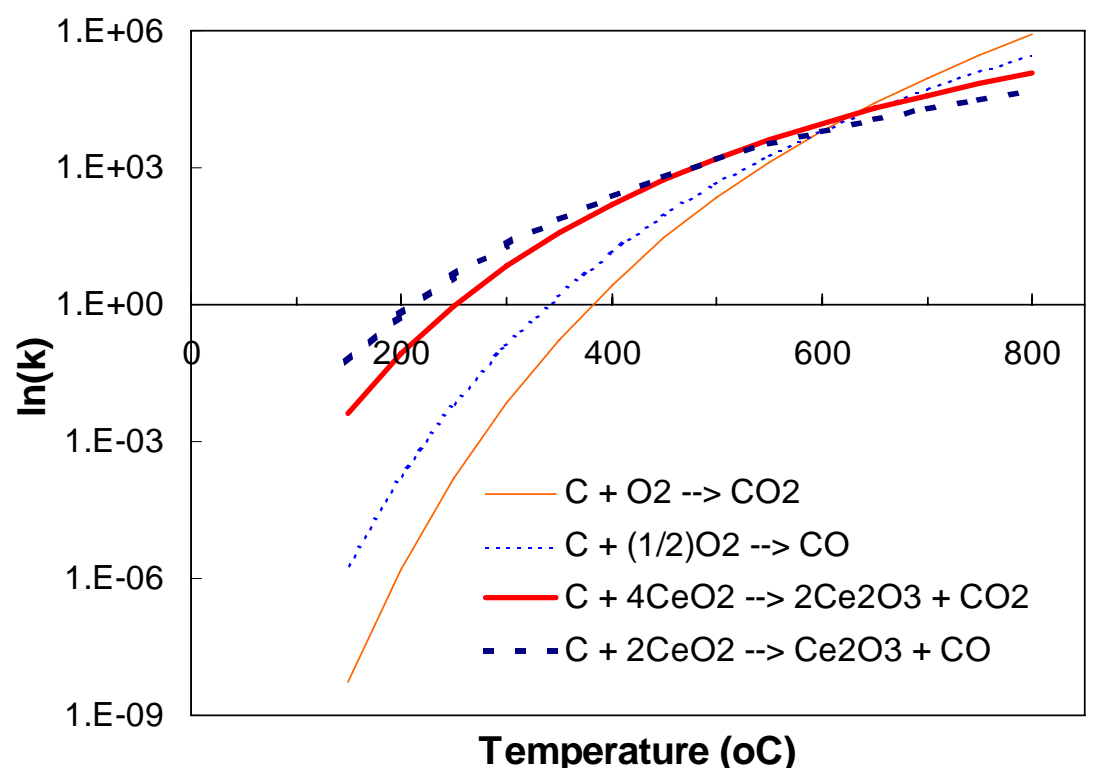

Figure 3.25 Comparison of thermal and catalytic regeneration reaction rates.

As a general rule of thumb, the catalytic regeneration kinetics (frequency factors) were tuned to represent the experimentally determined, low temperature (catalytic) regeneration behaviour of the filter. Then, the ability of the tuned model to predict the effects of exhaust mass flow rate, initial soot loading mass, volatile organic fraction of the soot and additive concentration in fuel was checked.

In principle, the values of frequency factors should keep close to certain reference values for each reaction of the scheme. However, one must bear in mind that the catalytic reactor in the case of a fuel additive assisted diesel filter, is made up of the soot layer, which contains the catalyst dispersed in it, in a close contact with the soot. This reactor is destroyed during regeneration and re-built during filter loading. The reactor's characteristics may change according to the prevailing exhaust gas conditions during loading.

The exact determination of the catalytic oxidation frequency factor values was proven sensitive to the ratio of $\mathrm{CO} / \mathrm{CO}_{2}$ in the exhaust gas during regeneration indicating dependence from the filter loading and regeneration conditions. This observation is confirmed by Aoki et al [108] and is more enhanced in the case of catalytic regeneration. The assessment of the experimental results was shown that the following parameters affect to the $\mathrm{CO} / \mathrm{CO}_{2}$ ratio:

- Soot loading: increase of soot loading mass over $6 \mathrm{~g} / \mathrm{l}$ filter causes a significant increase to the produced $\mathrm{CO}$ during regeneration.

- Soot VOF content: increase of VOF content results in a shift towards higher CO than $\mathrm{CO}_{2}$ production during regeneration.

- Exhaust gas mass flow rate: as the exhaust gas mass flow rate increases, more CO than $\mathrm{CO}_{2}$ is produced during regeneration also increases.

Based on the above reasoning, certain deviations from the reference kinetics of the catalytic reactions are observed in Table 3.9 and summarized below:

- It was found that the increase of the initial soot loading shifts the catalytic reaction kinetics to produce more $\mathrm{CO}$ and less $\mathrm{CO}_{2}$. The model can match this behaviour by a respective modification of the frequency factors of the respective reactions. 
- A severe decrease in the volatile organic fraction of the collected particulate, leads to a respective reduction of catalytic soot oxidation kinetics, which can be matched by a respective reduction of frequency factors of the catalytic oxidation reactions.

- As expected, the tuned 1D model does not successfully predict filter operation at low flowrates. This is due to the importance of complex 3D effects, related to exhaust gas flow and soot loading maldistribution across the filter face.

- On the other hand, by comparison of model predictions with measurements, it was observed that the catalytic activity is reduced at very high flowrates (very low residence times). This needs to be further investigated.

The above concern the soot oxidation kinetics behaviour.

Also the already known participation of $\mathrm{NO}_{2}$ in the soot oxidation reactions was demonstrated in our own experimental results, and suggested to insert in the extended reaction scheme presented in Pontikakis' Thesis [70]. A reduction to the filter exit $\mathrm{NO}_{2}$ concentration was recorded especially in the case of the low flowrate regeneration that is associated with lower exhaust gas temperatures indicating that the reaction with $\mathrm{NO}_{2}$ becomes more significant at low temperatures. Of course significant role to this play the parameters of engine $\mathrm{NO}_{2}$ emissions at the respective engine operation point the presence of oxidation catalyst or not in front of filter and the soot loading level.

As regards the pressure drop behaviour, a certain variation of soot permeability was allowed respectively, as function of the following factors:

- Effect of soot loading: thicker soot layer leads to lower permeability-density factor.

- Effect of Ceria concentration in fuel: higher Ceria concentration leads to lower soot permeability - density factor.

- Effect of VOF: higher VOF in soot leads to lower soot permeability- density factor.

Table 3.9 presents the wall permeability, soot density and soot permeability times density values inserted in the $1 \mathrm{D}$ model to simulate the pressure drop behaviour of the validation experiments. 


\begin{tabular}{|c|c|c|c|c|c|c|c|c|c|c|c|c|c|c|c|c|}
\hline \multirow[b]{3}{*}{$\begin{array}{c}\text { Name of studying } \\
\text { parameter }\end{array}$} & \multirow{3}{*}{ 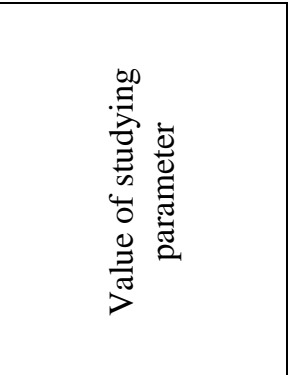 } & \multirow[b]{3}{*}{ 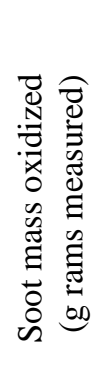 } & \multirow[b]{3}{*}{ 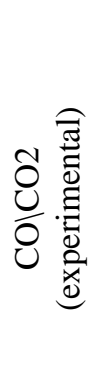 } & \multicolumn{3}{|c|}{ Pressure drop parameters } & \multicolumn{10}{|c|}{ Kinetic parameters } \\
\hline & & & & \multirow[b]{2}{*}{ 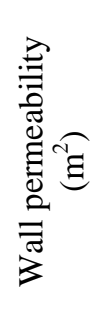 } & \multirow[b]{2}{*}{ 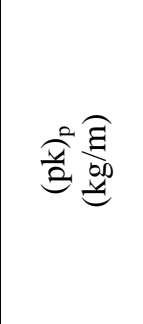 } & \multirow[b]{2}{*}{ 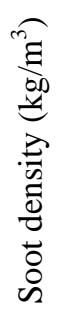 } & \multicolumn{4}{|c|}{ Thermal soot oxidation } & \multicolumn{6}{|c|}{ Catalytic soot oxidation } \\
\hline & & & & & & & 蛋 & 되 & 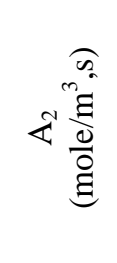 & 되 잉 & 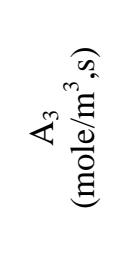 & 되 & 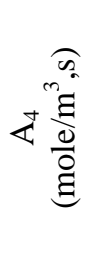 & 되 & 蛋 & 너 \\
\hline \multirow{3}{*}{$\begin{array}{c}\text { Catalyst } \\
\text { concentration }\end{array}$} & 0ppm & 20.5 & 0.02 & \multirow{3}{*}{$2 \mathrm{E}-13$} & $2.8 \mathrm{E}-13$ & \multirow{3}{*}{80} & $1 \mathrm{E} 13$ & $1.9 \mathrm{E} 5$ & 5.5E10 & $1.5 \mathrm{E} 5$ & - & - & - & - & - & - \\
\hline & 25ppm & 14.2 & 0.14 & & $2.2 \mathrm{E}-13$ & & \multirow{2}{*}{$1 \mathrm{E} 13$} & \multirow{2}{*}{$1.9 \mathrm{E} 5$} & \multirow{2}{*}{$5.5 \mathrm{E} 10$} & \multirow{2}{*}{$1.5 \mathrm{E} 5$} & \multirow{2}{*}{ 3.5E11 } & \multirow{2}{*}{$1.2 \mathrm{E} 5$} & \multirow{2}{*}{$6 \mathrm{E} 8$} & \multirow{2}{*}{$0.8 \mathrm{E} 5$} & \multirow{2}{*}{ 8E8 } & \multirow{2}{*}{$0.8 \mathrm{E} 5$} \\
\hline & 50ppm & 13.15 & 0.15 & & $2.0 \mathrm{E}-13$ & & & & & & & & & & & \\
\hline \multirow{3}{*}{ Soot mass loading } & $3 g / L$ & 8 & 0.01 & \multirow{3}{*}{$2 \mathrm{E}-13$} & $2.5 \mathrm{E}-13$ & \multirow{3}{*}{80} & \multirow{3}{*}{$1 \mathrm{E} 13$} & \multirow{3}{*}{$1.9 \mathrm{E} 5$} & \multirow{3}{*}{$5.5 \mathrm{E} 10$} & \multirow{3}{*}{$1.5 \mathrm{E} 5$} & 4.5E11 & \multirow{3}{*}{$1.2 \mathrm{E} 5$} & 3E8 & \multirow{3}{*}{$0.8 \mathrm{E} 5$} & & \\
\hline & $6 g / L$ & 14.2 & 0.02 & & $2.2 \mathrm{E}-13$ & & & & & & 3.5E11 & & $6 \mathrm{E} 8$ & & 8E8 & $0.8 \mathrm{E} 5$ \\
\hline & $9 g / L$ & 20.5 & 0.19 & & $2.0 \mathrm{E}-13$ & & & & & & $2.5 \mathrm{E} 11$ & & 9E8 & & & \\
\hline Filter accumulation & $\begin{array}{l}\text { 3000rpm x 40Nm } \\
\text { (VOF:10\%) }\end{array}$ & 14.2 & 0.14 & 12 & $2.2 \mathrm{E}-13$ & 80 & 1512 & 1055 & 55511 & 1555 & 4.5E11 & 1255 & 9E8 & 0.55 & 958 & 0955 \\
\hline condition & $\begin{array}{c}\text { 1800rpm x 90Nm } \\
\text { (VOF:2\%) }\end{array}$ & 13.8 & 0.08 & $2 E-13$ & $2.6 \mathrm{E}-13$ & 00 & $1 E 13$ & $1.9 \mathrm{E} 3$ & 5.JE10 & 1.JEJ & $2.5 \mathrm{E} 11$ & $1.2 \mathrm{E} 5$ & 3E8 & $0.8 \mathrm{EJ}$ & ОЕО & $0.8 \mathrm{E} 5$ \\
\hline & $\begin{array}{c}\text { 1500rpm x 80Nm } \\
(\mathrm{mfr}: 28 \mathrm{~g} / \mathrm{s})\end{array}$ & 14.2 & 0.07 & & $2.2 \mathrm{E}-13$ & & & & & & $1 \mathrm{E} 11$ & & $2 \mathrm{E} 8$ & & & \\
\hline Filter regeneration & $\begin{array}{c}\text { 2250rpm x 60Nm } \\
(\mathrm{mfr}: 52 \mathrm{~g} / \mathrm{s})\end{array}$ & 14.2 & 0.14 & $2 \mathrm{~F}-13$ & $2.2 \mathrm{E}-13$ & 80 & $1 \mathrm{~F} 13$ & 19F5 & 5.5E10 & $15 \mathrm{~F} 5$ & 3.5E11 & $12 F 5$ & $6 \mathrm{E} 8$ & 0855 & $8 \mathrm{~F} 8$ & 0855 \\
\hline condition & $\begin{array}{c}\text { 3000rpm x 60Nm } \\
\text { (mfr:70g/s) }\end{array}$ & 16 & 0.17 & $2 \mathrm{E}-13$ & $2.2 \mathrm{E}-13$ & 80 & & & & & 3.5E11 & $1.2 \mathrm{E} 5$ & $6 \mathrm{E} 8$ & 0.8Е5 & & \\
\hline & $\begin{array}{l}\text { 4000rpm x 30Nm } \\
(\mathrm{mfr}: 92 \mathrm{~g} / \mathrm{s})\end{array}$ & 13.3 & 0.19 & & $2.2 \mathrm{E}-13$ & & & & $6.5 \mathrm{E} 12$ & & 3.5E11 & & $6 \mathrm{E} 8$ & & & \\
\hline
\end{tabular}

Table 3.9 CATWALL 1D validation results based on Laboratory of Thermodynamics and Thermal engines experimental data. 
The measured filter exit temperatures at the central line (measurements taken by thermocouple T/C9) are compared with the predicted ones for each regeneration case, in Figures 3.26-3.34. The correlation observed is satisfactory. However a certain degree of inaccuracy in predicting the initial heating phase of the regeneration is observed. It is believed that this is a 3D phenomenon related to the change of average mass flowrate, due to the reduction in flow resistance in the central channels [106].

\subsubsection{Discussion}

The model is proven able to predict the effect of a significant number of design and operation parameters on the filter performance in a satisfactory way. In the following, certain important remarks from model validation are discussed in more detail. These remarks generally indicate directions for future, more refined experimental study.

\section{Catalyst concentration in fuel}

As a first step, the case of regular soot oxidation (no catalyst in fuel) is studied. The simulation of a regeneration of a filter loaded at 3000rpm, $40 \mathrm{Nm}$ with the engine operating on regular (not fuel additive-doped) is presented in Figure 3.26. The values of E determined from TGA experiments produce a satisfactory prediction of the full-scale process in the filter. However a certain discrepancy between the measured and the predicted filter backpressure is observed in the initial phase of regeneration.

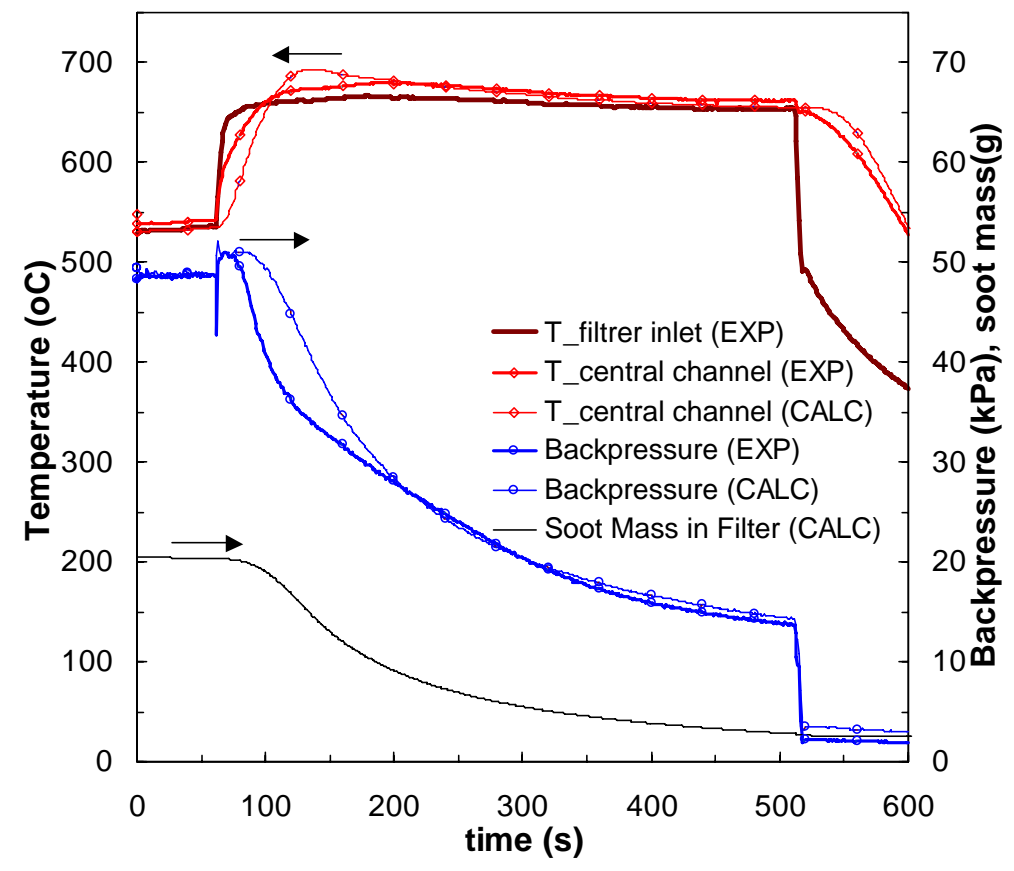

Figure 3.26 Simulation of regular soot (no Ceria added) filter regeneration. Measured and predicted trap temperatures near the exit of a central channel of a medium loaded filter $(6 \mathrm{~g} / \mathrm{l})$ with the engine running on 3000rpm, $40 \mathrm{Nm}$ without fuel additive-doped fuel. Also measurement and prediction of filter backpressure together with soot mass prediction are presented. Computation is made with $(\rho \mathrm{k}) \mathrm{p}=2.8 \mathrm{E}-13 \mathrm{~kg} / \mathrm{m}$, activation energy values: $E_{1}=1.9 \mathrm{E} 5, E_{2}=1.5 \mathrm{E} 5 \mathrm{~J} / \mathrm{mole}$ and frequency factor values: $\mathrm{A}_{1}=1 \mathrm{E} 13, \mathrm{~A}_{2}=5.5 \mathrm{E} 10 \mathrm{~mole} / \mathrm{m}^{3}$,s. The values for $\mathrm{E}$ and $\mathrm{A}$ are used as reference point for catalytic regeneration.

As it can be seen the model predicts a slower reduction to the pressure drop as the regeneration initiates. The reason could lie in the fact that this initial phase of the regeneration is associated with a VOF desorption process that is responsible for the rapid backpressure 
decrease. This situation is not predictable by the 1D pressure drop model, which considers an average soot permeability value during the whole regeneration process.

As a next step, two regeneration experiments with soot collected with 25 and 50ppm Cerium-doped fuel were performed. Starting from the simulation of the case with 25ppm Cerium doped fuel described in Figure 3.27, it should be mentioned that the fitted value of $(\rho k)_{\mathrm{p}}$ was necessary to be reduced compared to the one for the soot without catalyst. It seems that the presence of catalyst inside the particulate, modifies its permeability - density characteristics. However in this case a reduction to the collected soot mass is observed (14g vs $20 \mathrm{~g}$ in the case regular soot), indicating that also engine soot emissions are affected by the presence of the catalyst. This observation is confirmed by several reports in literature that give a reduction of the engine soot production of the order of $30 \%$ when a cerium additive is used [109].

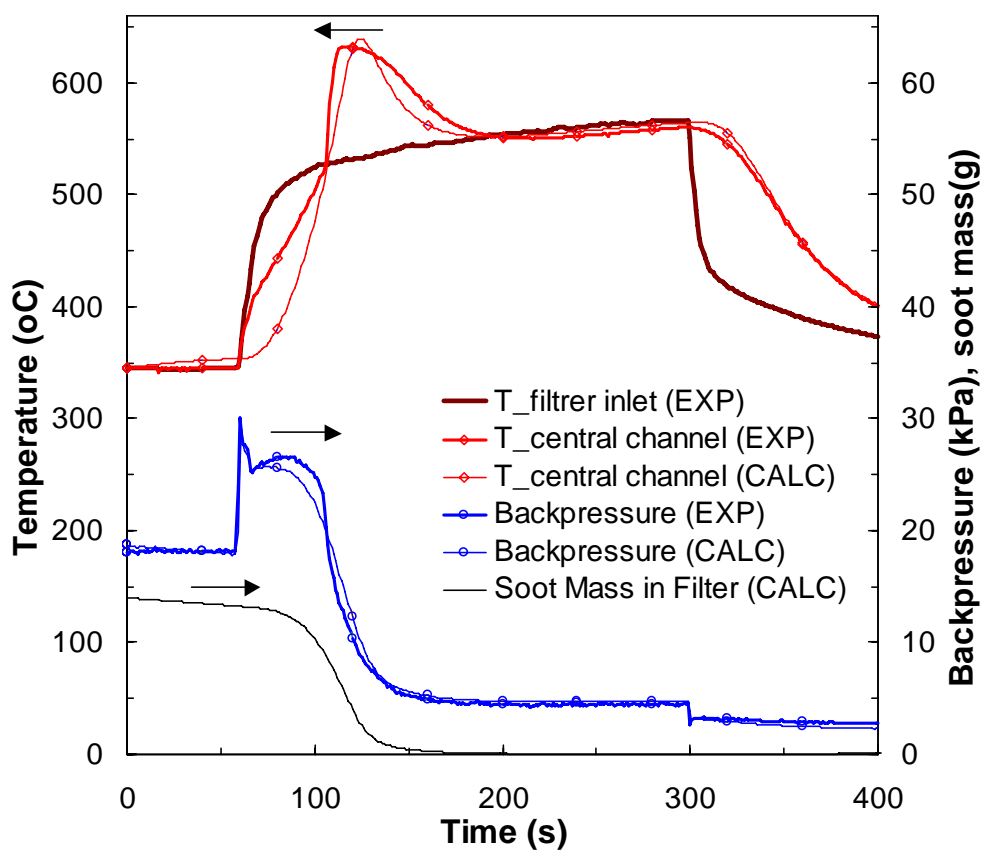

Figure 3.27 Simulation of regeneration of a filter loaded with soot emitted by the engine operating with 25ppm Cerium doped fuel.. Measured and predicted trap temperatures near the exit of a central channel of a medium loaded filter $(6 \mathrm{~g} / \mathrm{l})$ with the engine running on 3000rpm, 40Nm. Also measurement and prediction of filter backpressure together with soot mass prediction are presented. Computation is made with $(\rho \mathrm{k}) \mathrm{p}=2.2 \mathrm{E}-13 \mathrm{~kg} / \mathrm{m}$, activation energy values: $\mathrm{E}_{1}=1.9 \mathrm{E} 5, \mathrm{E}_{2}=1.5 \mathrm{E} 5, \mathrm{E}_{3}=1.2 \mathrm{E} 5, \mathrm{E}_{4}=0.8 \mathrm{E} 5, \mathrm{E}_{5}=0.8 \mathrm{E} 5 \mathrm{~J} / \mathrm{mole}$ and frequency factor values: $\mathrm{A}_{1}=1 \mathrm{E} 13, \mathrm{~A}_{2}=5.5 \mathrm{E} 10, \mathrm{~A}_{3}=3.5 \mathrm{E} 11, \mathrm{~A}_{4}=6 \mathrm{E} 8, \mathrm{~A}_{5}=8 \mathrm{E} 8 \mathrm{~mole} / \mathrm{m}^{3}$, s. The values for $\mathrm{E}$ and $\mathrm{A}$ are used as reference point for catalytic regeneration.

Continuing with the simulation of the case with 50ppm Cerium doped fuel the results of Figure 3.28 indicate that the regeneration behaviour is not significantly affected by the higher fuel additive concentration. . The effect is that the 50ppm fuel additive concentration leads to a somewhat lower peak temperature. If we examine the downstream filter CO concentration recordings (not presented in the figures, but summarized in Table 4) we will see that the 50ppm case is associated with somewhat higher CO concentrations (produced CO/CO2 ratio is 0.15 vs 0.14 in the $25 \mathrm{ppm}$ case). This hints to the, already reported by other researchers [110], effect of the Ceria in promoting the CO production. Overall, the increase of catalyst concentration in fuel from 25 to $50 \mathrm{ppm}$ seems not to reward with a higher catalytic effect. On the contrary, pressure drop behaviour is further affected, and a further decrease to the soot permeability is observed. Any attempt to explain these complex effects on the kinetics and pressure drop parameters of soot oxidation by Ceria must take into account the in-cylinder 
particulate formation mechanism that includes the injection and combustion of additive-doped fuel.

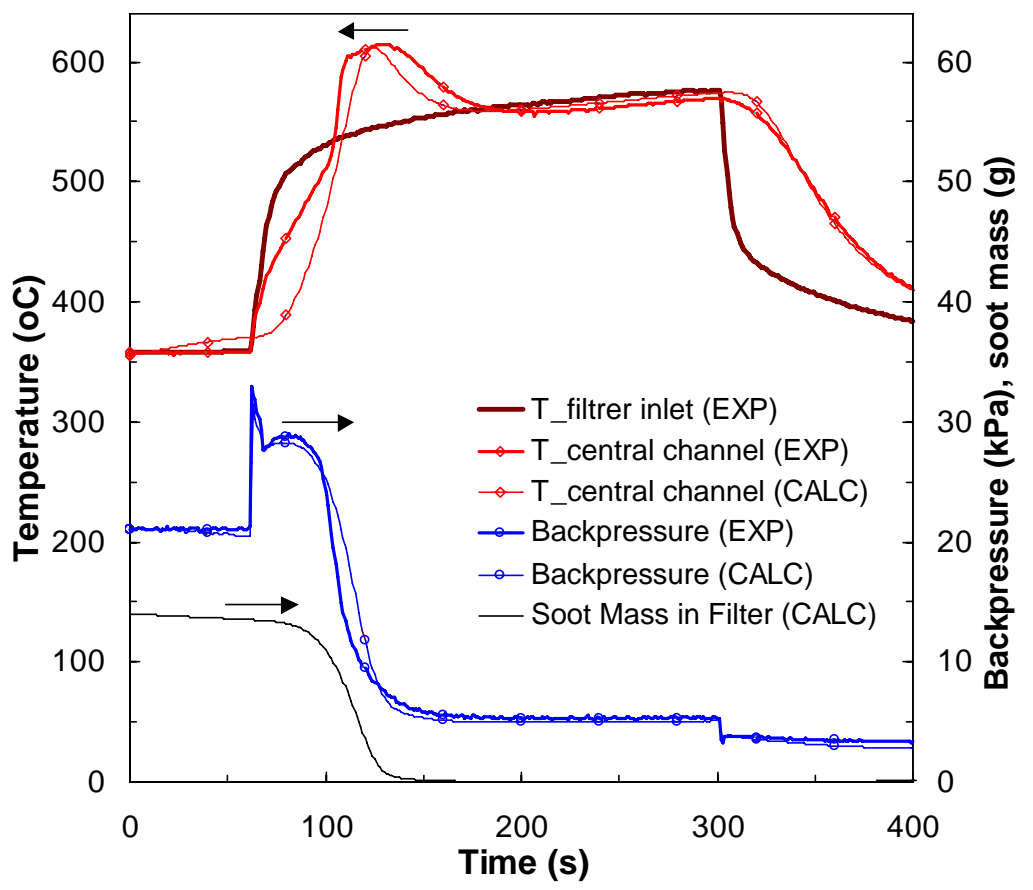

Figure 3.28 Simulation of regeneration of a filter loaded with soot emitted by the engine operating with 50ppm Cerium doped fuel.. Measured and predicted trap temperatures near the exit of a central channel of a medium loaded filter $(6 \mathrm{~g} / \mathrm{l})$ with the engine running on $3000 \mathrm{rpm}, 40 \mathrm{Nm}$. Also measurement and prediction of filter backpressure together with soot mass prediction are presented. Computation is made with $(\rho k) \mathrm{p}=2.0 \mathrm{E}-13 \mathrm{~kg} / \mathrm{m}$, activation energy values: $\mathrm{E}_{1}=1.9 \mathrm{E} 5, \mathrm{E}_{2}=1.5 \mathrm{E} 5, \mathrm{E}_{3}=1.2 \mathrm{E} 5, \mathrm{E}_{4}=0.8 \mathrm{E} 5, \mathrm{E}_{5}=0.8 \mathrm{E} 5 \mathrm{~J} / \mathrm{mole}$ and frequency factor values: $A_{1}=1 \mathrm{E} 13, \mathrm{~A}_{2}=5.5 \mathrm{E} 10, \mathrm{~A}_{3}=3.5 \mathrm{E} 11, \mathrm{~A}_{4}=6 \mathrm{E} 8, \mathrm{~A}_{5}=8 \mathrm{E} 8 \mathrm{~mole} / \mathrm{m}^{3}, \mathrm{~s}$.

\section{Initial soot loading}

The cases of 3, 6 and 9g/l, filter soot loading were studied. The simulations of the regeneration experiments performed at 2250rpm, $60 \mathrm{Nm}$ for a filter loaded at $3000 \mathrm{rpm}, 40 \mathrm{Nm}$ with the engine operating with 25ppm doped fuel until the respective loadings is achieved are presented in Figure 3.29, Figure 3.27 (reference experiment) and Figure 3.30. The following trends are observed:

From low -to medium -to high soot mass loading, a decrease to the $(\rho k)_{\mathrm{p}}$ product is observed. Although the increase of soot layer thickness is expected to cause an increase to the soot density due to the layer compression from the exhaust flow it seems that the decrease in soot permeability is more significant. This observation is confirmed from the results of previous experimental work with single channel filters [106].

As discussed in the previous section, higher frequency factors for the catalytic soot oxidation to $\mathrm{CO}$, and, respectively, lower frequency factors for catalytic soot oxidation to $\mathrm{CO}_{2}$ were inserted in the model to match the behaviour with higher initial soot mass in filter. The change is more severe for the $9 \mathrm{~g} /$ liter loading, where as it can be seen in Table 3.9 where the ratio of $\mathrm{CO} / \mathrm{CO}_{2}$ becomes about ten times higher. Any attempt to explain this behaviour must take into account the complex processes of exhaust gas oxygen diffusion through the soot layer. One could assume that in the initial phase of regeneration where the catalytic soot oxidation prevails over the thermal, the higher soot mass makes more difficult the diffusion of exhaust gas oxygen through the deposit layer thus preventing the oxidation of $\mathrm{Ce}_{2} \mathrm{O}_{3}$ by $\mathrm{O}_{2}$ to produce $\mathrm{CeO}_{2}$. 


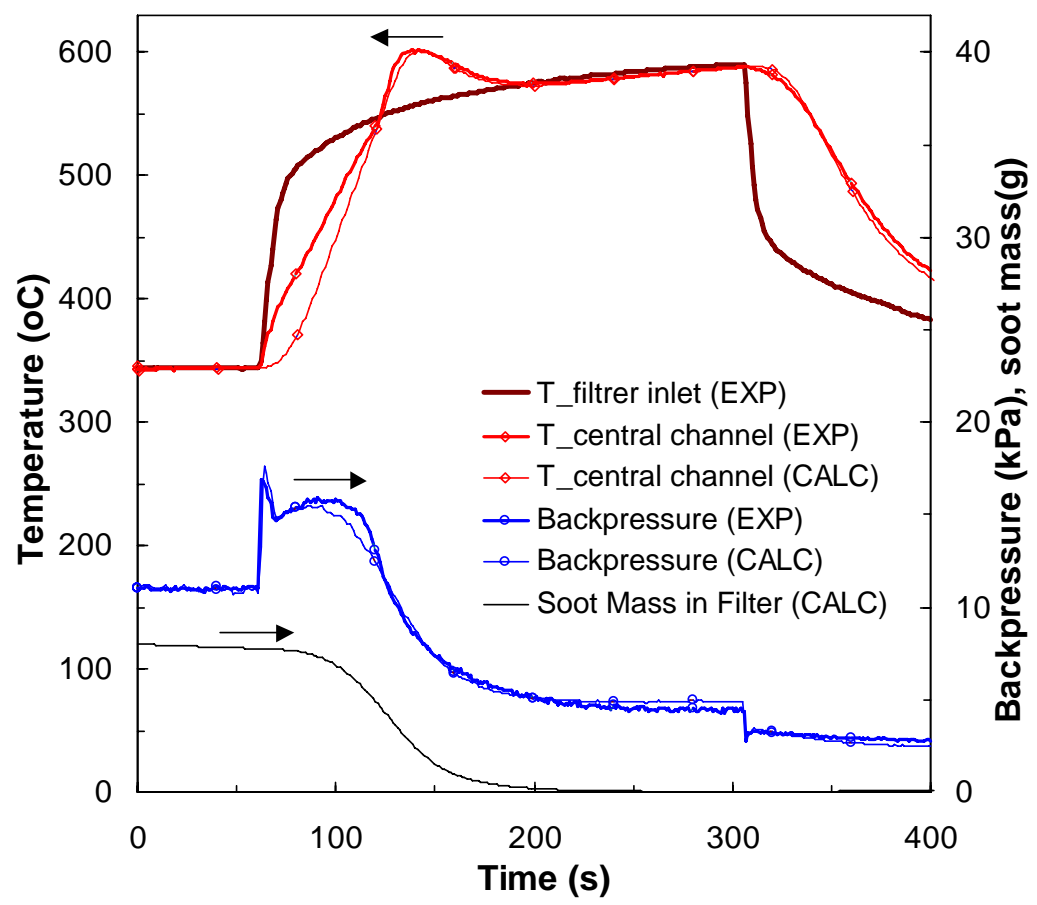

Figure 3.29 Simulation of a low soot mass loading (3g/l) filter regeneration. Measured and predicted trap temperatures near the exit of a central channel for a filter loaded with the engine running on 3000rpm, 40Nm with 25ppm Ce-doped fuel. Also measurement and prediction of filter backpressure together with soot mass prediction are presented. Computation is made with $(\rho k) \mathrm{p}=2.5 \mathrm{E}-13 \mathrm{~kg} / \mathrm{m}$, activation energy values: $\mathrm{E}_{1}=1.9 \mathrm{E} 5$, $\mathrm{E}_{2}=1.5 \mathrm{E} 5, \mathrm{E}_{3}=1.2 \mathrm{E} 5, \mathrm{E}_{4}=0.8 \mathrm{E} 5, \mathrm{E}_{5}=0.8 \mathrm{E} 5 \mathrm{~J} / \mathrm{mole}$ and frequency factor values: $\mathrm{A}_{1}=1 \mathrm{E} 13, \mathrm{~A}_{2}=5.5 \mathrm{E} 10$, $\mathrm{A}_{3}=4.5 \mathrm{E} 11, \mathrm{~A}_{4}=3 \mathrm{E} 8, \mathrm{~A}_{5}=8 \mathrm{E} 8 \mathrm{~mole} / \mathrm{m}^{3}$,s.

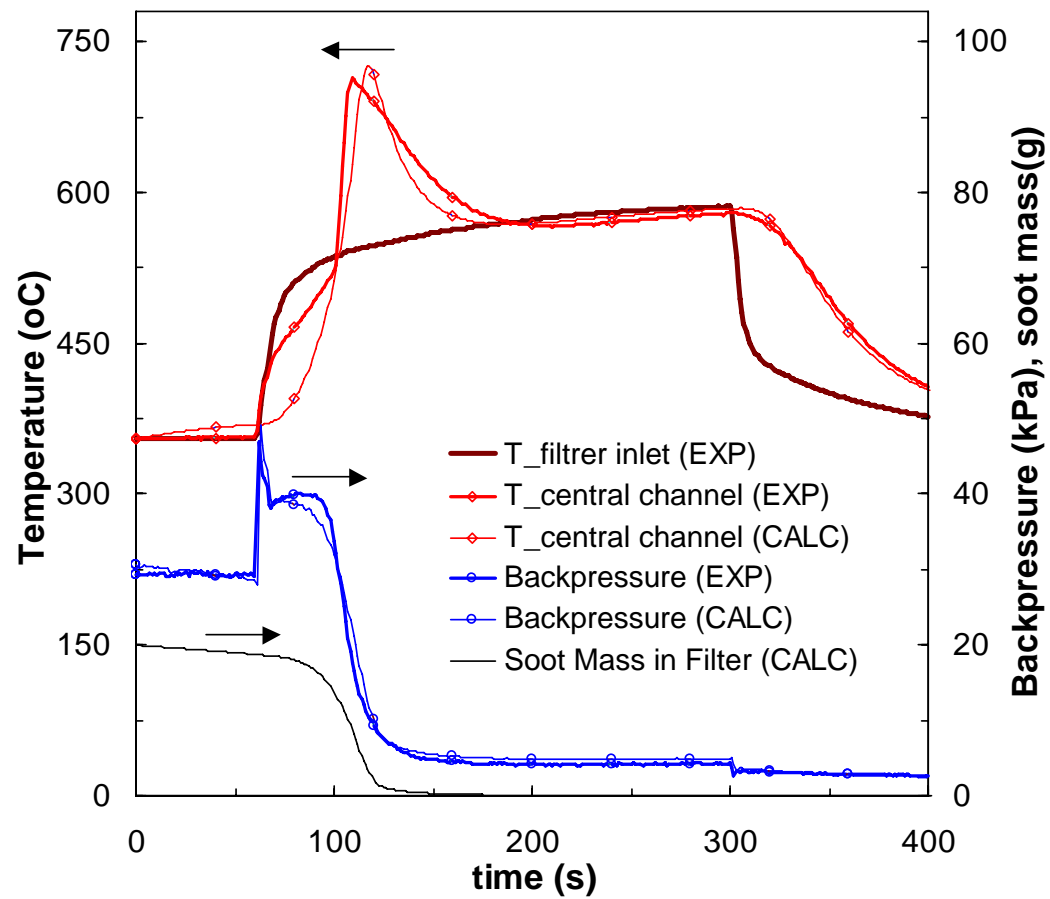

Figure 3.30 Simulation of a high soot mass loading (9g/l) filter regeneration. Measured and predicted trap temperatures near the exit of a central channel for a filter loaded with the engine running on 3000rpm, 40Nm with 25ppm Ce-doped fuel. Also measurement and prediction of filter backpressure together with soot mass prediction are presented. Computation is made with $(\rho k) \mathrm{p}=2.0 \mathrm{E}-13 \mathrm{~kg} / \mathrm{m}$, activation energy values: $\mathrm{E}_{1}=1.9 \mathrm{E} 5$, $\mathrm{E}_{2}=1.5 \mathrm{E} 5, \mathrm{E}_{3}=1.2 \mathrm{E} 5, \mathrm{E}_{4}=0.8 \mathrm{E} 5, \mathrm{E}_{5}=0.8 \mathrm{E} 5 \mathrm{~J} / \mathrm{mole}$ and frequency factor values: $\mathrm{A}_{1}=1 \mathrm{E} 13, \mathrm{~A}_{2}=5.5 \mathrm{E} 10$, $\mathrm{A}_{3}=2.5 \mathrm{E} 11, \mathrm{~A}_{4}=9 \mathrm{E} 8, \mathrm{~A}_{5}=8 \mathrm{E} 8 \mathrm{~mole} / \mathrm{m}^{3}$,s. 


\section{VOF content (soot accumulation condition)}

The effects of VOF content on the pressure drop and kinetic parameters could be assessed only indirectly, since the model considers only dry soot oxidation, without separately addressing adsorbed hydrocarbons oxidation by the catalyst. Thus, VOF oxidation kinetics must be lumped in the overall catalytic oxidation kinetics. It is known from the literature that VOF may be oxidized at temperatures as low as $200^{\circ} \mathrm{C}$, but also may desorb from the particulate at higher temperatures or readsorb at low temperatures. Further improvements by modelling of the adsorbed hydrocarbons oxidation are currently in progress, based on the detailed study of the oxidation and sorption behaviour of real diesel particulate produced by modern DI engines when run on catalyst-doped fuel [72]. The two regeneration cases considered in this validation study involve a filter loaded at 3000rpm, $40 \mathrm{Nm}$ (which is a high VOF point) and a filter loaded at 1800rpm, 90Nm (low VOF point).

The simulations of the regenerations performed at a medium flowrate engine operation point, are presented in Figure 3.27and Figure 3.31.

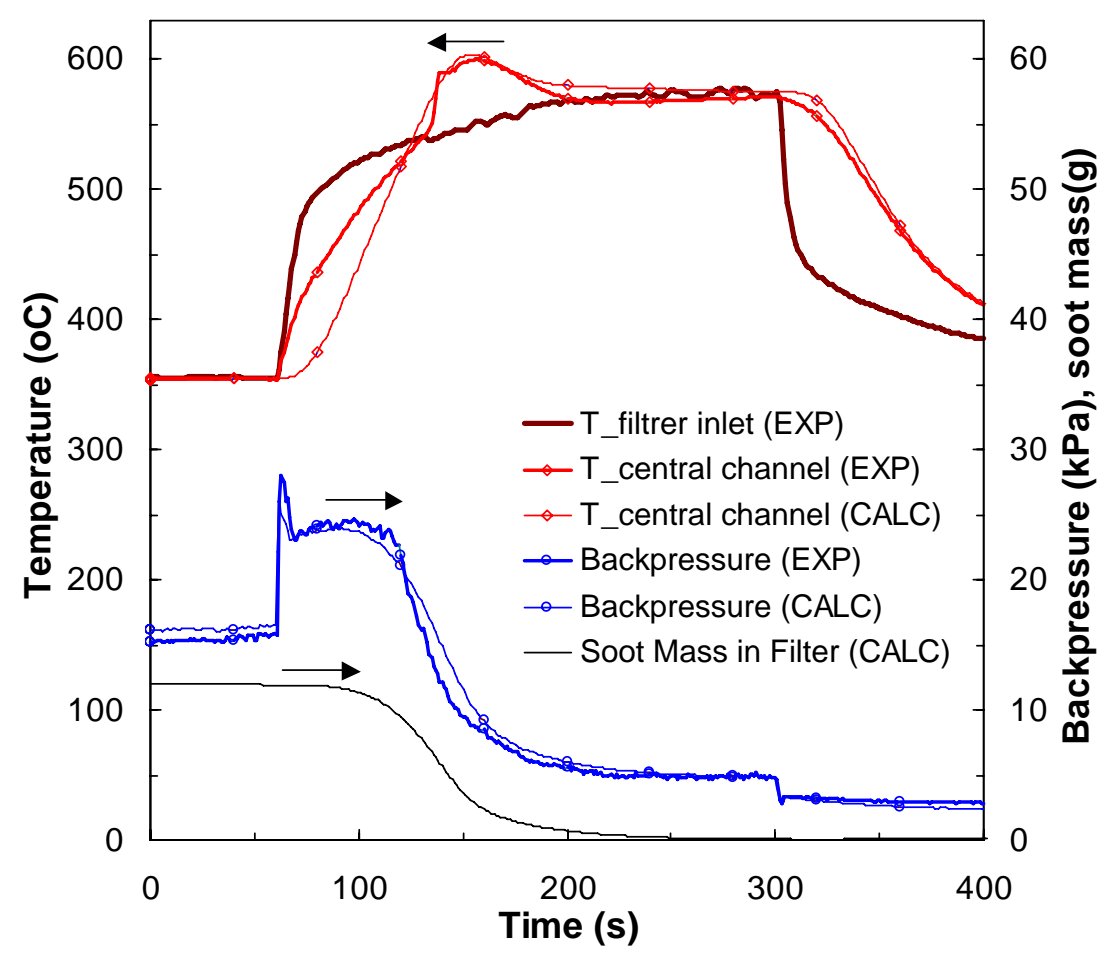

Figure 3.31 Simulation of a regeneration of a filter loaded at a low VOF operation point. Measured and predicted trap temperatures near the exit of a central channel for a medium loaded filter $(6 \mathrm{~g} / \mathrm{l})$ at a medium speed high load operation point (1800rpm, 90Nm) with 25ppm Ce-doped fuel resulting a low VOF content (2\%) in soot. Also measurement and prediction of filter backpressure together with soot mass prediction are presented. Computation is made with $(\rho k) \mathrm{p}=2.6 \mathrm{E}-13 \mathrm{~kg} / \mathrm{m}$, activation energy values: $\mathrm{E}_{1}=1.9 \mathrm{E} 5, \mathrm{E}_{2}=1.5 \mathrm{E} 5, \mathrm{E}_{3}=1.2 \mathrm{E} 5$, $\mathrm{E}_{4}=0.8 \mathrm{E} 5, \mathrm{E}_{5}=0.8 \mathrm{E} 5 \mathrm{~J} / \mathrm{mole}$ and frequency factor values: $\mathrm{A}_{1}=1 \mathrm{E} 13, \mathrm{~A}_{2}=5.5 \mathrm{E} 10, \mathrm{~A}_{3}=2.5 \mathrm{E} 11, \mathrm{~A}_{4}=3 \mathrm{E} 8, \mathrm{~A}_{5}=8 \mathrm{E} 8$ $\mathrm{mole} / \mathrm{m}^{3}, \mathrm{~s}$.

Fitting of model parameters to match the experimental behaviour indicates the following trends:

- From low to high VOF content, a decrease to the value of the $(\rho k)_{\mathrm{p}}$ product is observed. It seems that the VOF present in the thick particulate layer decreases the permeability of the soot layer. Furthermore secondary effects due to the adsorption- desorption processes produced from the temperature differences between the filter core and filter periphery 
(the VOF preferably condense on the colder outer channels of the filter) may differentiate the permeability characteristics and indicate that the mechanism is 3D.

- The lower VOF content hints to lower values for the frequency factors of catalytic soot oxidation. This could be attributed to the loss of the activity of VOF oxidation that accelerates the dry soot oxidation. According to previous experimental work performed in LTTE for the investigation of the role of VOF to the regeneration behavior [72] the higher soot mass is acossiated with a more rapid pressure drop and a higher heat release due to the higher exothermic nature of HC oxidation. On the other hand a relative increase to the frequency factor of catalytic soot oxidation to $\mathrm{CO} 2$ is necessary in order to match the decreased CO produced during regeneration (the unburned hydrocarbons are oxidized by ceria mainly to CO).

\section{Exhaust gas mass flow rate (regeneration condition)}

The effect of exhaust gas mass flow rate is studied in four regeneration experiments, conducted with 28, 52, 70 and $92 \mathrm{~g} / \mathrm{s}$ mass flow rate respectively. The results of the simulations are compared to the measurements in Figure 3.27 (52 g/s - reference experiment), Figure 3.32 (28 g/s), Figure 3.33 (70g/s) and Figure 3.34 (92 g/s).

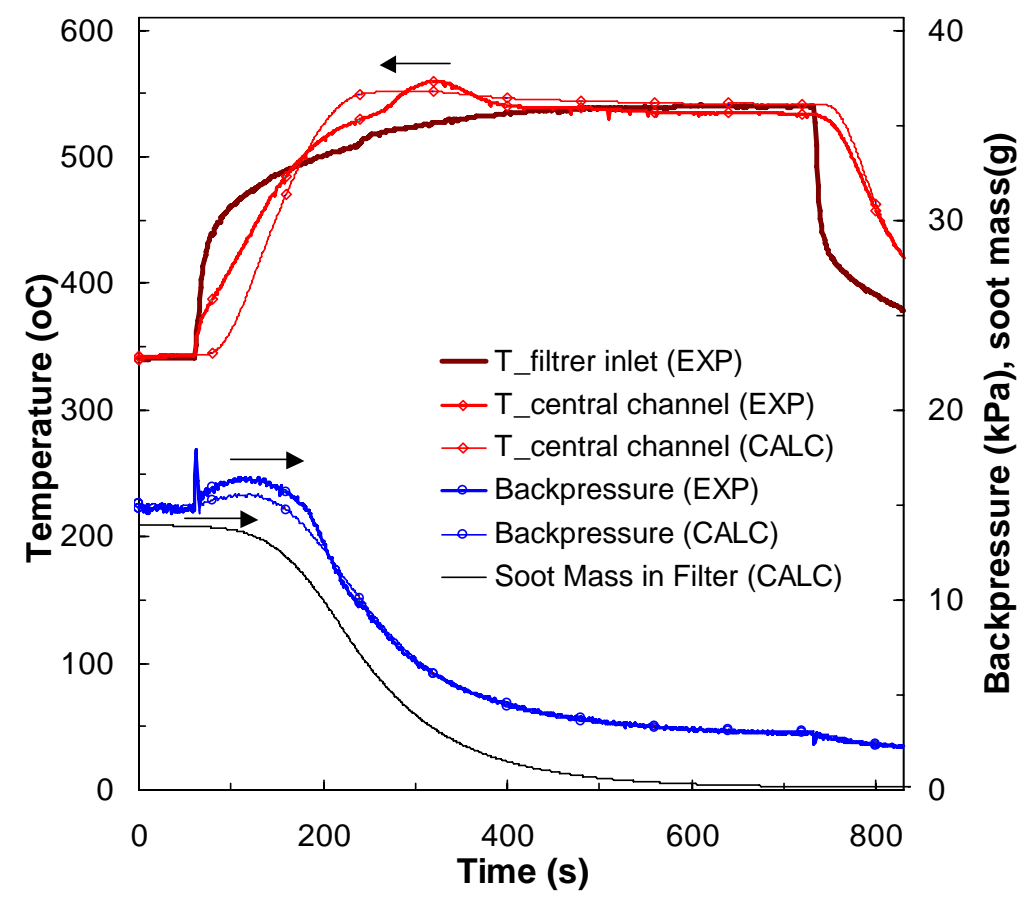

Figure 3.32 Simulation of a low mass flowrate regeneration scenario (28g/s). Measured and predicted trap temperatures near the exit of a central channel during a low mass flowrate regeneration performed at 1500rpm, $80 \mathrm{Nm}$ for a medium mass loaded filter at 3000rpm, 40Nm with 25ppm Ce-doped fuel. Also measurement and prediction of filter backpressure together with soot mass prediction are presented. Computation is made with $(\rho k) \mathrm{p}=2.2 \mathrm{E}-13 \mathrm{~kg} / \mathrm{m}$, activation energy values: $\mathrm{E}_{1}=1.9 \mathrm{E} 5, \mathrm{E}_{2}=1.5 \mathrm{E} 5, \mathrm{E}_{3}=1.2 \mathrm{E} 5, \mathrm{E}_{4}=0.8 \mathrm{E} 5, \mathrm{E}_{5}=0.8 \mathrm{E} 5 \mathrm{~J} / \mathrm{mole}$ and frequency factor values: $A_{1}=1 \mathrm{E} 13, A_{2}=5.5 \mathrm{E} 10, A_{3}=1 \mathrm{E} 11, \mathrm{~A}_{4}=2 \mathrm{E} 8, \mathrm{~A}_{5}=8 \mathrm{E} 8 \mathrm{~mole} / \mathrm{m}^{3}$,s.

The following trends were observed:

- The model is capable to predict the temperature and pressure drop of the filter at medium to high exhaust gas mass flow rate conditions (Figure 3.27 and Figure 3.33). The accuracy of the model is reduced at very low or very high mass flow rate conditions 
(Figure 3.32 and Figure 3.34). At low mass flow rate conditions, this is attributed to flow maldistribution phenomena [106]. At such conditions, the point within the filter where the regenerations initiates is stochastic and the propagation is expected to be slow. The model may not predict this stochastic behaviour, because of the assumption of uniform flow distribution at the filter inlet and the assumption of uniform soot mass distribution within the filter. Specifically, in Figure 3.32, it may be observed that there is a delay between the computed and the measured temperature in the central channel of the filter, despite that the pressure drop during regeneration is well predicted by the model. Moreover, the model also predicts the value of maximum temperature satisfactorily. This indicates that the regeneration did not initiate in the central region of the filter and propagated there with some delay.

- At medium to high mass flow rates, it is expected that the flow inside and at the inlet of the filter becomes more uniform. The assumption of 1-D modelling is more realistic at these conditions. This is also supported by the results of Figure 3.27 and Figure 3.33. where the computations match the measurements with higher accuracy.

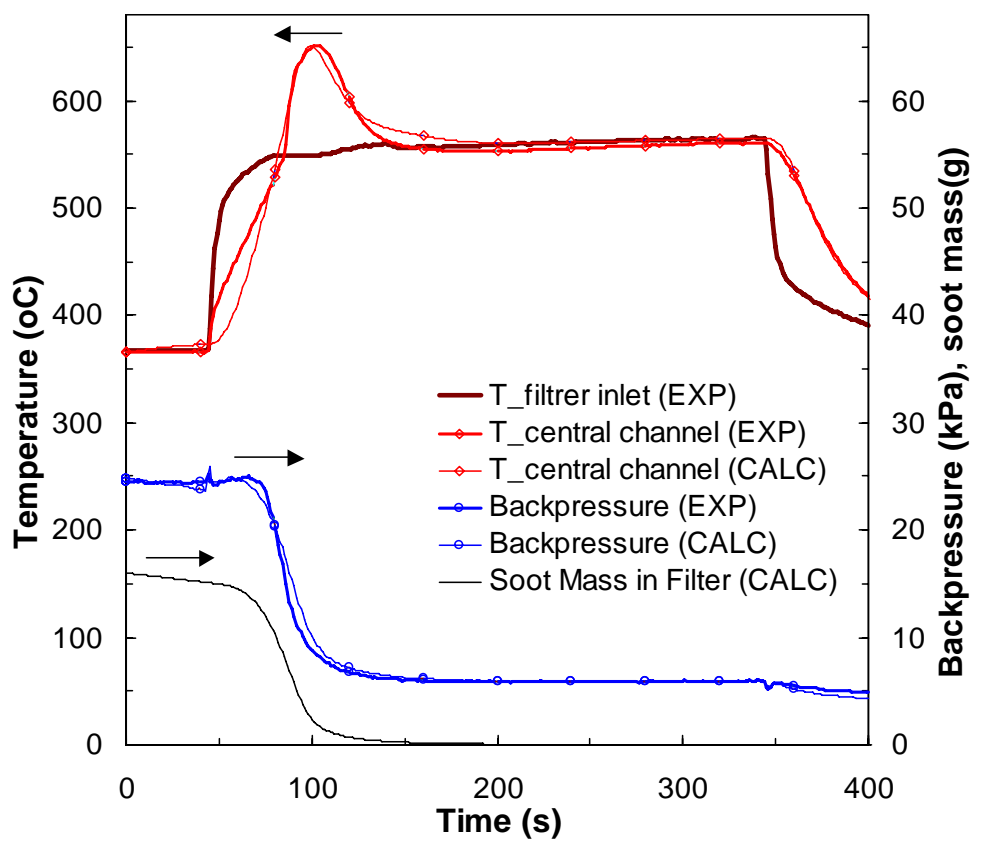

Figure 3.33 Simulation of a medium mass flowrate regeneration scenario (70g/s). Measured and predicted trap temperatures near the exit of a central channel during a low mass flowrate regeneration performed at 3000rpm, $60 \mathrm{Nm}$ for a medium mass loaded filter at 3000rpm, 40Nm with 25ppm Ce-doped fuel. Also measurement and prediction of filter backpressure together with soot mass prediction are presented. Computation is made with $(\rho k) \mathrm{p}=2.2 \mathrm{E}-13 \mathrm{~kg} / \mathrm{m}$, activation energy values: $\mathrm{E}_{1}=1.9 \mathrm{E} 5, \mathrm{E}_{2}=1.5 \mathrm{E} 5, \mathrm{E}_{3}=1.2 \mathrm{E} 5, \mathrm{E}_{4}=0.8 \mathrm{E} 5, \mathrm{E}_{5}=0.8 \mathrm{E} 5 \mathrm{~J} / \mathrm{mole}$ and frequency factor values: $\mathrm{A}_{1}=1 \mathrm{E} 13, \mathrm{~A}_{2}=5.5 \mathrm{E} 10, \mathrm{~A}_{3}=3.5 \mathrm{E} 11, \mathrm{~A}_{4}=6 \mathrm{E} 8, \mathrm{~A}_{5}=8 \mathrm{E} 8 \mathrm{~mole} / \mathrm{m}^{3}, \mathrm{~s}$.

- Finally, at very high mass flow rates (Figure 3.34), the amount of $\mathrm{CO}$ versus $\mathrm{CO}_{2}$ at the outlet of the filter because of soot oxidation increases. This is attributed to the lower residence time of exhaust gas within the soot layer because of the higher velocities of the exhaust gas. This effect is not included in the model, therefore, the pre-exponential factor of the thermal soot oxidation to $\mathrm{CO}$ had to be increased in order to match the temperature measurement. In addition, the model predicts some regeneration at the beginning of the measurement due to the high temperatures of the exhaust gas although no regeneration occurs. This suggests that, under high exhaust gas temperatures, the catalytic activity is 
diminishing as flow rate increases. This may also be connected to the residence time of the exhaust gas in the soot layer, but the phenomenon remains obscure and requires further investigation.

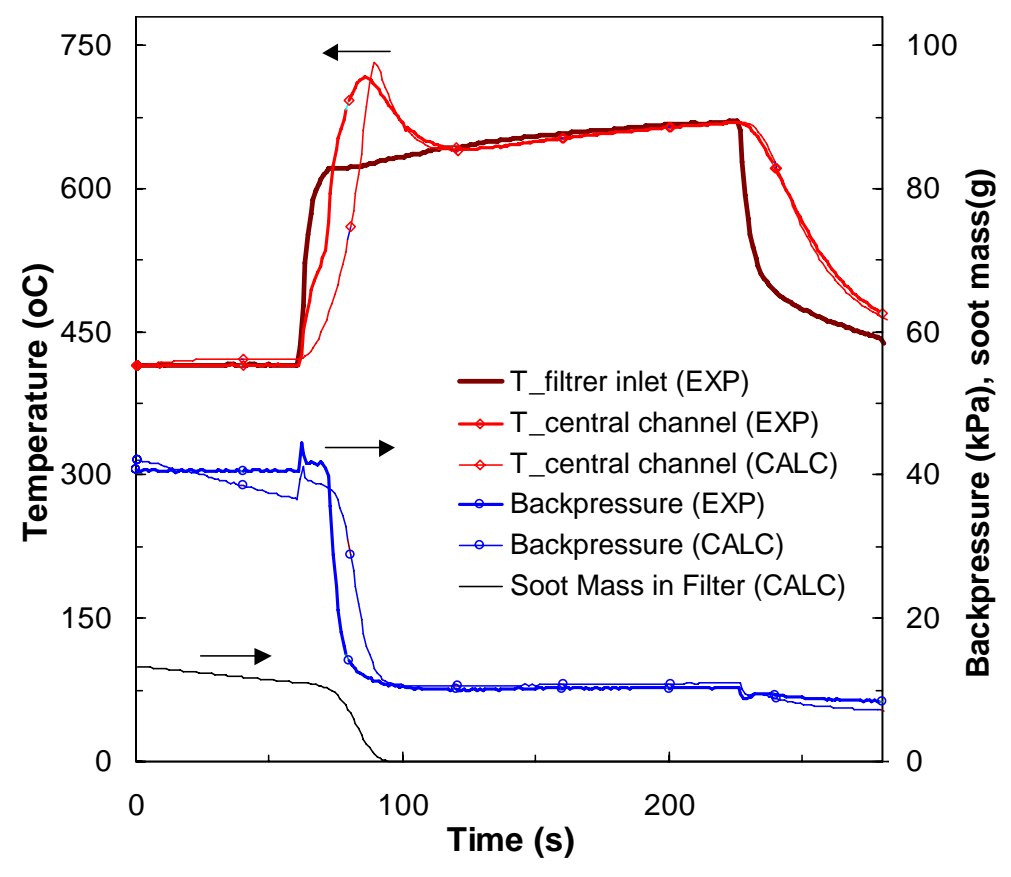

Figure 3.34 Simulation of a high mass flowrate-high temperature regeneration scenario (100g/s). Measured and predicted trap temperatures near the exit of a central channel during a high mass flowrate regeneration performed at $4000 \mathrm{rpm}, 40 \mathrm{Nm}$ for a medium mass loaded filter at 3000rpm, 40Nm with 25ppm Ce-doped fuel. Also measurement and prediction of filter backpressure together with soot mass prediction are presented. Computation is made with $(\rho \mathrm{k}) \mathrm{p}=2.2 \mathrm{E}-13 \mathrm{~kg} / \mathrm{m}, \mathrm{E}_{1}=1.9 \mathrm{E} 5, \mathrm{E}_{2}=1.5 \mathrm{E} 5, \mathrm{E}_{3}=1.2 \mathrm{E} 5, \mathrm{E}_{4}=0.8 \mathrm{E} 5, \mathrm{E}_{5}=0.8 \mathrm{E} 5 \mathrm{~J} / \mathrm{mole}$ and frequency factor values: $\mathrm{A}_{1}=1 \mathrm{E} 13, \mathrm{~A}_{2}=6.5 \mathrm{E} 12, \mathrm{~A}_{3}=3.5 \mathrm{E} 11, \mathrm{~A}_{4}=6 \mathrm{E} 8, \mathrm{~A}_{5}=8 \mathrm{E} 8 \mathrm{~mole} / \mathrm{m}^{3}$,s.

\subsubsection{Concluding remarks}

- A systematic experimental validation procedure was applied to an improved, comprehensive 1-D model of fuel additive assisted regeneration of a diesel filter. The model features separate consideration of Carbon oxidation to $\mathrm{CO}$ and $\mathrm{CO}_{2}$, which enhances its predictive ability. Also, it employs an improved kinetic submodel for the catalytic activity of the Cerium-based fuel additive employed.

- The validation was based on the results of a number of regeneration tests on the engine bench of a Silicon Carbide diesel filter fitted to a modern diesel engine run on catalyst (Cerium-based) doped fuel.

- First the thermal Carbon oxidation kinetics (frequency factors) were tuned to represent the experimentally determined thermal regeneration behaviour of the filter.

- As a second step, the catalytic regeneration kinetics (frequency factors) were tuned to represent the experimentally determined, low temperature (catalytic) regeneration behaviour of the filter. 
- The ability of the tuned model to predict the effects of exhaust mass flow rate, initial soot loading mass, volatile organic fraction of the soot and additive concentration in fuel was finally checked. In the following, the observed deviations from the reference kinetics of the catalytic reactions are summarized:

- It was found that the increase of the initial soot loading shifts the catalytic reaction kinetics to produce more $\mathrm{CO}$ and less $\mathrm{CO}_{2}$. The model can match this behaviour by a respective modification of the frequency factors of the respective reactions.

- A severe decrease in the volatile organic fraction of the collected particulate, leads to a respective reduction of catalytic soot oxidation kinetics, which can be matched by a respective reduction of frequency factors of the catalytic oxidation reactions.

- As expected, the tuned 1D model does not successfully predict filter operation at low flowrates. This due to the 1D nature of the model, since it is expected that, the complex 3D effects, related to exhaust flow maldistribution at filter inlet and soot loading maldistribution may significantly affect the regeneration of the DPF. This indicates that in this case a 3D approach should enhance our understanding about the variation of flow distribution at the inlet of the filter and the propagation of regeneration in general. This motivates the assessment of the 3D model that is presented in the following section under low flow rate conditions.

- On the other side, by comparison of model predictions with measurements, it was observed that the catalytic activity is reduced at very high flowrates (very low residence times). This needs to be further investigated.

- Along with the validation of the kinetic submodel, a validation of the pressure drop submodel was carried out. The study of the soot permeability variation in order to match the experimental curve of pressure drop indicated a significant influence of soot mass loading, engine operation point (VOF content) and catalyst concentration.

- The results of the systematic validation procedure are intended to demonstrate the scope and extents of applicability of the specific model category to real world design and optimization studies with diesel filters. The validation results hint to model improvements that are already under way.

\subsection{D ANSYS - CATWALL Model Validation}

In order to demonstrate the ability of the 3D DPF model developed in the frame of George Pontikakis' Thesis [70] to assist in the process of the detailed DPF design, simulations of the characteristic cases with various exhaust mass flow rates presented in section 3.3 and already used in the 1D validation, were performed. From the simulations the transient case of stochastic regeneration was excluded due to the lack of the model to include the mechanism of the VOF action. Three-dimensional temperature fields for any direction and inlet flow profile may be exited by the code and may be viewed for any filter section. The results provide excessive information for the regeneration process and the effects of the 3D phenomena.

The meshed finite element model of the DPF that was used in this work is illustrated in

Figure 3.35. It comprises different volumes for the main part of the SiC filter blocks, the filter blocks (where the plugs are), the adhesive of the filter blocks (cement), the insulation mat and the canning. Depending on the volume, different material properties are assigned. In 
this case, the material properties assigned were the thermal capacity, thermal conductivity and density.

The kinetic parameters of the rate expressions that were used in this work were obtained by the previous presented validation study of the 1D DPF model. The values for the preexponential factors and the activation energies employed in the simulations of the respective cases are already presented in Table 3.9.

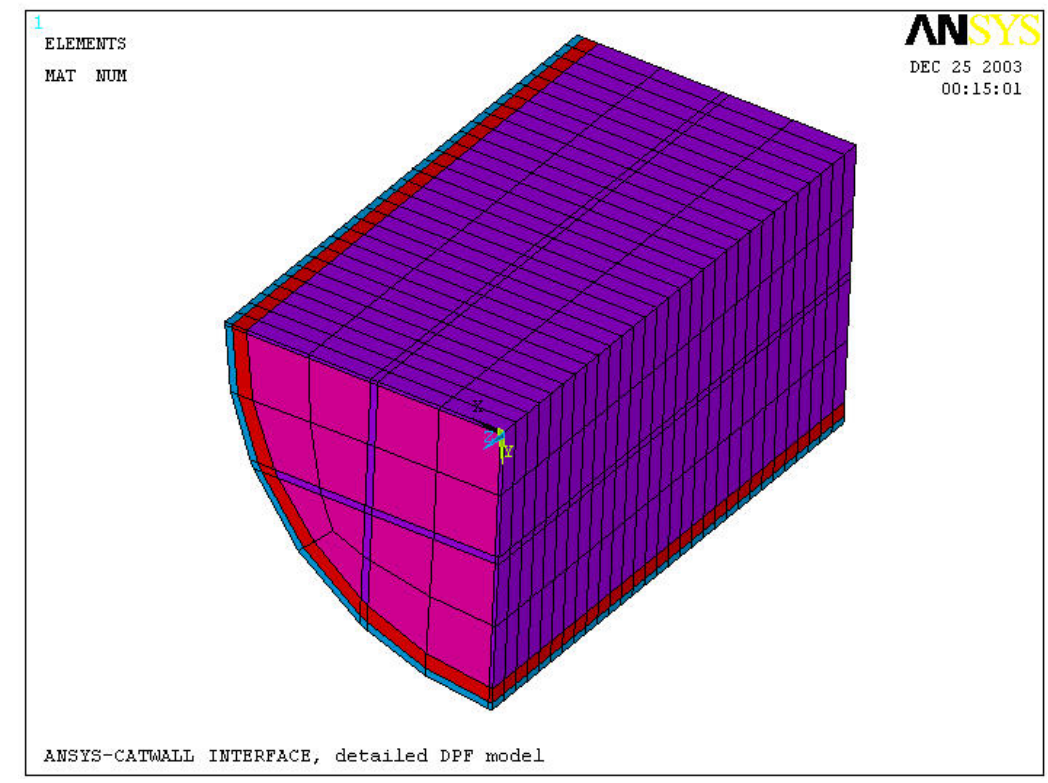

Figure 3.35 Solid model of the DPF. Different materials are designated by different colors. (a) Front view: blue=canning, red=insulation mat, purple=adhesive, magenta $=\mathrm{SiC}$

\subsubsection{D model validation results and discussion}

\section{High flowrate regeneration}

As a first step five snapshots of the predicted temperatures fields at the central and the outer section of the one-quarter of the filter in the case of the high flowrate regeneration scenario of Figure 3.21 is presented in Figure 3.36 The elements of the canning the insulation mat and the surrounding adhesive have been removed, to view the evolution of the regeneration process in the central and peripheric ceramic blocks. The results agree with the experimental measurements indicating that the model has no problem in matching the high flow rate behaviour. The regeneration has a uniform evolution through the central and peripheric ceramic blocks of the filter with the maximum temperatures to be predicted near the exit of the central blocks. Although as presented in [106] significant flow maldistribution exists during the high flowrate regeneration the short duration of the phenomena due to the higher oxidation rates make their effect to the prediction of the temperature fields negligible.

Another observation is related with the predicted propagation of regeneration in the outer blocks of the filter. From the outer shell snapshots of Figure 3.36 it becomes apparent that the evolution of regeneration in the small periphery block is significantly different than that of the neighboring big outer blocks. The regeneration in this block appears the higher delay, probably due to the combined effect of the external heat loses and the surrounding adhesive that acts as insulating material, inhibiting the propagation of the heat released from the central blocks. It is apparent that this delay is associated by temperature gradients that result to the appearance of higher thermal stresses in this filter area. 

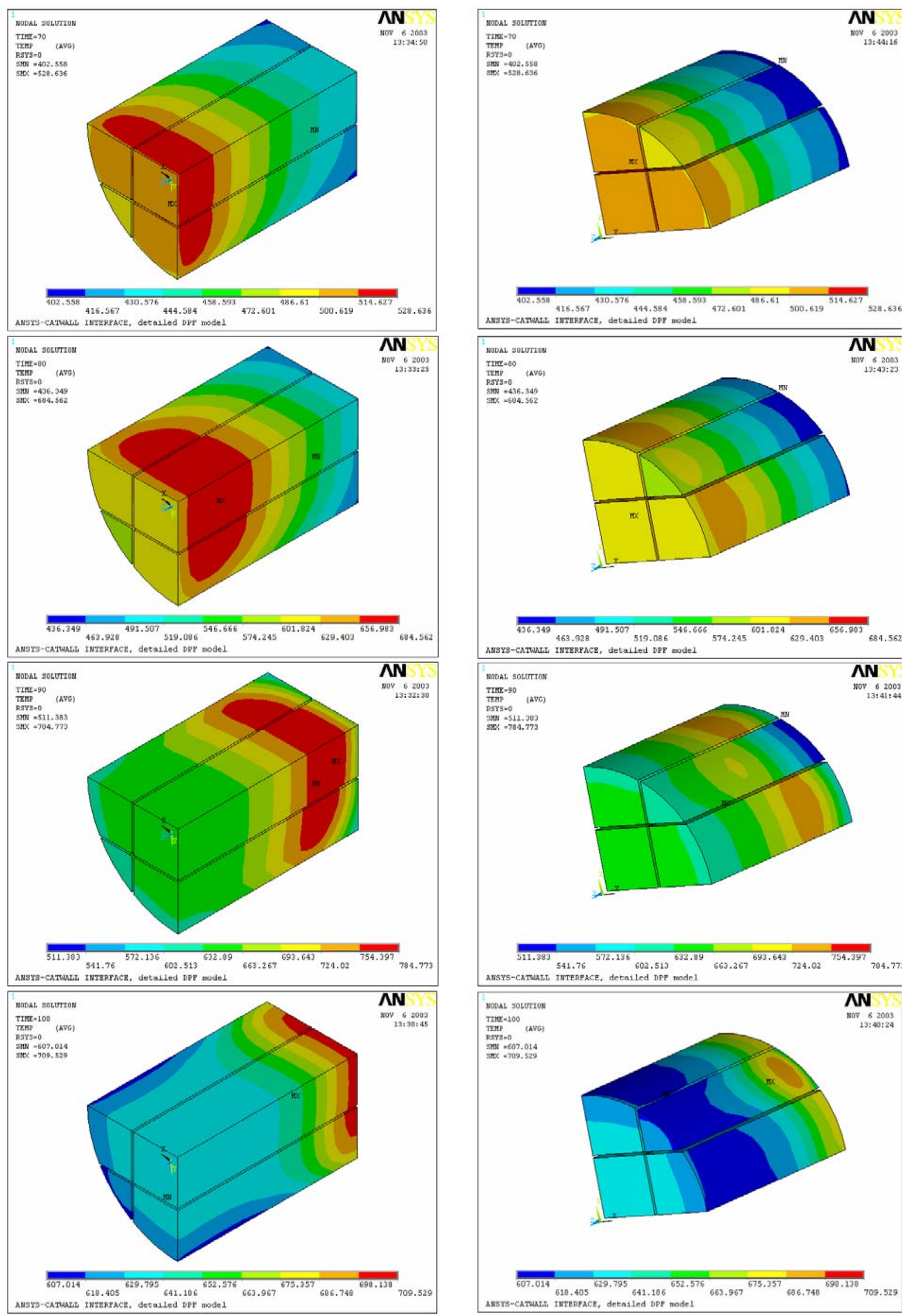

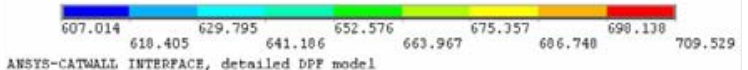

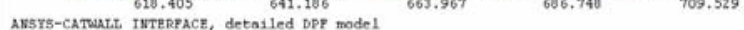

Figure 3.36 Sequence of snapshot views of the predicted temperature field in the interior and the outer shell of the filter with the ANSYS-CATWALL model during the high flowrate regeneration scenario of Figure 3.21. 
The simulations of the measured temperatures at 15mm from filter exit: TC/10 (near filter center) and TC/11 (near filter periphery) presented in Figure 3.37 confirm the above observations. The prediction of the central channel temperature by the $1 \mathrm{D}$ model is also shown and the results are compared with these of 3D model. A delay to the 1D model prediction is observed. This delay is due to the assumption of the high SiC thermal conductivity throughout the filter (that is, the insulation effect of the adhesive cement is not taken into account in the 1D model). Higher conductivity means higher thermal diffusivity that produces the delayed computed rise of temperature in the central part.

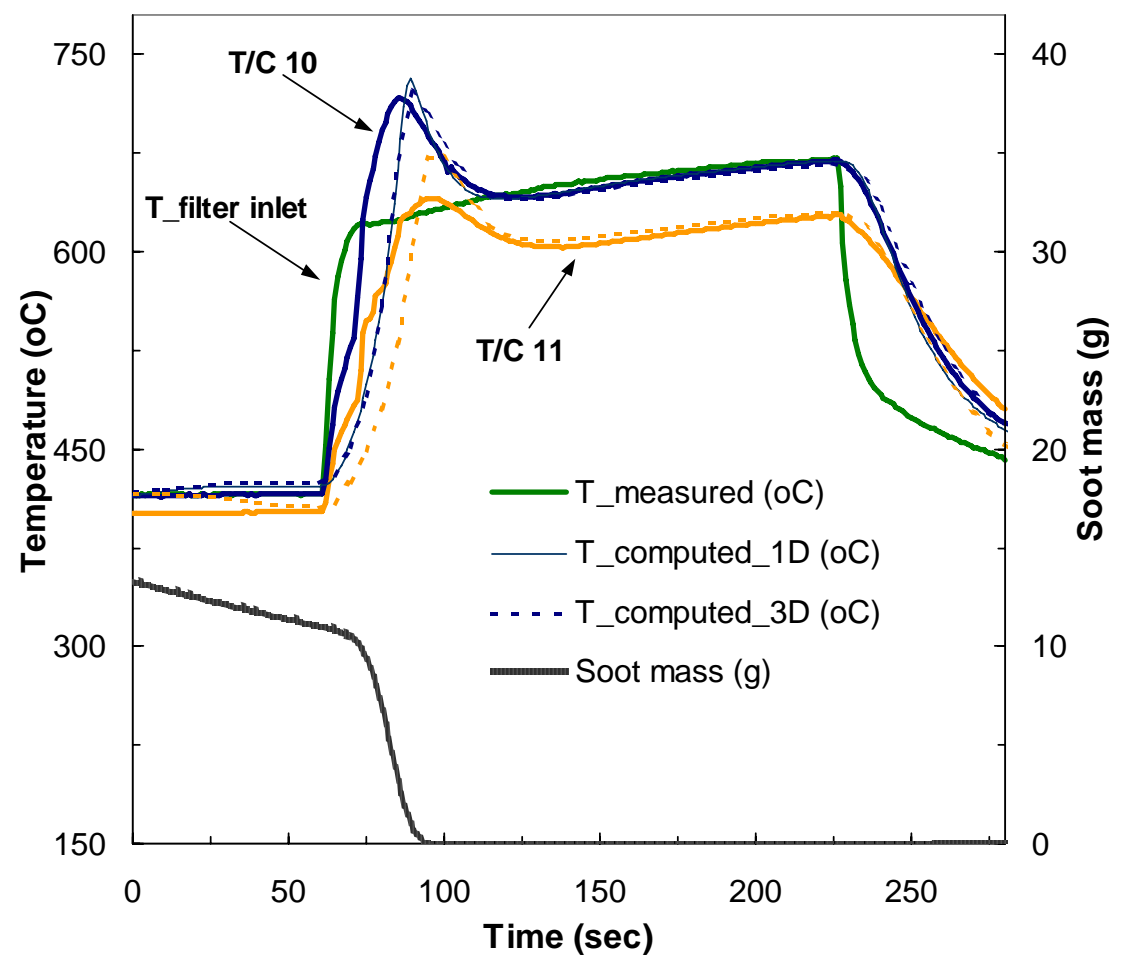

Figure 3.37 Computational simulation of the high flow rate regeneration of Figure 3.21by the Catwall 1-D and the 3D Ansys-Catwall interfacing. Predictions of measured temperatures by thermocouples T/C10 (filter central area) and T/C11 (near filter periphery) are presented. The prediction of the oxidized soot mass is also presented. $1 \mathrm{D}$ and $3 \mathrm{D}$ computations are made with activation energy values: $\mathrm{E}_{1}=1.9 \mathrm{E} 5, \mathrm{E}_{2}=1.5 \mathrm{E} 5, \mathrm{E}_{3}=1.2 \mathrm{E} 5, \mathrm{E}_{4}=0.8 \mathrm{E} 5$, $E_{5}=0.8 \mathrm{E} 5 \mathrm{~J} / \mathrm{mole}$ and frequency factor values: $A_{1}=1 \mathrm{E} 13, \mathrm{~A}_{2}=6.5 \mathrm{E} 12, \mathrm{~A}_{3}=3.5 \mathrm{E} 11, \mathrm{~A}_{4}=6 \mathrm{E} 8, \mathrm{~A}_{5}=8 \mathrm{E} 8 \mathrm{~mole} / \mathrm{m}^{3}, \mathrm{~s}$.

\section{Medium flowrate regeneration}

Five snapshots of the predicted temperatures fields at the central and the outer section of the one-quarter of the filter in the case of the medium flowrate regeneration scenario of Figure 3.22 is presented in Figure 3.38. The elements of the canning the insulation mat and the surrounding adhesive have been removed also in this case. The results generally agree with the measurements in the specific measurement points. Again, the maximum temperatures are predicted near the exit of the central blocks. However, matching of the filter behaviour is not the same quality as with the high flow rate case. The regeneration has a different evolution in the central and peripheric ceramic blocks of the filter. Although the regeneration starts from the central blocks where according to the measurement the higher inlet temperatures observed its propagation to the outer blocks is delayed. In this case, the effect of flow maldistribution becomes prominent. As presented in [106], the maldistributed flow that initially passes through the central channels of the filter lags to be transferred to the peripheric channels due to the lower oxidation rates resulted from the lower mass flow rate. 

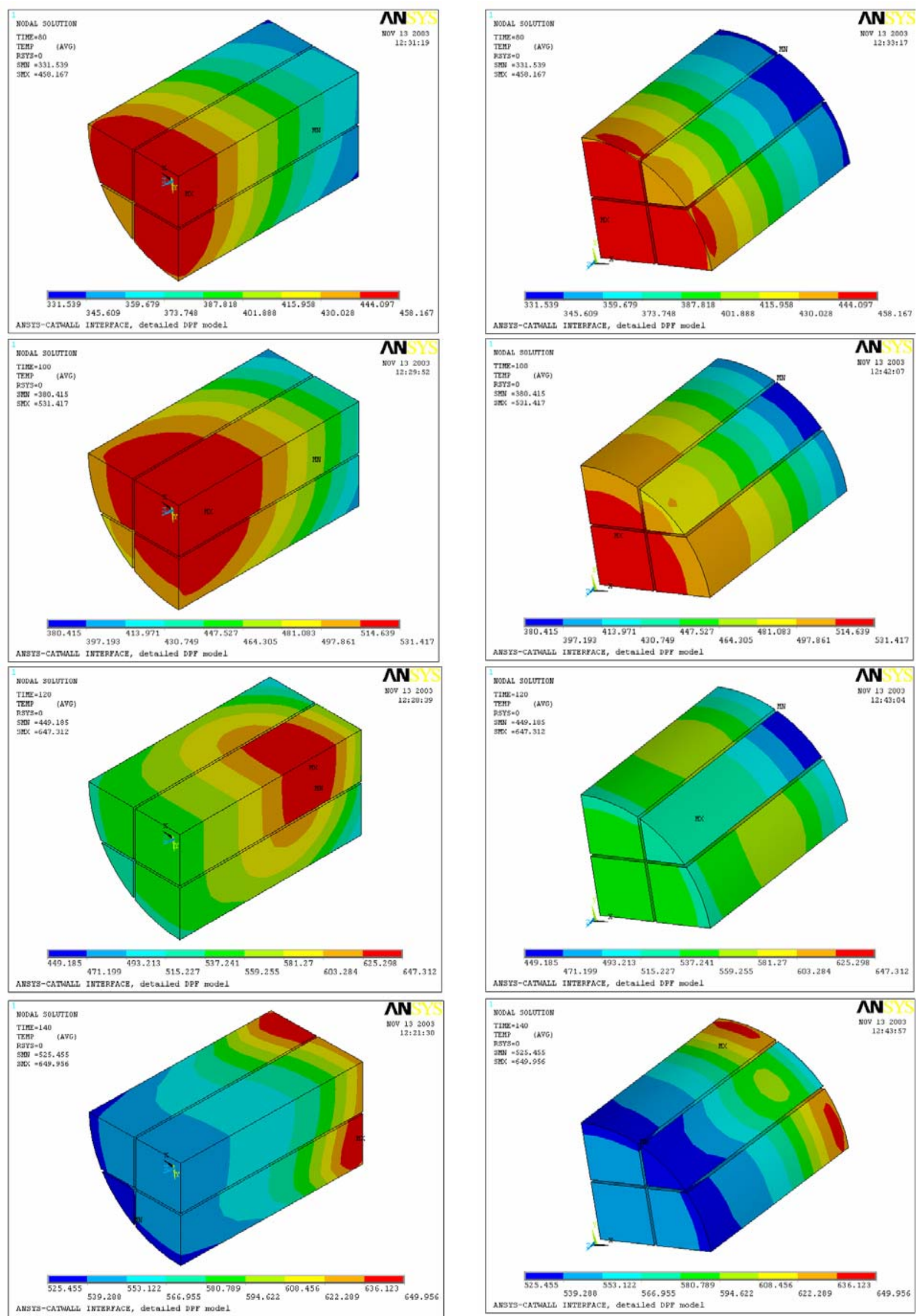

Figure 3.38 Sequence of snapshot views of the predicted temperature field in the interior and the outer shell of the filter with the 3D Ansys-Catwall model during the medium flowrate regeneration scenario of Figure 3.22. 
According to the simulation results of Figure 3.39 the computed delay in the regeneration of the outer blocks of the filter is somewhat less than that measured. Also the predicted temperature of the outer blocks after the end of regeneration is higher than the measured. This could be attributed to the remained flow maldistribution after the end of regeneration due to the inlet and soot maldistribution effects that result to a higher flow through the central blocks and thus the lower convective heating of the outer.

Again, the temperature prediction of the 1D model is observed to be somewhat delayed compared to the prediction of the 3D computation (Figure 3.39).

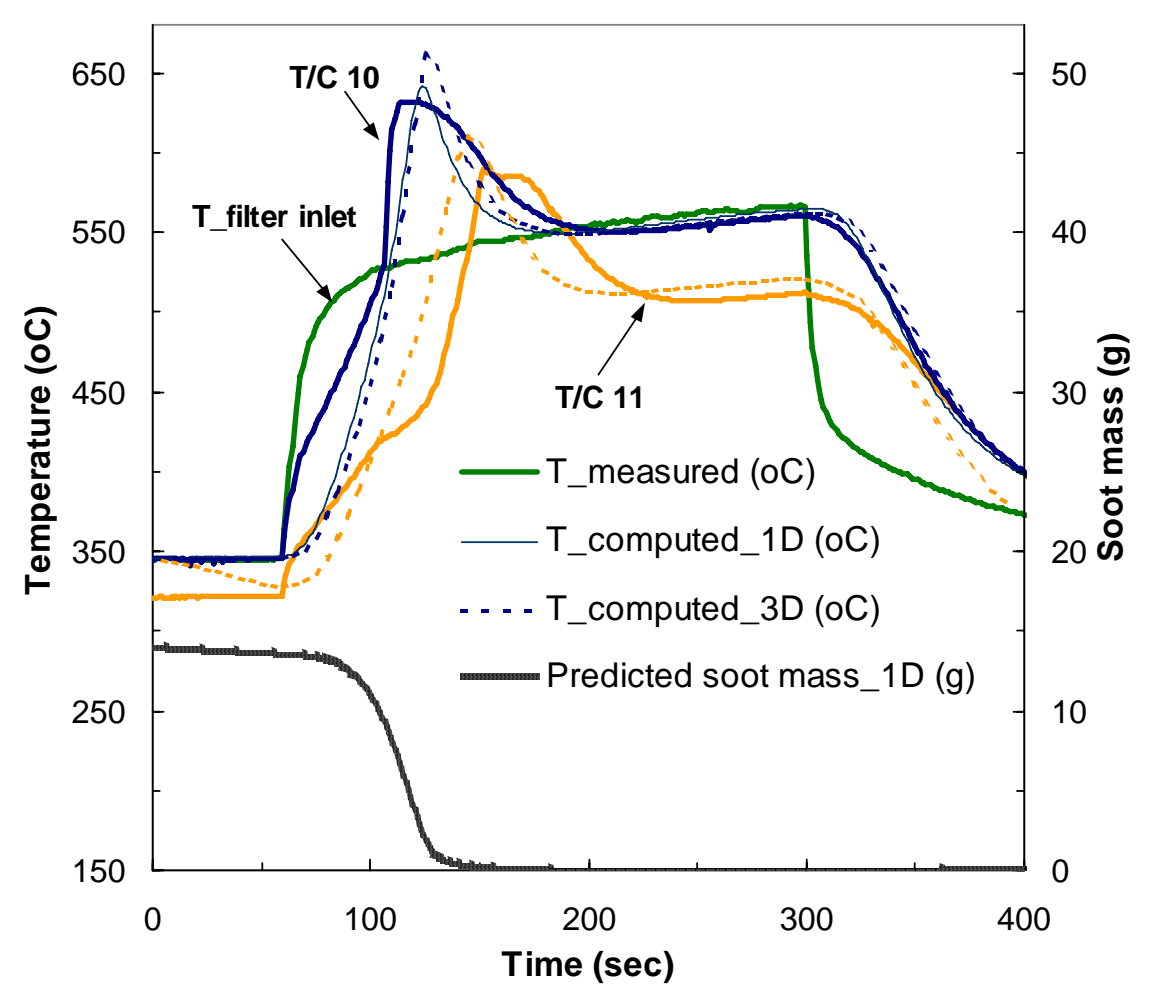

Figure 3.39 Computational simulation of the medium flow rate regeneration of figure 5 by the Catwall 1-D and the 3D Ansys-Catwall interfacing. Predictions of measured temperatures by thermocouples T/C10 (filter central area) and T/C11 (near filter periphery) are presented. The prediction of the oxidized soot mass is also presented. $1 \mathrm{D}$ and $3 \mathrm{D}$ computations are made with activation energy values: $\mathrm{E}_{1}=1.9 \mathrm{E} 5, \mathrm{E}_{2}=1.5 \mathrm{E} 5, \mathrm{E}_{3}=1.2 \mathrm{E} 5, \mathrm{E}_{4}=0.8 \mathrm{E} 5$, $E_{5}=0.8 \mathrm{E} 5 \mathrm{~J} / \mathrm{mole}$ and frequency factor values: $\mathrm{A}_{1}=1 \mathrm{E} 13, \mathrm{~A}_{2}=5.5 \mathrm{E} 10, \mathrm{~A}_{3}=3.5 \mathrm{E} 11, \mathrm{~A}_{4}=6 \mathrm{E} 8, \mathrm{~A}_{5}=8 \mathrm{E} 8 \mathrm{~mole} / \mathrm{m}^{3}, \mathrm{~s}$.

\section{Low flowrate regeneration}

Five snapshots of the temperature fields at the central and peripheric blocks of the filter obtained with application of 3D ANSYS-CATWALL are presented in Figure 3.40. A negligible regeneration of the periphery is observed which again is delayed due to the heat losses to the ambient air. The heat losses combined with the insulation effect of the adhesive result to the prediction of significantly lower temperatures at the small periphery block. Thus, the small size outer filter block in the specific filter design is characterized by incomplete regeneration due to the lower temperatures. The lower temperatures also mean the appearance of high thermal gradients at this block. The thermal gradients are associated with thermal stresses that under certain conditions may lead to filter failure [104]. 


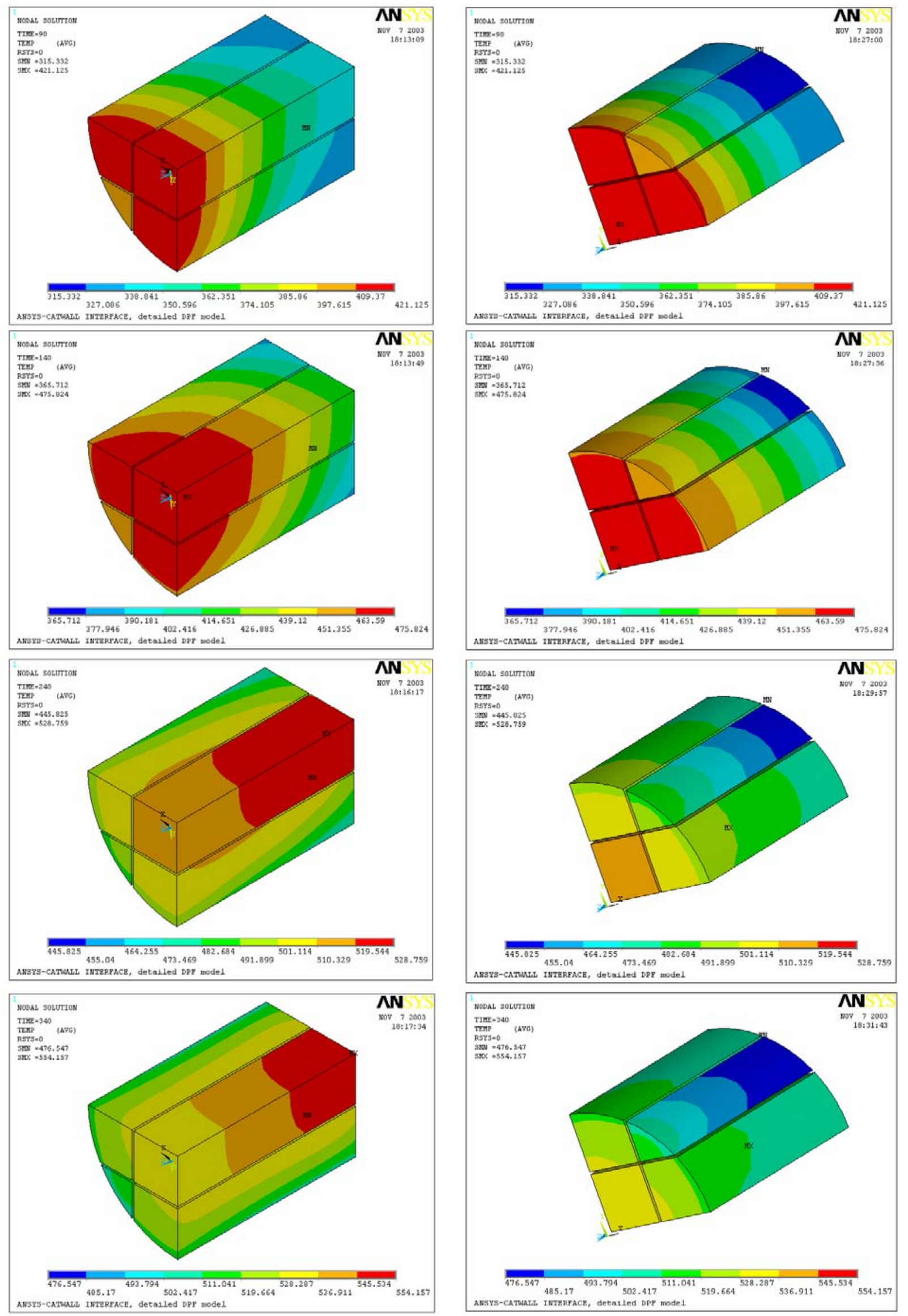

Figure 3.40 Sequence of snapshot views of the predicted temperature field in the interior and the outer shell of the filter with the 3D Ansys-Catwall model during the low flowrate regeneration scenario of Figure 3.23. 
In this case the predicted delay in the regeneration of the outer blocks of the filter is signifigantly less than the measured. As illustrated in Figure 3.41 the regeneration at the filter periphery starts more than 300 seconds after the regeneration of the central blocks while the predicted delay is not more than 100 seconds. It is beyond the capacity of the model in its current state to predict such a delay that could be attributed to phenomena that are not included in the model. In brief these phenomena could be summarized to the following:

- Flow maldistribution phenomena. The existence of complex flow maldistribution phenomena due to the design and operating characteristics of the system may have significant effects to the evolution of regeneration in the outer filter channels that are not taken in account by the model, which considers a uniform, flow distribution.

- Soot maldistribution phenomena. The accumulated soot distribution may be significant in such a scenario, while, for the time being the model considers a uniform distribution.

- Furthermore uncertainties regarding the thermophysical properties of the filter materials and especially the soot layer may influence the model's predictive ability.

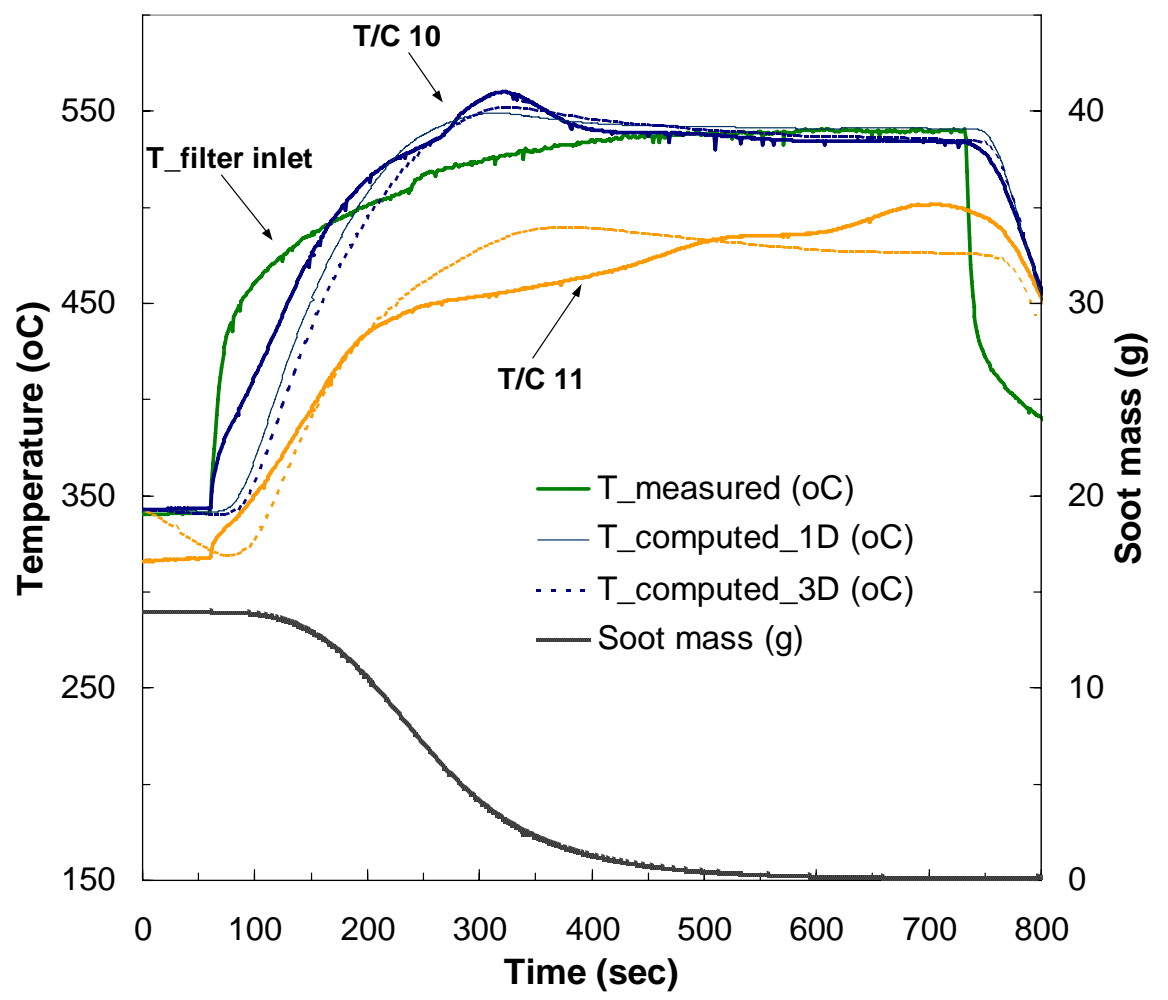

Figure 3.41 Computational simulation of the low flow rate regeneration of Figure 3.23by the Catwall 1-D and the 3D Ansys-Catwall interfacing. Predictions of measured temperatures by thermocouples T/C10 (filter central area) and T/C11 (near filter periphery) are presented. The prediction of the oxidized soot mass is also presented. $1 \mathrm{D}$ and $3 \mathrm{D}$ computations are made with activation energy values: $\mathrm{E}_{1}=1.9 \mathrm{E} 5, \mathrm{E}_{2}=1.5 \mathrm{E} 5, \mathrm{E}_{3}=1.2 \mathrm{E} 5, \mathrm{E}_{4}=0.8 \mathrm{E} 5$, $\mathrm{E}_{5}=0.8 \mathrm{E} 5 \mathrm{~J} / \mathrm{mole}$ and frequency factor values: $\mathrm{A}_{1}=1 \mathrm{E} 13, \mathrm{~A}_{2}=5.5 \mathrm{E} 10, \mathrm{~A}_{3}=1 \mathrm{E} 11, \mathrm{~A}_{4}=2 \mathrm{E} 8, \mathrm{~A}_{5}=8 \mathrm{E} 8 \mathrm{~mole} / \mathrm{m}^{3}, \mathrm{~s}$.

\section{Uncontrolled regeneration}

Obviously, the complexity of uncontrolled regeneration phenomena observed during transient (failure) scenarios, is prohibiting even to the 3D modeling. However, it is interesting to see what a 1D and a 3D model could predict for the situation of Figure 3.24. Figure 3.42 presents four snapshots of the predicted temperature field, as seen from a central and from a peripheric viewpoint. 


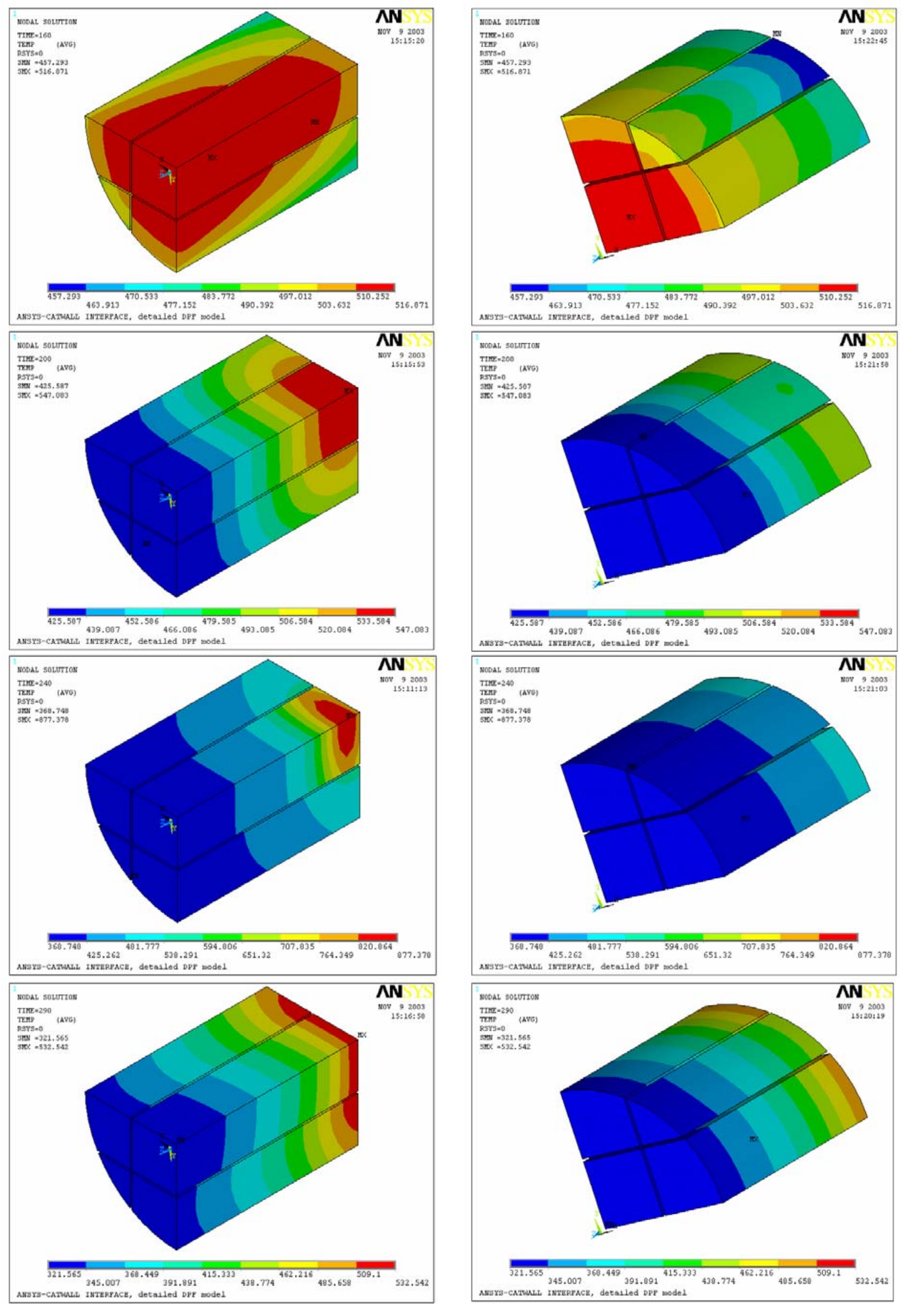

Figure 3.42 Sequence of snapshot views of the predicted temperature field in the interior and the outer shell of the filter with the 3D Ansys-Catwall model during the uncontrolled regeneration scenario of Figure 3.24. 
As it can be seen the regeneration starts near the exit of the inner filter block, where the maximum temperatures are also observed and is propagated only in the big outer blocks of the filter. According to the models prediction the small outer block is not regenerated.

Figure 3.43 summarized the $1 \mathrm{D}$ and $3 \mathrm{D}$ predictions and measurements at characteristic points. As reference point for the kinetics employed to the simulation was used the previous described low flow rate regeneration. However in order to match the experimentally observed behaviour in this case, it was necessary to significantly increase the frequency factors of the thermal regeneration. In this way, the $1 \mathrm{D}$ prediction comes close to the measured behaviour of the central channels.

Concerning the 3D prediction, again the center is close to the measured behaviour, however, the prediction for the small peripheric block fails completely. It should be noted that in contrast with the steady state regenerations this type of regeneration seems insensitive to the inlet gas temperature and seems more likely connected to the first regeneration occurring in the inner filter block. The role of soot VOF may be significant for such a scenario on the propagation of regeneration from the central to the outer filter blocks. Additional the initial temperature and soot distribution may largely influence the regeneration behavior. However for the time being the above phenomena are not taken in account by the model.

Obviously, significant further modeling improvements will be required in this direction, especially CFD modeling of flow maldistribution effects.

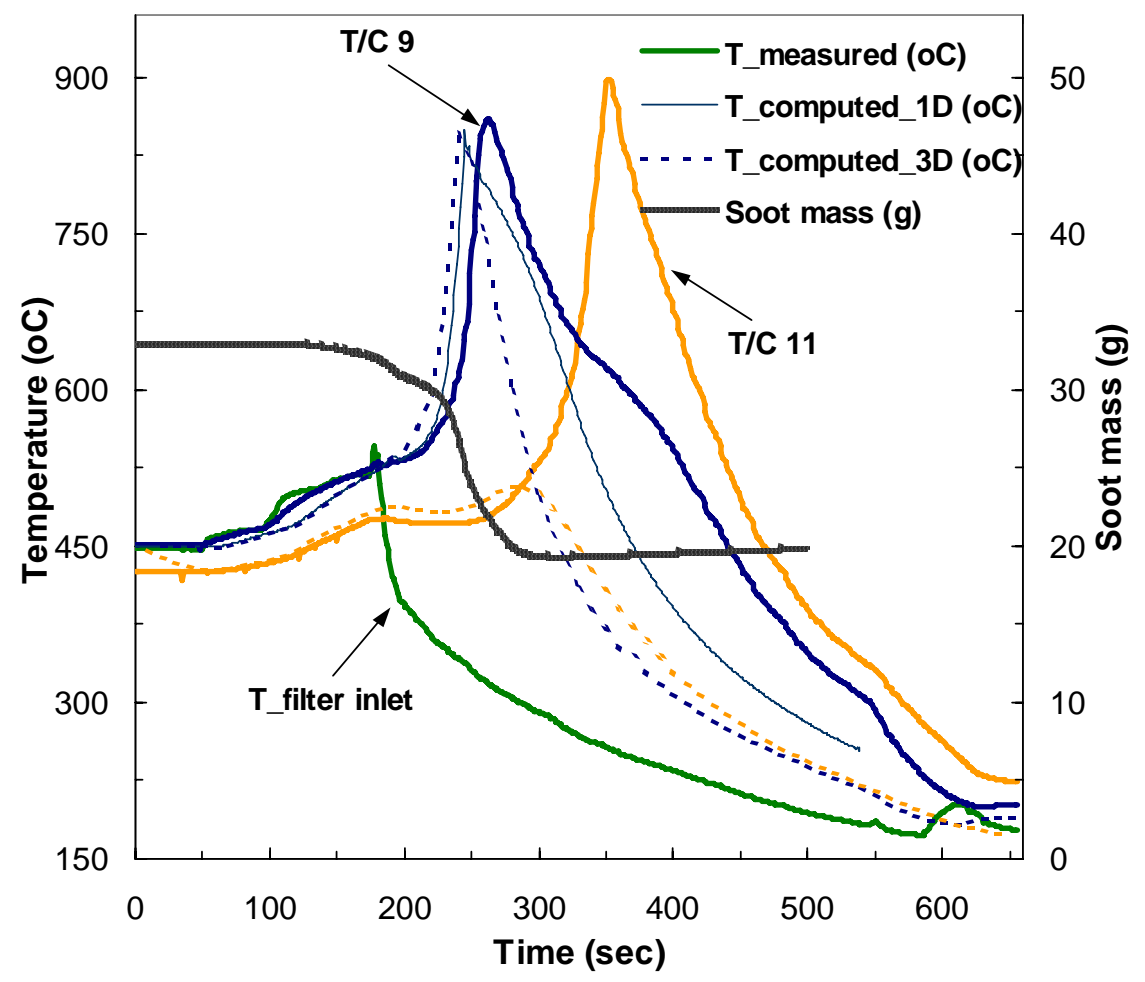

Figure 3.43 Computational simulation of the uncontrolled regeneration of figure 7 by the Catwall 1-D and the 3D Ansys-Catwall interfacing. Predictions of measured temperatures by thermocouples T/C9 (filter central area) and T/C11 (near filter periphery) are presented. The prediction of the oxidized soot mass is also presented. 1D and $3 \mathrm{D}$ computations are made with activation energy values: $\mathrm{E}_{1}=1.9 \mathrm{E} 5, \mathrm{E}_{2}=1.5 \mathrm{E} 5, \mathrm{E}_{3}=1.2 \mathrm{E} 5, \mathrm{E}_{4}=0.8 \mathrm{E} 5$, $E_{5}=0.8 \mathrm{E} 5 \mathrm{~J} / \mathrm{mole}$ and frequency factor values: $A_{1}=4 \mathrm{E} 14, \mathrm{~A}_{2}=5.5 \mathrm{E} 10, \mathrm{~A}_{3}=1 \mathrm{E} 11, \mathrm{~A}_{4}=1 \mathrm{E} 7, \mathrm{~A}_{5}=8 \mathrm{E} 8 \mathrm{~mole} / \mathrm{m}^{3}$,s.

\subsubsection{Concluding remarks}

- The experimental results obtained from three characteristic steady state regenerations with markedly different exhaust gas flow rates and one transient uncontrolled 
regeneration are employed together in a validation study of a 3D modeling approach of the catalytic regeneration developed in the Lab (interfacing with ANSYS commercial FEM software).

- The ability of the model to match the experimental behavior of the performed regeneration tests has been checked. The 3D model greatly extends the potential of its $1 \mathrm{D}$ counterpart towards the prediction of the full temperature and the three-dimensional propagation of the regeneration within the filter.

- The model's lack of a validated mechanism to account the effect of VOF is a marked weakness of the model especially in the study of the stochastic regeneration. Although the model predicts a delay to the evolution of regeneration between center and periphery due to the heat losses to the environment its ability is reduced at low flowrates due to the importance of the complex 3D effects, related to exhaust flow maldistribution and soot loading maldistribution across the filter face. Furthermore the uncertainties associated with the thermophysical properties of the filter materials and soot deposit greatly influences the performance of the model. More work is needed related to the above directions, that may probably further enhance the reliability and the accuracy of the model. However the stochastic nature of such phenomena will continue to be a limiting factor to the predictive ability of the model.

- Even such limitations the interaction between experimental findings and model predictions improves our understanding of the regeneration process and reveals the extents of 1D and 3D model applicability. 


\section{Experimental Part II: Pressure Drop, Flow and Soot Distribution}

Understanding of the mechanisms and phenomena affecting flow and soot distribution among the filter channels is an essential prerequisite for the design of reliable diesel exhaust aftertreatment systems based on ceramic filters. Additionally, the experimental determination of filter pressure drop parameters with respect to the specific design and operation characteristics of the system "engine + filter", is a prerequisite for the successful modeling of the loading and regeneration processes. In this chapter, the results of dedicated experimental investigation studies are discussed, and an attempt is made to determine ceramic wall and soot permeabilities, as well as soot density values in characteristics cases, as function of engine operation point during loading, with an emphasis on part-load operation. A novel device and methodology were developed in order to measure soot maldistribution in the different channels. The same device, supported by a simple computational model, was employed in the determination of the $(\rho k)_{\mathrm{p}}$ product (density times permeability) of the soot layer in specially constructed, single channel filters made of cordierite and SiC. The experimental study of flow maldistribution was carried out in two distinct phases: During the first phase, exhaust gas flow velocities at different channels (center and periphery) were measured with Pitot tubes under real engine operation conditions (loading and regeneration), aiming at the better understanding of the mechanisms affecting flow maldistribution, as function of engine and filter operating and design parameters. During a second phase, more detailed, cold flow velocity field measurements were carried out, with a specially designed test rig supplied with air by a roots blower with variable speed. Clean and loaded filters were measured in various layouts (with and without a catalytic converter between filter and inlet diffuser). The interaction between flow and soot maldistribution was also demonstrated by means of the specific test rigs. The experimental studies of this chapter are completed with an investigation of soot deposition morphology and characteristics, as well as soot density by means of SEM microscopy. A special procedure was developed for this task, with a sliced filter that was able to be dismantled after loading, in order to observe the soot layer in the SEM in different sections along the filter length.

\subsection{Experimental Investigation of the Pressure Drop Behavior of Porous Ceramic Filters.}

In this section the results of an experimental analysis of pressure drop as function of operation and geometric characteristics of $\mathrm{SiC}$ and Cordierite filters are presented. The product of soot permeability and density was experimentally determined by employing a special designed experimental apparatus. The same apparatus was used to the investigation of the soot loading distribution across the filter channels of a full-sized filter in various loading and partially regenerating conditions.

\subsubsection{Experimental}

\section{Single channel filter specimens}

Single channel filters were prepared by breaking up a full-sized one. As presented in Figure 4.1, a single channel filter consists of a central single inlet channel and the tangentially 
mounted four exit channels. The central channel is open in front and plugged backwards. On the contrary the four tangential channels are plugged in the front and open backwards.

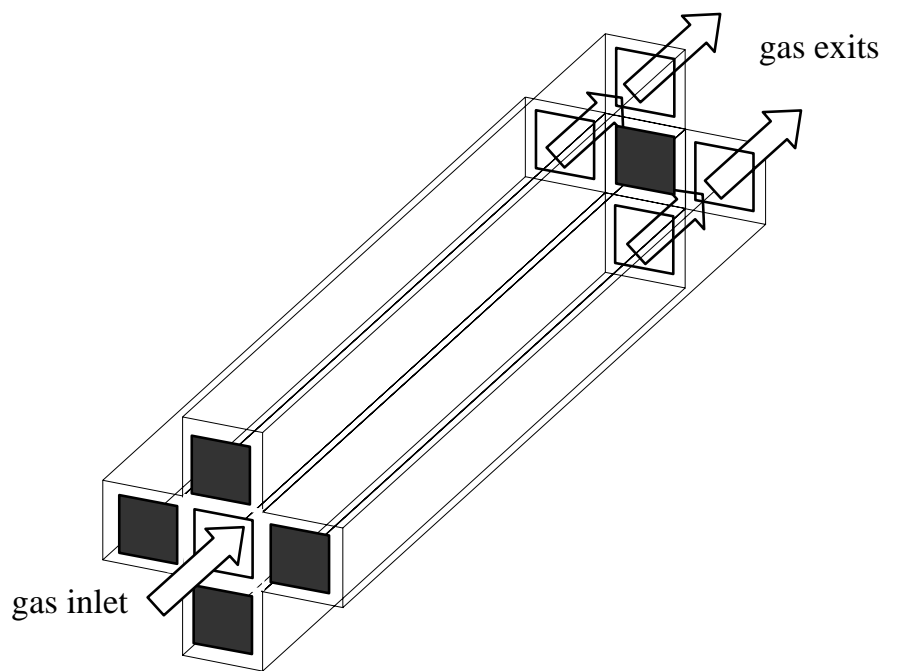

Figure 4.1 Principle of operation of a single channel filter

The single channel filters employed in this experimental study were prepared by breaking up two of the most frequently used filter types:

- a cordierite filter (17/100)

- a silicon carbide filter $(14 / 200)$

The properties of the two types of filter materials are presented in Table 4.1. The can at the inlet section of each single-channel filter is threaded and can be fitted to the engine exhaust pipe, as presented in Figure 4.2. In the specific experiments presented here, the mini-filters were mounted in parallel to the full-sized diesel filter into the exhaust system of a 2.0 litre HDI turbocharged passenger car engine, running on the engine test bed. The engine specifications are given in Table 4.2. Figure 4.3 presents the experimental layout of the filter loading experiment.

Table 4.1 Properties of single channel filters used for the experimental study.

\begin{tabular}{|l|l|l|}
\hline Property & Cordierite & SiC \\
\hline Material Density $\left(\mathrm{kg} / \mathrm{m}^{3}\right)$ & 2510 & 3080 \\
\hline Porosity $(\%)$ & 43 & 42 \\
\hline Cell Size $(\mathrm{mm})$ & $2.54 \times 2.54$ & $1.49 \times 1.49$ \\
\hline Wall thickness $(\mathrm{mm})$ & 0.6 & 0.4 \\
\hline Wall density $\left(\mathrm{kg} / \mathrm{m}^{3}\right)$ & 1000 & 1800 \\
\hline Mean pore size $(\mu \mathrm{m})$ & 33 & 9 \\
\hline Permeability $\left(\mathrm{m}^{2}\right)$ & $0.64 \mathrm{E}-12$ & $3.7 \mathrm{E}-13$ \\
\hline
\end{tabular}




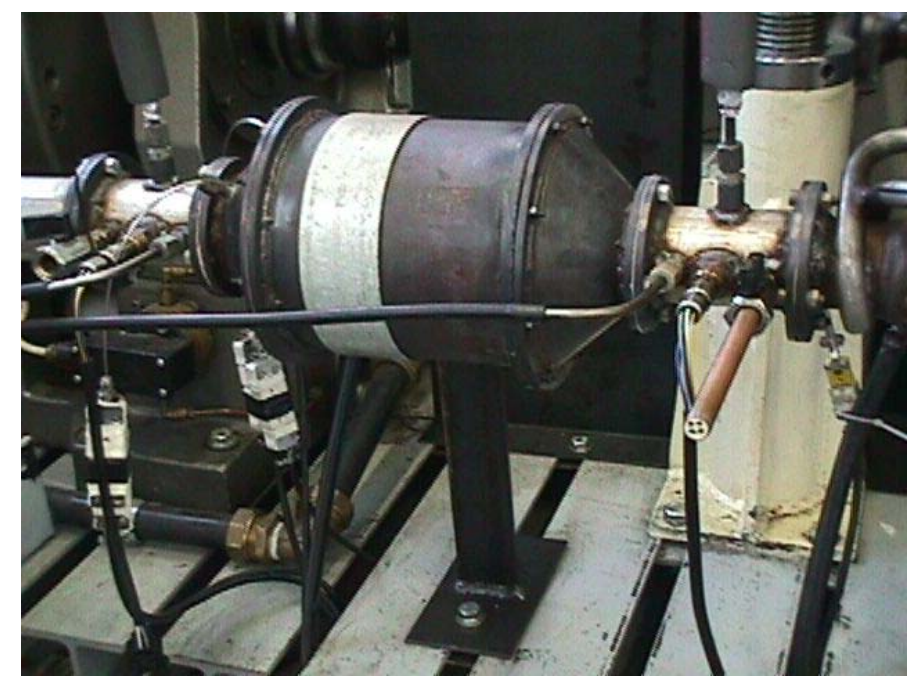

Figure 4.2 Mini scale filter assembly on engine exhaust system

Table 4.2 Diesel engine specifications

\begin{tabular}{|l|l|}
\hline Manufacturer & PSA \\
\hline Engine type & HDI turbocharged engine (DW 10 ATED) \\
\hline Cylinders & 4, in-line \\
\hline Displacement & $1997 \mathrm{~cm}^{3}$ \\
\hline Rated power /rated speed & $80 \mathrm{~kW} / 4000 \mathrm{rpm}$ \\
\hline Maximum torque/speed & $250 \mathrm{Nm} / 2000 \mathrm{rpm}$ \\
\hline Average fuel consumption & $5.5 \mathrm{l} / 100 \mathrm{~km}$ \\
\hline
\end{tabular}

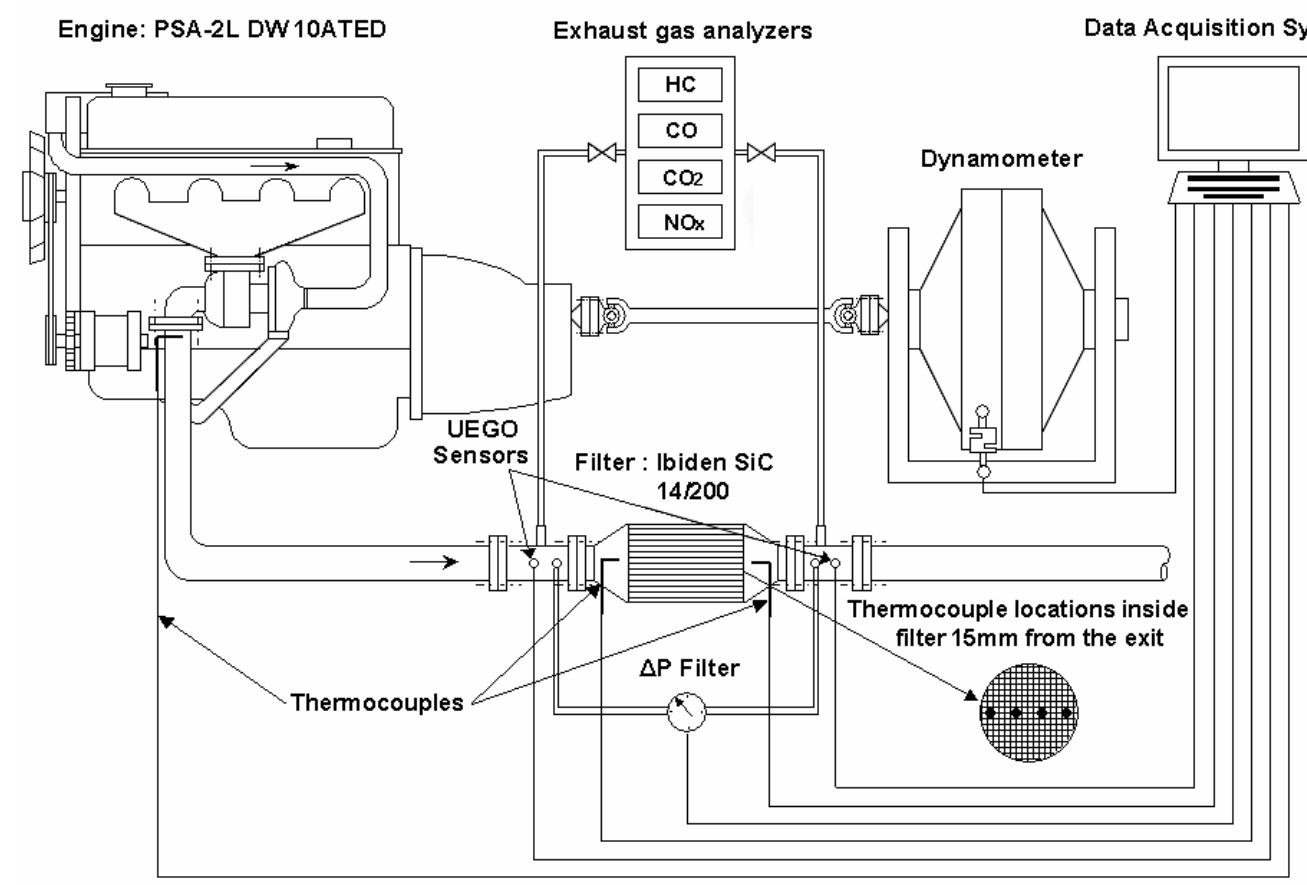

Figure 4.3 Experimental layout. Engine and digitally controlled dynamometer installation is shown along with exhaust gas analysers, main diesel filter measurement lines and data acquisition system. 
Each single channel filter, after being loaded with soot, maybe connected to a vessel, which contains air at 1 bar initial gauge pressure. The vessel's discharge pipe is controlled by a solenoid valve, and opens on demand by the data acquisition software, and triggers the expansion of the compressed air from the vessel through the channel walls to the atmosphere. Monitoring of the expansion process is carried out by means of recording the signal of a pressure sensor that is also mounted to the vessel. The layout of the measuring device is shown in more detail in Figure 4.4 and Figure 4.5a.

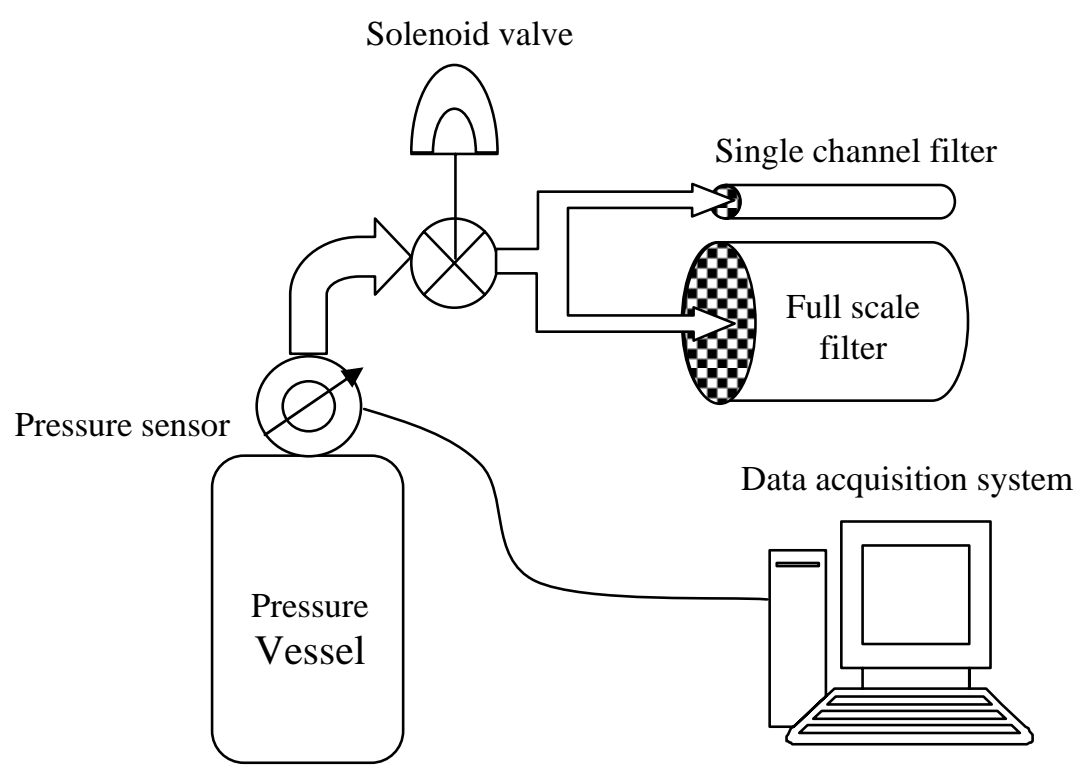

Figure 4.4 Schematic diagram of the measuring device

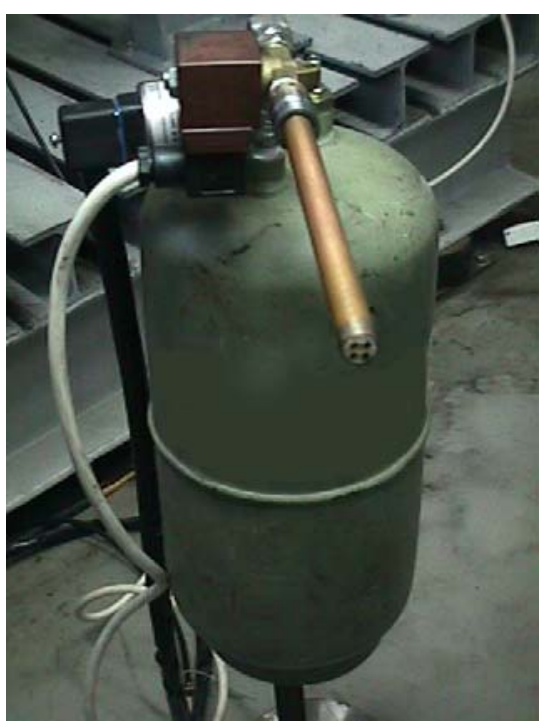

(a)

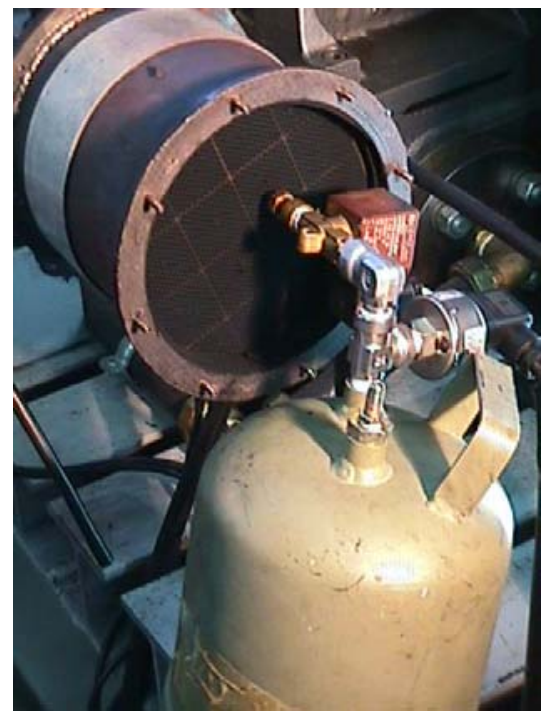

(b)

Figure 4.5 Use of measuring device for (a) single channel filter loading measurement, (b) full scale filter channel loading distribution measurement. 


\section{Experiments with a full-sized filter}

The full-sized filter employed in these experiments is a SiC14/200 filter with the characteristics shown in Table 4.3. In order to indirectly evaluate the soot loading distribution from filter centre to periphery, the design of the above device was improved in such a way that its nozzle being able to be tightly connected to selected inlet channels of the full-scale engine filter. The exhaust pipe of the vessel was carefully connected to the inlet of various trap channels and the pressure drop characteristic was recorded as shown in Figure 4.5b.

Table 4.3 Full scale diesel particulate filter specifications.

\begin{tabular}{|l|l|}
\hline Manufacturer & Ibiden \\
\hline Type & SiC $14 / 200$ \\
\hline Diameter (mm) & 143.8 \\
\hline Length (mm) & 152.4 \\
\hline Cell concentration (cells per square inch) & 200 \\
\hline Cell pitch (mm) & 1.89 \\
\hline Wall thickness (mm) & 0.4 \\
\hline
\end{tabular}

\section{Measurements' protocol}

The following strategy is applied in the determination of the exact protocol for filter loading tests:

Temperature and mass flowrate under full load and part load engine operating conditions, being more representative of city driving conditions are mapped in Figure 4.6 and Figure 4.7.

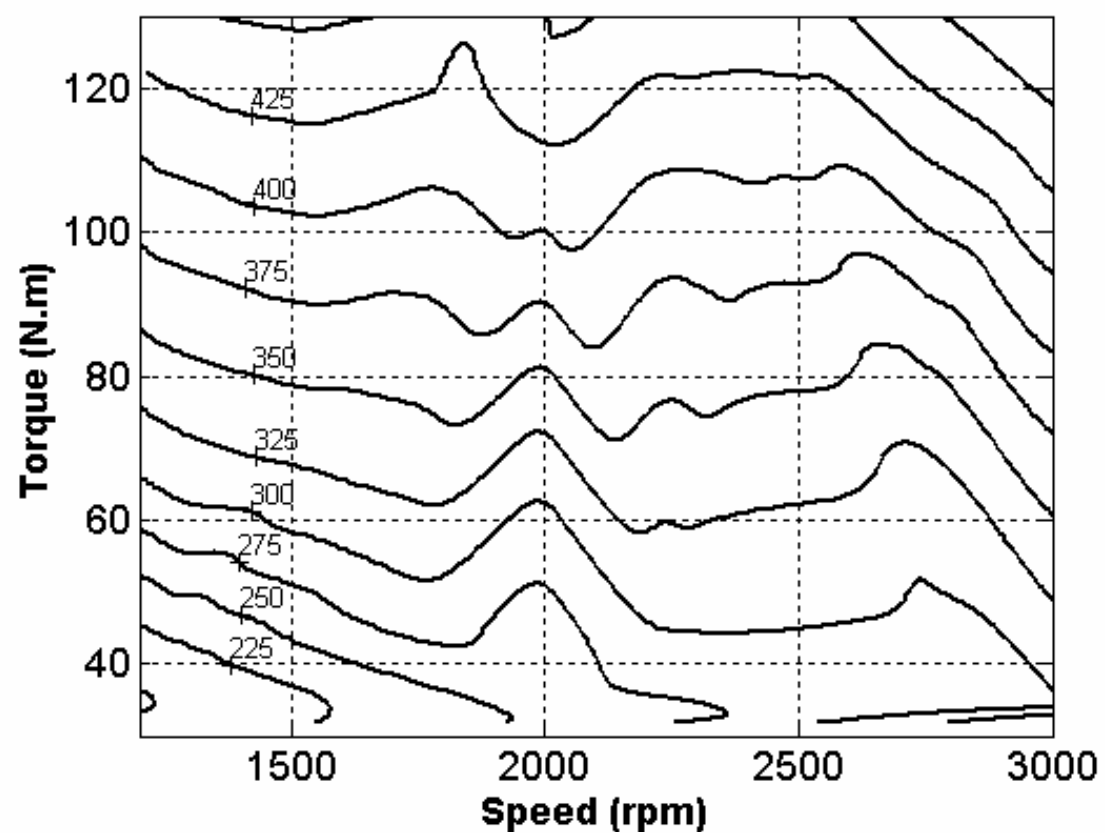

Figure 4.6 Map of filter wall temperature at the centreline, $15 \mathrm{~mm}$ from filter exit $\left[{ }^{\circ} \mathrm{C}\right]$. 


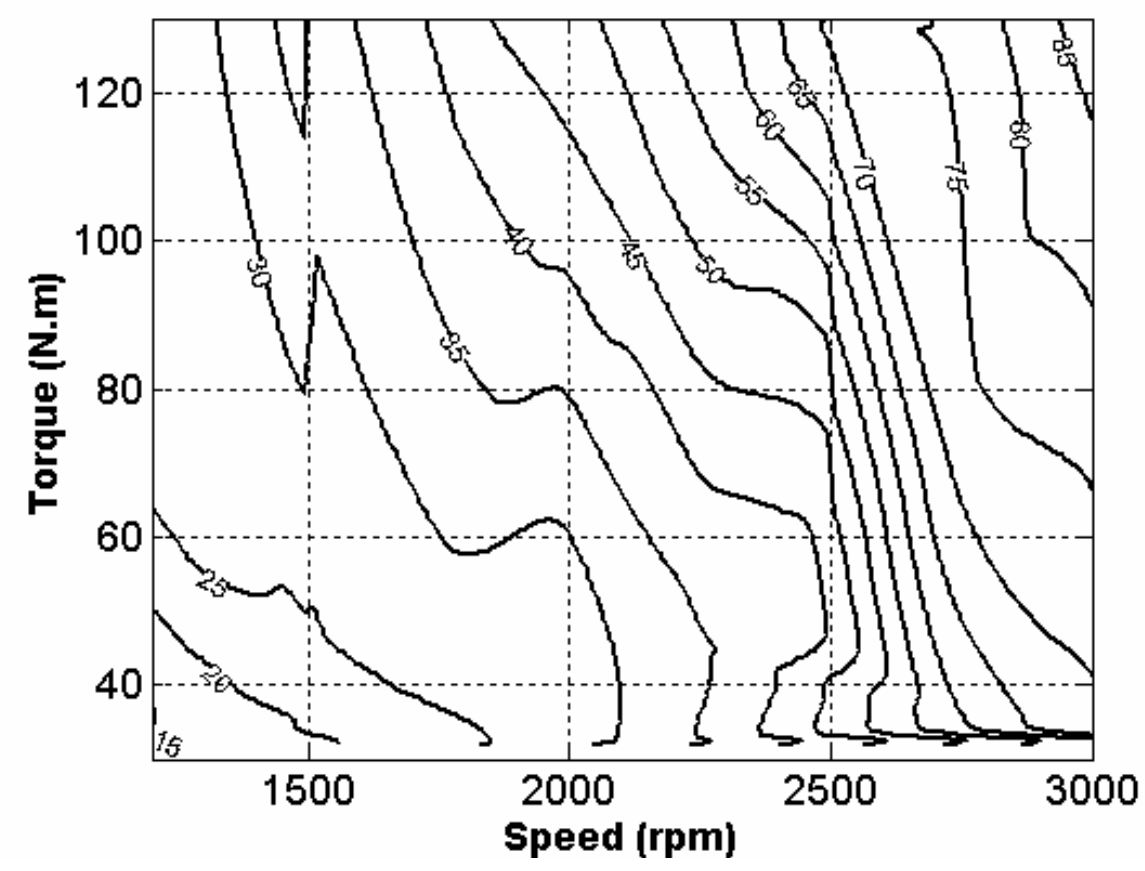

Figure 4.7 Map of engine exhaust gas mass flow rate [g/s].

The final design of the test protocol, based on the results of engine mapping, is presented in Table 4.4. The test protocol contains operation points lying at the lower left part of the engine map (low-to-medium speed by low-to-medium load) andat each operation point, both singlechannel and full-sized filters are loaded in parallel. Soot loading mass cannot be fixed in advance, due to the lack of knowledge of the function of mass versus $\Delta \mathrm{p}$.

Table 4.4 Experimental protocol

\begin{tabular}{|c|c|c|c|c|c|c|}
\hline $\begin{array}{l}\text { Speed } \\
\text { (rpm) }\end{array}$ & $\begin{array}{l}\text { Torque } \\
\text { (Nm) }\end{array}$ & $\begin{array}{c}\text { Mfr } \\
\text { (g/sec) }\end{array}$ & $\begin{array}{l}\text { T exhaust } \\
\left({ }^{\circ} \mathrm{C}\right)\end{array}$ & $\begin{array}{l}\text { T filter inlet } \\
\left({ }^{\circ} \mathrm{C}\right)\end{array}$ & $\begin{array}{l}\text { T filter wall } \\
\text { center }\left({ }^{\circ} \mathrm{C}\right)\end{array}$ & $\begin{array}{l}\text { Tfilter wall } \\
\text { side }\left({ }^{\circ} \mathrm{C}\right)\end{array}$ \\
\hline \multirow{4}{*}{1250} & 30 & 18 & 225 & 198 & 193 & 178 \\
\hline & 60 & 21 & 300 & 270 & 261 & 240 \\
\hline & 80 & 25 & 355 & 317 & 310 & 285 \\
\hline & 100 & 28 & 419 & 385 & 380 & 364 \\
\hline \multirow{4}{*}{1800} & 30 & 30 & 254 & 236 & 234 & 216 \\
\hline & 60 & 35 & 340 & 317 & 314 & 291 \\
\hline & 80 & 38 & 400 & 370 & 370 & 344 \\
\hline & 100 & 42 & 433 & 400 & 400 & 372 \\
\hline \multirow{4}{*}{2200} & 30 & 34 & 276 & 257 & 255 & 238 \\
\hline & 60 & 38 & 360 & 340 & 340 & 315 \\
\hline & 80 & 43 & 415 & 388 & 388 & 365 \\
\hline & 100 & 50 & 423 & 392 & 392 & 371 \\
\hline \multirow{4}{*}{3000} & 30 & 69 & 305 & 300 & 297 & 287 \\
\hline & 60 & 74 & 373 & 364 & 364 & 342 \\
\hline & 80 & 79 & 395 & 384 & 384 & 377 \\
\hline & 100 & 83 & 427 & 415 & 415 & 407 \\
\hline
\end{tabular}


After the completion of an approximate loading (order of 100 mbar independent of engine operation point), the expansion device can be fitted to both types of filter, in order to perform measurements to assess of soot loading. Especially for the case of the full-sized filter, the device is fitted to a number of characteristic channels' inlets (Figure 4.5b).

After the end of the recording of the single channel filter's expansion process, the singlechannel filter is weighed in order to get the amount of accumulated soot mass. Afterwards the filter is regenerated in order to be useable for the next experiment. Unfortunately, the weighing process is not practical with the full-sized filter, due to the large experimental error caused by the order-of-magnitude difference between filter mass ( $1 \mathrm{~kg}$ and more) and collected soot mass (10-30 g). Thus, in the case of full-sized filters, we do not have this possibility of accurately cross checking the collected soot mass.

\subsubsection{Computational assistance of experiments}

As explained above, aim of the single-channel filter experiments is the estimation of the product $(\rho \mathrm{k})_{\mathrm{p}}$ of soot collected in the filter channel. A calculation method, based on the simulation of the pressure drop curve obtained during the discharge process of vessel air through the filter channel was developed and employed in the estimation of $(\rho \mathrm{k})_{\mathrm{p}}$ product.

As first step the equation of Darcy is necessary in the calculation:

$$
\Delta p=\frac{\mu U E_{s}}{k_{s}}+\frac{\mu U m_{p}}{A_{f}(\rho k)_{p}}
$$

This formula allows in principle the calculation of $(\rho \mathrm{k})_{\mathrm{p}}$ product as function of the filter pressure drop, once the collected soot mass is known. Thus an accurate knowledge of the mass collected in the filter channel is necessary. To this end, the single-channel filter was weighed before and after each loading experiment, and the soot mass determined in this way was employed as input factor in the computer calculation.

As next step in order to correlate the filter pressure drop with the vessel pressure drop during the discharge process the equations used to describe the flow through the throat (or nozzle) connected to the filter are employed in the calculation. During discharging of the vessel air it is possible to have choked conditions of flow, or sonic velocity (Ma=1), in the throat. For that reason the computer calculation checks where there are choked or subcritical flow conditions and makes use of the corresponding formulas, at each calculation time step. In choked flow, the following formula is employed:

$$
\dot{m}_{\text {real }}=\frac{C_{D} A_{T} p_{0}}{\sqrt{R T_{0}}} \gamma^{1 / 2}\left(\frac{2}{\gamma+1}\right)^{(\gamma+1) / 2(\gamma-1)}
$$

on the other hand in subcritical flow condition, the following formula is employed:

$$
\dot{m}_{\text {real }}=\frac{C_{D} A_{T} p_{0}}{\sqrt{R T_{0}}}\left(\frac{p_{T}}{p_{0}}\right)^{1 / \gamma}\left\{\frac{2 \gamma}{\gamma-1}\left[1-\left(\frac{p_{T}}{p_{0}}\right)^{(\gamma-1) / \gamma}\right]\right\}^{1 / 2}
$$

The computer calculation continuously checks the pressure ratio and compares it to the critical pressure ratio $\frac{p_{T}}{p_{0}}$, which is taken equal to 0.528 (air at near-ambient pressure and temperature conditions can be assumed to behave like an ideal diatomic gas - $\gamma=1.4$ ). The discharge coefficient $C_{D}$ is a flow coefficient, which is mainly a function of the shape of the passage, and needs always to be experimentally determined. 
The vessel pressure at any time step is given by the equation of state:

$$
p_{0} V=m_{v} R T_{0}
$$

where $\mathrm{P}_{0}$ and $\mathrm{T}_{0}$ are the pressure and temperature inside the vessel or, in other words, the stagnation pressure and temperature. For the throat region, the following expression is valid:

$$
\dot{m}_{\text {real }}=\rho_{T} u_{T} A_{T}
$$

The filtration velocity $U$ is correlated with the throat velocity or the real mass flow $\dot{m}_{\text {real }}$ by means of the continuity equation:

$$
\begin{aligned}
\text { so, } & \dot{m}_{\text {real }}=\rho_{c h} U(4 L \ell) \\
\text { or, } & U=\frac{\dot{m}_{\text {real }}}{\rho_{c h}(4 L \ell)}
\end{aligned}
$$

The known input factors to the computer calculation are the initial vessel pressure, the vessel air temperature, the discharge coefficient, the dimensions of the single channel, the substrate thickness $\left(\mathrm{E}_{\mathrm{s}}\right)$ and permeability $\left(\mathrm{k}_{\mathrm{s}}\right)$, and the soot mass in the channel.

Two factors are unknown and must be tuned in this calculation process:

- the discharge coefficient $C_{D}$

- the second is the above-mentioned $(\rho \mathrm{k})_{\mathrm{p}}$ product.

These two factors govern the pressure drop characteristics of the experimental apparatus. The tuning process is based on the comparison of the experimentally measured and the calculated discharge characteristic of vessel pressure versus time.

The output of this calculation, which is written in Fortran 77, is a graph of vessel pressure as function of time, during the discharge process. The calculation can be done either for a single channel (made of Cordierite or SiC), clean or loaded with soot, or just for the discharge nozzle, without any filter channel connected, for validation purposes.

\subsubsection{Computer calculation procedure}

The computer calculation procedure consists of two discrete parts, as can be seen in the flowchart of Figure 4.8. In the first part, the real air mass outflow from the vessel up to the neck (converging nozzle of complex structure - see drawing in appendix) is calculated by the use of equation (4.2) for choked flow or (4.3) for subcritical flow. In the second part, the vessel pressure is calculated, by use of state equation (4.4). During the discharging process, two conditions of flow may prevail, choked or subcritical flow. The mass flowrate at each time step is computed according to the critical pressure ratio using the appropriate formula. From the second time step and on, the vessel pressure is iteratively determined, by means of the outgone mass for the ith time step (the $\dot{m}_{\text {real }}$ has already been calculated):

$$
d m_{(i)}=\dot{m}_{\text {real }(i)} \cdot d t
$$

and the remaining mass in the vessel:

$$
m_{v(i)}=m_{v(i-1)}-d m_{(i-1)}
$$

that updates the vessel pressure by means of the equation of state (4.4). Then the filtration (superficial) velocity $U$ is calculated by equation (4.8). The latter velocity is used in $(\Delta p)_{c}$ calculation with the aid of equation (4.1). 


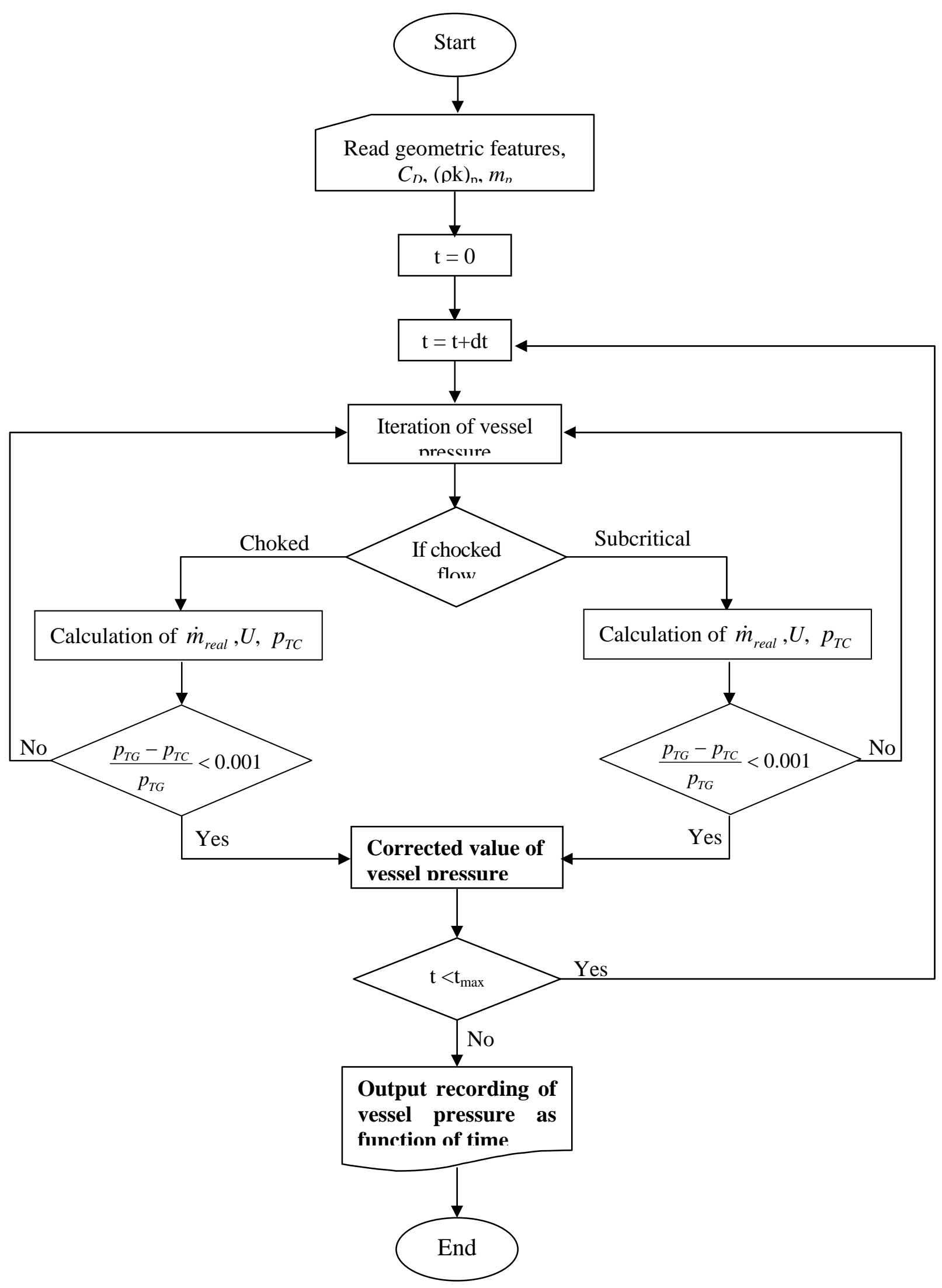

Figure 4.8 Computer calculation flowchart 
Also, because the filter is discharging to the free atmosphere, $\Delta \mathrm{p}$ can be assumed equal to the difference between pressure at throat and atmospheric pressure:

$$
\Delta p=p_{T}-p_{a t m}
$$

from equation (4.11)we get:

$$
p_{T c}=(\Delta p)_{c}+p_{a t m}
$$

Thereby two pressure values have been estimated for the throat region. This is a checkpoint for the code. The above values must satisfy the condition of equality in the same region (throat). If the difference between the two calculated pressures is small enough, or $\frac{p_{T G}-p_{T C}}{p_{T G}}<0.001$ then, the calculations are continued for the next time step. Otherwise the previously mentioned process is repeated with another guessed pressure value in the throat until the solution for this time step has been converged. Afterwards the code enters the next time step calculations and this procedure terminates when the vessel pressure has reached ambient pressure.

\subsubsection{Results and discussion}

\section{Single channel traps}

As already mentioned, the final objective of the combined experimental and computational method, is the determination of values for the product $(\rho \mathrm{k})_{\mathrm{p}}$. However, a validation of the computer calculation procedure is necessary. Thus, a set of validation tests was first performed. The validation procedure involved the determination of the discharge coefficient $\mathrm{C}_{\mathrm{D}}$ for both single-channel filter specimens (made from Cordierite and $\mathrm{SiC}$ respectively). Each validation test consisted of a particular experiment, in which each mini filter before being loaded (virgin filter) was connected to the vessel and the compressed air was discharged through the filter channel. Since the second term of equation (1) is equal to zero (no soot on the filter), we could tune the unknown value of discharge coefficient to match the experimental curve. The validation curves for both filter types (cordierite and SiC) are presented in Figure 4.9. It can be seen that the code behaves better with the SiC mini-filter, whereas in the cordierite case there is a small but observable deviation from the experimentally measured curve. Concerning the calculated values for $\mathrm{Cd}$ factor a higher statistical mean value was calculated in the case of SiC filter (0.96 vs 0.73 for Cordierite). The observed differences could be attributed to the lower hydraulic diameter of the SiC filter channel.

Afterwards, the single-filter specimens are loaded, with soot emitted by the engine operating on 25ppm DPX9- doped fuel at the previously described operation points (see Table 4.4). Subsequently, they can be connected to the measuring device and the experimental curve of the discharge process be recorded. The next step is to weigh the filter specimen, thus measuring the soot mass collected in the mini filter. The tuning process for the estimation of the $(\rho \mathrm{k})_{\mathrm{p}}$ is based on the fitting of the experimental discharge curve of the loaded mini-filter with the calculated curve with the computer calculation process. 


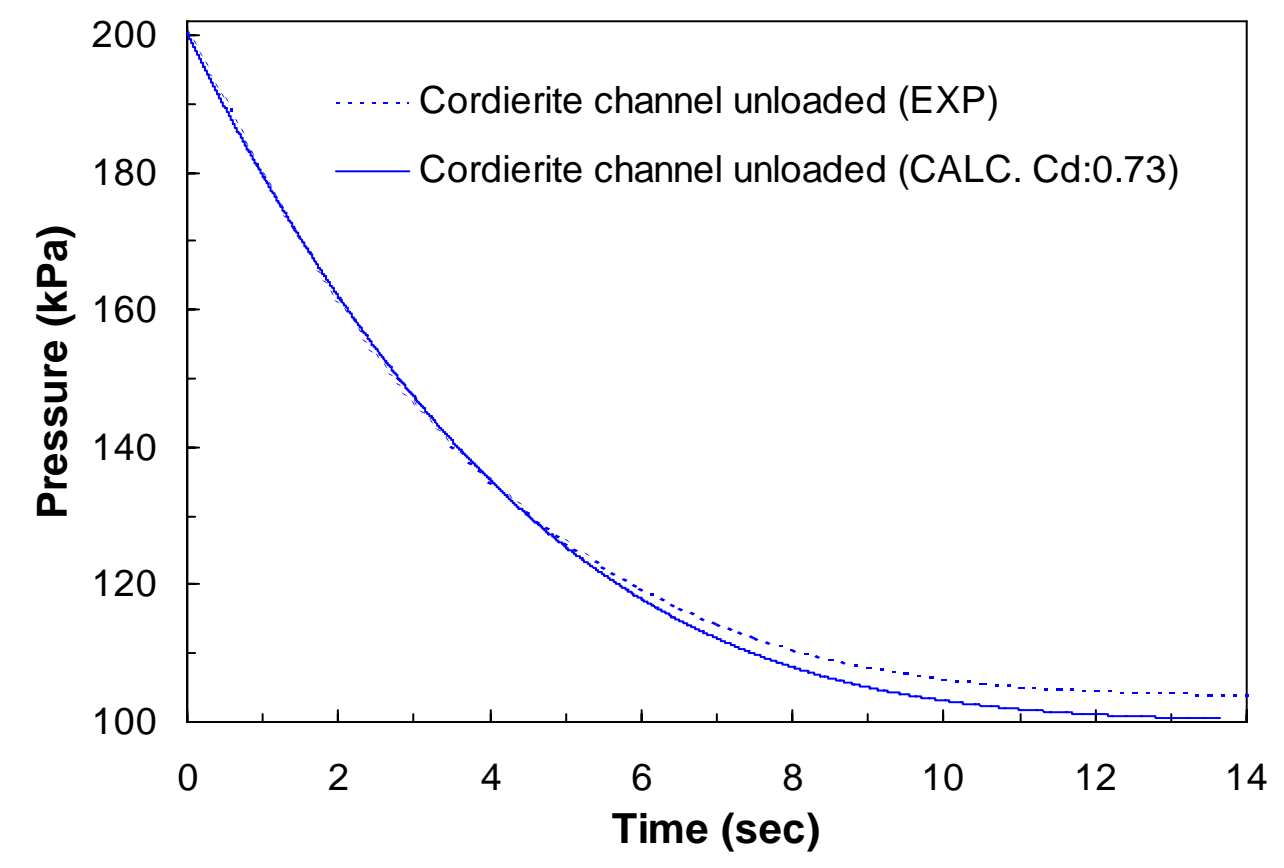

(a)

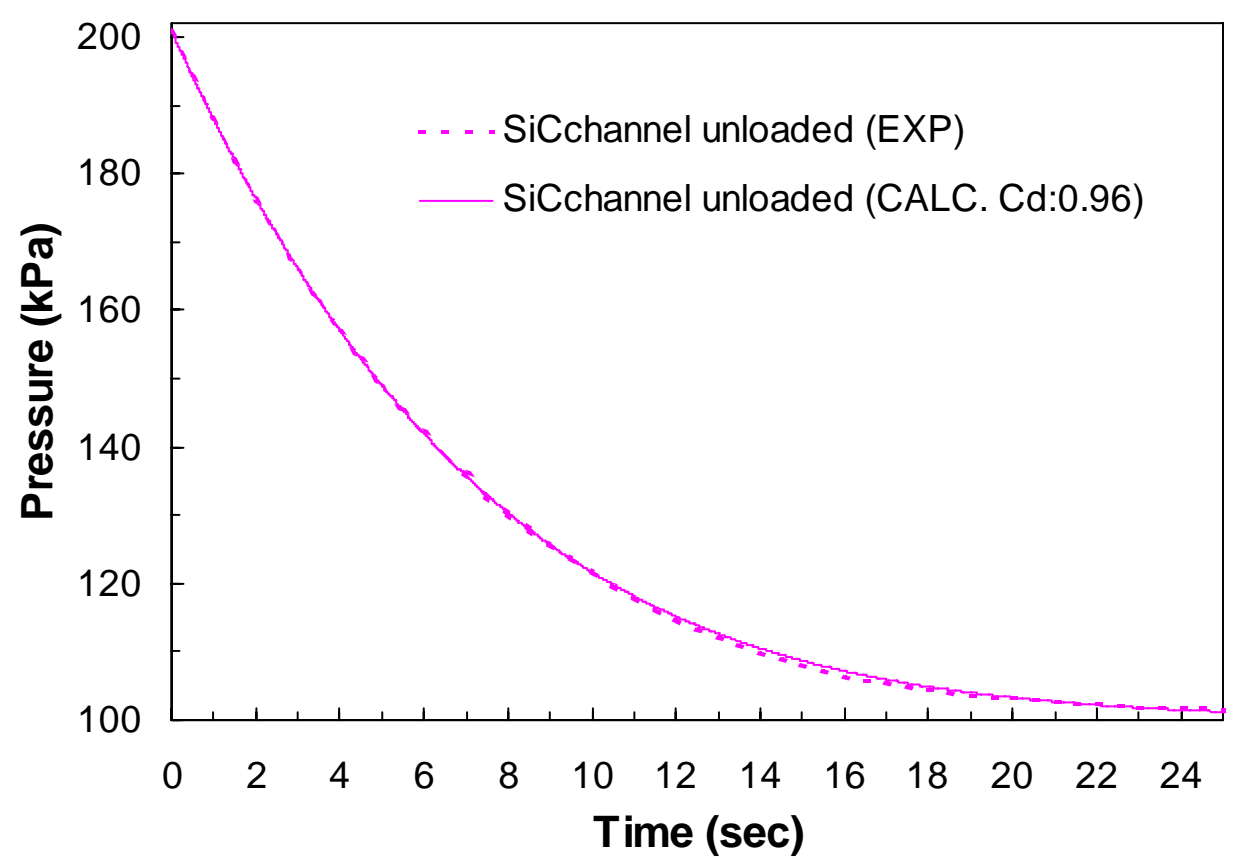

(b)

Figure 4.9 Code validation-Cd estimation curves for Cordierite (a) and SiC (b) single channel filters.

Two representative curves of vessel pressure- versus- time characteristics with a loaded Cordierite mini-filter that were employed in the tuning process are presented in Figure 4.10a. 


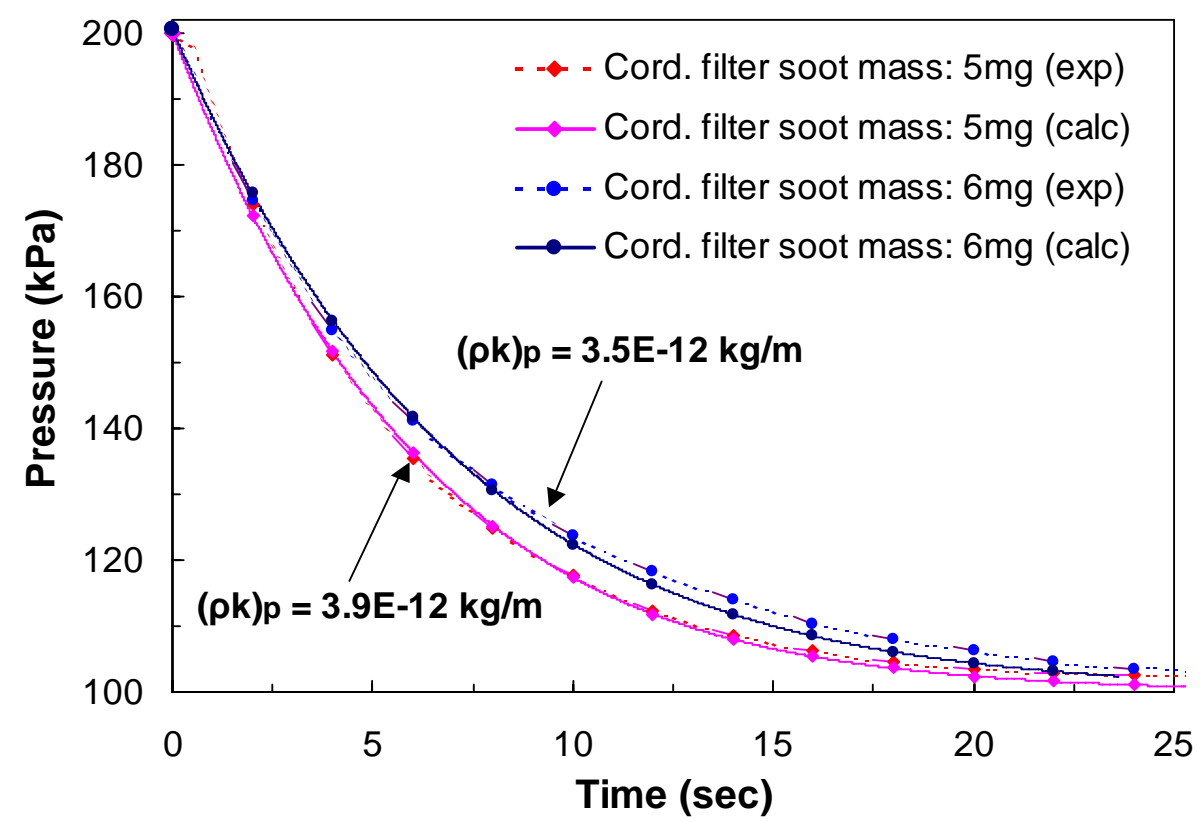

(a)

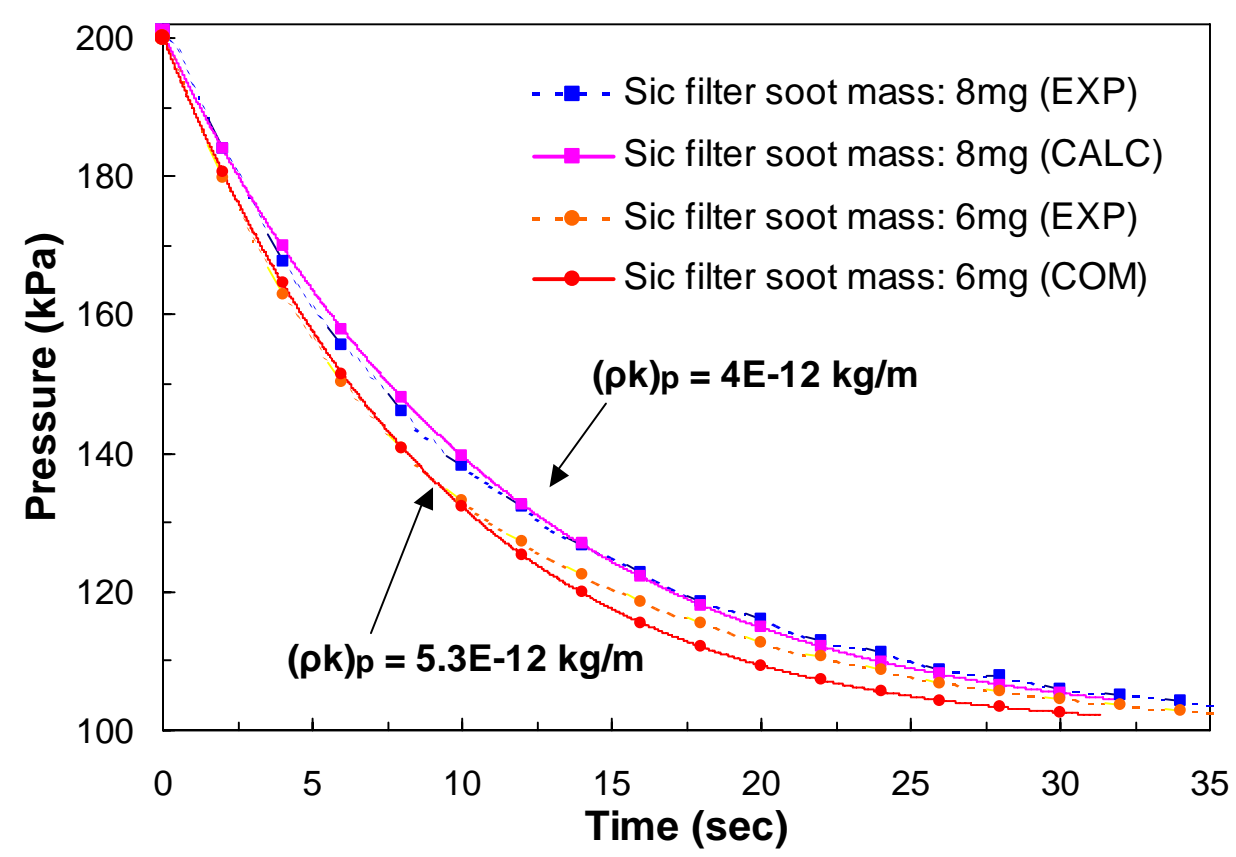

(b)

Figure 4.10 Representative curves of ( $\rho \mathrm{k}) \mathrm{p}$ parameter tuning in various filter loads and engine operational points. (a) cordierite single channel filter, (b) SiC single channel filter.

The tuning is implemented by comparison between the experimental and computational curve. The computation is seen to behave well with the simulation of the two experiments, resulting in different $(\rho \mathrm{k})_{\mathrm{p}}$ product values for the two different soot loading levels. For the Cordierite case it is observed that the greater soot mass retards the discharge process of the pressurized air, and the $(\rho \mathrm{k})_{\mathrm{p}}$ decreases as the mass of the accumulated soot increases. The results for a number of tuned values of $(\rho \mathrm{k})_{\mathrm{p}}$ as function of engine operation point are presented in Table 4.5 and Figure 4.11a. According to these results, the variation of $(\rho \mathrm{k})_{\mathrm{p}}$ with 
the engine operation point over the low and medium range of speed and load is measured in the range of $3.5 \mathrm{E}-12$ to $1.15 \mathrm{E}-11 \mathrm{~kg} / \mathrm{m}$.

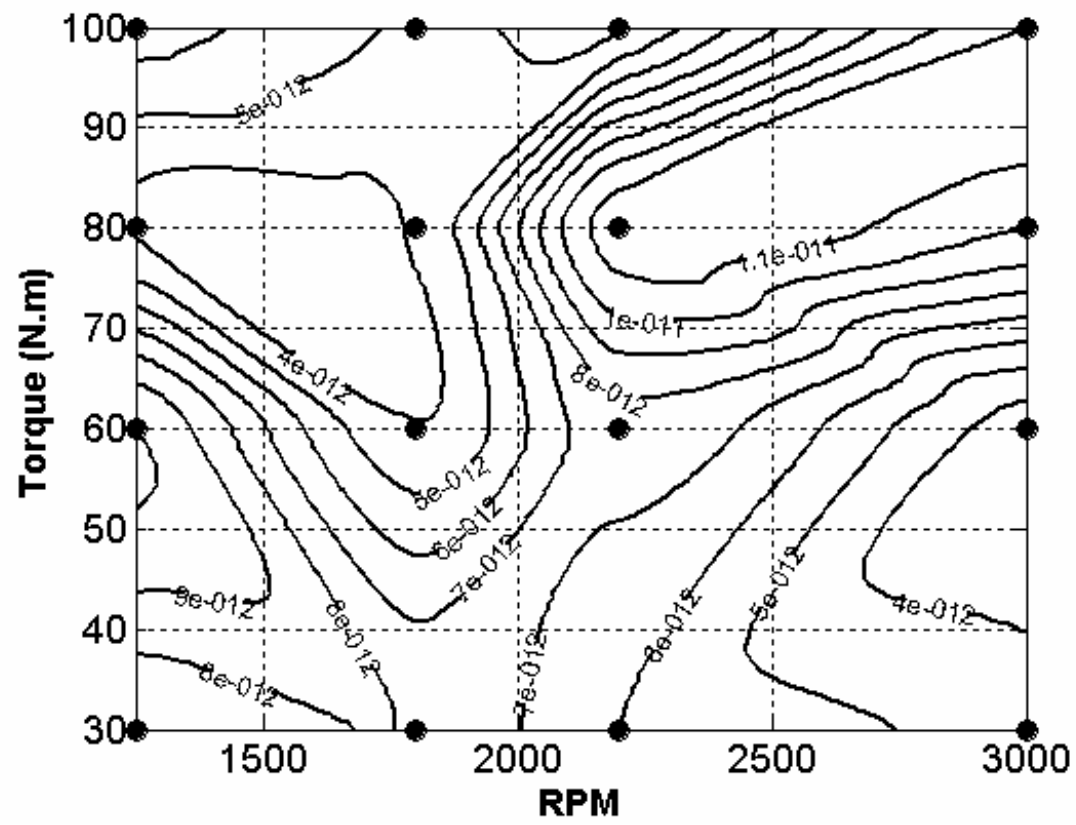

(a)

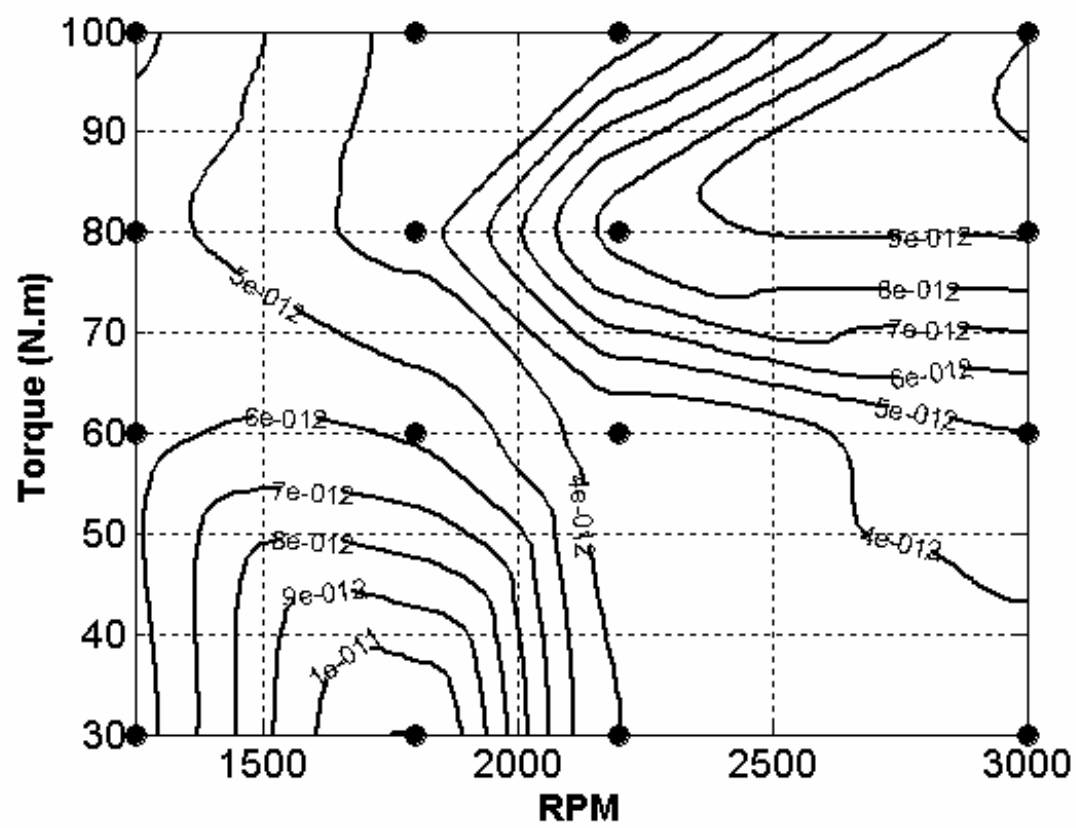

(b)

Figure 4.11 Map of ( $\rho \mathrm{k}) \mathrm{p}$ parameter as function of engine operation point. (a) cordierite single channel filter, (b) SiC single channel filter

In order to investigate the same effect with a different filter material, a new set of measurements was performed with the SiC single-channel filter. Two more coupled curves of experimental and calculated pressure discharge curves are presented in Figure 4.10b. Also in this case it is observed that for higher soot mass the air discharges slower and the $(\rho \mathrm{k})_{\mathrm{p}}$ value decreases, and the values determined lie in the same range as with the cordierite samples, as would be expected. The values of the determined $(\rho \mathrm{k})_{\mathrm{p}}$ product as function of engine 
operation point are presented in Figure 4.11b. The results for the two types of filter materials are summarized and presented in Table 4.5. Here it should be mentioned that a similar research carried out with an IDI engine by Sorenson et al as reported in [111], resulted in lower $(\rho \mathrm{k})_{\mathrm{p}}$ values. This could be attributed to the significantly higher VOF content of the exhaust gas of indirect injection diesel engines.

\begin{tabular}{|c|c|c|c|c|c|}
\hline \multicolumn{2}{|c|}{ Operation Point } & \multicolumn{2}{c|}{ Cordierite Single Channel } & \multicolumn{2}{c|}{ Sic Single Channel } \\
\hline Speed (rpm) & Torque (Nm) & $\begin{array}{c}\text { Soot mass } \\
\text { collected }(\mathrm{mg})\end{array}$ & $(\rho \mathrm{k}) \mathrm{p}(\mathrm{kg} / \mathrm{m})$ & $\begin{array}{c}\text { Soot mass } \\
\text { collected }(\mathrm{mg})\end{array}$ & $(\rho \mathrm{k}) \mathrm{p}(\mathrm{kg} / \mathrm{m})$ \\
\hline 1250 & 30 & 10 & $7.00 \mathrm{E}-12$ & 4 & $5.50 \mathrm{E}-12$ \\
\hline 1250 & 60 & 12 & $1.00 \mathrm{E}-11$ & 8 & $5.60 \mathrm{E}-12$ \\
\hline 1250 & 80 & 5 & $3.90 \mathrm{E}-12$ & 6 & $5.30 \mathrm{E}-12$ \\
\hline 1250 & 100 & 7 & $6.40 \mathrm{E}-12$ & 7 & $6.20 \mathrm{E}-12$ \\
\hline 1800 & 30 & 9 & $7.95 \mathrm{E}-12$ & 8 & $1.10 \mathrm{E}-11$ \\
\hline 1800 & 60 & 5 & $4.10 \mathrm{E}-12$ & 8 & $5.85 \mathrm{E}-12$ \\
\hline 1800 & 80 & 4 & $4.10 \mathrm{E}-12$ & 6 & $3.80 \mathrm{E}-12$ \\
\hline 1800 & 100 & 4 & $4.80 \mathrm{E}-12$ & 6 & $3.70 \mathrm{E}-12$ \\
\hline 2200 & 30 & 10 & $6.00 \mathrm{E}-12$ & 8 & $4.00 \mathrm{E}-12$ \\
\hline 2200 & 60 & 9 & $7.60 \mathrm{E}-12$ & 7 & $3.50 \mathrm{E}-12$ \\
\hline 2200 & 80 & 12 & $1.15 \mathrm{E}-11$ & 8 & $8.30 \mathrm{E}-12$ \\
\hline 2200 & 100 & 3 & $3.90 \mathrm{E}-12$ & 4 & $3.40 \mathrm{E}-12$ \\
\hline 3000 & 30 & 5 & $4.70 \mathrm{E}-12$ & 7 & $3.50 \mathrm{E}-12$ \\
\hline 3000 & 60 & 6 & $3.50 \mathrm{E}-12$ & 5 & $5.00 \mathrm{E}-12$ \\
\hline 3000 & 80 & 7 & $1.00 \mathrm{E}-11$ & 6 & $9.10 \mathrm{E}-12$ \\
\hline 3000 & 100 & 8 & $1.10 \mathrm{E}-11$ & 5 & $9.90 \mathrm{E}-12$ \\
\hline
\end{tabular}

Table 4.5 Summarised results for $(\rho \mathrm{k})_{\mathrm{p}}$ parameter for various single channel filter loads and engine operation points.

A more detailed analysis of the above- mentioned experimental results shows that $(\rho \mathrm{k})_{\mathrm{p}}$ product does not depend only on the accumulated soot mass, but it is also a function of engine operation point. That is, the pressure discharge versus time curve, which is employed in the indirect determination of the soot loading of each filter channel, is affected at least by the collected soot mass, the engine speed and torque, and the presence of a fuel additive component. Furthermore the role of adsorbed hydrocarbons on the soot particulate and the ceramic substrate must be considered [86] and research in this subject is underway in LTTE/UTh.

As a general conclusion for both types of filter material, it can be observed that the higher soot mass is associated with a decrease of the $(\rho \mathrm{k})_{\mathrm{p}}$ parameter value. This is apparent on Figure 4.12 at which the $(\rho \mathrm{k})_{\mathrm{p}}$ parameter is determined for various filter loading experiments at the same engine operation point. This could have the meaning that the decrease in soot permeability prevails over the increase in soot density during the assumed compression of the particle layer at higher soot loading levels. 


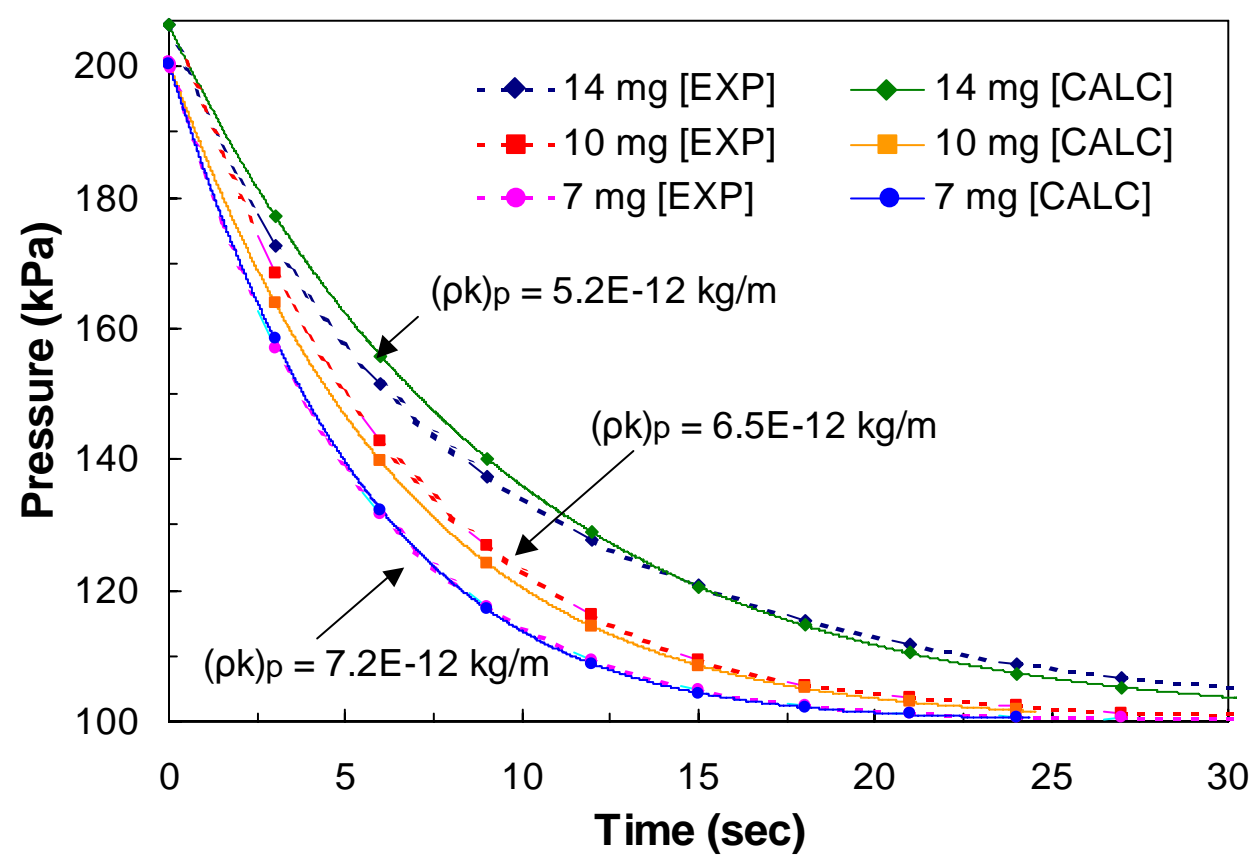

Figure 4.12 $(\rho \mathrm{k})_{\mathrm{p}}$ value calculation for various filter loading levels with engine running on the same operation point (3000 rpm - $40 \mathrm{Nm}$ ).

\section{Full-scale filters}

As reported above, experiments with full-scale filters were also performed in order to investigate the exhaust flow characteristics and soot mass distribution along the filter channels during the loading and regeneration procedure. Figure 4.13 presents the vessel pressure discharge curves obtained at selected channels equi-distantly positioned along a filter diameter, after loading at the following two characteristics points:

\begin{tabular}{|l|l|l|l|l|}
\hline Speed & load & $\begin{array}{l}\text { Final } \\
\text { backpressure }\end{array}$ & $\begin{array}{l}\text { Wall } \\
\text { temperature- } \\
\text { center }\end{array}$ & $\begin{array}{l}\text { Wall } \\
\text { temperature - } \\
\text { periphery }\end{array}$ \\
\hline $2000 \mathrm{rpm}$ & $90 \mathrm{Nm}$ & $150 \mathrm{mbar}$ & $390^{\circ} \mathrm{C}$ & $370^{\circ} \mathrm{C}$ \\
\hline $2000 \mathrm{rpm}$ & $90 \mathrm{Nm}$ & $450 \mathrm{mbar}$ & $410^{\circ} \mathrm{C}$ & $390^{\circ} \mathrm{C}$ \\
\hline
\end{tabular}

The coincidence of the curves in the case of filter loading to 150mbar backpressure, indicates a uniform distribution of soot mass (and possibly exhaust gas flow). This is not observed in the case of 450 mbar filter backpressure. As it can be seen, at least two different zones are observed as the measuring device is removed from the central (hotter during operation) to the outer (colder during operation) channels, since the pressurised air is shown to discharge faster through the central channels than through the circumferential. This could be attributed to the effect of VOF present in the thick particulate layer, which should preferentially condense on the colder outer channels after engine stop, thus differentiating permeability characteristics between filter core and periphery channels, that would be expected to be comparable during hot operation. 


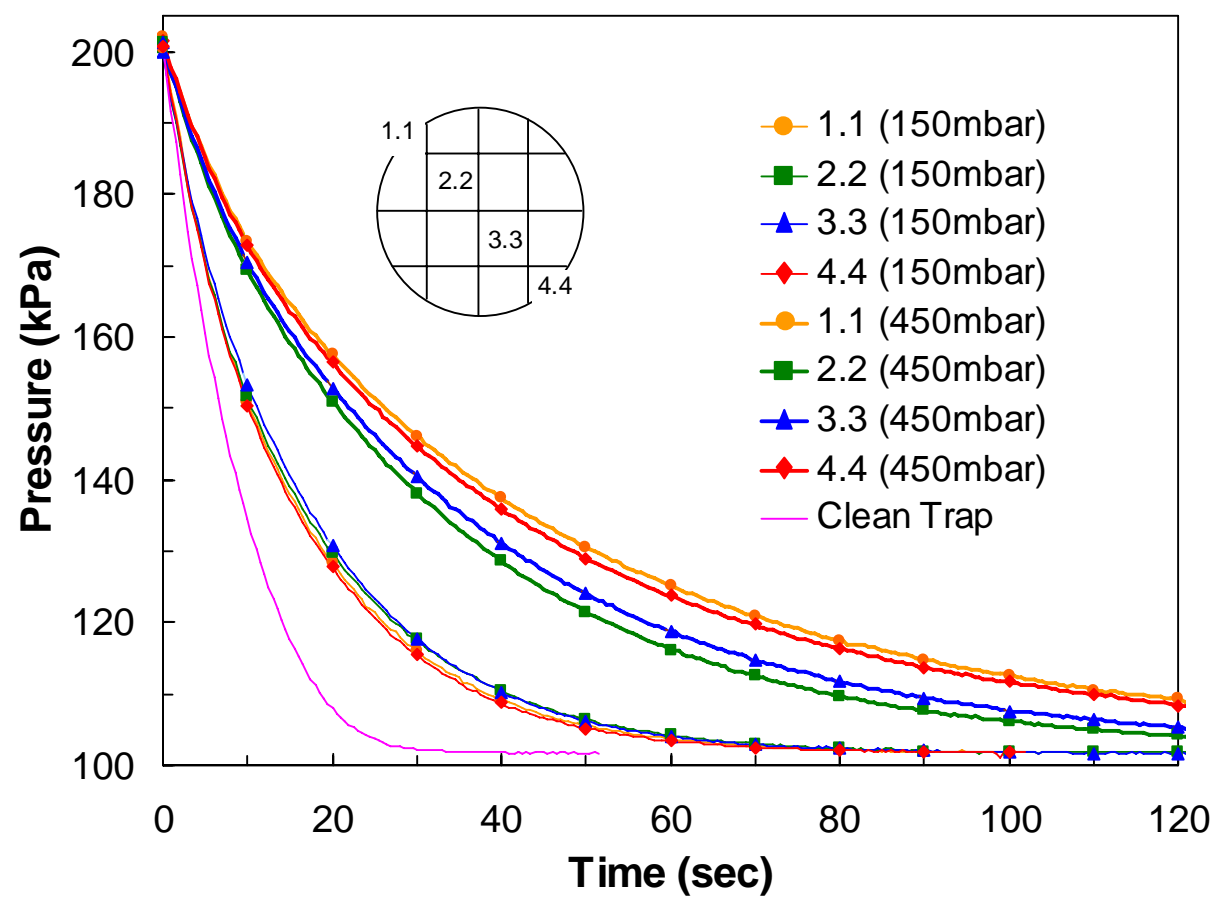

Figure 4.13 Pressure drop measurements along a diameter for a full-sized filter loaded up to 150mbar and up to 450mbar pressure drop (measured at $2000 \mathrm{rpm}-90 \mathrm{Nm}$ ).

The role of the wall temperature gradient during an incomplete regeneration to the soot mass distribution is further investigated with the experimental results of Figure 4.14 The resultant curves refer to the determination of the product $(\rho \mathrm{k})_{\mathrm{p}}$ in central and peripheric channels, after filter regeneration at engine speed of $2800 \mathrm{rpm}$ and $180 \mathrm{Nm}$ load, which results in filter wall temperature varying from $610^{\circ} \mathrm{C}$ (filter centre) to $580^{\circ} \mathrm{C}$ (filter periphery). In order to exclude any secondary effects from fuel additive residuals, the filter was loaded without a fuel additive at a speed of 2000rpm, and a load of $90 \mathrm{Nm}$ up to the backpressure of 150 mbar. The variation of the vessel discharge curves moving from the centre to the periphery indicates incomplete regeneration at the filter periphery. This is due to the lower wall temperature measured (thermal regeneration!). This behaviour during a partial regeneration would result, during a subsequent loading phase, to a significant differentiation of the exhaust flow distribution, with the exhaust flowing mainly through the central region of the filter. This mechanism could also explain the different loading behaviour between a new and a previously incompletely regenerated filter.

Finally the role of fuel additive was also investigated. After prolonged filter operation with the engine having consumed about 1000 lt of diesel fuel doped with 25 ppm Ce additive running in loading and regeneration modes at various operation points, a new set of measurements along a filter diameter was performed. At this case the filter was completely regenerated. The results of Figure 4.15 indicate that the pressurised air discharges faster through the circumferential channels than through the central. This means that the fuel additive ash is concentrated mainly on the central region of the filter. Again, such an observation can be explained by the previously proposed mechanism, because the central filter region tends to regenerate more completely than the outer region, thus attracting a higher total exhaust flow during a large period of time. 


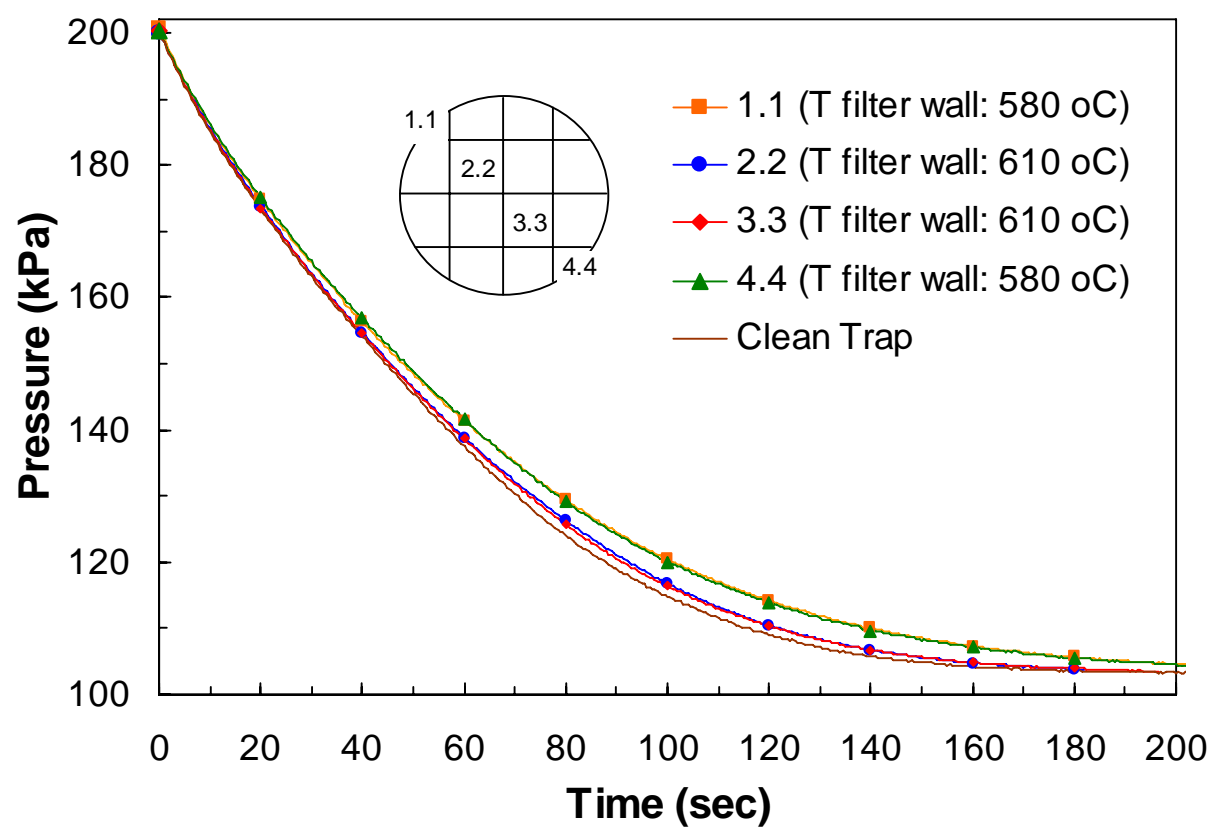

Figure 4.14 Pressure drop measurements along a diameter after the regeneration of a filter loaded until 150mbar at $2000 \mathrm{rpm} 90 \mathrm{Nm}$ with $0 \mathrm{ppm}$ fuel additive.

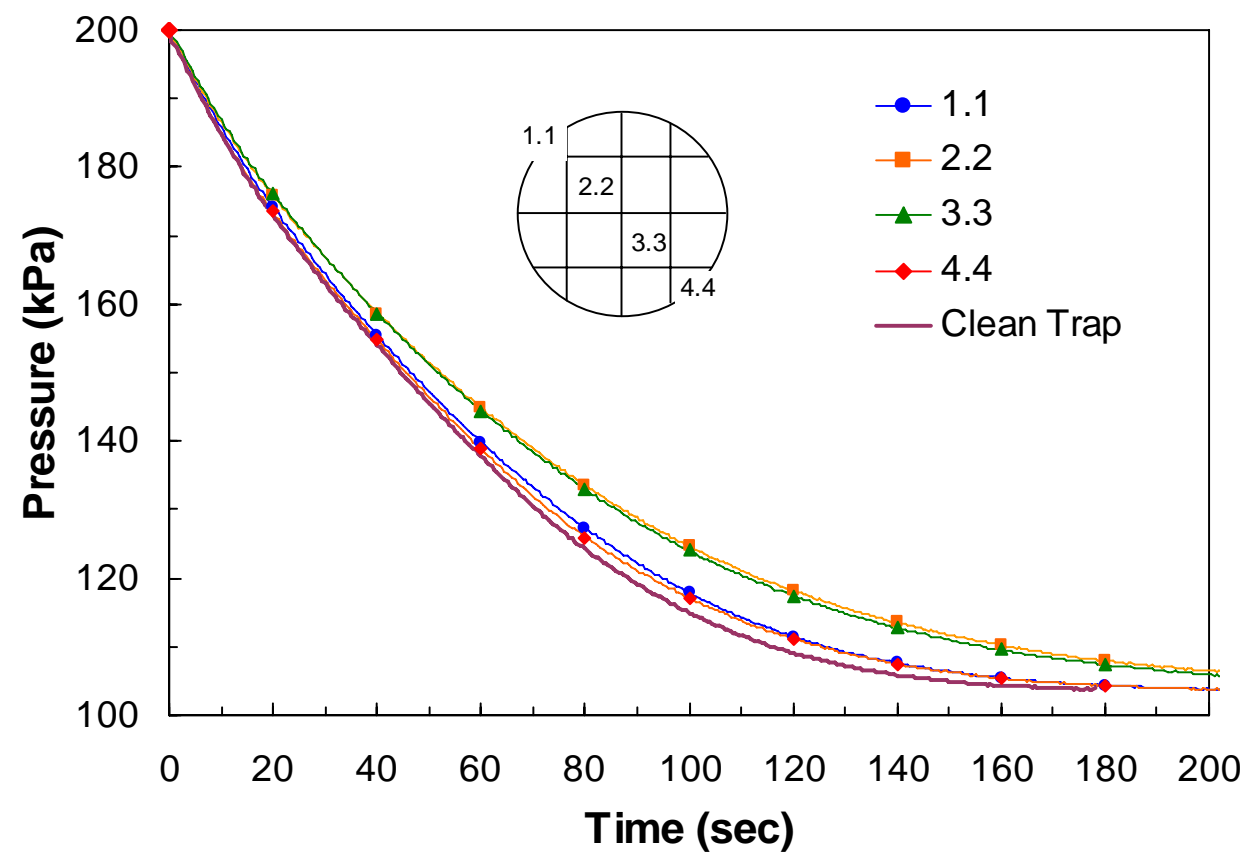

Figure 4.15 Pressure drop measurements for a complete regenerated filter after engine consumed about 1000lt diesel fuel doped with 25ppm additive (ash effect)

\subsubsection{Concluding remarks}

- A simple measuring device and a computer calculation procedure have been developed to assist the experimental study of soot loading distribution in wall flow diesel particulate filters.

- The measuring device records vessel pressure versus time as the pressurized air from a vessel discharges through selected channels of a particulate filter or through singlechannel specimens of Cordierite and SiC. 
- The pressure recordings taken by use of this simple and easily implemented device and methodology, are employed in the estimation of the product $(\rho \mathrm{k})_{\mathrm{p}}$ as demonstrated in this section.

- The results indicate that the product $(\rho \mathrm{k})_{\mathrm{p}}$, (soot layer density times permeability), for the specific engine and filters- combination, lies in the range of $3.5 \mathrm{E}-12$ to $1.15 \mathrm{E}-11 \mathrm{~kg} / \mathrm{m}$, both for a cordierite and a SiC single-channel filter specimen, fitted to the specific engine.

- Study of the soot layer density times permeability variation indicates a significant influence by engine operating point and also soot loading.

- The above simple $(\rho \mathrm{k})_{\mathrm{p}}$ estimation methodology may be extended to give also an indicative picture of the distribution of soot and fuel additive ash loading among the channels of a full-sized filter, as shown in selected cases.

- Further development of the above methodology is expected to improve our confidence to the accuracy of estimation of soot and additive ash loading in full sized filters.

\subsection{Flow Distribution Effects During Loading and Catalytic Regeneration (Pitot Tubes Experiments)}

In this section, the role of flow maldistribution is investigated. Loading and regeneration tests were performed at carefully selected engine operation points with markedly different levels of engine exhaust gas mass flowrate. The results indicate that there exist complex flow maldistribution phenomena that vary during the loading phase interacting with the soot maldistribution and VOF adsorption-desorption phenomena, which are not directly reflected to the behaviour of the pressure drop curve versus time. This work aims to present the importance of flow maldistribution in diesel filters and set the scene for the improvements should be done in the modelling of pressure drop and regeneration characteristics.

\subsubsection{Experimental}

The measurements were performed on a SiC filter fitted to the exhaust system of the 2.0 liter HDI turbocharged engine, running on the test bench, on carefully selected steady state operation points. The engine and diesel filter specifications are presented in Table 4.2 and Table 4.3. Figure 4.3 presents the experimental layout of the filter loading and regeneration experiments. Temperatures were measured simultaneously at the exhaust pipe $50 \mathrm{~mm}$ after turbine exit, at the inlet of the filter, and inside the filter, along a filter diameter $1.5 \mathrm{~cm}$ deep from the end.

The existence of flow maldistribution is investigated in this section by means of flow velocity measurements downstream filter (no catalyst in front), during:

- The loading phase

- The regeneration phase

The experimental determination of flow velocity is done with the use of Pitot tubes as the most appropriate method to meet the high temperature and corrosive conditions inside the 
exhaust system during the engine operation. The measurements are made at the filter exit for the following reasons:

- Pitot tubes could distort the velocity field upstream filter

- Pitot tubes would be quickly clogged with soot emitted by the engine (subsequently collected by the filter)

- Flow maldistribution downstream filter accounts also for the effects of in-channel flow variation that could not be measured with simple equipment.

Figure 4.16 presents the experimental layout of the exhaust gas velocity measurements. Two Pitot tubes were placed, the first one at the center and the second at the periphery of the filter. The distance between the measurement point and the channel exit was about twenty cell diameters as the theory demands for a complete development of the flow profile at the exit of the filter. The monitoring of the pitot tubes is carried out by means of recording the signal of a differential pressure sensor generated by the difference between the total or Pitot pressure and the static pressure. Assuming that the exhaust gas stream behaves as a steady-state onedimensional flow of an incompressible frictionless fluid, we may use the general relationship between the velocity and the pressure caused by the gas moved over the Pitot tube (total pressure minus static pressure) [112]:

$$
V_{g}=C \sqrt{\frac{2 \Delta p_{\text {pitot }}}{\rho_{g}}}
$$

Where $C$ is a Pitot tube constant (calculated during the calibration process presented in the Appendix as 0.97 for the first and 0.99 for the second pitot tube) and $\rho_{g}$ the exhaust gas density determined by measuring the temperature at the Pitot tube inlet.

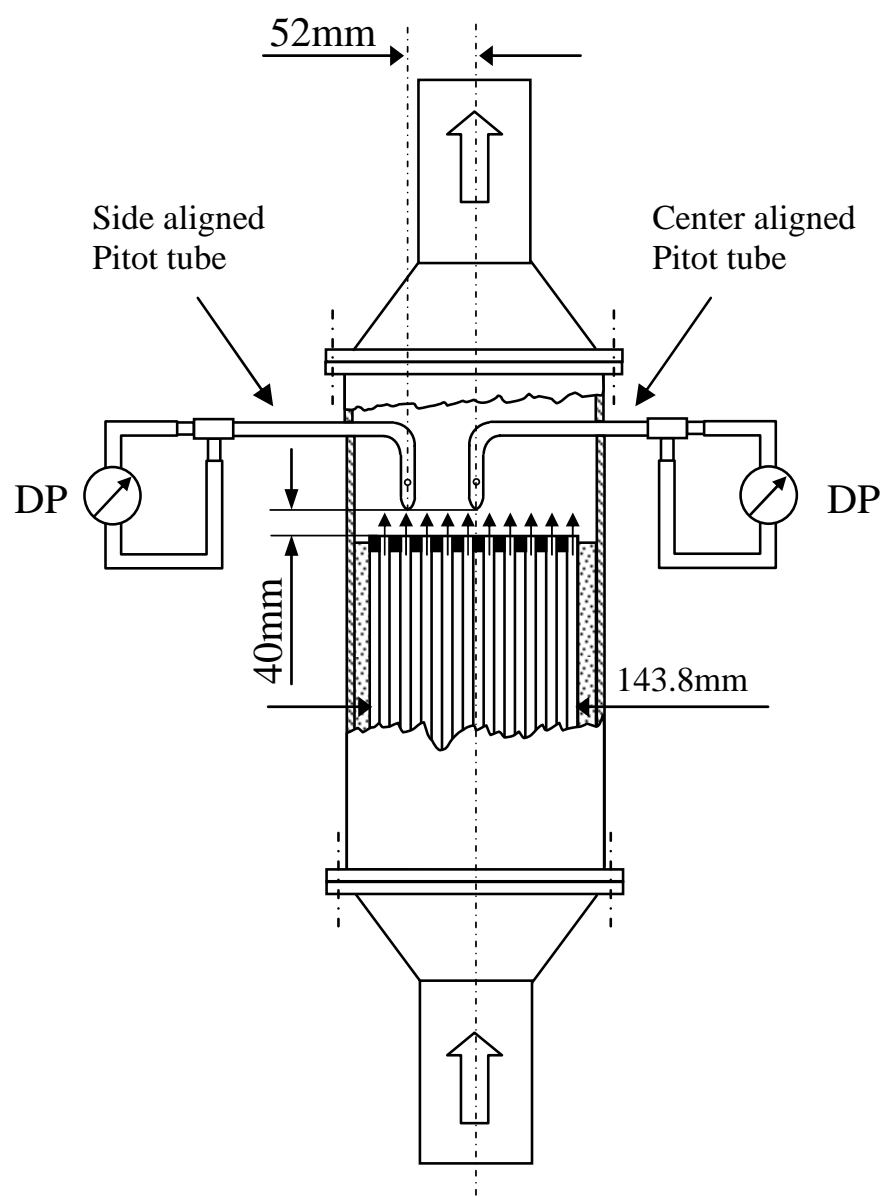

Figure 4.16 Schematic diagram of Pitot tubes assembly on engine exhaust system at the exit of the filter 


\section{Steady state loading experiments}

The steady state filter soot loading experiments were performed with the engine running with 25ppm Ce DPX9-doped fuel. Engine speed points were selected to cover a range between 2000 and 4000 rpm as more representative of the full range of City and Extra-Urban driving conditions, and engine load was varied to result in a range of filter wall temperatures from $300^{\circ} \mathrm{C}$ to $400^{\circ} \mathrm{C}$. As presented in [72] this range of filter wall temperatures results in varying VOF content of accumulated soot between 2 and 9 percent and allows the study of the effects of VOF adsorption-desorption and stochastic regeneration. After completion of each loading scenario the filter was regenerated and cleaned with a reverse flow of water in order to exclude any secondary effects from fuel additive ash residual. The loading test protocol, carefully designed based on the engine maps, is presented in Table 4.6.

\begin{tabular}{|c|c|c|c|c|c|c|c|}
\hline \multirow{2}{*}{ Process } & $\mathbf{N}(\mathbf{r p m})$ & $\begin{array}{c}\text { Torque } \\
\mathbf{( N m )}\end{array}$ & $\mathbf{m f r}(\mathbf{g} / \mathbf{s})$ & $\begin{array}{c}\text { T exhaust } \\
\left({ }^{\mathbf{0}} \mathbf{C}\right)\end{array}$ & $\begin{array}{c}\mathbf{T F} \text { inlet } \\
\left(\mathbf{}^{\circ} \mathbf{C}\right)\end{array}$ & $\begin{array}{c}\text { TF wall } \\
\text { center }\left({ }^{\circ} \mathbf{C}\right)\end{array}$ & $\begin{array}{c}\text { TF wall } \\
\text { side }\left({ }^{\mathbf{0}} \mathbf{C}\right)\end{array}$ \\
\hline \multirow{3}{*}{ Loading } & 1800 & 80 & 35 & 410 & 385 & 380 & 365 \\
\cline { 2 - 8 } & 3000 & 40 & 68 & 316 & 307 & 304 & 290 \\
\cline { 2 - 8 } & 4000 & 30 & 91 & 402 & 391 & 388 & 364 \\
\hline \multirow{2}{*}{ Regeneration } & 2000 & 180 & 58 & 533 & 516 & 510 & 490 \\
\cline { 2 - 8 } & 3000 & 160 & 91 & 600 & 584 & 580 & 550 \\
\hline
\end{tabular}

Table 4.6 Experimental protocol

\section{Regeneration experiments}

Two typical regeneration modes that appear under actual vehicle operating conditions were studied:

- The first one involves high speed and load engine operation associated with high exhaust gas mass flow rate and high exhaust gas temperature.

- The second involves medium speed and load engine operation resulting low flow rate and low exhaust gas temperature.

Each regeneration process was initiated after loading at the respective engine operation point, until the same level of filter loading is achieved by a step increase in engine load. The engine was left to run for 10 minutes on $2000 \mathrm{rpm}$ and $40 \mathrm{Nm}$ before the regeneration strategy is applied, duration considered long enough for thermal and chemical equilibrium to be reached. The regeneration engine operation points are presented in Table 4.6.

\section{Soot loading distribution measurements}

In order to confirm the measurements with the Pitot tubes and indirectly evaluate the soot loading distribution from filter centre to periphery, the expansion device described in the precious section and [69] was employed in a series of measurements at selected inlet channels of the engine filter after the end of the loading process. The exhaust pipe of the vessel was carefully connected to the inlet of typical, representative trap channels and the pressure drop characteristic was recorded as shown in Figure 4.5. 


\subsubsection{Results and discussion}

\section{Loading experiments}

As already mentioned, the steady state loading experiments were performed to investigate the flow and soot loading characteristics along the filter channels under various mass flow rates and filter wall temperatures. Initially a low mass flow rate $(35 \mathrm{~g} / \mathrm{s})$ loading experiment is performed with the engine running on speed: $1800 \mathrm{rpm}$ load: $80 \mathrm{Nm}$ resulting a filter wall temperature of the order of $370^{\circ} \mathrm{C}$ as measured at the center of the filter by thermocouple T/C9. The results presented in Figure 4.17.

We could observe three characteristic parts in the recording of the filter loading process:

- $\quad$ The first incorporates the phase of the beginning of loading until the accumulation of soot mass becomes uniform. During this initial short phase, the backpressure and velocity increase rapidly due to the filling of filter surface pores. After this initial period, the particulates start to accumulate on the wall surface and the pressure drop curve takes a more linear form. It can be observed that the exhaust gas velocity for all this period is higher in the central region of the filter, indicating that the flow passes mainly through the central channels of the filter. This behavior could be attributed to the effects of the inlet pipe and inlet diffuser geometry that enforces the flow through the central region. As the accumulated mass increases, the core velocity decreases and the flow is diverted towards the outer area of the filter, resulting in an increase of the peripheric channels' velocity.

- In the second part, the loading process is continued without any changes to the soot loading profile. The velocities at the centre and the periphery are equal and tend to decrease as a result of the increase of filter backpressure. This increase of filter backpressure due to the increase to the accumulated soot mass within the filter channels compensates the velocity differences between the center and periphery and makes the flow distribution more uniform.

- In the third part of the loading process, the effects of stochastic phenomena start to show up. A sudden decrease of the filter backpressure associated with an increase in the filter inlet temperature, exceeding $380^{\circ} \mathrm{C}$. This is shown to disturb the previously described uniform flow distribution. We could suspect that a VOF desorption process could take place in the central channels of the filter where the temperatures are higher. Comparison of wall temperatures at filter centre and periphery shows that periphery is systematically colder than the centre by about $25{ }^{\circ} \mathrm{C}$. Presumably, VOF desorption would cause a permeability increase of the soot layer of the central channels of the filter, resulting in a reduction of the flow resistance through this region. Thus, a marked increase of exhaust gas velocity at the central region of the filter is observed, indicating a nonuniform redistribution of the flow field over the filter channels. 


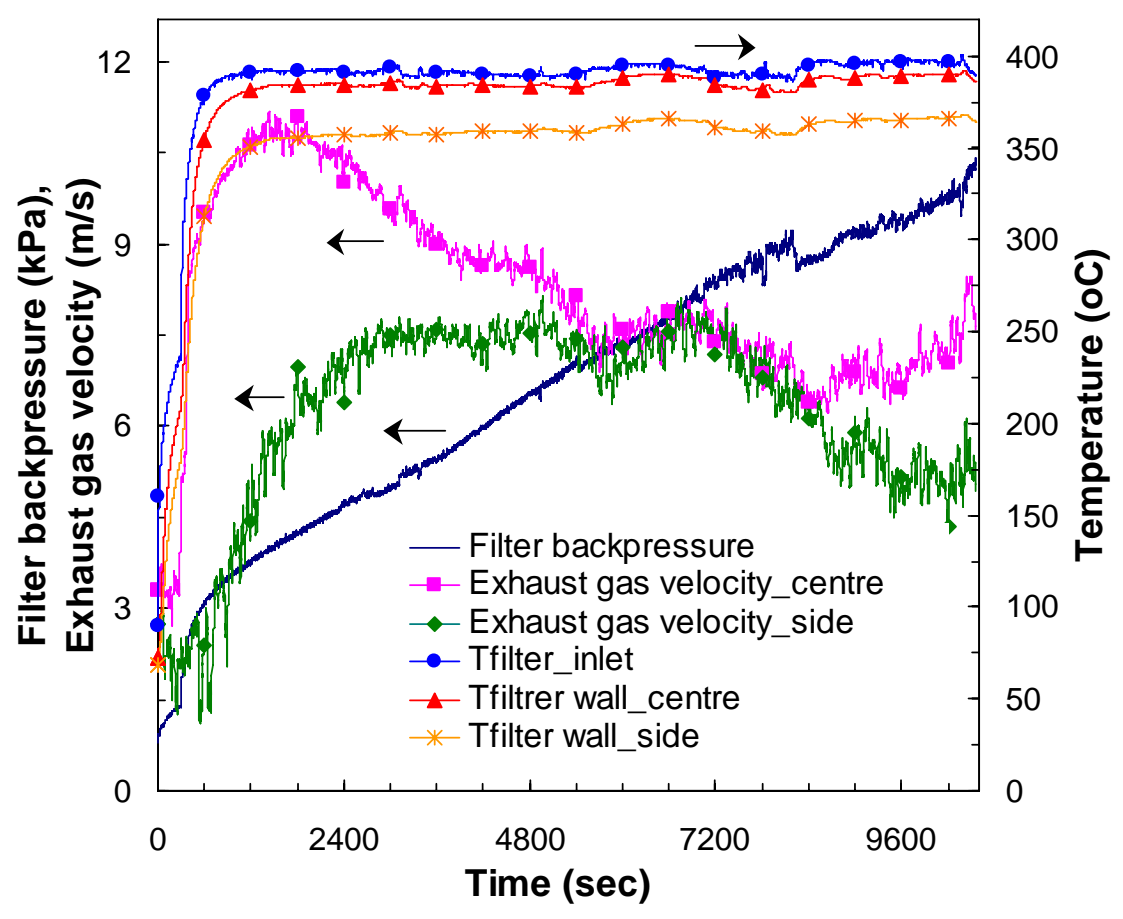

Figure 4.17 Low mass flow rate (35g/s) loading scenario. Exhaust gas velocity is measured simultaneously at the exit of exit of the central and peripheric channels of the filter during loading at 1800rpm, $80 \mathrm{Nm}$ with 25ppm additive. Evolution of filter backpressure is also monitored. The effects of filter wall temperature on the loading process are measured by thermocouple T/C9 (closer to the centre) and T/C8 (closer to the periphery).

The percentage of VOF that could eventually remain adsorbed on the dry soot accumulated in the filter, mainly depends on the filter temperature [72]. If the filter temperature exceeds the range of $380-420^{\circ} \mathrm{C}$, most VOF is vaporized and only dry soot remains accumulated on the filter wall. On the other hand, prolonged operation of a heavily loaded filter with dry soot under low load and speed conditions can lead to the readsorption of heavy hydrocarbons emitted in the particulate layer, thus increasing its VOF content.

In order to confirm the above observations, a set of measurements was carried out with the expansion device. The results are presented in Figure 4.18. Two different zones are observed as the measuring device is moved from the central (hotter during operation) to the outer (colder during operation). The pressurized air is shown to discharge faster through the central channels than those at the periphery indicating that the flow resistance due to the VOF desorption is lower. It must be mentioned that this type of measurements are performed after the engine stops, at filter wall temperatures that not exceed the $150^{\circ} \mathrm{C}$. Thus, a part of VOF that was present in the thick particulate layer could possibly condense on the colder outer channel walls after engine stop, resulting to a variation of permeability characteristics between filter core and periphery channels. 


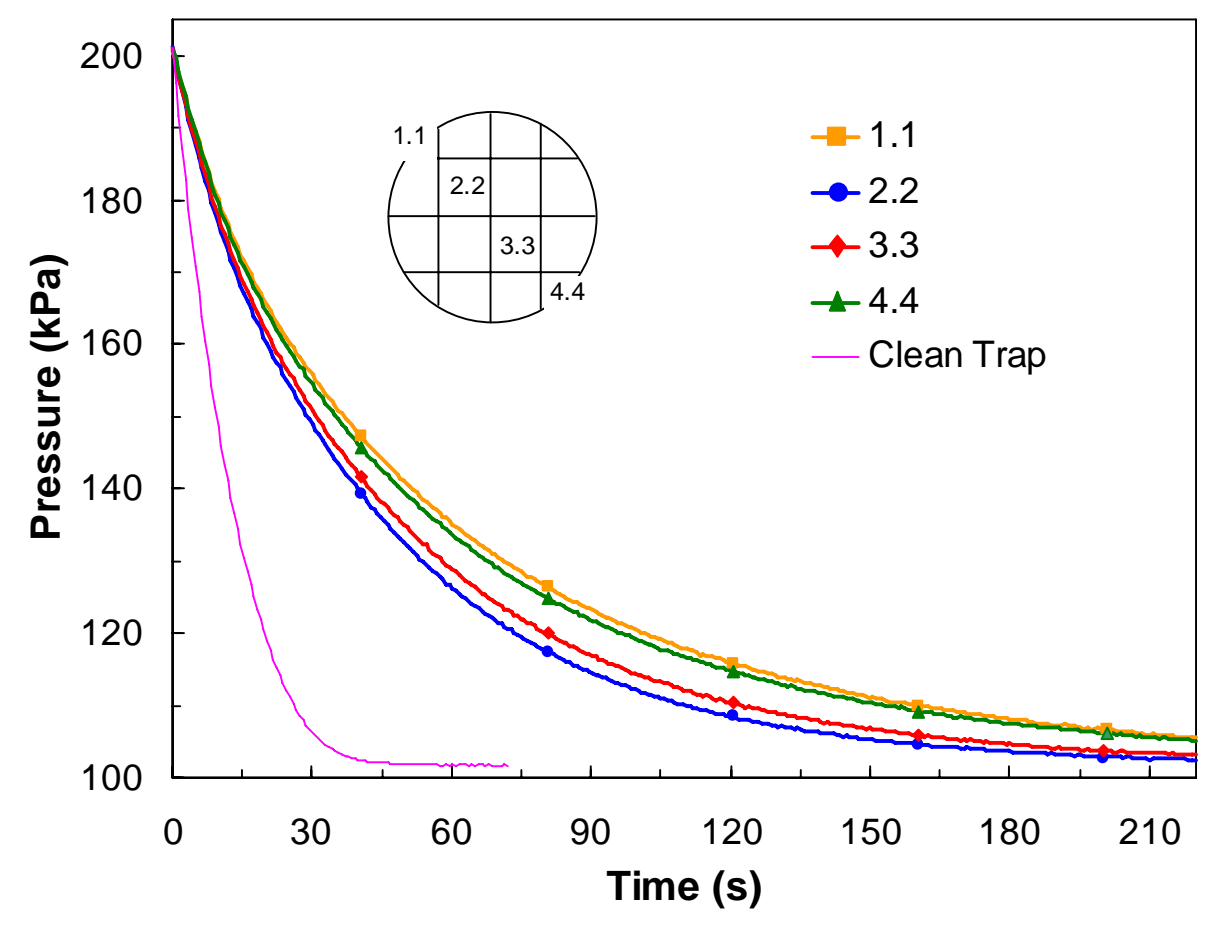

Figure 4.18 Estimation of soot mass distribution after the low mass flow rate loading scenario of Figure 4.17. Pressure drop measurements were performed with the expansion device described in Fig. 4 at specific channels along a diameter of the filter.

As a next step, the loading process was repeated with the engine running at 3000rpm$40 \mathrm{Nm}$ resulting in a medium exhaust gas mass flow rate of the order of $70 \mathrm{~g} / \mathrm{s}$ and a filter wall temperature of the order of $350^{\circ} \mathrm{C}$. The results are presented in Figure 4.19 Again, a higher exhaust gas velocity is initially observed at the central region of the filter. In comparison with the previous low mass flow rate loading point, the difference between the central and outer velocity is lower indicating that the increase of backpressure deviate a part of the flow through the outer channels. With increasing filter soot loading, this effect is counterbalanced, due to selective loading of the central part. After a period where the flow seems to be stabilized to a relatively homogenous distribution, a reversal of the flow distribution is observed. This could be attributed to stochastic regeneration phenomena at the soot of the filter periphery, which is expected to be VOF rich due to the lower wall temperatures. The VOF content on this operation point is varying from about $6 \%$ at the central to $9 \%$ at the circumferential channels and due to its very close contact with the catalyst oxides may be oxidized at temperatures bellow $250^{\circ} \mathrm{C}$. Thus we may assume that a slow regeneration process takes place at specific points of filter periphery where the soot loading, composition and temperatures are favorable. This slow regeneration reduces the flow resistance through the periphery allowing the increase of velocity through this region. Another phenomenon that could be involved in producing the above behavior is the appearance of significant inertia effects at high flowrates, due to the upstream piping and inlet diffuser causing a swirl flow component. 


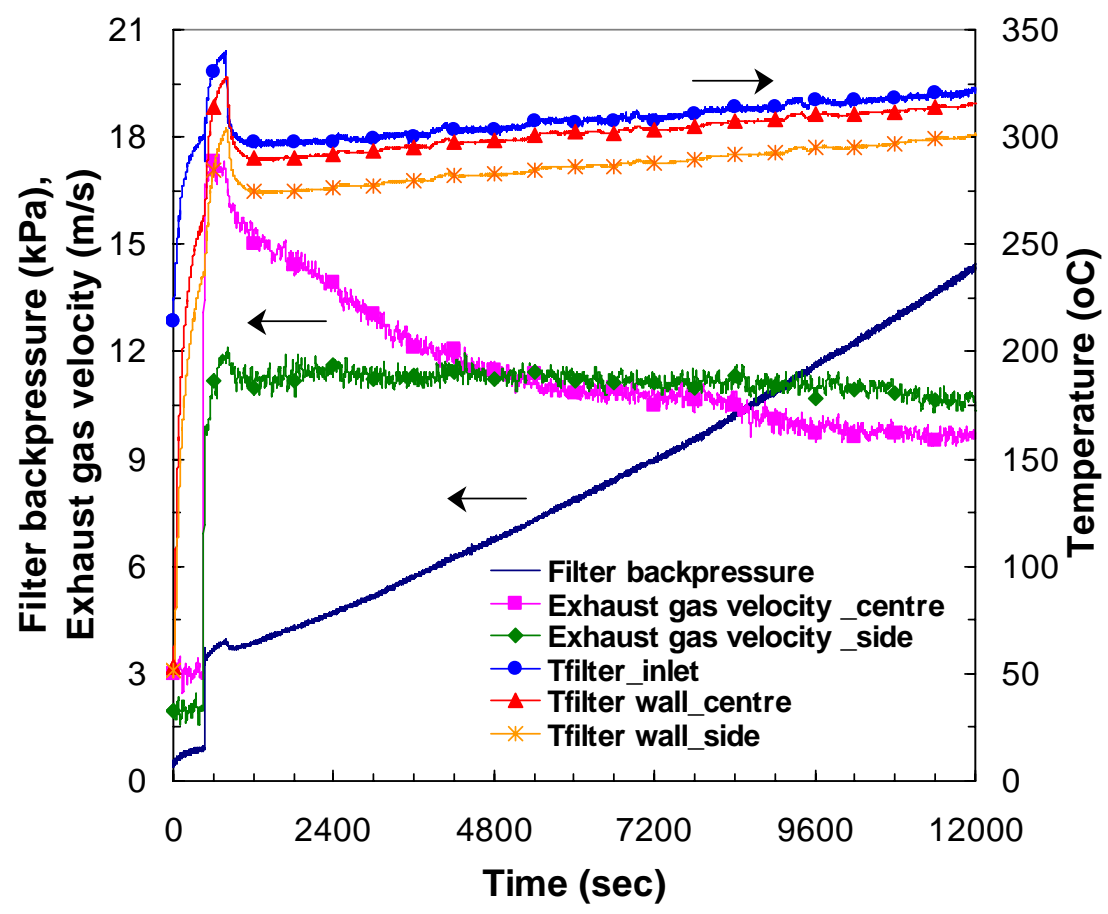

Figure 4.19 Medium mass flow rate $(68 \mathrm{~g} / \mathrm{s})$ loading scenario. Exhaust gas velocity is measured simultaneously at the exit of the central and peripheric channels of the filter during loading at 3000rpm, $40 \mathrm{Nm}$ with 25ppm additive. Evolution of filter backpressure is also monitored. The effects of filter wall temperature on the loading process are measured by thermocouple T/C9 (closer to the centre) and T/C8 (closer to the periphery).

The measurements with the expansion device confirm the above results. As shown in Figure 4.20 the vessel discharge curves indicate two distinct zones as the nozzle is moved from the central to the peripheric channels. The pressurized air discharges faster through the circumferential channels than through the central channels. This means that the stochastic regenerations happening in the VOF-rich peripheric channels, produce a lower soot loading zone. This could be responsible for the redirection of exhaust gas flow from the central towards the outer filter area.

The study of the effects of exhaust gas mass flow rate on flow and soot maldistribution during loading is completed with a high flow rate - high exhaust temperature loading test obtained with the engine running on $4000 \mathrm{rpm}-30 \mathrm{Nm}$. This point results to a mass flow rate of the order of $90 \mathrm{~g} / \mathrm{s}$ and a filter wall temperature of the order of $390^{\circ} \mathrm{C}$. The results are presented in Figure 4.21. As in the previous cases, the flow initially passes through the central core of the filter. In this case the velocity difference between center and periphery takes its lowest value and is compensated very early by the increase of backpressure as result of soot accumulation within the channels. After this period, a reversal of the flow is observed without any stabilization to a uniform flow distribution as in the previous cases. This could be attributed to the selective loading of the central part of the filter. Thus the previous described inertia phenomena may enforce the concentration of particulates at the central channels. After this phase a new reversal of the flow is observed which is associated with a continuous increase in the measured velocity at the filter center. 


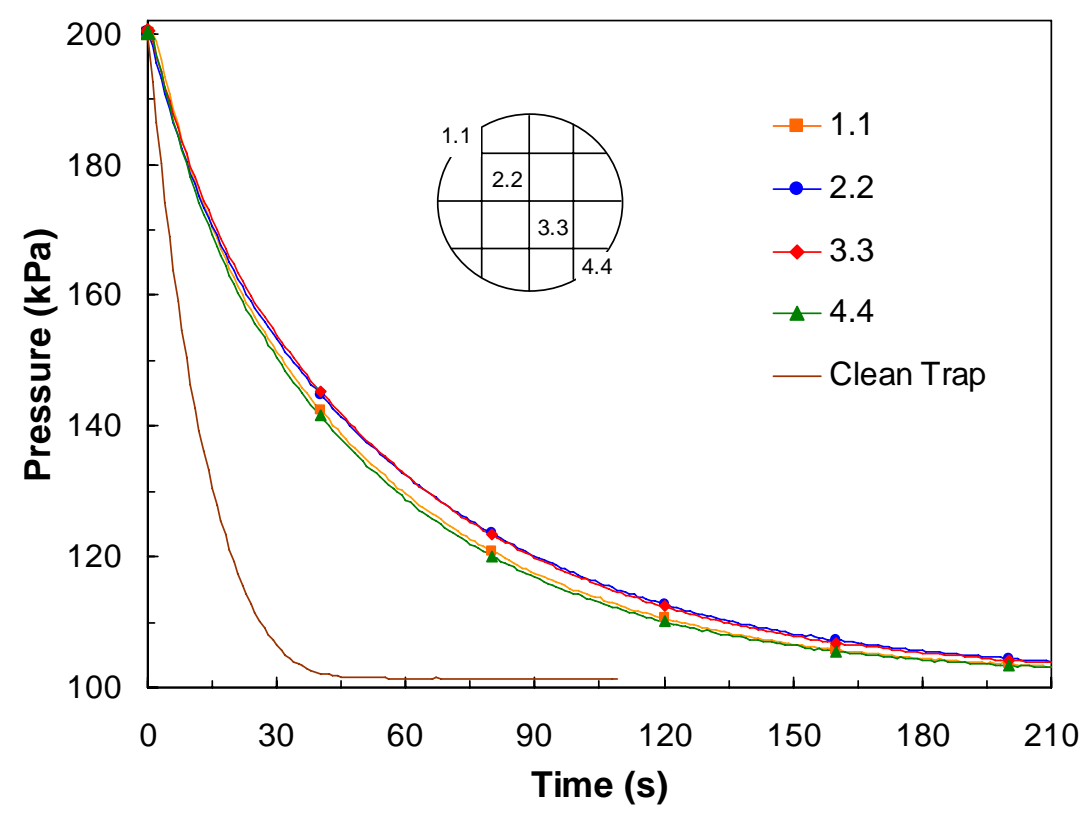

Figure 4.20 Estimation of soot mass distribution after the medium mass flow rate loading scenario of Figure 4.19. Pressure drop measurements were performed with the expansion device described in Fig. 4 at specific channels along a diameter of the filter.

This phase is characterized by a rapid increase of the filter wall temperature measured at the centerline, indicating that a regeneration process takes place at the center of the filter where the temperatures are higher. This mechanism results to a partial regeneration of filter and is responsible for the significant differentiation of the exhaust flow distribution and the subsequent loading maldistribution, with the exhaust flowing mainly through the central region of the filter.

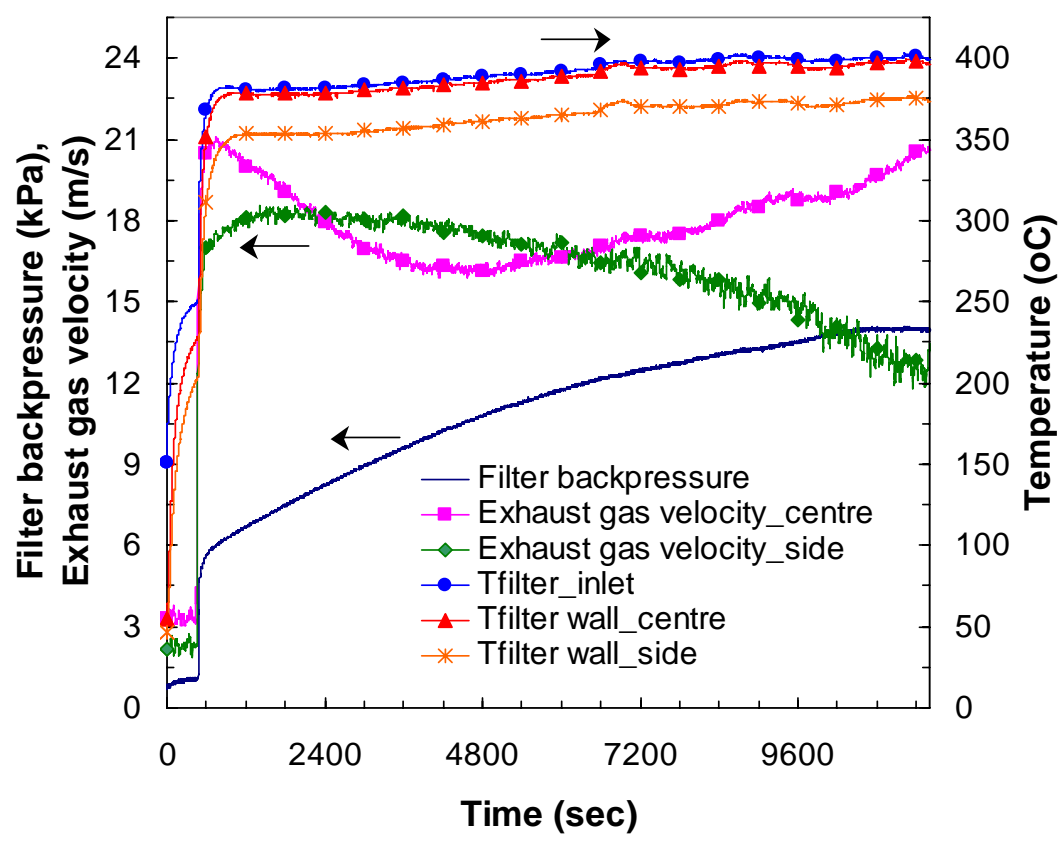

Figure 4.21 High mass flow rate (91g/s) loading scenario. Exhaust gas velocity is measured simultaneously at the exit of the central and peripheric channels of the filter during loading at 4000rpm, $30 \mathrm{Nm}$ with 25ppm additive. Evolution of filter backpressure is also monitored. The effects of filter wall temperature on the loading process are measured by thermocouple T/C9 (closer to the centre) and T/C8 (closer to the periphery). 
The test with the expansion device confirms the above observation. The results of Figure 4.22 indicate that the pressurized air discharges faster through the central channels than through the circumferential channels. This fact hints to a possible partial regeneration that took place at the central part of the filter.

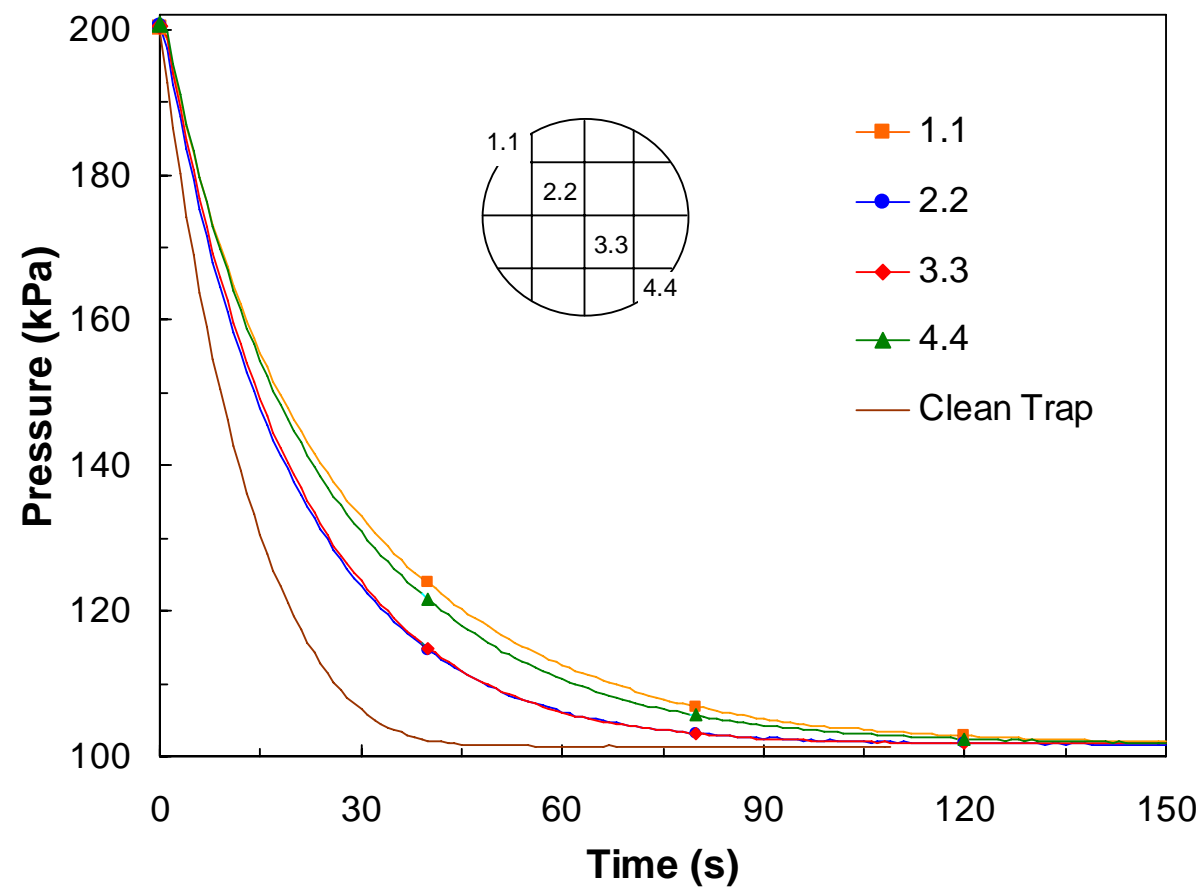

Figure 4.22 Estimation of soot mass distribution after the high mass flow rate loading scenario of Figure 4.21. Pressure drop measurements were performed with the expansion device described in Fig. 4 at specific channels along a diameter of the filter.

\section{Regeneration experiments}

The processes taking place during catalytic regeneration are quite complex and not yet well understood. The instability to the appearance and evolution of regeneration at the temperature range from $250^{\circ} \mathrm{C}$ (appearance of erratic regeneration) to $600^{\circ} \mathrm{C}$ (thermal regeneration) is one of the most significant problems since it is related with the filter durability. Monitoring of flow velocity at the core and periphery of the filter during regeneration can supply important information on the role of flow distribution. Two regeneration scenarios were investigated here: a low flow rate-medium exhaust gas temperature and a high flow rate-high exhaust temperature scenario.

In the first regeneration scenario, a filter previously loaded at $1800 \mathrm{rpm}-80 \mathrm{Nm}$ was subsequently regenerated at 2000rpm with a step increase from 40 to $180 \mathrm{Nm}$ (mass flow rate, $58 \mathrm{~g} / \mathrm{s}$ ) resulting in a temperature increase of the order of $250^{\circ} \mathrm{C}$. The recordings of flow velocities along with filter backpressure and filter wall temperatures are presented in Figure 4.23. It can been seen, that for specific levels of wall temperature and filter backpressure the regeneration is initiated and the shape of flow velocity curves directly correlates with the temperature. Initially due to the previous loading history the flow is higher through the central region of the filter indicating that a non-uniform soot loading distribution exists. After the step load increase, a slow increase in the flow velocity at the central region is observed indicating the initiation of regeneration due to the higher temperatures that accelerates the soot oxidation. The velocity through the periphery reduces during the regeneration of the central part as result of the flow deviation. However, as the regeneration process continues 
and the temperature at the periphery increases, the soot oxidation is transferred to the outer channels, thus increasing flow velocity until the completion of regeneration where steady state conditions are obtained again.

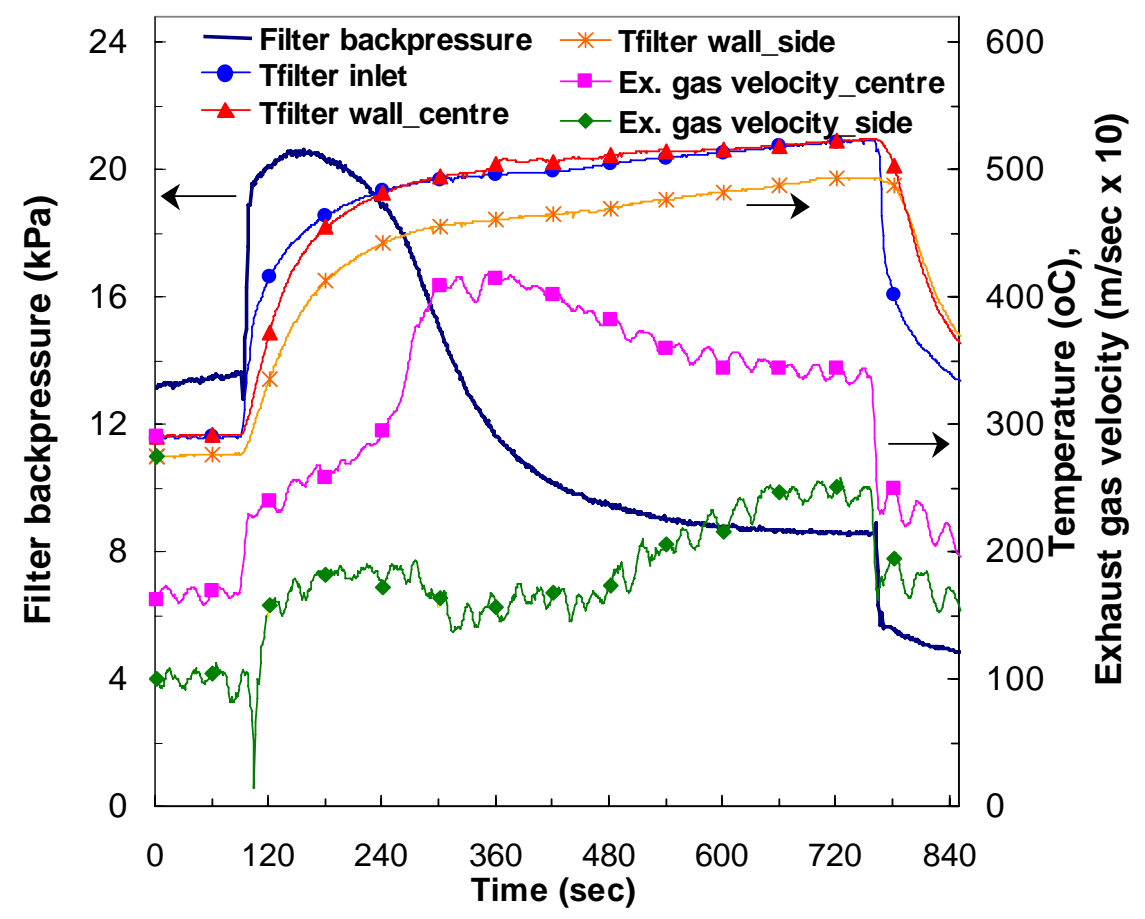

Figure 4.23 Low mass flow rate regeneration scenario of a previous loaded filter at 1800rpm 80Nm. Exhaust gas velocity is measured simultaneously at the exit of the central and peripheric channels of the filter during a step increase in filter inlet temperature, produced by changing the engine operation point from 2000rpm and $40 \mathrm{Nm}$ to $2000 \mathrm{rpm} 180 \mathrm{Nm}$. Evolution of filter backpressure is also monitored. The effects of filter wall temperature on the regeneration process are measured by thermocouple T/C9 (centre) and T/C8 (periphery).

In the case of high mass flow rate-high temperature regeneration mode a filter previous loaded at 3000rpm -40Nm was regenerated with a step increase from 2000rpm- $40 \mathrm{Nm}$ to $3000 \mathrm{rpm}-160 \mathrm{Nm}$ (mass flow rate $91 \mathrm{~g} / \mathrm{s}$ ) resulting in a temperature increase of the order of $320^{\circ}$. The results of Figure 4.24 show that initially the flow velocity at periphery is somewhat higher due to the previous loading history. Higher wall temperatures at the central part resulted in the initiation of regeneration there. The resulting soot depletion in this part, forced the flow to pass mainly from the centre, allowing the appearance of higher oxidation rates of soot by exhaust gas oxygen. Thus a rapid increase to the flow velocity at the centre associated with a higher heat release rate due to the higher exothermy is observed. At the same time, the flow velocity measured at the periphery is minimized indicating the existence of significant flow maldistribution. Afterwards, regeneration sets up also in the periphery and finally the flow is homogenized again, with a somewhat higher flow through the centre.

The above-described regeneration scenarios led us to conclude that the time lag in the regeneration of the outer filter channels observed in the experimental results could be attributed to flow maldistribution effects. 


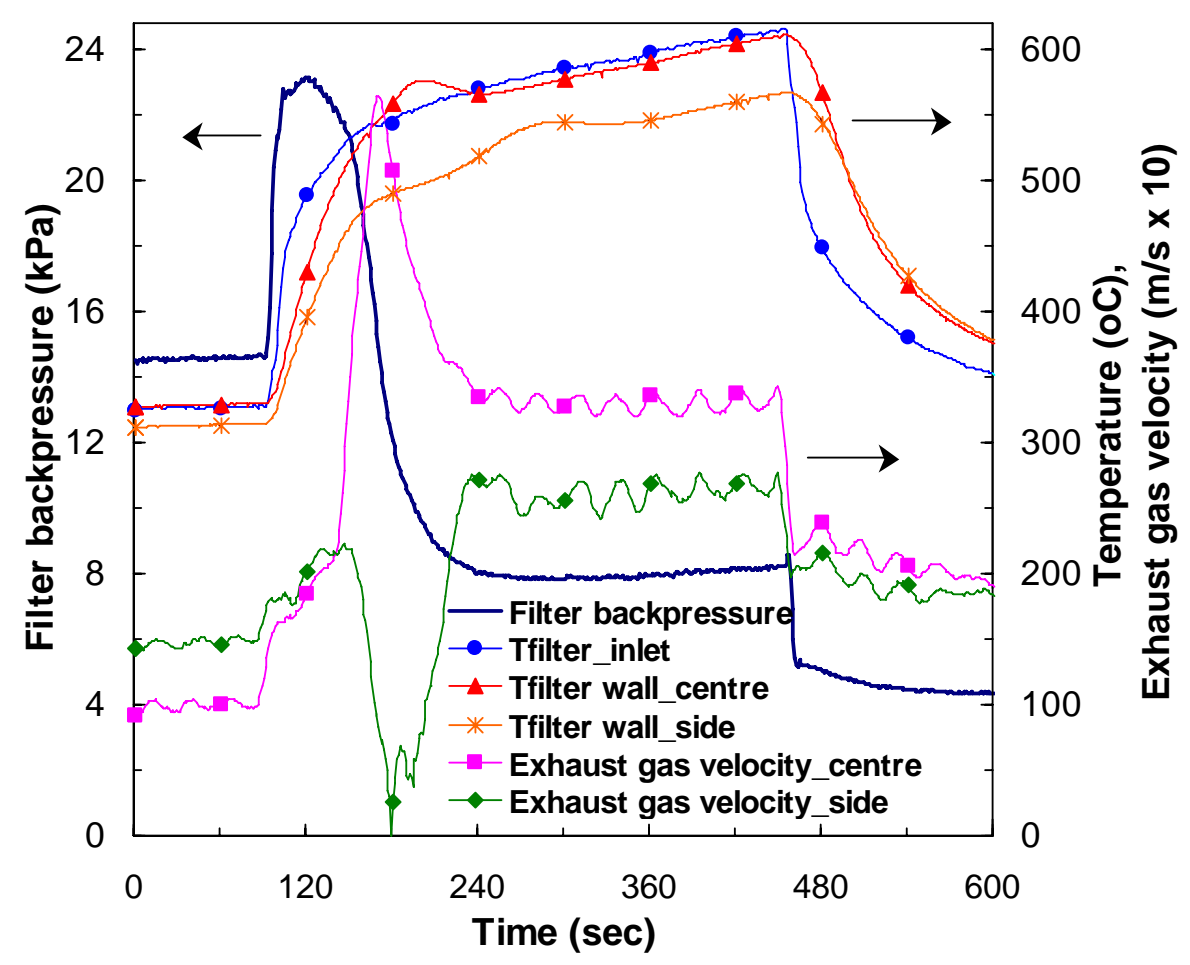

Figure 4.24 High mass flow rate regeneration scenario of a previous loaded filter at 3000rpm 40Nm. Exhaust gas velocity is measured simultaneously at the exit of the central and peripheric channels of the filter during a step increase in filter inlet temperature, produced by changing the engine operation point from 2000rpm and $40 \mathrm{Nm}$ to $3000 \mathrm{rpm} 160 \mathrm{Nm}$. Evolution of filter backpressure is also monitored. The effects of filter wall temperature on the regeneration process are measured by thermocouple T/C9 (closer to the centre) and T/C8 (closer to the periphery).

\subsubsection{Concluding remarks}

- A systematic effort was made to record and explain flow maldistribution effects across the channels of a diesel filter during loading and regeneration operation.

- Full- scale tests of the loading and regeneration behaviour of a particulate filter installed on a modern diesel engine run on catalyst doped fuel, are employed in this investigation.

- Loading tests were performed at 3 engine operation points with markedly different levels of engine exhaust gas mass flowrate. In these tests, it becomes apparent that there exist complex flow maldistribution phenomena that vary during the loading phase, which are not directly reflected to the behaviour of the pressure drop curve versus time. These phenomena are shown to affect the distribution of collected soot mass in the different channels of the filter, and, consequently, the regeneration behaviour.

- The evolution of flow maldistribution phenomena was also studied in a number of regeneration experiments. It was confirmed that the variation of volatile organic fraction in the filter and the associated partial catalytic regenerations at low temperatures are interacting with flow and soot maldistibution in a complex way. 
- A specially designed test rig for cold flow pressure drop and velocity distribution experiments will be employed in future, more detailed investigations of the flow maldistribution behavior of diesel filters.

\subsection{Cold Flow Distribution Experiments in a Wall Flow Diesel Particulate Filter}

In the previous presented experimental study it was apparent that tests under real engine operating conditions encounter problems to the understanding of the complex maldistribution phenomena due to the high temperature noisy environment of the exhaust system and the difficulties to control the operating conditions (particulate loading, mass flow rate in the filter, exhaust gas properties). In this work a more detailed investigation of flow maldistribution in diesel filters with the aid of a specially designed test rig for cold flow pressure drop and velocity distribution experiments is sought. Also a flow uniformity index was adopted, in order to quantify the measured flow maldistribution by a single number for each case.

\subsubsection{Experimental}

The existence of flow maldistribution is investigated in this section by means of flow velocity measurements. The measurements were performed on a specially designed cold flow test rig, capable of simulating engine-operating flow conditions using ambient air. It consists of an Eaton model 45 supercharger which is powered from a $5.5 \mathrm{HP}$, AC electric motor. The power is transmitted to the supercharger through a belt drive system capable of two different transmission ratios by use of two sets of interchangeable pulleys:

- The first ratio is 2 and results in a supercharger speed of $5600 \mathrm{rpm}$ and a corresponding mass flow rate of the order of $60 \mathrm{~g} / \mathrm{s}$ which represents a medium speed - medium load engine operation point.

- The second ratio is 1.75 and results in a supercharger speed of $4900 \mathrm{rpm}$ and a corresponding mass flow rate of the order of $45 \mathrm{~g} / \mathrm{s}$ which represents a low speed - low load engine operation point.

The low and medium flowrate test points were selected as more representative of city driving conditions. The particulate filter with the other components (diffuser and catalyst) is mounted on the test rig, about $500 \mathrm{~mm}$ downstream the supercharger and is supplied with ambient air.

The experimental determination of flow velocity is done with the use of a hot film sensor (TSI VelociCalc Air Velocity Meter Model 8346). The sensor is attached to an electronically controlled support device able to move with a pitch of $0.1 \mathrm{~mm}$ in the vertical and horizontal direction, scanning the face of the measurement area at each test. The layout of the measuring system is presented in detail in Figure 4.25.

Two of the most popular ceramic filter types were tested:

- a cordierite filter $(17 / 100)$

- a silicon carbide filter (14/200)

The properties and the geometrical characteristics of the two types of filters are presented in Table 4.7. Apart from the different material, the two types of filter represent two different design approaches: The $\mathrm{SiC}$ filter is made up from sixteen individual filter blocks, which are connected with a special impermeable cement while the cordierite filter is constructed as single monolith. These special characteristics are expected to demonstrate different flow field characteristics. In the case of $\mathrm{SiC}$ filter two different filter lengths were tested: 150 and 254 mm respectively. 


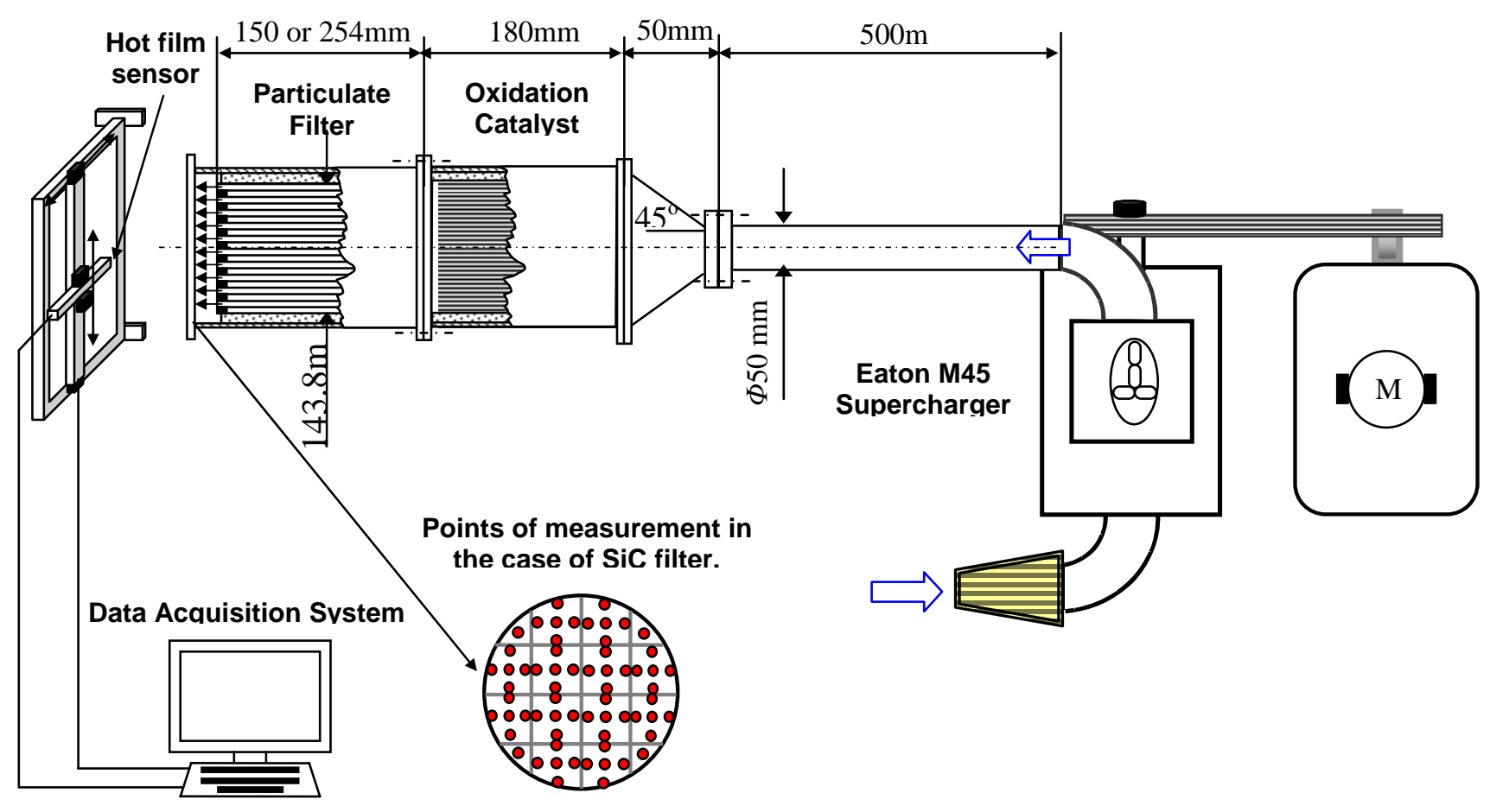

Figure 4.25 Schematic representation of the cold flow velocity measurements test rig. Supercharger installation and filter configuration are shown, along with the digitally controlled velocity scanning device and the data acquisition system.

In order to investigate the effect of catalyst to the flow distribution two configurations were tested:

- the first involves measurement of the velocity profile downstream the filter, when an oxidation catalyst with a cell density of 400 cells per square inch is installed upstream and

- the second involves measurement of the velocity profile downstream filter with the filter assembled directly downstream the inlet diffuser.

The velocity profiles at the exit of the inlet diffuser and the catalyst were also measured as a necessary step to the understanding of the interaction of flow fields in the various components.

Table 4.7 Properties of the full scale filters used in the study

\begin{tabular}{|l|l|l|}
\hline Property & Cordierite & SiC \\
\hline Diameter $(\mathrm{mm})$ & 143.8 & 143.8 \\
\hline Length $(\mathrm{mm})$ & 150 & 152.4 and 254 \\
\hline Cell concentration $\left(\mathrm{cells} / \mathrm{in}^{2}\right)$ & 100 & 200 \\
\hline Material Density $\left(\mathrm{kg} / \mathrm{m}^{3}\right)$ & 2510 & 3080 \\
\hline Porosity $(\%)$ & 43 & 42 \\
\hline Cell Size $(\mathrm{mm})$ & $2.54 \times 2.54$ & $1.49 \times 1.49$ \\
\hline Wall thickness $(\mathrm{mm})$ & 0.6 & 0.4 \\
\hline Wall density $\left(\mathrm{kg} / \mathrm{m}^{3}\right)$ & 1000 & 1800 \\
\hline Mean pore size $(\mu \mathrm{m})$ & 33 & 9 \\
\hline Permeability $\left(\mathrm{m}^{2}\right)$ & $0.64 \mathrm{E}-13$ & $3.7 \mathrm{E}-13$ \\
\hline
\end{tabular}


The velocity measurements were performed at carefully selected points for the investigation of the effects of the special structure characteristics of each type of filter material. In the case of the SiC filter the measurements were carried out at each block at the center and near the edges at a distance of about two cells from the cement borderline. In order to have comparable results the same measurements protocol was applied in the case of the cordierite filter.

The velocity profile for each type of filter material and configuration was measured at three different points downstream the channels' exit: $15 \mathrm{~mm}$ or about 10 cell diameters, $30 \mathrm{~mm}$ or about 20 cell diameters and $80 \mathrm{~mm}$, to understand the development of the flow profile by the mixing of the laminar jets exiting the channels. Comparison among the three profiles was also employed as a cross-checking method (a gradual damping of the flow field fluctuations should always be observed).

Measurements of a loaded SiC filter were also carried out, in order to understand differences in flow distribution in loaded filters. For this purpose, the $\mathrm{SiC}$ filter was fitted along with an upstream catalyst, to the exhaust of a 2.0 liter displacement HDI turbocharged passenger car engine, running on a steady state medium speed, medium load operation point with 25ppm Cerium doped fuel. As presented in [72] in this region the filter wall temperatures are varying in the range between 280 and $350 \mathrm{oC}$ resulting in a varying soot VOF content from 9 to 6 percent. This allows us to study VOF adsorption - desorption effects in the process. After the filter is loaded to an additional backpressure of the order of $40 \mathrm{mbar}$, (that is, $80 \mathrm{mbar}$ total backpressure), it is dismantled from the engine and fitted to the flow maldistribution measurements test rig. The process was repeated once more, up to a total filter backpressure of 120 mbar, that is, three times the backpressure of the clean filter. The experimental layout of the filter loading experiments is presented in Figure 4.26. The details of the measurement protocol are presented in Table 4.8.

Engine: 2L- HDI running on 3000rpm, $40 \mathrm{Nm}$

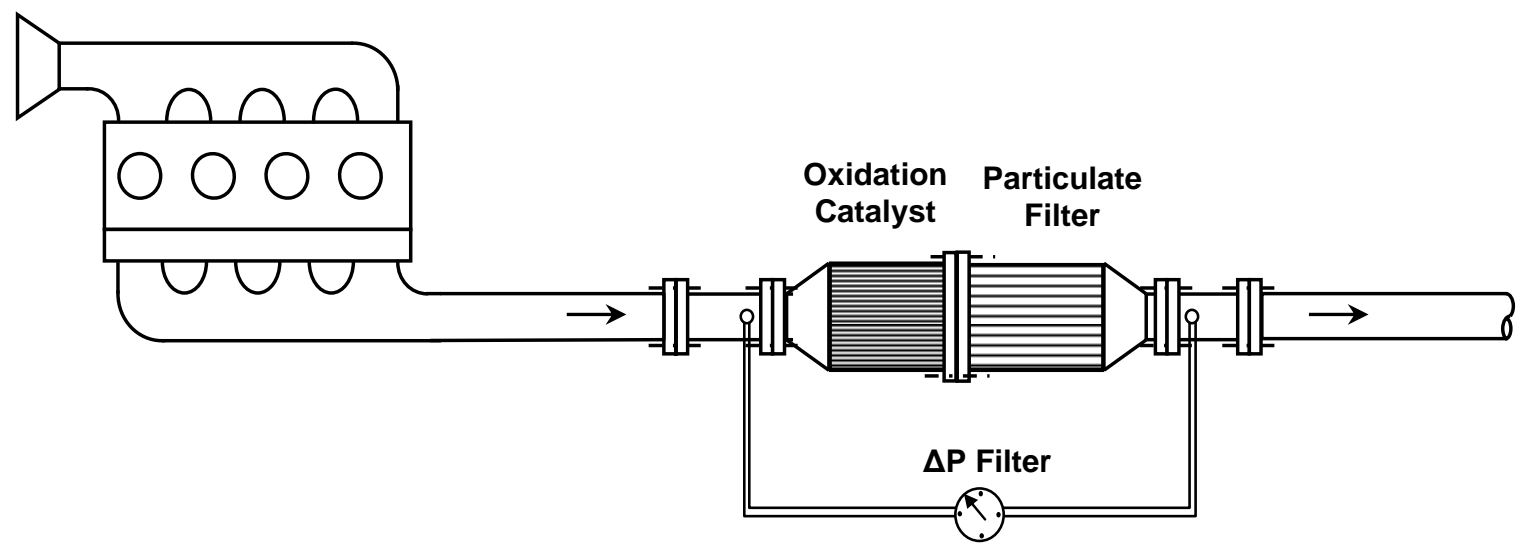

Figure 4.26 Experimental layout for the filter loading tests.

\section{Maldistribution Factor}

In order to quantify the uniformity of the flow velocity at the cross section of the filter monolith it is possible to use a quantity known as maldistribution factor (MF). In the case of cells with equal surfaces a MF can be defined based on the ratio $\sigma / \mu$ (standard deviation over mean value of velocity distribution) by the following equation [113]: 


$$
M F=1-\sqrt{\frac{1}{n} \sum_{n}\left(\frac{w_{i}-w_{o}}{w_{o}}\right)^{2}}
$$

Where $w_{i}$ is the local velocity for the $\mathrm{i}$-th area and $\mathrm{w}_{\mathrm{o}}$ is the mean velocity over the monolith exit defined as

$$
w_{o}=\frac{1}{n} \sum_{n} w_{i}
$$

and $n$ the total number of (equidistant) measurement points along a diameter. The expression is formulated in such a way that in the case of a completely uniform distribution, the value of the maldistribution factor takes the maximum value of 1 .

\begin{tabular}{|c|c|c|c|c|c|}
\hline \multirow[b]{2}{*}{$\begin{array}{l}\text { Name of } \\
\text { studied } \\
\text { parameter }\end{array}$} & \multicolumn{5}{|c|}{ Experimental Parameters } \\
\hline & 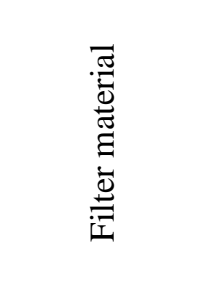 & 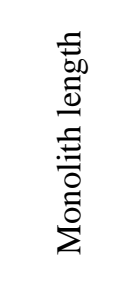 & 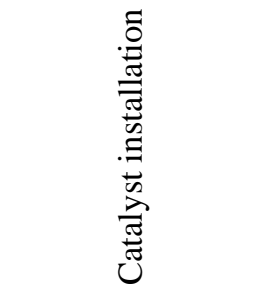 & $\begin{array}{l}\text { 节 } \\
3 \\
3 \\
0 \\
0 \\
0 \\
0 \\
\Xi \\
0 \\
0 \\
0\end{array}$ & 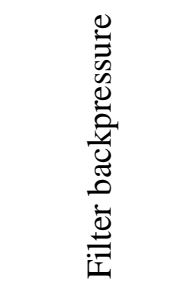 \\
\hline \multirow[t]{2}{*}{ Filter type } & $\begin{array}{c}\text { Cordierite } \\
\text { (17/100) }\end{array}$ & \multirow[t]{2}{*}{$150 \mathrm{~mm}$} & \multirow[t]{2}{*}{ Catalyst in front } & \multirow[t]{2}{*}{$45 \mathrm{~g} / \mathrm{s}$} & \multirow{2}{*}{$\begin{array}{c}40 \text { mbar } \\
\text { (clean filter }\end{array}$} \\
\hline & $\operatorname{SiC}(14 / 200)$ & & & & \\
\hline \multirow{2}{*}{ Filter geometry } & \multirow{2}{*}{$\operatorname{SiC}(14 / 200)$} & $150 \mathrm{~mm}$ & \multirow{2}{*}{ Catalyst in front } & \multirow{2}{*}{$45 \mathrm{~g} / \mathrm{s}$} & \multirow{2}{*}{$\begin{array}{c}40 \text { mbar } \\
\text { (clean filter) }\end{array}$} \\
\hline & & $254 \mathrm{~mm}$ & & & \\
\hline \multirow{2}{*}{$\begin{array}{l}\text { Presence of } \\
\text { catalyst }\end{array}$} & \multirow{2}{*}{$\operatorname{SiC}(14 / 200)$} & \multirow{2}{*}{$254 \mathrm{~mm}$} & No catalyst & \multirow{2}{*}{$45 \mathrm{~g} / \mathrm{s}$} & \multirow{2}{*}{$\begin{array}{c}40 \text { mbar } \\
\text { (clean filter) }\end{array}$} \\
\hline & & & Catalyst in front & & \\
\hline \multirow{2}{*}{$\begin{array}{c}\text { Gas mass flow } \\
\text { rate }\end{array}$} & \multirow{2}{*}{$\operatorname{SiC}(14 / 200)$} & \multirow{2}{*}{$254 \mathrm{~mm}$} & \multirow{2}{*}{ Catalyst in front } & $45 \mathrm{~g} / \mathrm{s}$ & \multirow{2}{*}{$\begin{array}{c}40 \text { mbar } \\
\text { (clean filter) }\end{array}$} \\
\hline & & & & $60 \mathrm{~g} / \mathrm{s}$ & \\
\hline \multirow{2}{*}{$\begin{array}{c}\text { Soot } \\
\text { accumulation }\end{array}$} & \multirow[b]{2}{*}{$\operatorname{SiC}(14 / 200)$} & \multirow[b]{2}{*}{$254 \mathrm{~mm}$} & \multirow[b]{2}{*}{ Catalyst in front } & \multirow[b]{2}{*}{$45 \mathrm{~g} / \mathrm{s}$} & 80 mbar \\
\hline & & & & & 120 mbar \\
\hline
\end{tabular}

Table 4.8. Summarized results of velocity measurements performed at a distance of $30 \mathrm{~mm}$ from filter exit.

\subsubsection{Results and discussion}

First a set of indicative measurements was performed to assess the flow distribution at the exit of the inlet diffuser and the oxidation catalyst. The velocity measurements were carried out with a gas flow rate of the order of $45 \mathrm{~g} / \mathrm{s}$ at a distance of $15 \mathrm{~mm}$, which is approximately equal to the usual distance between the diffuser exit and the catalyst or filter inlet. The resulting velocity profile is presented in Figure 4.27. As expected, the flow is concentrated in the central region of the diffuser resulting to the formation of a core where the velocities are high. The inertia forces of the flow in combination with the diffuser geometry are responsible for this behaviour. The mean velocity is $20 \mathrm{~m} / \mathrm{s}$ and the calculated Reynolds number is of the order of 35,000 indicating that the flow in the inlet of the catalyst or filter is turbulent. Outside the core of the incoming flow, recirculation zones are known to exist that affect the 
flow and pressure distribution at the inlet of the catalyst or the filter that can be sensed also with the specific hot film sensor and layout.

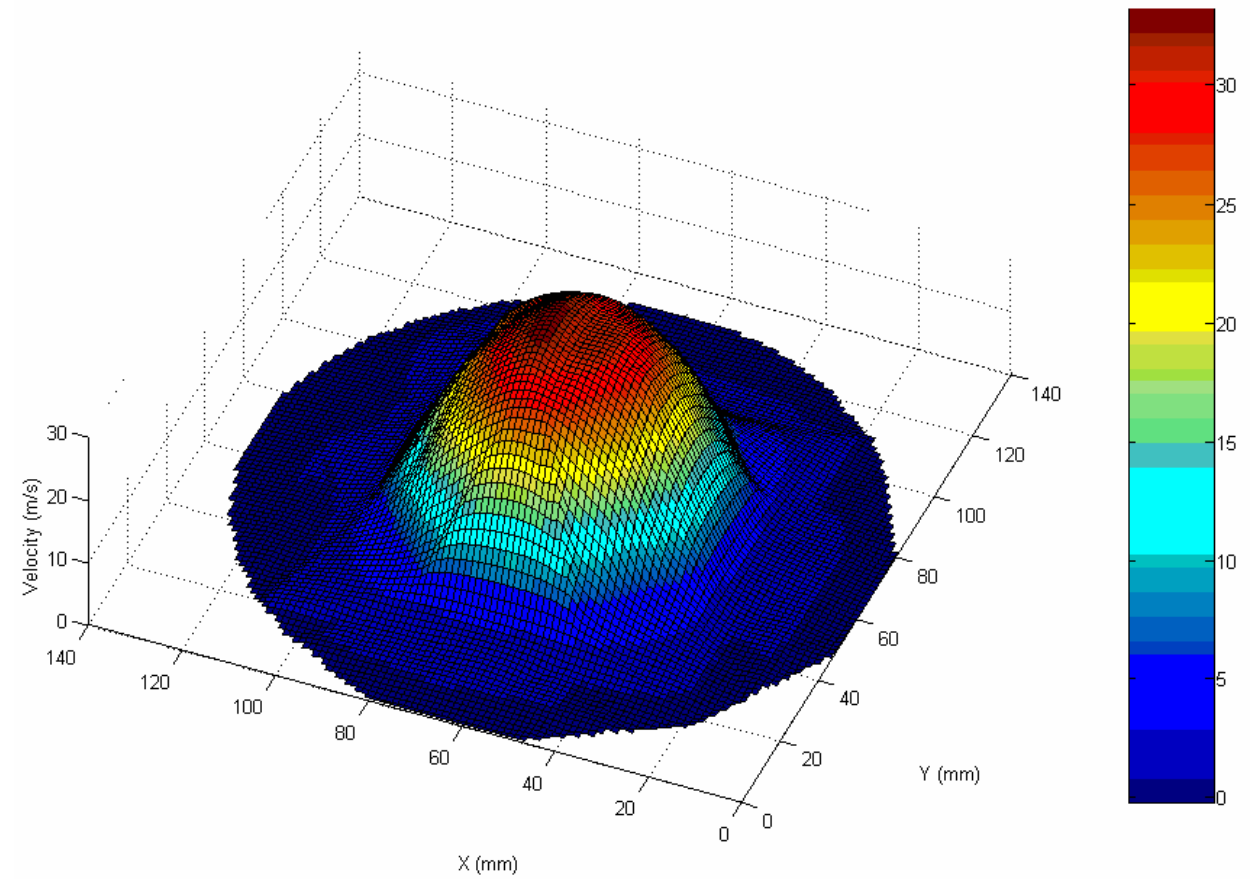

Figure 4.27 Velocity profile obtained at $15 \mathrm{~mm}$ downstream from exit of the diffuser with an exhaust gas mass flow rate of $45 \mathrm{~g} / \mathrm{s}$. Maldistribution factor: 0.12.

As next step the catalyst was assembled downstream the diffuser and velocity measurements downstream the catalyst exit, at a distance of $20 \mathrm{~mm}$ were performed with the same gas flow rate $(45 \mathrm{~g} / \mathrm{s})$. The resulting velocity profile is presented in Figure 4.28.

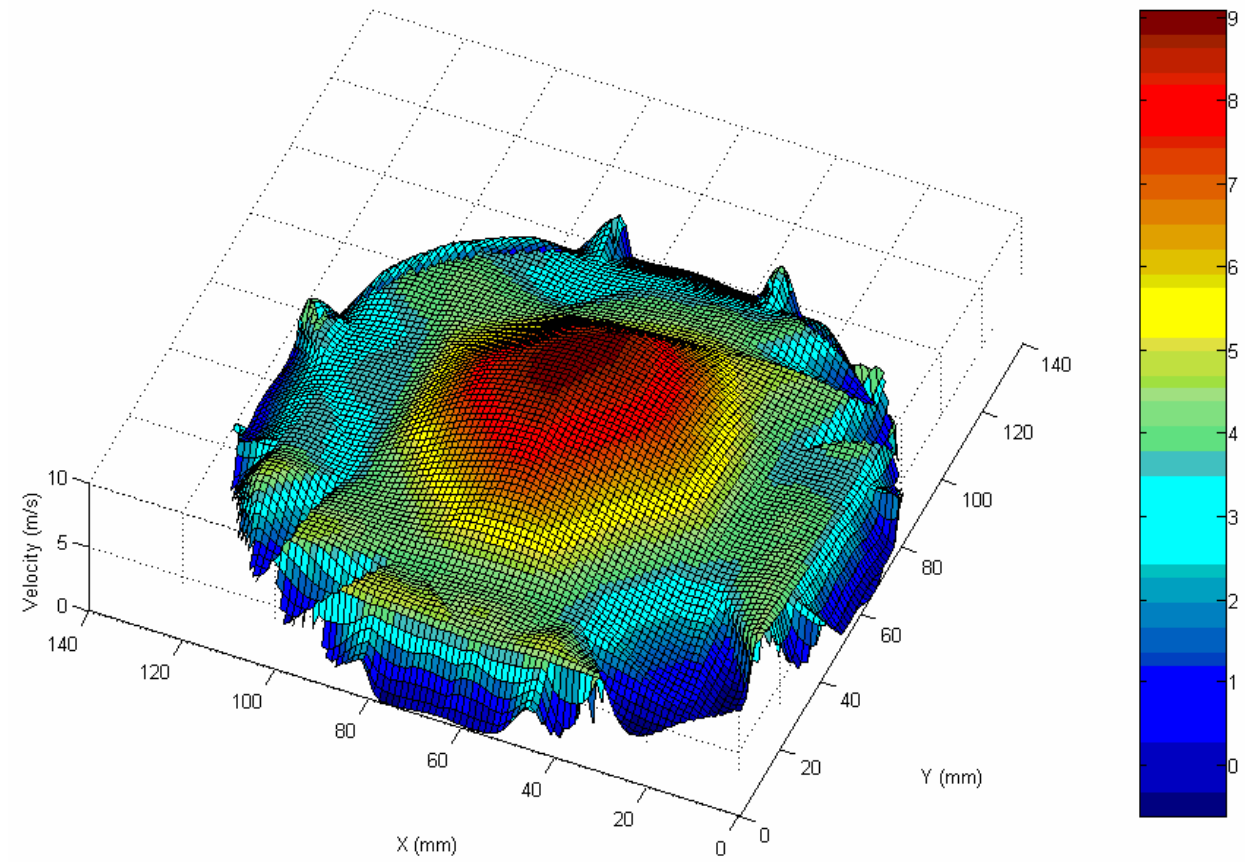

Figure 4.28 Velocity profile at $30 \mathrm{~mm}$ downstream from exit of a 180mm long catalyst. Exhaust gas mass flow rate: $45 \mathrm{~g} / \mathrm{s}$. Maldistribution factor: 0.71 . It should be mentioned that a certain degree of reverse flow is observed near the periphery. 
We could observe that the channels at the central part and at the periphery of the catalyst have higher mass flow rates than the channels in - between. The higher velocities are measured at the central region and the lowest at a radius about $80 \%$ of the catalyst radius. As mentioned above, this behavior is expected as a result of the upstream flow field that leads the incoming flow mainly through the central part of the monolith. Due to the interaction between the recirculating flow and the incoming flow a part of the gas is directed away from the center and is forced to flow through the outer channels of the catalyst. This may explain the higher velocities near the periphery of the catalyst. In the literature, the same type of behaviour was reported in catalytic converters by Holmgren et al [114] who report that the velocities near the periphery are not necessarily the lowest. Also the numerical study of Chacravarthy et al [115] predicts the appearance of high velocities at the outer channels of the catalyst as result of the recirculating flow at the inlet.

Flow measurements with the filter installed downstream a catalytic converter. Effect of filter material.

In this series of measurements, Cordierite and $\mathrm{SiC}$ filters were mounted alternately on the test rig with a catalytic converter in front. The measurements were carried out downstream the filters, at distances: 15, 30 and 80mm from filter exit face, with a mass flow rate of $45 \mathrm{~g} / \mathrm{s}$. The resulting velocity profiles for each type of filter at the distance of $30 \mathrm{~mm}$ are presented in Figure 4.29. Although the velocity profile obtained for each filter type is affected by the upstream catalytic converter, a more uniform flow is observed with case of a cordierite filter. The calculated maldistribution factors: 0.66 for cordierite versus 0.52 for SiC (see Table 4.9) confirm this observation.

In the case of a SiC filter, the design configuration with the separate blocks connected with cement, seems to be responsible for the severe flow maldistribution observed at the exit. It seems that each block behaves as an individual filter component with its flow field, which incorporates a region at the center of the block where the velocity is maximized while the lowest velocity is observed near the cement borderline. Thus, the total flow field of the SiC filter is composed of individual flow fields produced inside each filter block. Overall, the measured velocities with the Cordierite filter are lower than those measured with the SiC filter $\left(\mathrm{V}_{\text {mean }}=4.9 \mathrm{~m} / \mathrm{s}\right.$ for the case of cordierite filter vs $\mathrm{V}_{\text {mean }}=5.6 \mathrm{~m} / \mathrm{s}$ for the case of SiC filter). This could be attributed to the lower free flow area exposed by the SiC filter, due to the negligible permeability of the adhesive cement connecting the modules [60].

The results of velocity measurements along a filter diameter at 10 and $80 \mathrm{~mm}$ distance downstream filter outlet face are presented for Cordierite and SiC filters in the comparative charts of Figure 4.30. The velocity fields obtained very close to the filter exit demonstrate higher velocities near the periphery, indicating that the previously measured flow field at the exit of the catalyst is reversed as the flow passes through the filter channels. This could be attributed to the development of recirculation zones at the inlet section of the filter, which force the flow to the outer channels. However, as the measurement distance increases, the velocity differences between center and periphery are dampened and the flow profile becomes more uniform. The observation that the flow is made up of individual (laminar) jets that interact as the distance from the exit increases, seems to agree with of this behaviour. 

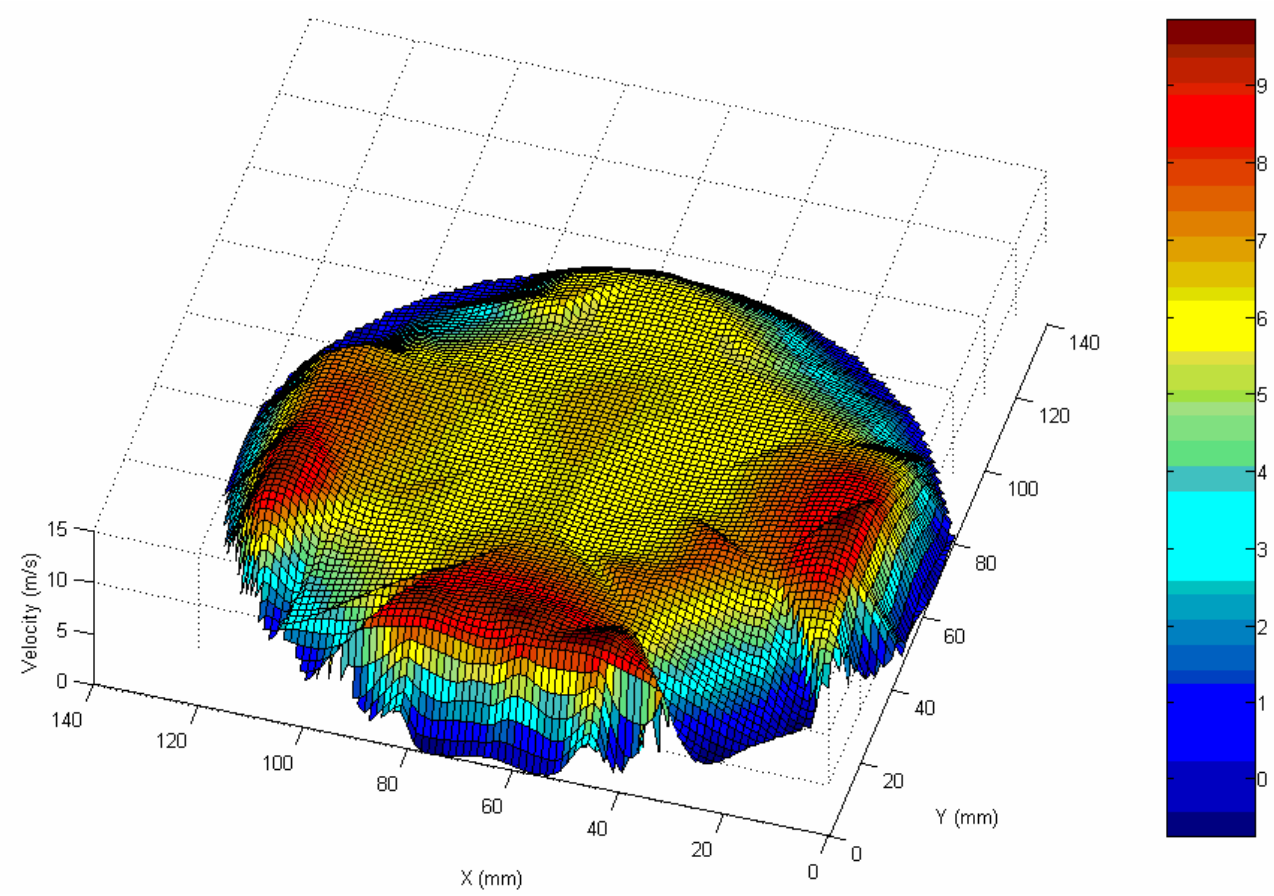

(a)
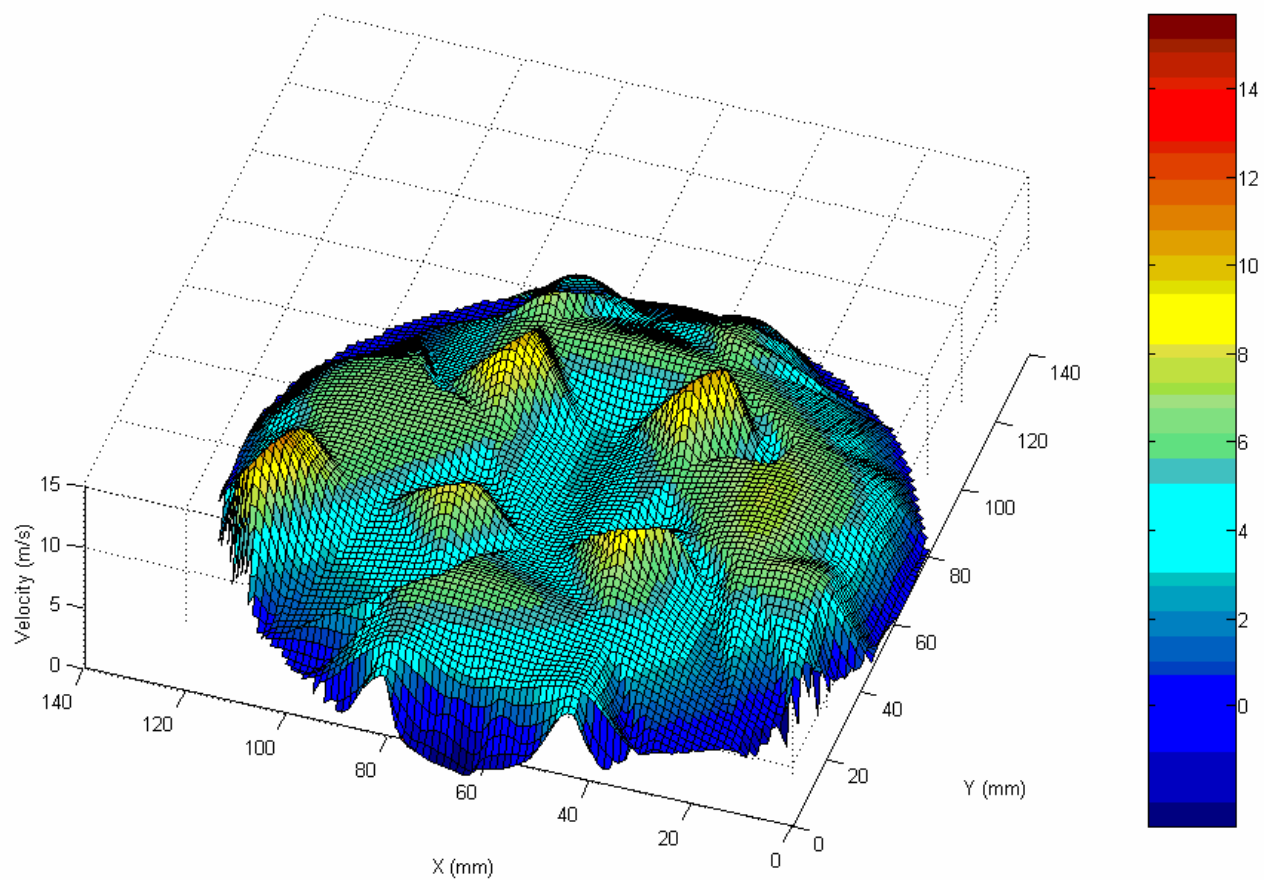

(b)

Figure 4.29 Velocity profiles at 30mm from exit of a $150 \mathrm{~mm}$ long cordierite (a) and a $152 \mathrm{~mm}$ long $\mathrm{SiC}$ filter (b). Exhaust gas mass flow rate: 45g/s. Maldistribution factor: 0.66 (a), 0.52 (b). A certain degree of reverse flow is observed near the periphery. 


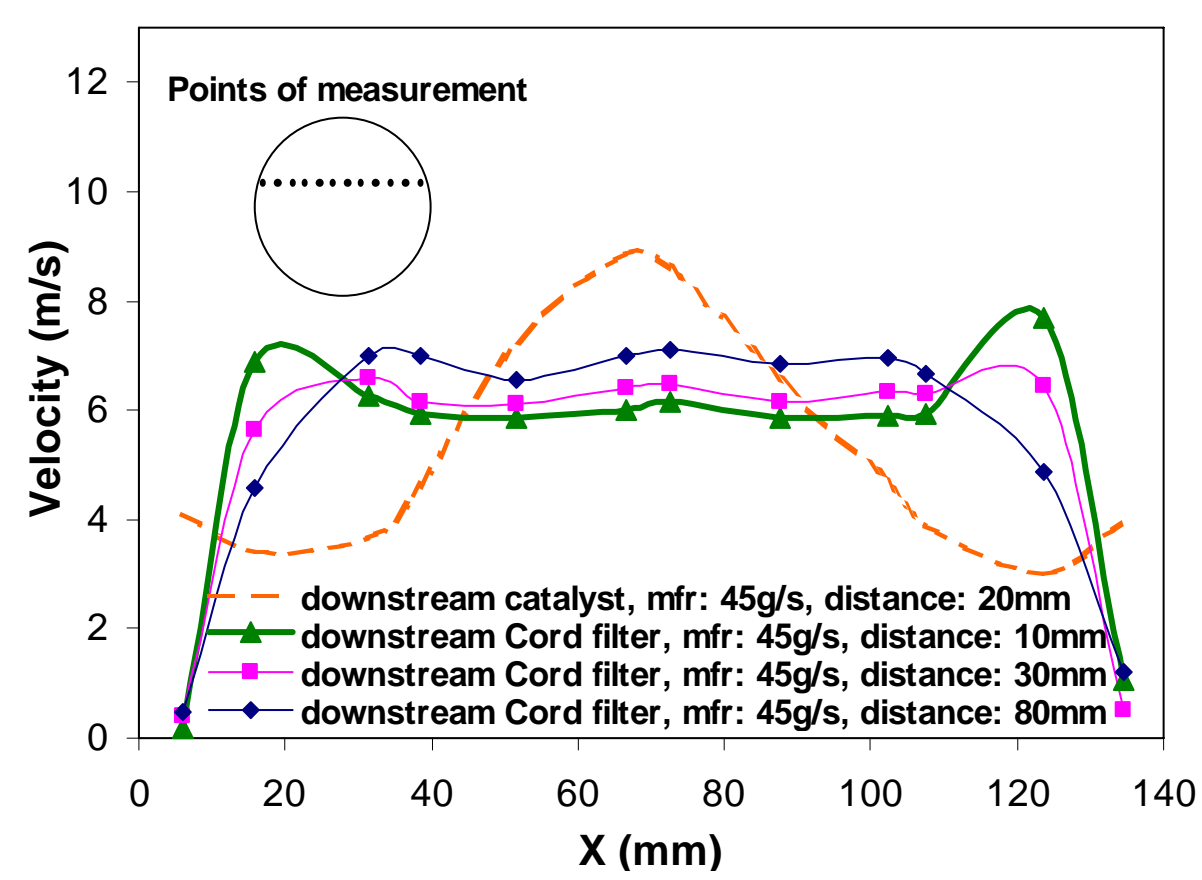

(a)

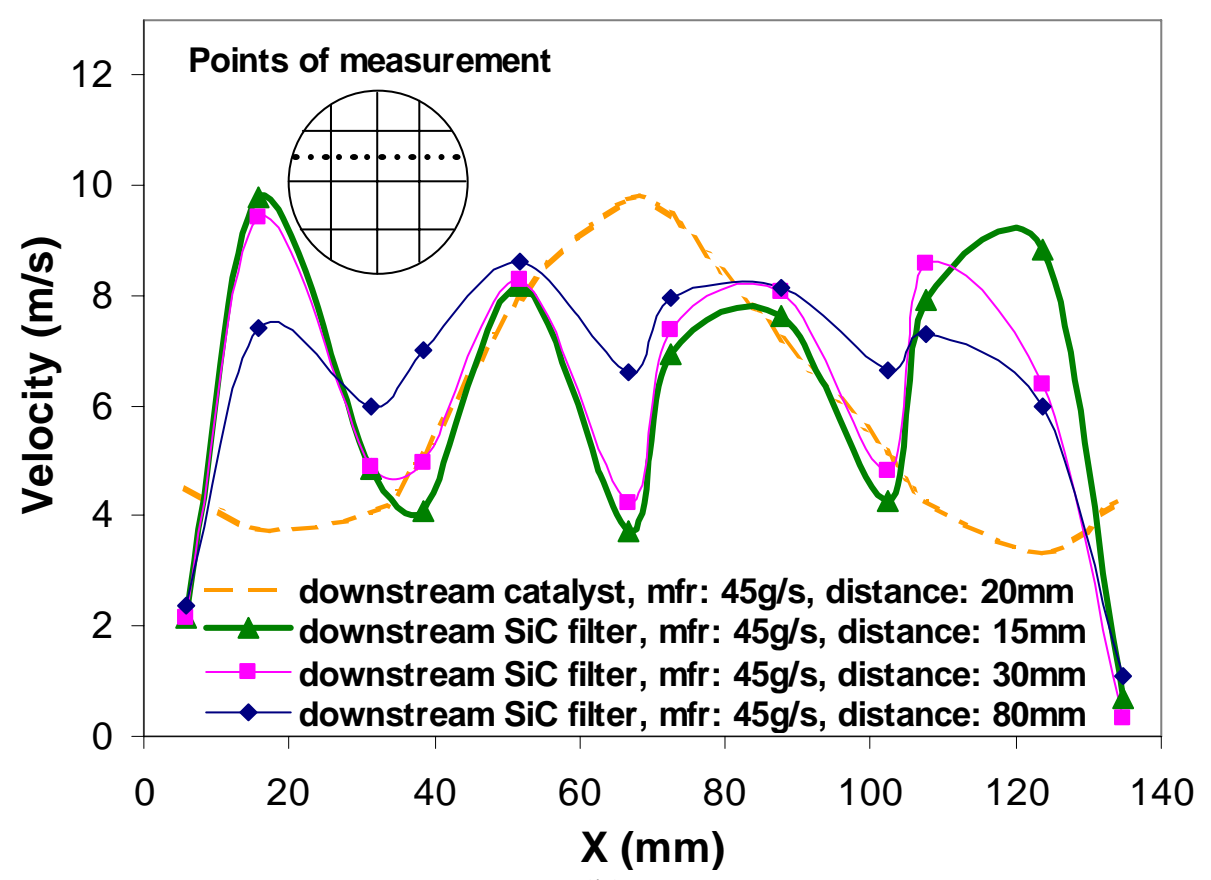

(b)

Figure 4.30 Comparative charts of the velocity measurements performed along a filter diameter at various distances downstream channels exit for the cases of cordierite (a) and SiC filter (b).

\section{Effect of filter length}

For the study of the effect of filter length, the velocity profile downstream a longer SiC filter with $254 \mathrm{~mm}$ length was measured. The other geometric characteristics of the filter were the same and the gas mass flow rate was kept unchanged $(45 \mathrm{~g} / \mathrm{s})$. The velocity profile measured is presented in Figure 4.31. 


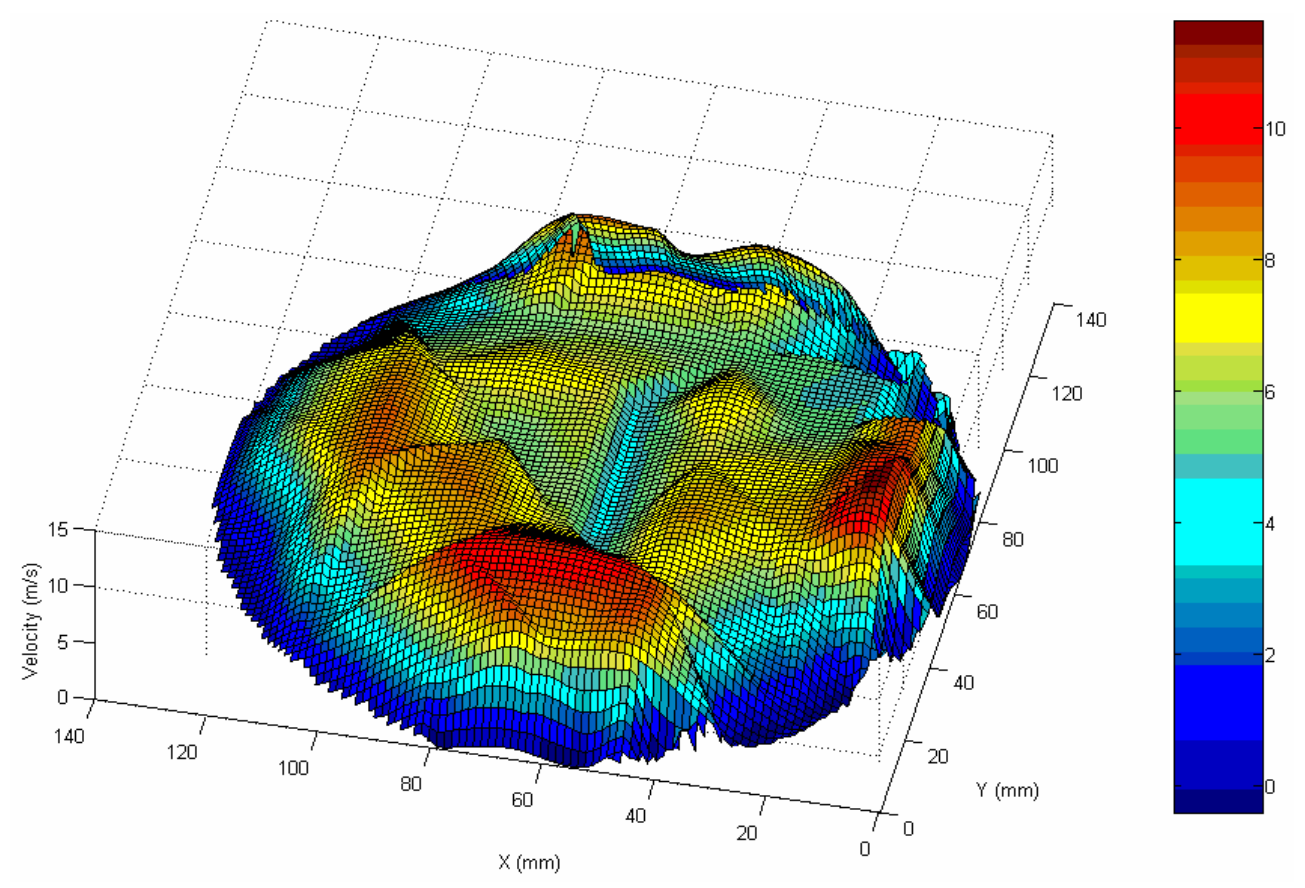

Figure 4.31 Velocity measurements at the exit of a $254 \mathrm{~mm}$ - long filter with a mass flow rate of $45 \mathrm{~g} / \mathrm{s}$. Maldistribution factor: 0.60. It should be noted that, as expected, the longer filter makes the flow more uniform (that is, it demonstrates lower maldistribution factor than the $150 \mathrm{~mm}$ filter).

By comparing it with the respective of Figure 4.29b for the $152 \mathrm{~mm}$ length filter, it can be observed that the shorter filter demonstrates higher velocities at the centers of the blocks. The more detailed comparative chart of Figure 4.32 confirms this observation, indicating that the maximum velocities measured at the center of each block are higher in the case of the shorter filter.

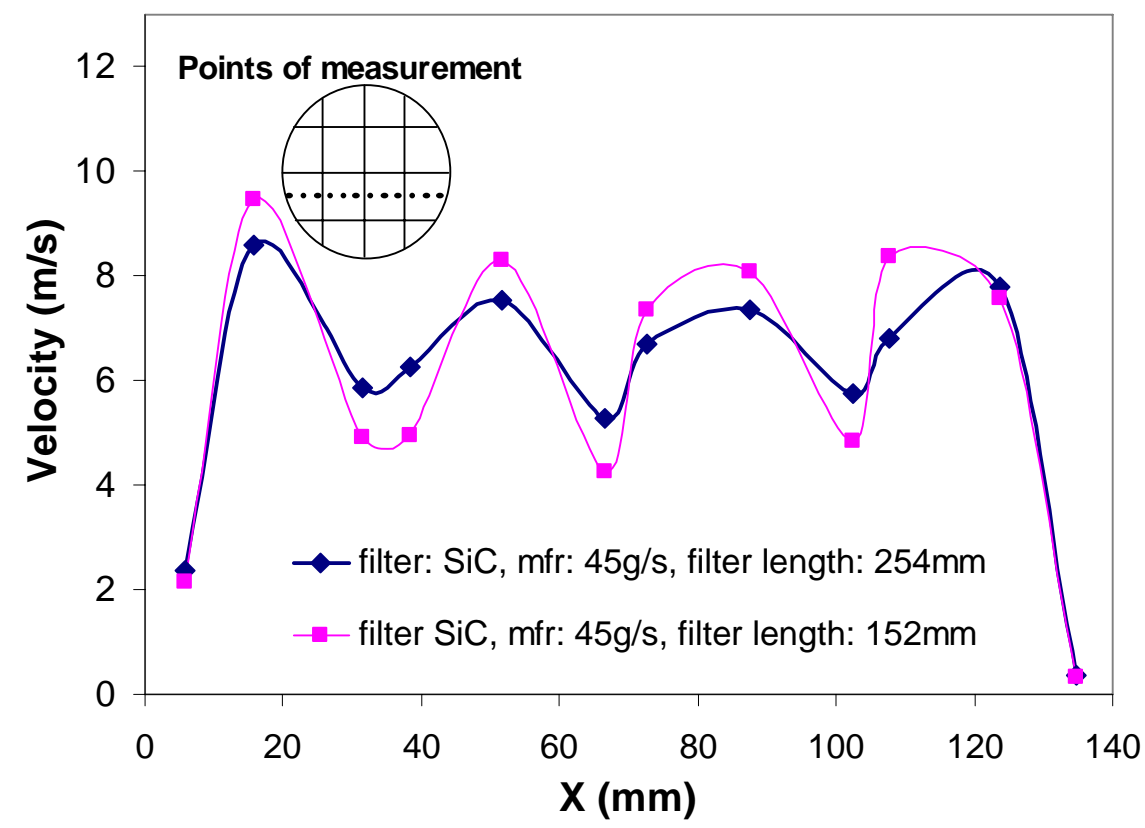

Figure 4.32 Comparative presentation of the velocity measurements performed along a filter diameter, for the cases of a short $(150 \mathrm{~mm})$ and a long $(254 \mathrm{~mm}) \mathrm{SiC}$ filter. The effect of filter length on the flow maldistribution is obvious in this figure. 
The calculated maldistribution factors: 0.52 for the case of $150 \mathrm{~mm}$ length filter vs 0.60 for the case of $254 \mathrm{~mm}$ length filter (Table 4.9) indicate that, as expected, the long filter produces more uniform flow. This behaviour could be attributed to the increased pressure drop as the channel length increases. The differences to the flow velocity are reduced due to the higher friction-losses and the pressure field causes the redistribution of the flow through the channels with lower pressure drop (channels with lower mass flow rate). Of course, the optimum channel length would depend also on the type of filter, geometry of channels as well as the material permeability etc.

\section{Effect of catalyst}

In order to investigate the effect of catalyst to the flow distribution inside the filter, the catalyst was removed and a new series of velocity measurements were performed with the $\mathrm{SiC}$ filter assembled directly downstream the inlet diffuser. The measurements were performed at a distance of $30 \mathrm{~mm}$ from channels exit, with a gas mass flow rate of $45 \mathrm{~g} / \mathrm{s}$. The resulting velocity profile is presented in Figure 4.33. By comparing it with the one obtained with the upstream catalyst installed (Figure 4.31), it is observed that in this case the flow is preferably driven through the central region of the filter with the maximum velocities measured at the center of the central blocks. A secondary zone with high velocities is observed near the filter periphery. Presumably due to recirculation zones at the inlet section, a part of the incoming flow is directed through the outer cells of the filter. In the intermediate area, reduced velocities are observed, with the minimum values measured near the cement borderlines between the outer and the central blocks.

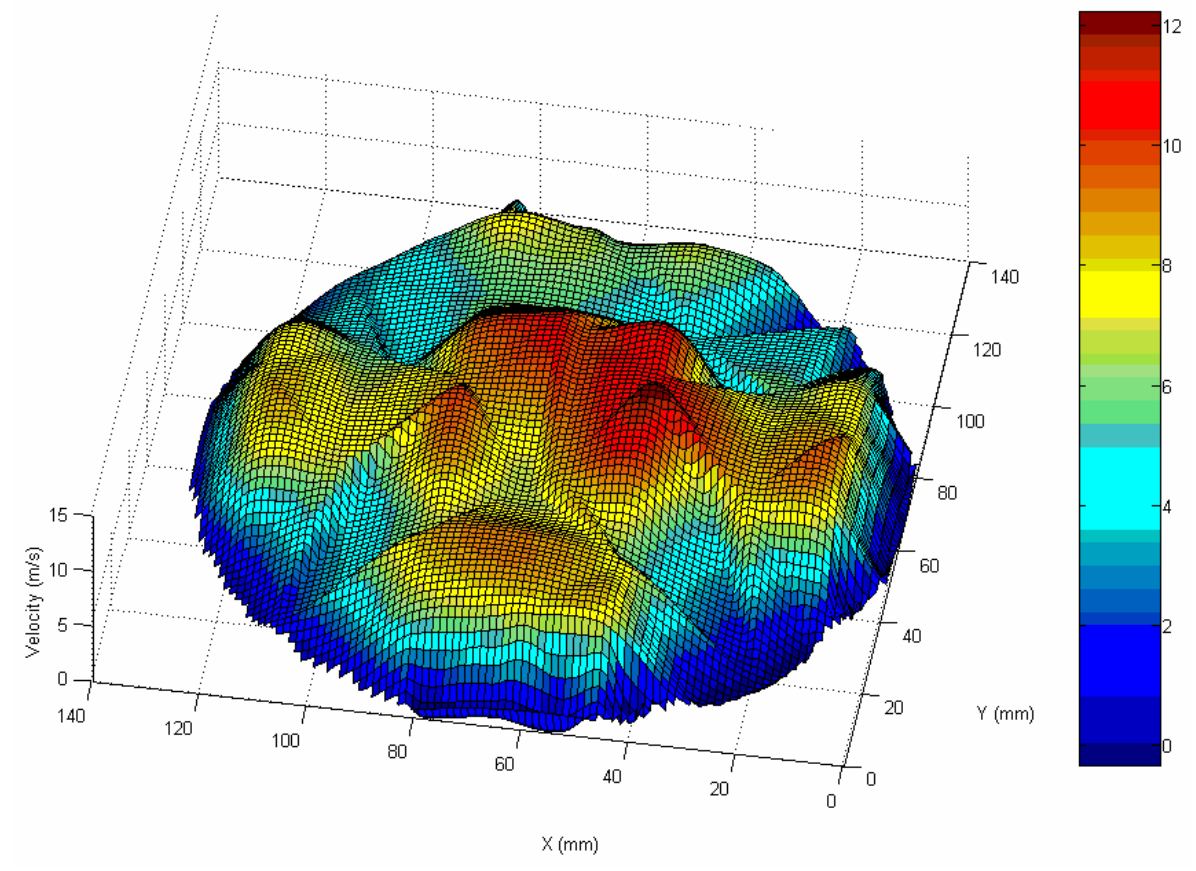

Figure 4.33 Velocity profile obtained at a distance of $30 \mathrm{~mm}$ from exit face of a $254 \mathrm{~mm}$ SiC filter. In this case, there is no catalyst in front of the filter. The maldistribution index takes a lower value than with the catalyst in front of the filter (0.54 instead of 0.6). Higher velocities are measured in the central core region. Exhaust gas mass flow rate: $45 \mathrm{~g} / \mathrm{s}$.

The calculated maldistribution factor 0.54 , versus that of 0.6 for the case of catalyst in front, (Table 4.9), indicates that the presence of the catalyst distributes the flow more uniformly. As mentioned above, the interaction between the pressure and the flow field inside the catalyst channels results to an equalizing effect to the flow distribution. Thus, the initial 
flow maldistribution due to the inlet diffuser effect may be partly compensated by the use of the catalyst in-between. However, as presented in the comparative chart of Figure 4.34, the presence of the catalyst has also a reverse effect on the velocity distribution. The velocities are maximized near the periphery in contrast with the no-catalyst configuration where the maximum velocities are appeared at the central region.

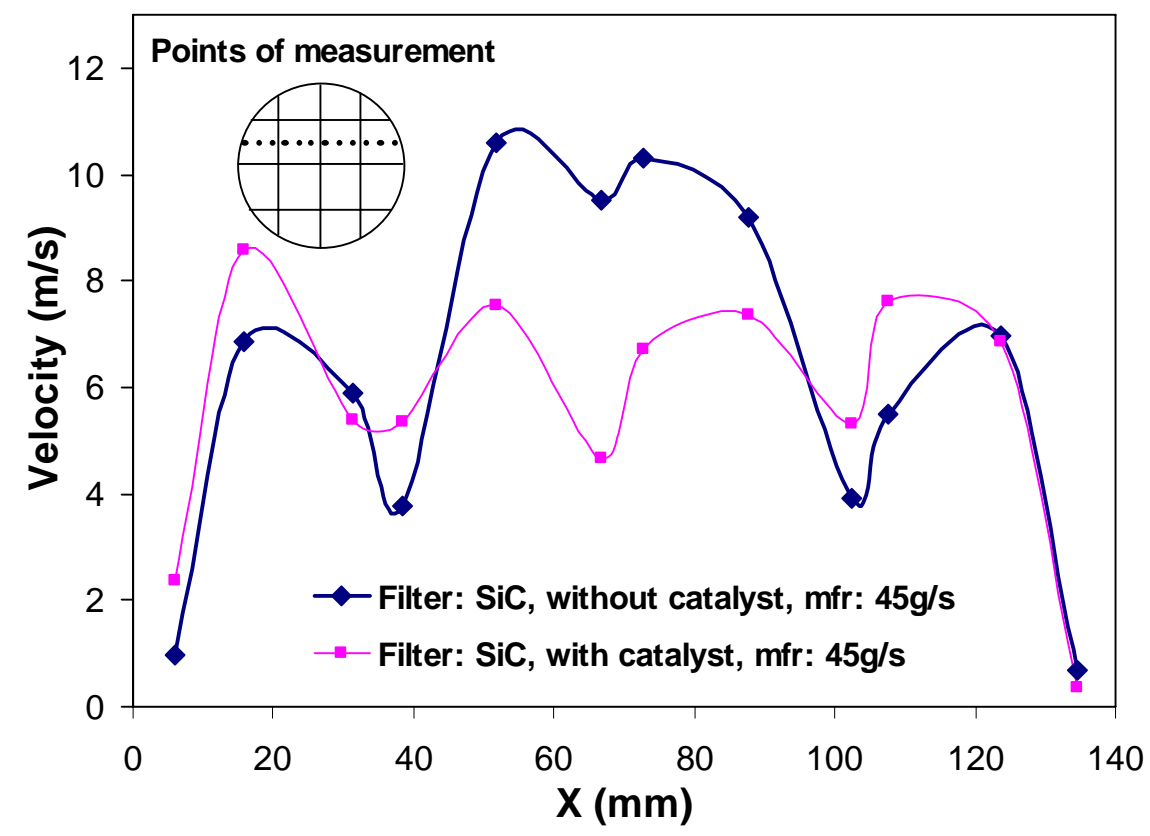

Figure 4.34 Comparative presentation of velocity measurements performed across the exit channels of a SiC filter: with and without catalyst installed between diffuser and filter. The effect of the catalyst in the flow equalization is obvious in this figure. However, there continue to exist significant velocity variations across each one of the four unit filters.

\section{Effect of gas mass flow rate}

The measurements of the flow velocity at the exit of the SiC filter (with the catalyst in front) were repeated with the increased mass flow rate of $60 \mathrm{~g} / \mathrm{s}$. The resulting velocity profile at the distance of $30 \mathrm{~mm}$ is presented in Figure 4.35 . By comparing it with the velocity profile obtained with the flow rate of $45 \mathrm{~g} / \mathrm{s}$ (Figure 4.31) it is concluded that the qualitative characteristics of the flow field is not affected by this variation of the gas mass flow rate. However, the resulting value of the calculated maldistribution factor is now lower: 0.57 vs 0.6 (Table 4.9), indicating that the flow maldistribution becomes worse with the increased flow rate. Based on the processing of the experimental results presented in the comparative chart of Figure 4.36, the differences between the maximum and the minimum velocities appeared to be larger for the case of the increased flow rate. The increased inertial forces of the flow are considered to be responsible for this behavior. 


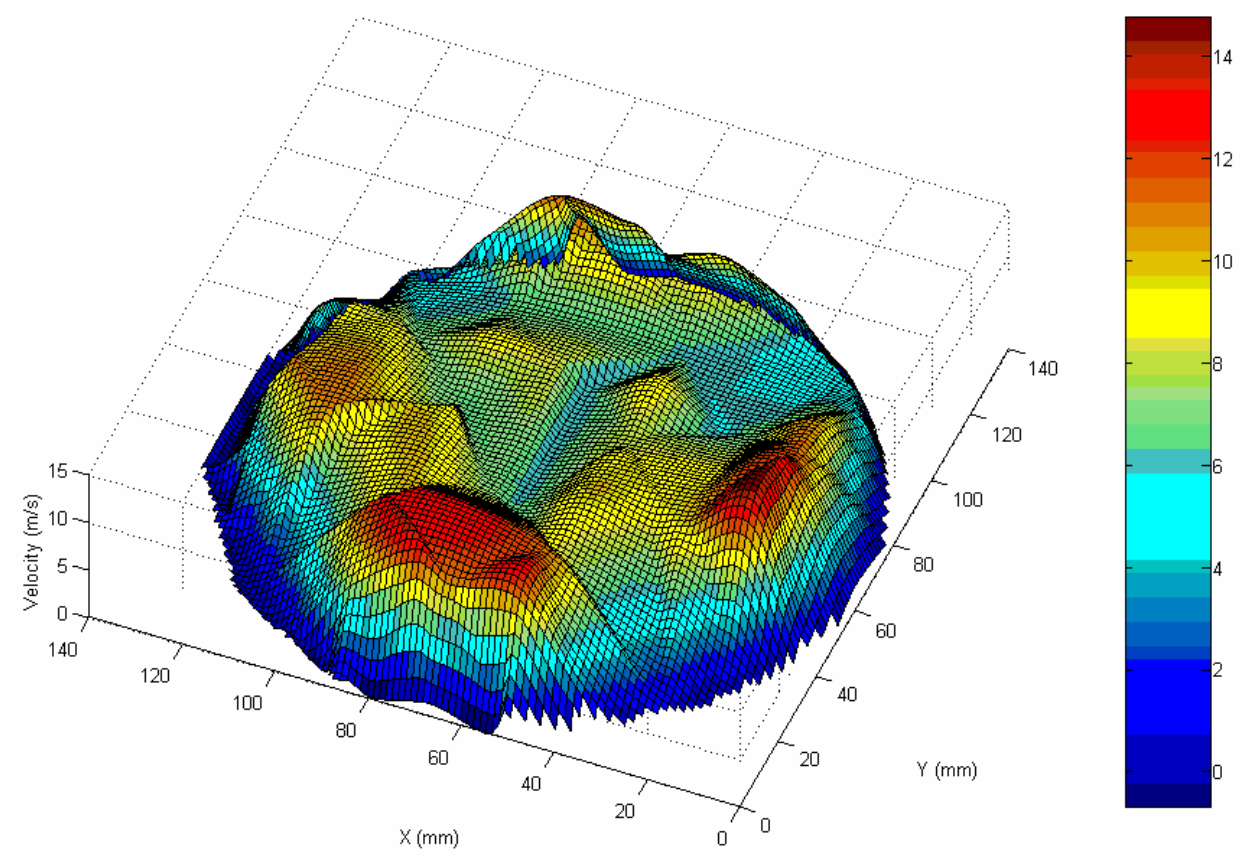

Figure 4.35 Velocity profiles at $30 \mathrm{~mm}$ from exit of a $254 \mathrm{~mm} \mathrm{SiC} \mathrm{filter} \mathrm{with} \mathrm{increased} \mathrm{exhaust} \mathrm{gas} \mathrm{mass} \mathrm{flow}$ rate $(60 \mathrm{~g} / \mathrm{s})$. Maldistribution index:0.57 A certain maldistribution deterioration with exhaust flowrate increase is observed.

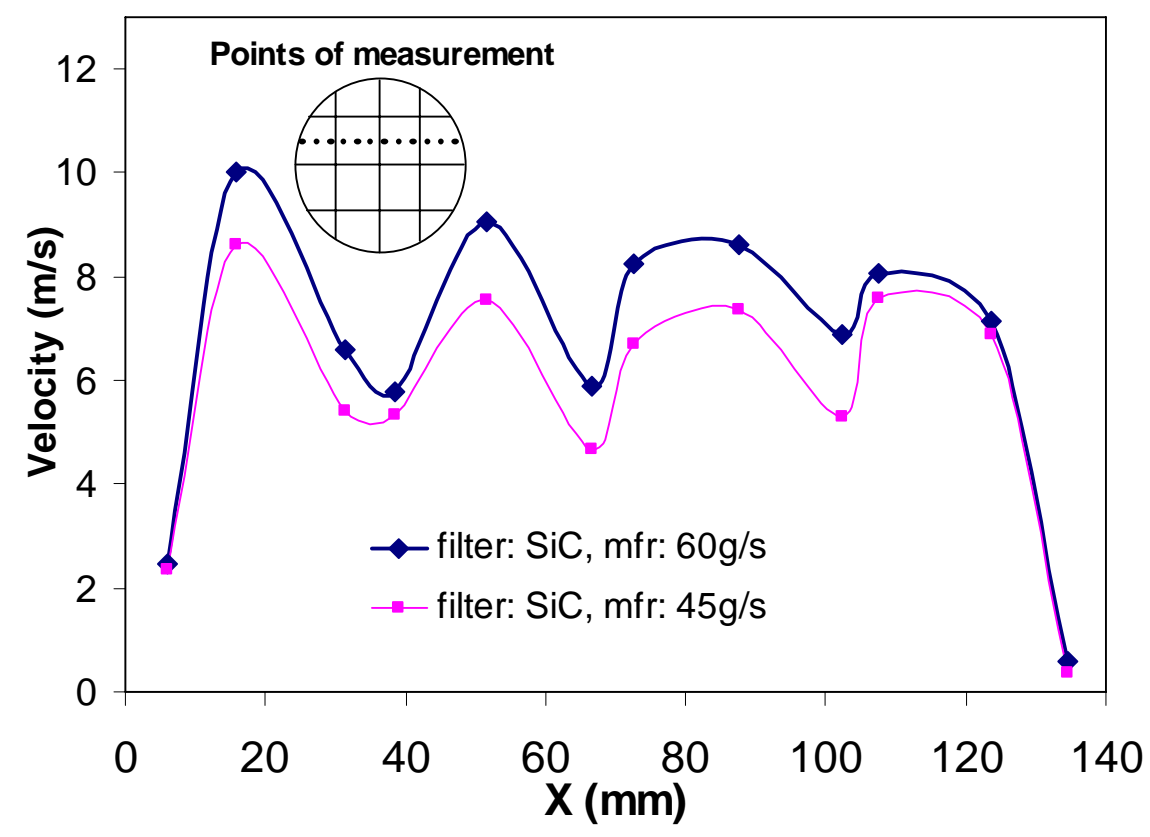

Figure 4.36 Comparative presentation of velocity measurements performed along a filter diameter with a medium $(45 \mathrm{~g} / \mathrm{s})$ and an increased $(60 \mathrm{~g} / \mathrm{s})$ exhaust gas mass flow rate.

\section{Effect of soot loading level}

The study of the parameters affecting the flow distribution inside the filter is completed with measurements performed to a loaded $\mathrm{SiC}$ filter. The filter is loaded with the engine running on a medium operation point (speed: 2600rpm, load: 40Nm, exhaust gas mass flowrate: $45 \mathrm{~g} / \mathrm{s}$ ), up to the following two soot loading levels: 


\begin{tabular}{|l|l|l|}
\hline Final back pressure (mbar) & Wall temperature, center & Wall temperature, periphery \\
\hline 80 & 280 & 260 \\
\hline 120 & 300 & 285 \\
\hline
\end{tabular}

The velocity measurements where performed on the test rig with the same gas mass flow rate $(45 \mathrm{~g} / \mathrm{s})$ at the distance of $30 \mathrm{~mm}$ from channels exit. The resulting profiles for both cases are presented in Figure 4.37. As can be seen, a crater appears at the central region of the filter flow field in the case of loading up to $80 \mathrm{mbar}$. This crater indicates that the flow resistance through the central region is higher, presumably due to the higher accumulated soot mass in the central channels. However, according to the measured flow field in the case of the clean filter it would be expected that due to the higher velocities near the periphery, the soot loading in this region would be higher. The observed behavior could be partly attributed to the role of the temperature field inside the filter that is not taken in account here (the measurements are performed with ambient air instead of exhaust gas) and may differentiate the shape of the flow field due to the variations to the fluid viscosity between center and periphery. Under real operating conditions the higher temperatures in the central region of the filter lead to an increase in viscosity that affects (increases) the flow velocity. The opposite phenomenon may be observed near the colder periphery leading to a reduction of flow velocity. The volatile organic fraction (VOF) of the thick particulate layer could also affect this behavior. The VOF of the particulate layer has been calculated to vary from about $6 \%$ at the central to $9 \%$ at the circumferential channels [72] and may be oxidized at temperatures below $250^{\circ} \mathrm{C}$. Thus, stochastic regenerations could take place at specific points of filter periphery where the soot loading, composition and temperatures are favorable. These slow regenerations could decrease flow resistance through the periphery allowing the observed increase of velocity through this area.

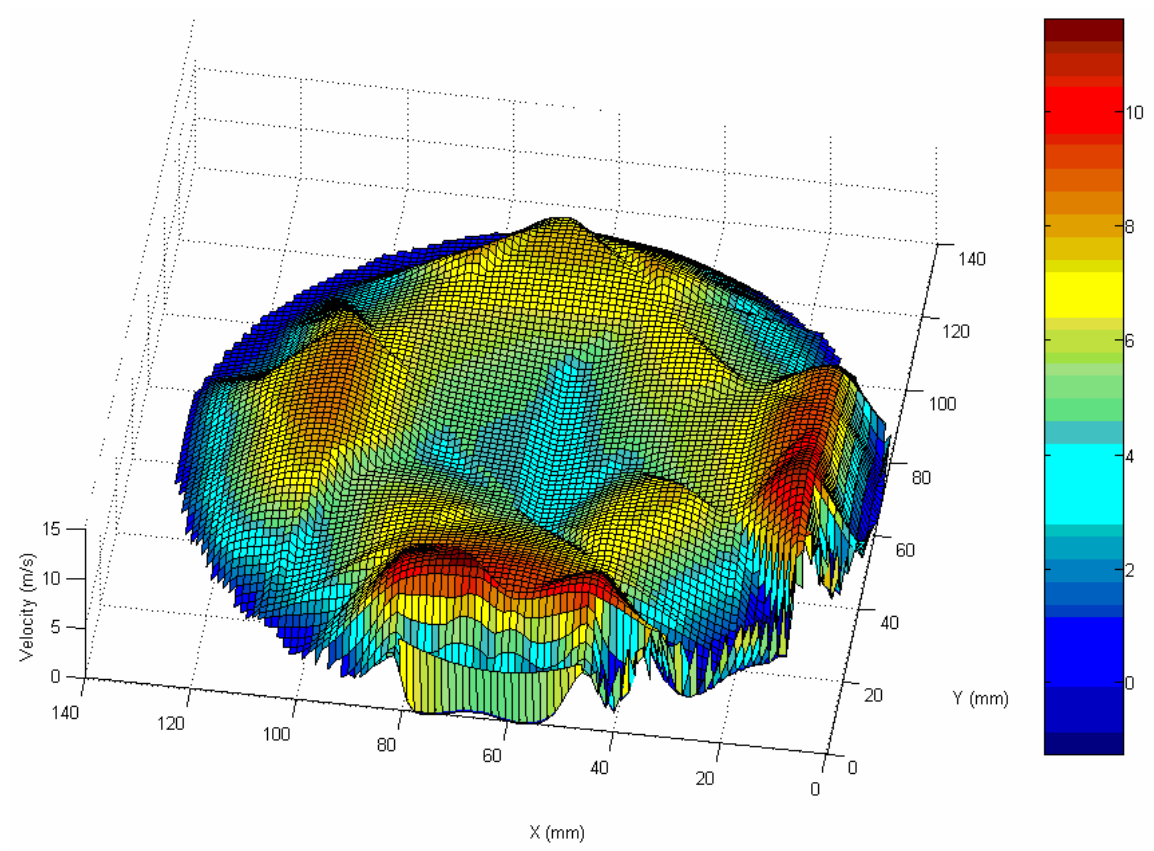

(a) 




(b)

Figure 4.37 Velocity profiles at 30mm from exit of a loaded $254 \mathrm{~mm} \mathrm{SiC} \mathrm{filter.} \mathrm{Flow} \mathrm{maldistribution} \mathrm{is}$ evaluated with two different loading levels: 80 (a) and 120 mbar (b). The measurements were performed with a mass flow rate of 45g/s. Maldistribution factors (0.61 (a) and 0.63 (b)) are improved compared to the clean filters.

With increasing filter soot loading up to the level of $120 \mathrm{mbar}$ (Figure 4.37b), the previously observed crater disappears and a more uniform flow field is established. The continuous redistribution of soot and flow profile that takes place as the accumulated mass increases and is triggered by the effects of the temperature field [106] is considered to be responsible for this complex behavior.

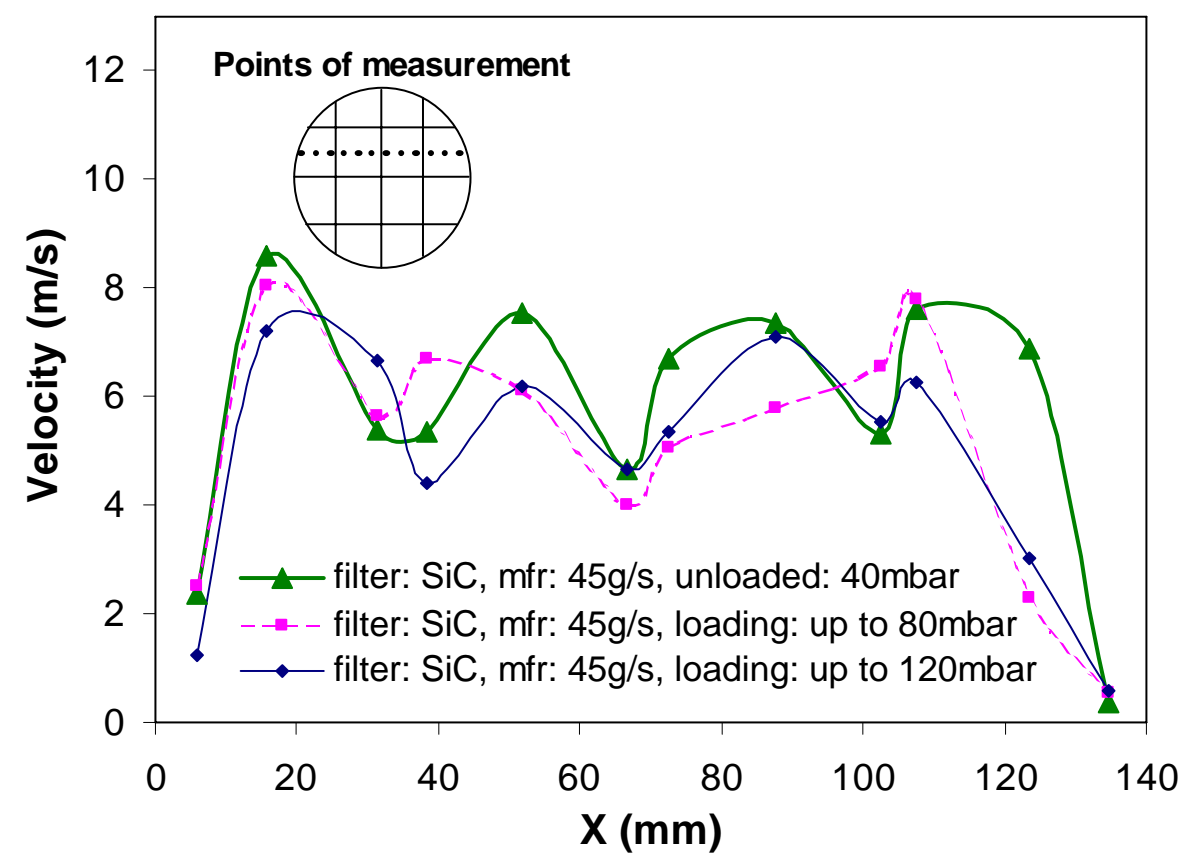

Figure 4.38 Comparative presentation of the velocity measurements performed along a filter diameter in the cases of: clean filter, filter loaded up to $80 \mathrm{mbar}$ and filter loaded up to $120 \mathrm{mbar}$ (250mm SiC filter in all cases). 
The velocity measurements along a filter diameter presented in the comparative chart of Figure 4.38 indicate that the velocities near the periphery are systematically higher than those at the central area, which is mainly influenced by the variations to the soot loading profile. The reduction of the mean flow velocity (Table 4.9) depends on the pressure drop characteristics of the soot layer formed on the channel wall (layer thickness - permeability.

Based on the calculated maldistribution factors for both cases ( 0.61 for the $80 \mathrm{mbar}$ - loading case and 0.62 for the 120 mbar - loading case) we could conclude that soot accumulation partly compensates the initially observed maldistribution. But the overall exhibited behavior is quite complex.

Table 4.9 Summarized results of velocity measurements performed at a distance of $30 \mathrm{~mm}$ from filter exit.

\begin{tabular}{|c|c|c|c|c|}
\hline $\begin{array}{c}\text { Name of studied } \\
\text { parameter }\end{array}$ & 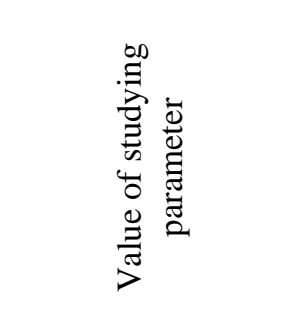 & 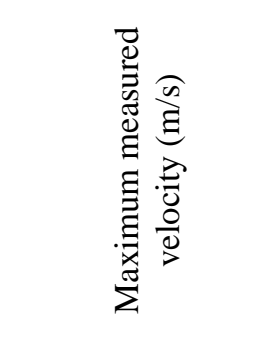 & 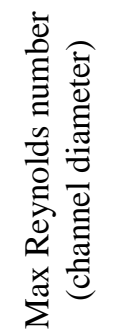 & 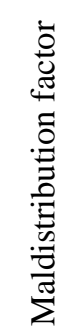 \\
\hline \multirow{2}{*}{ Filter type } & Cordierite (17/100) & 9.2 (Periphery) & 0.93E3 & 0.66 \\
\hline & $\operatorname{SiC}(14 / 200)$ & 13.8 (Periphery) & $1.41 \mathrm{E} 3$ & 0.52 \\
\hline \multirow{2}{*}{ Filter geometry } & $150 \mathrm{~mm}$ & 13.8 (Periphery) & $1.41 \mathrm{E} 3$ & 0.52 \\
\hline & $254 \mathrm{~mm}$ & 11.0 (Periphery) & $1.12 \mathrm{E} 3$ & 0.60 \\
\hline \multirow{2}{*}{ Presence of catalyst } & No catalyst & 12.2 (Centre) & $1.24 \mathrm{E} 3$ & 0.54 \\
\hline & Catalyst in front & 11.0 (Periphery) & $1.12 \mathrm{E} 3$ & 0.60 \\
\hline \multirow{2}{*}{ Gas mass flow rate } & $45 \mathrm{~g} / \mathrm{s}$ & 11.0 (Periphery) & $1.12 \mathrm{E} 3$ & 0.60 \\
\hline & $60 \mathrm{~g} / \mathrm{s}$ & 13.7 (Periphery) & $1.40 \mathrm{E} 3$ & 0.57 \\
\hline \multirow{2}{*}{ Soot accumulation } & 80 mbar & 10.9 (Periphery) & 1.11E3 & 0.61 \\
\hline & 120 mbar & 10 (Periphery) & $1.02 \mathrm{E} 3$ & 0.62 \\
\hline
\end{tabular}

\subsubsection{Concluding remarks}

- A specially designed test rig for cold flow velocity distribution experiments has been developed in order to measure flow maldistribution phenomena in wall flow ceramic diesel particulate filters.

- The effects of the following design parameters are tested: filter material, (SiC vs Cordierite), filter length, (150mm vs $250 \mathrm{~mm}$ ), presence of catalyst in front of the filter.

- The effects of the following operation variables were also tested for the SiC filter: Exhaust gas mass flow rate (45 vs $60 \mathrm{~g} / \mathrm{s}$ ) and soot loading level. The results indicate that the flow field at the exit of the filter is complex and presents significant flow 
maldistribution that must be taken into account in any attempt to model filter loading and regeneration behaviour. This is true not only for the clean filter but also for the loaded one.

- As expected, the flow maldistribution is significantly influenced by the presence of a diffuser and a catalytic converter upstream the filter. The influence depends on the specific design of each component.

- The specific geometry and design of the filter significantly influence flow maldistribution. Thus, a more uniform flow was observed in the case of a monolithic cordierite filter. On the other hand, a SiC filter made up from 16 individual blocks connected with cement demonstrated a worsened flow distribution.

- An increase in the length of the SiC filter from 150 to $250 \mathrm{~mm}$ resulted in a better flow distribution.

- The presence of a catalytic converter in front of the filter resulted in a more uniform flow distribution at the filter level. Moreover, a certain degree of reversal of the velocity profile, with somewhat higher velocities near the periphery was observed, especially with the cordierite filter.

- An increase of the gas mass flowrate did not affect the qualitative velocity profile. However, it resulted in a marked decrease of the calculated maldistribution factor (worsening of flow distribution).

- The accumulation of soot inside the filter channels tends to make the flow more uniform. This process is slower and more complex than usually anticipated. During the process, the flow is continuously redistributed. The same is true for the soot profile across the filter channels.

- Better understanding of these phenomena will require significant additional experimental study. Future investigations should include the following additional measurements: Hot flow measurements, pressure field measurements and turbulence intensity measurements

\subsection{Soot Deposition Characteristics as Function of Engine Operation Point and Filter Loaded History.}

In this section the soot deposition morphology and characteristics inside the channel on $\mathrm{SiC}$ filters are investigated with the use of electronic microscopy (SEM). A novel procedure was developed in order to be able to study the filter in the SEM at various points along the channels and measure the soot layer thickness. Based on the soot layer thickness measurements, a calculation of the density of the collected soot for the specific engine operation point and loading was attempted.

\subsubsection{Experimental}

A novel procedure has been developed for the microscopic study of soot deposition characteristics on SiC filters. The procedure is schematically described in Figure 4.39. 

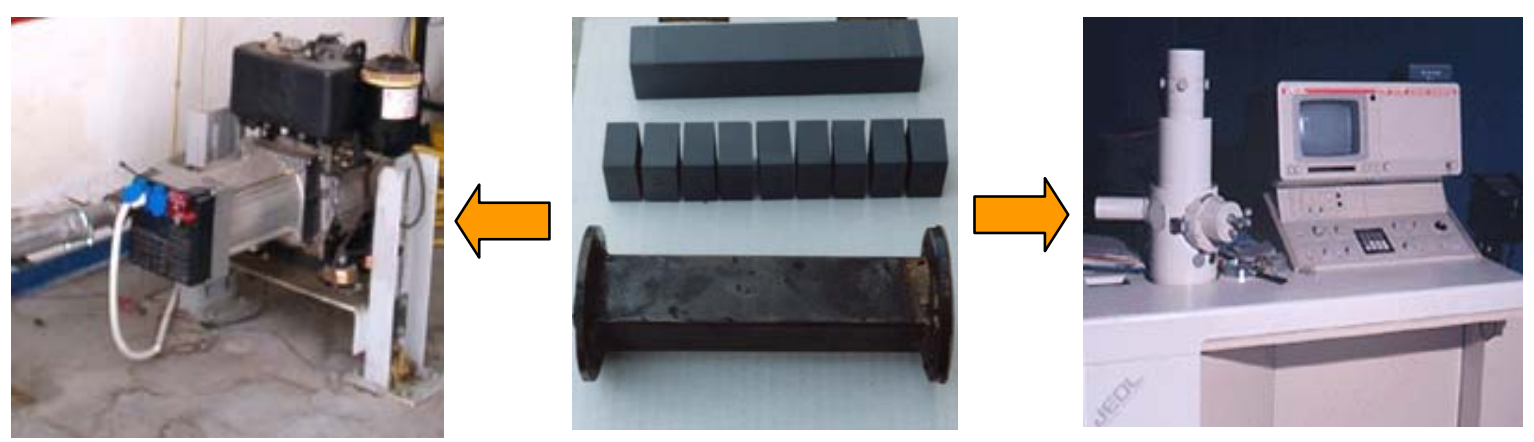

Figure 4.39 Experimental procedure.

The procedure is based on the slicing of a unit filter in 9 individual pieces of equal length. The pieces are numbered and fitted together again, in a special canning device that ensures their hermitic connection and the minimization of the gas leaks during operation. Thus, a filter is produced that can be easily dismantled to pieces that can be inspected on the SEM. The specially engineered unit filter is installed on a single cylinder DI diesel engine (0.5 l displacement), where the loading and regeneration experiments can be carried out. The engine and filter specifications are presented in Table.4.10.

The filter was loaded with the engine running on the medium operation point of speed: 2400rpm, load: $12 \mathrm{Nm}$ with 25ppm Ce doped fuel. The presence of the catalyst inside the particulate made the soot layer observable in the SEM without the need of other type of processing that may change the chemical or deposition characteristics of the layer. On the other hand the presence of the catalyst was expected to give us a lot of information about the catalysed soot deposition characteristics. SEM images were acquired with a Jeol 5310 Scanning Electron Microscope in the secondary electron mode with an accelerating voltage in the range of 10 to $25 \mathrm{KV}$.

Table.4.10 Engine technical data and diesel filter specifications

\begin{tabular}{|l|l|}
\hline Engine manufacturer & RUGGERINI \\
\hline Engine type & DI 1cylinder engine \\
\hline Displacement & $497 \mathrm{~cm}^{3}$ \\
\hline Rated power /rpm & $5.5 \mathrm{~kW} / 4000 \mathrm{rpm}$ \\
\hline Rated torque/rpm & $25 \mathrm{Nm} / 2400 \mathrm{rpm}$ \\
\hline Diesel Filter Manufacturer & Ibiden \\
\hline Filter Type & SiC $14 / 200 \mathrm{cpsi}$ (cells per square inch) \\
\hline Filter Dimensions (WxHxL) & $34 \mathrm{~mm}$ x 34mm x $150 \mathrm{~mm}$ \\
\hline Cell pitch & $1.89 \mathrm{~mm}$ \\
\hline Filter Wall thickness & $0.4 \mathrm{~mm}$ \\
\hline
\end{tabular}

\subsubsection{Results and discussion}

As first step SEM photos of a virgin filter piece were taken in order to be employed as reference point to the study of soot deposition. The photos of Figure 4.40 show a cross section of the virgin filter and a close up of the ceramic wall substrate where the $\mathrm{SiC}$ microstructure is observed. The characteristic uniform pore distribution of the SiC material reported by other researchers is also confirmed here [111, 116]. This is the main reason for the lower pressure losses of $\mathrm{SiC}$ filters in comparison with Cordierite, which is characterized by a more 
amorphous structure. However as it can be seen the passages through many of the large pores are very narrow indicating that the pores have small contribution to the filtration. This means that depth filtration mechanism is weakened in this filter material.

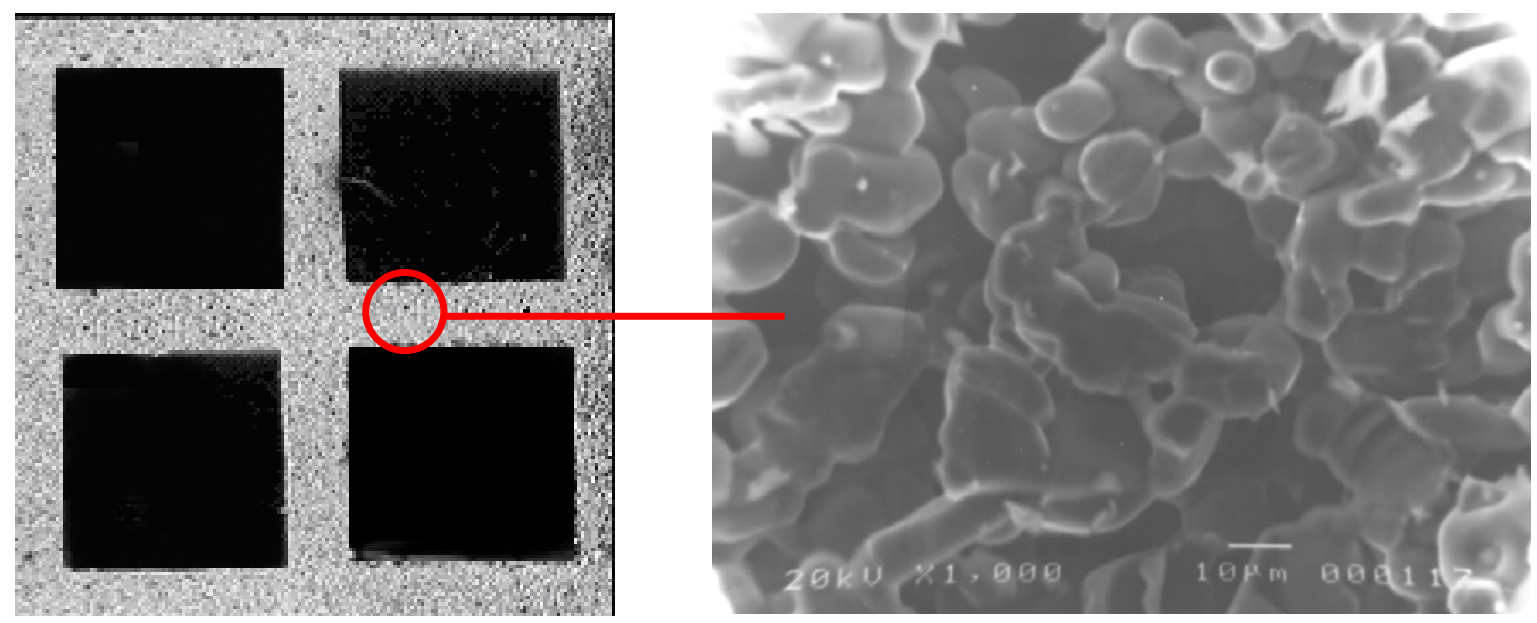

Figure 4.40 Scanning electron micrographs of a virgin filter piece

As next step loaded filter pieces of the filter device were inspected in the SEM. The loading was performed at the specific engine operation point with a total duration of 9 hours divided in 3 equal phases with the engine and filter to be cooled before the beginning of each phase. According to the particulate emissions of the engine at this point ( $3 \mathrm{~g} / \mathrm{hr}$ ) an approximate mass of 25 grams was collected. After the end of the loading phase, the filter was dismantled from the exhaust system, and the nine pieces have been separated and examined in the SEM. A number of photographs have been taken, that are presented below. Figure 4.41 presents a typical photo of a loaded central channel taken near the middle section of the ceramic. The following could be observed:

- The soot layer has a uniform distribution across the four edges of the channel wall. This indicates that the gas flow is equally divided to the four walls of the channel. However as presented below the same is not observed in the case of channels placed near the filter periphery revealing the role of the 3D effects.

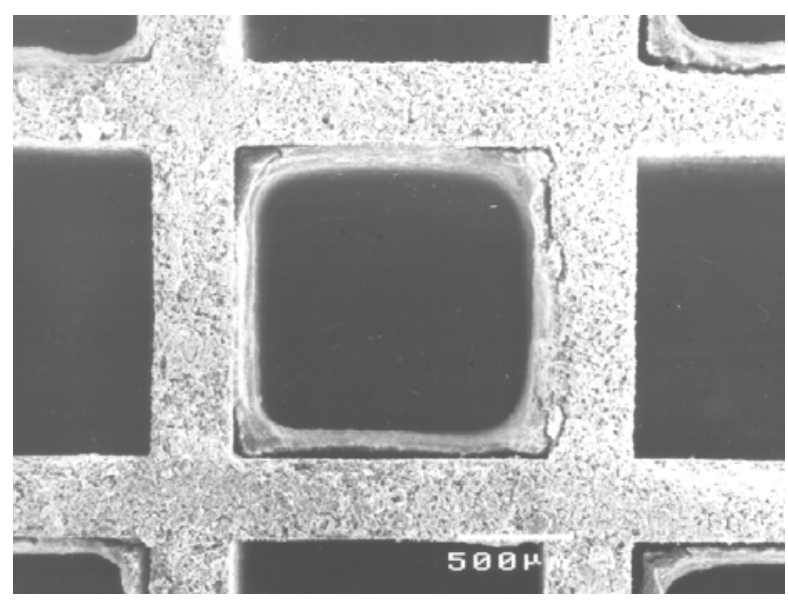

Figure 4.41 SEM micrograph of a loaded central channel of a middle piece of the segmented filter. 
- The shape of the soot layer becomes oval at the corners. This results to the increase of the accumulated mass at the corners. This information may be useful in the design and the modelling.

- The soot layer seems to be detached from the wall. This finding is in contrast with other published SEM photos. For example, we mention, for comparison, a scanning electron micrograph of a slice of a loaded DPF published by PSA and IFP researchers (Figure 4.42 [103]). One could assume that the detachment happens during the cooling of the filter due to the drying of the soot that makes the layer stiffer.

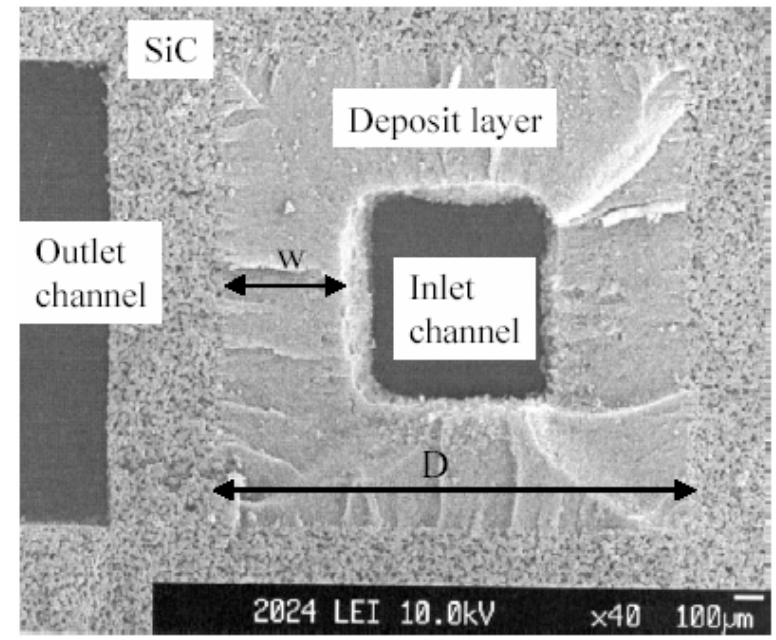

Figure 4.42 SEM micrograph of a slice of a heavily loaded DPF (reproduced from [[103]]).

One more micrograph of a peripheric channel located near the filter corner is presented in Figure 4.43. A non-uniform distribution of the soot across the four channel edges could be observed. This could indicate that 3 dimensional effects of the flow maldistribution are important even in this small sized filter.

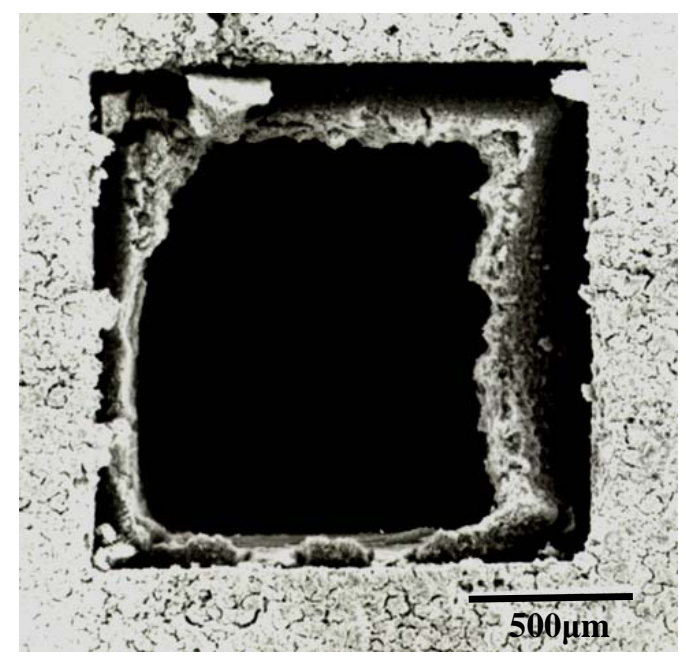

Figure 4.43 SEM micrograph of a loaded peripheric channel of a middle piece of the segmented filter.

A higher flowrate through the central region of the filter could be the cause of the increase of the accumulated soot mass on the two adjacent channel walls, which are oriented in the 
direction of the central core. As a general conclusion, the special characteristics of the exhaust flow can be traced on the deposited soot layer, differentiating its shape and thickness. Again, a certain degree of detachment between the soot layer and the ceramic wall is observed.

A close up of a layer cross section is presented in Figure 4.44. In this figure, three distinct, consecutive soot layers can be observed. They have been produced by the carrying out of the loading in the three consecutive phases. The separation of the layers indicates that the quality of the soot differentiates from the one to the other loading phase. This is attributed to the stochastic nature of the engine particulate emissions that among others are influenced by the ambient conditions (air temperature and humidity). The variation to the engine emissions results to a variation to the catalyst concentration of the particulate that makes observable the separate layers.

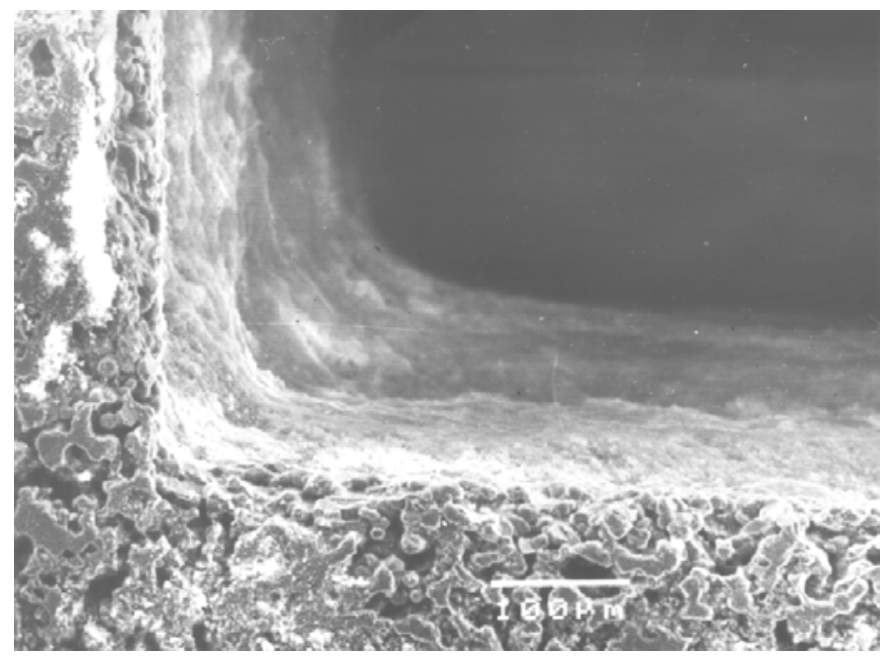

Figure 4.44 Soot layers (various loadings)

The interface between the ceramic wall (left) and the soot layer (right) can be observed in the micrograph of Figure 4.45. A certain degree of intrusion of the soot particles inside the pores of the ceramic can be observed. However the soot is mainly accumulated on the cell wall indicating that the prevalent mechanism is the surface filtration, which is the largest contributor to the pressure losses during loading.

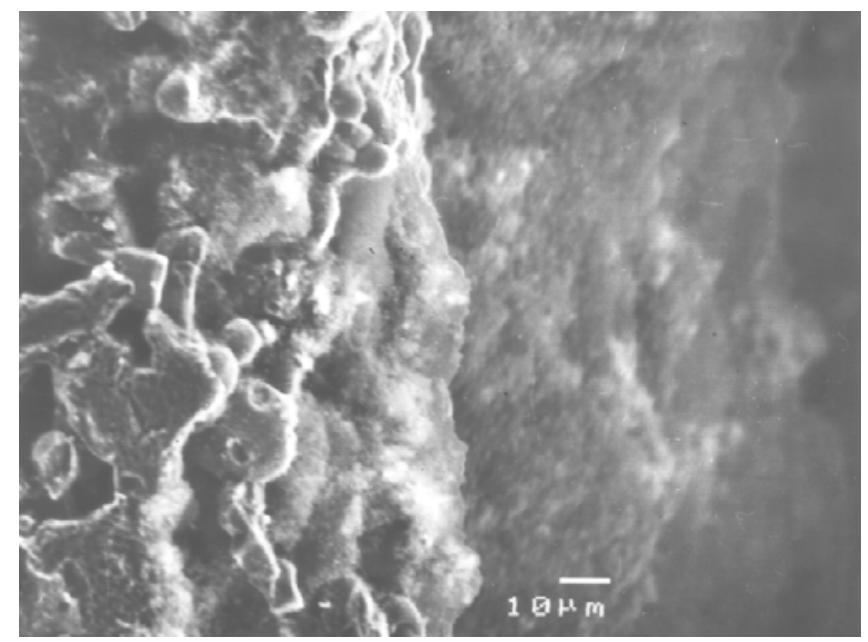

Figure 4.45 Boundary soot layer-ceramic substrate 
The soot particles that have intruded in the SiC structure can be observed in Figure 4.46 (in bright white colour). They are attached on the much bigger amorphous porous ceramic granular structure. Responsible for this is the flow that pushes the particles on the stationary ceramic particles. The degree of intrusion depends on the morphology characteristics of the ceramic substrate. The correlation between the soot particles size distribution and the porosity and pore size of the ceramic is thought to have significant role to the filter pressure drop. Although the increase of the pore size results to lower pressure drop for a clean filter, this effect may be counterbalanced in the case of the loaded filter due to the lower porosity of the soot layer.

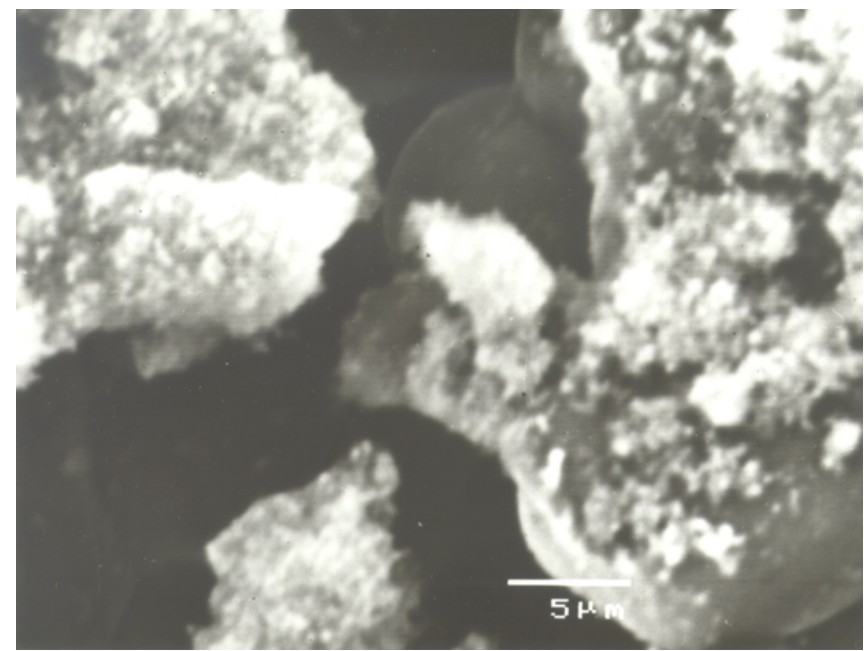

Figure 4.46 Soot intrusion inside the ceramic pores.

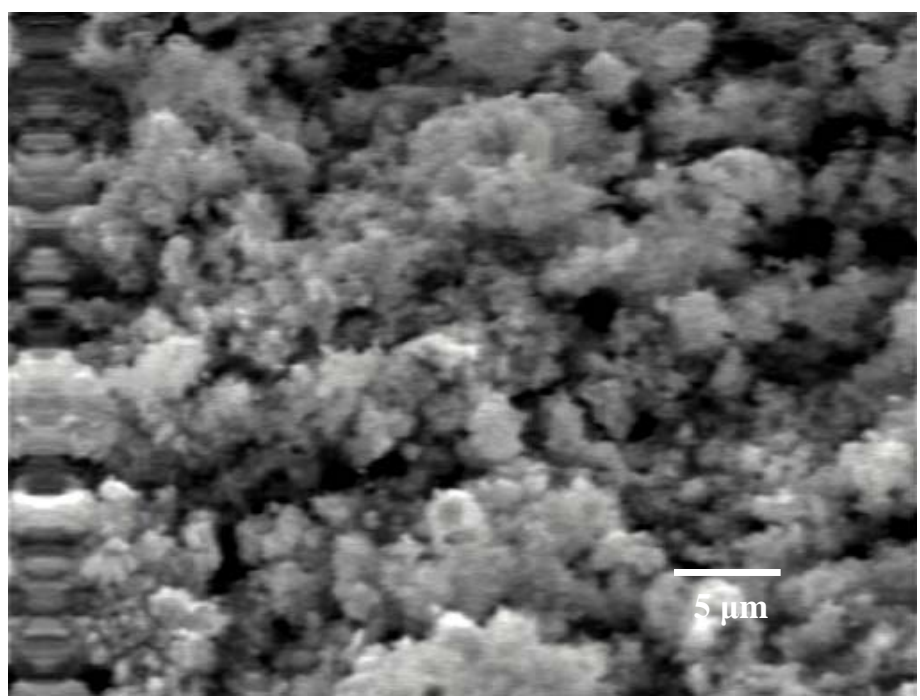

Figure 4.47 SEM micrograph of the soot deposited on the channel wall

The soot layer morphology observed with SEM presented in Figure 4.47 reveals two main classes of particulates: 
- The first class consists of fine mode particles that are almost spherical with an approximate diameter of the order of $0.5 \mu \mathrm{m}$. These particles originate from the nucleation of the primary carbon and volatilised fuel and oil species in the combustion chamber.

- The second class consists of clusters of catenulate aggregates that in turn composed of the previous described spherical fine mode particles. The primary particles are adhered together constituting agglomerated clusters with an approximate size of the order of 2.5 $\mu \mathrm{m}$. These agglomerates are usually broken off after a certain degree of growing from the deposition of the small primary particles onto the surface.

By comparing the micrographs of figure 4.47 and figure 4.46 it is concluded that the pores of the soot layer are smaller than those of the ceramic substrate. This fact is responsible for the lower permeability of the soot layer which as mentioned in the previous section is affected by the accumulated soot mass. The higher soot mass results to lower permeability indicating that the pores become narrower due to the compression of the layer.

Finally, the soot layer distribution along the filter channel is presented. The thickness of the layer along a central channel was measured by processing the micrographs of the nine individual segments. The results are presented in Figure 4.48. It can be seen that the soot layer thickness increases near the inlet and the plugged end of the channel. The maximum value was measured at the end of the channel while the minimum was measured at the middle section of the channel. The results indicate that the soot distribution along the filter channel is nonuniform. We are not aware of analogous investigations in the literature to compare with.

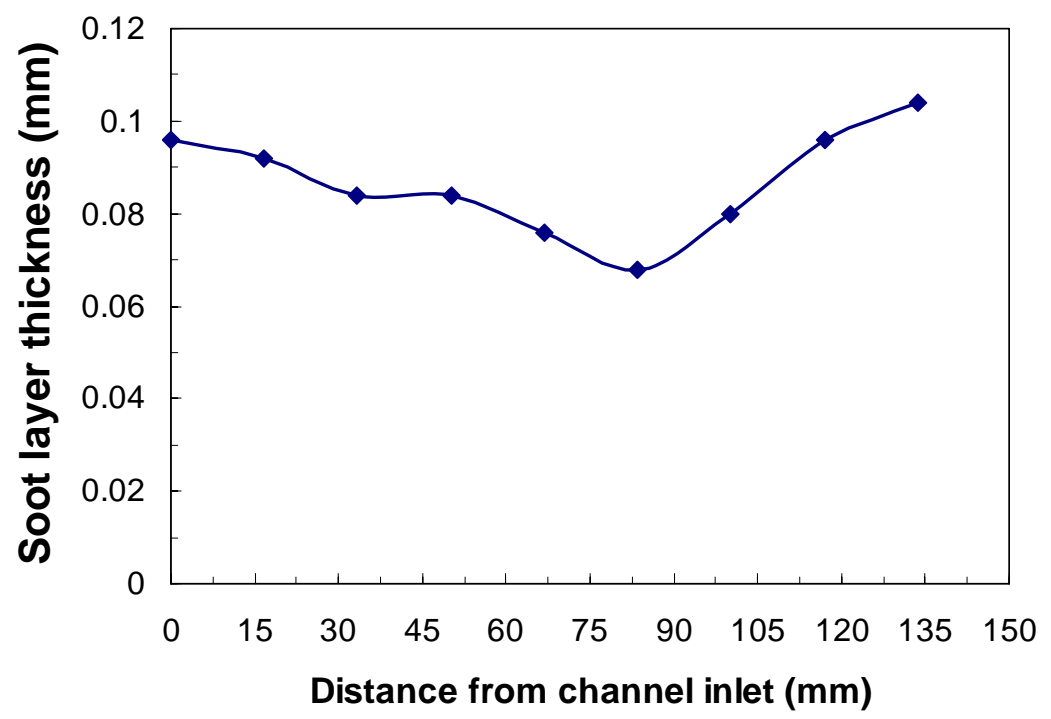

Figure 4.48 Soot layer thickness along a central filter channel.

Based on the soot layer thickness measurements, a calculation of the density of the collected soot was attempted. The mass of the accumulated soot is measured by weighing the filter pieces. The volume of the accumulated soot mass can be defined from the measurement of the soot layer thickness assuming linear variation along the filter channels. However the accurate calculation of soot density makes necessary the measurement of the soot layer thickness in all the channels due to the existence of soot maldistribution between the centre and periphery. Here we present this calculation for the third filter piece, which, as can be seen from the diagram of figure 4.48, appears equal distribution at the central channels. It is assumed that the layer thickness does not significantly vary from the centre to periphery. This 
is a realistic assumption for our experimental case due to the low filter dimensions that limit the effects of the $3 \mathrm{~d}$ phenomena. The results are presented in the next table:

\begin{tabular}{|c|c|c|c|c|c|}
\hline $\begin{array}{c}\text { Soot mass } \\
(\mathrm{kg})\end{array}$ & $\begin{array}{c}\text { Layer thickness } \\
(\mathrm{m})\end{array}$ & $\begin{array}{c}\text { Layer length } \\
(\mathrm{m})\end{array}$ & $\begin{array}{c}\text { Number of } \\
\text { channels }\end{array}$ & $\begin{array}{c}\text { Volume } \\
\left(\mathrm{m}^{3}\right)\end{array}$ & $\begin{array}{c}\text { Density } \\
\left(\mathrm{kg} / \mathrm{m}^{3}\right)\end{array}$ \\
\hline $0.5 \mathrm{E}-3$ & $0.08 \mathrm{E}-3$ & $16.7 \mathrm{E}-3$ & 162 & $5.34 \mathrm{E}-6$ & 93.6 \\
\hline
\end{tabular}

\subsubsection{Concluding remarks}

The SEM findings can be summarized as follows:

- The soot layer is not uniform across the different channels. However, this could be affected by the specific (segmented filter) layout employed in the loading.

- A longitudinal variation of the soot layer thickness was also found: The inlet and outlet sections present a higher layer thickness than the interior sections.

- Detachment of the layer from the wall was observed in many cases. It must be investigated whether this resulted from the segments dismantling procedure, or from shrinking of the soot layer after cooling out and drying of the filter.

- The accurate measurements of soot thickness taken from the SEM pictures are employed in the more accurate determination of the density of collected soot. 


\section{Comparative Discussion}

This thesis concerns further investigation on yet unsolved issues in catalytic regeneration and pressure drop characteristics of wall-flow diesel filters. It focuses on the catalytic regeneration with Ceria fuel additive, which is currently in use in hundreds of thousands of diesel passenger cars, but its main findings could be useful in the study of other fuel additives (Manganese, Ferrocene, Copper etc). The investigations carried out in the frame of this thesis are focused to assist the design optimisation of the ceramic filter itself, the fuel additive dosing device and the post-injection system.

Now, detailed physical models of the diesel filter operation, especially the regeneration process, are increasingly employed as analysis and design tools in this process. This is the reason why the main findings of the experimental work carried out in the frame of this thesis, are always cross-checked with our in-house diesel filter modelling software, in order to improve understanding of the processes involved and also improve the capacity of our computational tools. One main goal of this chapter is to demonstrate the synergy between the various experimental investigations carried out in this work in order to develop improved engineering models that enhance our understanding of the diesel filter operation and can be used as component, system and control design aids.

By now, it should have become clear to the reader that the filter is a very complex multi-phase chemical reactor working in transient conditions, which is possibly the most demanding and challenging application in heterogeneous catalysis. The soot has to be filtered while at the same time the formed layer is by itself a filter medium causing the continuous change of the reactor characteristics and subsequently it has to be oxidized bringing the reactor back to its initial state. Its performance is significantly affected by the following:

- Time series of exhaust gas properties (temperature, flowrate and velocity filed, species concentrations). For a specific engine, these depend on the succession of engine operating points (driving cycle etc). On the other hand, the effect of engine type (size, speed range, combustion chamber, injection system, turbocharging, EGR etc) can also be of profound importance.

- Time series of particulate (mass) emissions, size distribution and characterization. The physical structure of the particulate depends on a variety of variables like fuel injection and combustion chamber characteristics, engine operation point, weather conditions, turbocharging and EGR.

- Exhaust gas flow field upstream the filter. The filters inlet flow field is affected by the design characteristics of the engine exhaust system (exhaust manifold geometry, downpipe geometry, presence of catalytic converter, inlet diffuser etc).

- Flow through the various channels, (variable property) soot layer and channel walls (porous media).

- Soot accumulation in the different channels (3D - variable reactor geometry and conditions, dictated by variable soot layer and catalyst particle size distribution). 
- Soot oxidation by catalyst particles (produced by fuel additive combustion). Catalysts are used to lower the soot oxidation temperature.

- Soot Volatile Organic Fraction oxidation by catalyst particles.

- Soot oxidation by exhaust gas oxygen.

In this complex reactor environment, we spotted the following main points where the regeneration process lacks a good understanding and modeling capability:

- Reaction scheme and kinetics of the catalytic regeneration process

- The role of the volatile organic fraction of the particulate (adsorption, desorption, stochastic regeneration)

- 3D effects during filter loading (flow maldistribution, instabilities during soot loading, soot maldistribution).

- 3D effects during catalytic regeneration (flow maldistribution at filter inlet, soot maldistribution, effect of filter material and design).

- Correlation between pressure drop and accumulated soot mass.

(The last point is essential for the design of a reliable regeneration onset control system).

In the following, we summarize the main findings of this work with regard to each one of the above issues.

\subsection{Reaction Scheme and Kinetics of the Catalytic Regeneration Process}

The extensive TGA experiments with soot samples taken directly form the filter channel, supplied us with valid kinetics for the thermal and catalytic regeneration of the soot and VOF. This information is not yet fully exploited. Thus, the 1D model version validated in this work lumps the VOF effect on the kinetics parameters of the carbon oxidation.

Another important area investigated by means of the validation experiments, was that of the Ceria oxidation - reduction cycling during regeneration. In this context, it is of interest to understand how the lower state Ceria (that has reacted with the neighbouring soot) could be brought again to the higher (oxidized) state by reacting with the exhaust gas oxygen. This fundamental question has not yet been answered by the fundamental researchers [70]. During our validation procedure, we comparatively tested two possibilities

- Continuous oxidation of the lower state Ceria, (as indicated in our reaction scheme)

- Deletion of the Ceria oxidation from the reaction scheme

The second option, that corresponds to the assumption that only the oxygen initially stored in the Ceria is available for catalytic soot oxidation, did not work. That is, it was indirectly reconfirmed that the lower state Ceria can be oxidized and used again for the oxidation of further soot in the neighbourhood, until it completely loses contact with the soot and becomes ash that is deposited in successive layers on the wall, and new soot is deposited above during the subsequent loading of the filter. The re-oxidation of the lower state Ceria was first suggested by de Soete [110], and first employed in DPF modelling in [66].

As regards the effect of Ceria concentration in soot (and indirectly, of Cerium additive in fuel), it was found that, above $25 \mathrm{ppm}$, the effect on the kinetics of soot oxidation by Ceria 
was proved to be negligible, both in the TGA experiments and in the engine bench experiments. This confirms the findings of other researchers [103].

\subsection{The Role of the Volatile Organic Fraction of the Particulate.}

The VOF content of the soot collected on a specific filter layout was measured on the TGA, as function of the engine operation point during loading, and the central or peripheric position of the specific channel in the filter. Useful desorption kinetics information was extracted from the TGA recordings. However, this information has not yet fully exploited in the modeling. VOF oxidation kinetics are exploited in the updated reaction kinetics. However, for the time being, lumping of VOF is made on the soot oxidation kinetics. That is, in the apparent kinetics parameters presented in the 1D code validation runs, it is obvious that increased VOF content reduces the activation energy of catalytic soot oxidation. Attempts to model stochastic regeneration behaviour are not yet successful.

\subsection{D Effects During Filter Loading}

Flow maldistribution in diesel filters had not yet been studied before this work. The experimental investigations carried out here (hot and cold flow experiments), indicated significant maldistribution, at least in the specific filter layouts examined. Also, instabilities during the soot loading process were recorded, for the first time. We have been able to measure, indirectly, soot maldistribution, based on a novel methodology. Soot maldistribution was also found to be significant. This could not be assessed by means of the pressure drop signal, due to the nature of pressure drop dependence on the parallel flow resistance of all channels. In addition to the soot maldistribution, we also noticed a maldistribution of VOF content in soot, between central and peripheric channels. Furthermore, a correlation of soot with flow maldistribution was attempted, with promising results.

\subsection{D Effects During Catalytic Regeneration}

The validation work of the 3D ANSYS-CATWALL interfacing developed by other group members, allowed a better assessment of the effects of flow maldistribution, soot maldistribution, the effect of filter material and design. More specifically, certain design problems of the specific SiC filter tested were revealed and reported to the manufacturer. These problems relate to the 3D structure of the specific filter (made up of rectangular filter blocks, bound together by means of adhesive cement), and could not be studied with 1D or 2D models. Flow maldistribution was found to be responsible for the significant delay between the onset of regeneration of central and peripheric channels, which reaches sometimes the order of several minutes.

\subsection{Correlation Between Pressure Drop and Accumulated Soot Mass.}

As already discussed, the pressure drop signal carries important information on the accumulated soot mass on the filter that cannot be ignored. On the other hand, this information is encoded in the pressure drop signal in a very complex way, interacting with soot distribution between channels, VOF content, exhaust gas thermodynamics etc.

The experimental investigations presented in Chapter 4 revealed the existence of significant soot and flow maldistribution. 


\section{Conclusions and Future Outlook}

- Aim of this work is to improve our understanding of diesel filter regeneration reaction scheme and kinetics parameters, as well as pressure drop characteristics and their dependence on 3D effects on flow, soot and VOF distribution.

- A secondary objective is to improve in-house engineering models of diesel filter regeneration and pressure drop, as well as their employment in design, in order to move, also in this interdisciplinary area, from "trial and error" to "engineering".

- Although the diesel filter is in series production and installation today and in near future by an increasing number of automotive manufacturers, an extensive literature search shows that there is a lack of fundamental knowledge on the regeneration reaction scheme, kinetics and the complex phenomena determining filter pressure drop characteristics.

- The reported lack of knowledge could be the result of the interdisciplinary nature of diesel filter studies: On the one hand, the chemist tries to research fundamental kinetics in soot produced artificially, or collected in layouts not typical of the real reactor. On the other hand, the emissions control engineer tests the full-scale performance of the real system, which deprives him of the opportunity to focus in the details of the process.

- The application of mathematical modeling during the last decade, allow us today, in a certain extent, to bridge the gap between the two distant perspectives, and design experiments of fundamental nature, but also in a typical reactor environment, close to the real one. The results of the application, in this PhD work of such a unified approach are summarized below.

- As regards the regeneration kinetics, the novel device and process for soot sampling directly from the channel developed in this work, allowed, for the first time, the reliable determination of real soot kinetics.

- As regards the soot maldistribution, the novel experimental device and process for indirect determination of flow resistances of the different channels is already employed by other researchers and developers as a test aid for better understanding of individual channels performance in specific filter system design.

- As regards the determination of the $(\rho k)_{\mathrm{p}}$ product (density times permeability of the collected soot), a novel device based on a single channel filter, in combination with a soot morphology characterization by means of scanning electron microscopy is expected to lead to a new test procedure for better assessment of soot deposition characteristics in specific filter system design versions, and their effect on the pressure drop characteristics.

- As regards the assessment of flow maldistribution, the novel device and methodology developed, for cold flow velocity measurements downstream filters, is a simple but effective testing technique that gives a wealth of information on the loading and regeneration behaviour of different channels in various filters design versions. 
- The above improvements in our understanding of the filter operation are mirrored in improvements in our in-house computational tools. An extensive validation of the updated 1D and 3D codes (CATWALL and ANSYS-CATWALL) is reported in Chapter 3. Of course, we cannot state that we can model diesel filter regeneration, especially the low flowrate and stochastic one, in a level comparative with our catalytic converter modeling. But this is also due to the complexity of the diesel filter operation process, which is mainly due to the significantly more complex reactor conditions discussed above. The modeling improvements that have been done in parallel with the experimental work by other researchers of the same group based on the feedback of this experimental work, along with the improved knowledge on the kinetics and pressure drop parameters that came out of this $\mathrm{PhD}$ work, significantly improved the accuracy, predictive ability and scope of application of our models. Furthermore, due to the lack of published experimental results that could be employed in real world model validation, the experiments employed in the validation work are available for benchmarking with models developed by other researchers.

- Another important category of improvements refers to the testing procedures employed as a support to diesel filter systems design. Some of them are already adopted by other researchers and developers, after their publication or communication.

- Last but not least, during the evolution of this $\mathrm{PhD}$ thesis work, the significant advances made in new components that are integrated into automotive powertrain systems are accelerating. These include, for the case of the diesel engine, diesel filters and diesel NOx traps, electronic throttles, advanced common rail injection systems. In parallel, the on board computers embodied in the ECU are becoming faster and better equipped with memory and networking capabilities. Thus, more detailed physical models of complex systems, like the diesel filter, are now demonstrating real time capability, and control system tools and theories are becoming more applicable to these complex systems.

- A significant challenge for the automotive engineer is to determine how the total system, comprising aftertreatment devices, sensors, actuators and ECU, can be optimized to deliver increased efficiency and durability at reduced cost and increased reliability and robustness. 


\section{Appendix: Experimental}

During this Ph.D. study, a variety of measurement equipment was used. Also, a number of experimental devices and test rigs, have been designed and constructed in-house, and were employed in the testing of the filter loading and regeneration behaviour, the extraction of soot and VOF oxidation kinetics and the investigation of flow and soot distribution phenomena. The above-mentioned equipment and experimental devices are briefly described here. Some more details are given in the appropriate chapters.

\subsection{Equipment}

\section{Engine benches}

The loading and regeneration tests described in this thesis were performed on two test benches. The first one is presented in Figure 7.1 and comprises a large scale commercial Diesel engine manufactured by PSA (model: DW10ATED). It is a high pressure, direct injection (HDI), 1997 cc, 4-cylinder (in line), 4-stroke turbocharged diesel engine rating a maximum power of $80 \mathrm{~kW}$ at $4000 \mathrm{rpm}$ and a maximum torque of $250 \mathrm{Nm}$ at $2000 \mathrm{rpm}$. The engine is equipped with an electronically managed high-pressure common rail injection system. With the common rail system, an extremely fine spray of fuel is possible, thus ensuring an excellent quality mixture with combustion air. This result is attributable to the system's extremely high operating pressure (1,350 bar), the injector's extremely small orifices (roughly 200 microns in diameter) and an ideal injection curve ensured by continuous monitoring of each component in the system with an electronic control unit.
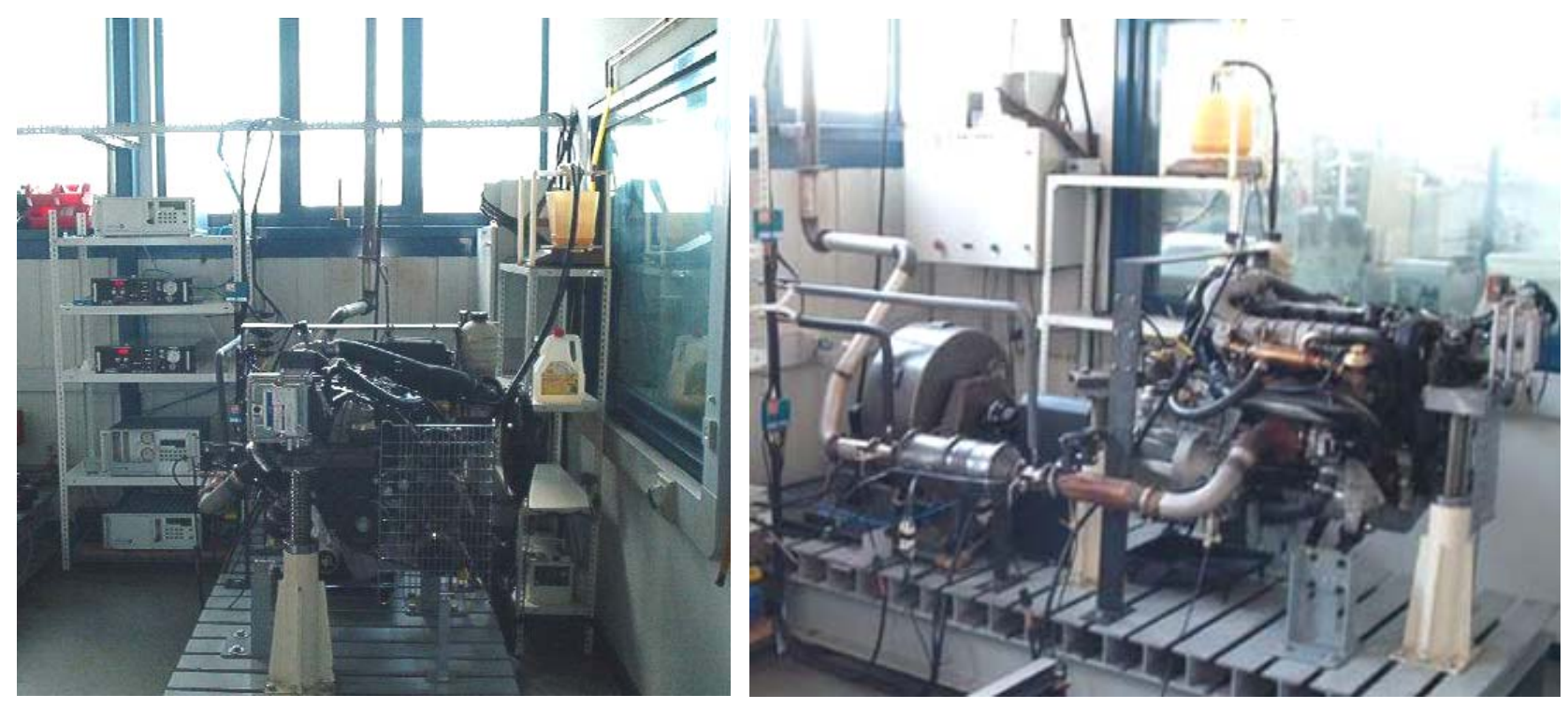

Figure 7.1 Large scale engine test bed

The engine is coupled with a Froude-Consine (model: AG 150) eddy current dynamometer rating a maximum brake torque of $250 \mathrm{Nm}$ (measured at the shaft) and a maximum speed of $8000 \mathrm{rpm}$. The power and torque capacity diagram of the dynamometer is presented in Figure 7.2. Dynamometer load measurement is carried out via a strain gauge load cell and speed measurement is carried out via a magnetic pulse pick up that monitors the rotation of a sixty- 
tooth wheel mounted on the dynamometer shaft. The load cell specifications are presented in Table 7.1.

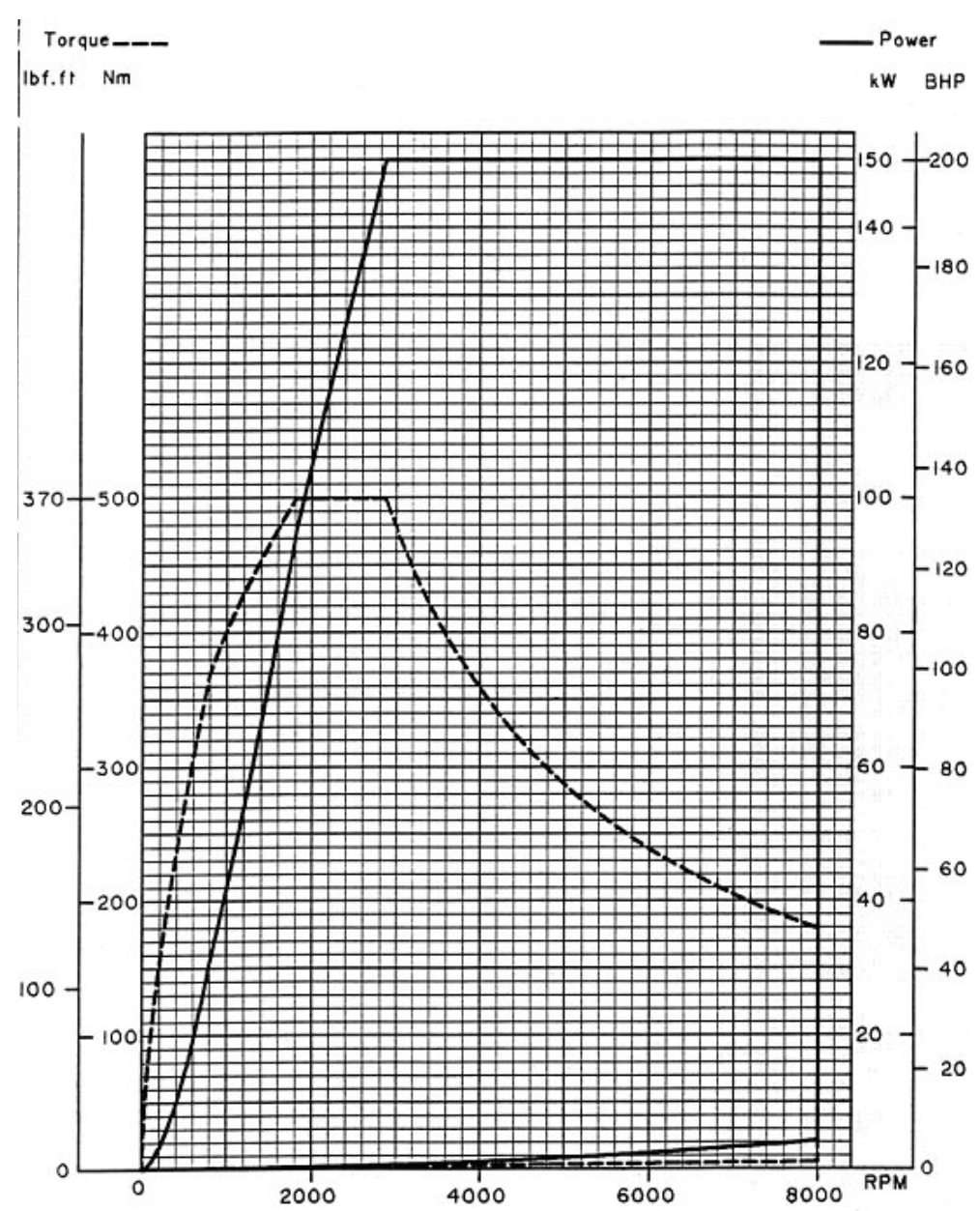

Figure 7.2 Power and Torque capacity diagram of the dynamometer

Table 7.1 Load cell specifications

\begin{tabular}{|l|l|l|}
\hline Non-Linearity & $\% \mathrm{FSO}$ & $+/-0.03$ \\
\hline Hysterisis & $\% \mathrm{FSO}$ & $+/-0.03$ \\
\hline Thermal Zero Shift & $\% \mathrm{FSO} /{ }^{\circ} \mathrm{C}$ & $+/-0.002$ \\
\hline Thermal Sensitivity Shift & $\%$ Reading $/{ }^{\circ} \mathrm{C}$ & $+/-0.002$ \\
\hline Operating Temperature Range & $0-60{ }^{\circ} \mathrm{C}$ \\
\hline
\end{tabular}

The dynamometer is controlled via a direct digital controller (Froude-Consine, model: Texcel 100) housed in the control room (Figure 7.3). The torque controller accuracy is $\pm 1.25 \mathrm{Nm}$. A throttle actuator designed and manufactured in the lab for the specific engine is used for the control of engine throttle. The actuator comprises a geared motor (DC 12V) driving an output shaft carrying a pulley that drives the engine throttle pulley, through a belt system that includes a torque-limiting clutch for safety purposes. A potentiometer informs the controller for the exact position of the throttle. The resolution of the throttle actuator is 0.1 degree and the response was measured to $0.5 \mathrm{~s}$ for full travel. 


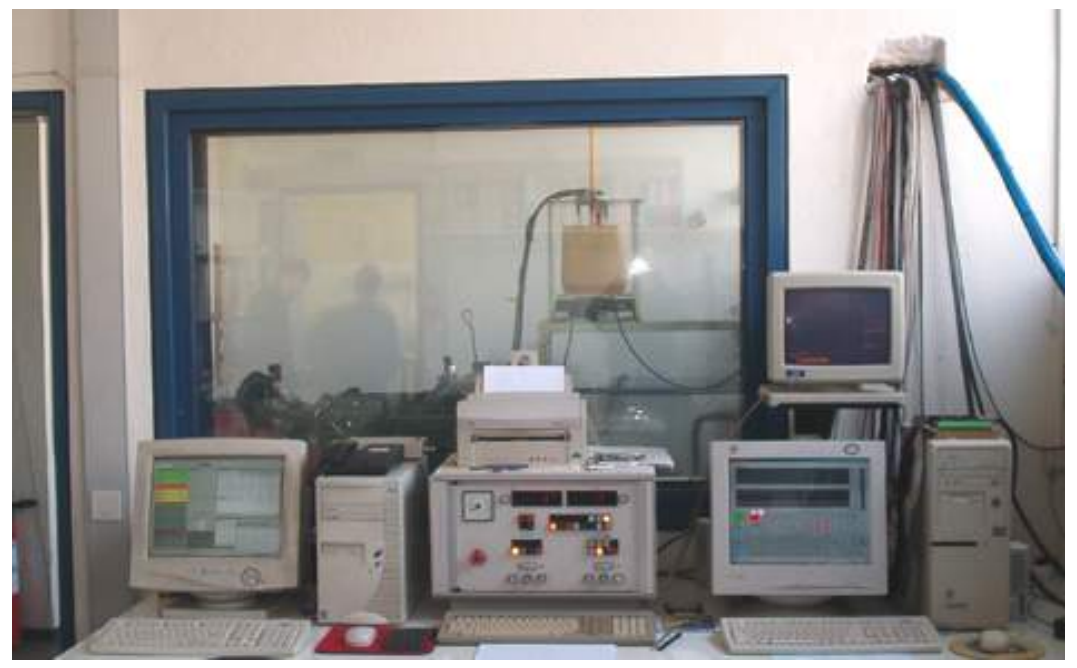

Figure 7.3 Engine and dynamometer control section

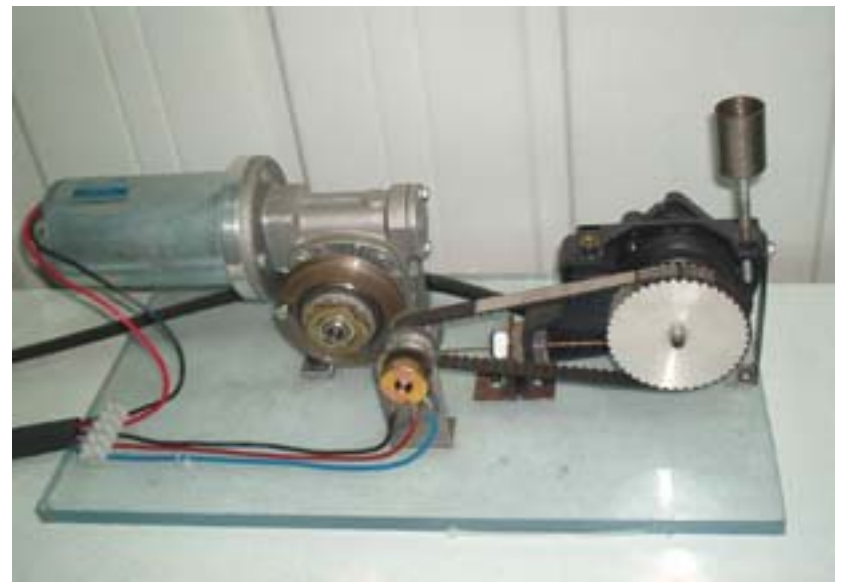

Figure 7.4 Engine throttle actuator

The second engine bench is presented in Figure 7.5 contains a small-scale diesel engine installed as a soot generator for the loading of small-scale filters according to the process described in chapter 4. It is a direct injected 497 cc Rugerrini diesel engine (model: brio 91) with a maximum power rating of $5.5 \mathrm{~kW}$ at $4000 \mathrm{rpm}$ and a maximum torque rating of $25 \mathrm{Nm}$ at $2400 \mathrm{rpm}$. The engine is coupled with a generator (MS100LG by MSM generators) with a maximum power rating of $6 \mathrm{~kW}$ loaded with a set of 4 resistances with a maximum capacity of $1.5 \mathrm{~kW}$ for each resistance. During this experimental work the engine was always operated at a constant speed of $2400 \mathrm{rpm}$ and a constant load of $3 \mathrm{~kW}$, which is about $50 \%$ load. 


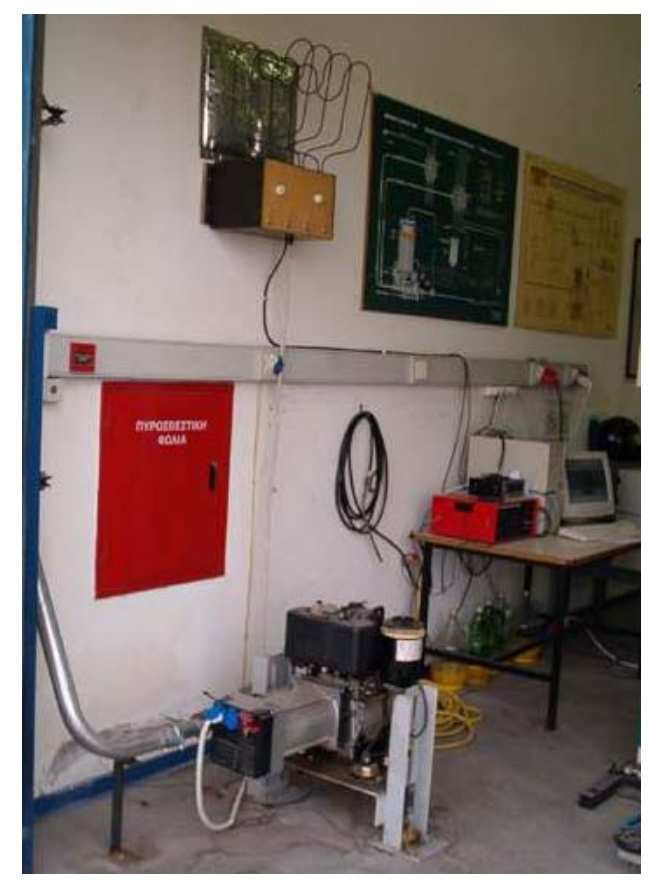

Figure 7.5 Small scale engine test bed

\section{Exhaust gas analysers}

The engine emissions tests include simultaneous measurement of $\mathrm{HC}, \mathrm{CO}, \mathrm{CO}_{2}$ and $\mathrm{NO}_{\mathrm{x}}$ concentrations in exhaust gas with a set of separate analysers. The analysers are placed near the exhaust pipe (sampling line length: $0.5 \mathrm{~m}$ ) in order to increase the response of the measurements and avoid any secondary effects due to the condensation of the sample in the sampling pipes. Especially in the case of HC emissions measurement in order to avoid the condensation of the high boiling components the sampling line is heated.

For the Hydrocarbons measurement, a couple of flame ionisation analyzers (J.U.M model: HFID 3300A) are used. This is a heated analyser that includes a flame ionisation detector (fuel gas: Hydrogen) designed to continuously measure the concentration of total organic hydrocarbons in a gaseous sample. The analyzer is calibrated to measure Hydrocarbons as $\mathrm{C}_{3} \mathrm{H}_{8}$. The specifications of the analyser are presented in Table 7.2.

Table 7.2 Hydrocarbons analyser specifications

\begin{tabular}{|l|l|}
\hline Detector type & FID \\
\hline Measuring ranges & $0-10 / 100 / 1.000 / 10.000 / 100.000 \mathrm{ppm}$ \\
\hline Sensitivity & $1 \mathrm{ppb}$ \\
\hline Response & $<1.2 \mathrm{sec}(95 \% \mathrm{FSD})$ \\
\hline Linearity & $1 \%$ \\
\hline Sampling rate & $25 \mathrm{cc} / \mathrm{min}$ \\
\hline
\end{tabular}

The measurement of CO concentration is performed with a Signal (model 2200) analyser that uses for the detection the property of the gases to selectively absorb infared radiation in a narrow range of wavelength (NDIR method). The specifications of the analyser are presented in Table 7.3. 
Table 7.3 CO analyser specifications

\begin{tabular}{|l|l|}
\hline Detector type & NDIR \\
\hline Measuring ranges & $0-100 / 500 / 1000 \mathrm{ppm}, 0-1 / 5 / 10 \%$ \\
\hline Sensitivity & $0.7 \%$ (flow sensitivity) \\
\hline Response & $15 \mathrm{sec}(90 \%$ FSD) \\
\hline Linearity & $0.50 \%$ \\
\hline Sampling rate & $1000 \mathrm{cc} / \mathrm{min}$ \\
\hline
\end{tabular}

The measurement of $\mathrm{CO}_{2}$ concentration is also performed with a Signal (model 2200) analyser with the infared detector controlled to recognise the wavelength that $\mathrm{CO}_{2}$ absorbs infared radiation. The specifications of the analyser are presented in Table 7.3.

Table 7.4 $\mathrm{CO}_{2}$ analyser specifications

\begin{tabular}{|l|l|}
\hline Detector type & NDIR \\
\hline Measuring ranges & $0-1000 / 5000 / 10000 \mathrm{ppm}, 0-1 / 5 / 10 \%$ \\
\hline Sensitivity & $0.9 \%$ (flow sensitivity) \\
\hline Response & $7.5 \mathrm{sec}(90 \% \mathrm{FSD})$ \\
\hline Linearity & $0.60 \%$ \\
\hline Sampling rate & $1000 \mathrm{cc} / \mathrm{min}$ \\
\hline
\end{tabular}

The measurement of $\mathrm{NO}_{\mathrm{x}}$ concentration is performed with a Signal (model 4000) analyser that uses the chemiluminescent method for the detection. As only NO can be detected by chemioluminescence a converter that consists of a heated tube is incorporated for the conversion of $\mathrm{NO}_{2}$ to NO. The specifications of the analyser are presented in Table 7.5.

Table 7.5 $\mathrm{NO}_{\mathrm{x}}$ analyser specifications

\begin{tabular}{|l|l|}
\hline Detection method & Chemioluminescence \\
\hline Measuring ranges & $0-4 / 10 / 40 / 100400 / 1000 / 4000 / 1000 \mathrm{ppm}$ \\
\hline Sensitivity & 0.9 \\
\hline Response & $5 \mathrm{sec}$ \\
\hline Linearity & $0.50 \%$ \\
\hline Sampling rate & $200 \mathrm{cc} / \mathrm{min}$ \\
\hline
\end{tabular}

\section{UEGO sensors}

For the measurement of exhaust gas oxygen concentration before and after the filter (needed for the calculation of oxygen consumed during regeneration) two wide range oxygen sensors so called UEGO (Universal Exhaust Gas Oxygen sensor) made by NGK (model: TL-6312W1) was installed and linked each one via a controller (model: TC-6110C) to the data acquisition system. The controller was calibrated by the manufacturer to measure in the range of $\mathrm{A} / \mathrm{F}=14.57(\lambda=1)$ to $\mathrm{A} / \mathrm{F}=50$ (Figure 1.1). The specifications of the sensor and controller are summarized in Table 7.6. 
Table 7.6 UEGO sensor specifications

\begin{tabular}{|l|l|}
\hline UEGO sensor TL-6312-W1 & \\
\hline $\mathrm{I}_{\mathrm{p}}$ (pumping current) & -0.05 to $0 \mathrm{~mA}(\lambda=1), 3.859$ to $5.314\left(\mathrm{O}_{2} 16 \%\right)$ \\
\hline $\mathrm{V}_{\mathrm{p}}$ (pumping voltage) & -2 to $2.5 \mathrm{~V}$ \\
\hline Sensor element tip Temperature & 750 to $950^{\circ} \mathrm{C}$ \\
\hline Controller TC-6110C & \\
\hline Measuring range & $\mathrm{A} / \mathrm{F}=14.57$ to $\mathrm{A} / \mathrm{F}=50(\mathrm{H} / \mathrm{C}: 1.85)$ \\
\hline Output & $1(\mathrm{~A} / \mathrm{F}=14.57)$ to $5.34 \mathrm{~V}(\mathrm{~A} / \mathrm{F}=50)$ \\
\hline Correction Error & $\left(\mathrm{V}_{\text {out }}-1\right) \times 0.02 \mathrm{~V}$ \\
\hline Amplifier Error & $\pm 0.02 \mathrm{~V}$ \\
\hline Temperature Error & $\pm 0.03 \mathrm{~V}$ \\
\hline
\end{tabular}

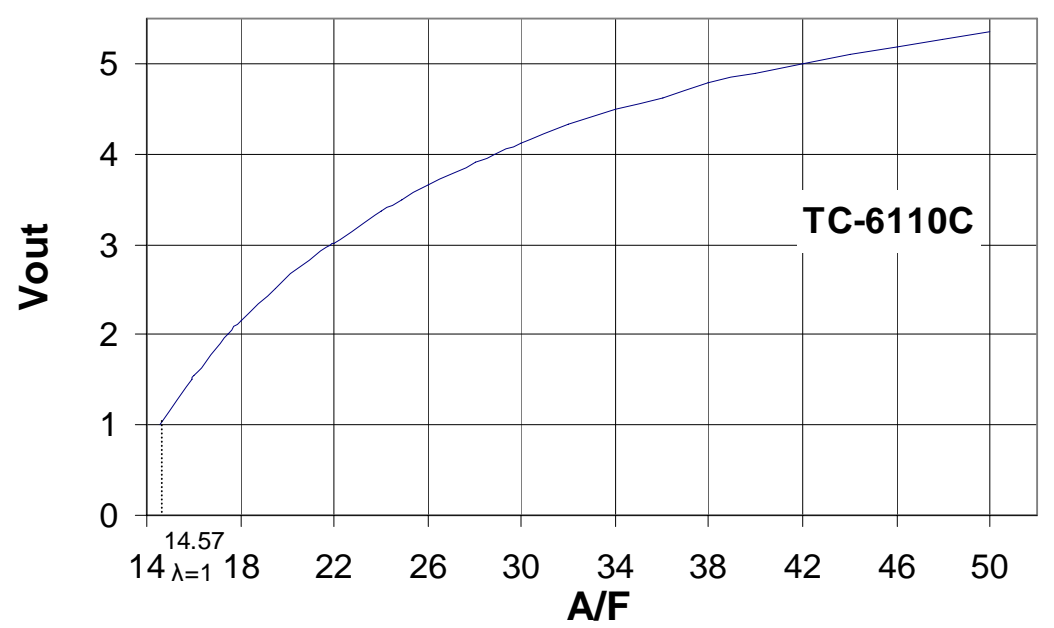

Figure 7.6 UEGO sensor characteristic curve

\section{Pressure transducers}

During this experimental work absolute and differential pressures were measured. Absolute pressure measurements were taken at the engine inlet pipe before and after the valve that regulates the EGR flow and at the engine exhaust pipe before and after the turbocharger. Also an absolute pressure transducer was used for the measurement of pressure drop during the expansion process of the vessel used for the determination of ( $\rho \mathrm{k}$ )p product (chapter 4). All the transducers were piezoresistive WIKA (model: ECO-TRONIC) measuring at the ranges 00.5/1/2/4/6bar with the specifications of Table 7.7.

Table 7.7 Absolute pressure transducers specifications

\begin{tabular}{|l|l|l|}
\hline Transducer type & Piezoresistive & $<5$ \\
\hline Response & $\mathrm{ms}(10-90 \%)$ & $<1$ \\
\hline Hysterisis & $\% \mathrm{FSO}$ & $<0.3$ \\
\hline Reproducibility & $\% \mathrm{FSO}$ & $0.4 / 10 \mathrm{~K}$ \\
\hline Thermal Zero Shift & $\% \mathrm{FSO} / \mathrm{K}$ & $0.3 / 10 \mathrm{~K}$ \\
\hline Thermal Sensitivity Shift & $\%$ Reading/K & \\
\hline
\end{tabular}


The diferential pressure transducer used for the filter backpressure measurements was a JUMO 0-500 mbar delta pressure range (4 ADI-55), calibrated from the manufacturer with a full-scale error of 0.01 . The specifications of the transducer are presented in Table 7.8. The pressure transducer was verified on a weekly basis, using a Druck (Model DPI 510) dual range pressure controller. The DPF pressure drop was validated at ten independent points from 0-500mbar.

Table 7.8 Differential pressure transducer specifications

\begin{tabular}{|l|l|l|}
\hline Transducer type & Inductive displacement transducer \\
\hline Error (under standard conditions) & $\%$ FSO & $<1 \%$ \\
\hline Response & $\mathrm{ms}(10-90 \%)$ & $<20$ \\
\hline Hysterisis & $\% \mathrm{FSO}$ & $<0.1$ \\
\hline Ambient temperature error $\left(10-50^{\circ} \mathrm{C}\right)$ & $\% \mathrm{FSO} /{ }^{\circ} \mathrm{C}$ & $0.05 /{ }^{\circ} \mathrm{C}$ \\
\hline
\end{tabular}

\section{Thermocouples}

During this experimental work temperatures were measured at various engine and filter system points. The measurements were performed with $\mathrm{K}$ type thermocouples with the following characteristics: material: $\mathrm{NiCr}-\mathrm{Ni}$, measuring range: $-65 /+1150{ }^{\circ} \mathrm{C}$, dimensions: $\varnothing 1 \mathrm{x} 200 \mathrm{~mm}$.

\section{Data acquisition system}

The DAQ system allows graphical presentation and storage of real-time measurement data. The systems hardware incorporates:

- a model 6024 National Instruments PCI card (analog input channels: 16 single ended, digital I/O channels: 8 , measurements accuracy: $\pm 0.074 \%$ ) which obtains the signals from the pressure and flow transducers mounted on the engine and filter systems, the load and speed sensors of the dynamometer and finally the exhaust gas analysers.

- a model 6033 National Instruments PCI card with (analog input channels: 64 single ended, digital I/O channels: 8, measurements accuracy: $\pm 0.052 \%$ ) which is linked in a multiplexing way with a model SCXI 1100 card (analog input channels: 32 differential, measurements accuracy: $\pm 0.03 \%$, linearity: $\pm 0.008 \% \mathrm{FS}$ ) used for the collection and amplification of the temperature signals of the thermocouples mounted at various engine and filter points.

For the acquisition a virtual instrument (VI) was designed in LABVIEW 5 environment. The VI is separated in the front panel (Figure 7.7) that serves as the user interface and the block diagram (graphical source code) designed with the synthesis of the functions and structures called from the built-in libraries of the program. As can been seen in Figure 7.7 at the bottom of the front panel are presented the numerical values of all the recording data in bar graphs shape and optical alarms for the case of engine coolant overheating and depletion or outpouring of the fuel tank. At the center of the front panel there are two real-time waveform graphs showing the evolution of record data in time. The entering of data such as the path and filename of the recording file, the scanning rate and the buffer size, data for SCXI and acquisition card, thermocouples types and analysers measurement ranges is also needed for the operation of the VI. 


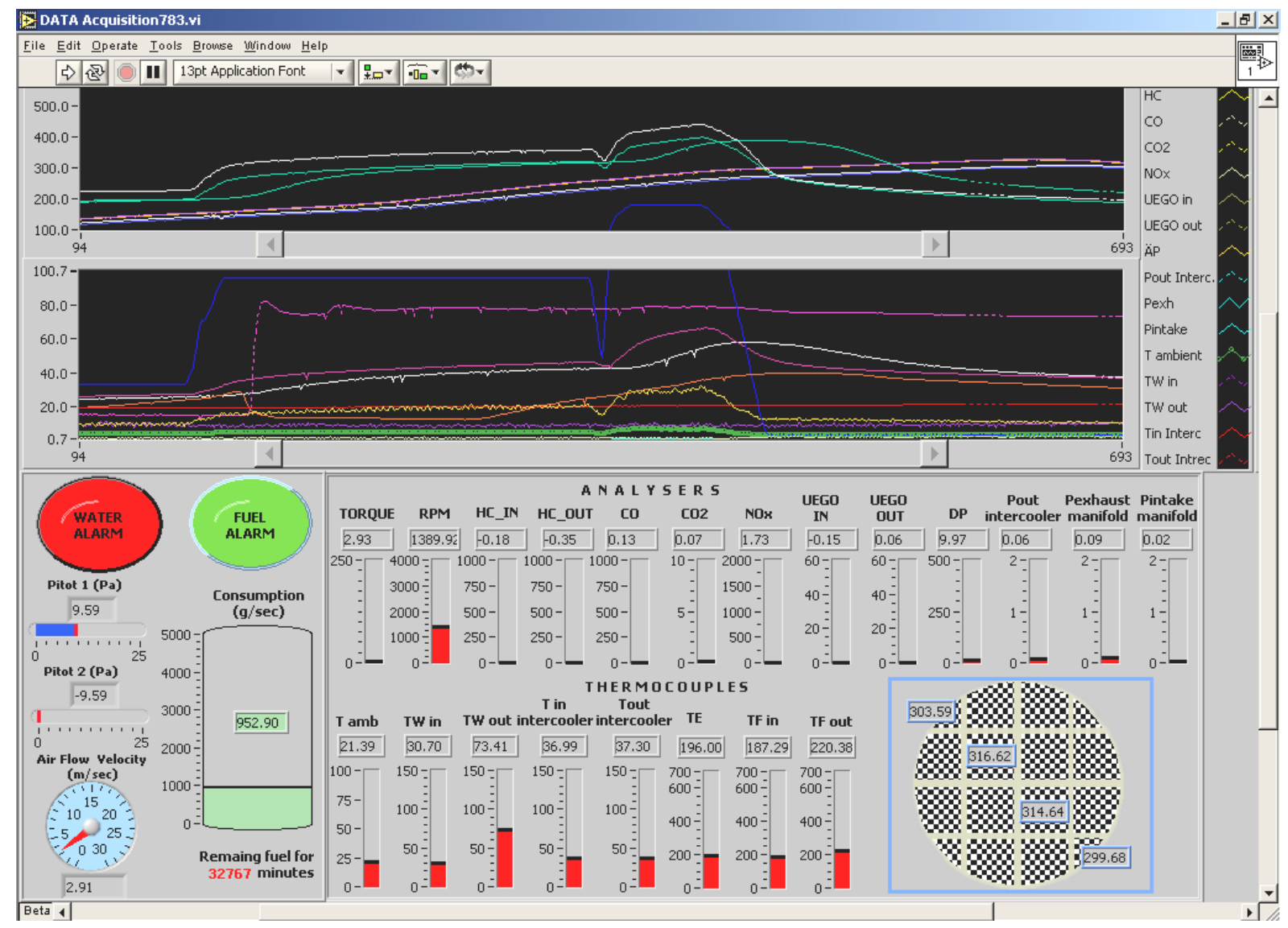

Figure 7.7 Graphical presentation of data acquired by the cards in Labview environment.

The connection diagram of the VI is separated in two sequences. In the first sequence are defined as strings of constant values the names of each column in the record file. The second sequence initializes the acquisition cards, acquires, post-process and saves data in the record file. The VI hierarchy is shown in Figure 7.8.
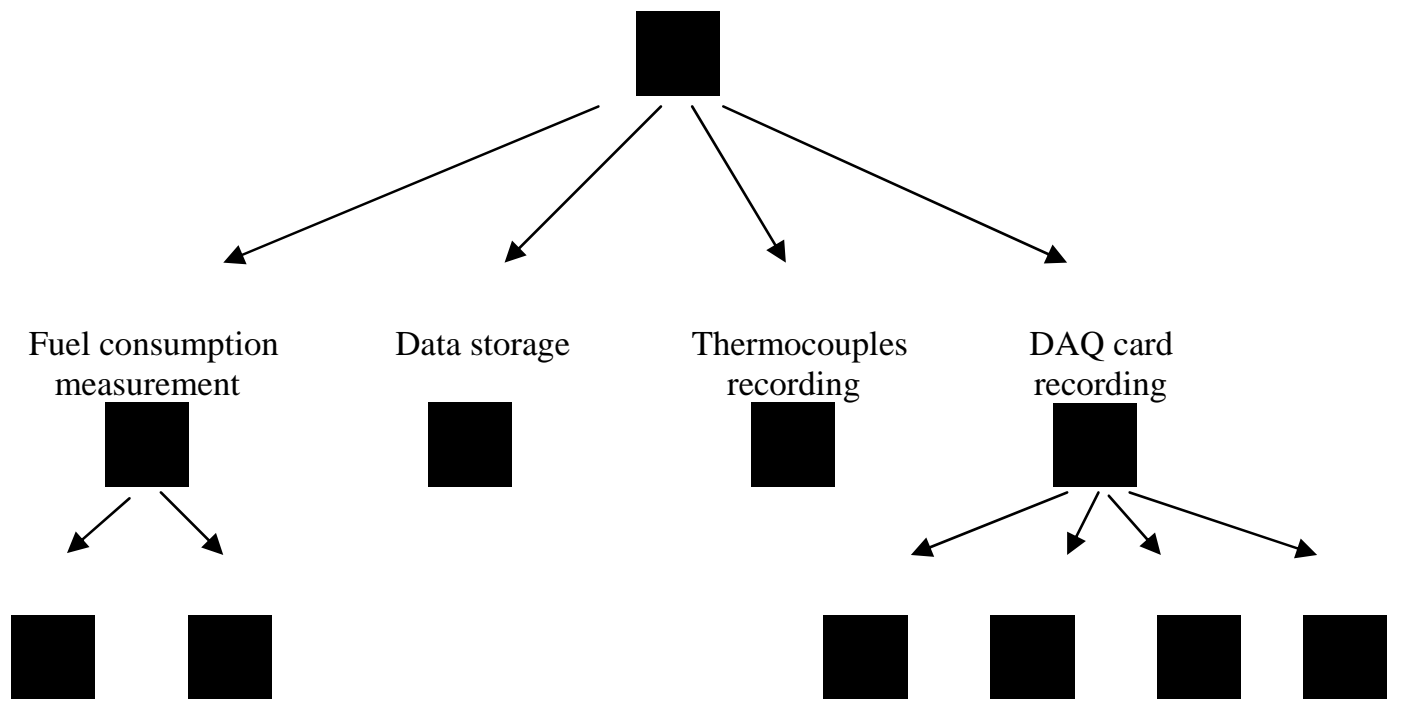

Figure 7.8 Hierarchy of the VI connection diagram 
During this experimental work the sampling rate of the cards was controlled to $80 \mathrm{kS} / \mathrm{s}$ (defined according Nyquist theory) and the obtained data was subjected to a moving average process resulting to a final sampling rate in the range between 0.1 and $1 \mathrm{~Hz}$.

The obtained accuracy for each type of measurement can be defined based on the ratio $\sigma / \mu$ (standard deviation over mean value of measurement). The value of this ratio for each type of measurement for a set of 600 measurements obtained under a typical steady state experiment with engine operating at the medium point of speed: $3000 \mathrm{rpm}, 40 \mathrm{Nm}$ is presented in Table 7.9.

Table $7.9 \sigma / \mu$ ratio for all the measured quantities under a typical steady state experiment

\begin{tabular}{|l|c|c|c|}
\hline Type of measurement & Mean value & $\begin{array}{c}\text { Standard } \\
\text { deviation }\end{array}$ & $\sigma / \mu$ \\
\hline Engine speed (rpm) & 3017 & 2.54 & 0.0008 \\
\hline Engine torque (Nm) & 41.12 & 0.16 & 0.004 \\
\hline Pressure (bar, measured at exhaust) & 0.87 & 0.01 & 0.012 \\
\hline Temperature $\left({ }^{\circ} \mathrm{C}\right.$ measured at exhaust) & 264.58 & 0.45 & 0.001 \\
\hline $\mathrm{A} / \mathrm{F}$ & 43.32 & 0.23 & 0.005 \\
\hline $\mathrm{HC}$ concentration (ppm) & 84.83 & 0.86 & 0.011 \\
\hline $\mathrm{CO}$ concentration $(\mathrm{ppm})$ & 814.82 & 12.48 & 0.015 \\
\hline $\mathrm{CO}_{2}$ concentration $(\%)$ & 4.85 & 0.01 & 0.003 \\
\hline NO concentration $(\mathrm{ppm})$ & 200 & 3.25 & 0.016 \\
\hline Air velocity $(\mathrm{m} / \mathrm{s})$ & 10.84 & 0.05 & 0.005 \\
\hline
\end{tabular}

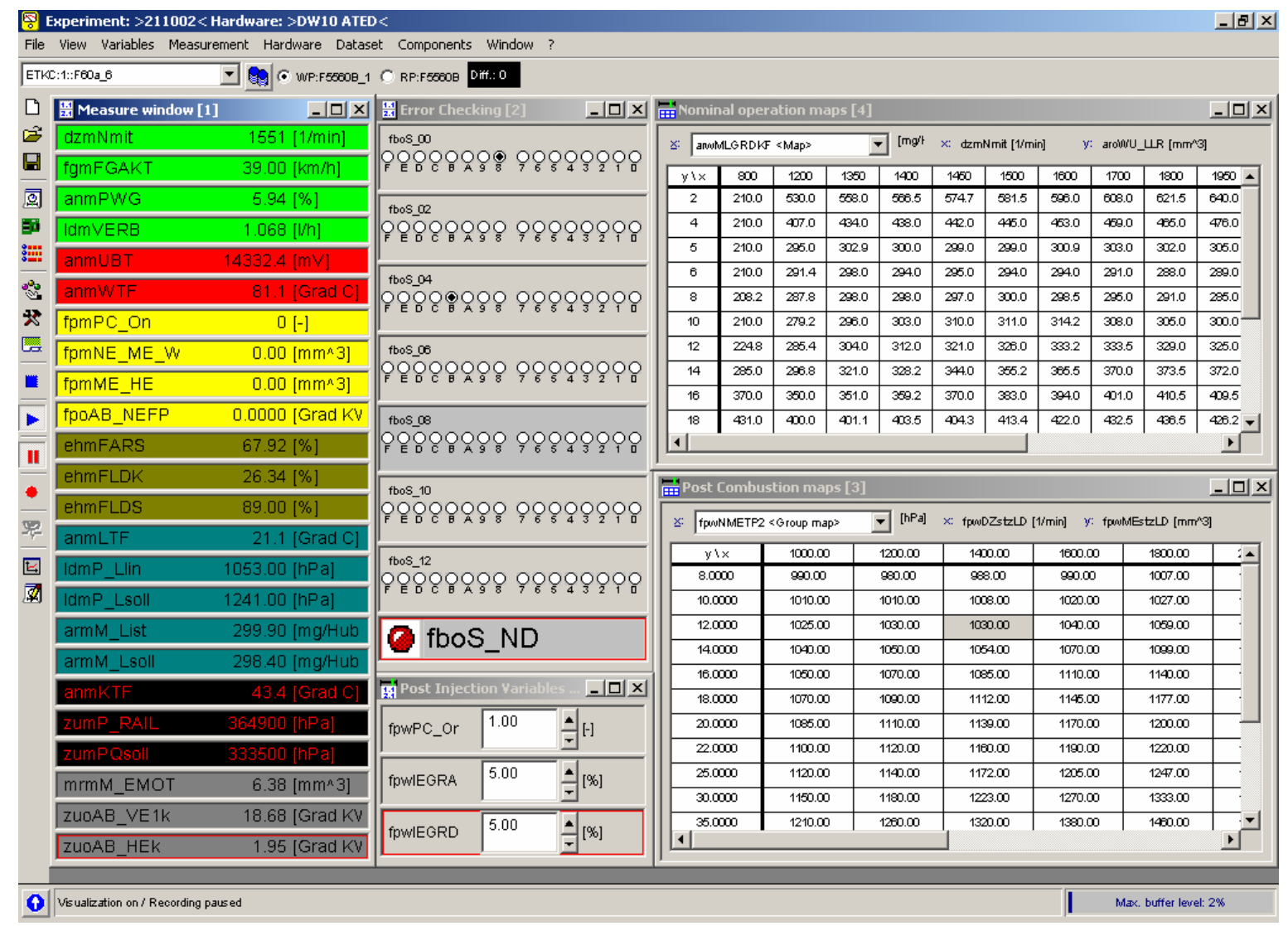


Figure 7.9 INCA measurement and calibration environment

The more sophisticated system of ETAS that allows access to ECU measurement data as well as request changes to internal ECU calibration variables was also established. The system incorporates an intermediate module to load the programme and the maps stored in the EPROM of engine ECU and an interface module (ETK) to link the ETK module with the PCbased measurement and calibration software (INCA). Required measure and calibration variables and their parameter settings are called from the engine ECU with the use of ASAM interface and stored and easily assigned to the same workspace. The configuration of the workspace used in our experimental work is presented in Figure 7.9.

\section{Thermal analysis}

Thermal analysis of soot samples was performed on a Rheometric Scientific STA 1500 simultaneous TGA-DSC analyser, which consists of a microbalance with a hangdown that is placed in a very accurately controlled electrical oven with a maximum temperature of 1500 . Two ceramic crucibles (volume: $100 \mu \mathrm{l}$ ) a first one containing the sample and a second one empty as reference can be placed on the hangdown as presented in the figure. The temperature under each crucible is measured with $\mathrm{K}$ type thermocouples and the temperature difference is employed to the calculation of the heatflow (mcal/s). The combined monitoring of heatflow (DSC analysis) and sample mass variation (TGA analysis) makes possible the identification of the processes, which can occur during soot oxidation. Non-isothermal tests were performed with each sample, with a heating rate of $10^{\circ} \mathrm{C}$ per minute. The experiments were carried out in air (for the characterization of the catalytic soot oxidation processes and the extraction of soot and VOF oxidation kinetics) and also in nitrogen environment (for the determination of VOF content). A gas flow of $10 \mathrm{ml} / \mathrm{min}$ in the case of synthetic air experiments and of $35 \mathrm{ml} / \mathrm{min}$ in the case of nitrogen was maintained over the receptacle chamber. The analysis data was recorded by the data acquisition system with a scan rate adjusted to $1 \mathrm{sec}$.

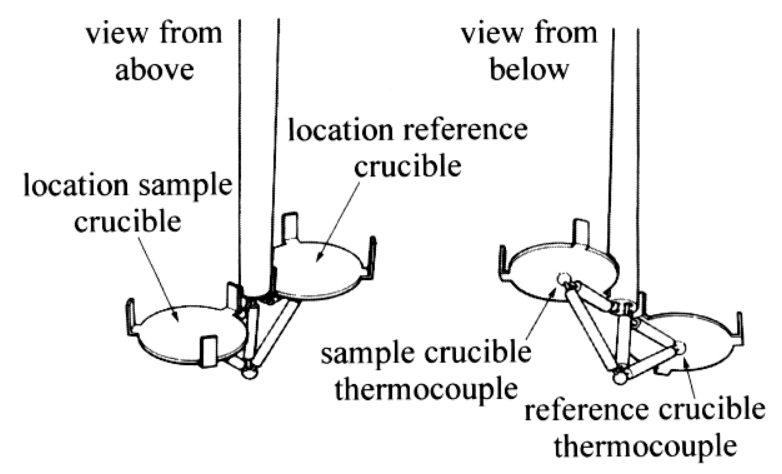

Figure 7.10 Illustration of TGA-DSC device hangdown

\section{SEM}

Sem images were acquired with a Jeol 5310 Scanning Electron Microscope in the secondary electron mode with an accelerating voltage of $25 \mathrm{KV}$.

\section{Electronic balance}

An electronic balance Kern (model: 822-43) with a precision of $0.1 \mathrm{~g}$ and a weighing range of 0.1 to $6200 \mathrm{~g}$ is used for the monitoring of engine fuel consumption (Figure 7.11) and the 
other weighing processes (e.g. weighing of filters after loading for the determination of accumulated soot mass) performed during this work. The balance is linked to the PC via the RS232 serial port, which is read by the LabVIEW software allowing the real-time collection and graphical representation of the measurements data.

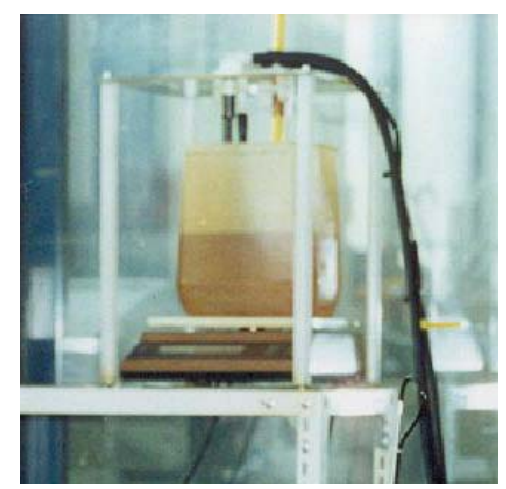

Figure 7.11 Use of electronic balance to measure engine fuel consumption.

\subsection{Materials}

Wall flow filters

The wall flow filters used in this work were SiC 14/200 provided by Ibiden. The design characteristics of this filter type are presented in Figure 7.12. It consists of separate blocks (four central and twelve peripheric) connected each other with the use of impermeable cement. The thermal and mechanical properties of the materials and the resulted filter structures are presented in Table. 7.10.

$\begin{array}{llll}\text { Ceramic substrate } & \text { Unit filter } & \text { Filter block } & \text { Full filter structure } \\ \begin{array}{l}\text { Mean pore diameter: } 9 \mu \mathrm{m} \\ \text { Porosity : } 42 \%\end{array} & \begin{array}{l}\text { Cell pitch: } 1.89 \mathrm{~mm} \\ \text { Wall thickness: } 0.4 \mathrm{~mm}\end{array} & \begin{array}{l}\text { Length: } 150,254,380 \mathrm{~mm} \\ \text { Cell density: } 200 \mathrm{cpsi}\end{array} & \begin{array}{l}\text { Diameter } 143.8 \mathrm{~mm} \\ \text { Adhesive thickness: } 2 \mathrm{~mm}\end{array}\end{array}$
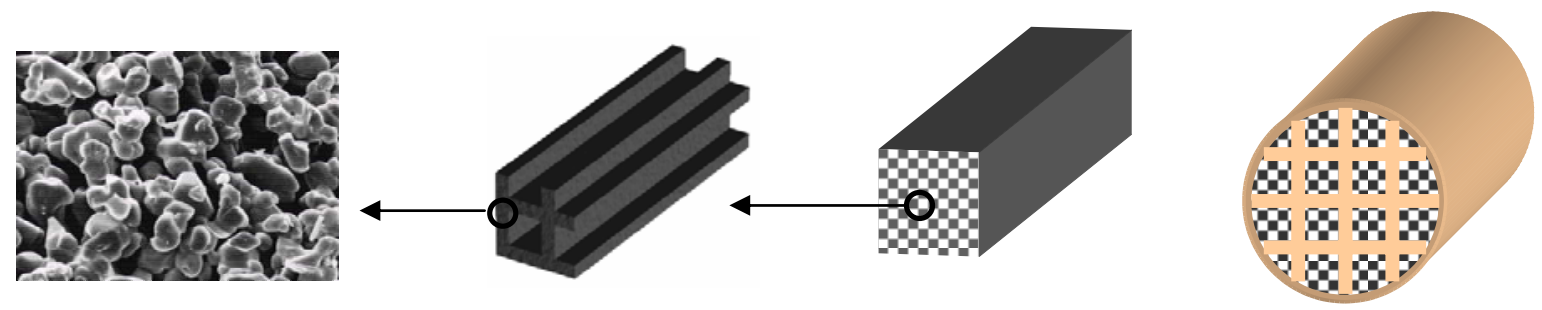

Figure 7.12 Design characteristics of Ibiden 14/200 SiC filters

3M Interam expansion ceramic fibre mat with a thickness of $5 \mathrm{~mm}$ was wrapped around the filters and the resulted structures was placed inside steel shells (St 37) so that the mat to be compressed about $3 \mathrm{~mm}$ to avoid gas leaks. 
Table 7.10 Thermal and mechanical properties of Ibiden 14/200 SiC filters

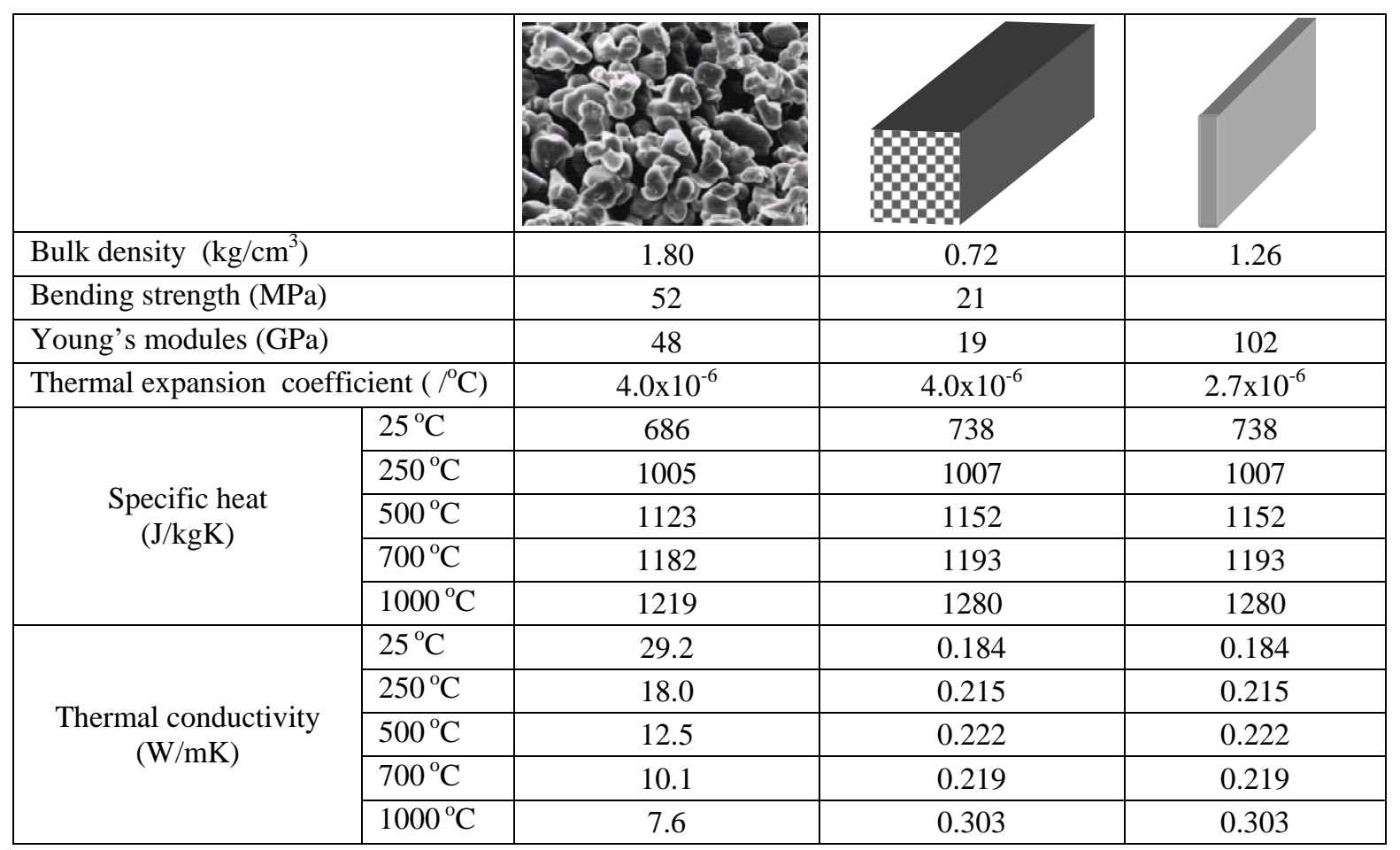

\section{Chemicals}

The diesel fuel used in this experimental work is in conformance with the 2000 European standard of EN590 containing less than $350 \mathrm{mg} / \mathrm{kg}$ sulfur.

The fuel additive used in the loading and regeneration experiments is based on Cerrium and provided by Rhodia with the commercial name of Eolys DPX9. The fuel containing the additive was prepared in batches of 4 litres using volumetric tubes for the precise measurement of the fuel and additive quantities. The additive was very well mixed with the fuel before entering the engine fuel pan with the use of an electric mixer.

\subsection{Experimental Devices}

\section{Single channel filters}

Single channel filter specimens were prepared by breaking up a full sized filter to be employed to the measurements of $(\rho \mathrm{k}) \mathrm{p}$ as described in chapter 4 . The canning of the filters was configured to be connected directly to the engine exhaust for loading or the expansion device for the $(\rho \mathrm{k}) \mathrm{p}$ measurement. The construction characteristics of these filters are presented in Figure 7.13. 


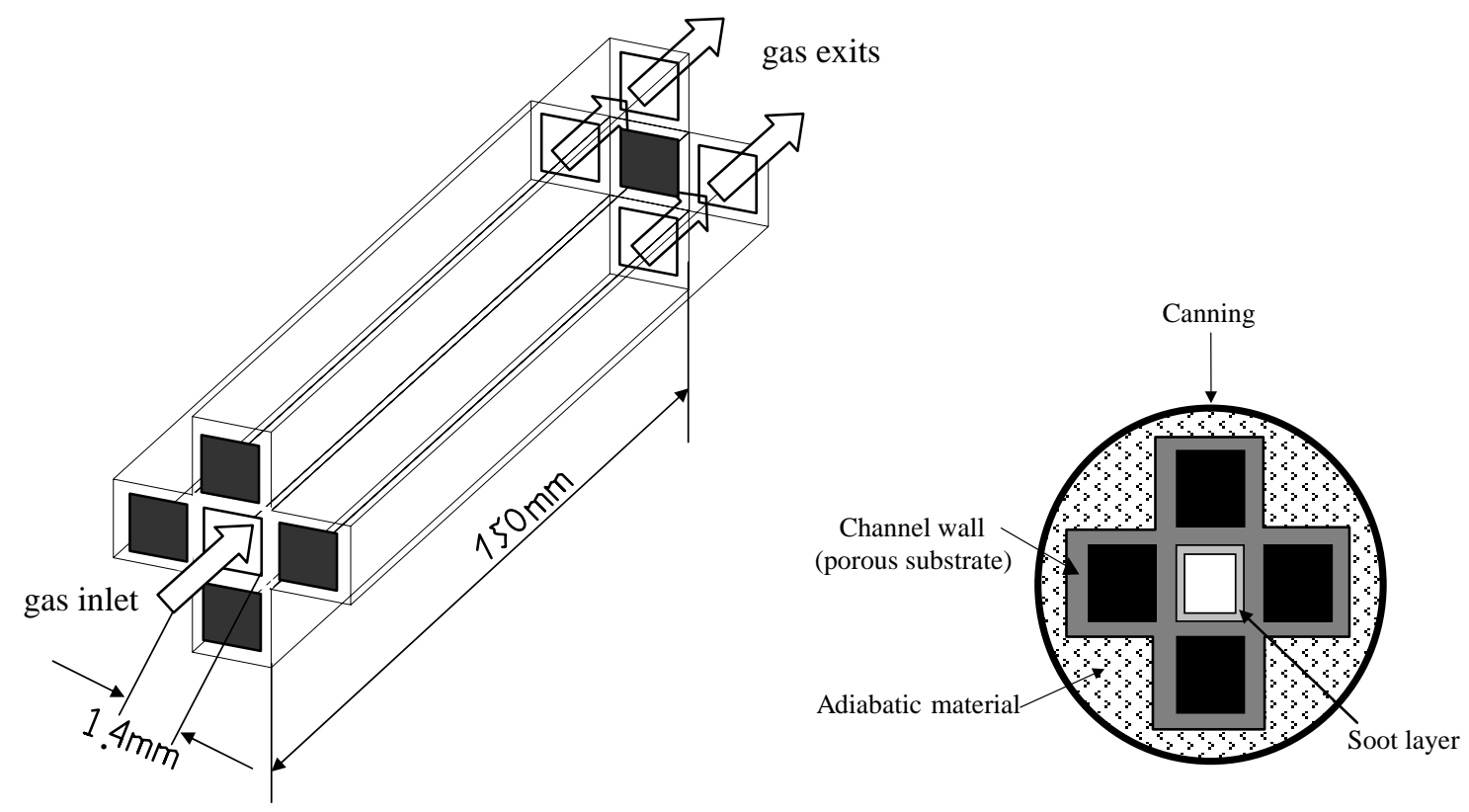

Figure 7.13 Single channel filter specimens

\section{Expansion device for ( $\rho$ k)p estimation and soot maldistribution measurements}

A novel experimental device designed and employed to the estimation process of $(\rho \mathrm{k}) \mathrm{p}$ product in single channel filters and the measurement of soot maldistribution in full scale filters. The configuration of the device is presented in Figure 7.14.

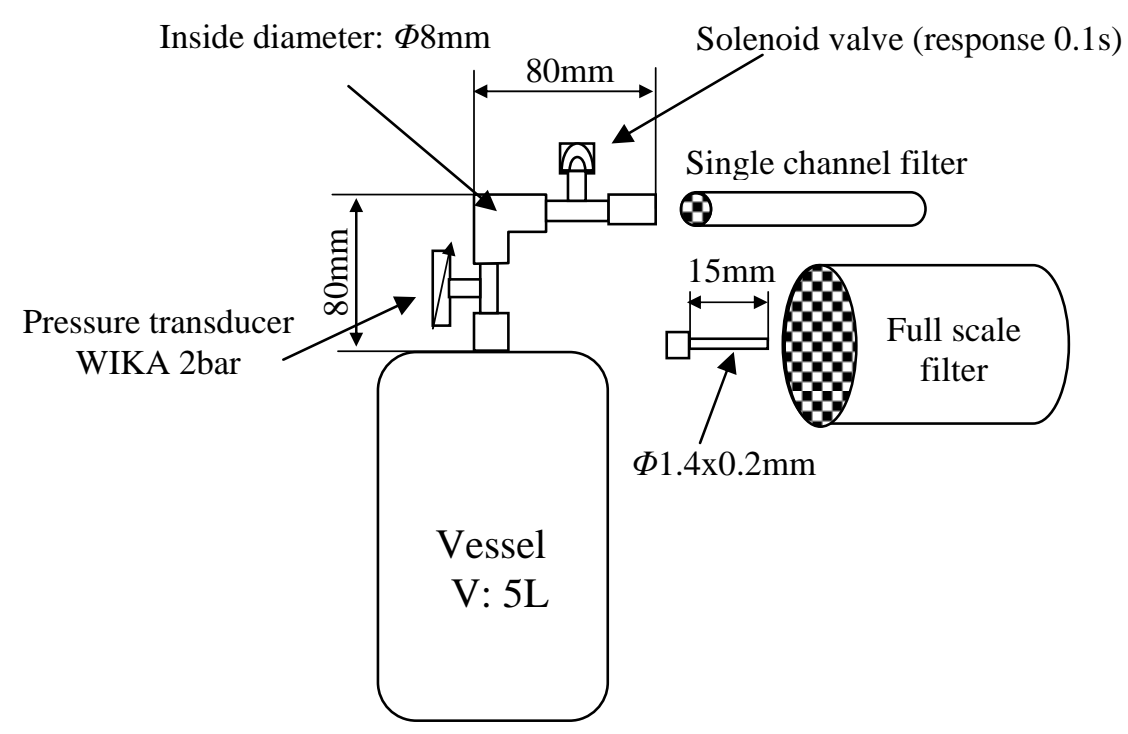

Figure 7.14 Expansion device design characteristics

The device consists of a pressure vessel with a volume of 5lt, which initially contains about 2 bar of pressurized air. Monitoring of the expansion process is carried out by means of recording the signal of a pressure transducer (WIKA 2 bar) that is also mounted to the vessel. A solenoid valve opens on demand by the data acquisition system, and triggers the expansion of the compressed air from the vessel through the channel walls to the atmosphere. Two 
alternative types of discharge nozzles were designed and machined on the lathe, to allow the measuring device to be fitted to mini-scale filters and to specific channels of full-scale filters.

\section{Pitot tubes}

During this work Pitot-static tubes were prepared in order to measure the flow velocities in the high temperature corrosive environment of engine exhaust pipe. Two L-shaped Pitot tubes were constructed from co-axial tubing according to the specifications presented in Figure 7.15.

Total or Pitot Pressure Port

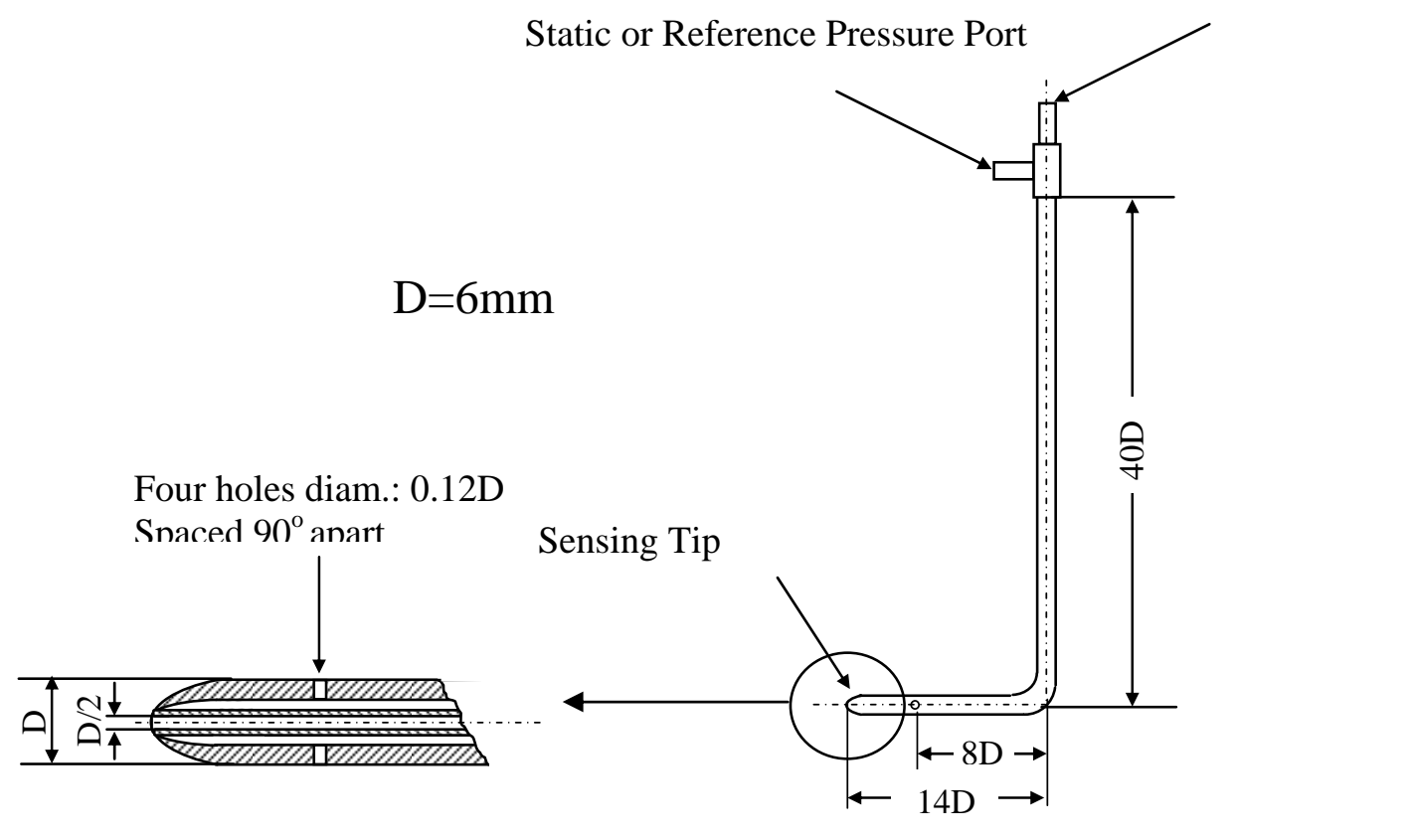

Figure 7.15 Basic Pitot-static tube construction

According to the theory the inner tube conducts the total pressure from the sensing tip to the total or Pitot pressure port whereas the outer tube provides a path from the static pressure tap to the static or reference pressure port.

The calibration of the pitot static tubes was carried out by placing them at the exit of a pipe connected to an electric blower that supplies air at various mass flowrates controlled to result in a variation to the air velocity in the range of 3 to $30 \mathrm{~m} / \mathrm{s}$ (Figure 7.16). The pipe was quite long (about 3m) so that the air stream at the pipe exit to be fully developed. As reference instrument for the velocity measurement was used a hot film sensor (TSI model 8345) that also used for the control of the blower mass flowrate to result to the desired flow velocity. After the measurement of velocity the pitot-static tube was placed at the exit of the pipe and the differential pressure between the total and static pressure was measured with the use of a differential pressure transducer (Cole\&Palmer piezoresistive transducer, measuring range: 0$1 \mathrm{kPa}$ ). According to the theory this pressure is correlated with the air flow velocity according to the relationship:

$$
V_{g}=C \sqrt{\frac{2 \Delta p_{\text {pitot }}}{\rho_{g}}}
$$

Where $C$ is a Pitot tube constant and $\rho_{g}$ the exhaust gas density set equal to 1.2 for the ambient conditions of the calibration experiments. 


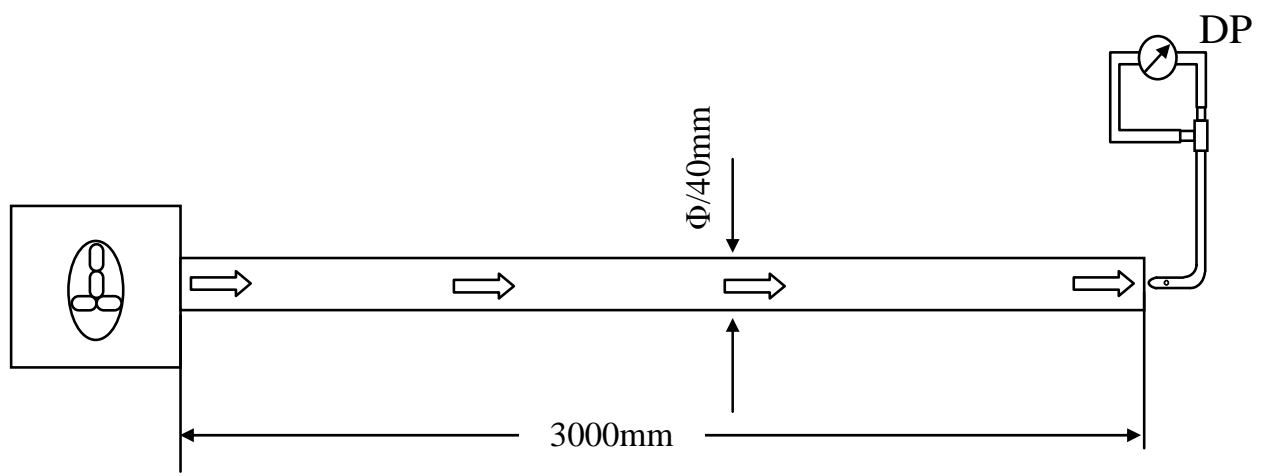

Figure 7.16 Pitot-static tubes calibration experiment

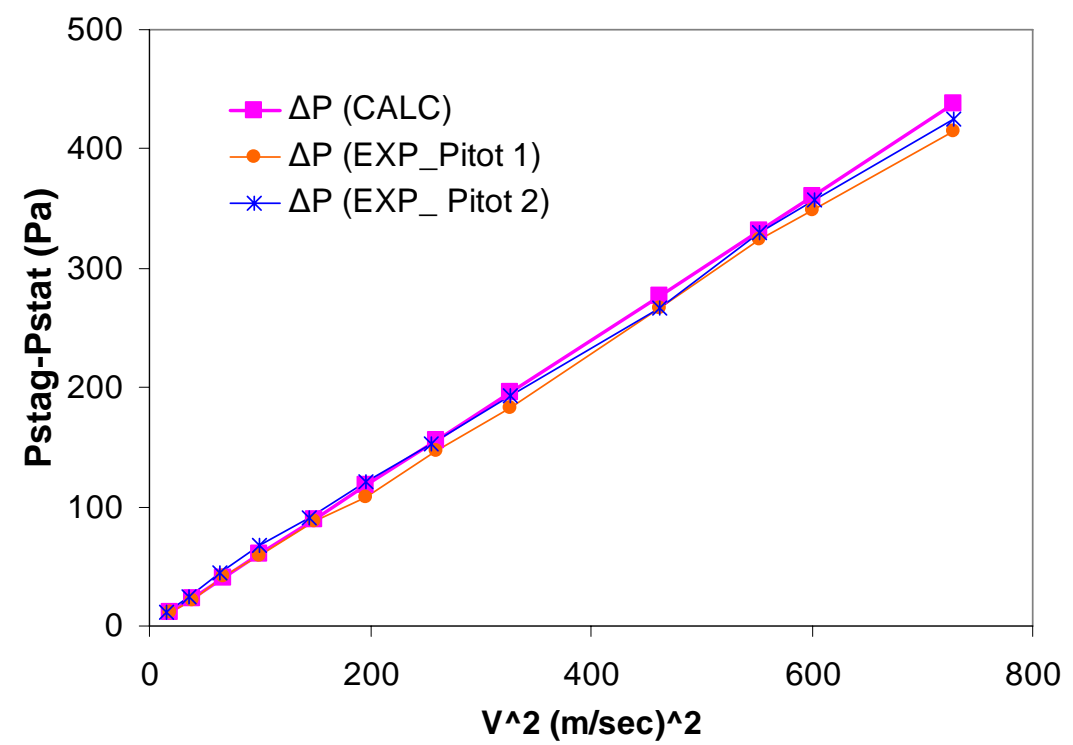

Figure 7.17 Calibration curves of the Pitot-static tubes used for the exhaust gas flow velocity measurements. The calibration performed at ambient conditions with a TSI hot wire velocity sensor to be used as reference for the flow velocity measurements.

The differential pressure was validated at twelve independent velocity points. The results for each pitot-static tube are presented in Figure 7.17. Based on the processing of the experimental data the value for the constant $\mathrm{C}$ was defined as 0.97 for the first and 0.99 for the second pitot. The mean error calculated in the range of 3 to $30 \mathrm{~m} / \mathrm{s}$ is $4 \%$ for the first and $2 \%$ for the second pitot.

\section{Cold flow measurements test bed}

A cold flow test rig for the investigation of flow maldistribution phenomena in wall flow Diesel filters by means of flow velocity measurements was designed and manufactured. The layout of the test rig is presented in Figure 7.18. It consists of a positive displacement Eaton model 45 supercharger (displacement: $0.75 \mathrm{l} / \mathrm{rev}$ ) which is powered from a $5.5 \mathrm{HP}$, AC electric motor (speed:2850rpm, frequency: $50 \mathrm{~Hz}$ ). The characteristic curves of the supercharger are presented in Figure 7.19. The power is transmitted to the supercharger through a belt drive system with an electromagnetic clutch to be included in the supercharger section. 


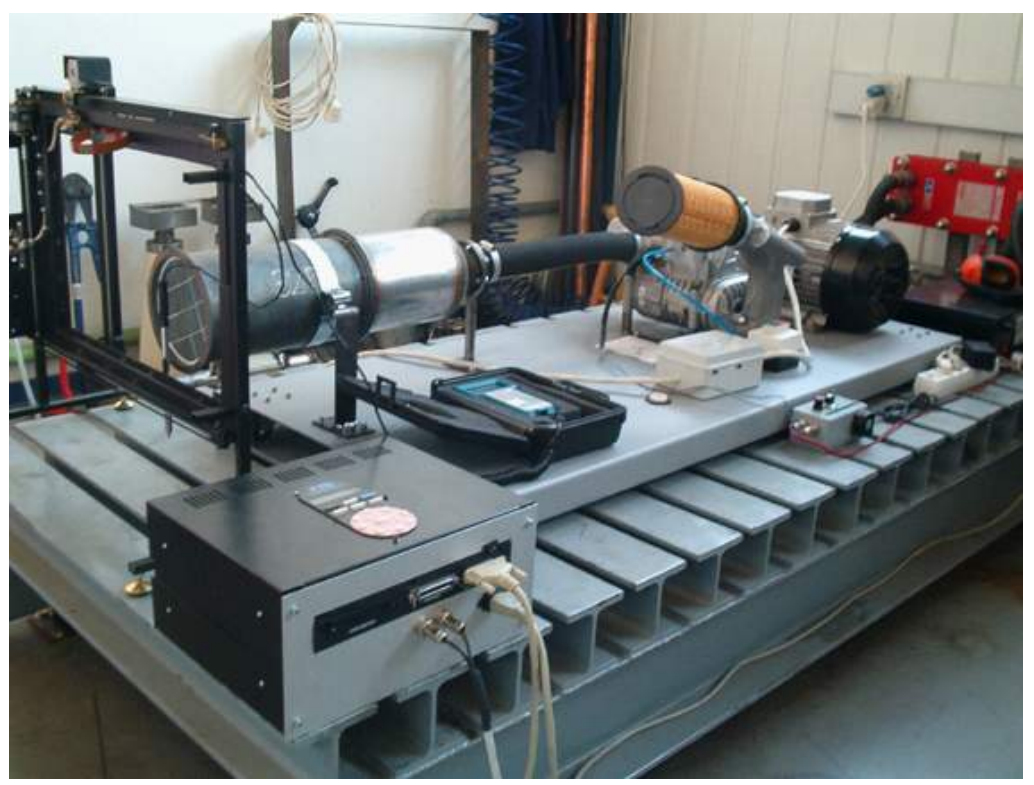

Figure 7.18 Cold flow test rig

The system of the particulate filter (diffuser + catalyst + filter) is mounted on the test rig, about $500 \mathrm{~mm}$ downstream the supercharger and is supplied with ambient air under various mass flowrates. The experimental determination of flow velocity is done with the use of a hot film sensor (temperature compensated, TSI VelociCalc Air Velocity Meter Model 8346). The specifications of the sensor are presented in Table 7.11.

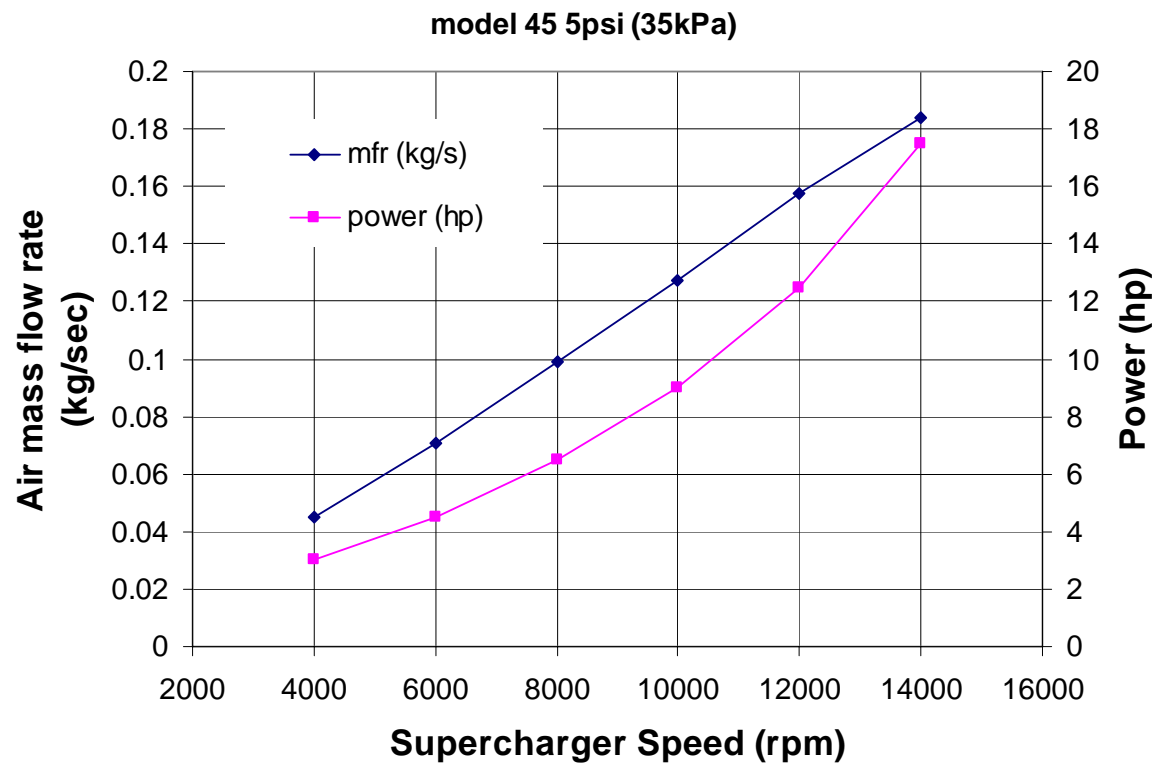

Figure 7.19 Power and mass flowrate curves of the supercharger us function of rotating speed for a pressure drop of $35 \mathrm{kPa}$.

The sensor is attached to an electronically controlled support device able to move with a pitch of $0.1 \mathrm{~mm}$ in the vertical and horizontal direction, scanning the face of the measurement area at each test. The mechanical and electronic parts used for the construction of the moving device were taken from a typical plotter. 
Table 7.11 Air velocity transducer specifications

\begin{tabular}{|l|l|l|}
\hline Transducer type & Hot film & 0 to 30 \\
\hline Measuring range & $\mathrm{m} / \mathrm{s}$ & $<3 \%$ \\
\hline $\begin{array}{l}\text { Accuracy (temperature compensated in the } \\
\left.\text { range 5 to 65 }{ }^{\circ} \mathrm{C}\right)\end{array}$ & $\% \mathrm{FSO}$ & $<200$ \\
\hline Response & $\mathrm{ms}(10-90 \%)$ & $<1$ \\
\hline Repeatability & $\% \mathrm{FSO}$ & $0-93$ \\
\hline Operating temperature & ${ }^{\circ} \mathrm{C}$ & \\
\hline
\end{tabular}

The moving device is linked to the PC via the parallel port and the coordinates of the measuring positions are programmed in HPGL language. 


\title{
SHORT CURRICULUM VITAE
}

\author{
PERSONAL DATA
}

Date and place of birth

Marital status

Chania, Crete, February $25^{\text {th }}, 1974$

Single

\section{EDUCATION}

Secondary Education, Chania

1979-1991

University of Thessaly (Volos)

Faculty of Engineering,

Diploma in Mechanical Engineering

1992-1997

Grade Point Average: 8.10 ( $1^{\text {st }}$ Class)

Graduation ranking: $4^{\text {th }}$ out of 43 graduates in 1997

$\mathrm{PhD}$ in Mechanical Engineering

University of Thessaly

2000-2003

Thesis title: 'Experimental investigation of Catalytic Soot Oxidation and

Pressure Drop Characteristics in Wall-Flow Diesel Particulate Filters’

\section{AREAS OF SCIENTIFIC INTEREST}

Internal Combustion Engines (exhaust treatment technologies on automotive spark-ignition and Diesel engines)

Fuel Injection \& Ignition Systems (investigation into fuel transport phenomena at the engine fuel entry system)

Thermal Systems Design

\section{WORK EXPERIENCE}

Technician Engineer in marine and agricultural engines

1990-1994

Public Power Corporation - Steam Power Station (SPS) Chania, Crete (sandwich placement)

1995

West Crete Development Organisation-(sandwich placement)

1996

Greek Army Service- Corps of Engineers

(NAMFI Design Office)

1998-1999

Research Assistant, Laboratory of Thermodynamics and Thermal

Engines, University of Thessaly, Mechanical Engineering Department

2000-Present

\section{PARTICIPATION IN RESEARCH PROGRAMMES}

- "Development of design techniques for automotive gas after-treatment systems"

Provider: PSA PEUGEOT CITROEN

Program Duration: Jan. 2000- Dec. 2002

Program involvement: Researcher 


\section{LIST OF PUBLICATIONS}

1. Recent developments in spark-ignition engine technology (In Greek) "Anosi" Publications, ISBN 960-86339-9-0, 2000

2. G. A. Stratakis and A. M. Stamatelos: Thermogravimetric Analysis of Soot Emitted by a Modern Engine Run on Catalyst-Doped Fuel. Combustion and Flame, Volume 132, 2002, pp. 157-169

3. G. A. Stratakis, G. S. Konstantas and A. M. Stamatelos: Experimental Investigation of the Role of Soot Volatile Organic Fraction in the Regeneration of Diesel Filters. Proc. Instn Mech Engrs, Volume 217 Part D: J Automobile Engineering, 2003, pp.307-317

4. G. A. Stratakis, D. L. Psarianos and A. M. Stamatelos: Experimental Investigation of Pressure Drop in Porous Ceramic Filters. Proc. Instn Mech Engrs, Volume 216, Part D: J Automobile Engineering, 2002, pp.773-784

5. G. A. Stratakis and A. M. Stamatelos: Flow distribution Effects in the Loading and Catalytic Regeneration of Wall-Flow Diesel Particulate Filters, In press, Proc. Instn. Mech. Engs, Part D: J Automobile Engineering, 2003

6. G. A. Stratakis and A. M. Stamatelos: Flow Maldistribution Measurements in WallFlow Diesel Filters, Submitted, Proc. Instn. Mech. Engs., Part D: J Automobile Engineering, 2003

7. G. A. Stratakis, G. N. Pontikakis and A. M. Stamatelos: Experimental Validation of a Fuel Additive Assisted Regeneration Model in SiC Diesel Filters. Submitted, Proc. Instn. Mech. Engs., Part D: J Automobile Engineering, 2003

8. G. A. Stratakis and A. M. Stamatelos: Experimental and Computational Study of the Fuel Additive Assisted Regeneration Process in SiC Diesel Particulate Filters, Submitted, Combustion and Flame, 2003 


\section{REFERENCES}

1 M. P. Walsh, Global Trends in Diesel Emissions Regulation-A 2001 Update. SAE Paper 2001- 01-0183.

2 J. Abthoff, H. D. Schuster, C. Noller: Concept of catalytic exhaust emission control for Europe, SAE Paper 94047, 1994.

3 K. Pattas, Z. Samaras, N. Patsatzis, C. Michalopoulou, O. Zogou, A.M. Stamatelos and M. Barkis. On-Road Experience with Trap Oxidizer Systems Installed on 5 Urban Buses, SAE paper 900109, 1990.

4 K. Pattas., A. Stamatelos, The Effect of Exhaust Throttling on the Diesel Engine Operation Characteristics and Thermal Loading, SAE paper 890399, 1989.

5 J.C. Clerc, Catalytic Diesel exhaust aftertreatment. Applied Catalysis B: Environmental 10 (1996) 99-115

6 H. Watanabe, T. Tahara, M. Tamanouchi, J. Iida, Study of the effects on exhaust emissions in direct injection diesel engines: Effects of fuel injection system, distillation properties and cetane number, JSAE Review 19 (1998) 21-26

7 S.Hori, NK. Narusawa, Fuel Composition Effects on SOF and PAH Exhaust Emissions from DI Diesel Engine, SAE paper 980507, 1998.

8 S. J.Jelles, Development of catalytic systems for diesel particulate oxidation, PhD Thesis TU Delft 1999.

9 D.B. Kittelson, Engines and Nanoparticles, A Review. J. Aerosol Sci. Vol. 29, No 5/6, pp.575-588, 1998

10 Chemical Methods for the Measurement of Non-Regulated Diesel Emissions. 1993 SAE Handbook, Vol.3, Engines, Fuels Lubricants, Emissions and Noise, Society of Automotive Engineers 1993.

11 M. Kasper, U. Matter, H. Burtscher, On-line Characterization of Particle Size and Composition, SAE Paper 2000-01-1998.

12 J.H. Johnson, S.T. Bagley, L.D. Gradz, D.G. Leddy, A Review of Diesel Particulate Control Technology and emissions effects. SAE paper 94023, 1994.

13 J. Pagan, Study of Particle Size Distributions Emitted by a Diesel Engine, SAE paper 1999-01-1141

14 K. Baumgard, J. Johnson, The Effect of fuel and Engine Design on Diesel Exhaust Particle Size Distributions. SAE paper 960131, 1996.

15 J. B. Heywood, Internal Combustion Engine Fundamentals, McGraw-Hill, ISBN 0-07-100499-8, pp. 635643, 1988

16 K Froelund,. J. Schramm, PAH-Transport in Diesel Engines, SAE paper 972960, 1997.

17 J. Kagawa, Health Effects of Diesel Exhaust Emissions-a Mixture of Air Pollutants of Worldwide Concern, Toxicology, Vol. 181-182, No.1, pp. 349-353, 2002.

18 P. Zelenka, K. Ostgathe, E. Lox: Reduction of Diesel Exhaust Emissions by Using Oxidation Catalysts, $S A E$ paper 902111, 1990.

19 W. Held, A. Koenig, A., T. Richter, L. Puppe: Catalytic $\mathrm{NO}_{\mathrm{x}}$ Reduction in Net Oxidizing Atmosphere, $S A E$ paper 920496, 1994.

20 Y. Kintaichi, H. Hamada, M. Tabata, M. Masato, T. Ito, Selective Reduction of Nitrogen Oxide with Hydrocarbons over Solid Acid Catalysts in Oxygen Rich Atmosphere. Catal. Lett. 6, 239 (1990).

21 N. Miyoshi, S. Matsumoto, K. Katoh, N. Takahashi, K. Yokota, M. Sugiura, K. Kasahara, Development of New Concept Three Way Catalyst for Automotive Lean Burn Engines, SAE paper 950809, 1995.

22 J. Howitt, M. Montierth, Cellular Ceramic Diesel Particulate Filter, SAE paper 810114, 1981.

23 C. N. Davis, (Editor), Aerosol Science, Academic Press, 1966.

24 A. M. Stamatelos, A Review of the Effect of Particulate Traps on the Efficiency of Vehicle Diesel Engines Energy Conversion \& Management, Vol. 38, No.1, pp. 83-99, 1997.

25 R.J. Farrauto, K.E. Voss, Monolithic Diesel Oxidation Catalysts, Applied Catalysis B: Environmental 10 (1996) 29-51. 
26 J.A.A. van den Tillaart, J. Leyrer, S. Eckhoff, E.S. Lox, Effect of Support Oxide and Noble Metal Precursor on the Activity of Automotive Diesel Catalysts. Applied Catalysis B: Environmental 10 (1996) 53-68.

27 J.P.A. Neeft, M. Makkee, J.A. Moulijn, Catalysts for the Oxidation of Soot from Diesel Exhaust gases, An exploratory study, Applied Catalysis B: Environmental 8 (1996) 57-78

28 J.P.A. Neeft, W. Schiper, M. Makkee and J.A. Moulijn, Feasibility Study towards a Cu/K/Mo/(Cl) soot Oxidation Catalyst for Application in Diesel Exhaust Gases, Applied Catalysis B: Environmental 11 (1997) 365382

29 T. V. Johnson, Diesel Emission Control in Review SAE paper 2000-01-0184, 2000.

30 M. Kasper, K. Sattler, K. Siegmann, U. Matter and H. C. Siegmann: The Influence of Fuel Additives on the Formation of Carbon During Combustion, Journal of Aerosol Science, Vol. 30, February 1999, Pages 217-225

31 K. Ohno, T. Komori, T. Ninomiya, N. Taoka, S. Hong, Regeneretion System for an Exhaust Gas Cleanining Device. US Patent 6,447,564B1, Sep. 10, 2002.

32 P. Tallec, O. Salvat : System for Assisting the Regeneration of a Particle Filter Integrated into an Exhaust Line of a Motor Vehicle Diesel Engine. US Patent 6,484,496B2, Nov. 26, 2002.

33 M. W. Vincent, P. J. Richards, S. L. Cook, Fuel Additives. US Patent 6,488,725B1, Dec. 3, 2002

34 K. Pattas, Z. Samaras, A. Roumbos, J. Lemaire, W. Mustel, P. Ruveirolles: Regeneration of DPF at Low Temperatures with the use of a Cerium Based Fuel Additive, SAE paper 960135, 1996.

35 T. Seguelong, G. Blanchard, J. Michelin, F. Terres, H. Weltens, Ceria-Based Fuel-Borne Catalysts for Series Diesel Particulate Filter Regeneration, SAE paper, 2003-01-0378.

36 B. Stanmore, J. F. Brihlac, P. Gilot, The Ignition and Combustion of Cerium Doped diesel Soot, SAE paper 1999- 01-0115.

37 J. Zhang, C. M. Megaridis: Soot Suppression by Ferrocene in Laminar Ethylene/ Air Nonpreximed Flames. Combustion and Flame 105:528-540 (1996).

38 K. Ritrievi, J. Longwell, A. Sarofim: The Effects of Ferrocene Addition on Soot Particle Inception and Growth in Premixed Ethylene Flames, Combustion and Flame, Vol. 70, Oct. 1987, Pages 17-31.

39 A. Gantawar, C. Opris, J. Johnson, A Study of the Regeneration Characteristics of Silicon Carbide and Cordierite Diesel Particulate Filters Using a Copper Fuel Additive, SAE paper 970187.

40 D. Daly, D. McKinnon, J. Martin, D. Pavlich, Diesel Particulate Regeneration System using a Copper Fuel Additive, SAE paper, 930131, 1993.

41 M. Hasan, M. Zaki, K. Kumari, L. Pasupulety: Soot Deep Oxidation Catalyzed by Molybdena and Molybdates: a Thermogravimetric Investigation, Thermochimica Acta, Vol. 320, 1998, Pages 23-32.

42 O. Salvat, P. Marez, G. Belot, Passenger Car Serial Application of a Particulate Filter System on a Common Rail Direct Injection Diesel Engine, SAE paper 2000-01-0473.

43 K. Ohno, K. Shimato, N. Taoka, S. Hong, T. Ninomiya, T. Komori, O. Salvat, Characterization of SiC-DPF for Passenger Car. SAE paper 2000-01-0185.

44 Diesel particulate filters as standard on VW Passat, http://www.dieselnet.com/news/0311vw.html accessed at 12 Nov. 2003.

45 Guido Saracco, Nunzio Russo, Michele Ambrogio, Claudio Badini and Vito Specchia Diesel Particulate Abatement via Catalytic Traps, Catalysis Today, Volume 60, Issues 1-2, 10 July 2000, Pages 33-41.

46 Debora Fino, Nunzio Russo, Claudio Badini, Guido Saracco and Vito Specchia, Effect of Active Species Mobility on Soot-Combustion over Cs-V Catalysts, AIChE Journal, Volume 49, Issue 8, August 2003, Pages 2173-2180.

47 Barry van Setten, ‘ Development of a Liquid Catalysts for Diesel Soot Oxidation’, PhD Thesis TU Delft 2001.

48 Coen van Gulijk, ‘ Rational Design of a Robust Diesel Particulate Filter’, PhD Thesis TU Delft 2002.

49 S. Sasaki, (Toyota (JP)), Device for Purifying the Exhaust Gas of an Internal Combustion Engine, US Patent 6,490,857, Dec 10, 2002. 
50 I. Kolmanovsky, A. Stefanopoulou, Control Method for Turbocharged Diesel Engine Aftertreatment System, US Patent 6,568,173, May 27,2003.

51 K. Nakatani, S. Hirota, S. Takeshima, K. Itoh, T. Tanaka, K. Dohmae, Simultaneous PM and NOx Reduction System for Diesel Engines, SAE paper 2002-01-0957.

52 J. Gieshoff, M. Preifer, A. Schafer-Sindlinger, U. Hackbarth, O. Teysset, C. Colignon, C. Rigaudau, O. Salvat, H. Krieg, B.W. Wenclawiak, Regeneration of Catalytic Diesel Particulate Filters, SAE paper 2000-010907.

53 B.J. Cooper, H.J. Jung, J.E. Thoss, Treatment of Diesel Exhaust Gas, US Patent 4,902,487, 1990.

54 Y. Levendis, C. Larsen, Use of Ozone-Enriched Air for Diesel Particulate Trap Regeneration, SAE paper 1999-01-0114.

55 M. V. Twigg: System and Method for Purifying Exhaust Gases. US patent 6,557,340 May 6, 2003.

56 S. Thomas, et al, Non Thermal Plasma Aftertreatment of Particulates - Theoretical Limits and Impact on Reactor Design, SAE paper 2000-01-1926.

57 M. Vincent, P. Richards, D. Catterson, A Novel Fuel Borne Catalyst Dosing System for Use with a DIesel Particulate Filter, SAE paper, 2003-01-0382.

58 A. Oliveira, M. Kaviany, Nonequilibrium in the transport of heat and reactants in combustion in porous media, Progress in Energy and Combustion Science, 27 (2001) 523-545.

59 D. E. Rosner, Transport Processes in Chemically Reacting Flow Systems, Butterworth - Heinemann , Stoneham, MA 1986

60 N. Taoka, K. Ohno, S. Hong, H. Sato, Y. Yoshida, T. Komori, Effect of SiC-DPF with High Cell Density for Pressure Loss and Regeneration, SAE paper 2001-01-0191.

61 G. Merkel, D. Beall, D. Hickman, M. Vernacotola, Effects of Microstructure and Cell Geometry on Performance of Cordierite Filter Particulate Filters, SAE paper 2001-01-0193.

62 B. Wiedemann, U. Doerges, W. Engeler, B. Poettner, Regeneration of Particulate Filters at Low Temperatures, SAE paper 830086, 1983.

63 G. C. Koltsakis, A. M. Stamatelos, Catalytic Automotive Exhaust Aftertreatment. Prog. Energy Combust. Sci. Vol. 23, pp.1-39, 1997

64 E. Bissett, F. Shadman, Thermal Regeneration of Diesel-Particulate Monolithic Filters. AlChE J. 1985, 31 (5), May, 753-765.

65 G. C. Koltsakis, A. M. Stamatelos, Modeling Thermal Regeneration of Diesel Particulate Traps AIChE Journal, Vol.42, No.6, pp.1662-1672, June 1996.

66 G. C. Koltsakis, A. M. Stamatelos: Modeling Catalytic Regeneration of Diesel Particulate Traps. Industrial \& Engineering Chemistry Research, 1996, (35), pp. 2-13.

67 G. C. Koltsakis, A. M. Stamatelos, Modes of Catalytic Regeneration in Diesel Particulate Filters Ind. Eng. Chem. Res. 1997, 36, 4155-4165.

68 University of Thessaly / LTTE, CATWALL Diesel Particulate Filter Modeling Software. User's Manual. Version v4r1 Volos, December 2002.

69 G. A. Stratakis, D. L. Psarianos and A. M. Stamatelos: Experimental Investigation of the Pressure Drop in Porous Ceramic Diesel Particulate Filters. Proc Instn Mech Engrs, Part D: J Automobile Engineering, 2002, 216, 773-784.

70 G. Pontikakis: Modeling, Reaction Schemes and Parameter Estimation in Catalytic Converters and Diesel Filters. PhD Thesis, Mechanical Engineering Dept., University of Thessaly, Volos, June 2003.

71 H. O. Herrmann, O. Lang I. Miculic, V. Scholz, Particelfiltersysteme fur Diesel-Pkw, MTZ Motortechnische Zeitscrift 2001, 62, 1, 652-660.

72 G. A. Stratakis, G. S. Konstantas and A. M. Stamatelos, Experimental Investigation of the Role of Soot Volatile Organic Fraction in the Regeneration of Diesel Filters. Proc Instn Mech Engs, Part D, J Automobile Engineering, 2003, 217, pp.307-317. 
73 G. Bella, V. Rocco, M. Maggiore, A Study of Inlet Flow Distortion Effects on Automotive Catalytic Converters, J. Eng. Gas Turbines Power, 1991, 13, 419-426.

74 A. Martin, N. Will, A. Bordet, P. Cornet, C. Gondoin, X. Mouton, Effect of Flow Distribution on Emissions Performance of Catalytic Converters, SAE paper 980936, 1998.

75 Y. S. Cho, D. S. Kim, M. Han, Y. Joo, J. H. Lee, K. D. Min, Flow Distribution in a Close-Coupled Catalytic Converter SAE paper 982552, 1998.

76 R. Durakhiev, C. Dodev, Gas Flow Distribution in Packed Columns, Chemical Engineering and Processing, 2002, 41, 385-393.

77 S. C. Sorenson, Jacob W. Hoj, Per Stobbe, Flow Characteristics of SiC Diesel Particulate Filter Materials, SAE paper 940236, 1994.

78 S. Ebener, P. Florchinger, Drukverlustmodel fur Keramische Dieslpartikelfilter, MTZ Motortechnische Zeitscrift 2000, 61, 6, 414-422.

79 H. Schulz, G. Bandeira De Melo, F. Ousmanov, Volatile Organic Compounds and Particulates as Components of Diesel Engine Exhaust Gas, Combustion and Flame, Volume 118, Issues 1-2, July 1999, Pages 179-190

80 S. Hori, K. Narusawa, Fuel composition Effects on SOF and PAH Exhaust Emissions from DI Diesel Engines, SAE paper, 980507, 1998.

81 S. Sidhu, J. Graham, R. Striebich, Semi-Volatile and Particulate Emissions from the Combustion of Alternative Diesel Fuels, Chemosphere, Volume 42, (2001), Pages 681-690.

82 M. Tanaka, Y. Tsujimoto, T. Miyazaki, M. Warashina, S. Wakamatsu, Peculiarities of Volatile Hydrocarbon Emissions from Several Types of Vehicles in Japan, Chemosphere - Global Change Science 3 (2001), 185-197.

83 J.C. Summers, S. Van Houtte, D. Psaras, Simultaneous Control of Particulate and NOx Emissions from Diesel Engines, Applied Catalysis B: Environmental 10 (1996) 139-156.

84 G. Lepperhoff, H. Lueders, P. Barthe, J. Lemaire, Quasi-Continuous Particle Trap Regeneration by Cerium Additives. SAE paper 950369, 1995.

85 P.J.Richards, M.W.Vincent, S.L.Cook, Operating Experience of Diesel Vehicles Equipped with Particulate Filters and Using Fuel Additive for Regeneration, SAE paper 2000-01-0474.

86 G. A. Stratakis, A. M. Stamatelos: Thermogravimetric Analysis of Soot Emitted by a Modern Engine Run on Catalyst-Doped Fuel, Combustion and Flame, Volume 132, 2002 pp. 157-169.

87 J. Kaerger; D. M. Ruthven, Diffusion in Zeolites and Other Microporous Solids, John Wiley \& Sons, 1992

88 Wesley WM. Wendlandt, Thermal Analysis, 3rd ed., J. Wiley \& Sons New York 1986, pp.12-38, $227-264$.

89 M. Arai, Thermal Analysis for the Evaluation of SOF Oxidation Temperature by Diesel Catalyst, SAE paper 930134, 1993.

90 J. P. A. Neeft, F. Hoornaert, M. Makkee, J. A. Moulijn The effects of heat and mass transfer in thermogravimetrical analysis. A case study towards the catalytic oxidation of soot. Thermochimica Acta (1996) 287:261.

91 P. Ciambelli, P. Corbo, M. Gambino, V. Palma, and S. Vaccaro: Catalytic combustion of carbon particulate. Catalysis Today (1996) 53:99.

92 K. Otto, MH. Sieg, M. Zinbo and L. Bartosiewz, The Oxidation of Soot Deposits from Diesel Engines, SAE paper 1981-80-0336.

93 J.P.A Neeft, T. X. Nijhuis, M. Makkee, and J. A. Moulijn, Kinetics of the oxidation of diesel soot Fuel (1997) $76: 1129$

94 B. Stanmore, J. F. Brihlac, P. Gilot, The Oxidation of Soot, A review of Experiments, Mechanisms and Models. Carbon, Vol. 39, 2001, 2247:2268.

95 G. Neri, L. Bonaccorsi, A. Donato, C. Milone, M.-G. Musolino, A.-M. Visco: Catalytic Combustion of Diesel Soot over Metal Oxide Catalysts, Applied Catalysis B: Environmental (1997) 11: 217. 
96 G. De Soete, Catalysis of Soot Combustion by Metal Oxides, In: Western States section meeting. Salt Lake City, 21-22 March 1988, The Combustion Institute.

97 J.C. Summers, S. Van Houtte, D. Psaras, Simultaneous control of Particulate and NOx Emissions from Diesel Engines, Applied Catalysis B: Environmental (1996) 10:139.

98 J. Lahaye, S. Boehm, PH. Chambrion and P. Ehrburger, Influence of Cerium Oxide on the formation and oxidation of Soot, Combustion and Flame (1996) 104:199.

99 P. Ciambelli, P. Corbo, M. Gambino, V. Palma, and S. Vaccaro: Catalytic combustion of carbon particulate, Catalysis Today (1996) 53:99.

100 S.J.Jelles, R.R.Krul, M. Makkee, J.A. Moulijn, The influence of NOx on the Oxidation of Metal Activated Diesel Soot, Catalysis Today (1999) 53:623.

101 A.F. Ahlstrom, C.U.I Odenbrand, Combustion Characteristics of Soot Deposits from Diesel Engines. Carbon (1989) 27:475.

102 M. Zinbo, L.M. Skewes, C.E. Hunter and D. Shuetzle, Thermogravimetry of Filter-Borne Diesel Particulates, Thermochimica Acta, (1990) 166:267.

103 C. N. Millet, P. Menegazzi B. Martin, H. Colas, C. Bourgeois, Modeling of Diesel Particulate Filter Regeneration: Effect of Fuel-Borne Catalyst, SAE paper 2002-01-2786.

104 G.Pontikakis, A. Stamatelos, K. Bakasis, N. Aravas, 3-D Catalytic Regeneration and Stress Modeling of Diesel Particulate Filters by ABAQUS FEM Software, SAE paper 2002-01-1017.

105 B. Wiedemann, Neumann, K. Vehicular Experience with Additives for Regeneration of Ceramic Diesel Filters. SAE Paper 850017 (1985).

106 G. A. Stratakis and A. M. Stamatelos: Flow distribution Effects in the Loading and Catalytic Regeneration of Wall-Flow Diesel Particulate Filters, In press, Proc. Instn Mech Engrs, Part D, 2003

107 S. C. Sorenson, Jacob W. Hoj, Per Stobbe, Flow Characteristics of SiC Diesel Particulate Filter Materials, SAE paper 940236, 1994.

108 H. Aoki, A. Asano, K. Kurazono, K. Kobashi and H. Sami, Numerical Simulation Model for the regeneration Process of a Wall-Flow Monolith Diesel Particulate Filter, SAE paper 930364, 1993.

109 N. Ladegaard, S. C. Sorenson, J. Schramm, L. Gratz, P. Stobbe, Fuel Additive Effects on Particulate Emissions from a Diesel Engine, SAE paper 970181, 1997.

110 George de Soete: Catalysis of soot combustion by metal oxides, in: Western states Section Meeting: The Combustion Institute, Salt Lake City, 21-22 March, 1988.

111 S. C. Sorenson, J. W. Hoj, P. Stobbe, Flow Characteristics of SiC Diesel Particulate Filter Materials, SAE paper 940236, 1994.

112 R. J. Klopfenstein, Air Velocity and Flow Measurement Using a Pitot Tube, ISA Transactions, 1998, 37, 257-263.

113 R. Durakciev, C. Dodev: Gas Flow Distribution in Packed Columns, Chemical Engineering and Processing, 2002, 41, 385-393.

114 A. Holmgren, T. Grontedt, B. Andersson, Improved Flow Distribution in Automotive Monolith Converters, React. Kinet. Catal. Lett, 1997, 60 (2), 363-371.

115 V.K. Chakravarthy, J.C. Conklin, C.S. Daw, E.F. D’Azevedo, Multi-dimensional Simulations of Cold-start Transients in a Catalytic Converter under Steady Inflow Conditions. Applied Catalysis A (General), 2003, 241, 289-306.

116 A. Itoh, K. Shimato, T. Komori, H. Okazone, T. Yamada, K. Niimura, Y. Watanabe: Study of SiC Application to Diesel Particulate Filter (Part1): Material Development. SAE paper 930360, 1993. 